\title{
User's Manual for QWGRAF, Computer Programs for Water-Quality Graphics
}

By James C. Schornick and Michael C. Place

U.S. GEOLOGICAL SURVEY

Water-Resources Investigations Report 97-4226

Reston, Virginia

1998 


\section{U.S. DEPARTMENT OF THE INTERIOR \\ BRUCE BABBITT, Secretary \\ U.S. GEOLOGICAL SURVEY \\ Mark Schaefer, Acting Director}

The use of trade, product, industry, or firm names is for descriptive purposes only and does not imply endorsement by the U.S. Government.

For additional information write to:

U.S. Geological Survey

Chief, Hydrologic Analysis Support Section

437 National Center

Reston, VA 20192

Electronic Mail: h2osoft@usgs.gov
Copies of this report can be purchased from:

U.S. Geological Survey

Branch of Information Services

Box 25286

Denver, CO 80225-0286 


\section{CONTENTS}

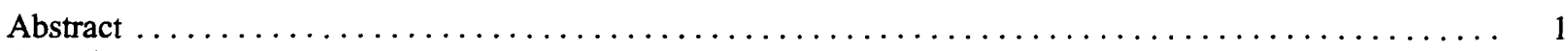

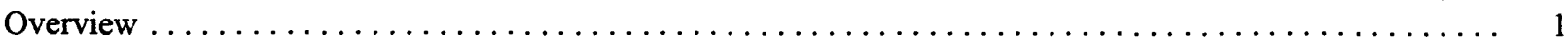

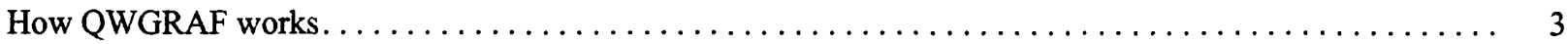

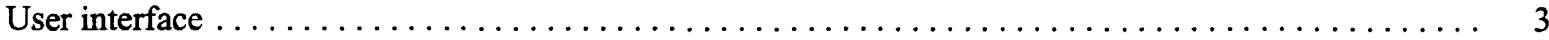

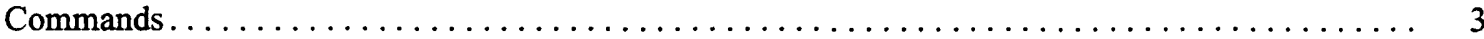

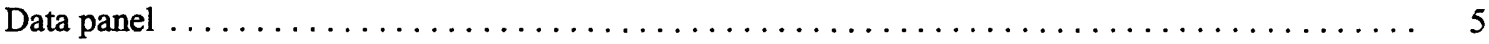

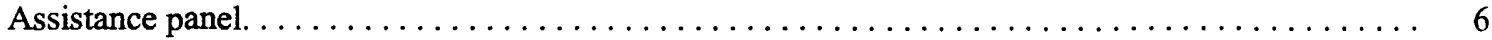

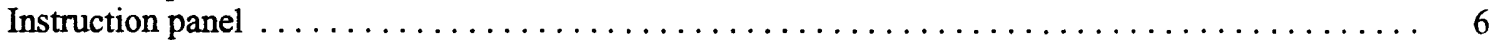

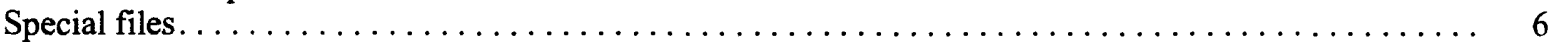

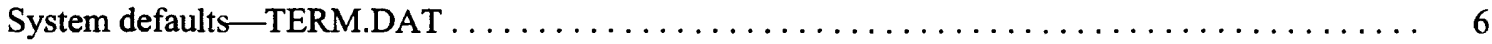

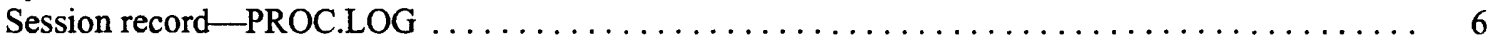

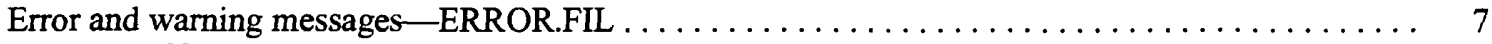

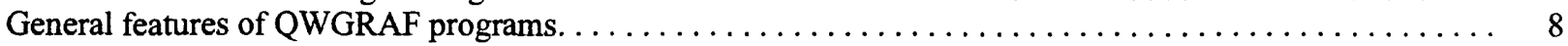

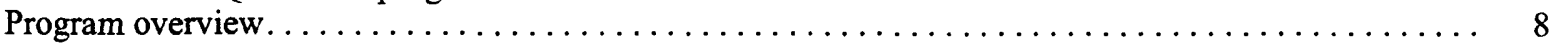

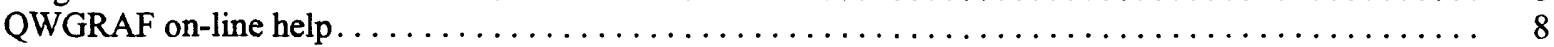

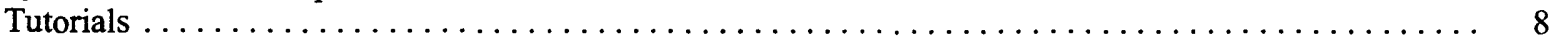

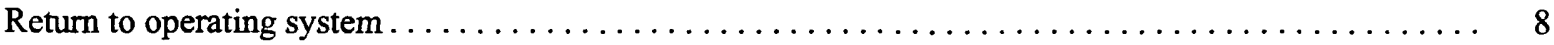

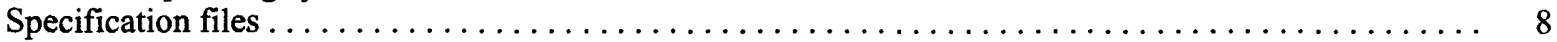

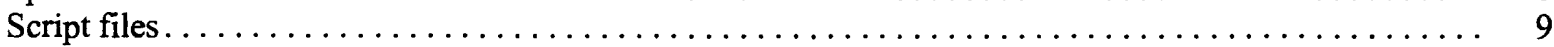

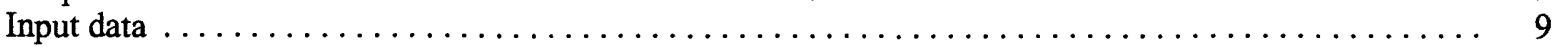

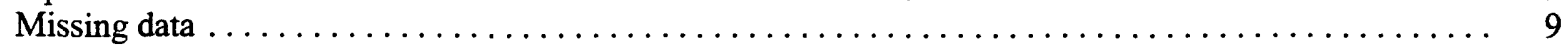

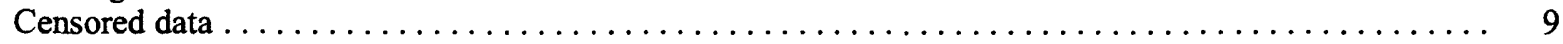

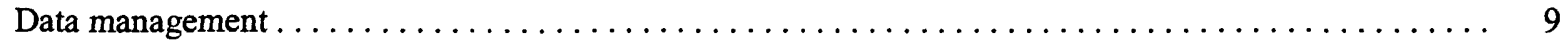

Conversion of concentration data to equivalents. . . . . . . . . . . . . . . 10

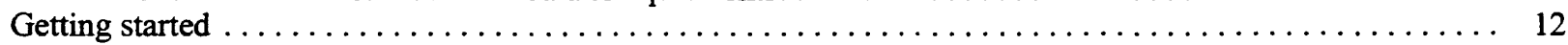

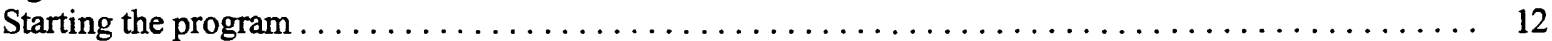

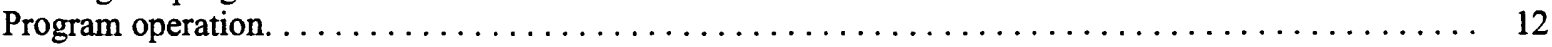

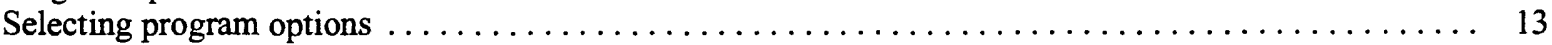

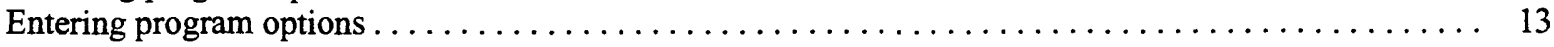

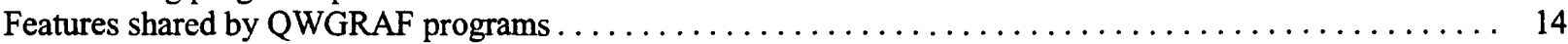

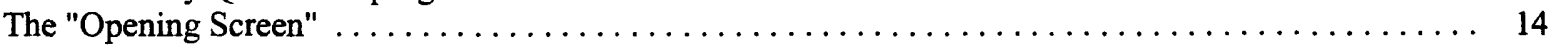

Open input data file. $\ldots \ldots \ldots \ldots \ldots \ldots \ldots \ldots \ldots \ldots \ldots \ldots \ldots \ldots \ldots \ldots \ldots \ldots \ldots$

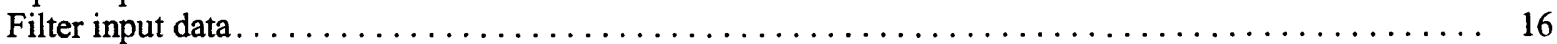

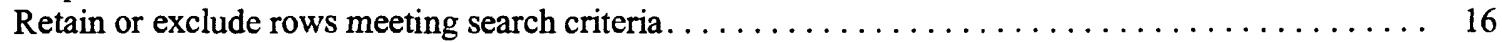

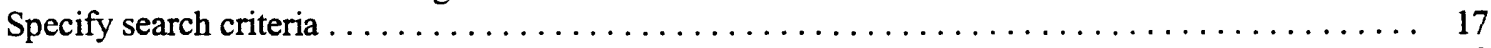

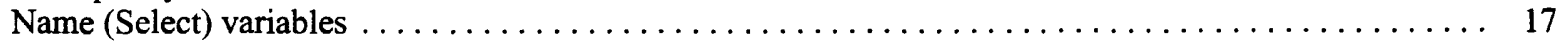

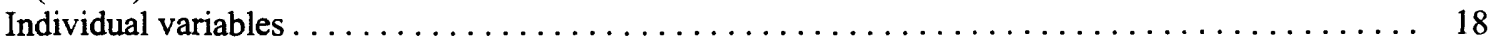

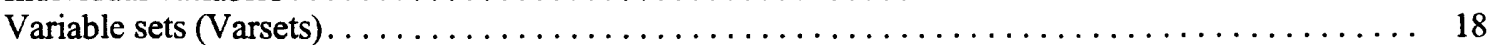

Add a variable $s e t . \ldots \ldots \ldots \ldots \ldots \ldots \ldots \ldots \ldots \ldots \ldots \ldots \ldots \ldots \ldots \ldots \ldots$

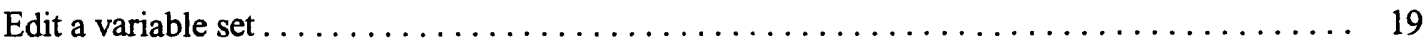

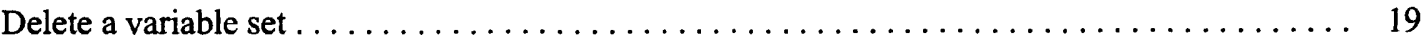

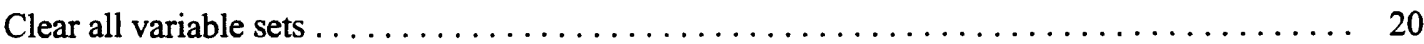

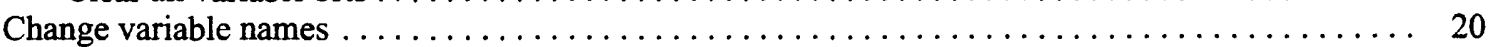

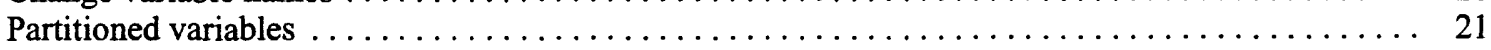

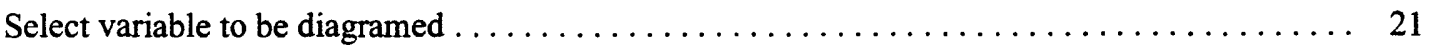

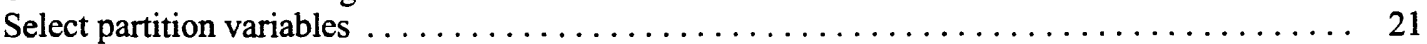

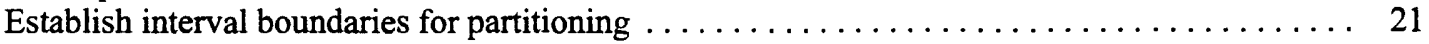

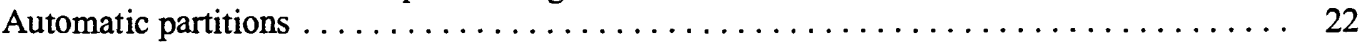

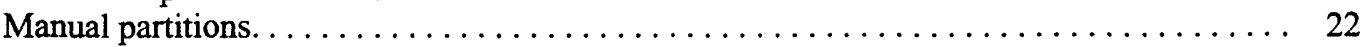

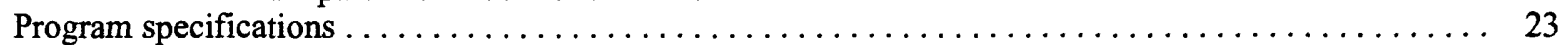

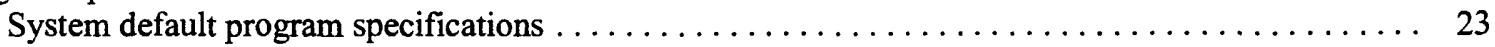

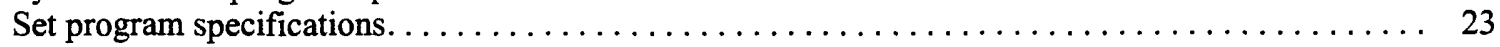

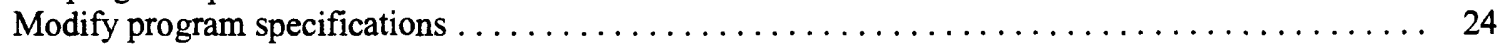

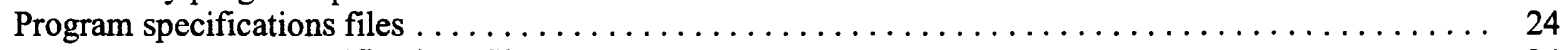

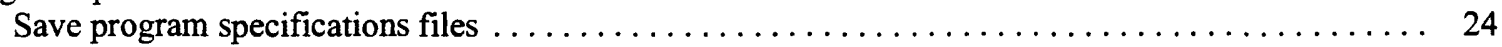

"Latest" specifications file . . . . . . . . . . . . . . . . . . . . . . . . . . . 24 
"User-defined" specifications file. . . . . . . . . . . . . . . . . . . . . . . . . 25

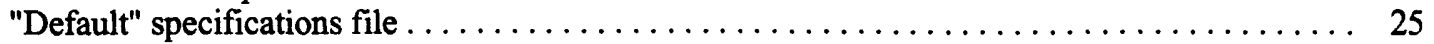

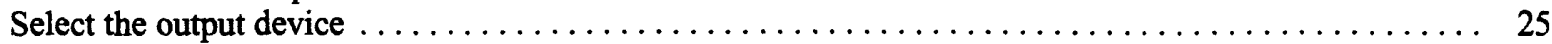

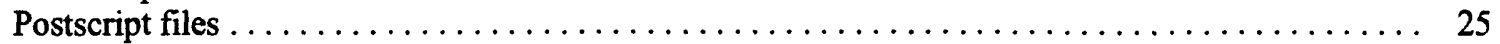

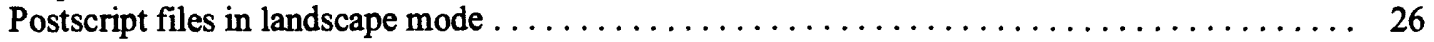

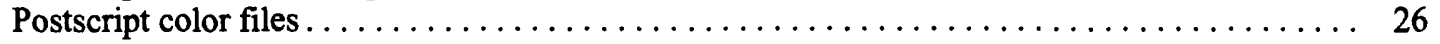

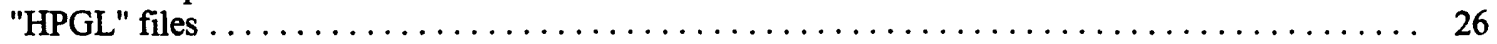

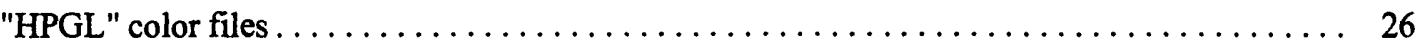

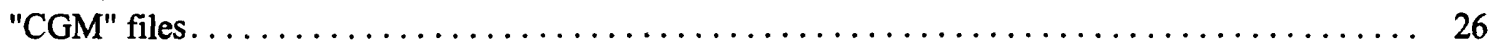

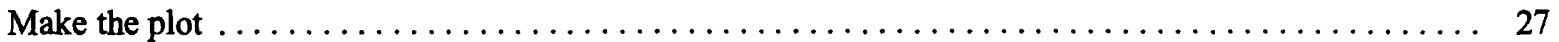

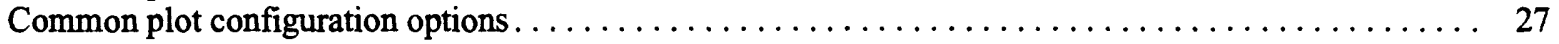

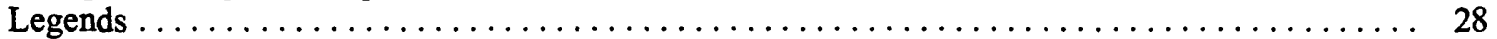

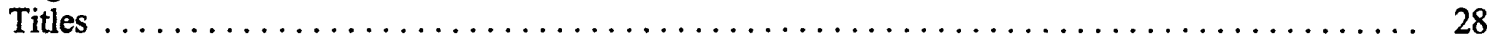

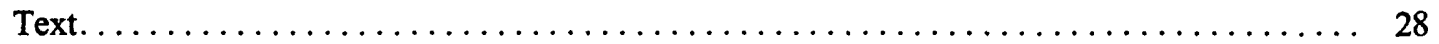

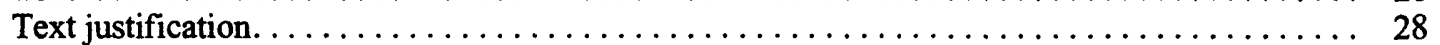

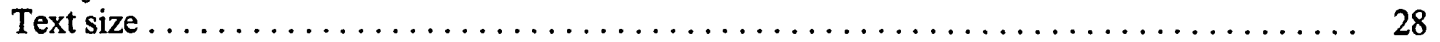

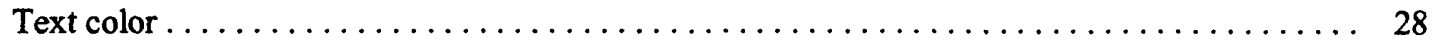

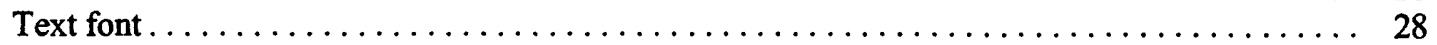

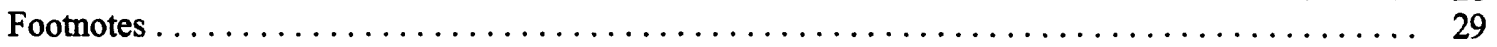

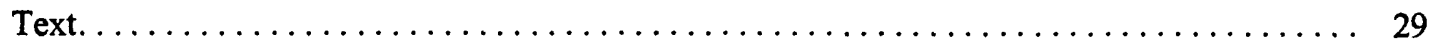

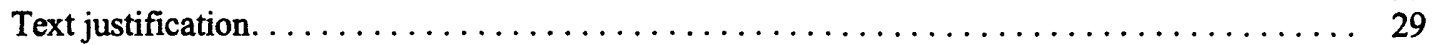

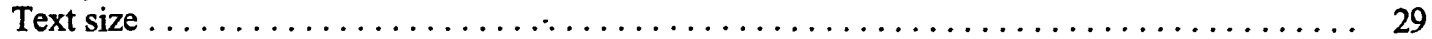

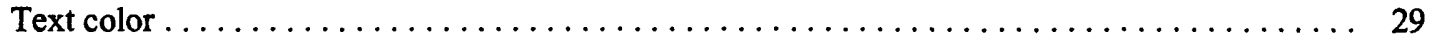

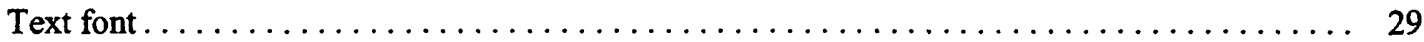

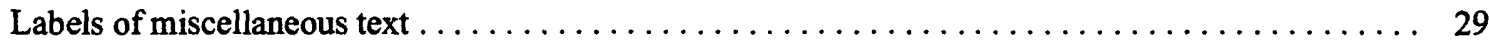

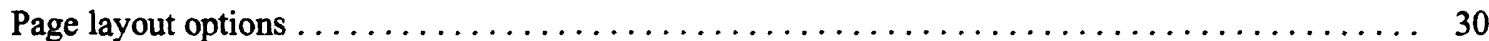

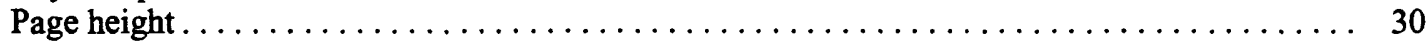

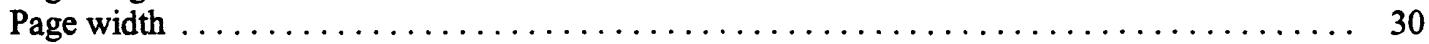

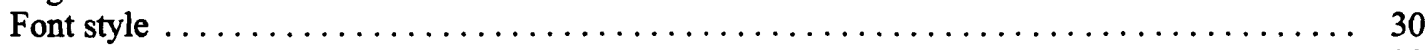

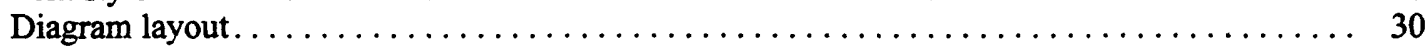

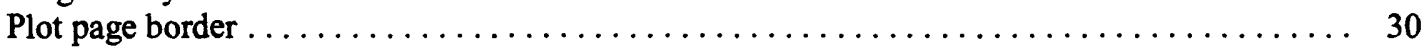

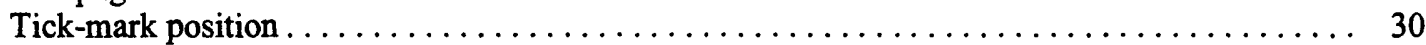

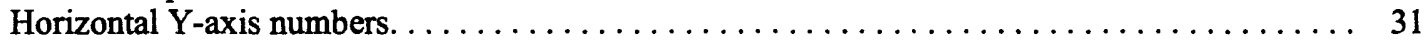

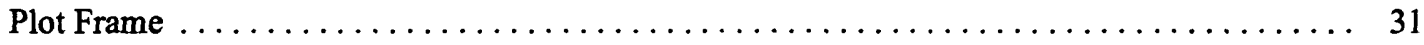

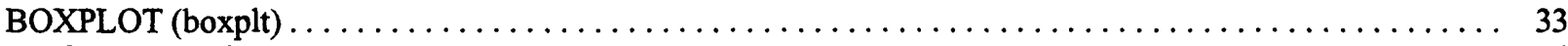

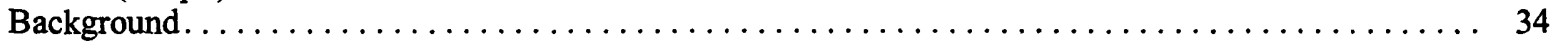

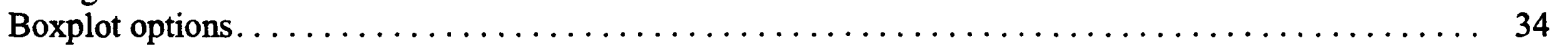

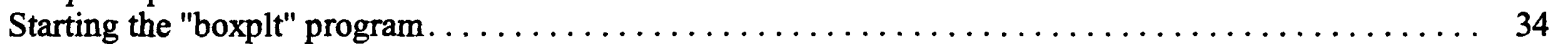

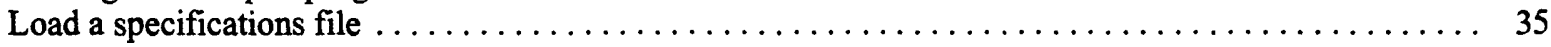

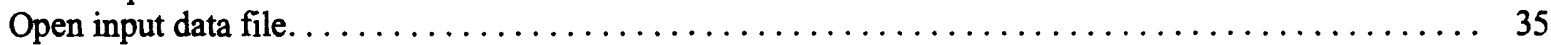

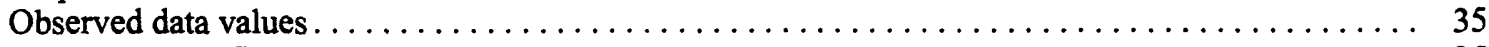

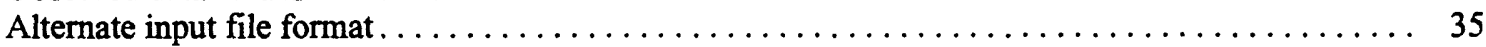

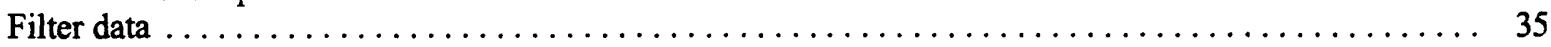

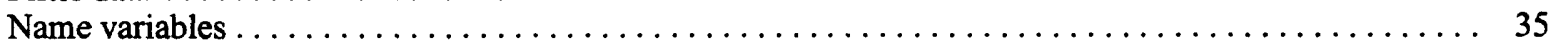

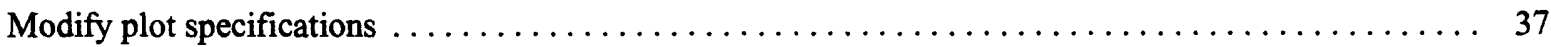

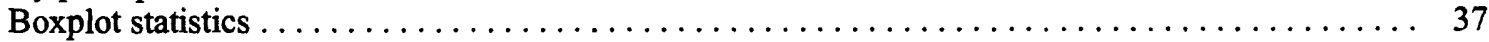

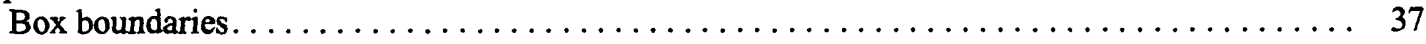

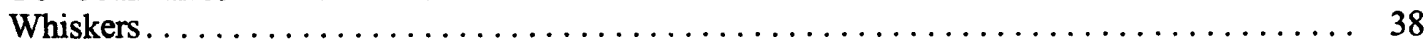

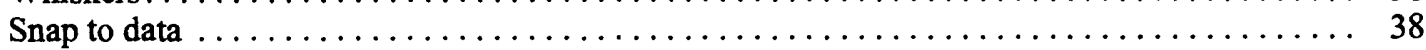

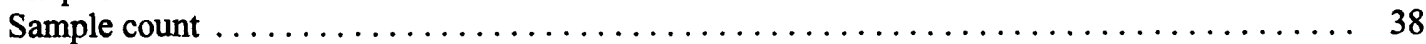

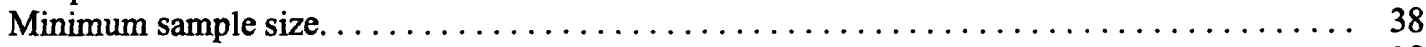

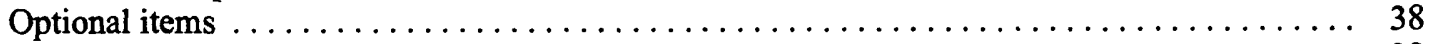

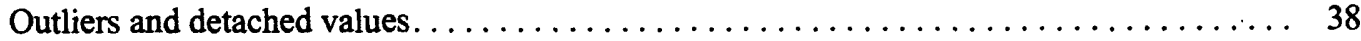

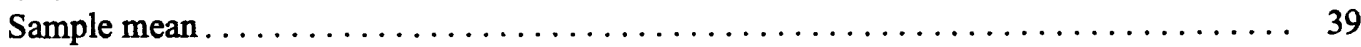

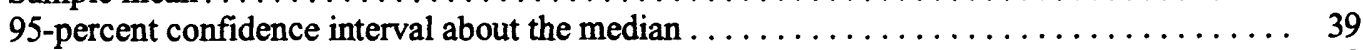

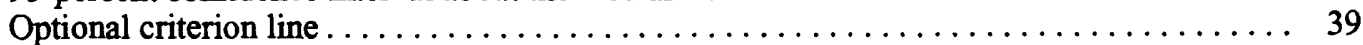

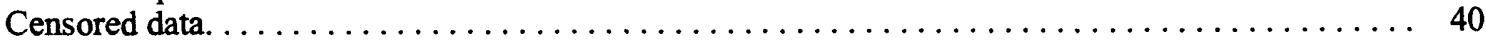

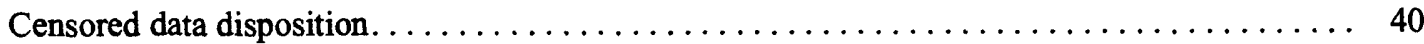

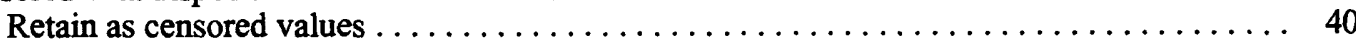




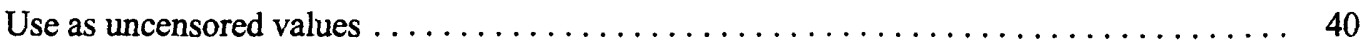

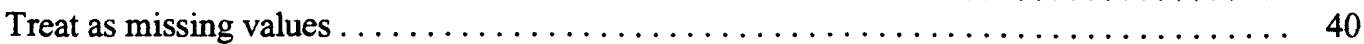

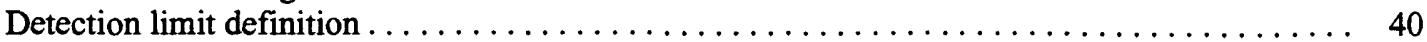

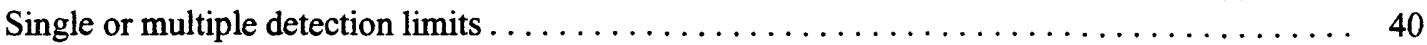

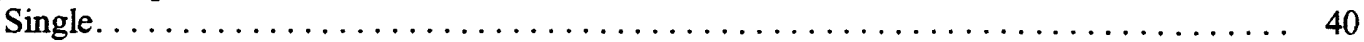

Multiple....................................... 40

Box and whisker line type below detection limit line $\ldots \ldots \ldots \ldots \ldots \ldots \ldots \ldots \ldots \ldots, 41$

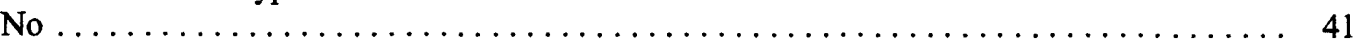

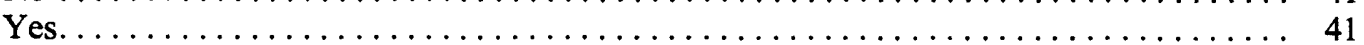

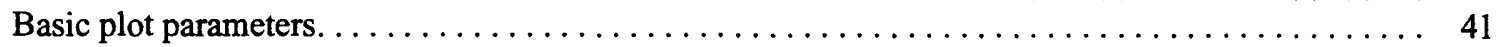

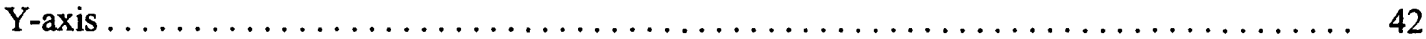

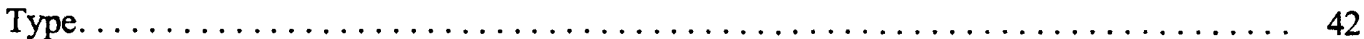

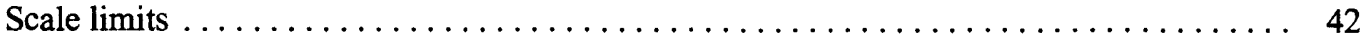

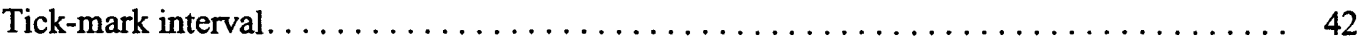

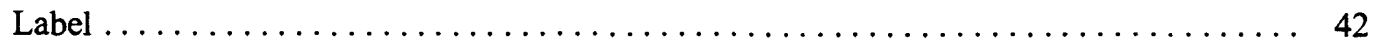

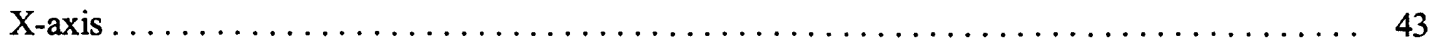

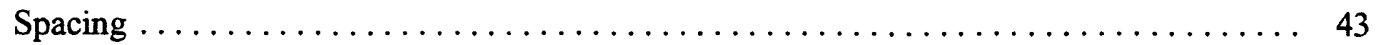

Box labels $\ldots \ldots \ldots \ldots \ldots \ldots \ldots \ldots \ldots \ldots \ldots \ldots \ldots \ldots \ldots \ldots \ldots \ldots \ldots \ldots \ldots \ldots \ldots \ldots, 43$

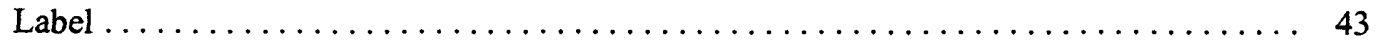

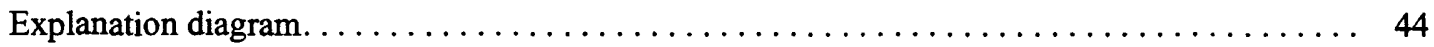

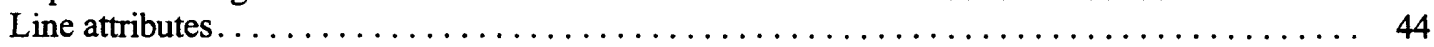

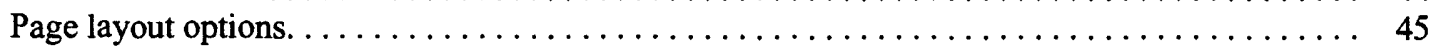

Maximum number of diagrams per page $\ldots \ldots \ldots \ldots \ldots \ldots \ldots \ldots \ldots \ldots \ldots \ldots \ldots$

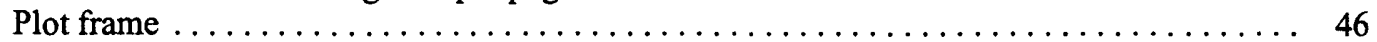

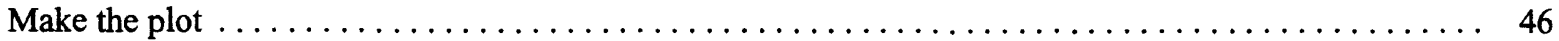

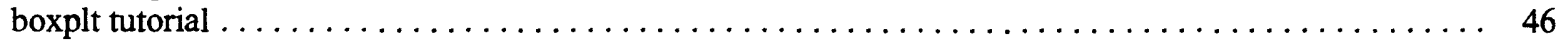

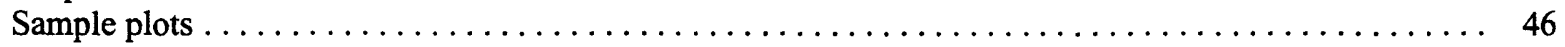

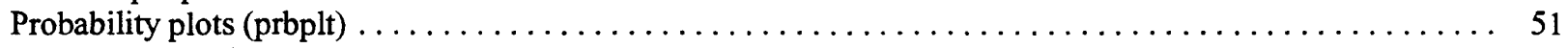

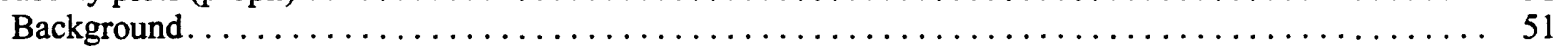

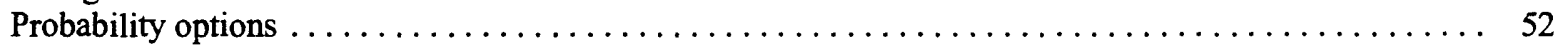

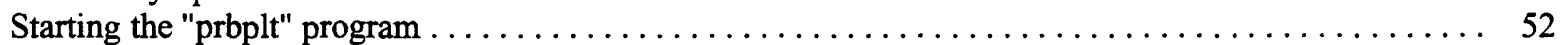

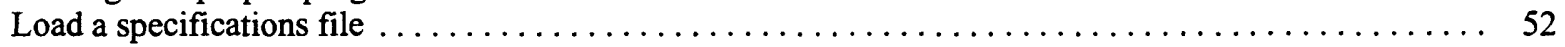

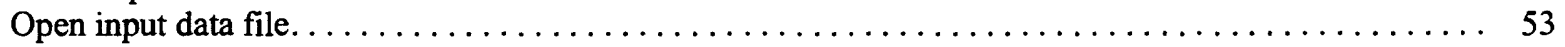

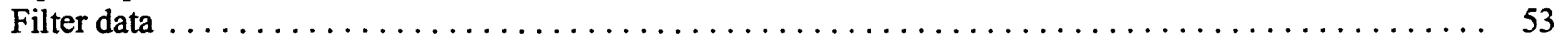

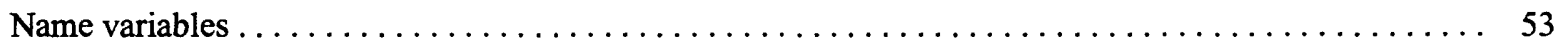

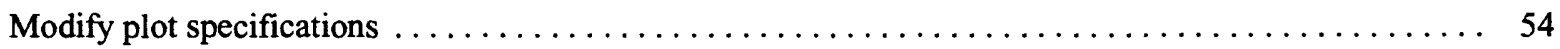

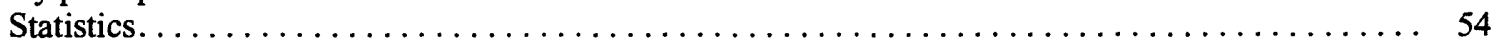

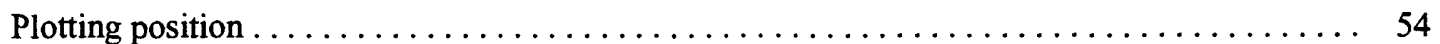

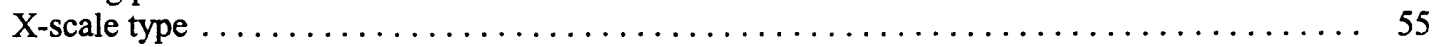

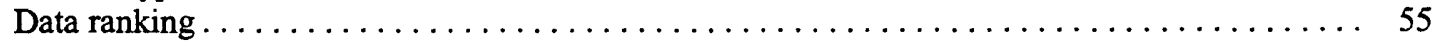

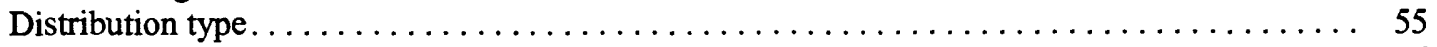

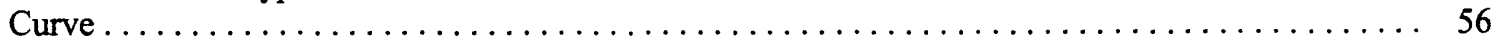

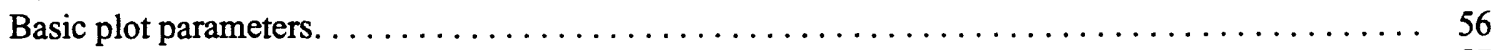

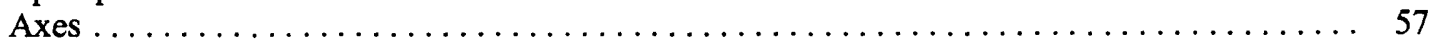

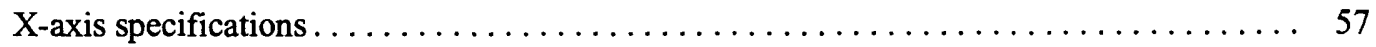

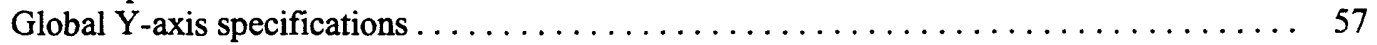

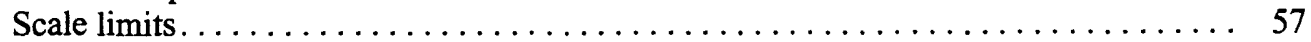

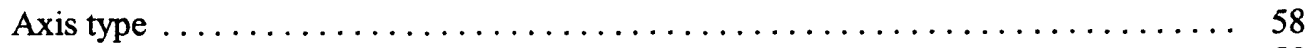

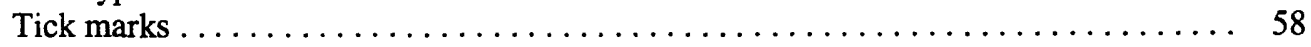

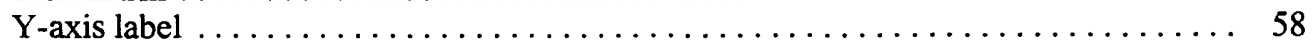

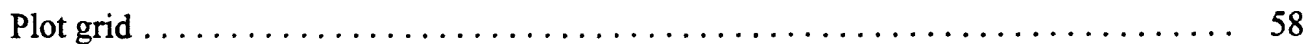

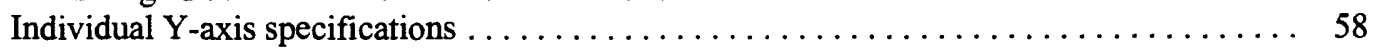

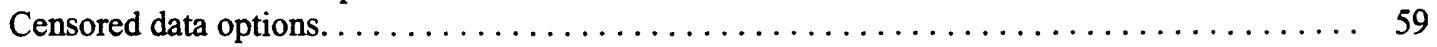

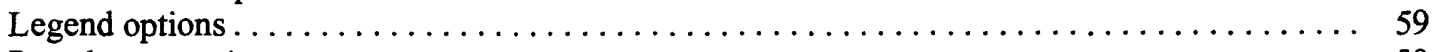

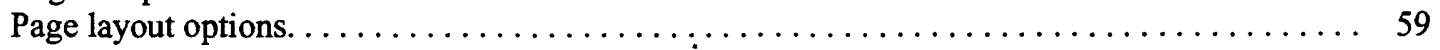

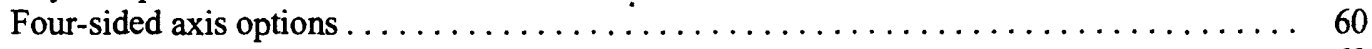

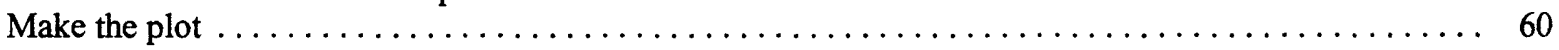

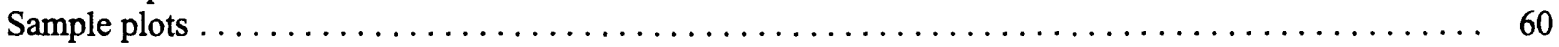




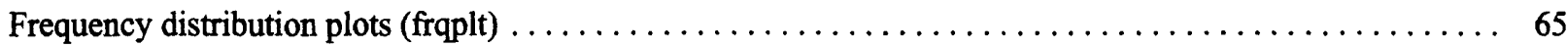

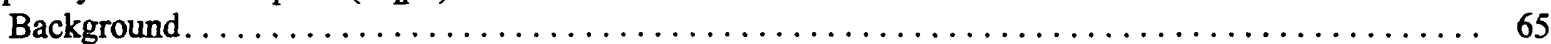

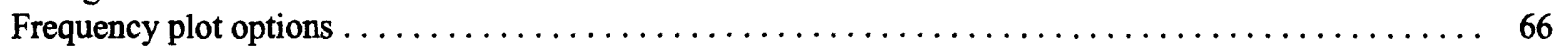

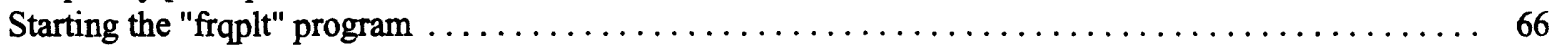

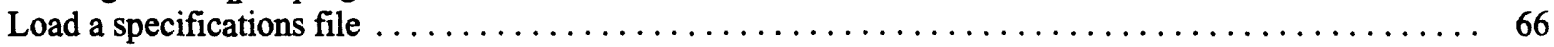

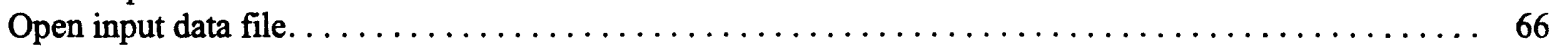

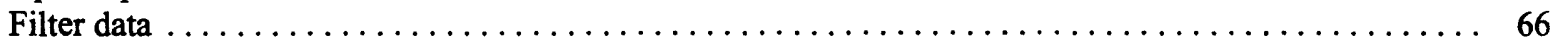

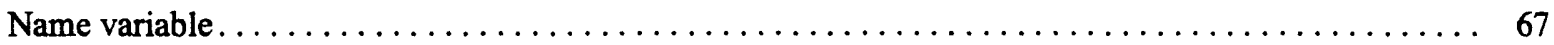

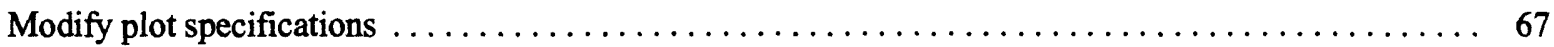

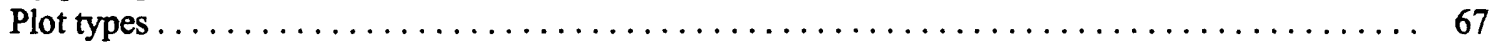

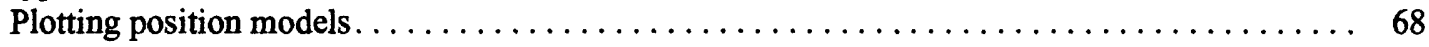

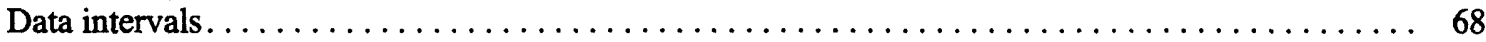

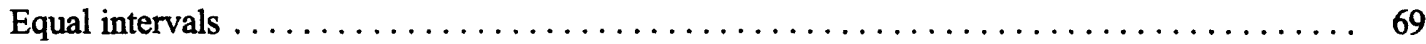

Quantile intervals . . . . . . . . .

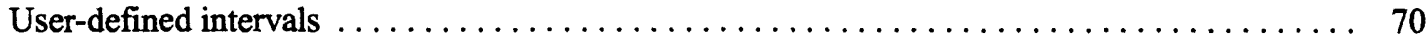

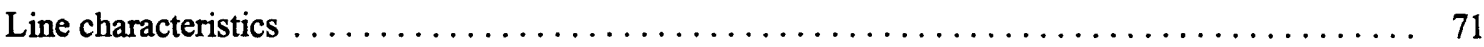

Basic plot parameters. . . . . . . . . . . . . .

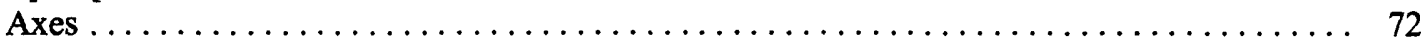

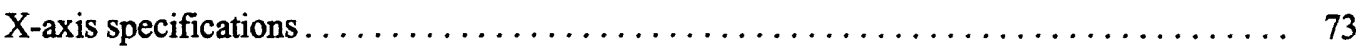

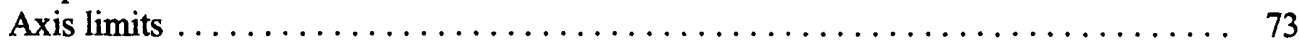

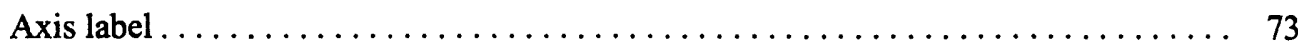

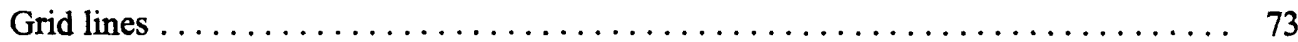

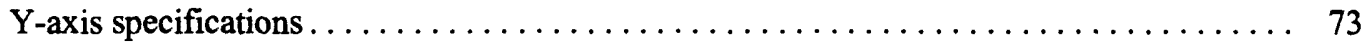

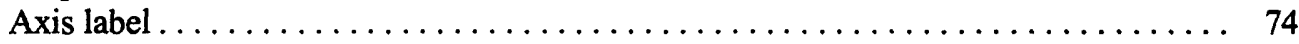

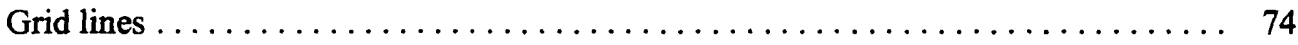

Censored data options. . . . . . . . . . .

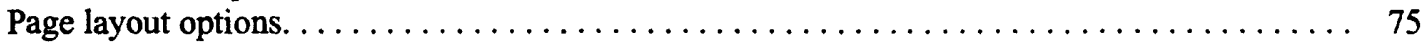

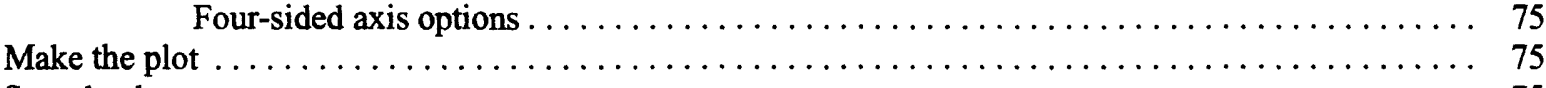

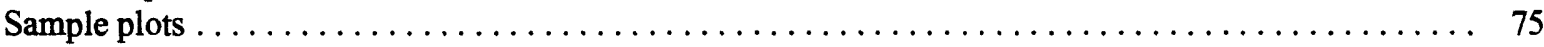

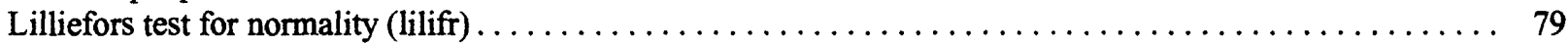

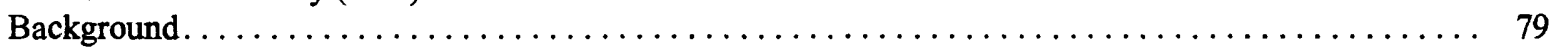

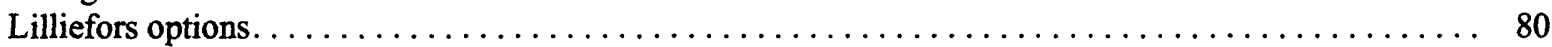

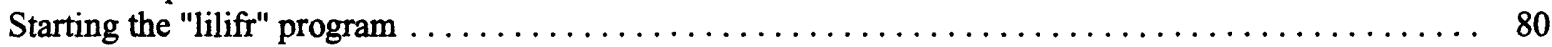

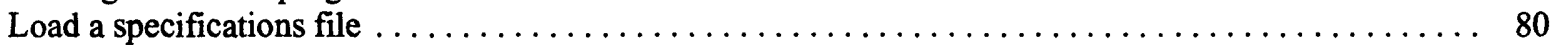

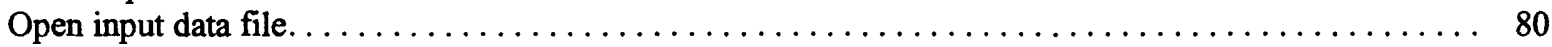

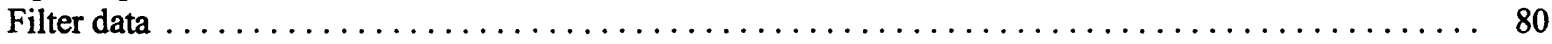

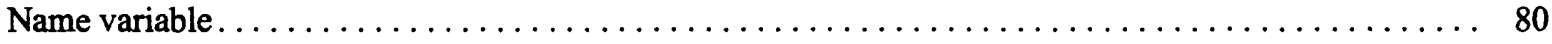

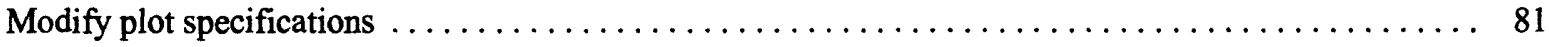

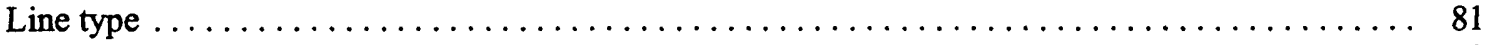

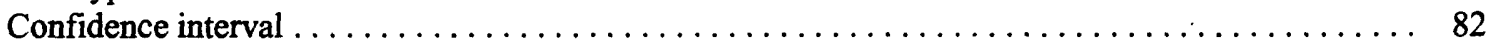

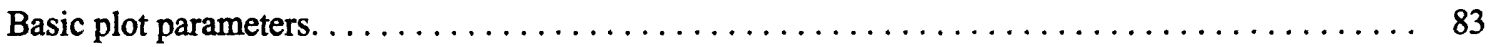

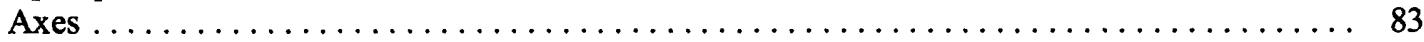

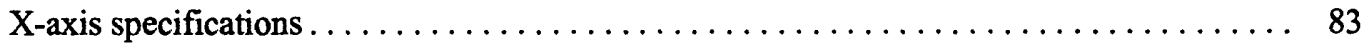

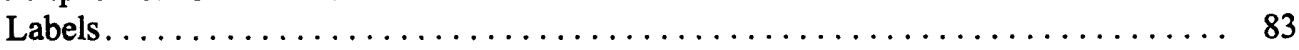

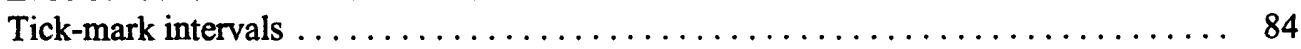

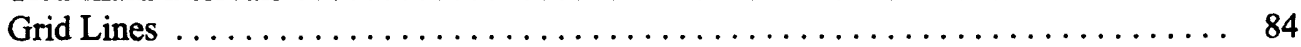

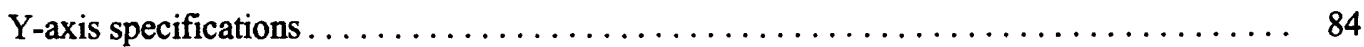

Label. .............................................. 84

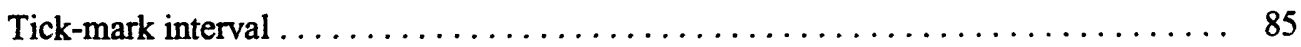

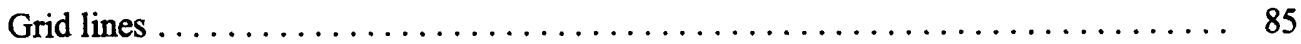

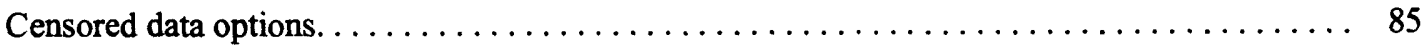

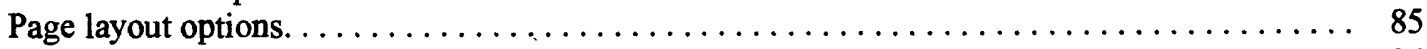

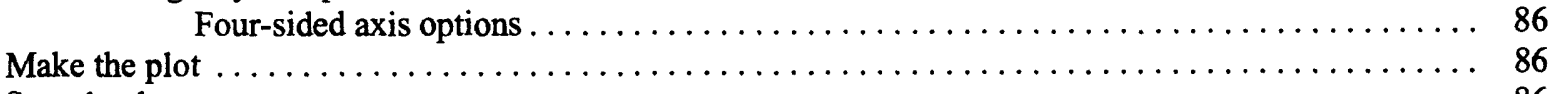

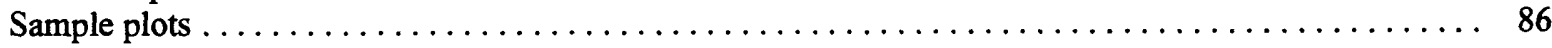




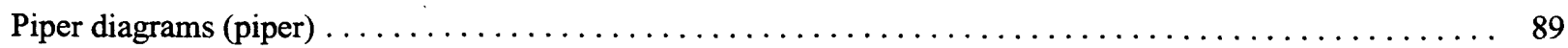

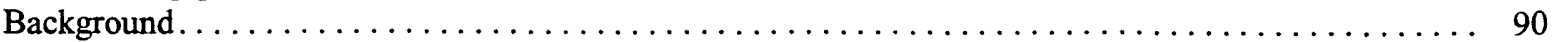

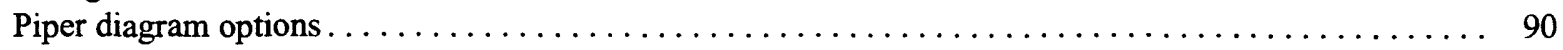

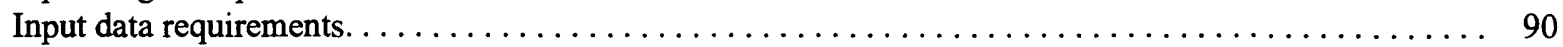

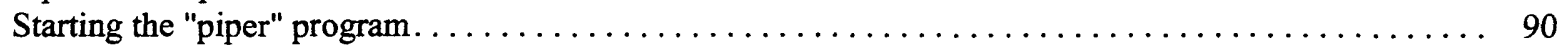

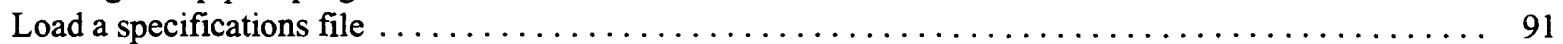

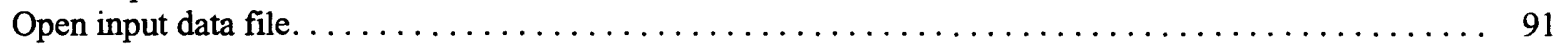

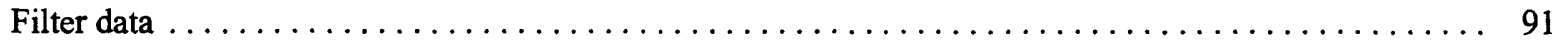

Name variables . . . . . . . . . . . . . . . . . . . . . . . . 91

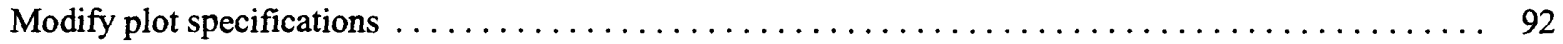

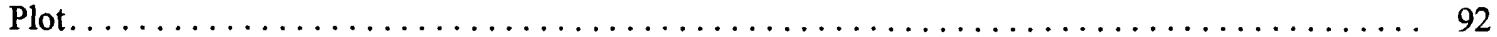

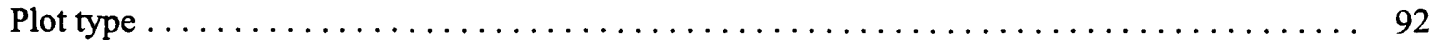

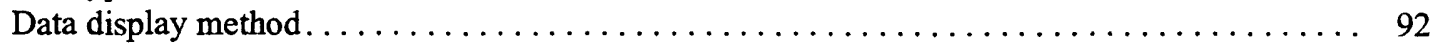

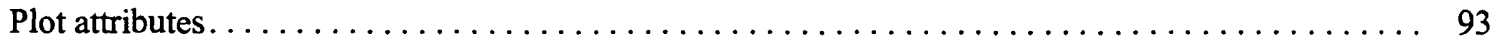

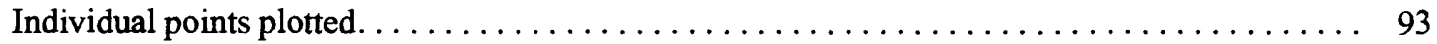

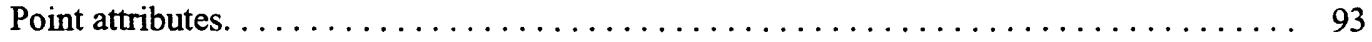

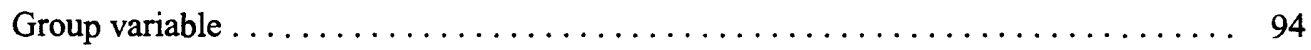

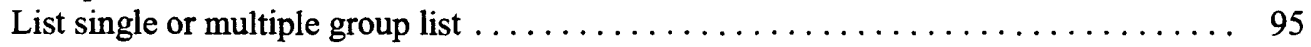

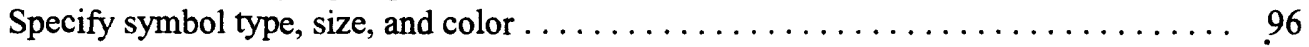

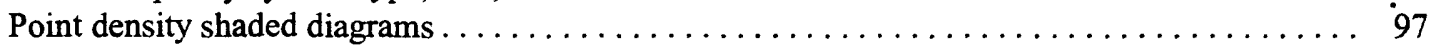

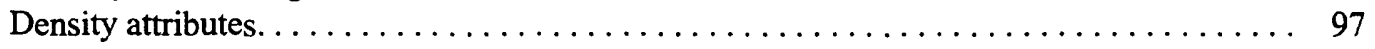

Axis divisions. . . . . . . . . . . . . . . . . . 98

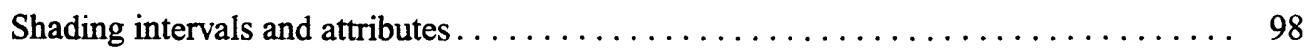

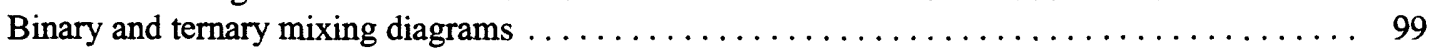

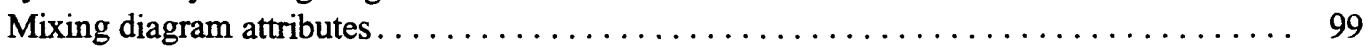

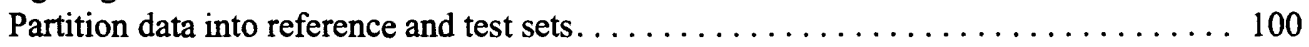

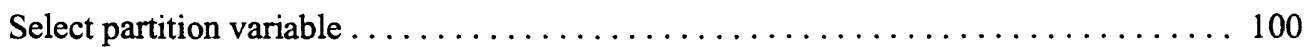

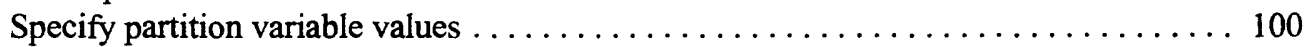

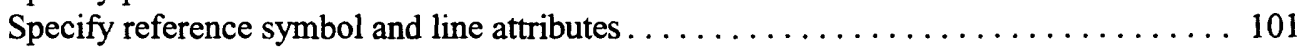

Basic plot parameters. . . . . . . . . . . . . . . . . . . . . . . . . . . . . . 102

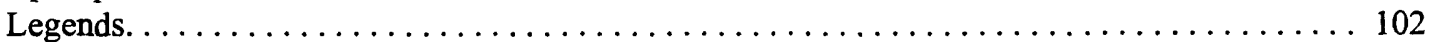

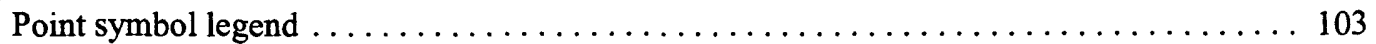

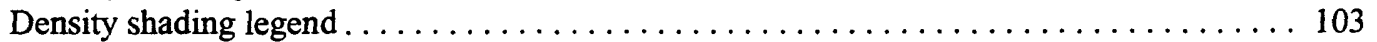

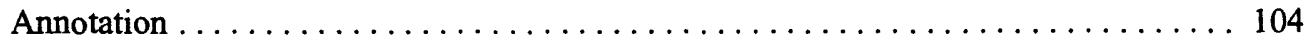

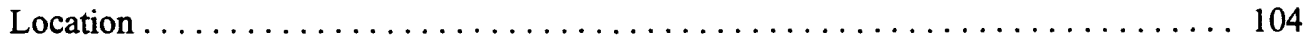

Statistical explanation block .................................. 104

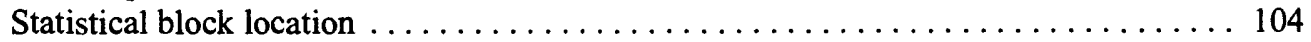

User-specified period of record . . . . . . . . . . . . . . . . . . . . . 104

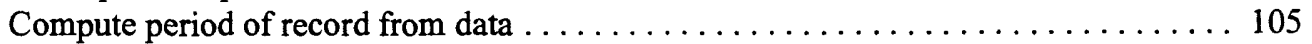

Select statistical legend variables. . . . . . . . . . . . . . . . . . . . 105

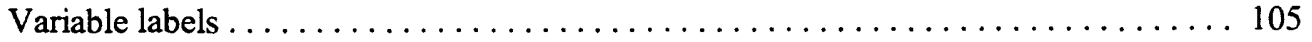

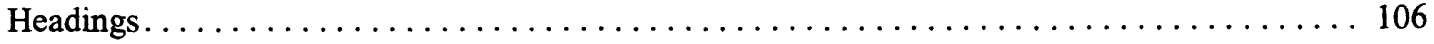

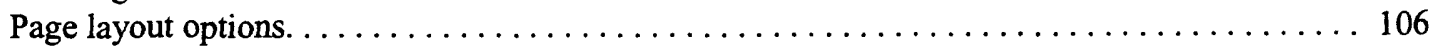

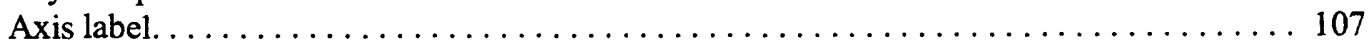

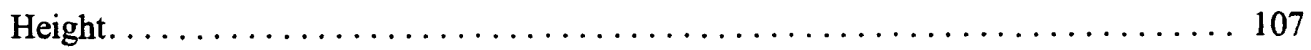

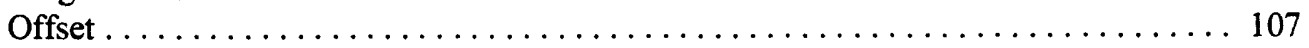

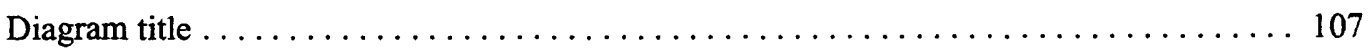

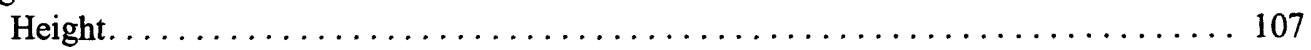

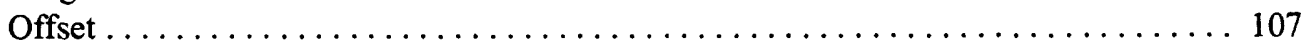

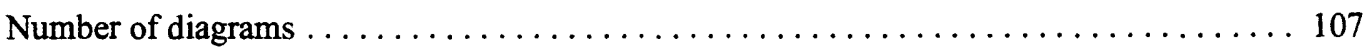

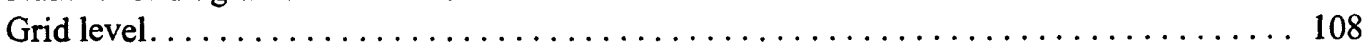

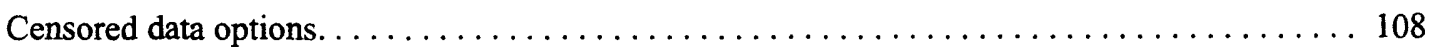

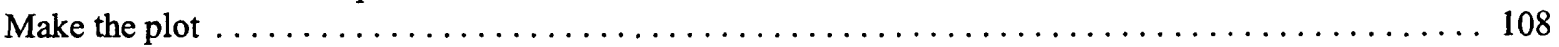

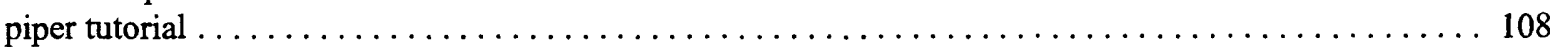

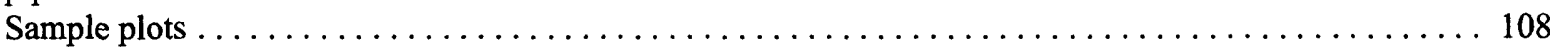




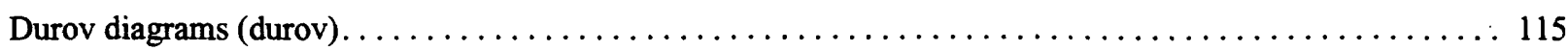

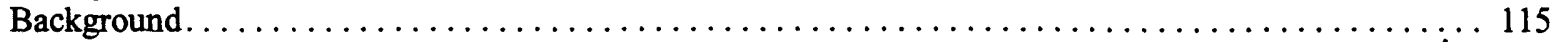

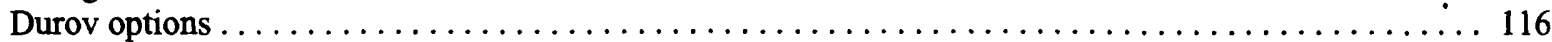

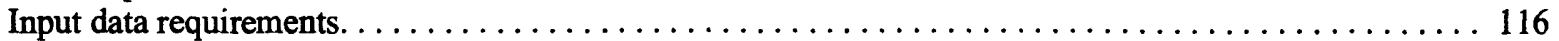

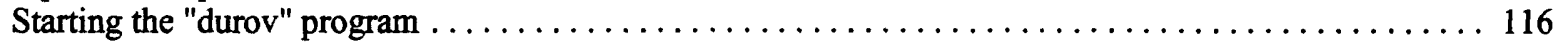

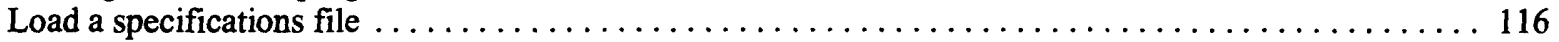

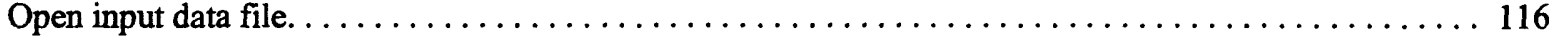

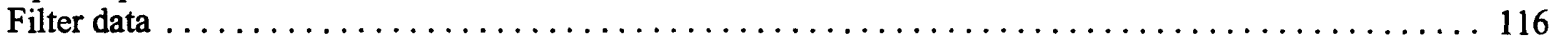

Name variables .................................................... 117

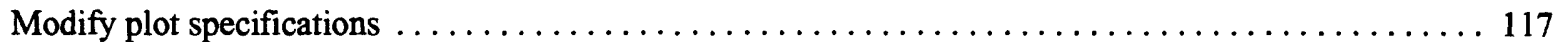

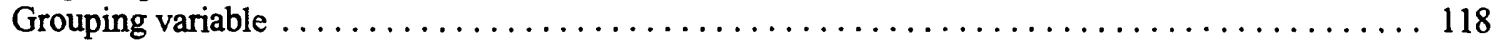

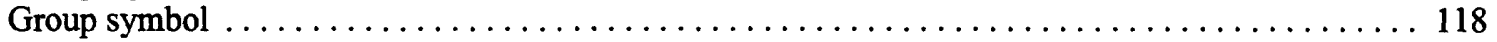

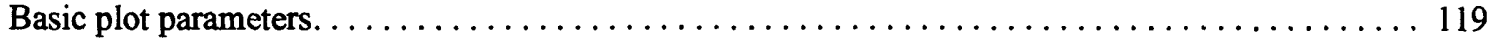

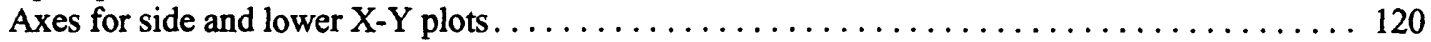

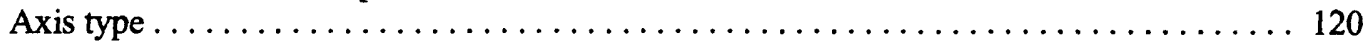

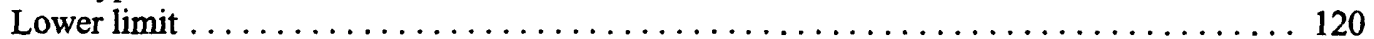

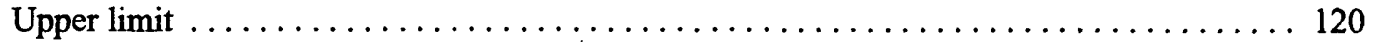

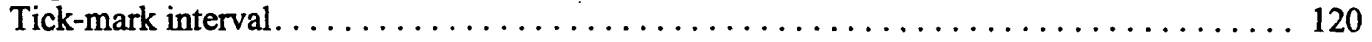

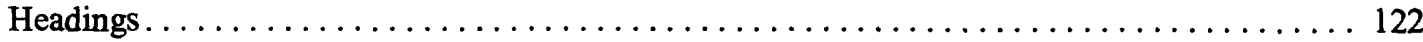

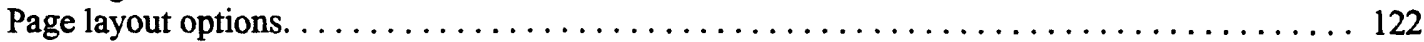

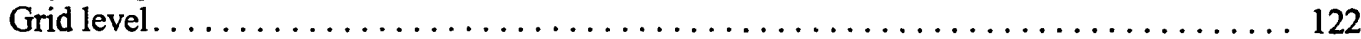

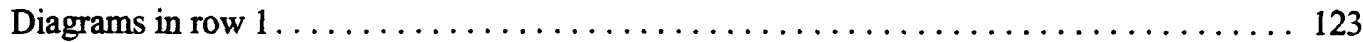

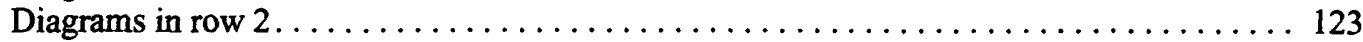

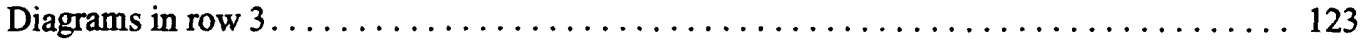

Censored data options. . . . . . . . . . . . . . . . . 123

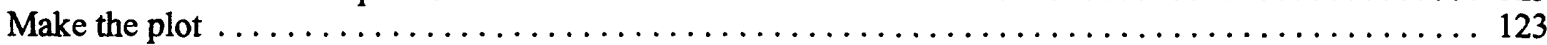

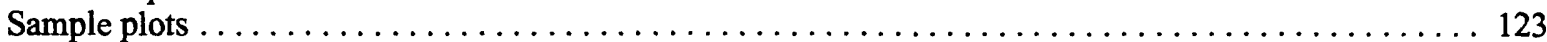

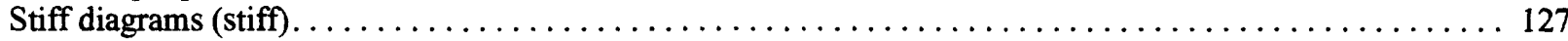

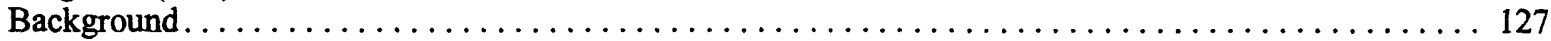

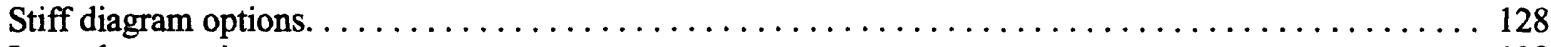

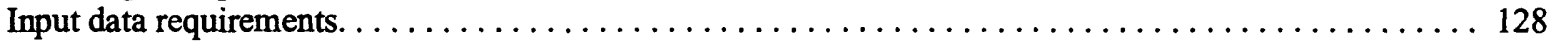

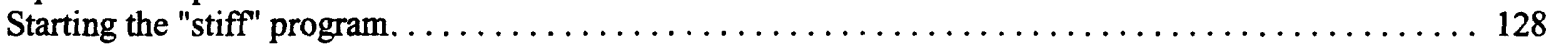

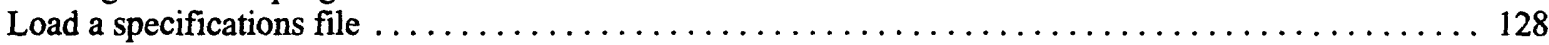

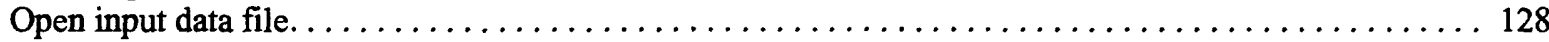

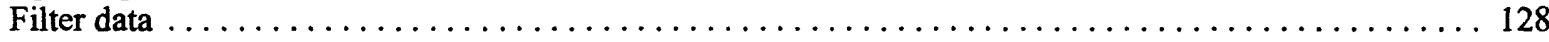

Name variables . . . . . . . . . . . . . . . . . . . . . . . . . . . . . . . . 129

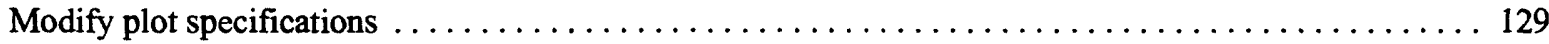

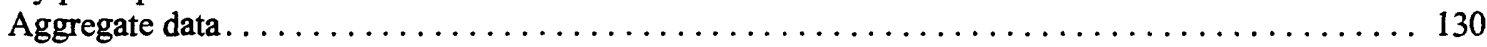

Basic plot parameters. . . . . . . . . . . . . . . . . . . . . . . . . . . .

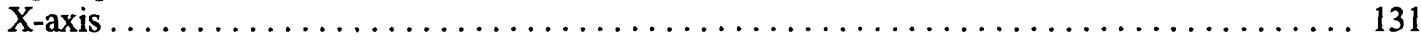

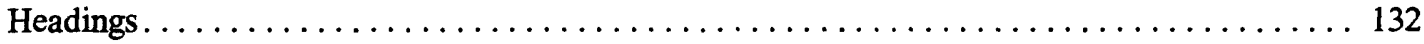

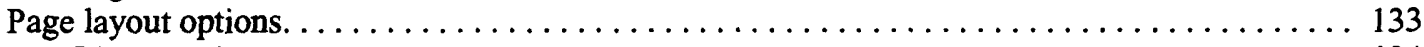

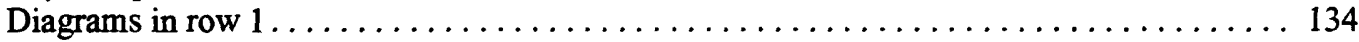

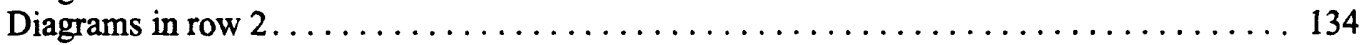

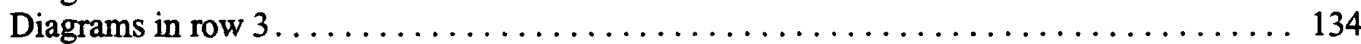

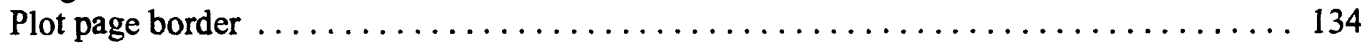

Censored data options. . . . . . . . . . . . . . . . . . . . . . . . . 134

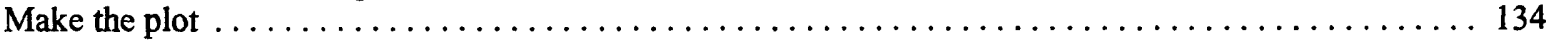

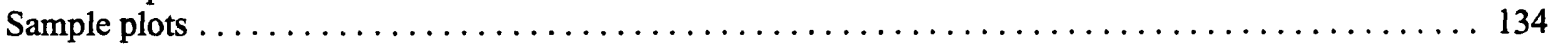

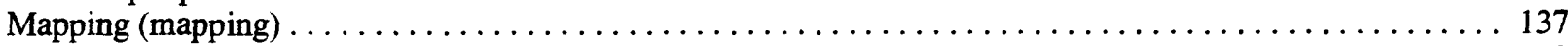

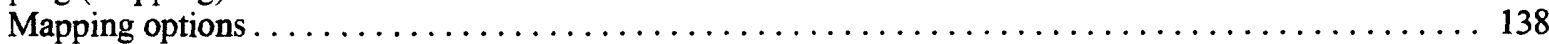

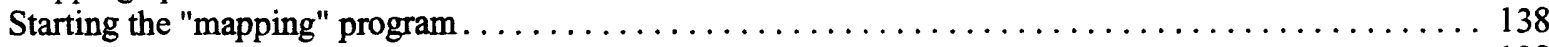

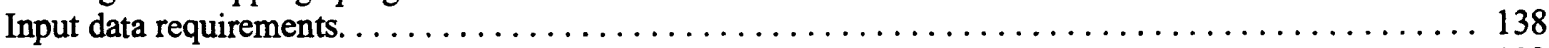

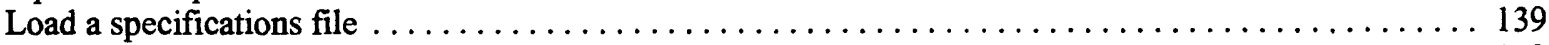

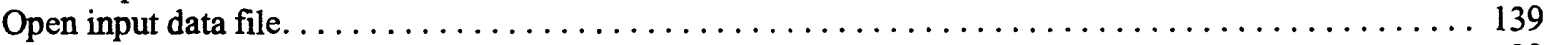

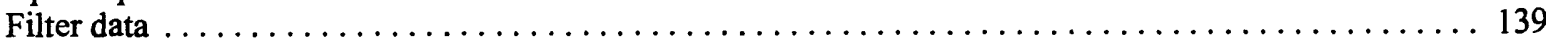

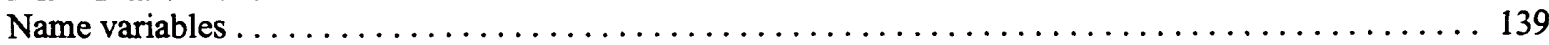

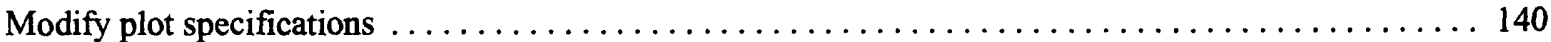

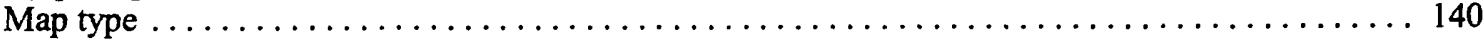




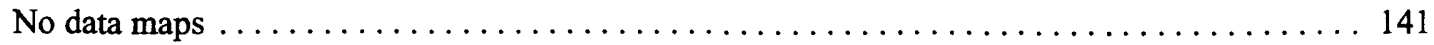

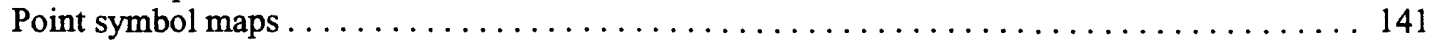

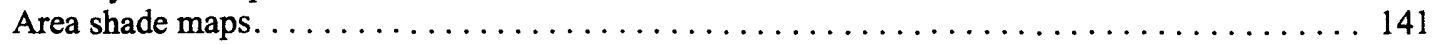

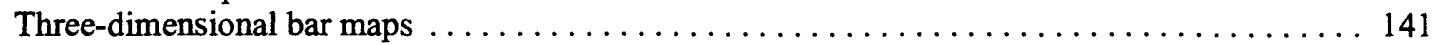

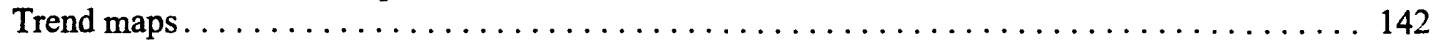

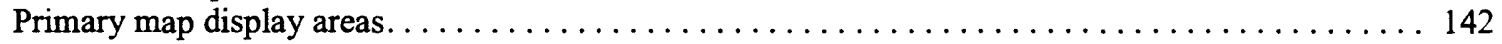

Contiguous 48 States or contiguous 48 States plus Alaska, Hawaii, and Puerto Rico. . . . . . 142

Latitude/longitude rectangle. . . . . . . . . . . . . . . . . . . . . . . . . 143

Lists of States, counties, and hydrologic units . . . . . . . . . . . . . . . . . . . 143

Xref - Cross-reference names of political and hydrologic units with FIPS codes . . . . . . 143

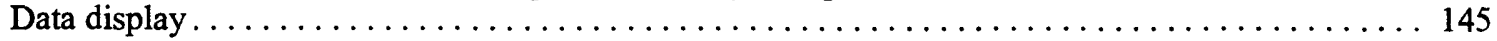

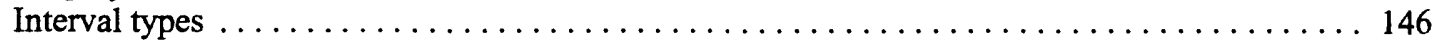

Equal intervals. . . . . . . . . . . . . . . . . . . . . . . . . 146

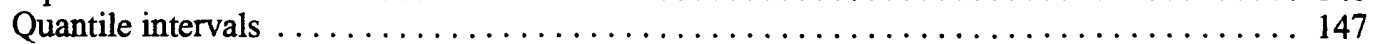

User-defined intervals . . . . . . . . . . . . . . . . . . . . . . . . . . . 148

Interval attributes . . . . . . . . . . . . . . . . . . . . . . . . . . . . . . . 149

Aggregation options . . . . . . . . . . . . . . . . . . . . . . . . . 149

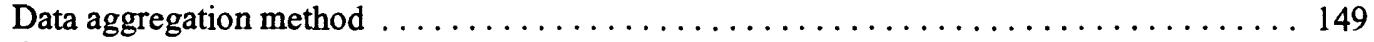

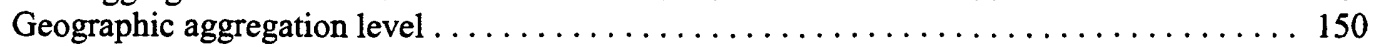

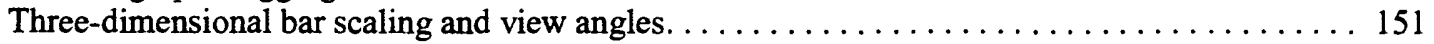

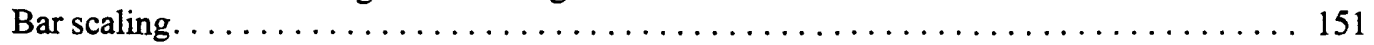

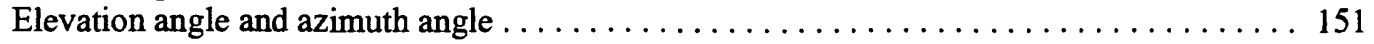

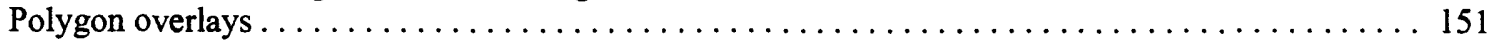

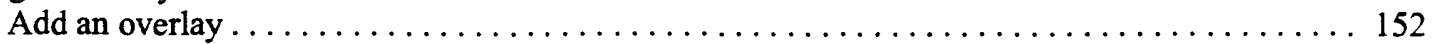

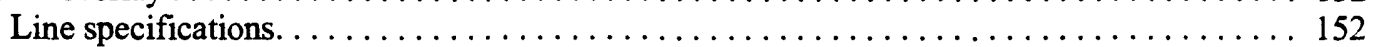

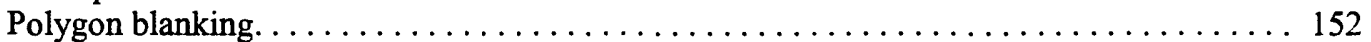

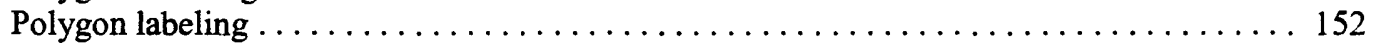

Selective polygons within a given geographic level.................... 152

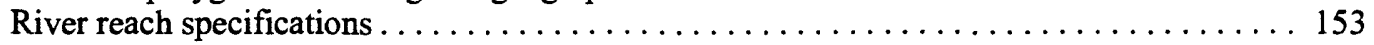

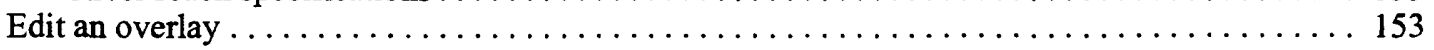

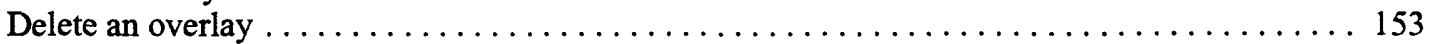

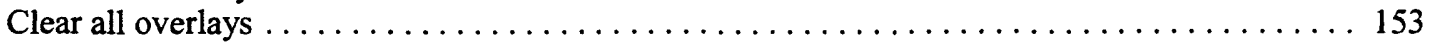

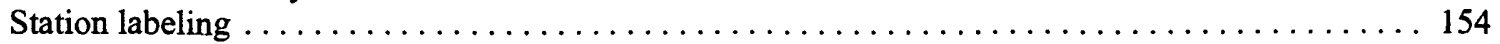

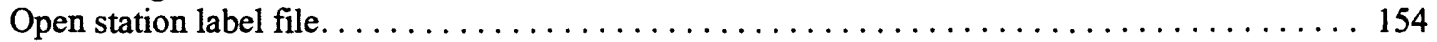

Filter data in label variable file. . . . . . . . . . . . . . 154

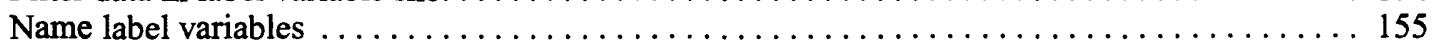

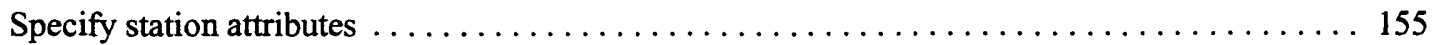

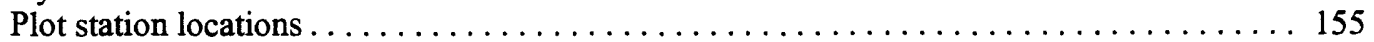

Label stations. . . . . . . . . . . . . . . . . . . . . . . . . . . 155

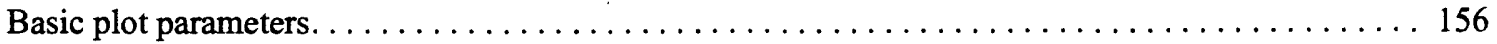

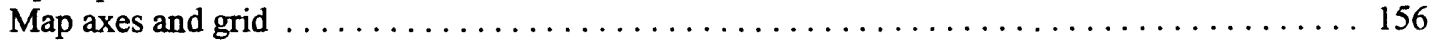

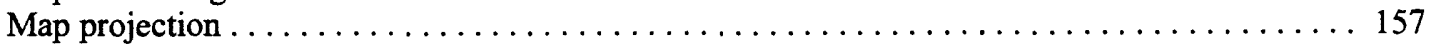

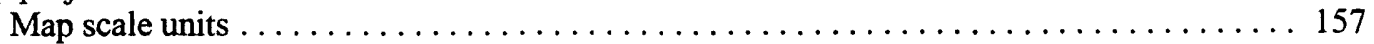

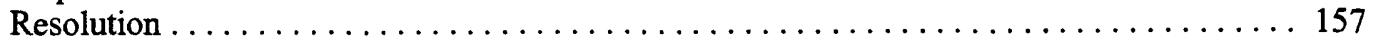

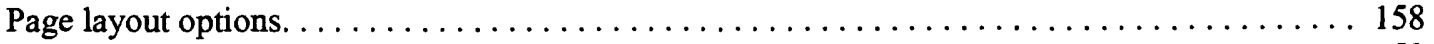

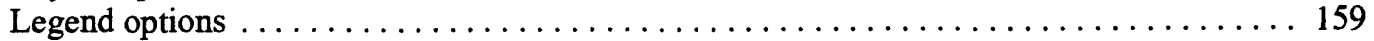

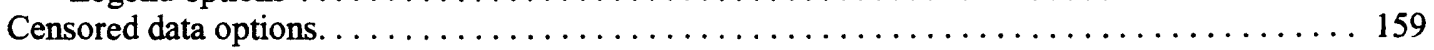

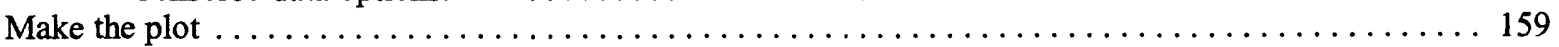

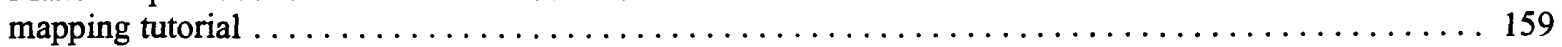

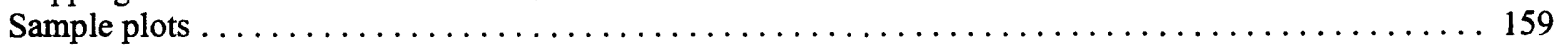

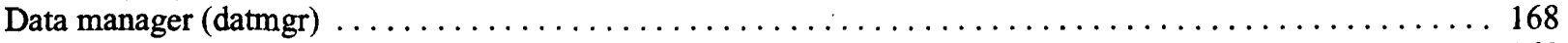

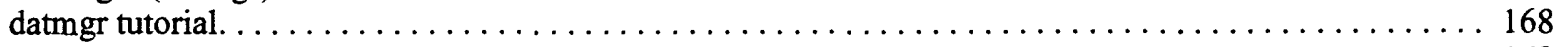

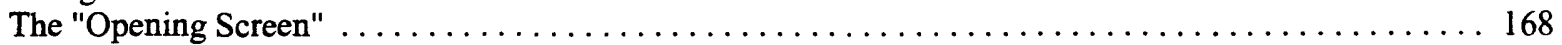

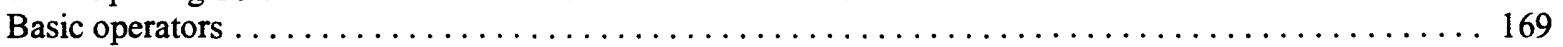

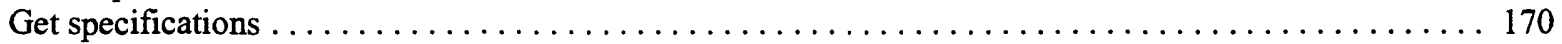

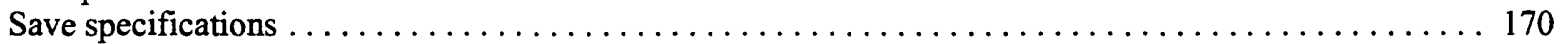

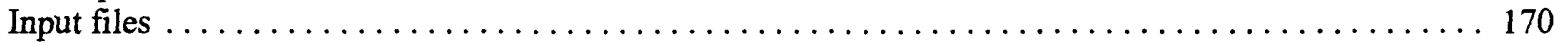

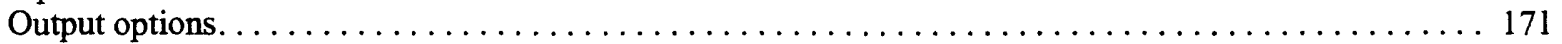

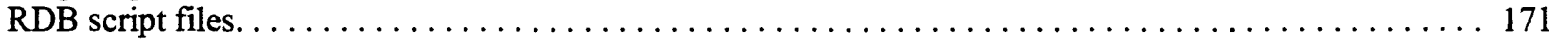




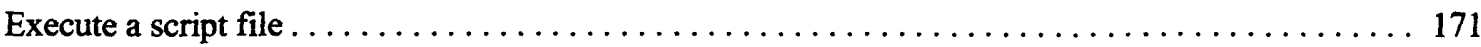

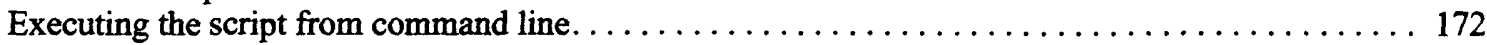

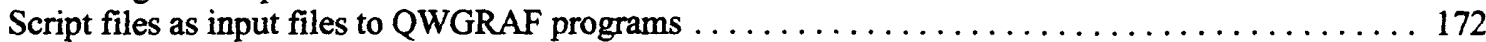

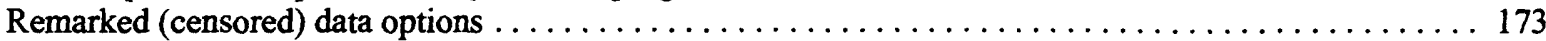

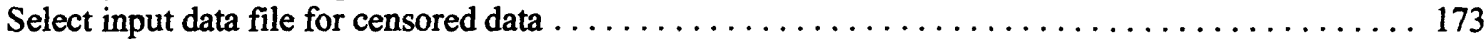

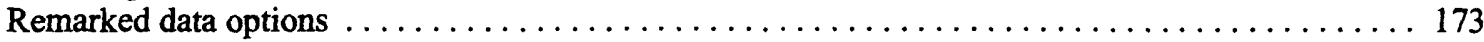

Save an RDB data file from "Remarked Data" option . . . . . . . . . . . . . . . . . 174

Save an RDB script file from "Remarked Data" option $\ldots \ldots \ldots \ldots \ldots \ldots \ldots \ldots \ldots \ldots \ldots \ldots$

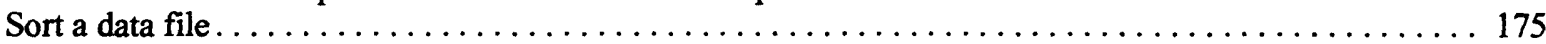

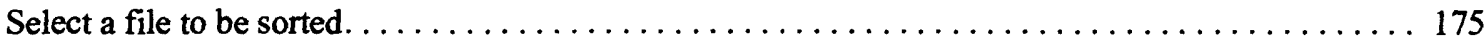

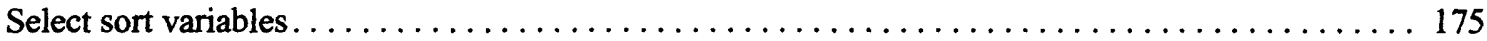

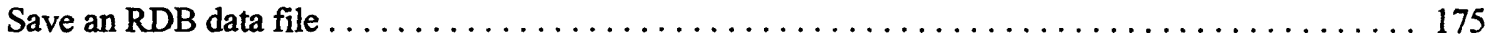

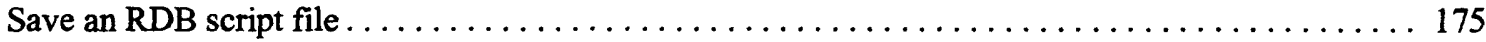

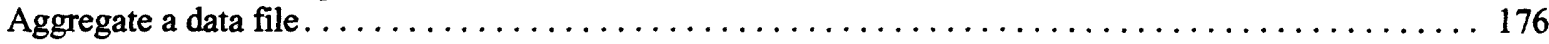

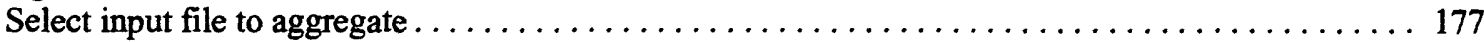

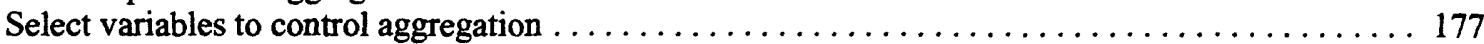

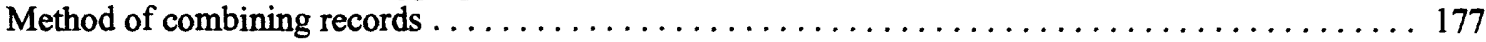

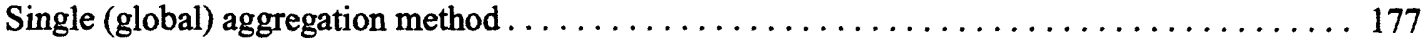

Individual variable aggregation method $\ldots \ldots \ldots \ldots \ldots \ldots \ldots \ldots \ldots \ldots \ldots \ldots \ldots \ldots \ldots \ldots \ldots \ldots$

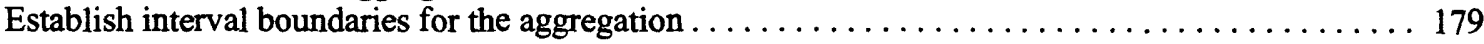

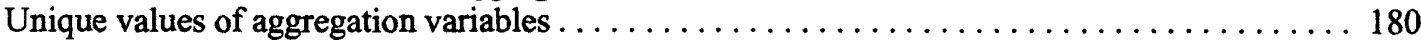

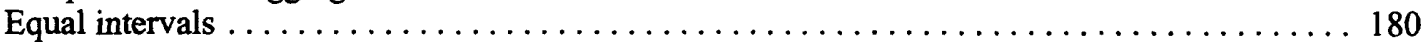

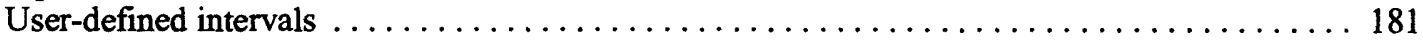

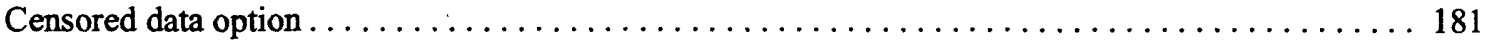

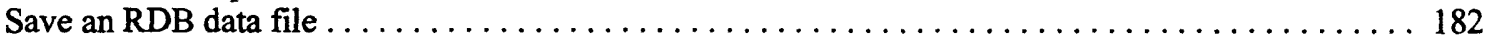

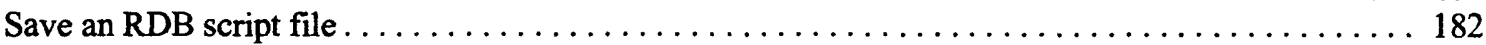

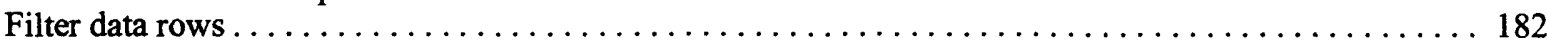

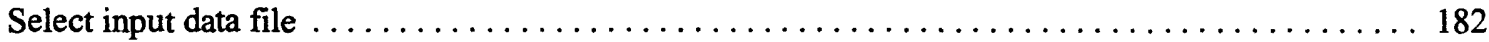

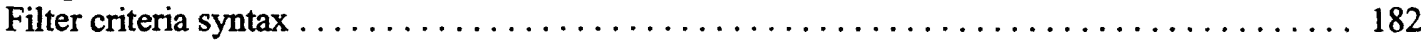

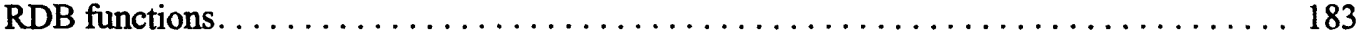

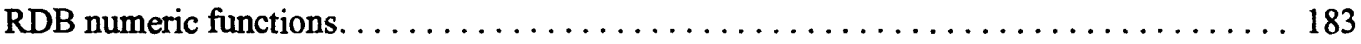

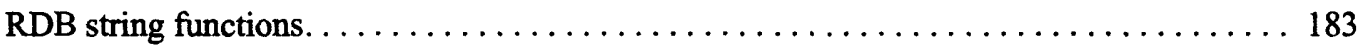

Date/time functions . . . . . . . . . . . . . . . . . . . . . . . . . . . . 184

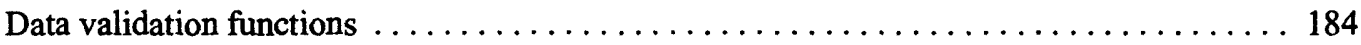

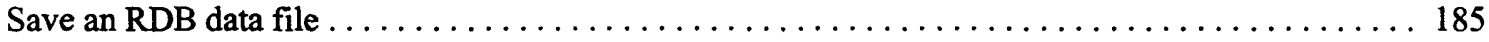

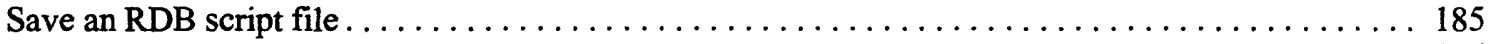

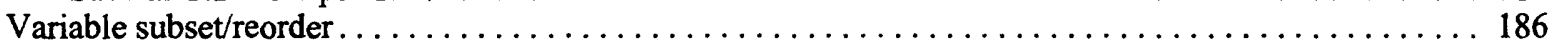

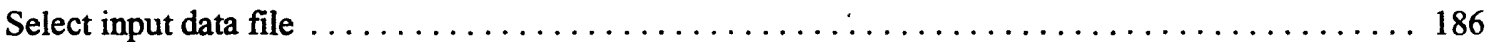

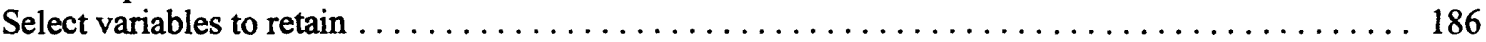

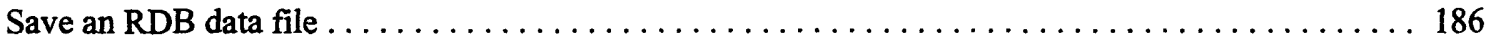

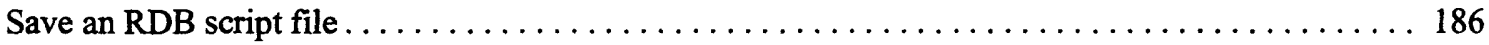

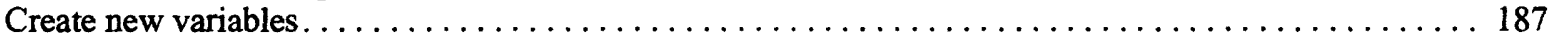

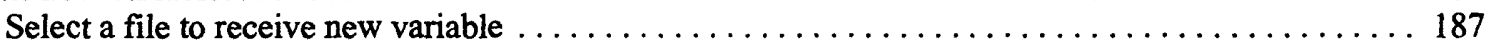

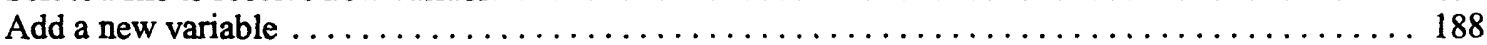

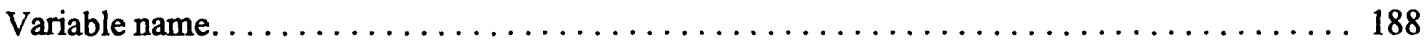

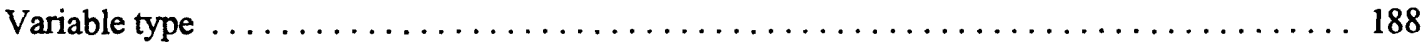

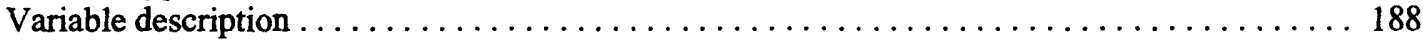

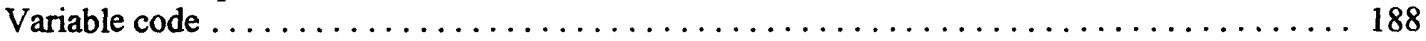

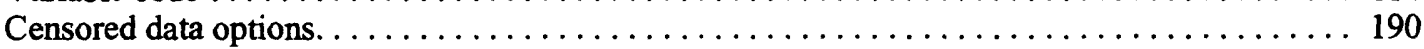

Edit a new variable . . . . . . . . . . . . . . . . . . . . . . . . . . . . . . . . . 190

Delete a new variable. . . . . . . . . . . . . . . . . . . . . . . . . . . . . 191

Clear all new variables. . . . . . . . . . . . . . . . . . . . . . . . . . . . 191

Save an RDB data file . . . . . . . . . . . . . . . . . . . . . . 191

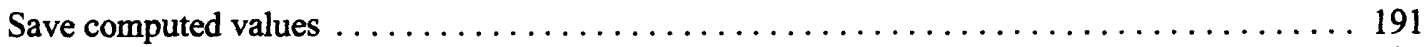

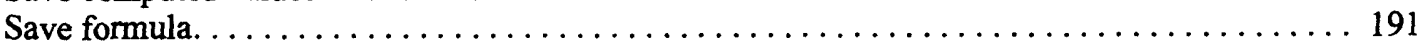

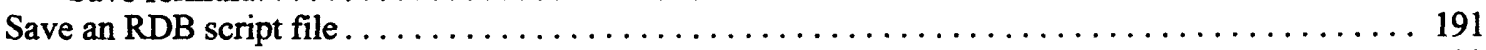

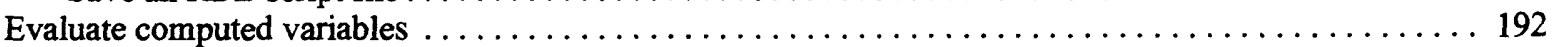

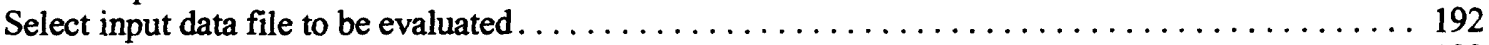

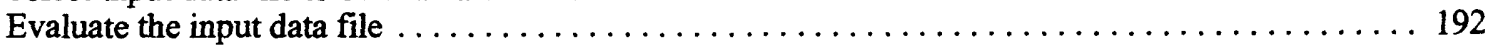




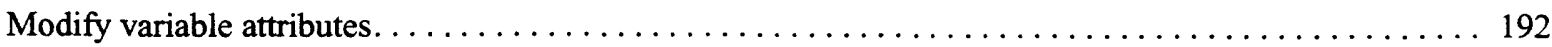

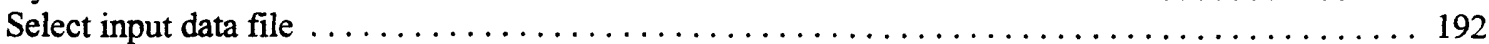

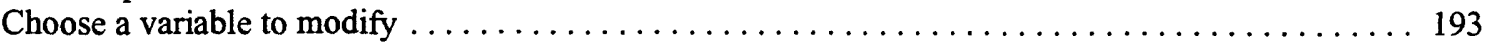

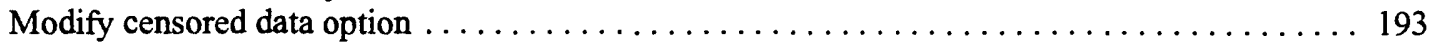

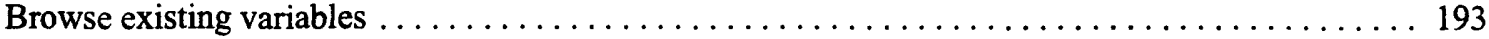

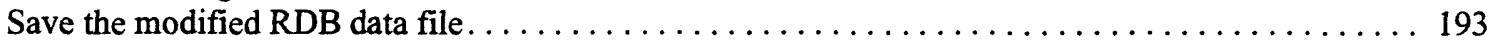

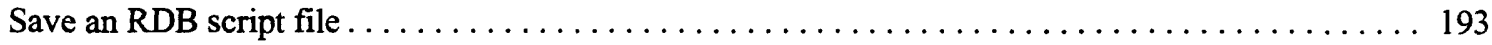

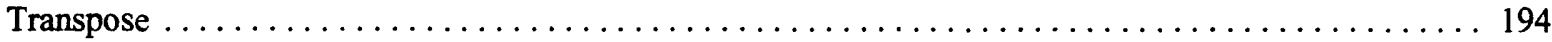

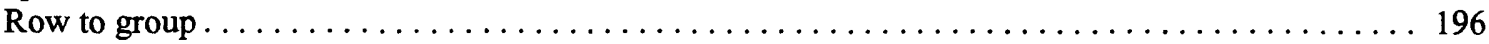

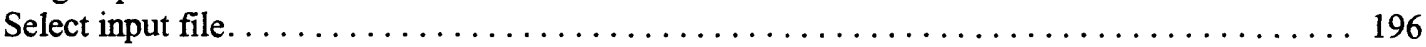

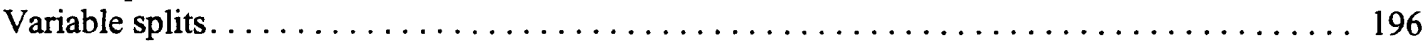

Add a new group of variables . . . . . . . . . . . . . . . . . . . . . 197

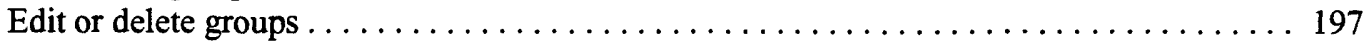

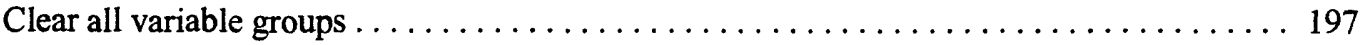

Pattern splits . . . . . . . . . . . . . . . . . . . . . . . . . . . . . . . 198

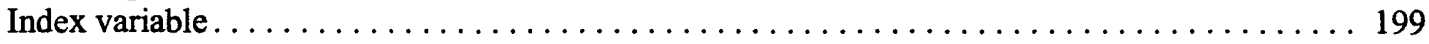

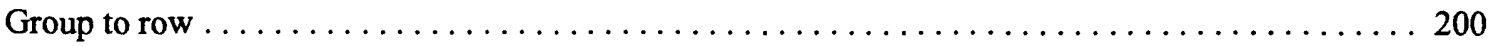

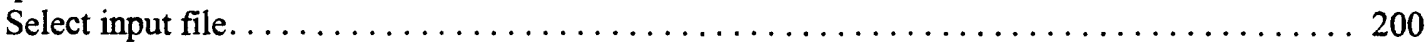

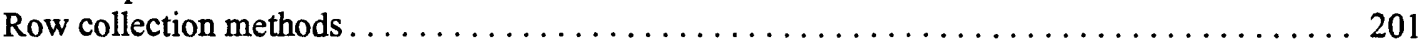

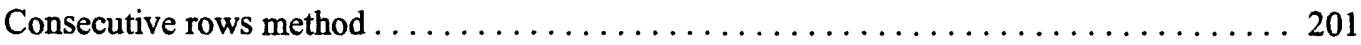

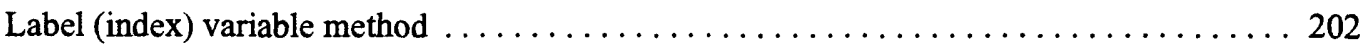

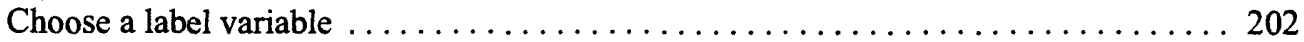

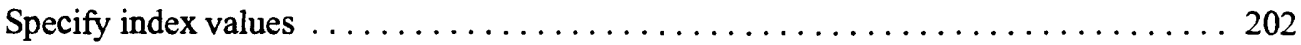

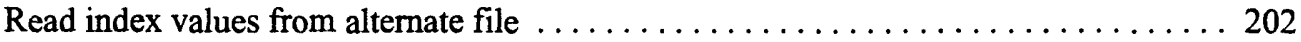

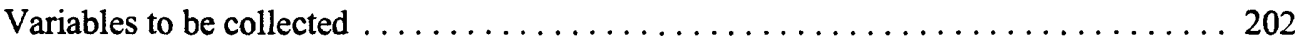

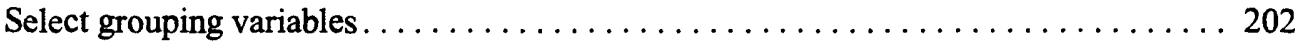

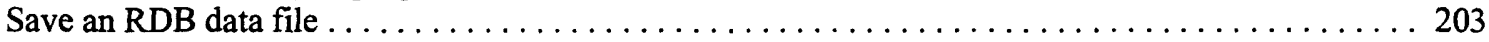

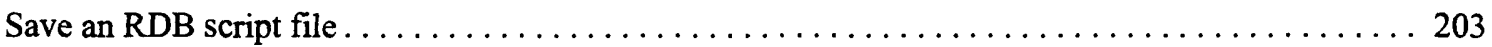

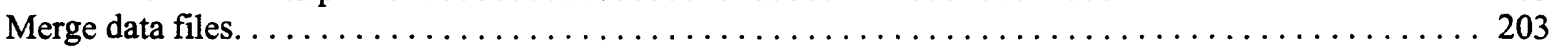

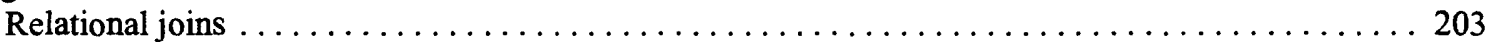

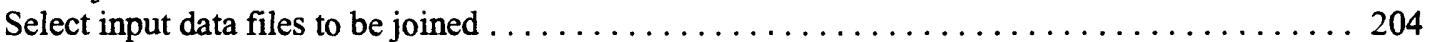

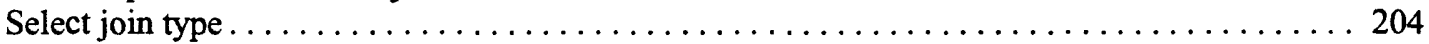

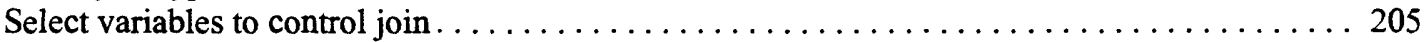

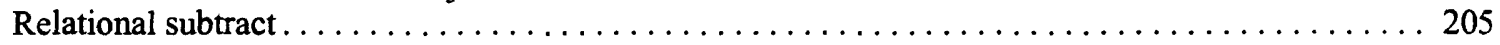

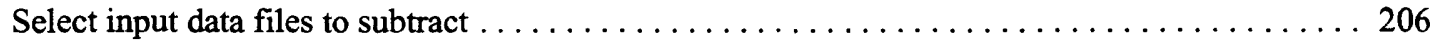

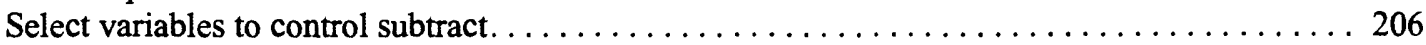

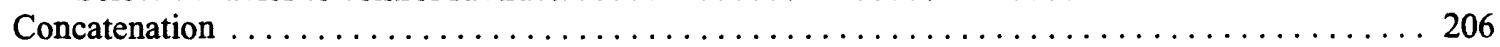

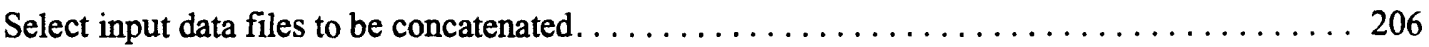

Options for concatenation. . . . . . . . . . . . . . . . . . . . . . . . . . . . . 207

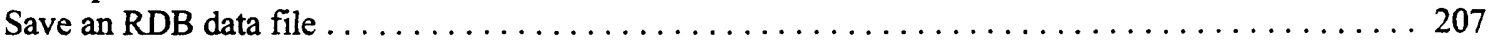

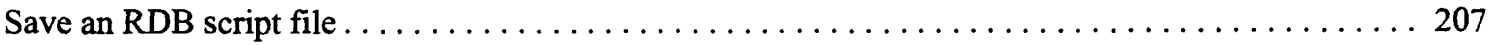

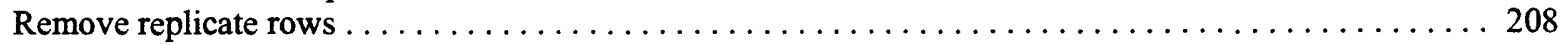

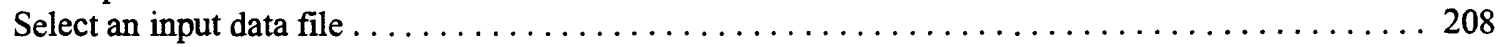

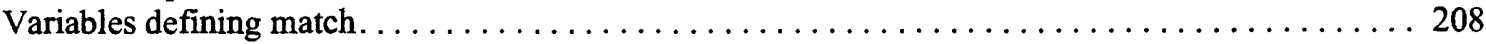

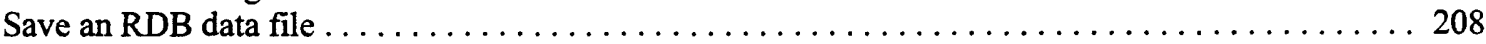

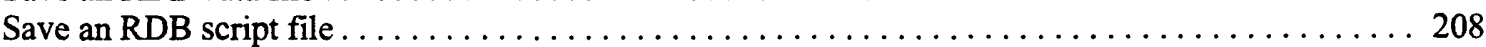

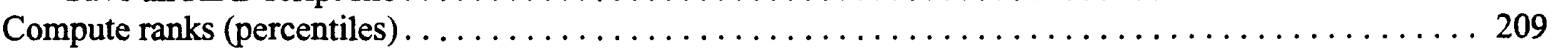

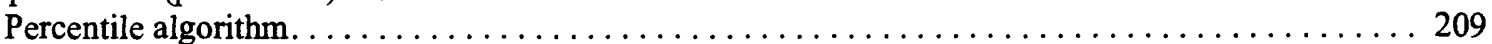

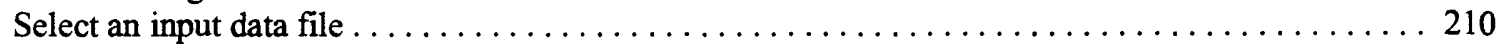

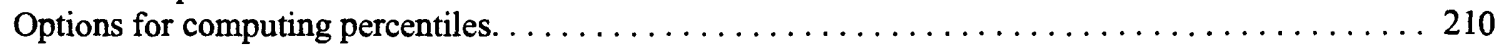

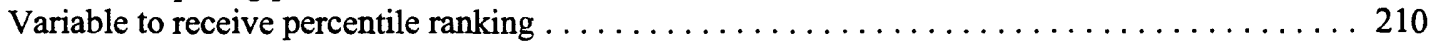

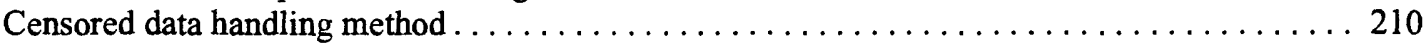

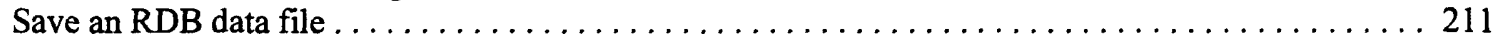

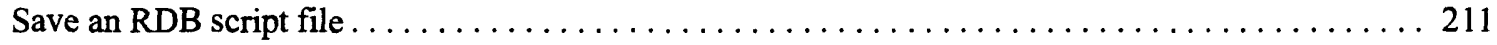

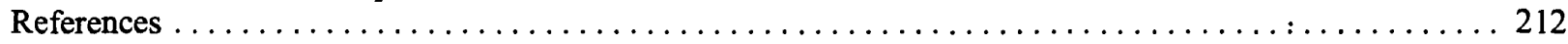

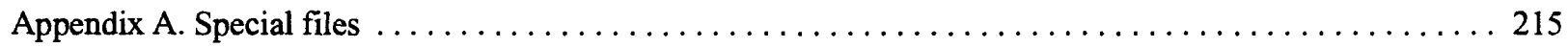

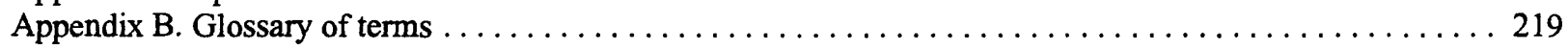




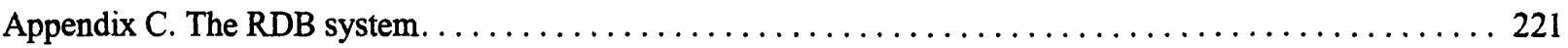

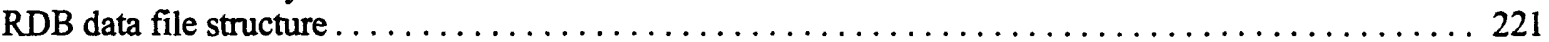

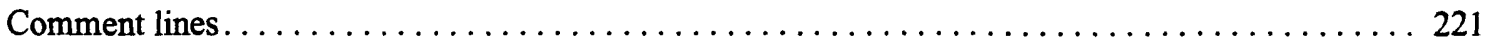

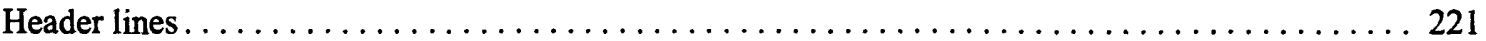

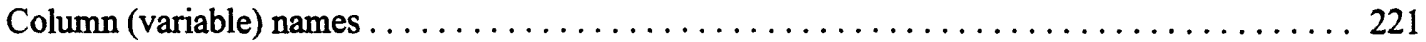

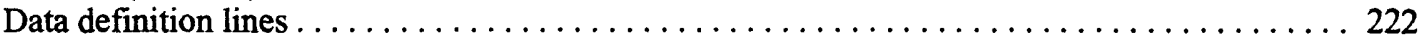

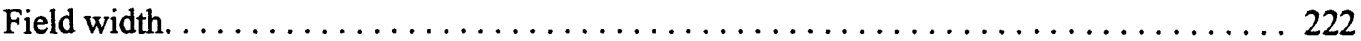

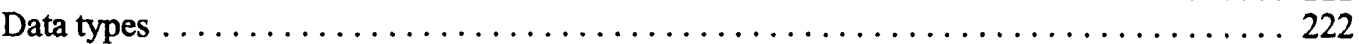

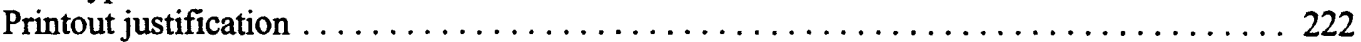

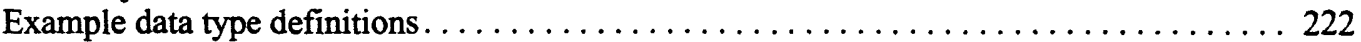

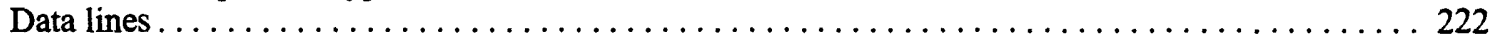

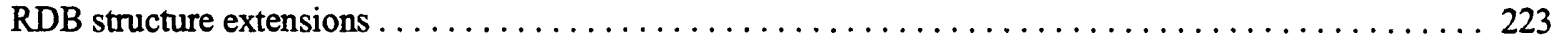

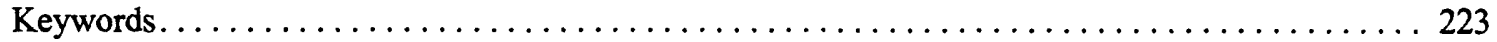

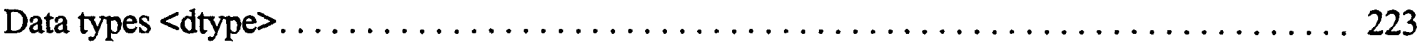

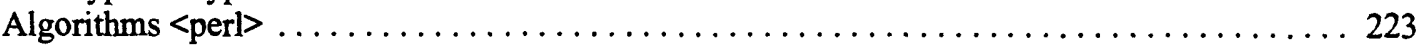

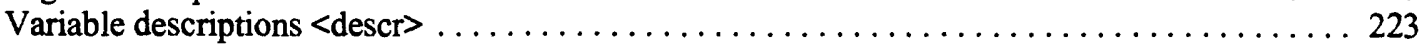

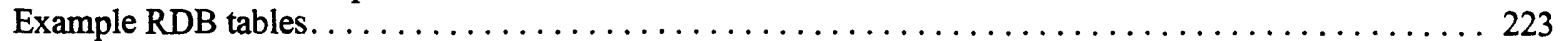

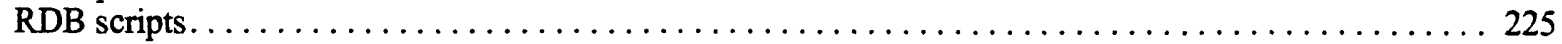

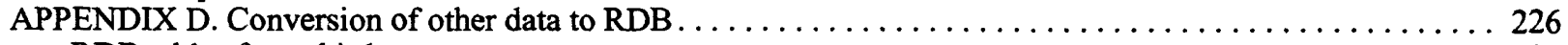

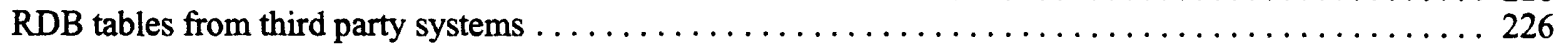

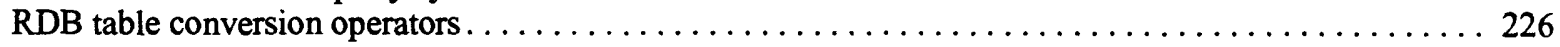

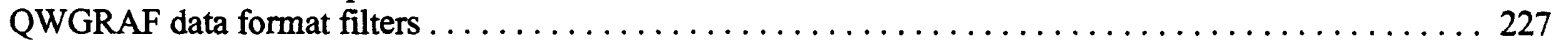

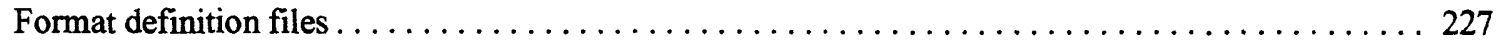

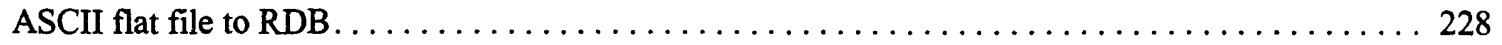

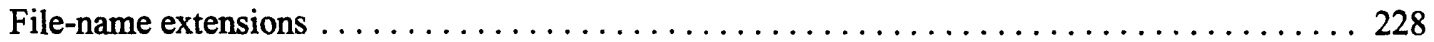

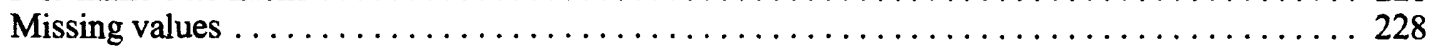

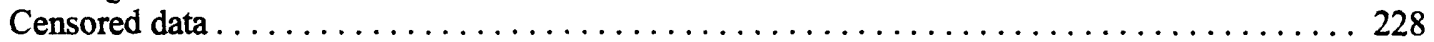

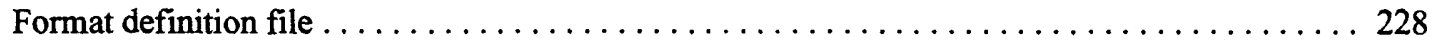

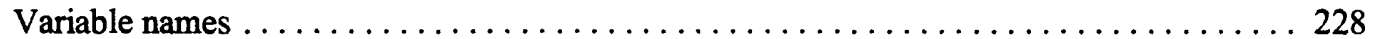

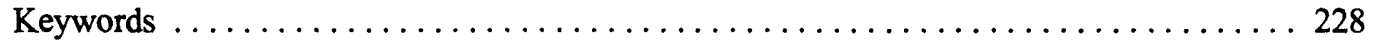

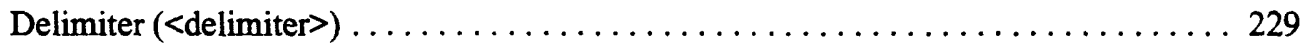

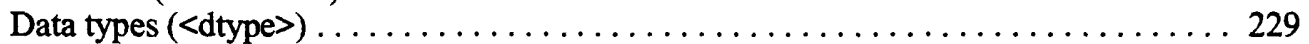

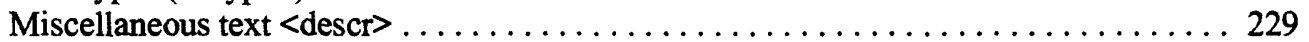

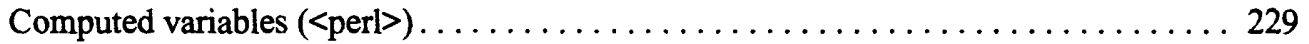

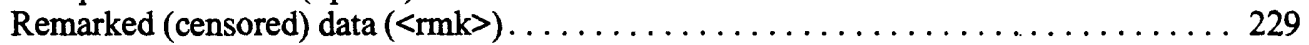

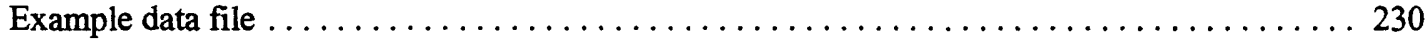

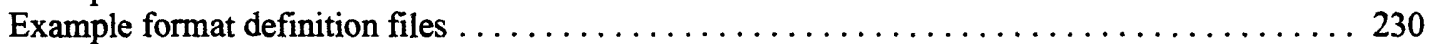

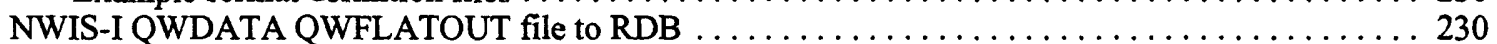

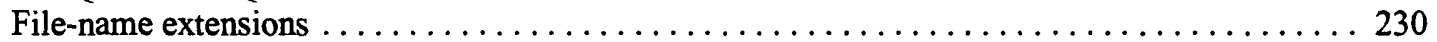

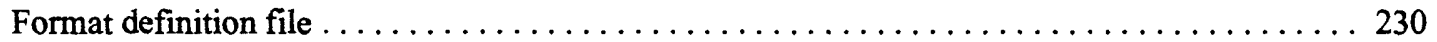

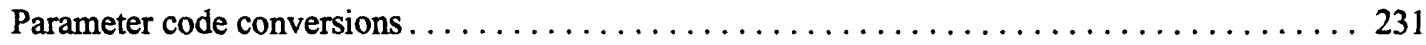

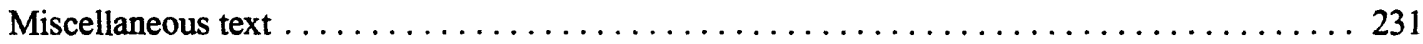

Data file format. . . . . . . . . . . . . . . . . . . . . . . . . . . . . . . 232

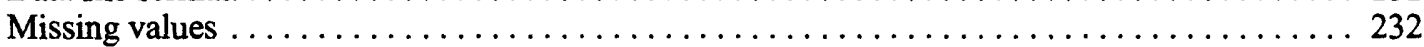

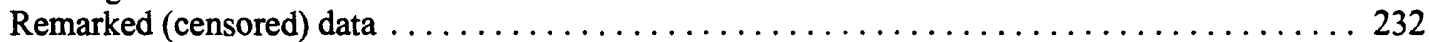

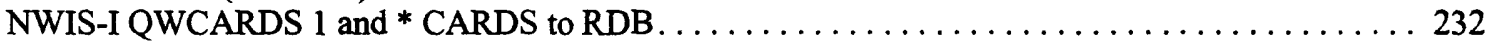

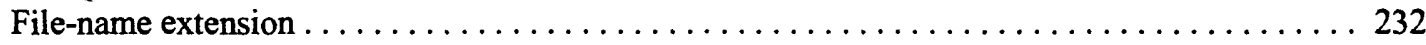

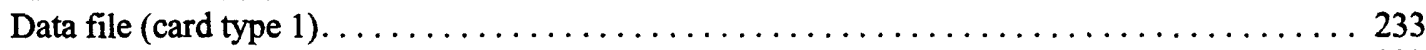

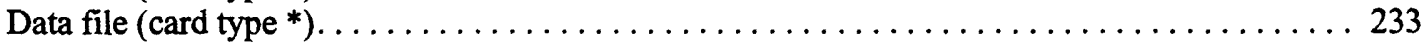

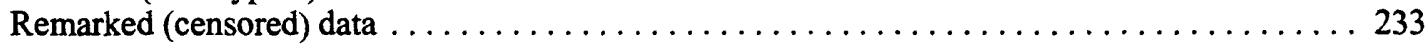

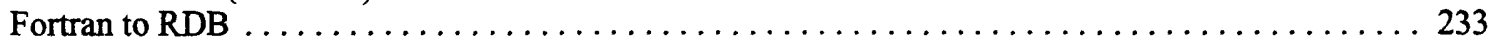

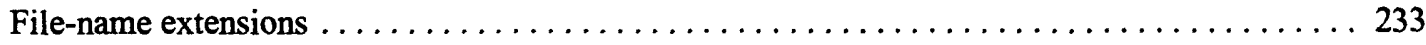

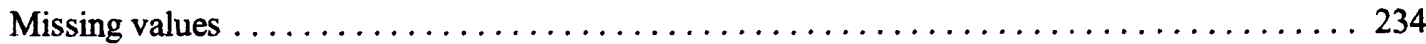

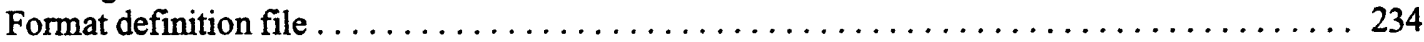

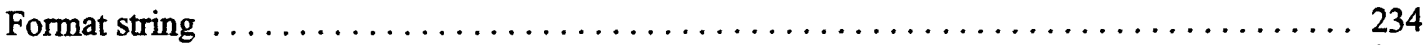

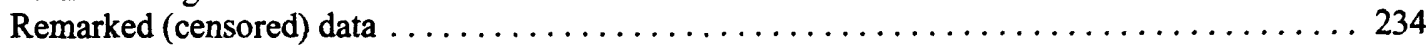

Data file format. . . . . . . . . . . . . . . . . . . . . . . . . . . . . . . 235

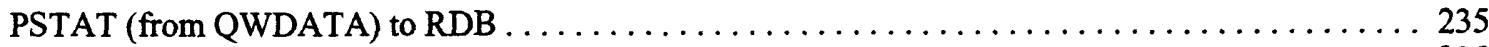

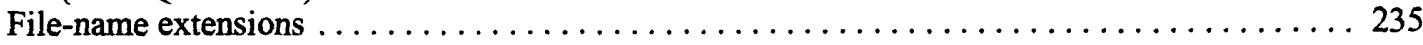




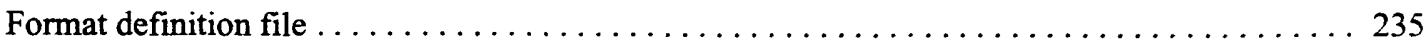

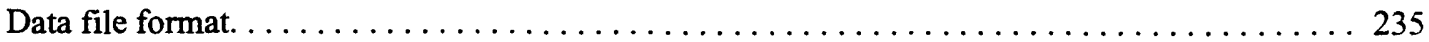

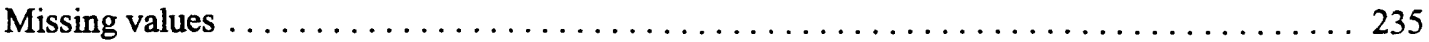

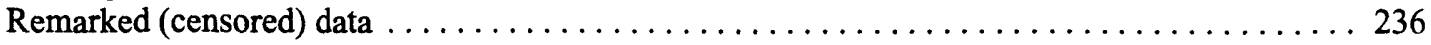

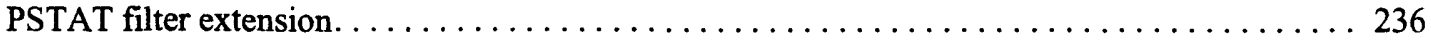

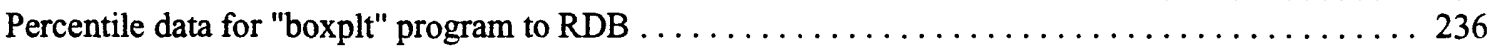

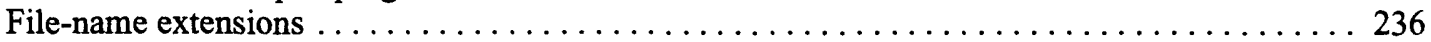

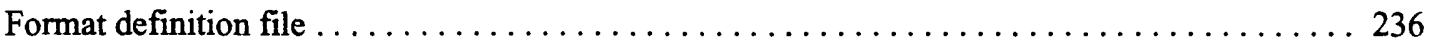

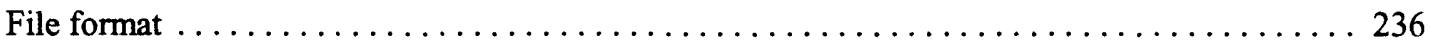

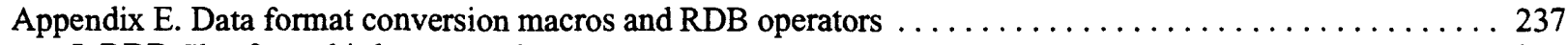

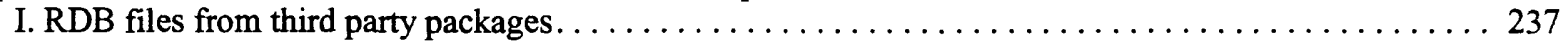

II. Standard RDB operators for data format conversions $\ldots \ldots \ldots \ldots \ldots \ldots \ldots \ldots \ldots \ldots \ldots \ldots \ldots \ldots \ldots \ldots \ldots$

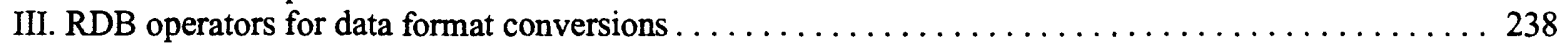

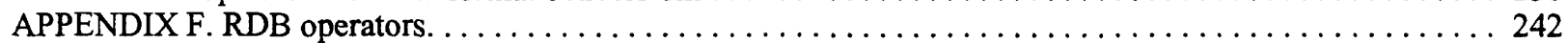

APPENDIX G. Importing QWGRAF graphics into FrameMaker $\ldots \ldots \ldots \ldots \ldots \ldots \ldots \ldots \ldots \ldots \ldots \ldots 26$

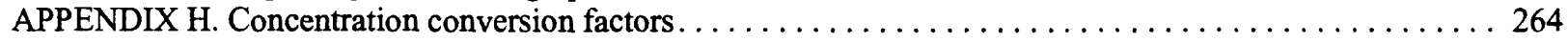

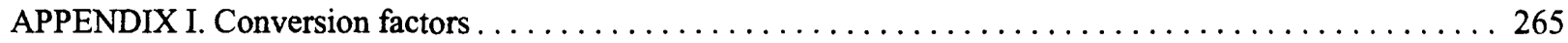

\section{FIGURES}

Figure 1. Basic screen layout and commands for QWGRAF programs $\ldots \ldots \ldots \ldots \ldots \ldots \ldots \ldots \ldots \ldots$

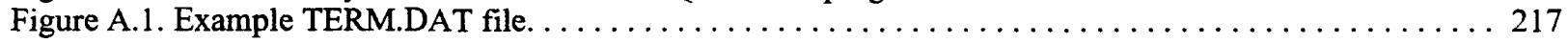

\section{TABLES}

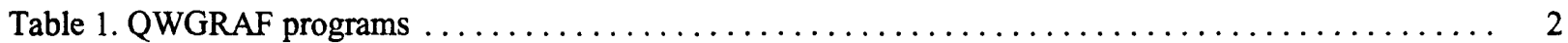

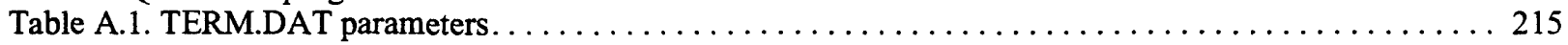

Table A.2. MENCRA values and corresponding program response $\ldots \ldots \ldots \ldots \ldots \ldots \ldots \ldots \ldots \ldots \ldots$

Table A.3. TERM.DAT parameters for color display (DOS-based computers) $\ldots \ldots \ldots \ldots \ldots \ldots \ldots \ldots 217$

Table A.4. Codes used for nonprinting characters in a $\log$ file $\ldots \ldots \ldots \ldots \ldots \ldots \ldots \ldots \ldots \ldots \ldots \ldots \ldots \ldots \ldots$

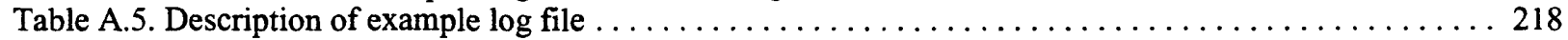

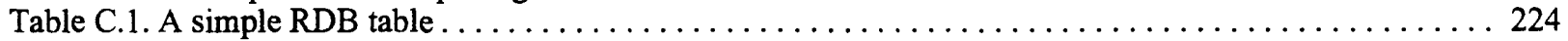

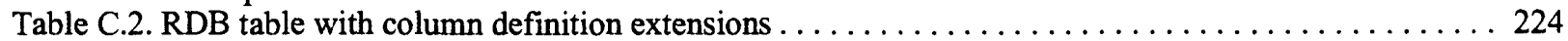

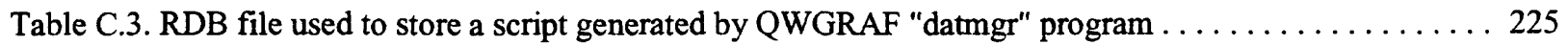

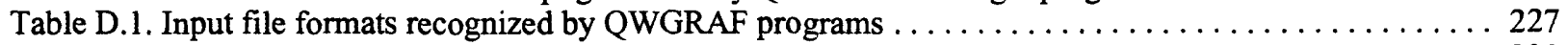

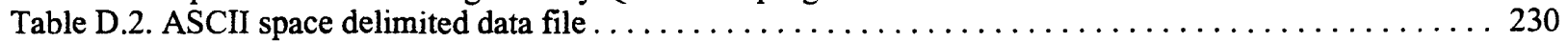

Table D.3. Format definition file for use with data file in table D. $\ldots \ldots \ldots \ldots \ldots \ldots \ldots \ldots \ldots \ldots \ldots 230$

Table D.4. Conversion criteria for QWDATA alpha parameter codes $\ldots \ldots \ldots \ldots \ldots \ldots \ldots \ldots \ldots \ldots 231$

Table D.5. Output format from QWDATA FLATOUT program for data in a QWDATA data file

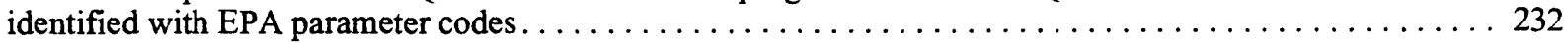

Table D.6. Output format from QWDATA FLATOUT program for alpha parameter code text variables . . . 232

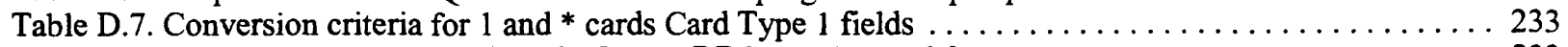

Table D.8. Example of a single analysis in QWCARDS 1 and *-card format $\ldots \ldots \ldots \ldots \ldots \ldots \ldots \ldots \ldots \ldots$

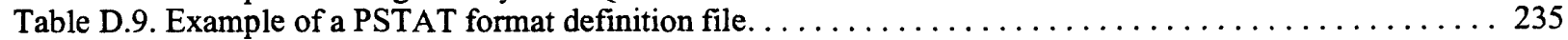

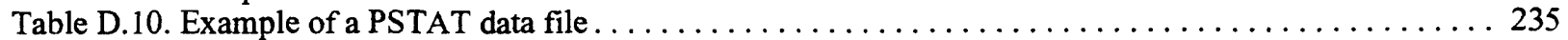

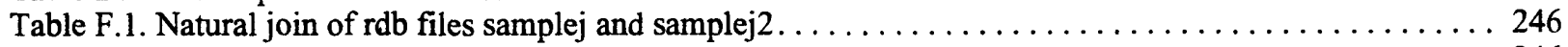

Table F.2. Master-detail join of rdb files samplej and samplej $2 \ldots \ldots \ldots \ldots \ldots \ldots \ldots \ldots \ldots \ldots \ldots \ldots \ldots$

Table F.3. One-to-one join of $\mathrm{rdb}$ files samplej and samplej2 $\ldots \ldots \ldots \ldots \ldots \ldots \ldots \ldots \ldots \ldots \ldots \ldots \ldots \ldots$

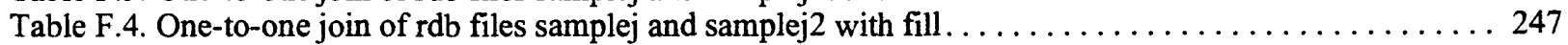




\title{
User's Manual for QWGRAF, Computer Programs for Water-Quality Graphics
}

\author{
By James C. Schornick and Michael C. Place
}

\section{Abstract}

QWGRAF is a collection of interactive programs with a common interface that provides statistical and graphical tools for management, review, analysis, quality assurance, and presentation of water-quality data. The software is written in ANSI Fortran and C and is designed for portability to UNIX workstations and personal computers. The programs that make up QWGRAF provide (1) Box and Whisker plots (boxplt); (2) probability plots (prbplt); (3) frequency plots (frqplt); (4) graphical means of performing the Lilliefors test for normality (lilifr); (5) Piper diagrams (piper); (6) Durov plots (durov); (7) Stiff diagrams (stiff); (8) boundary outline maps of the United States and its territories with quantitative representation of data on an areal or site specific basis (mapping); and (9) data-management capability for modifying data sets (datmgr). QWGRAF programs read and operate on files formatted in RDB. The programs also read and convert to RDB format other data formats, such as flat files, Fortran formatted files, PSTAT files, and U.S. Geological Survey National Water Information System 1 and * cards and QWDATA FLATOUT files. Flat files and Fortran formatted files can be accompanied by a file that defines the variable names, data types, variable descriptions, column delineation, and algorithms for calculating new variables. Censored data (data remarked with "less than" codes) and missing values are accommodated.

This document is a user's guide for QWGRAF programs.

\section{Overview}

QWGRAF is a group of selected graphics and data-management procedures for the management, review, analysis, quality assurance, and presentation of water-quality data. Input data can come in a variety of formats, which are converted automatically to the $\mathrm{RDB}$ format used internally by the programs. QWGRAF programs share a common user interface and many functional features to facilitate the learning curve (table 1).

Each program has a robust list of options (specifications) available for uniquely configuring a plot. Values for program options, once entered or selected by the user, are retained throughout each program session but can be changed at any time. This provides for rapid multiple scenario runs during a given session. Complex configuration scenarios can be saved in "Specifications" files, which can be read into the program in subsequent QWGRAF sessions, thus saving time in setting up similar program runs.

QWGRAF offers an extensive data-management capability, which includes the use of filters to convert other data formats to the $\mathrm{RDB}$ data formatting system used by QWGRAF programs. No special action is required of the user, other than appending the file name with an appropriate three-character extension, in order for a QWGRAF 
program to read one of the other formats. The program automatically invokes the filter associated with the file-name extension.

The data-management program "datmgr" provides an interactive interface to many commonly used data manipulation functions, which are normally accessed from the command line by using the complex syntax of a scripting language. The program provides functions for such operations as Boolean expressions for filtering data rows; selecting and ordering variables; creating new variables; sorting; grouping and aggregating rows of data; relational joins to other data sets; and concatenation of data sets. Script files, which contain instructions for processing primary input and intermediate files, can be created and used as input files to any QWGRAF program. Censored data (data remarked with "less than" codes) and missing values are accommodated.

Table 1 provides a brief description of the purpose and capability of each of the programs currently in QWGRAF.

Table 1. QWGRAF programs

\begin{tabular}{|c|c|}
\hline $\begin{array}{c}\text { Program } \\
\text { name }\end{array}$ & Description \\
\hline boxplt & $\begin{array}{l}\text { Skeletal box and whisker diagrams (box plots). Options available for Quartiles or } \\
\text { Fourths Box boundaries, and Tukey, Min-Max, or 10-90 Percentile Whisker placement. } \\
\text { Special options for using censored data. Data partitioning by groups. Arithmetic and } \\
\text { logarithmic y-axes scales. }\end{array}$ \\
\hline prbplt & $\begin{array}{l}\text { Probability curves for up to six variables against one of the following probability } \\
\text { distribution functions: plotting position, quantile deviates, exceedance and } \\
\text { nonexceedance cumulative probability percentiles, and recurrence interval. Multiple } \\
\text { arithmetic or logarithmic y-axes scales. }\end{array}$ \\
\hline frqplt & $\begin{array}{l}\text { Frequency, relative frequency, cumulative frequency, cumulative relative frequency } \\
\text { distributions, and the empirical distribution function (EDF). }\end{array}$ \\
\hline lilifr & $\begin{array}{l}\text { Graphical means to perform the Lilliefors test for sample normality. The procedure } \\
\text { compares a standard normal distribution function with the empirical distribution } \\
\text { function of the sample. Cumulative relative frequency is plotted on the } \mathrm{Y} \text {-axis and the } \\
\text { normalized sample values on the X-axis. Confidence level bounds are used to indicate } \\
\text { if the data are normal. }\end{array}$ \\
\hline piper & $\begin{array}{l}\text { Trilinear and quadlinear Piper hydrochemical facies diagrams used to characterize or } \\
\text { compare the chemical character of water bodies. User-defined nodes, including } \\
\text { composite nodes. Partitioning of data with symbols within diagrams. Multiple diagrams } \\
\text { on a page where each diagram represents a different set of variables or each diagram } \\
\text { represents a partitioned group of values of a single set of variables. Binary and ternary } \\
\text { mixing diagrams. Shaded density diagrams. }\end{array}$ \\
\hline durov & $\begin{array}{l}\text { Durov diagrams - hydrochemical facies diagrams similar to quadlinear Piper diagrams } \\
\text { where the intersection of points from two Piper triangles are projected to one or two } \\
\text { additional variables in adjacent scaled rectangles. }\end{array}$ \\
\hline stiff & $\begin{array}{l}\text { Stiff diagrams for comparing water type based on user-defined cation-anion pairs. User- } \\
\text { defined nodes, including composite nodes. Multiple diagrams on a page representing } \\
\text { either a unique set of variables for each diagram or a diagram for each subgroup of a } \\
\text { single set of variables based on composite values of up to four partitioning variables. }\end{array}$ \\
\hline mapping & $\begin{array}{l}\text { Outline maps of the United States, individual States, counties, and hydrologic unit } \\
\text { boundaries with multiple overlay capability, including river reaches. Data is grouped } \\
\text { into intervals and mapped to point locations or the data can be aggregated and } \\
\text { represented as shaded areas. Extensive station labeling capability. }\end{array}$ \\
\hline datmgr & $\begin{array}{l}\text { Provides interactive capability to modify the data in an input data file. Functions include } \\
\text { sorting, creating new variables, aggregating rows, filtering selected rows, selecting and } \\
\text { reordering columns, joining and concatenating files, transforming rows to columns and } \\
\text { columns to rows, and computing of percentiles. Also provides for concatenating a series } \\
\text { of operations in a script that can be used as the input file to QWGRAF programs. }\end{array}$ \\
\hline
\end{tabular}




\section{How QWGRAF Works}

\section{User Interface}

Program interaction takes place in a screen 80 characters wide by 24 characters high. Figure 1 shows the basic screen layout. Each screen consists of a list of available commands displayed at the bottom of the screen and one or more boxed-in areas that are referred to as panels. Commands are used to obtain additional information and to move between screens. There are three types of panels - data, assistance, and instruction. The data panel displayed at the top of the screen is always present. Data panels contain menus, forms, tables, and text to permit user interaction with the program. An assistance panel may be present depending on user or program assignments. When present, the assistance panel is displayed below the data panel (usually as the middle panel) and contains textual information, such as help messages, valid range of values, and details on program status. The instruction panel is displayed above the available commands when the user is expected to interact with the program. When present, the instruction panel contains information on what keystrokes are required to interact with the program.

Each screen can be identified by a name and the path selected to reach the screen. The screen name appears in the upper left corner of the data panel, where the words "screen name" appear in figure 1. The first screen displayed by the program is named "Opening Screen." All subsequent screens are named on the basis of menu option or program sequence that caused the current screen to be displayed. Screen names are followed by "(path)," a string of characters that consist of the first letter(s) of the menu options selected in order to arrive at the current screen. In some cases, descriptive text may follow the path to further help identify the screen. The path can aid in keeping track of the position of the current screen in the menu hierarchy. For example, "Open (FO)" indicates that the menu option Open was selected previously and that the path to this screen from the "Opening Screen" consisted of two menu selections-File and Open.

\section{Commands}

The screen commands and their associated keystrokes are described in figure 1. A subset of the screen commands is available for any given screen. Most commands can be executed by pressing a single function key. (The designation for a function key is "F\#" where \# is the number of the function key.) All of the commands can be executed in "command mode." Command mode is toggled on and off by pressing the semicolon (;) $\mathrm{key}^{1}$. In command mode, any command can be executed by pressing the first letter of the command name; for example, "o" or "O" for the Oops command. When commands are discussed in this report, the command name is spelled out with the function key or keystroke given in parentheses. For example, Accept (F2) is the most frequently used command.

Use Help (F1) and Limits (F5) to obtain additional information about the current screen and use Status (F7) to obtain information on the state of the program. Cmhlp (;c) will display information on the available commands. Use Quiet (F8) to close the assistance panel. To move between screens, use Accept (F2), Prev (F4), Intrpt (F6), Dnpg (;d), Uppg (;u), or Top (F10). To reset the values in the data panel, use Oops (;0). Xpad (F9) is used to save typed information in a file called XPAD.DAT.

\footnotetext{
${ }^{1}$ On some systems the F3 key and (or) the escape key (Esc) may also work.
} 
Assistance panel

instruction type

Instruction panel

Help:F] Accept:F2 Prev:F4 Limits:F5 Intrpt:F6 Status:F Quiet:F8 Cmhlp Oops

\begin{tabular}{|c|c|c|}
\hline Command & $\begin{array}{l}\text { Associated key- } \\
\text { strokes }^{\text {a }}\end{array}$ & Description \\
\hline Help & F1 $($ or $; \mathrm{h})$ & $\begin{array}{l}\text { Displays help information in the assistance panel. The help information is updated as the user } \\
\text { moves from field to field in the data panel or to a different screen. The program automatically clos- } \\
\text { es the assistance panel if a screen is displayed for which no help information is available. }\end{array}$ \\
\hline Accept & F2 $($ or $; \mathrm{a})$ & $\begin{array}{l}\text { Indicates that you have "accepted" the input values, menu option currently highlighted, or text mes- } \\
\text { sage in the data panel. Selection of this command causes program execution to continue. }\end{array}$ \\
\hline Cmhlp & $\mathrm{F} 3 \mathrm{c}($ or $; \mathrm{c})$ & Displays brief descriptions of the commands available on the screen. \\
\hline Oops & F3o $($ or $; 0)$ & Resets all data fields in an input form to their initial values. \\
\hline Dnpg & F3d $(o r ; d)$ & $\begin{array}{l}\text { Displays next "page" of text in data panel. Available when all of the text cannot be displayed at one } \\
\text { time. }\end{array}$ \\
\hline Uppg & F3u $($ or $; \mathrm{u})$ & Redisplays previous "page" of text in data panel. Available after execution of Dnpg (F3d). \\
\hline Prev & F4 (or;p) & $\begin{array}{l}\text { Redisplays a previous screen. Any modifications in the data panel are ignored. Which screen is the } \\
\text { previous one may be ambiguous in some cases. }\end{array}$ \\
\hline Limits & F5 $($ or $; 1)$ & $\begin{array}{l}\text { Displays valid ranges for numeric fields and valid responses for character fields. As with the Help } \\
\text { command, information on field limits is updated as the user moves from field to field in the data } \\
\text { panel or to a different screen by using the arrow keys or the Enter (Return) key. }\end{array}$ \\
\hline Intrpt & F6 (or;) & $\begin{array}{l}\text { Interrupts current processing. Depending on the process, returns the program to the point of } \\
\text { execution prior to the current process or advances to the next step in the process. This key also } \\
\text { activates the State/County/Hydrologic Unit cross reference function in the mapping program (see } \\
\text { Area and Overlay screens). }\end{array}$ \\
\hline Status & F7 (or;s) & Displays program status information. \\
\hline Quiet & F8n (or ;q) & Closes the assistance panel. Available when the assistance panel is open. \\
\hline Xpad & F9 $(o r ; x)$ & $\begin{array}{l}\text { Opens the assistance panel as a "scratch pad." Text entered in the scratch pad is saved in a file } \\
\text { called "XPAD.DAT." }\end{array}$ \\
\hline Top & F10 & Returns to the Opening Screen \\
\hline
\end{tabular}

a. The function keys will execute the commands on most computer systems. On all computer systems, the semicolon key (";") followed by the first letter (upper or lower case) of the command can be used to execute the commands. The F3 function key may not be available on some systems.

Figure 1. Basic screen layout and commands for QWGRAF programs. 
There are four types of data panels-menu, form, table, and text. Menus offer a choice of two or more options. A form or table allows data values to be entered or modified in one or more data fields. General or specific information, program progress, messages, and results of analyses may be displayed in a text data panel. The data panel appears at the top of the screen, as shown in figure 1 . There are 16 rows in the data panel when the assistance panel is closed and 10 rows when the assistance panel is open.

A single option is selected from a menu that consists of two or more options. There are two ways to select a menu option. Either press the first letter (not case sensitive) of the menu option (if more than one menu option begins with the same letter, then press in sequence enough characters to uniquely identify the option) or use the arrow keys to move the cursor to the option and then execute Accept (F2).

Forms may contain any number and combination of character, numeric, file-name, or option fields. Character fields may be a variable entry, such as a descriptive text string (case sensitive), or they may require a specific entry, such as "yes" or "no" (not case sensitive). The text string "none" in a field indicates that the field is currently undefined.

Option fields are activated and deactivated by positioning the cursor in the option field and pressing any key, such as the space bar. Use arrow keys to move up, down, and laterally among fields. The Enter (Return) key is used to move forward through fields. Use Accept (F2) to accept the entered and modified data and continue with the program. The use of Oops $(; 0)$ sets all fields in the current screen to their initial values. The use of Prev (F4) will cause the data values entered on the current screen to be ignored and the previous screen to be displayed again.

Tables may contain any number and combination of character, numeric, and file-name columns. As with forms, character fields may require a specific entry or a variable entry. Use arrow keys to move up, down, and laterally among fields. The Enter (Return) key is used to move forward across rows and to the next row. Some tables may contain more rows than can be displayed in the 10 or 16 rows of the data panel. In these cases, the table is divided into multiple screens. Use Accept (F2) to move forward through each of the screens for the table and to continue with the program after the last screen of the table. The use of Oops $(; 0)$ sets all fields in the current screen to their initial values. The use of Prev (F4) will cause the data values entered on the current screen to be ignored and the previous screen to be displayed again. The use of Intrpt (F6) will cause the data values entered on the current screen to be ignored and the remaining screens in the table to be skipped. Use Quiet (F8) to close the assistance panel and view the 16 lines of the data panel.

A text data panel may contain a warning or error message, a tabular list of data, a progress message for an activity that may take more than a few seconds, or other general information. Use Accept (F2) to continue to the next screen. In cases where the displayed text requires more lines than the number available in the data panel, the Prev (F4), Dnpg (;d), and Uppg (;u) commands may be available to move forward and backward (scroll) through the screens. Note that the up and down arrows also may be used to move through the screens. Intrpt (F6) may be available to permit skipping the remaining screens of text. 


\section{Assistance Panel}

The assistance panel provides information to help the user enter data in the data panel or to allow note taking during a program session. The panel appears in the middle of the screen below the data panel. A name corresponding to the type of assistance being provided is displayed in the upper left corner of the panel, where the words "assistance type" appear in figure 1. The Help (F1), Limits (F5), Status (F7), Cmhlp (;c), and Xpad (F9) commands open the assistance panel. Programs may open the assistance panel to display status information. Help (F1) and Limits (F5) provide information about the current screen and data fields; Status (F7) provides information about the current process; Cmhlp (;c) provides information about the available commands; and Xpad (F4) provides a "note pad" for making notes in the file XPAD.DAT. Use Quiet (F8) to close the assistance panel.

Assistance panels display four lines at a time. In cases where the assistance information is more than four lines, the assistance panel becomes the active panel when one of the assistance type commands is invoked. Use the up and down arrow keys to scroll through the information. If the Page Down and Page Up keys are available, then they may be used to page through the information. To reactivate the data panel, toggle the command mode by pressing (;) or (F3).

\section{Instruction Panel}

The instruction panel provides information on how to interact with the current screen, such as how to enter data or how to advance to another screen. This panel appears at the bottom of the screen just above the screen commands (fig. 1). The instruction panel is present whenever the program requires input from the user. Up to four lines of text are displayed in an instruction panel. If an invalid keystroke is entered, then the information in the instruction panel is replaced with an error message and the panel name (upper left corner) changes from "INSTRUCT" to "ERROR." Once a valid keystroke is entered, the Instruct panel is displayed again.

\section{Special Files}

Three files are associated with the interaction between the user and the program. System defaults that control how the program operates can be overridden by setting parameters in the optional TERM.DAT file. A session record is written to the PROC.LOG file, for example, PIPER.LOG, each time the program is run; all or portions of this file can be used as input to the program at a later time. Error and warning messages, as well as some additional information, may be written to the file ERROR.FIL.

\section{System Defaults-TERM.DAT}

Certain aspects of the appearance and operation of the program are controlled by parameters within the program. These parameters specify information such as the computer system type, graphic output type, terminal type, program response to the Enter key, and colors. Each parameter is set on the basis of preferences of users who tested the program. The preset values can be overridden by creating a TERM.DAT file in the directory where the program is initiated (the current working directory). The available parameters and the format of the TERM.DAT file are described in Appendix A. If a TERM.DAT file does not exist in the current directory, then the message "optional TERM.DAT file not opened, defaults will be used" is displayed briefly when the program starts. If the TERM.DAT file is present, then the message "reading users system parameters from TERM.DAT" is displayed. 


\section{Session Record PROC.LOG}

The keystrokes entered during a program session are recorded in the PROC.LOG file. Each time the program is run, a PROC.LOG file is created; if one already exists in the current directory, then it is overwritten. All or part of this file can be used as input to the program as a means of repeating the same or similar tasks. To do this, first save the PROC.LOG file under a different name. Modify the file to contain only the sequence of commands that need to be repeated. Then, at the appropriate point in a subsequent program session, press "@"; a small file-name panel appears. Type the name of the log file and press the Enter key. Appendix A describes the use and format of the PROC.LOG file.

\section{Error and Warning Messages_ERROR.FIL}

Any error or warning messages produced during a program session are written to the ERROR.FIL file. Each time the program is run, an ERROR.FIL file is created; if one already exists in the current directory, then it is overwritten. Diagnostic and summary reports also may be written to this file. Examine ERROR.FIL if an unexpected program response is encountered. 


\section{General Features of QWGRAF Programs}

\section{Program Overview}

For a general overview of a program, select and bring up the "Query for general information" option from the "Opening Screen." If the overview has more than 16 lines, then use the Page Down key or type ";D" on the keyboard to scroll the next 16 lines. Use the Page Up key or type "; U" on the keyboard to scroll back. Use Accept (F2) to return to the "Opening Screen." If the word "ERROR" appears on the instruction screen, then toggle the Command Mode (press "F3" or ";") to reactivate the Data panel.

\section{QWGRAF On-line Help}

When a program is initially started, only the "Data Panel" and the "Instruction Panel" are presented on the screen. Use the Help (F1) key to activate the center "Assistance Panel." With the "Help" panel activated, appropriate information about the option selected (highlighted) on the "Data Panel" is presented. If additional lines of help need to be screened, then use the Help (F1) key again to put the assistance panel in scroll mode whereby the "Page Down" and "Page Up" keys can be used to scroll through the entire help message. Use the "F3" or ";" key to reactivate the "Data Panel" for data entry. The "Limits (F5)" key is sometimes used in conjunction with the Help (F1) key to display allowable values for an option, such as the list of colors, line types, and fonts.

\section{Tutorials}

The programs in the QWDATA system share many similar functions and the interface has been designed to present these functions to the user in a consistent manner. However, the number of options available in the programs and the flexibility afforded the user in configuring a particular plot can initially make the programs appear to be difficult to learn and use. Therefore, interactive tutorials have been created for selected programs to guide the user through the basic elements of the programs. Instructions for using the tutorials are given in the discussions for "boxplt," "piper," "mapping," and "datmgr."

\section{Return to Operating System}

To exit from any program, return to the "Opening Screen" by using a combination of Accept (F2) and the "Return" option found in most screens. Use the arrow keys in the "Opening Screen" to select the "Return to operating system" option and press Accept (F2) or the Enter key.

\section{Specification Files}

Specification files are presaved lists of programs options that can be loaded (from "Get Specifications" option in "Opening Screen") at any time during a program session. These options overwrite any existing options, including default options, which produces a plot with a particular configuration. Options loaded in this manner can still be changed to meet user requirements. The Specifications file includes the name of the input data file open at the time the file was created. The use and creation of Specifications files is described in detail in the "Programs Specifications Files" discussion in the "Features Shared by QWGRAF Programs" section. 


\section{Script Files}

Script files contain instructions for modifying an input data file and are generated by the QWGRAF "datmgr" program. Because the script includes the name of the original data file and keeps track of any subsequent files generated in the data modification process, a script file can be used as an input data file for any QWGRAF program. Script files are discussed in detail in the "datmgr" program section and in Appendix $\mathrm{C}$ under "The RDB System."

\section{Input Data}

QWGRAF programs operate internally on RDB files. RDB files are basically ASCII files and can be created either manually in a text editor or by macros from spreadsheet or statistical programs. Data files in a variety of other formats (ASCII flat files, NWIS-I QWDATA FLATOUT files, 1 and * cards, PSTAT files, and FORTRAN formatted files) are read and converted to RDB by QWGRAF filter programs, which are transparent to the user. The appropriate filter is activated when a program attempts to read a file whose root file name has been appended with a special three-character extension. RDB files normally have the extension ".rdb"; however, any input file with no recognizable extension is assumed to be an RDB file.

See Appendix C for a discussion of "The RDB System" and Appendix D for a discussion of "Conversion of Data to RDB."

\section{Missing Data}

QWGRAF programs support missing data. With the exception of RDB files, all data file formats supported by the QWGRAF system may use any of the following as a missing data indicator:

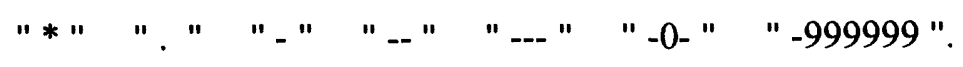

Missing data in RDB files are indicated by consecutive TABS.

\section{Censored Data}

Censored data are indicated by the presence of the "less than" $(<)$ remark code that precedes the value in the data file. With the exception of the "boxplt" and "datmgr" programs, QWGRAF programs, by default, exclude censored data from the analysis. The "boxplt" and "datmgr" programs, on the other hand, include censored data by default, as each program has an option for computing percentile values from the observed data. In the presence of censored values these programs activate a routine that simulates percentile values below the designated "detection" level. The "boxplt" and "datmgr" programs each have unique pathways to the screens used to select the method by which censored data are treated. In the other programs the censored data options are found in the "Censored (MBC)" screen accessed by first selecting the "Modify" option in the "Opening Screen" followed by the "Basic" option in the "Modify (M)" screen, and then the "Censored" option in the "Basic (MB)" screen.

\section{Data Management}

In some instances the input data file will be in a form suitable for use by a given QWGRAF program. In other cases, it may be necessary to modify the data before use, such as sorting the data or creating the normal form of cation and anion concentrations. Because RDB files are basically text files, they can be modified with text editors, statistical packages, spreadsheet packages, or by using a scripting language, such as PERL or PYTHON. To learn the complex syntax for these various approaches ion order to modify 
data files can be quite tedious. QWGRAF provides an alternative approach for modifying data files to be used with QWGRAF programs or any other programs that utilize RDB files or simple ASCII files. The QWGRAF "datmgr" program provides a robust capability to modify input data files by using the same user interface as the other QWGRAF programs. Little knowledge of $\mathrm{RDB}$ or a scripting language syntax is required to use the "datmgr" program, which provides options for sorting, filtering data rows with Boolean expressions, aggregating rows, creating new variables, selecting and reordering variables, relational joins and concatenation of data files, and row to column and column to row transformations.

The data-management process can result in the creation of either a new RDB file, a script, or both. Scripts are strings of data-management operations that can be executed to carry out the operations. Scripts are stored in special RDB files identified by the extension ".rqs" appended to the file name. Script files contain no data but rather the information necessary to find the appropriate input data file, make any required modifications, and then make the data available to the program. Thus, script files can be used as input files to QWGRAF programs.

Selected data-management functions also are provided by individual QWGRAF programs. For example, all programs have a data filtering option that allows the user to specify a Boolean expression to retain or exclude selected rows from the input data file.

Some programs also have an option for grouping (partitioning) observations on the basis of composite values of up to four partitioning variables. Each of these logical groups is then the basis of a diagram on the plot page. These grouping operations do not result in a permanent change to the input data file; only the temporary RDB file created internally for use with the program is changed.

\section{Conversion of Concentration Data to Equivalents}

One of the most common data-management operations required for using data in QWGRAF programs is the conversion of cation/anion data, which is typically reported in units of milligrams per liter, to the milliequivalent form. The "piper," "durov," and "stiff" programs are based on the assumption that the data are in the form of milliequivalents, and it is the user's responsibility to create the input data file with the data in the proper form. This can be done with a text editor and a calculator, command line use of a scripting language accessing RDB files, a spreadsheet program, or by using the facilities available in the QWGRAF system. The following scenario demonstrates the use of QWGRAF facilities to convert concentration data to milliequivalents (see discussion in Appendices C and D on the "RDB System" and "Conversion of Data to RDB," respectively, for more background information).

If the input data file is an ASCII flat file of rows and columns and has a file-name extension of either ".dta" and "ftr" as described in Appendix D, then it can be accompanied by a "format definition file" ("dtv" or "ftv" file-name extension). One of the functional features of a format definition file is the ability to define algorithms for the creation of new variables. For example, the following line added to the end of a format definition file would (1) define a new variable called "CA_MEQ," (2) declare it a "real" variable, (3) indicate it has some values remarked (censored), and (4) define an algorithm to compute its values as milliequivalents of calcium on the basis of concentration values of variable "CA."

$$
\text { CA_MEQ <dtype }>\text { real }<\text { rmk }><\text { perl }>\$ C A * 0.04990 .
$$

The variable creation process using a format definition file occurs on the fly as the file is being read (and converted to RDB) by the program. The RDB file that is created is only a temporary file that disappears when the program session ends or a new input file is opened. The original input file is not changed. 
For the other data formats that can be read by QWGRAF programs, but that do not provide the algorithm definition option (qwd, str, pst file-name extensions), the "Create" operator in the "datmgr" program can be used to create the milliequivalent form of the required variables. This process does result in the creation of a new file (or script), which then is opened by one of the other QWGRAF programs.

In either case, the algorithms can be as complex as necessary; for example, new variables may be composites of two or more variables. See Appendix F (perltbl operator) for a discussion of the Perl syntax used for algorithm development. Appendix G provides a list of commonly used equivalent conversion factors for use in the algorithms. 


\section{Getting Started}

\section{Starting the Program}

QWGRAF programs are started by typing the name of the program from the command line:
piper
prbplt
fraplt
lilifr
piper
durov
stiff
mapping
datmgr

Each program consists of several screens in which the data panel at the top of the screen is used to present options to the user. These options can be fields where program arguments are entered, or they can be triggers that tell the system to go to another screen where program arguments can be entered, or control can be passed on to still other screens.

Many of the options available to users of the QWGRAF system are common to all of the programs. These are discussed as a block in the section "Shared Features." Functions unique to each program are discussed in the appropriate program sections and are generally found in the "Modify" option accessible from the "Opening Screen" of each program.

\section{Program Operation}

There are eight steps routinely used to create plots with QWGRAF programs, but the system provides a great deal of flexibility as to which steps are used and in what order.

Step

1. Type program name

2. Load a Specifications file

3. Open an input file

4. Select variables to display

5. Modify default plot specifications

6. Make the plot on the screen

7. Make and save plot to a file

8. Save plot specifications

\section{Program Access Point}

From command line.

"Get - specifications from file" option (from "Opening Screen").

"Open - input data file" option (from "Opening Screen"). "Name - variables" option (from "Opening Screen").

"Modify compute/plot" option (from "Opening Screen").

"Make - the plot" option (from "Opening Screen"). "Device" option (from "Modify (M)" screen) followed by the "Make - the plot" option (from "Opening Screen").

"Save - current specifications" option (from "Opening Screen").

Simple exploratory plots with default options and minimal annotation can be generated by using steps $1,3,4$ and 6 or steps 1,2 and 6 . Step 2 is used only as needed and usually obviates the need for steps 3,4 or 5; however, it is permissible to modify the specifications from a loaded Specifications file. The input file (step 3) can be a data file or a script 
file. Step 5 is used to configure a plot to meet more specific and detailed needs. Step 7 is used to create plot files for use in displaying on alternate devices or for importing into drawing or word-processing programs. Step 8 involves the creation of Specifications files for use in future program sessions.

\section{Selecting Program Options}

QWGRAF screens usually present several options. An option can be either a data field for which a value must be entered, or a gateway to additional screens where program options are entered. Although there is usually a logical path from one screen to another, the system, in most cases, does not force the user to follow a particular path. In general, any option on a screen can be selected and processed in any order.

To select an option that initiates movement to a new screen, use either of the following steps:

1. Type the first letter or as many letters as required to form a unique text string (not case sensitive) of an option name. The next screen will appear directly.

or

2. Use the Tab and arrow keys to select (highlight) the option required. Then use the Accept (F2) or the Enter (Return) key to view the next screen.

\section{Entering Program Options}

Once a screen that contains fields for selecting options is displayed, use the arrow keys, the Enter key, or the Tab key to select (highlight) the option desired. A data entry field will be either blank or populated with a default value. After selecting a field, an alternative value can be entered in the field. For some options, the space bar is used to toggle the option "On," as indicated by the presence of an "X" in the field to the left of the option name, or "Off," as indicated by a "blank" field.

When selecting the list of variables from the input data file for use in a plot, the variables are usually selected in their intended plotting order by placing the appropriate sequence number in the "Order" field. The numbers need not be contiguous. A selected variable can be deselected by entering a " 0 " (zero).

When all options in a given screen are populated with appropriate values, use the Accept (F2) key to accept the selections for use in the program. All options, default or user modified, remain current throughout the program session until changed. If selected sets of program options need to be saved for use in other program sessions, then they should be saved in a "specifications" file (see the section "Program Specifications"). The system proceeds to the next screen, if applicable, or returns control to the previous screen. 


\section{Features Shared by QWGRAF Programs}

QWGRAF programs share the same consistent user interface. In addition, the programs share many common functions for setting specific program arguments. The first screen to appear after typing the program name is the "Opening Screen" and the user will notice that it is essentially the same for all QWGRAF programs. The "datmgr" program presents a slightly different "Opening Screen" that substitutes the "Basic operators" option for the "Open input data file" option. Common functions, such as selecting input files, naming variables, setting axis limits, and creating titles and footnotes, share the same procedural steps and similar screens from program to program. The pathway to these common features generally is consistent between programs. The following discussions describe the functions and associated screens common to all programs. They generally are not repeated in the individual program sections, which concentrate on the features unique to the program, but there are exceptions when minor variations in program functionality dictate clarification.

\section{The "Opening Screen"}

The first screen to appear after entering the program name is the "Opening Screen," which presents options for the following functions:

$\begin{array}{ll}\text { Open } & \text { - input file } \\ \text { Name } & \text { - variables } \\ \text { Get } & \text { - specifications from file } \\ \text { Save } & \text { - current specifications file } \\ \text { Modify } & \text { - compute/plot specifications } \\ \text { Make } & \text { - the plot } \\ \text { Query } & \text { - for general information } \\ \text { Return } & \text { - to operating system. }\end{array}$

The general procedure is to "Open" a data file, "Name" the variables to be used, "Modify" the default plot specifications, and "Make" the plot. The alternative is to use (and possible modify) a "Specifications file and then "Make" the plot. Type in the first one or two characters of the function name to bring up the next screen, or use the Tab and arrow keys followed by the Accept (F2) key to select a function.
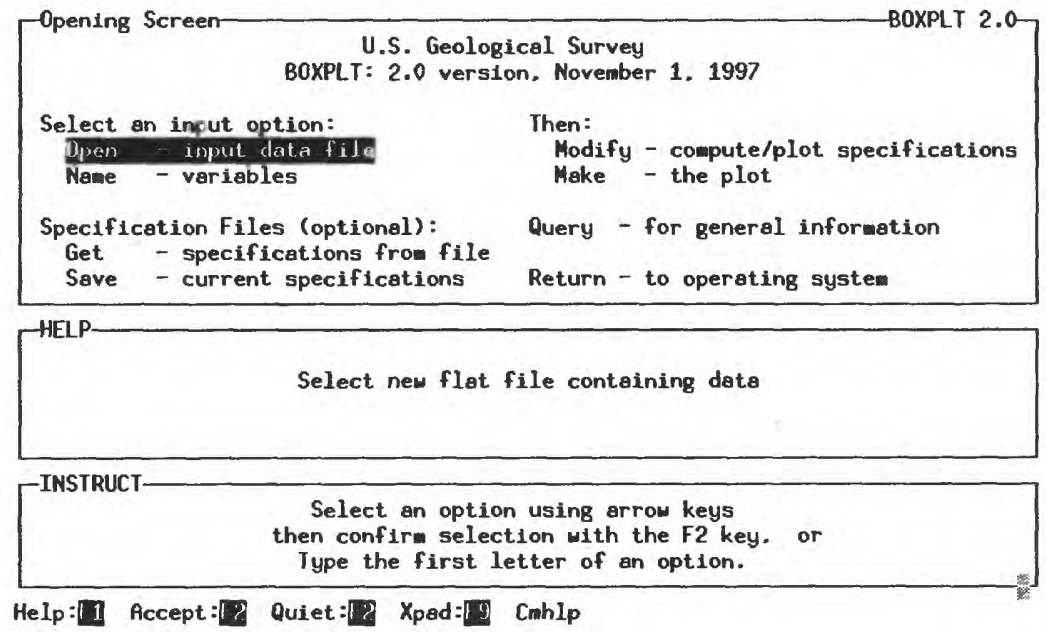


\section{Open Input Data File}

The "Opening Screen" of each QWGRAF program has an option for "Open input data file." Use the arrow keys to select this option and then use Accept (F2) or the Enter key. The "Open (O)" screen lists an option for selecting a data file and another option for filtering the data before use in the program. Select the "Select a data file" option to bring up the "Select (OS)" screen.

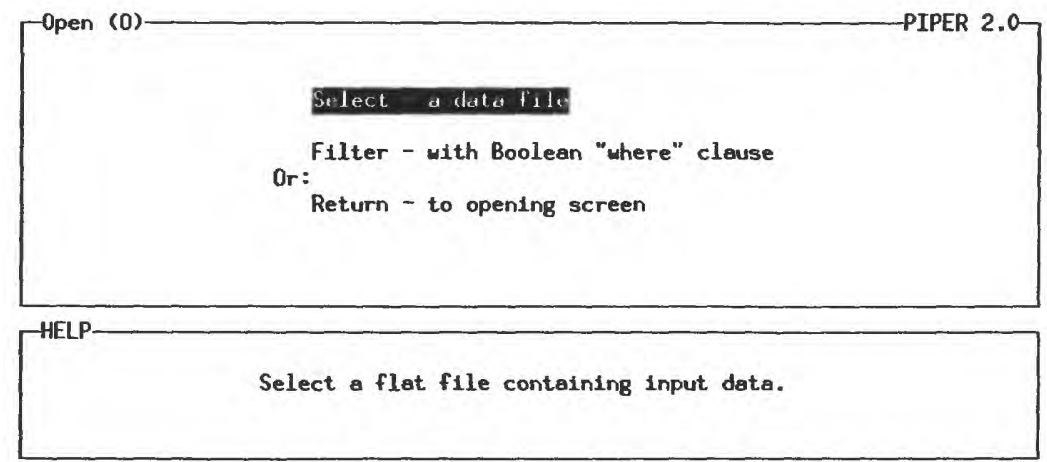

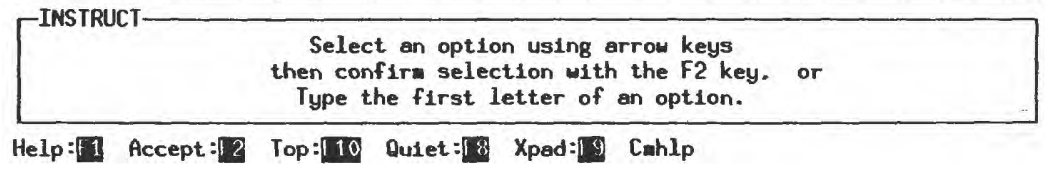

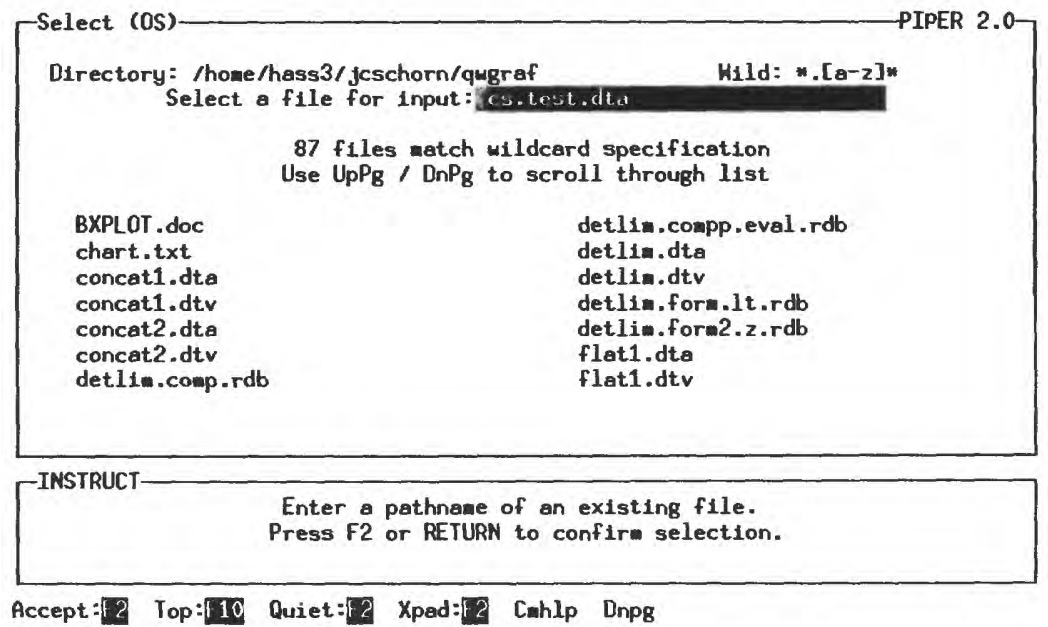

In the "Select (OS)" screen, check the "Wild" option in the upper right corner to ensure that the wildcard specification presents the files of interest. The default is to show all files in the directory. If necessary, edit this option by using the "regular expression" syntax of your system to create a new wildcard search criteria set. Press Enter and a new list of files will appear. Some examples of wildcard search criteria for files are as follow:

$\underline{\text { Regular Expression }}$

$$
\text { *. }[\mathrm{a}-\mathrm{z}]^{*}
$$

\section{Will Present on the Screen}

All files whose name includes a period (".") followed by any alphabetic character. 


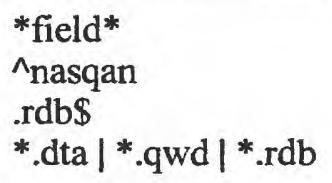

All file names that contain "field".

All file names that begin with "nasqan".

All file names that end in ".rdb".

All ".dta" or ".qwd" or ".rdb" files (the "|" acts as an "or" operator).

If an alternative directory is required, then use the arrow keys to select the "Directory" option and edit this field. Press Enter and a new list of file names appears.

Either type in the file name or use the arrow keys to select the file required. Use either Accept (F2) or the Enter key to accept the selection and return control to the "Opening Screen."

\section{Filter Input Data}

Once the input data file has been selected, the user can select the "Filter" option from the "Open (O)" screen, which provides for specifying a Boolean logic expression to include or exclude selected rows from the active data file. This action does not change the input data file permanently, but results in the creation of a temporary file used by the program. As with any other option, the "Filter" option can be returned to as many times as necessary during a given program session to create new variations of the input data file. Note that each time the "Filter" option is activated the search criteria is applied against the original data file, not the current temporary file. New search criteria will produce a new temporary file.

\section{Retain or Exclude Rows Meeting Search Criteria}

From the "Open (O)" screen, select the "Filter (OF)" screen. Use the arrow keys to select the "Filter Type" option and then use the space bar to toggle on either "Exclusion" or "Retention," as indicated by the appearance of an "X" in the field to the left of the option name. If "Exclusion" is selected, then rows that meet the criteria specified in the "Criteria" box will be excluded from the working data file; selection of the "Retention" option will result in the rows that meet the criteria being retained in the working file.
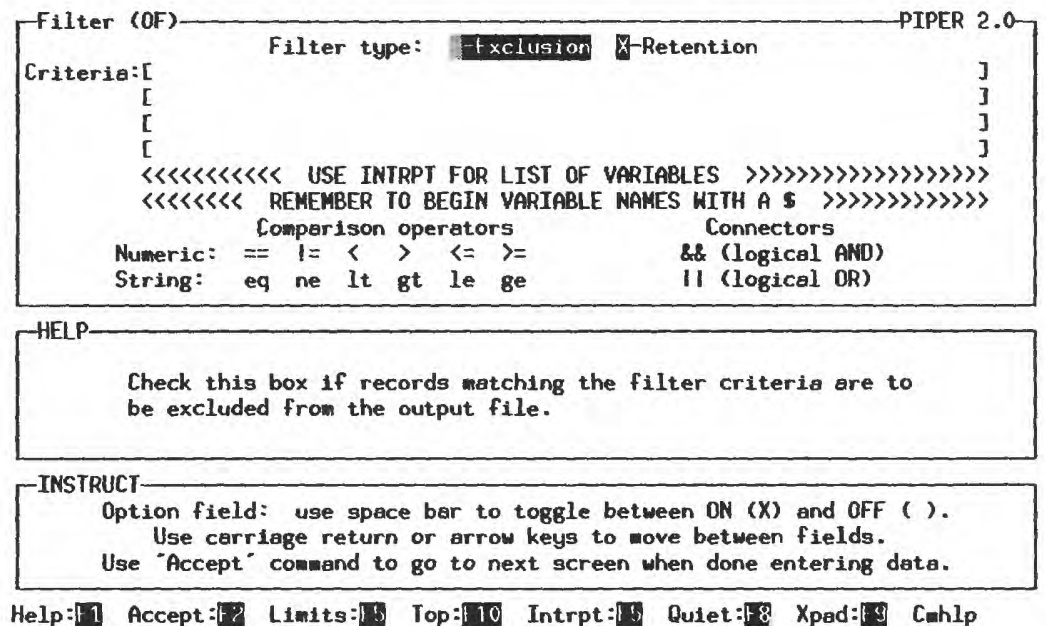


\section{Specify Search Criteria}

Use the arrow keys to select the "Criteria" option. Details of the syntax used to specify the search criteria are given in the "FILTER DATA ROWS" section of the "datmgr" program discussion. Begin each variable name with a " $\$$ " and precede function names with an "\&." Use of operators and the "II" (OR) and "\&\&" (AND) logical connectors in combination with the available RDB functions permits complex search criteria to be specified based on the values of the variables in the input data file.

$$
\text { Example: } \$ C A L C I U M>=5.0 \& \& \text { SSTCODE }=42 \text {. }
$$

When finished, use Accept (F2) to accept the criteria and return to the "Opening Screen."

For details on the syntax to be used in specifying the search criteria, see the individual program documentation and Appendix C on "The RDB System," Appendix D on "Converting Data Formats to RDB," and Appendix F on the "Perltbl" operator. See also the textbook Programming Perl (Wall and Schwartz, 1991).

\section{Name (Select) Variables}

After the input data file has been selected (and filtered, if necessary), the "Name variable" option in the "Opening Screen" is highlighted. Use (F2) or the Enter key to accept this option. The form of the next screen to be displayed will depend on the number of variables that the program can use and whether the data can be partitioned into groups for the purpose of creating a diagram for each group. If the program requires just a simple list of one or more variables, then the next screen to appear is the "Name $(\mathrm{N})$ " screen, which lists the variables in the input data file. To select a variable, enter a "1" in the "Select" column opposite the variable name. For more than one variable, enter sequential numbers in the order the variables are to be presented. Deselect a variable by entering a " 0 " (zero).

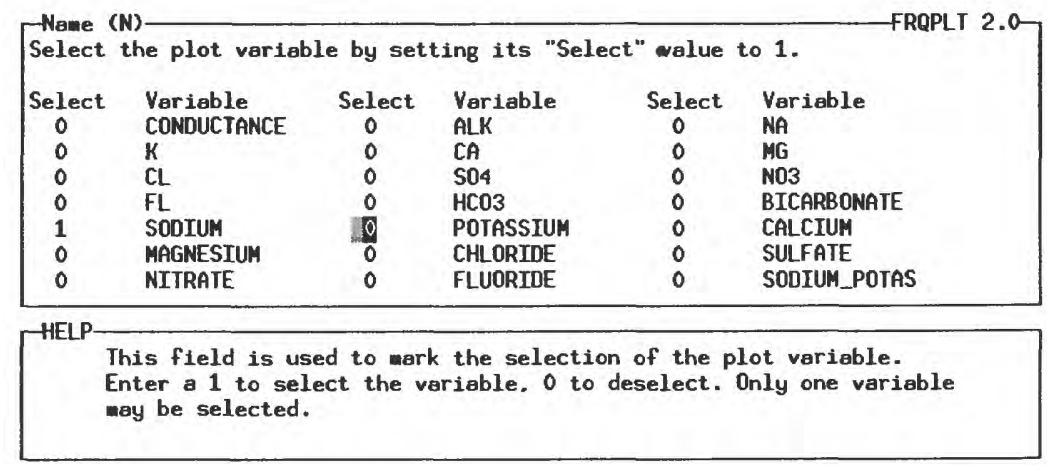

\footnotetext{
INSTRUCT- Enter data in highlighted field(s).
Use carriage return or arrow keys to enter data and wove between fields.
Use "Accept" comand to go to next screen when done entering data.
}

The "prbplt" and "boxplt" programs can produce a diagram for each of one or more individual variables or a single variable can be the basis of several diagrams, where each diagram represents a partitioned subgroup of values of the designated variable. For these programs the "Name $(\mathrm{N})$ " screen presents the following options:

Individual - Select one or more variables.

Partition - Group data by a partition variable. 


\section{Individual Variables}

Selection of the "Individual" option brings up the "Individual (NI)" screen, which lists the variables in the data file. Use the Tab and arrow keys to select a variable for each diagram to be plotted. Select the variables in the order they are to be plotted by entering the appropriate sequence number in the "Order" column. A variable can be deselected by entering a "zero $(0)$ " in the field.
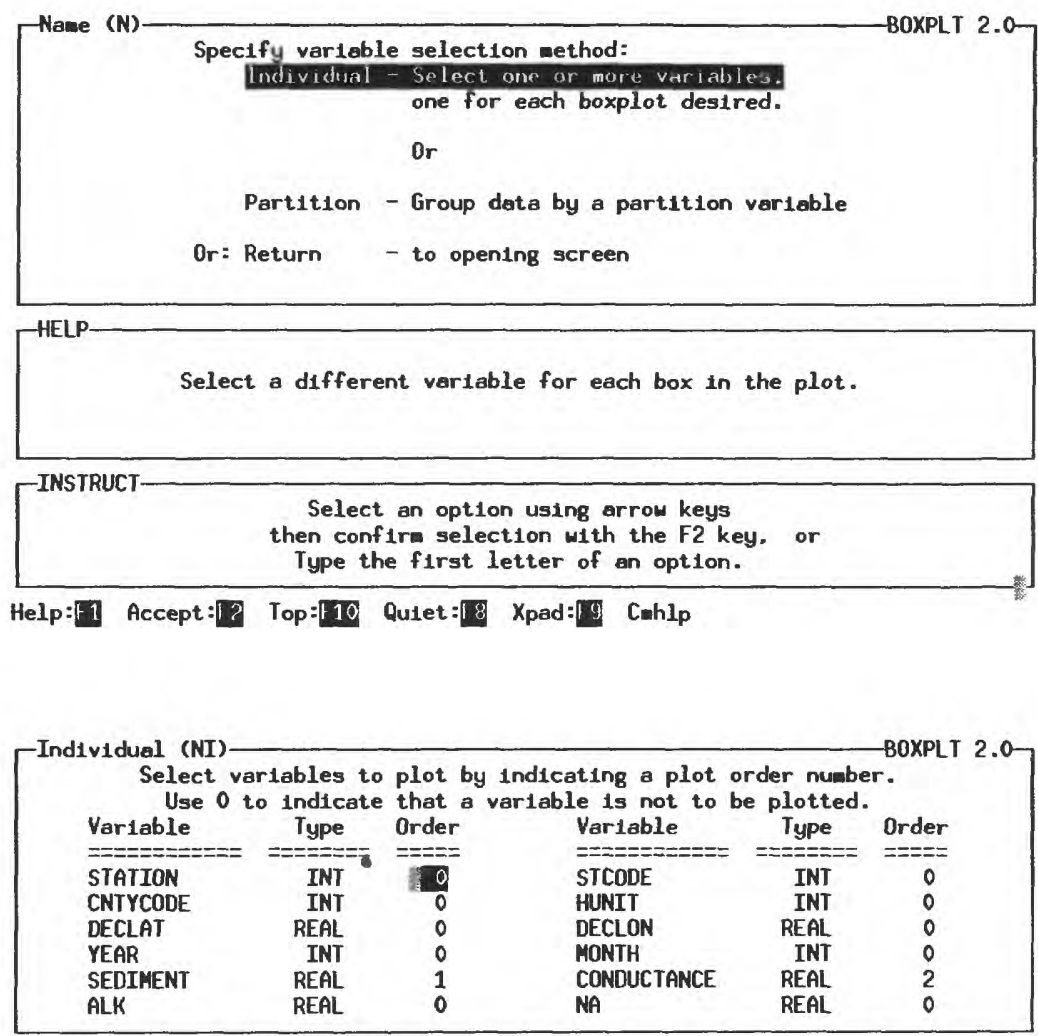

This field indicates plotting order from left to right.
Zero indicates that the variable is not to be plotted.

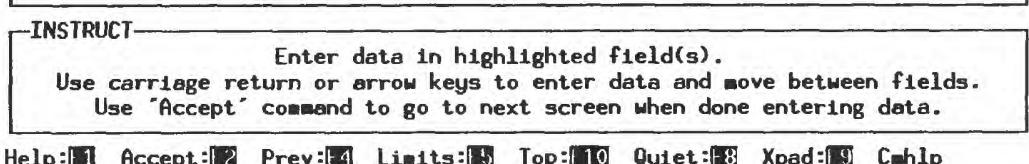

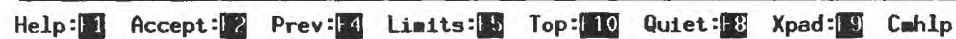

\section{Variable Sets (Varsets)}

If the program provides for establishing one or more sets of variables, such as the cations and anions used in piper, durov, and stiff diagrams, then the "Name (N)" screen will present the "Varsets" option instead of the "Individual" option. Selection of the "Varsets" option brings up the "Varsets (NV)" screen and presents options for adding, editing, deleting, or clearing sets of variables. Normally, only one varset is required, but QWGRAF provides for as many as required. A diagram is produced for each variable set. Multiple diagrams can be placed on a plot page and multiple plot pages can be used. 
From the "Name (N)" screen, select the "Varsets" option to display to the "Varsets (NV)" screen. The following options are listed:

$\begin{array}{ll}\text { Add } & \text { - a variable set } \\ \text { Edit } & \text { - a variable set } \\ \text { Delete } & \text { - a variable set } \\ \text { Clear } & \text { - all variable sets } \\ \text { Return } & \text { - to previous menu. }\end{array}$

From the "Varsets (NV)" screen, either type "A" (or "a") or use the arrow keys to select the "Add a variable set" option. Use the Enter key to display the "Add (NIA)" screen. This screen may be used as many times as necessary to create the number of variable sets needed, one set for each diagram.

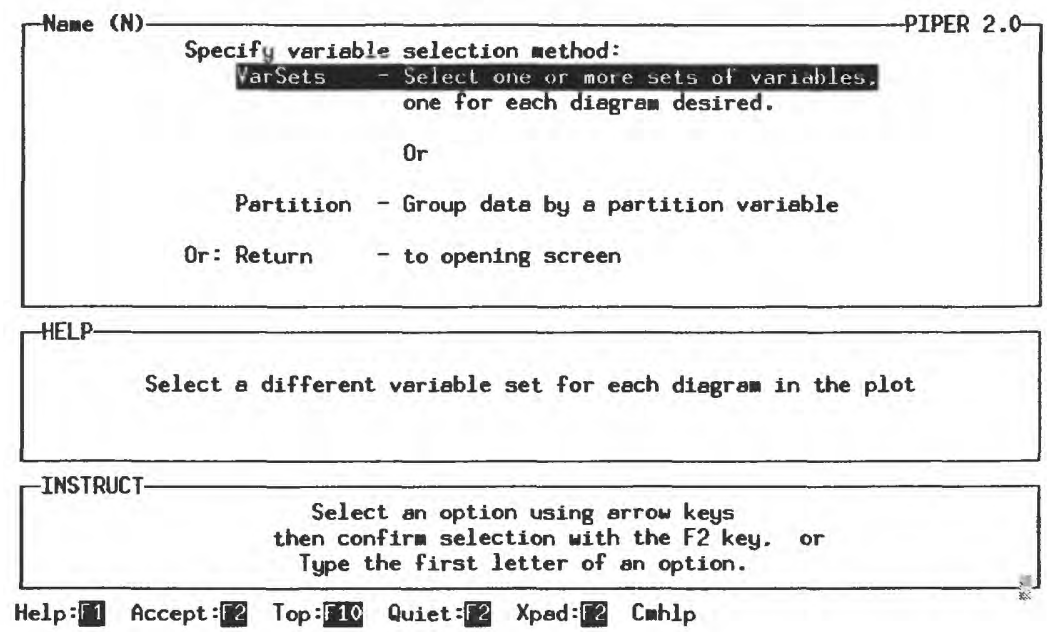

\section{Add a Variable Set}

The "Add (NIA)" screen lists the variables contained in the input data file. The variables should be selected in the order to be presented in the diagram. Use the Enter and arrow keys to select a variable and place the appropriate sequence number in the "Order" field. Deselect a variable by entering a " 0 " in the "Order" field.

The variable name fields also can be edited to produce a more suitable label for the variable on the diagram. Highlight the field and type in the new name (12 characters or less). Note: This does not change the name of the variable in the data file, only the label used on the diagram. When finished, use Accept (F2) to return control to the "Individual (NI)" screen.

\section{Edit a Variable Set}

A variable set can be edited during the current session. From the "Individual (NI)" screen, type "E" (or "e") or use the arrow keys to select the "Edit a variable set" option and use the Enter key. The user is prompted for the number of the variable set to edit if there is more than one variable set. Make the necessary changes to the variable list and use Accept (F2) to accept the changes.

\section{Delete a Variable Set}

Unneeded variable sets can be deleted with the "Delete a variable set" option. From the "Individual (NI)" screen, type "D" (or "d") or use the arrow keys to select the "Delete a variable set" option and use the Enter key. From the "Delete (NID)" screen the user is 
prompted for the number of the variable set to delete. Use the Enter key to delete that variable set and return control to the "Individual (NI)" screen. If you elect not to delete a variable set, then blank out the number field before using the Enter key.

\section{Clear All Variable Sets}

To delete all defined variable sets, type "C" (or "c") or use the arrow key to select the "Clear all variable sets" option and use the Enter key. Use the "Return to the previous menu" function to return control to the "Name $(\mathrm{N})$ " screen.

\section{Change Variable Names}

For those programs where the variable names are used as labels (boxplt, piper, durov, stiff), the names can be changed for presentation purposes. Select the field in the "Label" column and replace the default label (the input variable name) with a preferred label (12 characters or less). This does not change the name of the variable in the data file; it changes only the form of the name used as a label by the program.
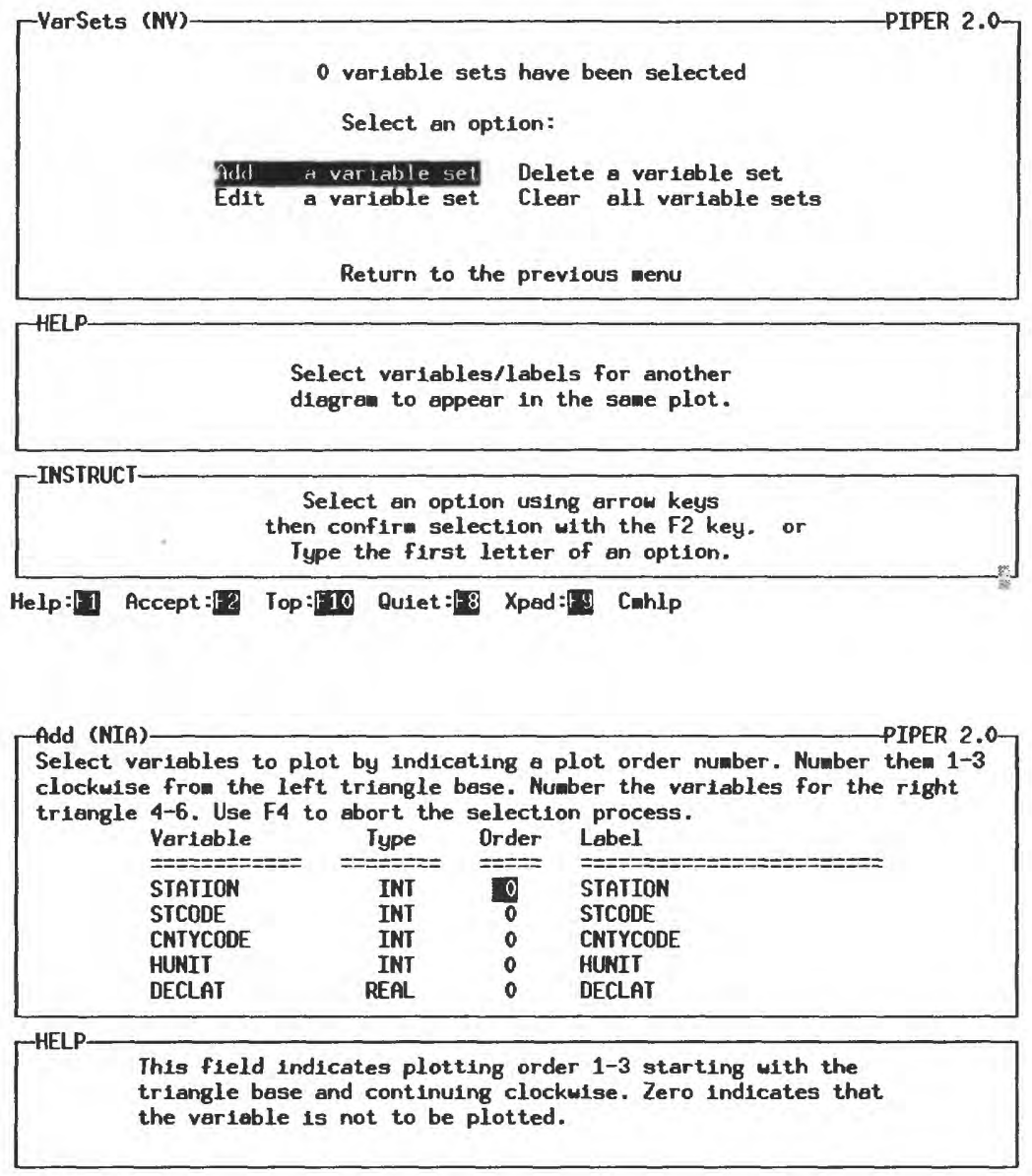

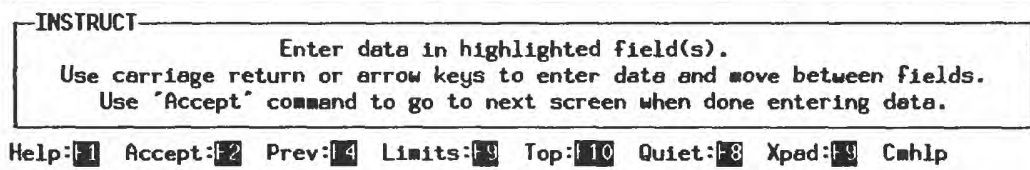




\section{Partitioned Variables}

The alternative to creating a single diagram for each variable selected is to create a diagram for each subset of values from a single variable based on composite values of up to four "Partitioning" variables; for example, create boxplots of magnesium by State and by year.

From the "Name (N)" screen, select and bring up the "Partition (NP)" screen. The "Partition (NP)" screen lists the following options:

Select:

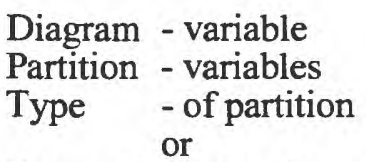

Return to the previous menu.

Type the first letter of the option (not case sensitive) to view the required option screen or use the arrow keys to select the option and use the Enter key.

\section{Select Variable to be Diagramed}

The "Diagram variable" option brings up the "Data (NPD)" screen, which lists the variables in the input data file. Use the arrow keys and the Enter key to select the variable to be represented in the diagrams. Place a " 1 " in the appropriate "Select" field. A variable can be deselected by entering a " 0 " (zero).

The variable name field can be edited to produce a more suitable label for the variable on the diagram. Highlight the field and type in the new name (12 characters or less). Note: This does not change the name of the variable in the data file, only the label used on the diagram. Use Accept (F2) to return to the "Partition (NP)" screen.

\section{Select Partition Variables}

The "Partition variable" option brings up the "Partition (NPP)" screen, which lists the variables in the input data file. Use the arrow keys and the Enter key to select up to four variables. Each unique composite value of these variables forms the basis of a diagram. Nest the variables in appropriate order by entering 1, 2,3, or 4 in the "select" column field to the left of the appropriate variable names. For example, STATE would receive a value of " 1 " and COUNTY a value of " 2 ." A variable can be deselected by reentering a "0" (zero).

Use Accept (F2) to return to the "Partition (NP)" screen.

Note: The selection of more than one partitioning variable is only appropriate if the

"Automatic partitions" option is selected in the "Type (NPT)" screen.

\section{Establish Interval Boundaries for Partitioning}

The "Type of partition" option brings up the "Type (NPT)" screen, which is used to select the method by which the values of the partition variable(s) are used to group the values of the data variable. There will be one diagram for each group.

The methods are as follow:

$$
\begin{array}{ll}
\text { Automatic } & \text { - partitions } \\
\text { Manual } & \text { - partitions. }
\end{array}
$$




\section{Automatic Partitions}

From the "Type (NPT)" screen, select the "Automatic partition" option if an interval is to be created for each unique value of a single partition variable or for each unique composite value of up to four partition variables. Text and real variables can be chosen as multiple partition variables.

In most cases, the unique values of the partitioning variables adequately define the number of intervals. However, it is possible to extract a higher level partitioning from the partition variables. For example, in the case of the Hydrologic Unit Code, there are four accounting levels imbedded in the eight-digit integer number. If unique values of the entire number are used with the "Automatic partition" option, then the intervals would be based on the Hydrologic Cataloging Unit. By using a special extension of the "Automatic partition" option, it is possible to have the interval based on Hydrologic Region (the first two digits of the code), Hydrologic Subregion (the first four digits in the code), or the Hydrologic Accounting Unit (the first six digits in the code).

Acceptance of the "Automatic partition" option (use Accept (F2)) brings up the "Auto (NPTA)" screen in which an integer divisor is specified that is used by the system to truncate the partition variable to form the higher level partitioning factor. For example, in the Hydrologic Unit Code case, use 1000000 for Region; 10000 for Subregion; and 100 for Accounting Unit. A divisor may be specified for each of the partition variables. After entering a divisor(s), if any, use Accept (F2) to return control to the "Partition (NP)" screen.

\section{Manual Partitions}

From the "Type (NPT)" screen, select the "Manual (NPTM)" screen. Use the arrow keys and the Enter key to enter minimum and maximum values of the partition variable for as many intervals as required. The intervals need not be contiguous and may overlap. Use Accept (F2) to return control to the "Partition (NP)" screen. Select the "Return to previous menu" option to return to the "Opening Screen."

Note: The "Manual" option is only applicable when a single partition variable has been selected.
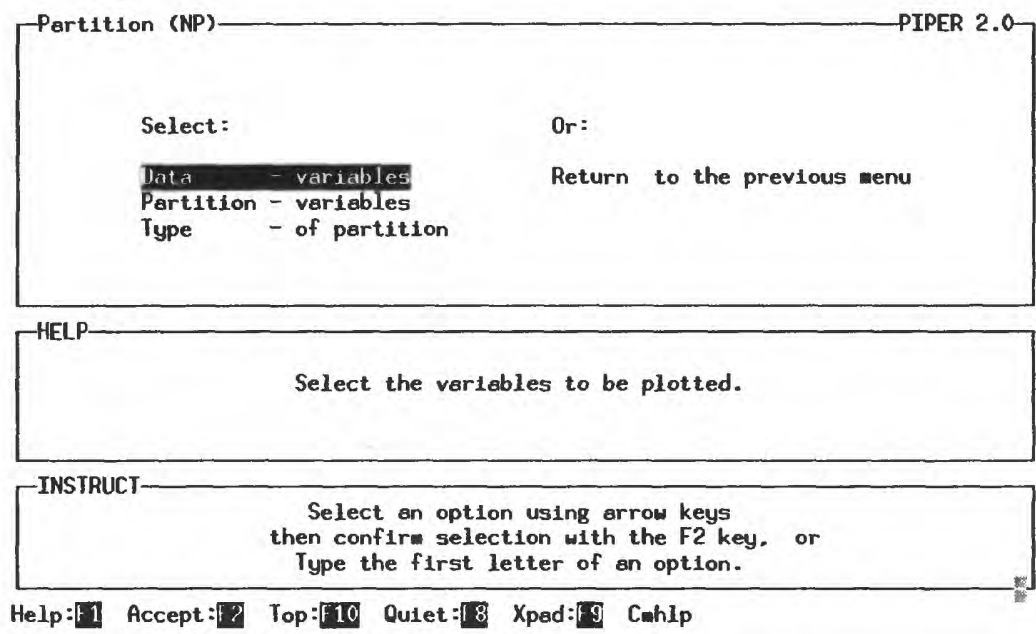

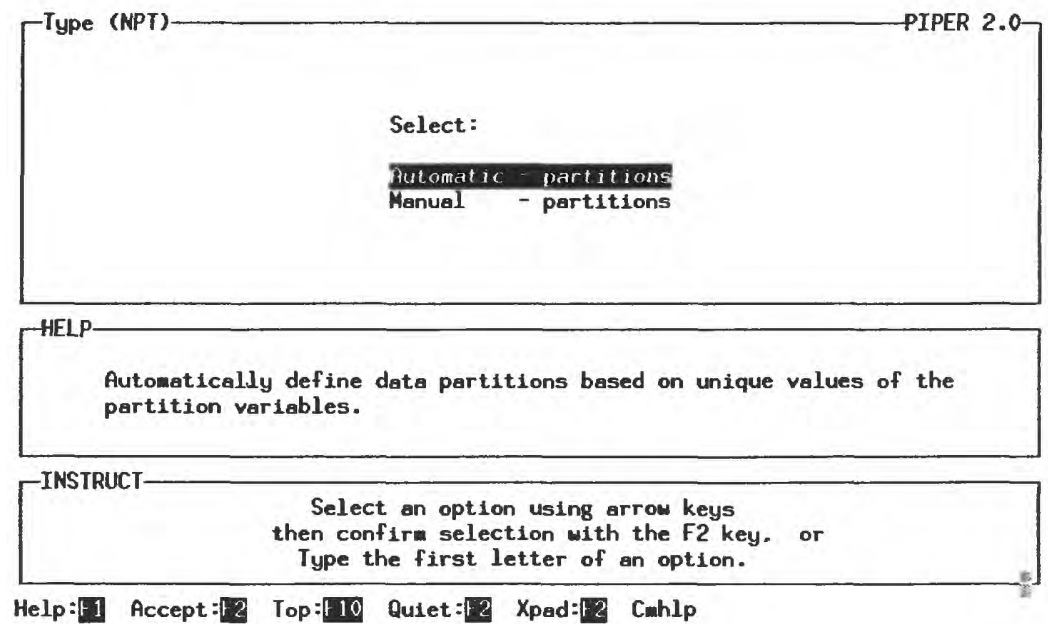

Help:t1 Accept: 12 Top: 10 Quiet:
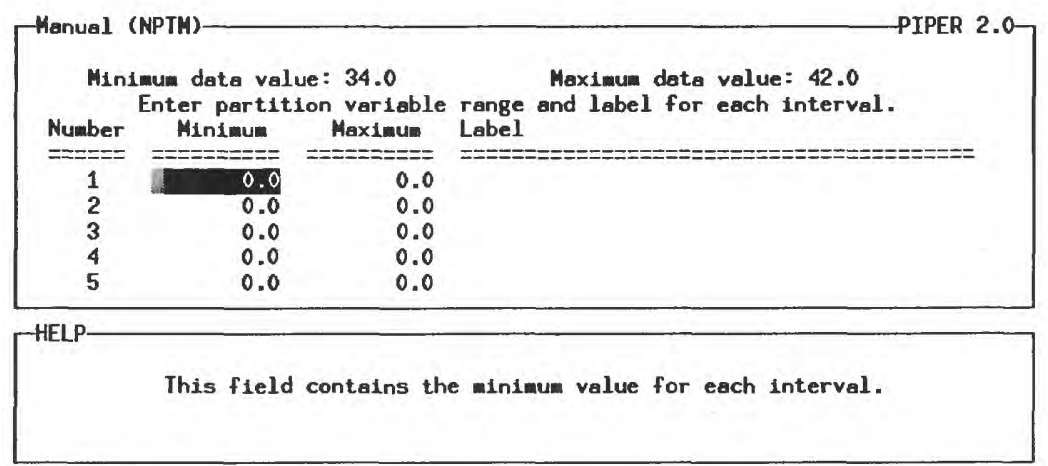

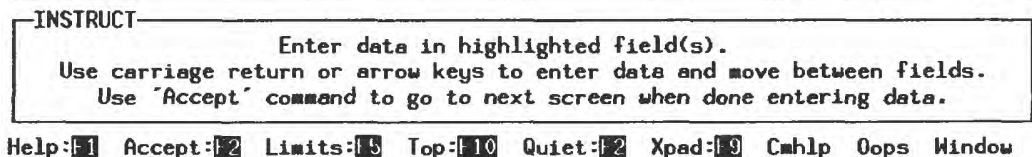

\section{Program Specifications}

\section{System Default Program Specifications}

For exploratory plots, the user needs only to (1) select an input data file; (2) name and order the variable(s) to be used; and (3) select the "Make the plot" option from the "Opening Screen" to execute the program. System default arguments are used for program variables unless alternatives are entered by the user, either manually or by loading a specifications file.

\section{Set Program Specifications}

QWGRAF programs provide many options for specifying program specifications, such as plot types, variable selection, line types, symbols, titles, labels, and axes. There is no set path through the programs, and the user can navigate the option tables in any direction and as many times as needed to configure the program to produce the required output. Any option not specified by the user utilizes a system default value. 


\section{Modify Program Specifications}

After a QWGRAF program has been run with a given set of specifications, selected specifications can be changed and the program run again. This process can be repeated as often as necessary to produce the required plot variations.

\section{Program Specifications Files}

QWGRAF programs provide for saving and reusing sets of program specifications in files that can be recalled at any time during any QWGRAF session (see section below on "Saving Program Specifications Files"). Specifications files can save time in setting up and reusing complex program scenarios. Thus, as an alternative to manually entering program specifications as described above, the user can select the "Get specifications from file" option in the "Opening Screen" to load a set of previously saved specifications. These specifications include the name of the input data file in use at the time the specifications file was saved. The user can then invoke the "Make the Plot" option (see subsequent discussion) to execute the program. Loaded specifications can be changed at any time. Once a specifications file has been loaded and any modifications made, the new set of specifications can be saved as a new file or it can overwrite an existing specifications file, which includes the file just loaded.

\section{Save Program Specifications Files}

To save the current set of specifications, select the "Save specifications file" option in the "Opening Screen." The "Save (S)" screen is brought up and a file name (and pathname, if necessary) is entered into the highlighted field. The system appends a program's unique three-character extension to the file name.

There are three types of specifications files: Latest, User-Defined, Default.

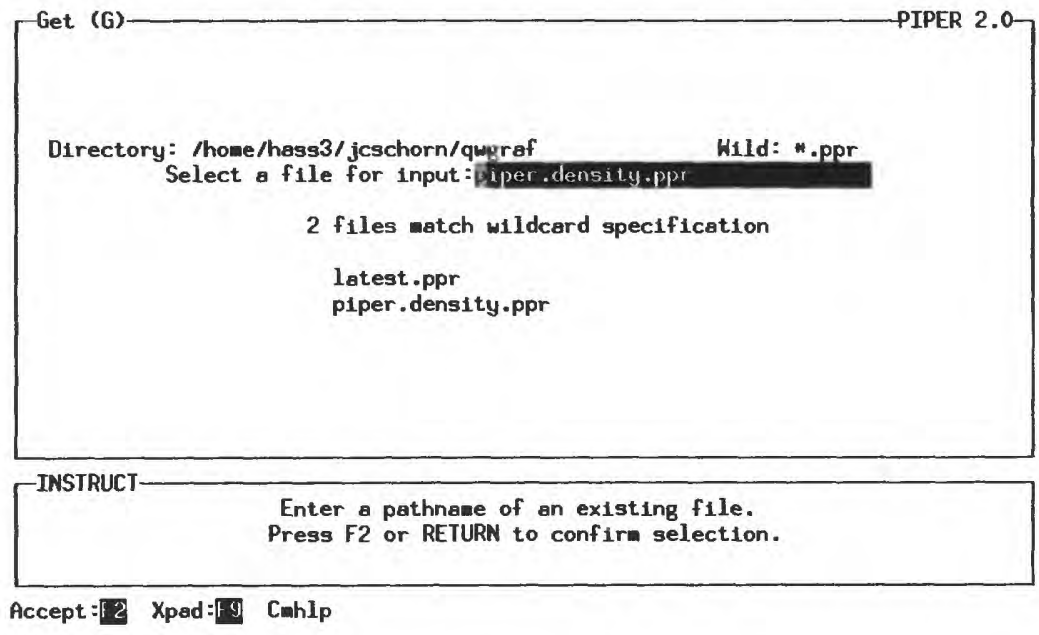

\section{"Latest" Specifications File}

Upon execution, a QWGRAF program saves the "latest" set of specifications in a specifications file of the form latest.ppr, latest.prb, or latest.mpp. Thus, the user can invoke the program and recall the last set of specifications used for the program. The "latest" file is a safety valve in case the user fails to save a "user-defined' specifications file. 


\section{"User-Defined" Specifications File}

Any number of "user-defined" specifications files can be created for a given QWGRAF program and the "Save specifications file" option can be selected at any time during a program session, even multiple times if different sets of specifications are to be saved. User-defined specifications files would have the form user_file_name.bxp, user_file_name.ppr, or user_file_name.mpp. The system supplies the appropriate threecharacter extension when saving the file.

\section{"Default" Specifications File}

If the user wants a specific set of specifications loaded each time a given program is selected, then there is a third type of specifications file available. The user creates specifications and then simply names the specifications file "default." The program appends the appropriate three-character extension for the program; for example, default.frq, default.prb, default.stf. Each program automatically looks for a "default" file each time it is invoked. If a default file is not located (it is not required to have one), then a file of system defaults is loaded.
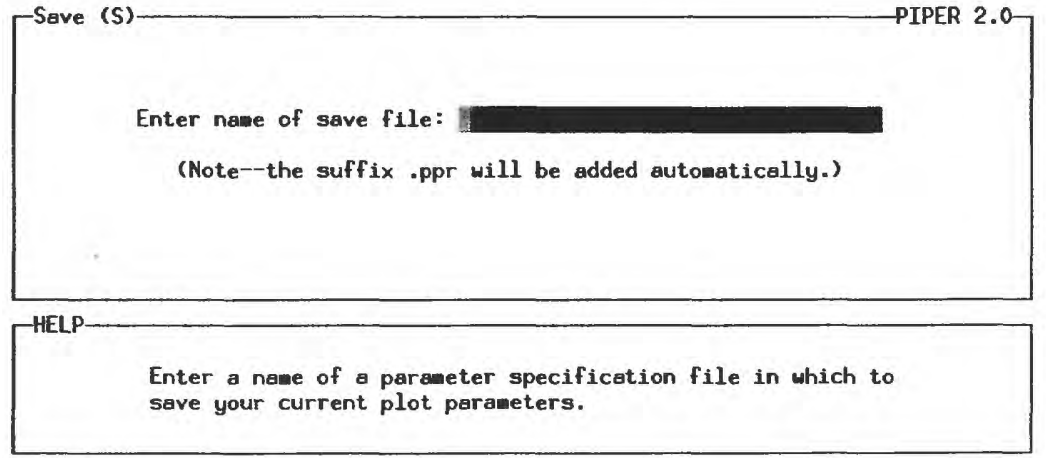

TINSTRUCT- Enter data in highlighted field(s).

Use carriage return or arrow keys to enter data and wove between fields.

Use "Accept" comand to go to next screen when done entering data.

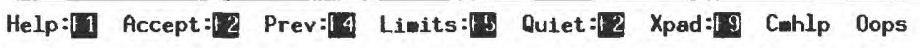

\section{Select the Output Device}

The "Device" option, which is found in the "Modify (M)" screen, allows the user to select the output device for presenting the graphic. The screen is the default device. Selection of an output file rather than the "screen" activates another screen that requests the user to supply a file name (a pathname can be included in the file name). The file types supported by QWGRAF programs include "postscript," "hpgl," and "cgm."

\section{Postscript Files}

Postscript files are generally used for printing on graphics supported printers such as laser printers. A postscript file can be converted to an "encapsulated" postscript file, which can facilitate the importing of the graphic into certain drawing and word-processing programs, but not FrameMaker. Utilities are available to read postscript files and present the graphic on the screen. See the "README" file supplied with the program for information on utilities applicable to your platform. 


\section{Postscript Files in Landscape Mode}

Utilities may be available for converting a portrait mode postscript file to landscape mode. See the "README" file supplied with the program for information on utilities applicable to your platform. When outputting the graphic to a postscript file, the "page_height" and "page_width" options in the "Page (MBP)" screen might have to be adjusted to obtain the desired size and position of the plot on the plot page.

\section{Postscript Color Files}

When the graphic output to a postscript file includes color, it will be necessary to modify the TERM.DAT file so that the appropriate color related code is included in the file. If a TERM.DAT file has not been created in the current directory, then create one with an editor and add the appropriate lines of code. See the "README" file supplied with the program for information on the "GKSPRT" keyword parameter that controls color for postscript files.

\section{"HPGL" Files}

An "hpgl" file can be used for directing the plot to an on-line plotter as well as importation to drawing and word-processing programs, including FrameMaker. See Appendix $G$ for an explanation of how to import "hpgl" files into FrameMaker documents.

\section{"HPGL" Color Files}

When the graphic output to a "hpgl" file includes color, it will be necessary to modify the TERM.DAT file so that the appropriate color related code is included in the file. See the "README" file supplied with the program for information on the "GKSPLT" keyword parameter that controls color for "hpgl" files.

\section{"CGM" Files}

"cgm" files can also be imported into drawing and word-processing programs. See Appendix G for an explanation of how to import "cgm" files into FrameMaker documents.
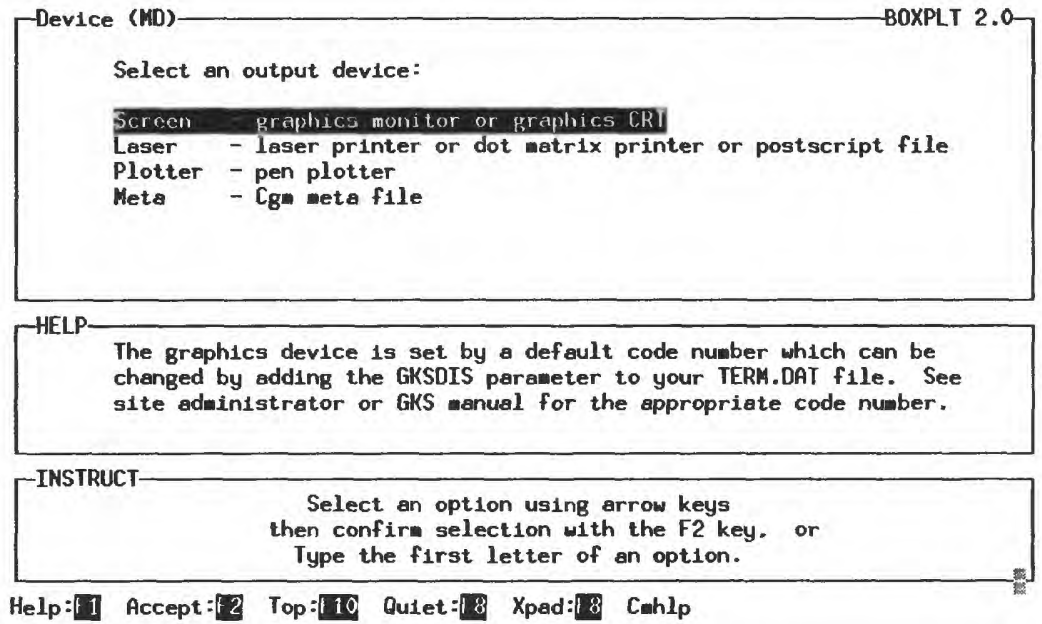
The "Make plot" option, usually found in the "Opening Screen," but also found in selected other screens, is used to formally submit the program for execution. When this option is selected, the plot is generated on the device specified at that time. The plot can be regenerated on an alternative device (screen or file) without resetting any of the specifications as they remain active until they are changed during the current session of the program.

\section{Common Plot Configuration Options}

The following options relate to the general configuration of a plot, such as titles, axes, labels, and so forth, and are generally available in all QWGRAF programs. The gateway to the screens that sets the specifications for these options, as well as those options specific to a given program, is through the "Basic" option found in the "Modify (M)" screen. The "Page" option from the "Basic (MB)" screen is also a gateway to selected - page layout options, such as page size, borders, and diagram placement.
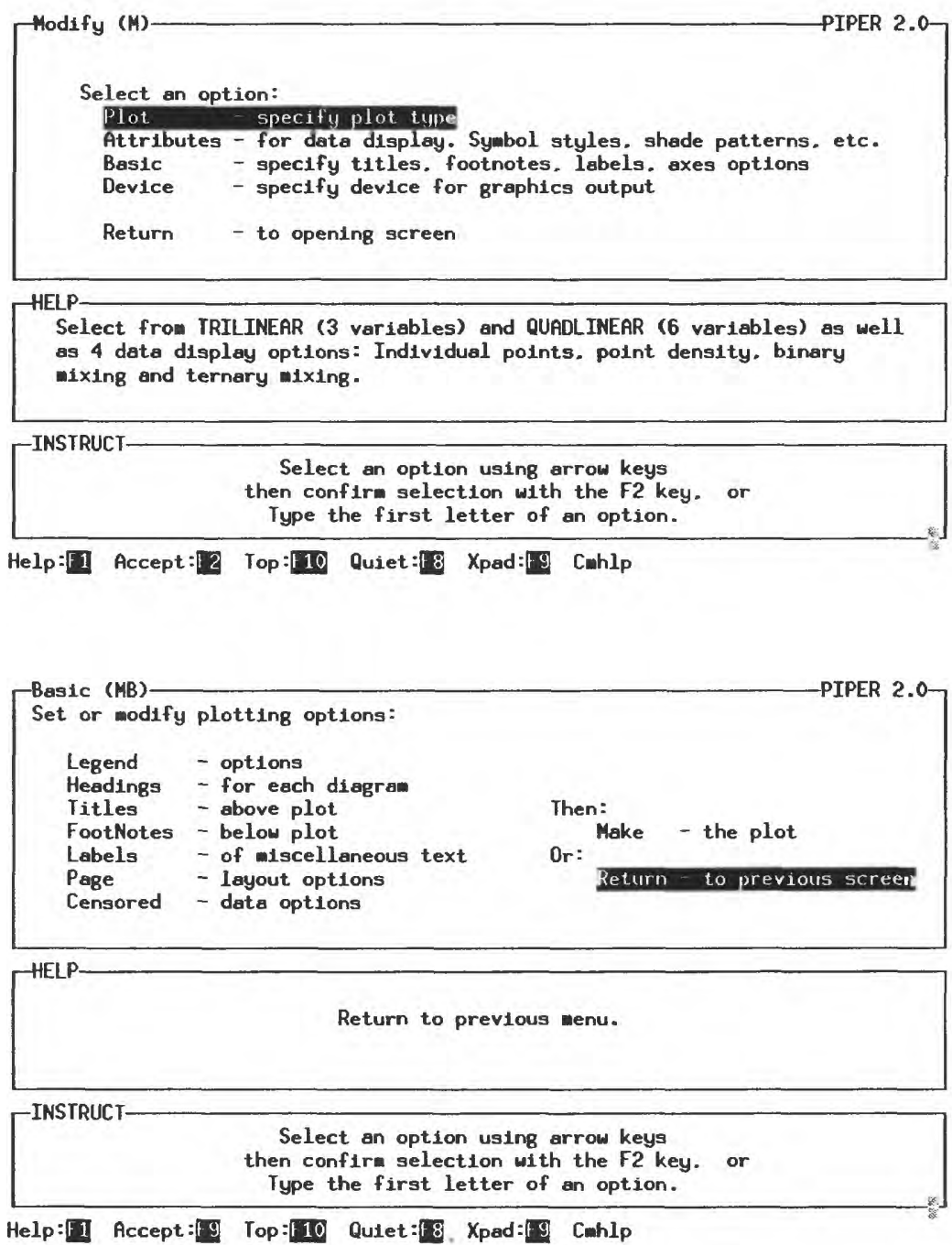
Legends

For those programs where multiple symbols and lines can be presented in a given diagram, legends (explanation blocks) relate the symbol and line characteristics (type, size, color) presented in the plot to the variables and (or) the values of the variables they represent. Use the Enter and arrow keys followed by using the space bar to toggle the desired option "On" as indicated by the appearance of a "X" in the selected option field.

The "Legend" option in the "piper" program is more involved than in the other programs and is discussed in more detail in the piper program documentation. Unlike the other programs, the piper "Legends" option is accessed directly from the "Basic (MB)" screen.

\section{Titles}

The "Titles" option provides for specifying up to three titles to be placed above the plot. From the "Basic (MB)" screen, type."T" (or "t") or use the arrow keys to select the "Title (MBT)" screen. The "Title (MBT)" screen provides options for specifying the length, text justification, text size, text color, and text font.

Text

A title may be up to 80 characters.

\section{Text Justification}

Titles are centered by default. To left justify a title, preface text with a " $<$ " sign. To right justify a title, preface text with a ">" sign.

\section{Text Size}

Use the arrow keys to select the text size specification field for each title. Enter the text size in decimal inches.

\section{Text Color}

Use the arrow keys to select the text color specification field for each title. Enter a color code for each title. Use Help (F1) to bring up a list of colors and associated color codes.

\section{Text Font}

Use the arrow keys to select the text font specification field for each title. Enter a font code for each title. Use Help (F1) to bring up a list of fonts and associated codes.

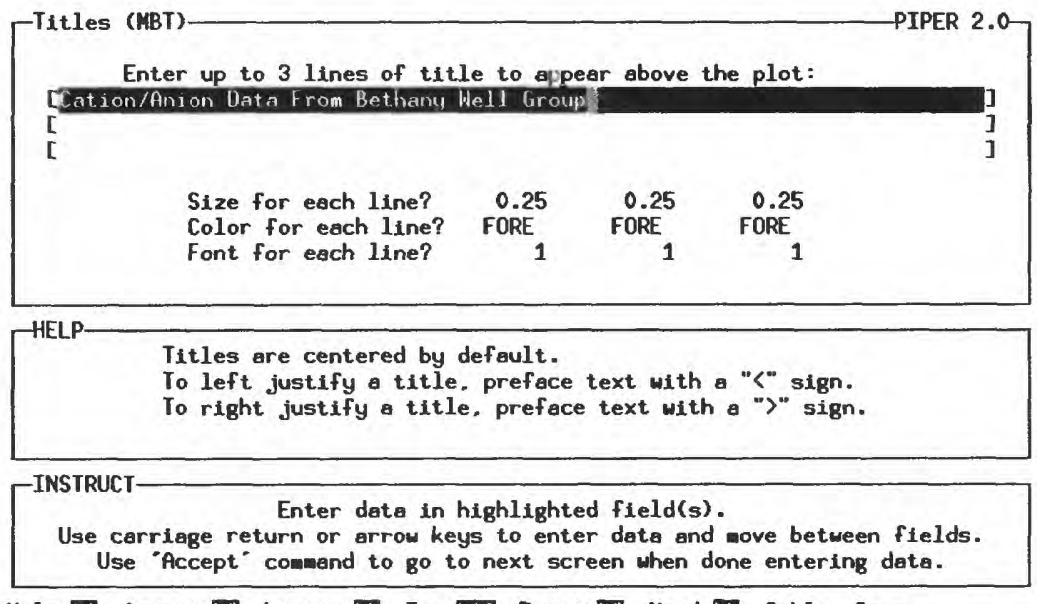

Help: 1 Accept: 12 Limits: 
Footnotes

The "Footnotes" option provides for specifying up to two footnotes to be placed below the plot. From the "Basic (MB)" screen type "F" (or " $\mathrm{f}$ ") or use the arrow keys to bring up the "Footnote (MBF)" screen. The "Footnote (MBF)" screen, which is similar to the "Title (MBT)" screen, provides options for specifying the footnote length, footnote text justification, footnote text size, footnote text color, and footnote text font.

Text

A footnote may be up to 80 characters.

Text Justification

Footnotes are centered by default. To left justify a footnote, preface the text with a "<" sign. To right justify a footnote, preface the text with a " $>$ " sign.

\section{Text Size}

Use the arrow keys to select the text size specification field for each footnote. Enter the text size in decimal inches.

\section{Text Color}

Use the arrow keys to select the text color specification field for each footnote. Enter a color code for each footnote. Use Help (F1) to bring up a list of colors and codes.

\section{Text Font}

Use the arrow keys to select the text font specification field for each footnote. Enter a font code for each footnote. Use Help (F1) to bring up a list of fonts and codes.

\section{Labels of Miscellaneous Text}

From the "Basic (MB)" screen, select the "Labels (MBL)" screen to enter up to five labels that can be placed at user-defined coordinates on the plot. The labels can be up to 40 characters long and each can be broken into two or more lines by inserting carets (" $\wedge$ ") into the text. The "X" and "Y" coordinates of the lower left corner of the text block are specified in decimal inches relative to the lower left corner of the plot page (not the plot frame), which has coordinate values of 0.0 and 0.0 , respectively. Enter text size, color, font, and orientation angle (counterclockwise from the horizontal). Use the Enter and arrow keys to select the appropriate field. Use Accept (F2) to return control to the "Basic (MB)" screen.
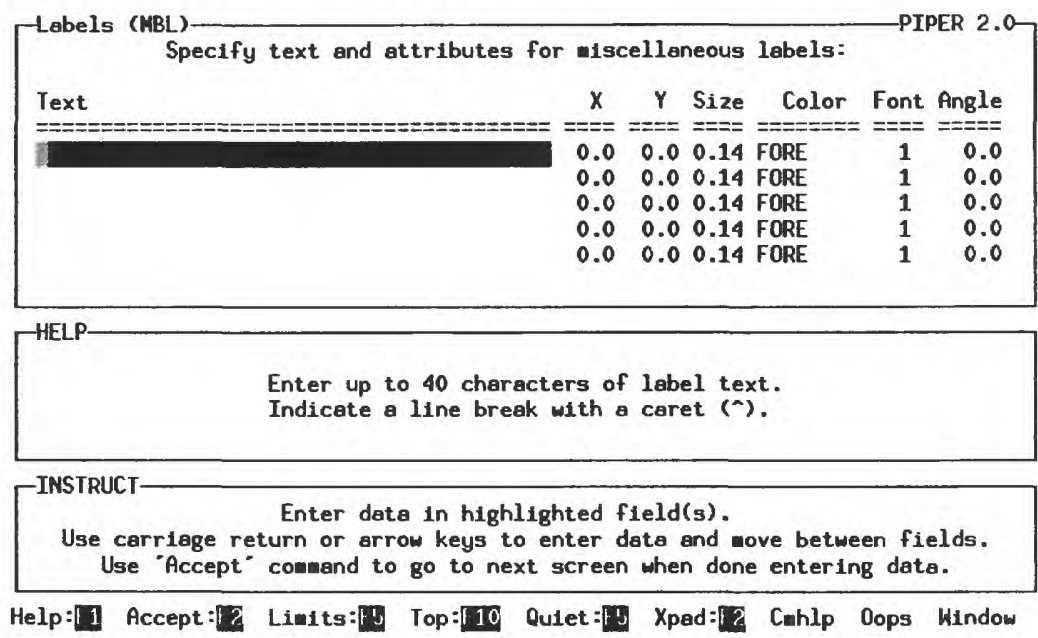


\section{Page Layout Options}

From the "Basic (MB)" screen, select the "Page layout options" option to bring up the "Page (MBP)" screen. The options available in the "Page (MBP)" screen can vary to meet any unique requirements of a program, but in general, the following categories are available:

Page Height

Page Width

Font Style

Diagram Layout

Plot Page Border

Tick Marks Location

Horizontal Y-Axis Numbers

Plot Frame

Legends.

\section{Page Height}

Use the Enter and arrow keys to select the "Page height" option. Specify the plot page height in decimal inches. The default page height is set at 8 inches.

\section{Page Width}

Use the Enter and arrow keys to select the "Page width" option. Specify the plot page width in decimal inches. The default width is set at 10.0 inches.

\section{Font Style}

Use the Enter and arrow keys to select the "Font style" option. If the "Help" panel is not already activated, then use Help (F1) to bring up the "Help" panel, which presents the available fonts and their associated codes. Enter the code in the "Font style" field. This font is applied to all text on the plot, with the exception of titles, footnotes, and the miscellaneous text labels.

\section{Diagram Layout}

Most QWGRAF programs provide for presenting multiple diagrams on a page. The number of lines and the number of diagrams per line permitted on a page is program dependent, but the user can specify how many lines and diagrams per line are utilized, up to the maximum. The template defined for the first page of output is carried over to each subsequent page.

When multiple pages of output are created and the plots are displayed on the screen, use the Enter key to view subsequent plot screens. When a multiple page plot is output to a file (Postscript, HPGL, or CGM), the individual pages are printed.

\section{Plot Page Border}

To have a page border drawn around the plot, select this option by using the Enter and arrow keys followed by using the space bar to toggle "On" the option as indicated by the appearance of an "X" in the option field.

\section{Tick-Mark Position}

By default, tick marks for X-and Y-axes are placed on the inside of the axis scale line. To place tick marks on the outside of the axis scale line, select this option by using the Enter and arrow keys followed by using the space bar to toggle the option "Off" as indicated by the appearance of a "space" in the option field. 


\section{Horizontal Y-Axis Numbers}

For those programs where Y-axes are appropriate, Y-axis scale numbers are, by default, plotted horizontally on the plot. To plot these numbers vertically, select this option by using the Enter and arrow keys followed by using the space bar to toggle the option "Off" as indicated by the appearance of a "space" in the option field.

\section{Plot Frame}

"Plot Frame" options are available in those programs with an X-and (or) Y-axis. The options available generally determine whether a partial frame (one X-axis and one $\mathrm{Y}$-axis) or a full frame (top $\mathrm{X}$-axis and right side $\mathrm{Y}$-axis added) is drawn and how the axes are to be annotated relative to tick marks and scaling.

To select an alternative framing, select the appropriate option by using the Enter and arrow keys followed by using the space bar to toggle the option "On" as indicated by the appearance of a "X" in the selected option field.

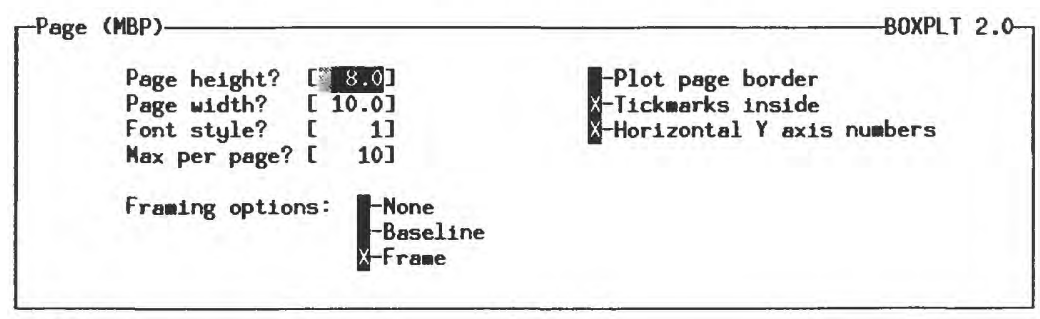

\footnotetext{
Enter the height of the plot page in inches. For Postscript landscape
use 8.5 . For Postscript portrait use 11.

INSTRUCT- Enter data in highlighted field(s).

Use carriage return or arrow keys to enter data and move between fields.

Use "Accept" comand to go to next screen when done entering data.

Help: 11 Accept: 2 Limits:
} 


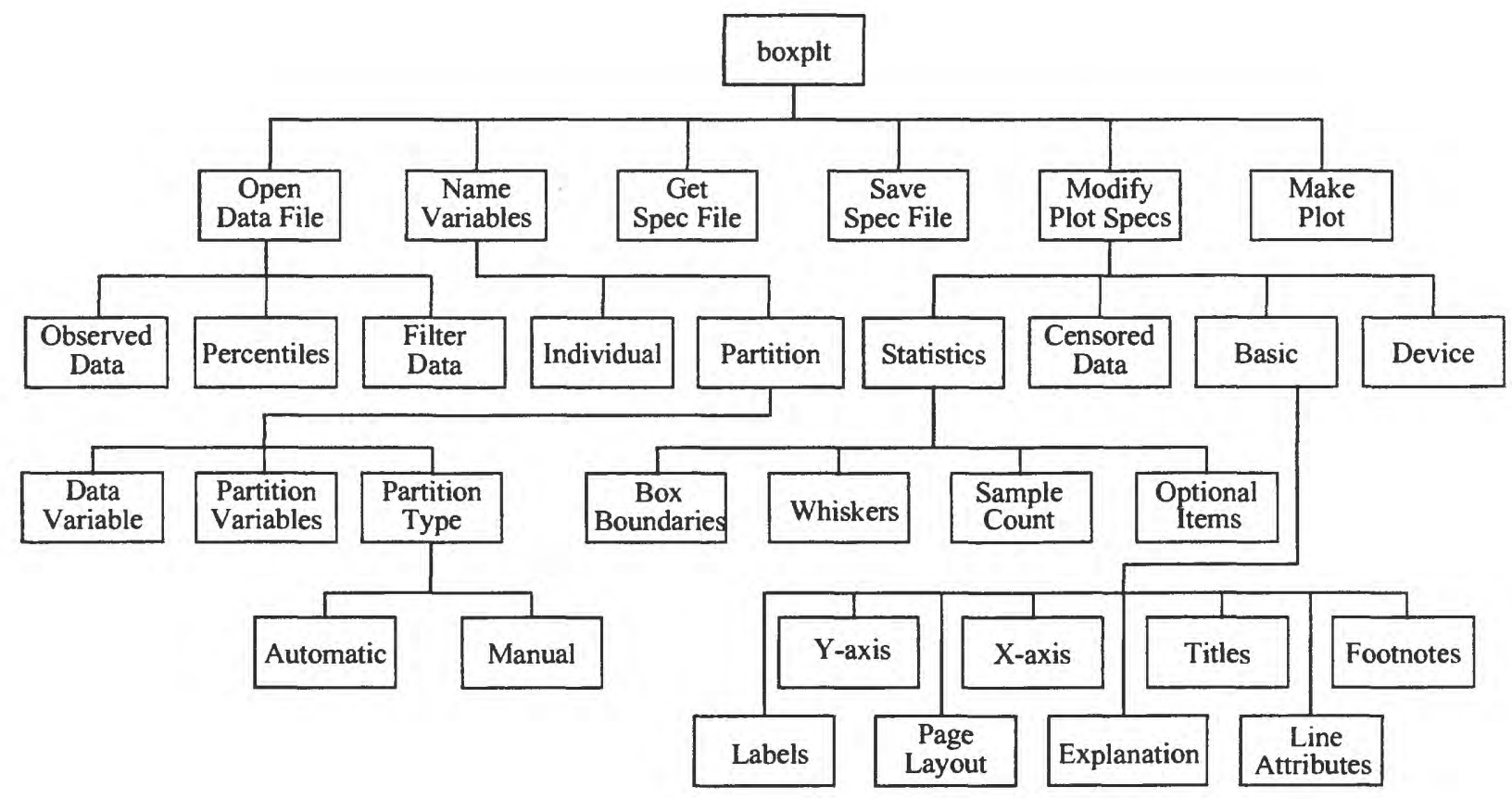

The "boxplt" program produces skeletal box and whisker diagrams (boxplots). The program provides for up to 90 boxplots on multiple pages, where the diagrams can represent individual variables or subsets of values from a single variable that has been partitioned based on the unique values of up to four user-selected partitioning variables. The spacing between boxes can be equal or proportional, that is, based on the range of values in the partition interval. Options are available for displaying the 95-percent confidence interval about the median, outliers, detached values, and the sample count. The whiskers can be Tukey ( $+/-1.5$ times box height), the minimum/maximum data limits, or 10/90 percentiles. The box limits can be computed on the basis of quartiles or fourths.

Remarked (or censored) data values can be included or excluded from the data set. If the data set includes remarked data and the quartiles box limit option is selected, then the percentile statistics for the remarked data are estimated by a routine that uses robust probability and distributional maximum likelihood estimate (MLE) techniques. The portion of the box and whisker below the detection limit can be blanked out or distinguished with a unique line type. The Y-axis scaling can be either linear or logarithmic and can be set automatically by the program or user defined. Tick-mark intervals are user defined. Options are provided for plot titles (up to three lines), footnotes (up to two lines), box labels, and X-and Y-axis labels. Up to five user-defined labels (up to 40 characters on multiple lines) can be positioned anywhere on the plot. Any number of plot specification files can be saved and used to facilitate plot specification in other "boxplt" sessions. 
A boxplot is a graphical summary of the basic statistics of a data variable. The core of a boxplot is a rectangle whose top and bottom edges are referred to as hinges. Hinges can be defined as either fourths or quartiles, depending on the method of calculation relative to the median value. The median splits an ordered (ranked) batch of data in half and the fourths represent the middle summary values of each half of the data. The fourths are calculated from the formula $\mathrm{d}(\mathrm{H})=([\mathrm{d}(\mathrm{M})]+1) / 2$ where $[\mathrm{d}(\mathrm{M})]$ is the integer part of the depth of the median value. For example, if the batch of data has nine values, then the depth of the median is five, that is, the fifth value in the ordered list of numbers; if the number of values in the data batch is eight, then the depth of the median is four and onehalf. The above formula then produces either an integer or an integer plus a half. In the latter case the hinge is the average of the two data values on either side of the hinge depth values; for example, the average of the second and third values and the average of the sixth and seventh values, respectively, in the eight-value batch example. Quartiles are similar to fourths, but are defined so that one quarter of the data lies below the lower quartile (25th percentile) of the data values and one quarter lies above the upper quartile ( 75 th percentile). The differences between fourths and quartiles is small, with fourths lying somewhat closer to the median.

Extending from each hinge (or quartile) is a whisker, whose end point is computed on the basis of one of three models supported in the "boxplt" program. In the Tukey model the whiskers are extended to the data point on or just within one and one-half times the $\mathrm{H}$-spread (the difference between the hinge values). In the MinMax model the whiskers are extended to the extremes of the data. In the percentile model, which requires the "QUARTILES" box boundary limits option, the whiskers are extended to the 10/90 percentile values. In the Tukey model, outliers are data values that fall beyond one and one-half times the $\mathrm{H}$-spread (the inner fences) but short of three times the $\mathrm{H}$-spread (the outer fences). Data values that fall beyond the outer fences are called detached values.

When the input data file contains censored data values, as indicated by the " $<$ " sign, computation of the summary statistics, including the percentile parameters, is based on a routine developed by Helsel (1990) that uses regression and a combination of robust probability and distributional MLE procedures for computing the mean, standard deviation, median, and interquartile range (IQR).

\section{Boxplot Options}

The "boxplt" program provides options for the following boxplot related functions:

Function

Box boundaries

Whiskers

Boxplot annotation

Estimated parameters

Axes

Boxplot explanation

\section{Screen Sequence}

Opening Screen, Modify (M), Statistics (MS)

Opening Screen, Modify (M), Statistics (MS)

Opening Screen, Modify (M), Statistics (MS)

Opening Screen, Modify (M), Censored Data (MC)

Opening Screen, Modify (M), Basic (MB),

$\mathrm{Y}$-Axis (MBY), $\mathrm{X}$-axis (MBX)

Opening Screen, Modify (M), Basic (MB),

Explanation (MBE).

\section{Starting the "boxplt" Program}

The "boxplt" program is invoked by typing "boxplt" on the command line. The "Opening Screen" displays the options that serve as gateways to the screens used to set or modify the plot configuration options. 


\section{Load a Specifications File}

To use specifications from a previous "boxplt" session, load a "Specifications" file. From the "Opening Screen," select the "Get (G)" screen. A list of specifications files (extension ".bxp") and a field for specifying the file name are presented. Type in a file name or use the arrow keys to select the required file and use either Accept (F2) or the Enter key to accept the selection. The "latest.bxp" file contains the program specifications used during the most recent execution of the "boxplt" program, whether in the current or previous session. Once the specifications file has been loaded, the "Make the Plot" option can be used to create the plot.

If any program options, including the input file name or output device, need to be changed, then proceed to the appropriate screens to make the changes. As appropriate, save sets of program options in specifications files using the "Save specifications" option in the "Opening Screen."

\section{Open Input Data File}

The "boxplt" program can read regular QWGRAF data files that contain raw observed data values or a specialized file format unique to the "boxplt" program.

\section{Observed Data Values}

For a data file that contains observed values, select the "Open" option in the "Opening Screen" followed by the "Observed data values" option from the "Open (O)" screen to bring up the "Observed (OO)" screen. Type in a file name or use the arrow, Page Down, or Page Up keys to select a data file. Use either Accept (F2) or the Enter key to accept the file selection and return to the "Open (O)" screen.

\section{Alternate Input File Format}

The alternate file type available for "boxplt" consists of data already converted to percentiles. Also included in this file are a plot title, $\mathrm{X}$ - and $\mathrm{Y}$-axis labels, and a detection limit. This format of this file, which is identified by the ".pct" file-name extension, is described in Appendix D. From the "Open (O)" screen select the "Percentiles - of data values" option to display the "Percentile (OP)" screen. Select or enter a file name with the ".pct" file-name extension and use Accept (F2) to accept the selection.

\section{Filter Data}

To subset the rows to be used in the analysis, select the "Filter" option from the "Open

$(\mathrm{O})$ " screen and create the row selection criteria statement in the "Filter (OF)" screen.

\section{Name Variables}

The program will create one boxplot for each variable selected or multiple boxplots based on subsets of values of a single variable whose values have been partitioned into groups based on unique composite values of up to four partitioning (grouping) variables. From the "Opening Screen," select and display the "Name (N)" screen and choose from the following options:

$$
\begin{array}{ll}
\text { Individual } & -\quad \begin{array}{l}
\text { Select one or more variables, } \\
\text { one for each boxplot desired. }
\end{array} \\
\text { Partition } & -\quad \begin{array}{c}
\text { or } \\
\text { Group data by partition variable. }
\end{array} \\
\text { Return } & -\quad \text { Return control to opening screen. }
\end{array}
$$


Selection of the "Individual" option brings up the "Individual (NI)" screen from which the list of variables (boxplots) will be selected in the order they are to be plotted. Start with " 1 " and enter the plot order numbers in the "Order" column. Use Accept (F2) to accept the screen and bring up the "Labels (NIL)" screen, which provides for entering alternative labels for the diagrams (the input variable name is the default label). The labels can be up to 40 characters, and carets $\left(^{\wedge}\right)$ can be placed in the field to force line breaks. The angle of the label, as presented below the X-axis, is 0.0 (horizontal) by default. Alternative angles (specified in a counterclockwise direction from the horizontal), font and label size are set in the "X-Axis (MBX)" screen accessed from the "Basic (MB)" screen. Use Accept (F2) to accept the "Labels (NIL)" screen and return control to the "Opening Screen."
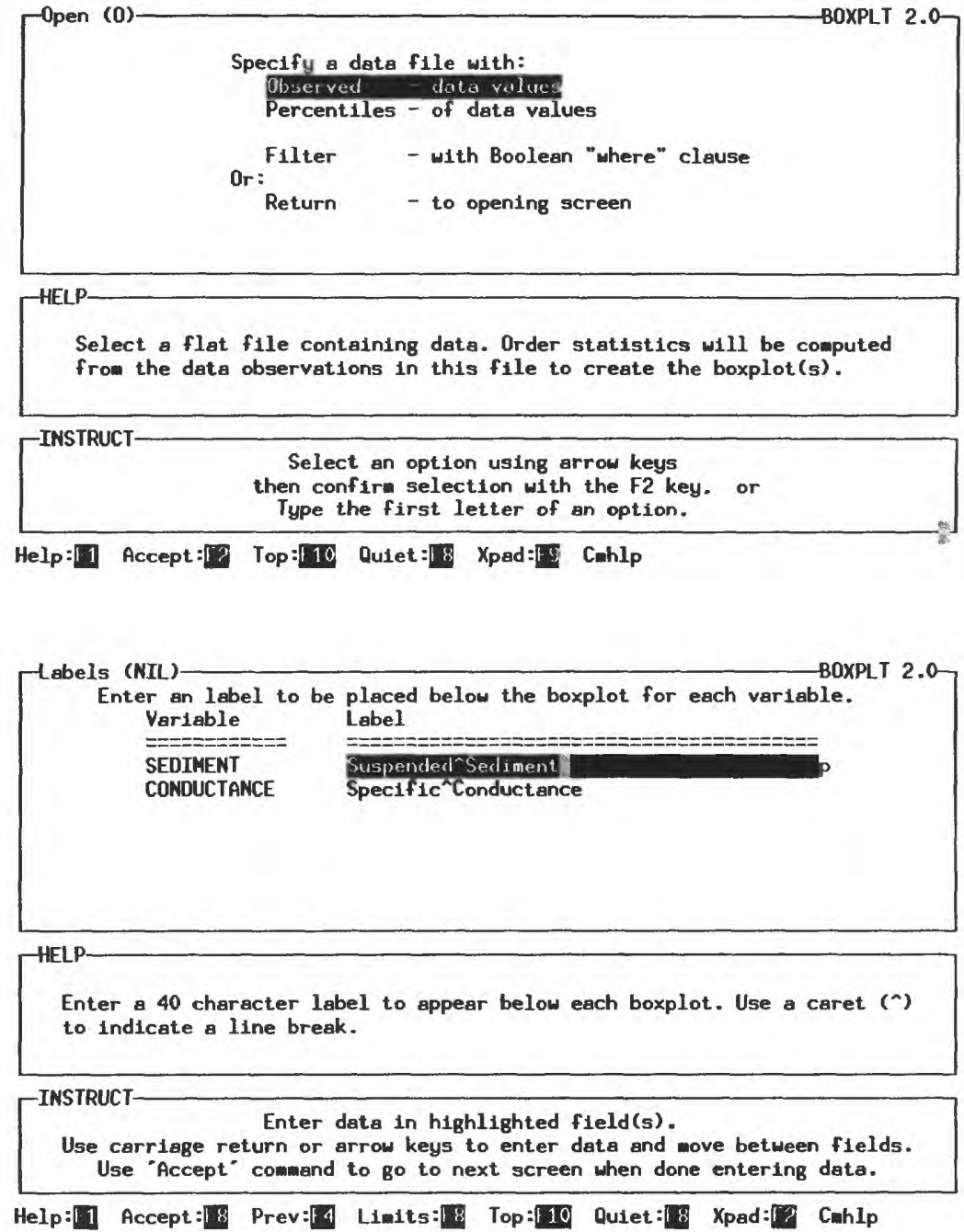


\title{
Modify Plot Specifications
}

After the data file has been opened and the variables of interest named, the "Make the plot" option can be selected to generate the plot by using system default options. To annotate the plot or activate other options, select the "Modify (M)" screen by typing "M" (or "m"), or use the Tab and arrow keys to select the "Modify compute/plot specifications" option followed by pressing Accept (F2) or the Enter key. The "Modify (M)" screen lists the following options:

Statistics - specify boxplot statistical options

Censored - specify censored data, detection limit options

Basic - specify title, footnotes, labels, and axes options

Device - specify device for graphics output.
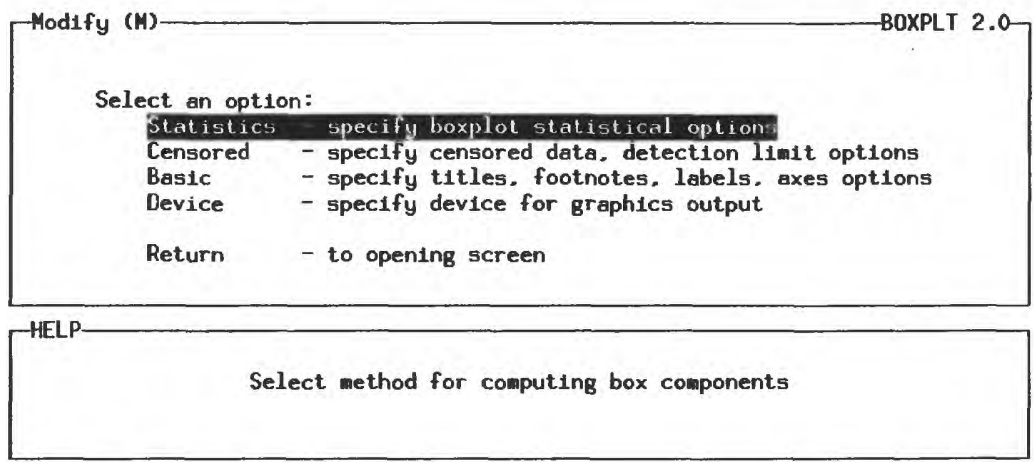

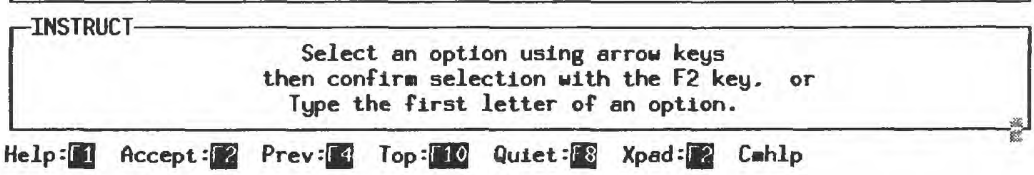

\section{Boxplot Statistics}

From the "Modify (M)" screen, select the "Statistics (MS)" screen, which lists the following items relevant to boxplot statistics:

\author{
Box Boundaries \\ Whiskers \\ Sample Count \\ Snap to data \\ Optional Items \\ Minimum Sample Size.
}

The default option for each item is indicated by the presence of an " $\mathrm{X}$ " in the field to the left of the option name. Use the arrow keys, Tab key, or the Enter key to select an alternate option for any item and then use the space bar to toggle "On" the new option. Use the space again to toggle "Off" a selected option as indicated by a space in the field.

\section{Box Boundaries}

The options for "Box Boundaries" are as follow:

Quartiles

Fourths. 
For sample sizes greater than about 35 , the two options produce essentially the same hinge values. The "Quartiles" option is required if the "Percentiles" option under

"Whiskers" is selected. The default setup for a new session of boxplt is "Quartiles," "Percentiles," and "Retain censored data."

If censored data are retained in the analysis (see "Censored data options" in the "Modify (M)" screen), then selection of the "Quartiles" option and either the "Tukey" or "Percentiles" whisker option invokes the Helsel distributional MLE routine that estimates the box and whisker statistics that correspond to the portion of the diagram below the detection limit value.

\section{Whiskers}

Whiskers are drawn to a point on or just within a limiting value beyond the hinges as defined by one of the following methods:

$$
\begin{array}{ll}
\text { Tukey } & -1.5 \text { Multiple of Hinge Value } \\
\text { Min/Max } & - \text { Minimum and Maximum Data Value } \\
\text { Percentiles } & \text { - 10th and 90th Percentile Values. }
\end{array}
$$

Note: Only the "Tukey" or "Min/Max" whisker options are permitted if "Fourths" is selected as the "Box Boundary."

\section{Snap to Data}

This option is only relevant for Tukey or $10 / 90$ percentile whiskers. The default for this option is "On" as indicated by an " $\mathrm{X}$ " in the field to the left of the option name. Each whisker will end at the actual data point closest to but within the computed whisker endpoint relative to the hinge. If this item is toggled "Off" by pressing the space bar key, then a blank will replace the "X" in the field and the whisker end point will be plotted at the calculated end point, whether or not it represents an actual data point.

\section{Sample Count}

The number of data values for each variable can be displayed on the plot in two locations.

The options available are as follow:

Omit

Over the Maximum Value (Default)

Above the Plot Frame.

\section{Minimum Sample Size}

The default minimal number of data values required for a boxplot is 10 . This value can be set higher or as low as 9 . If the number of data values in the data set is less than the specified minimum, then the box and whiskers are not plotted; but the individual data values are plotted as pluses $(+)$.

\section{Optional Items}

In addition to the sample median, the following items can be added to the diagram:

Outliers and Detached Values

Sample Mean

95-percent Confidence Interval About the Median

Criteria Line.

\section{Outliers and Detached Values}

Data values that fall beyond the whiskers are represented on the plot as either asterisks or circles, depending upon the whisker model selected. The Min/Max whisker model has no values beyond the whiskers, which represent the extremes of the data. In the 10/90 percentile whisker model, all data values beyond the whiskers are represented by asterisks. In the Tukey model, data values that fall between the whiskers and three times the $\mathrm{H}$-value are called outliers and are represented by asterisks. Data values that fall outside three times the $\mathrm{H}$-value are called detached values and are represented by circles. 
The default for this option is "On" as indicated by the presence of an " $\mathrm{X}$ " in the field to the left of the option name. This option is turned off by using the space bar to replace the "X" with a blank.

\section{Sample Mean}

The sample mean is represented on the plot by a dotted line within the box.

The default for this option is "Off" as indicated by a blank in the field to the left of the option name. Use the space bar to turn this option "On" as indicated by the appearance of an "X" in the field.

\section{5-Percent Confidence Interval About the Median}

If this option is selected, then 95-percent confidence interval lines will be drawn on the plot as solid lines that extend slightly beyond the left and right boundaries of the box.

The default for this option is "Off" as indicated by a blank in the field to the left of the option name. Use the space bar to turn this option "On" as indicated by the appearance of an " $\mathrm{X}$ " in the field.

\section{Optional Criterion Line}

If it is desired to indicate some other criterion value on the plot, then a horizontal line across the plot frame with its own unique attributes of line type, color, and thickness, can be drawn on the plot, along with an optional 20-character label.

The default for this option is "Off" as indicated by a blank in the field to the left of the option name. Use the space bar to turn this option "On" as indicated by the appearance of an " $\mathrm{X}$ " in the field.

If this option is selected, then the "OC Line_type (MSO)" screen will be displayed after the "Statistics (MS)" screen is completed and accepted.

The "OC Line_type (MSO)" screen presents fields for entering the following attribute values for the optional criteria line:

Y intercept value

Line label (up to 20 characters)

Label Size.

Use the arrow keys or the Tab key to select an option. The label can be broken into multiple lines by placing carets at appropriate places in the text; for example, Criteria^ Line. Use the "Line" option in the "Basic (MB)" screen to assign the other line attributes (style, color, thickness). Use Accept (F2) to return to the "Modify (M)" screen.

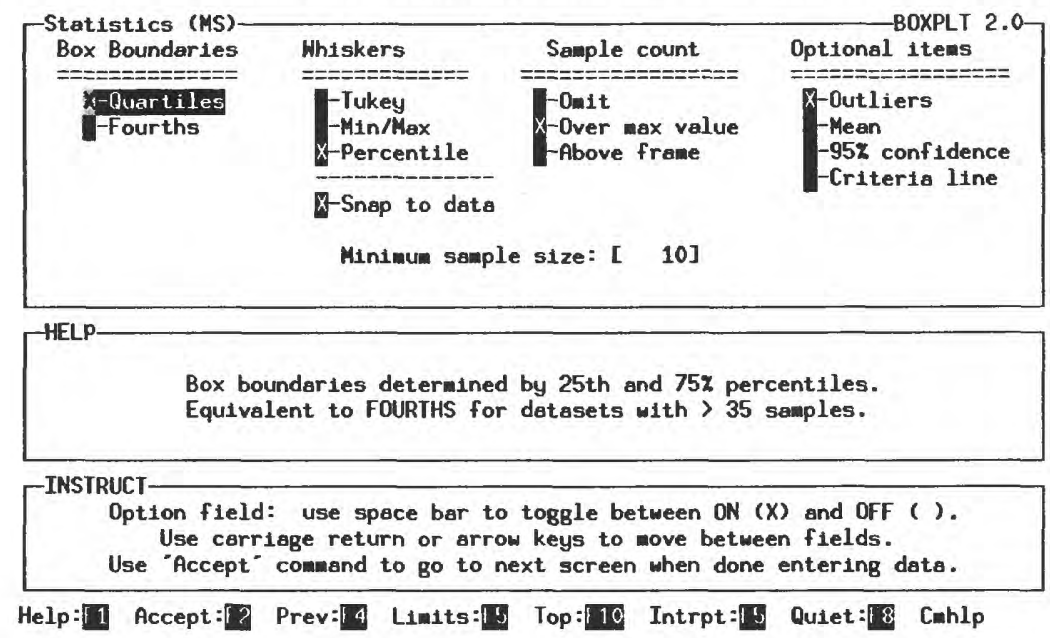




\section{Censored Data}

The "boxplt" program provides for treatment of censored data in the creation of a boxplot. By default, the program assumes censored values in the input data file and selects the "Quartiles" option for the box borders in the "Statistics (MS)" screen. Under these conditions the program activates the MLE technique, which estimates the boxplot statistics based on simulated replacements of the censored data values. The maximum detection limit value is used as the threshold value for the estimated percentile values that form the basis of the sample median and applicable hinge and whisker values.

The options available for treating censored data values are displayed by selecting the "Censored" option in the "Modify (M)" screen. Use the arrow keys or the Tab key to select the options desired and then the space bar to turn an option "On" as indicated by an "X" in the field to the left of the option name.

\section{Censored Data Disposition}

The following options are available:

Retain as Censored (Default)

Use as Uncensored

Treat as Missing.

\section{Retain as Censored Values}

Data values remarked with the "<" remark code are retained as censored and are used in the computation of the box statistics. Selection of this option is permitted only when the "Quartiles" option is selected in the "Statistics (MS)" screen.

\section{Use as Uncensored Values}

Remarked data values are treated as actual values without regard to the remark code.

\section{Treat as Missing Values}

When the "Missing" option is selected, remarked data values are converted to "missing" value indicators; thus they are not used in the analysis.

\section{Detection Limit Definition}

Water-quality data often have multiple detection limits that result from new or improved analytical methods. When the box statistics are estimated, a detection limit line is drawn on the diagram. The user has the option of displaying this line as representing either the highest detection value (default) or the minimum.

Highest (Default)
Lowest.

\section{Single or Multiple Detection Limits}

If the plot consists of multiple diagrams, either multiple variables or a single variable partitioned into several data intervals, then the user may elect to display either a single detection limit line for all diagrams or a detection limit line for each diagram.

Single (Default) Multiple.

\section{Single}

With the "Single" option, the maximum and minimum detection limit values are selected from the population of all values from all variables.

\section{Multiple}

With the "Multiple" option, a maximum and minimum detection limit value is selected from the population of values from each variable. 


\section{Box and Whisker Line Type Below Detection Limit Line}

When box statistics are estimated on the basis of censored data, it may be desirable to distinguish that part of the diagram associated with these computations from the rest of the diagram. The first option is to display that part of the diagram, possibly with line characteristics different from the rest of the diagram. The second option is to the eliminate that part of the diagram from the plot. The following options are available:

$$
\begin{aligned}
& \text { No } \\
& \text { Yes. }
\end{aligned}
$$

No

Do not plot that part of the boxplot diagram based on estimated values, that is below the highest detection limit value.

Plot the entire boxplot diagram. If it is desired to distinguish that part of the diagram based on estimated values from the rest of the diagram that uses alternate line characteristics, then select the "Line" option in the "Basic (MB)" screen in addition to the "Yes" option here.

Use Accept (F2) to return to the "Modify (M)" screen.

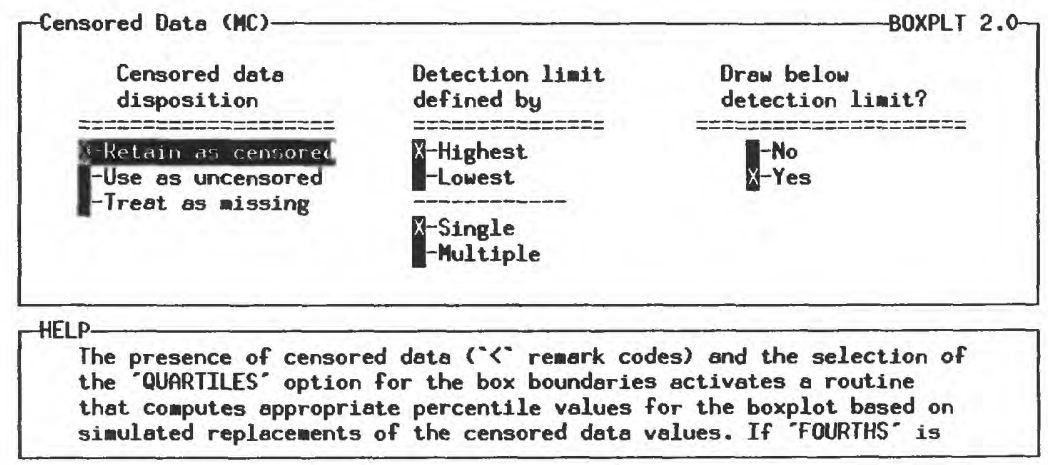

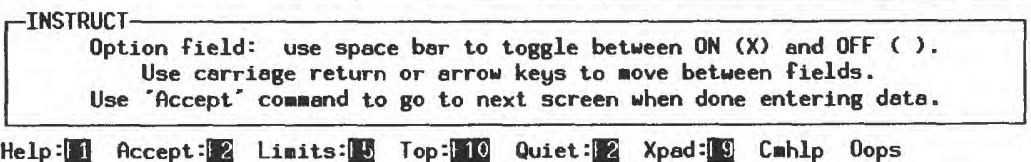

\section{Basic Plot Parameters}

From the "Modify (M)" screen, select the "Basic (MB)" screen, which lists the following options for modifying plot specifications:

$$
\begin{array}{ll}
\text { Y-Axis } & \text { - type, limits, label } \\
\text { X-Axis } & \text { - spacing, label, variable label angle } \\
\text { Titles } & \text { - above plot } \\
\text { Footnotes } & \text { - below plot } \\
\text { Labels } & \text { - of miscellaneous text } \\
\text { Page } & \text { - layout options } \\
\text { Explanation } & \text { - block options } \\
\text { Line } & \text { - attribute options } \\
\text { Make } & \text { - the plot } \\
\text { Return } & \text { - to previous screen. }
\end{array}
$$

Type the first letter of any option or use the arrow keys or Enter key to select the screen for the option required. 
$\underline{\text { Y-Axis }}$

From the "Basic (MB)" screen, type "Y" (or "y") or use the arrow and Enter keys to select the "Y-Axis (MBY)" screen. The "Y-Axis" option provides for specifying the axis type, the upper and lower limits of the scale, the tick-mark interval, and a label for the Y-axis.

Type

The Y-axis scale can be either "linear" or "log;" the default is "linear." Use the arrow keys to select this option and then use the space bar to toggle "On" the required option as indicated by the appearance of an " $\mathrm{X}$ " in the field to the left of the option name.

\section{Scale Limits}

"Auto" scaling is the default; the system computes and displays the upper and lower boundaries based on the range of the data. If the "Manual" scaling option is used, then the user can specify alternate upper and lower boundaries. Note: The initial scale limits listed for the "Manual" option do not correspond to the extremes of the data in the input data file. To set manual scale limits, select the "Lower limit" and "Upper limit" fields under the "Manual" option and enter appropriate values. In subsequent uses of the "Y-axis (MBY)" screen in the same session of "boxplt," scale limit values listed in the "Manual" option fields will be the last scale limits used by the procedure regardless of whether the "Auto" or "Manual" option was selected in the previous execution of "boxplt."

\section{Tick-Mark Interval}

A default tick-mark interval is determined based on the range of the data. It also can be specified when the "Manual" option is selected.

\section{Label}

Use the arrow keys to select the Y-axis label field. Enter a label of up to 60 characters in this field. Use Accept (F2) to return control to the "Basic (MB)" screen.
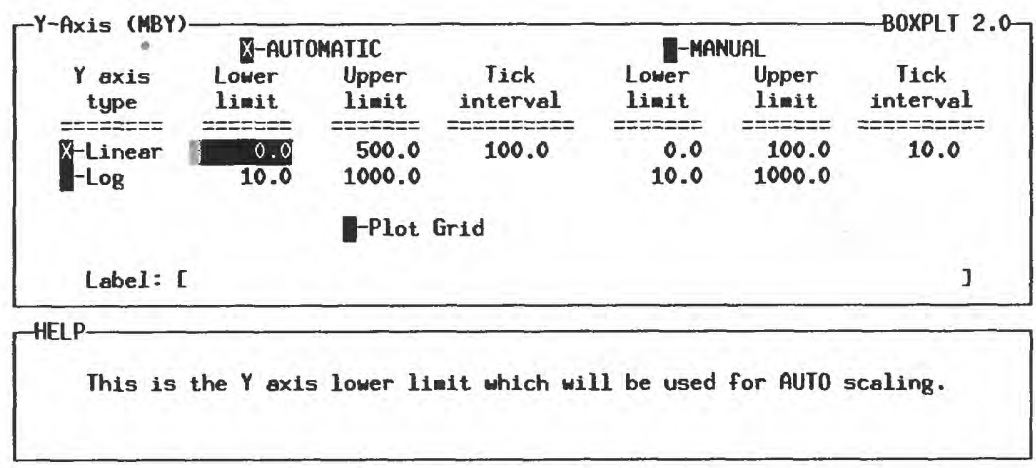

\footnotetext{
ГINSTRUCT- Enter data in highlighted field(s).

Use carriage return or arrow keys to enter data and sove between fields. Use "Accept" comend to go to next screen when done entering data.

Help: 11 Accept:F2 Limits: Top $=10$ Quiet: 18 Xpad:10 Cahlp Dops
} 


\section{$\underline{X-A x i s}$}

From the "Basic (MB)" screen, type "X" (or "x") or use the arrow keys to select the "X-Axis (MBX)" screen. The "X-axis" option provides for specifying the spacing between diagrams, attributes for the labels to be placed beneath each diagram on the plot, and a label for the $\mathrm{X}$-axis.

\section{Spacing}

Use the arrow keys to select either the "Equal" or "Proportional" options. Then use the space bar to toggle "On" the desired option as indicated by the appearance of an " $\mathrm{X}$ " in the field to the left of the option name. If the "Equal" option is selected, then the multiple boxes will be spaced equally along the X-axis. If the "Proportional" option is selected, then a boxplot diagram will be spaced along the $\mathrm{X}$-axis at the midpoint value of each partition variable interval. The "Proportional" option is only applicable when the "Partition" option is selected in the "Name Variables" screen.

\section{Box Labels}

The following attributes can be specified for the labels beneath each diagram:

\section{Text Size \\ Text Angle \\ Text Font.}

Use the arrow keys to select the option desired. Use Help (F1) or Limits (F5) to display allowable values for these attributes. Enter any new values to replace the default values. The default text angle is " 0 " degrees (horizontal). Changing the angle forces the text to rotate counterclockwise on its pivot point, which is the right edge of the text.

The default variable labels, which originate as the variable names from the input data file, can be changed for plotting purposes. To change any variable name, first select the "Name" option from the "Opening Screen" followed by selecting either the "Individual" or "Partition" options. Then from either the "Individual (NI)" screen or the "Data (NPD)" screen, select a variable name in the "Label" column and enter a variable name of up to 12 characters.

\section{Label}

Enter up to 60 characters for a label to be placed centered below the X-axis. Use Accept (F2) to return control to the "Basic (MB)" screen.

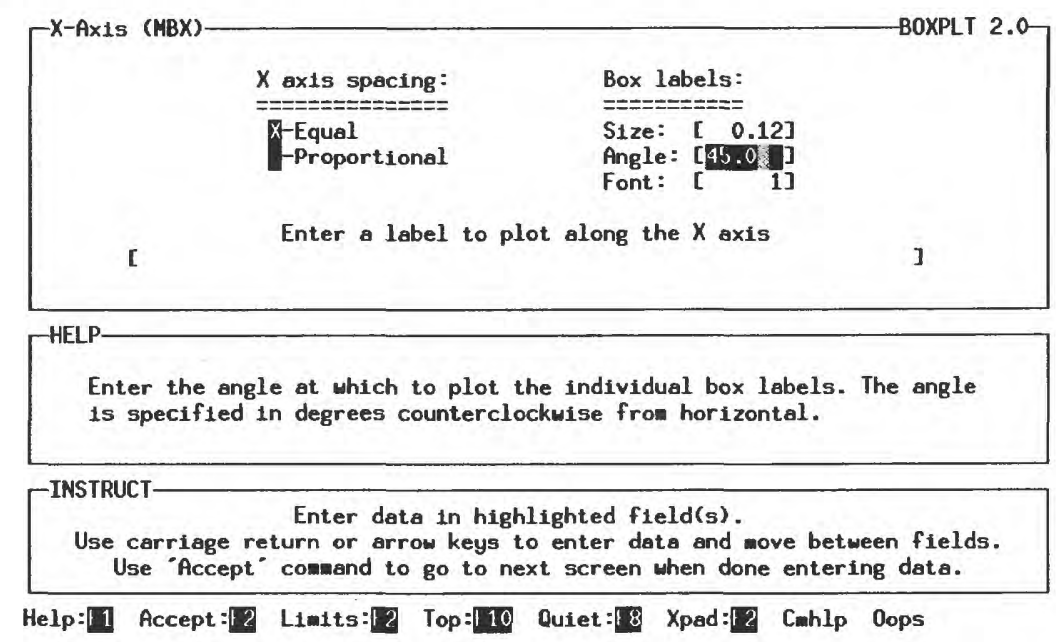




\section{Explanation Diagram}

From the "Basic (MB)" screen select and bring up the "Explanation (MBE)" screen, which provides for placing an explanatory boxplot on the plot. The options are as follow:

$\begin{array}{ll}\text { Box Placement } & \text { Text Format } \\ \text { Omit (Default) } & \text { Brief (Default) } \\ \text { Right Side of Plot } & \text { Verbose } \\ \text { Below Plot. } & \end{array}$

Selection of the "Right" option for "Box Placement" will place the explanatory diagram to the right side and top of the plot frame. Selection of the "Below" option will center the explanatory diagram below the plot frame.

Selection of the "Verbose" text format option produces a more detailed definition of the boxplot components, but takes considerably more space.

The default box placement is "Omit" and the default text format is "Brief." To change the defaults, use the arrow keys, Tab key, and Enter key to select the option desired and toggle "On" this selection by pressing the space bar.

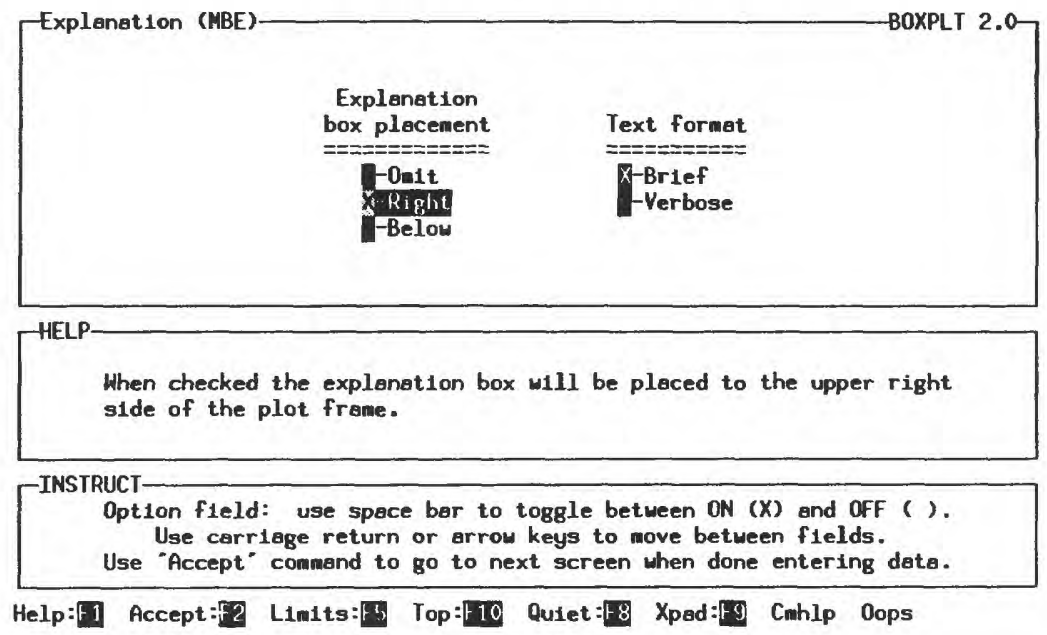

\section{Line Attributes}

From the "Basic (MB)" screen select the "Line" option and bring up the "Line (MBL)" screen, which provides options for specifying line attributes (color, style, thickness) for the following boxplot components:

Box \& Whiskers

Median

Mean

Confidence Interval

Below Detection Limit

Detection Limit

Optional Criteria.

Use the arrow keys or the Tab key to select the attribute. Use "Limits (F5)" to display the allowable values for that attribute in the Help panel. Type in an alternate value as required. 


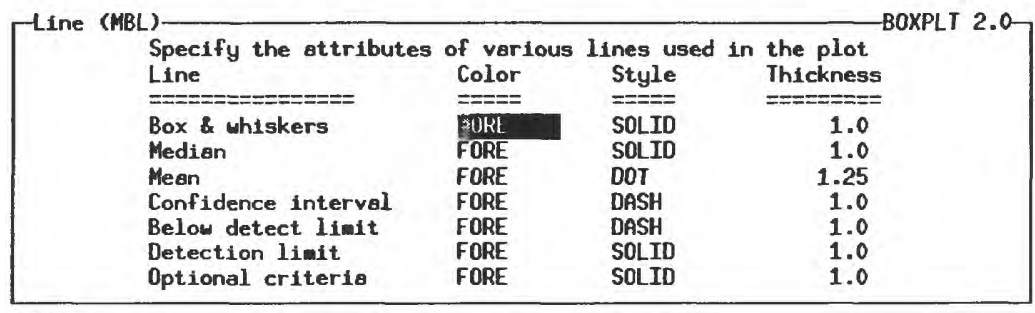

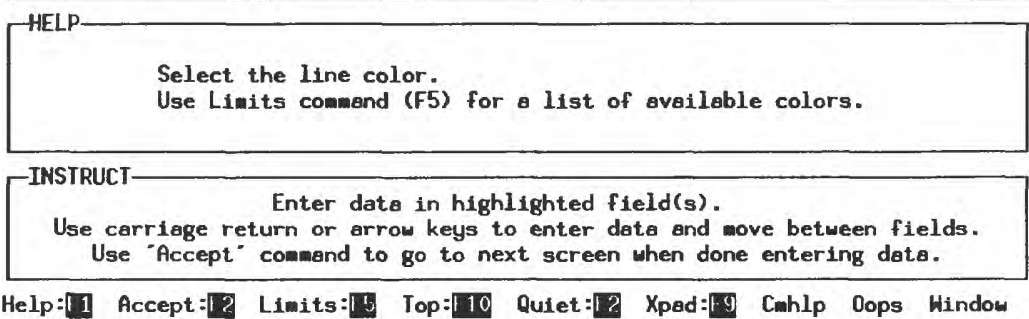

\section{Page Layout Options}

From the "Basic (MB)" screen, select the "Page (MBP)" screen. The "Page (MBP)" screen provides the following options:

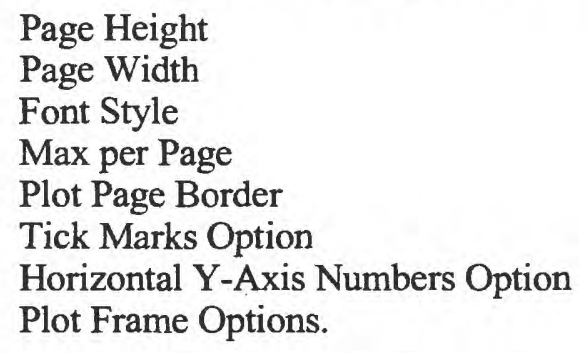

Most options in this list are discussed in the section "Page Layout Options" under "Steps to Using QWGRAF Programs."
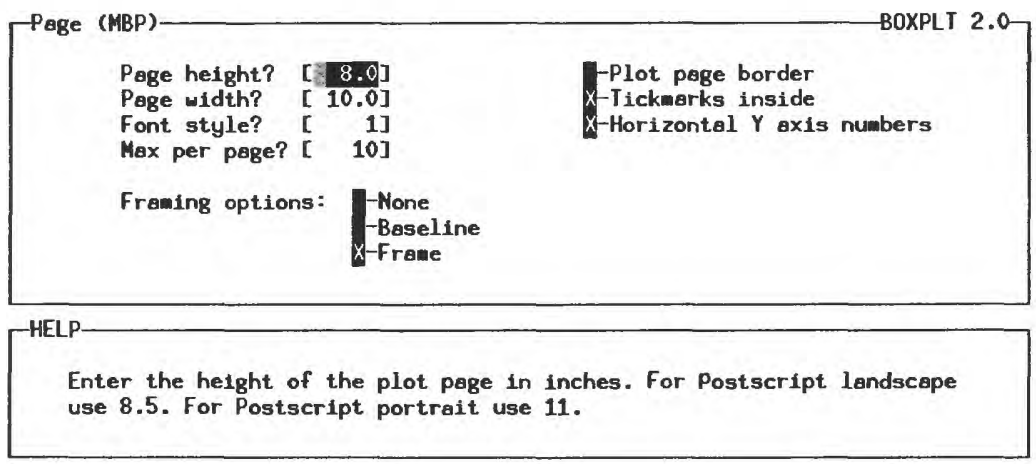

\footnotetext{
TINSTRUCT- Enter dato in highlighted field(s).

Use carriage return or arrow keys to enter data and move between flelds.

Use "Accept" comand to go to next screen when done entering data.

Help: 11 Accept: 2 Limits: Top $: 10$ Quiet: 8 Xpad: 9 Cahlp Dops
} 


\section{Maximum Number of Diagrams per Page}

"Boxplt" provides for distributing the diagrams on multiple screens or output pages as needed. Crowding can become a problem when the "Explanation" option is selected, especially the "Verbose Explanation." The order of the boxes to be presented is determined when selecting the variables to be plotted in the "Individual (NI)" screen accessed from the "Name" option in the "Opening Screen."

Use the Enter and arrow keys to select the "Max per Page" option. Enter the maximum number of boxes to appear on a single page. The system may reduce this number if it determines that the plot page size is too small to accommodate the maximum.

When the multiple page option is selected, the output will be presented on two or more screens or output pages in a file. Use the Enter key to view the subsequent plot screens. A multiple page plot can be output to a file (Postscript, HPGL, or CGM), which will produce the individual pages when printed.

\section{Plot Frame}

Three options are available for framing the boxplot:

None - Y-Axis line only

Baseline - X-Axis baseline added to Y-Axis line

Frame - Full rectangular frame (Default).

The "Full rectangular frame" is the default framing for the plot. To select an alternative framing, select the appropriate option by using the Enter and arrow keys followed by using the space bar to toggle the option "On" as indicated by the appearance of a " $\mathrm{X}$ " in the selected option field.

\section{Make the Plot}

When all arguments for the boxplot have been specified and the appropriate device selected, return control to the "Opening Screen," select the "Make the plot" option, and use the Enter key. If a screen output was selected, then a graphics window opens and the boxplot is presented. If a file output was selected, then a message screen indicates that the output from the procedure has been saved.

\section{boxplt Tutorial}

An interactive tutorial is available to guide the user through the basic options of the "boxplt" program. The user first generates a basic plot with minimal annotation. The tutorial then continues to guide the user through a series of enhancements, including saving the specifications for use in another "boxplt" session and creating an output postscript file for printing on a laser printer. The tutorial is activated by first typing "Shift @" in the "Opening Screen" of a new "boxplt" session. When the dialog box comes up, enter the file name found in the tutorial.doc file distributed with the QWGRAF programs. This file is located in the doc subdirectory, which provides specific details on using this and other QWGRAF tutorials.

The tutorial prompts the user for specific keystrokes. The program will only respond when the correct keystroke sequence is entered; that is, incorrect information cannot be entered. Text for labels, titles, and so forth, can be entered in either upper or lower case. The system converts everything to upper case.

\section{Sample Plots}

The following are examples of the types of plots that can be created with the QWGRAF "boxplt" program. These examples are intended to illustrate various options available in the program and do not necessarily conform to publication standards of the Geological Survey. 


\section{Program: BOXPLT}

Plot Specifications: Individual Variables; Quartile Hinges; 10/90 Percentile Whiskers; Outliers; Observation Count; Censored Data Estimations; Detection Limit Indicator; Y-Axis Label; Automatic Y-Axis Scaling; Angled Diagram Labels; Grid Lines; Two Titles; Brief Explanation Block

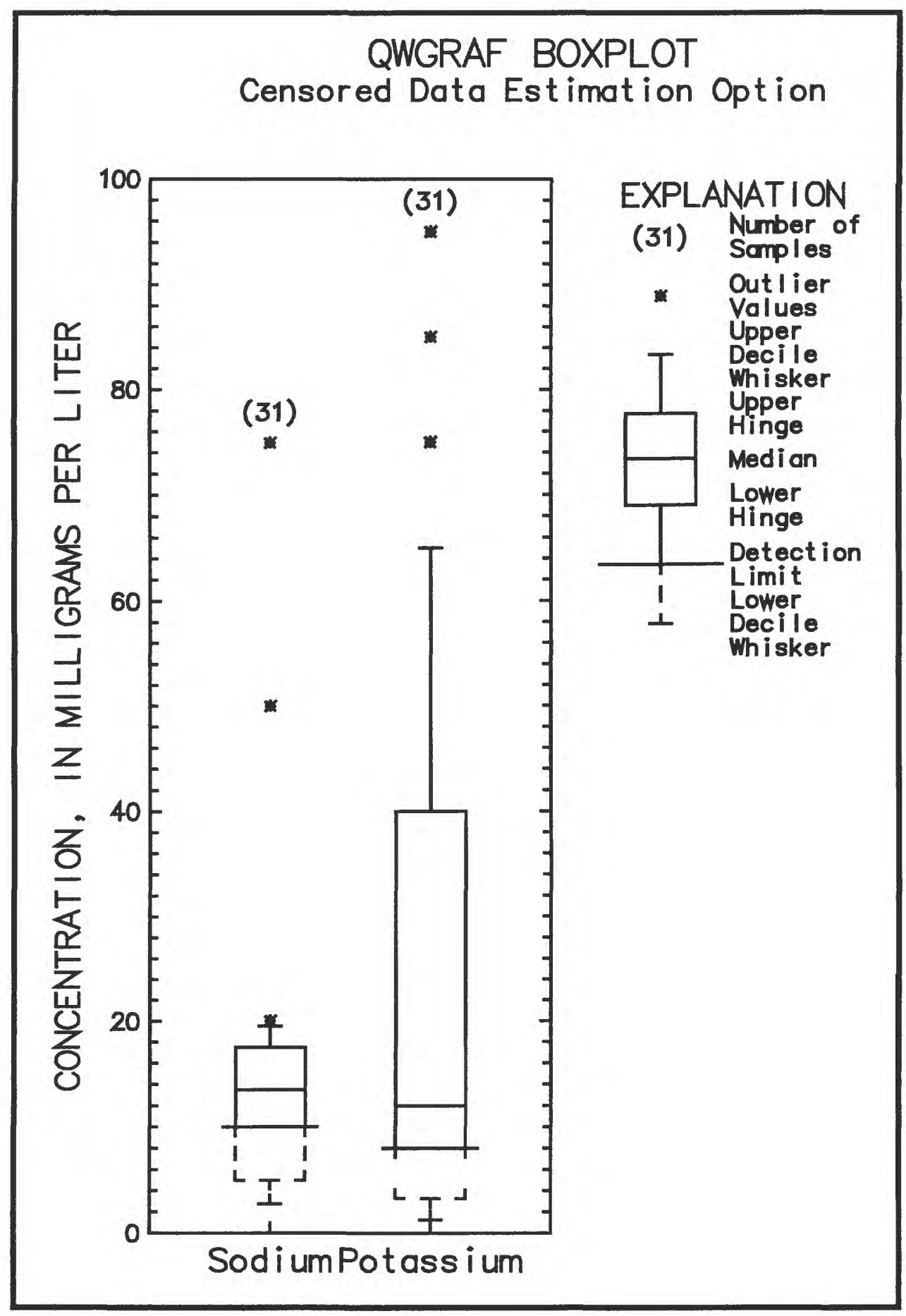


Program: BOXPLT

Plot Specifications: Individual Variables; Quartile Hinges; 10/90 Percentile Whiskers; Outliers; Observation Count; Automatic Y-Axis Scaling; Y-Axis Label; Sample Count; Snap to Data

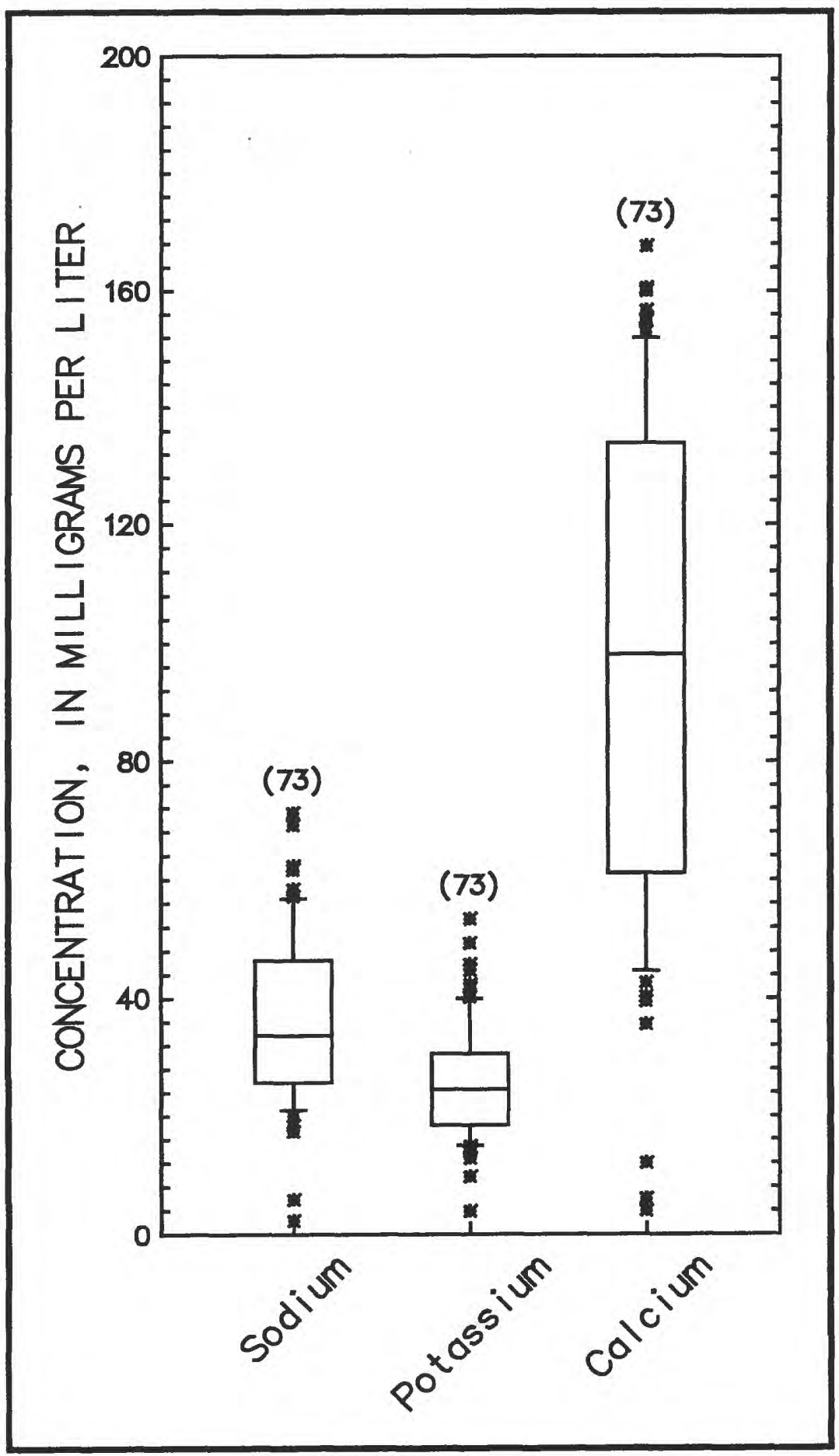


Program: BOXPLT

Plot Specifications: Individual Variables; Quartile Hinges; 10/90 Percentile Whiskers; Outliers; Mean; 95-percent Confidence Interval; Observation Count; Snap Whisker to Data; Optional Criterion Line; Random Labels; Manual Y-Axis Scaling; Y-Axis Label; Y-Axis Grid Lines; X-Axis Label; 3 Titles; 2 Footnotes; Brief Explanation

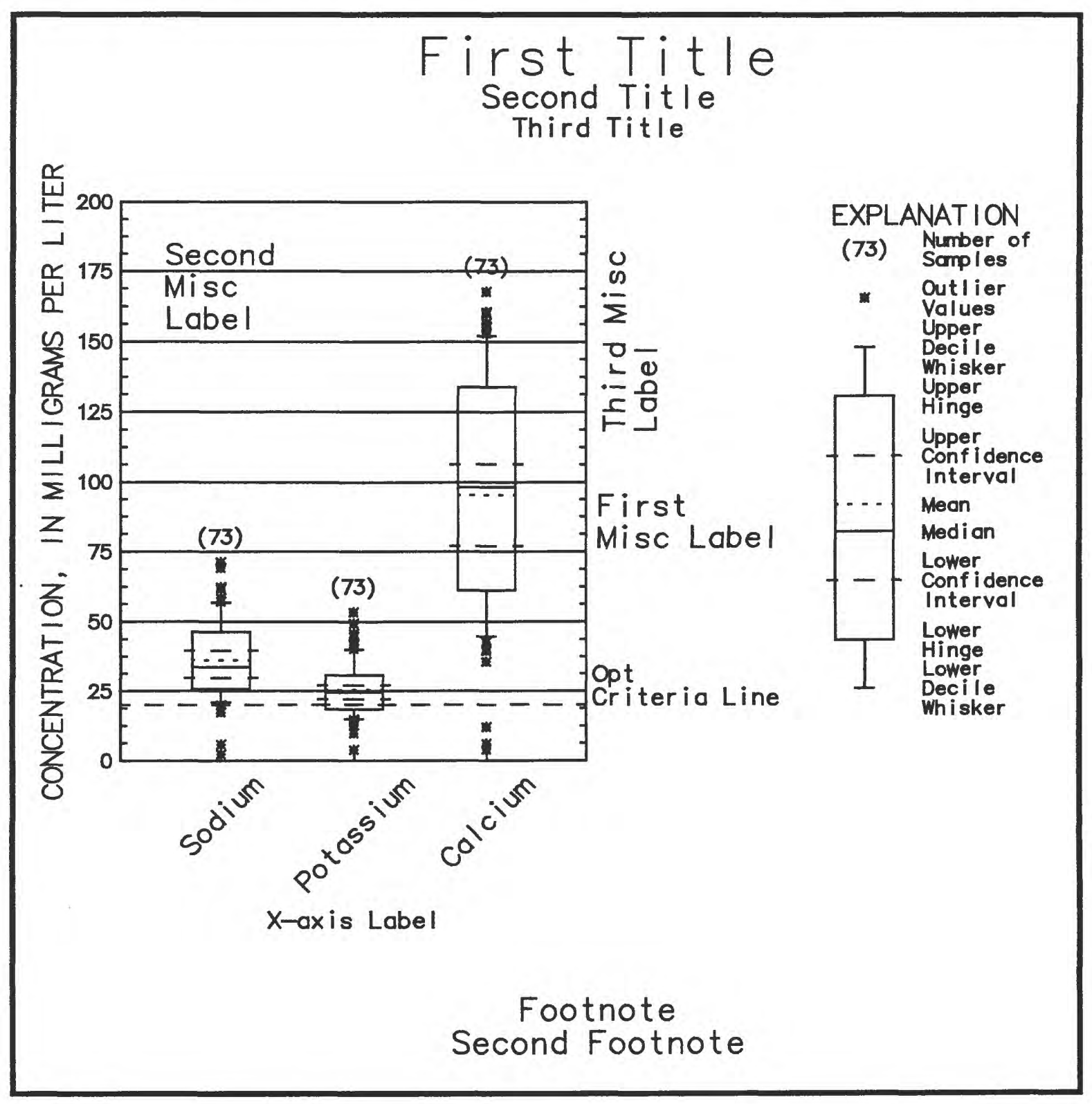


Program: BOXPLT

Plot Specifications: Single Variable Partitioning; Fourths Hinges, Tukey Whiskers; Outliers and Detached Values; Observation Count; 2 Titles; Manual Y-Axis Scaling and Tick-Mark Interval; Y-Axis Grid Lines; Labels for Partitioning Variable Values

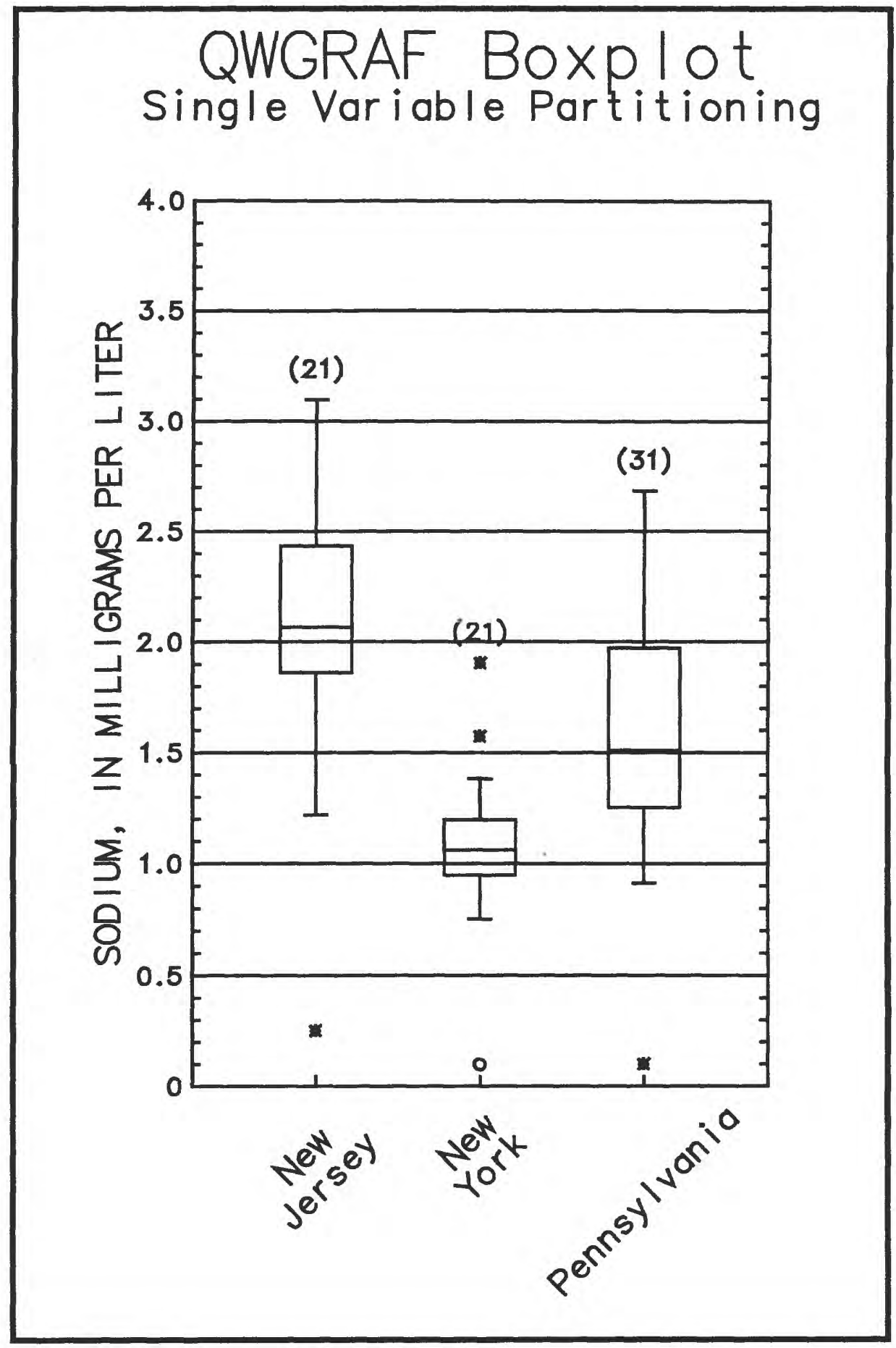




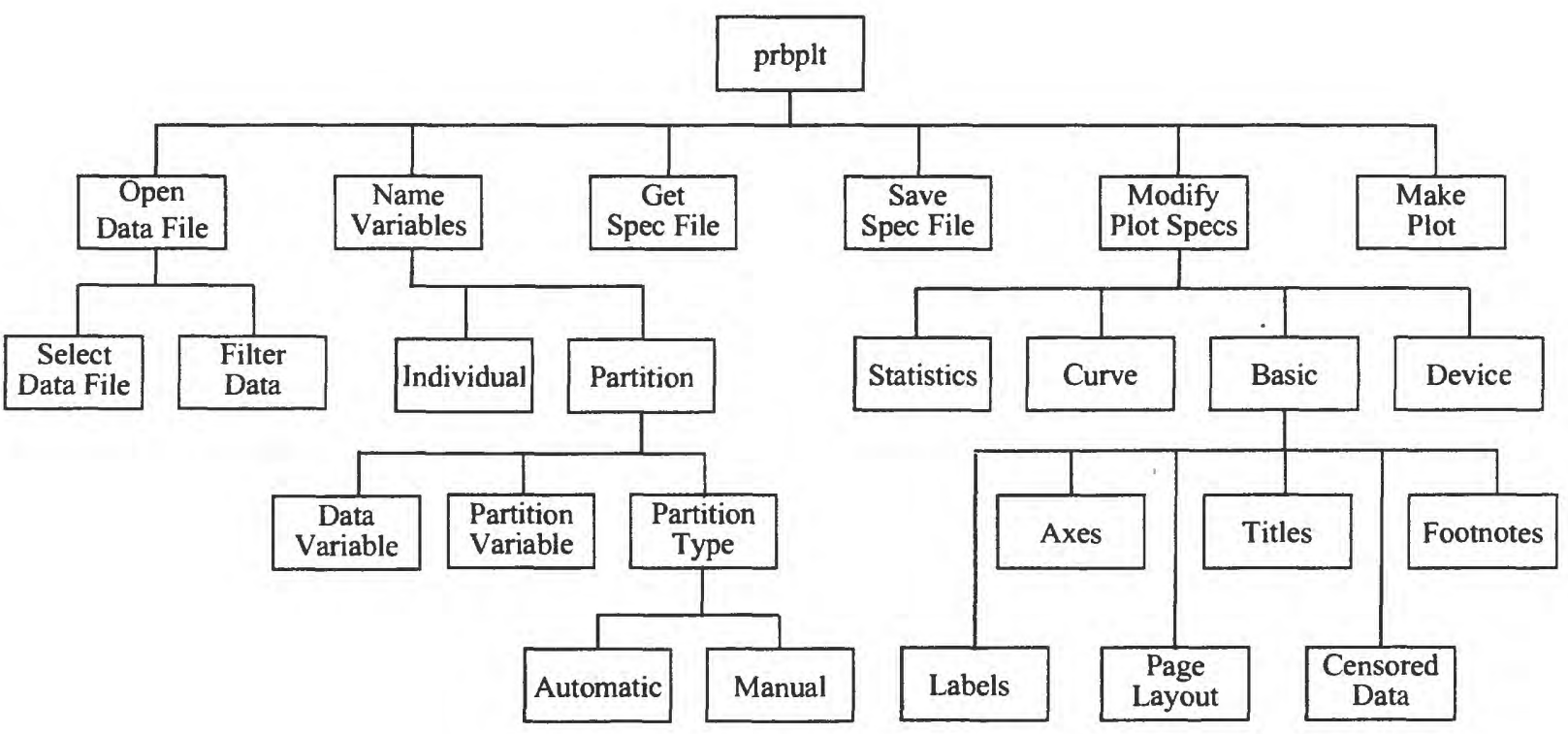

PROBABILITY PLOTS (prbplt)

The "prbplt" program plots values for one to six variables on multiple Y-axis scales versus a probability distribution function on the $\mathrm{X}$-axis. The $\mathrm{X}$-axis probability scale types include Plotting Positions, Quantile Deviates, Exceedance and Nonexceedance Cumulative Probability Percentiles, and Recurrence Intervals. Six plotting position models and three distribution types are available for computing the Plotting Positions, Quantile Deviates, and Cumulative Probability Percentiles. The Recurrence Interval scale type can be presented as either a linear or log scale. Remarked (censored) data values reported as the method detection or reporting limit can be included or excluded from the plotted data. Data may be ranked in ascending (nonexceedance) or descending (exceedance) order before the plotting position is computed. The Y-axes can be linear or logarithmic and can be scaled by the program or by user-defined limits. All Y-axis variables may use the same scale or each variable may have its own scale. For the multiple Y-axis scale option, a given Y-axis scale may be placed on elther side of the plot frame in any order relative to the other Y-axis scales on that side of the plot frame. Tick-mark intervals are user defined. Grid lines for a single Y-axis scale and (or) the X-axis can be plotted. Each Y-axis scale can have its own label. There can be up to three title lines and two footnote lines each definable as to size, font, and centering. Up to five user-defined labels (up to 40 characters on multiple lines) can be positioned anywhere on the plot. Any number of plot specification files can be saved and used to facilitate plot specification in other "prbplt" sessions.

\section{Background}

The relative distribution of the values or groups of values of a measured or observed variable defines a frequency distribution, which usually approximates one of several theoretical distributions. A given value or group number will have a finite probability of occurrence relative to other values. A plot of the data values on the Y-axis versus the computed probabilistic statistic on the $\mathrm{X}$-axis provides a means of comparing the 
distributions of different samples or a given sample with a theoretical distribution. The distribution function forms include:

Plotting Position

Quantile Deviates

Exceedance Cumulative Probability Percentiles

Nonexceedance Cumulative Probability Percentiles

Recurrence Interval (Linear or Logarithmic).

The probability distribution form can be computed by one of the following plotting position models:

$$
\begin{array}{llc}
\text { Standard } & - & \mathrm{I} / \mathrm{N} \\
\text { Weibull } & - & \mathrm{I} /(\mathrm{N}+1) \\
\text { Blom } & -(\mathrm{I}-0.375) /(\mathrm{N}+0.25) & \text { (Default) } \\
\text { Cunnane } & - & (\mathrm{I}-0.4) /(\mathrm{N}+0.2) \\
\text { Gringorten } & - & (\mathrm{I}-0.33) /(\mathrm{N}+0.12) \\
\text { Hazen } & - & (\mathrm{I}-0.5) / \mathrm{N} \\
\text { where: } \mathrm{I} & =\text { Rank of the Observations, and } \\
& \mathrm{N} & =\text { Total Number of Observations. }
\end{array}
$$

The result from the plotting position model is passed to a function representing one of three distributions supported in "prbplt"-Normal (Gaussian), Gumbel Extreme, and Log-Normal - where the Quantile Deviates and Cumulative Probability Percentiles are computed. The Quantile Deviates usually range from -3.0 to +3.0 and the Cumulative Probability Percentiles from 0.01 to 99.99 (Exceedance Probability) or 99.99 to 0.01 (Nonexceedance Probability). If the data fit the distribution, then the resulting plot will be a straight line. Left skewness in a sample produces a convex plot and right skewness in a sample produces a concave plot.

\section{Probability Options}

The options pertaining to the probability functions supported in the "prbplt" program are found in the following screens:

$\begin{array}{ll}\text { Function } & \text { Screen Sequence } \\ \text { Plot Position } & \text { Opening Screen, Modify (M), Statistics (MS) } \\ \text { X-Scale Type } & \text { Opening Screen, Modify (M), Statistics (MS) } \\ \text { Ranking } & \text { Opening Screen, Modify (M), Statistics (MS) } \\ \text { Distribution } & \text { Opening Screen, Modify (M), Statistics (MS) } \\ \text { Axes } & \text { Opening Screen, Modify (M), Basic (MB), Axes (MBA). }\end{array}$

\section{Starting the "prbplt" Program}

The "prbplt" program is invoked by typing "prbplt" on the command line. The "Opening Screen" displays the options that serve as gateways to the screens used to set or modify the plot configuration options.

\section{Load a Specifications File}

To use specifications from a previous "prbplt" session, load a "Specifications" file. From the "Opening Screen," select the "Get (G)" screen. A list of specifications files (extension ".prb") and a field for specifying the file name are presented. Type in a file name or use the arrow keys to select the required file and use either Accept (F2) or the Enter key to accept the selection. The "latest.prb" file contains the program specifications that were 
used during the most recent execution of the "prbplt" program, whether in the current or previous session. Once the specifications file has been loaded, the "Make the Plot" option can be used to create the plot.

If any program options, including the input file name or output device, need to be changed, then proceed to the appropriate screens to make the changes. As appropriate, save sets of program options in specifications files using the "Save specifications" option in the "Opening Screen."

\section{Open Input Data File}

To load a data file, select the "Open - input data file" option in the "Opening Screen" to display the "Open $(\mathrm{O})$ " screen. From the "Open $(\mathrm{O})$ " screen, select the "Select - a data file" option to display the "Select (OS)" screen. Type in a file name or use the arrow, Page Down, and Page Up keys to select a data file. Use either Accept (F2) or the Enter key to accept the file selection and return control to the "Open $(O)$ " screen.

\section{Filter Data}

If it is necessary to restrict the data to be applied to the probability plots, then select the "Filter" option from the "Open (O)" screen to display the "Filter (OF)" screen. Specify a criteria statement for selecting the rows to be retained for the plot.

\section{Name Variables}

The "prbplt" program can produce a curve for each variable selected or a curve for each subset of values from a single variable that has been partitioned into subsets of values based on the composite values of up to four partitioning (grouping) variables. From the "Opening Screen," select the "Name (N)" screen and choose from the following options:

Individual - Select a set of individual variables (one for each probability curve desired).

or

Partition - Select a data variable, a partition variable, and interval limits. One curve for each partition interval.

or

Return - Return control to opening screen.
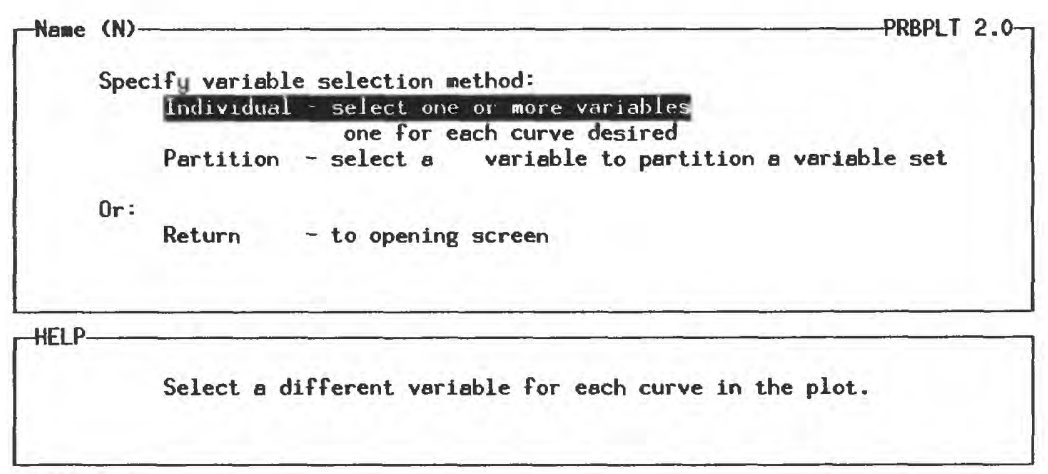

\footnotetext{
INSTRUCT- Select an option using arrow keys

then confirm selection with the F2 key. or

Type the first letter of an option.

Help: 11 Accept: 2 Top: 10 Quiet $: 18$ Xpad: 19 Cahlp
} 


\section{Modify Plot Specifications}

After the data file has been opened and the variables of interest named, select the "Modify (M)" screen either by typing "M" (or " $\mathrm{m}$ ") or use the Tab and arrow keys to select the "Modify compute/plot specifications" option followed by using Accept (F2) or the Enter key. The "Modify (M)" screen lists the following options:

Statistics - specify the statistical options for the probability plot

Curve - specify the curve type options for each variable

Basic - specify title, footnotes, labels, and axes options

Device - specify device for graphics output.

Type the first letter (not case sensitive) of the option desired to bring up the next screen immediately or use the arrow keys to select the option. Use Accept (F2) or the Enter key to accept the selection.
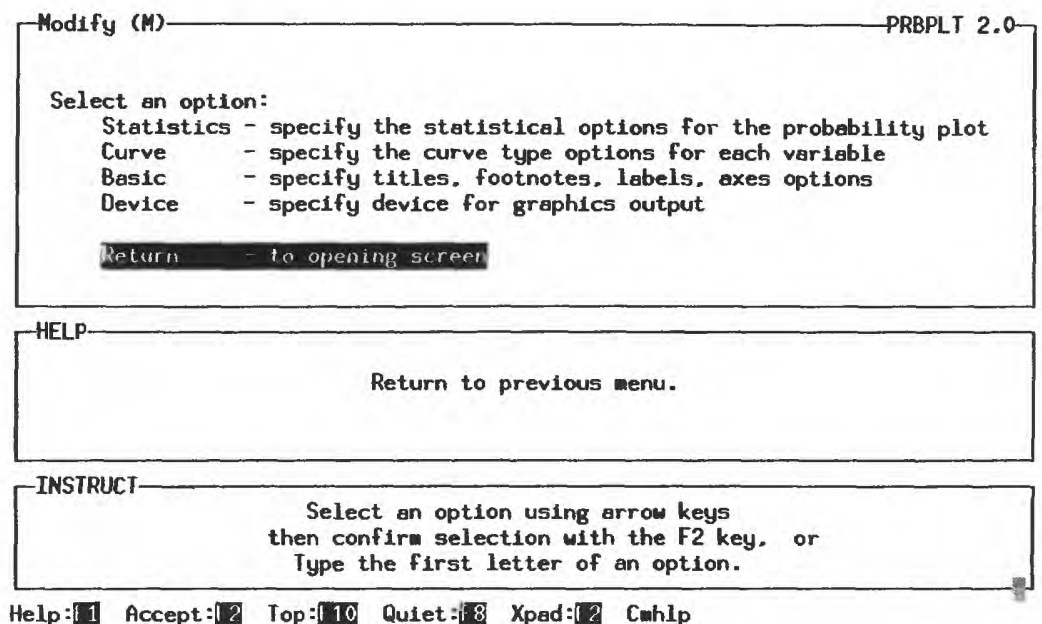

\section{Statistics}

The "Statistics" option provides for the following probability related functions:

Plotting Position

X-Scale Type

Data Ranking

Distribution Type.

\section{Plotting Position}

The "Plotting Position" option supports the following models:

$\begin{array}{llc}\text { Standard } & - & \mathrm{I} / \mathrm{N} \\ \text { Weibull } & - & \mathrm{I} /(\mathrm{N}+1) \\ \text { Blom } & -(\mathrm{I}-0.375) /(\mathrm{N}+0.25) & \text { (Default) } \\ \text { Cunnane } & -(\mathrm{I}-0.4) /(\mathrm{N}+0.2) \\ \text { Gringorten } & -(\mathrm{I}-0.33) /(\mathrm{N}+0.12) \\ \text { Hazen } & -(\mathrm{I}-0.5) / \mathrm{N} \\ \text { where: } \mathrm{I} \quad=\text { Rank of the Observations, and } \\ \quad \mathrm{N}=\text { Total Number of Observations. }\end{array}$


Use the arrow keys to select the plotting position model desired and toggle this selection "On" by using the space bar. An "X" will appear in the field to the left of the selected option.

\section{X-Scale Type}

Six X-axis scale types are supported by the "prbplt" program:

Plotting Position

Quantile Deviate

Percentile Scale - Low to High $(0.01$ - 99.99) (Default)

Percentile Scale - High to Low (99.99 - 0.01)

Recurrence Interval with linear scale

Recurrence Interval with logarithmic scale.

Use the arrow keys to select the X-axis scale type desired and toggle this selection "On" by using the space bar. An "X" will appear in the field to the left of the selected option.

\section{Data Ranking}

The data can be ranked in ascending or descending order before the plotting position computations are made. Use the arrow keys to select the ranking order desired and toggle this selection "On" by using the space bar. An "X" will appear in the field to the left of the selected option.

\section{Distribution Type}

Three distribution models are supported:

Gaussian Normal

Gumbel Extreme Value

Log-Normal.

Use of these models is only applicable for the following "X-scale" types:

Quantile Deviate

Percentile Scale - Low to High (0.01 - 99.99)

Percentile Scale - High to Low (99.99 - 0.01).

Use the arrow keys to select a distribution model and then use the space bar to toggle the selection "On" as indicated by the appearance of an "X" in the field to the left of the option name.

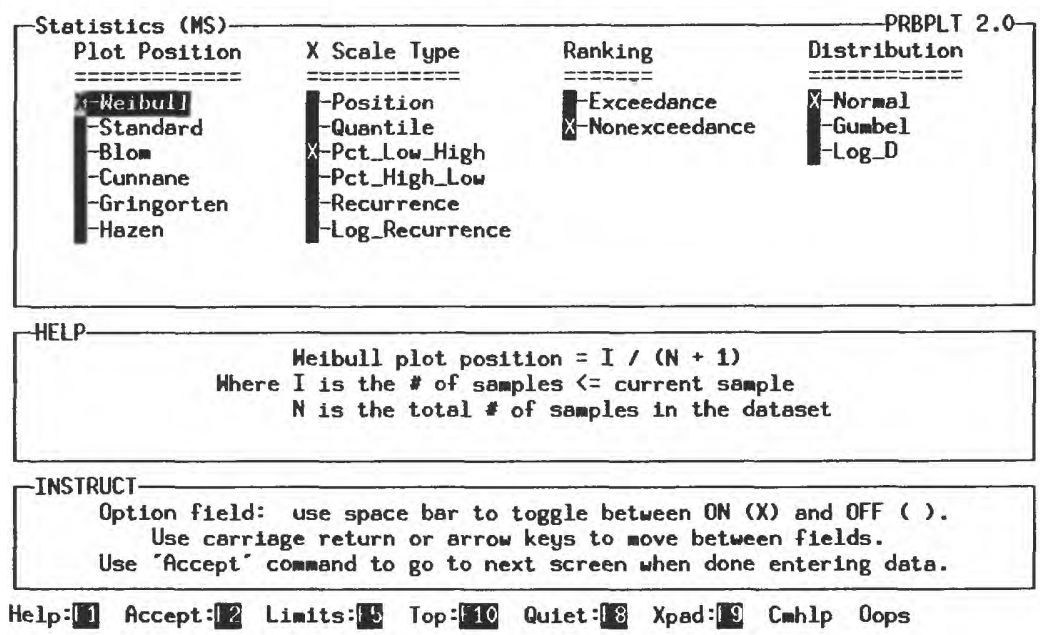




\section{Curve}

The "Curve" option in the "Modify (M)" screen displays the "Curve (MC)" screen used to define the characteristics of the plotted curve lines. The following characteristics can be defined for each curve:
Type
Line Color
Line Pattern
Symbol Type
Symbol Size
Line Width
Legend Label.

Use the arrow and Enter keys to select the variable and curve option desired. Use Help (F1) to bring up the "Help" panel, which lists the allowable values for the curve option selected. Use Limits (F5) to bring up the "Limits" panel, which presents any limits for the codes or values to be entered. Enter the appropriate value or code in the highlighted field. Note that the default legend label is the variable name or the values of the partitioning variable. Enter an alternative label (up to 16 characters) for each curve if you intend to display a legend for the plot.

When finished, use Accept (F2) to return control to the "Modify (M)" screen.

\begin{tabular}{|c|c|c|c|c|c|c|c|}
\hline Number & $\begin{array}{l}\text { Curve } \\
\text { Type }\end{array}$ & $\begin{array}{l}\text { Plot } \\
\text { Color }\end{array}$ & $\begin{array}{l}\text { lect opt } \\
\text { Line } \\
\text { Style }\end{array}$ & $\begin{array}{l}\text { ons for } \\
\text { Symbol } \\
\text { Type }\end{array}$ & $\begin{array}{l}\text { each curv } \\
\text { Symbol } \\
\text { Size }\end{array}$ & $\begin{array}{l}\text { e: } \\
\text { Line } \\
\text { Hidth }\end{array}$ & $\begin{array}{l}\text { Legend } \\
\text { Label }\end{array}$ \\
\hline $\begin{array}{l}1 \\
2\end{array}$ & $\frac{1}{1}$ & $\begin{array}{l}\text { FORE } \\
\text { FORE }\end{array}$ & $\begin{array}{l}\text { SOLID } \\
\text { SOLID }\end{array}$ & $\begin{array}{l}2 \\
3\end{array}$ & $\begin{array}{l}0.1 \\
0.1\end{array}$ & $\begin{array}{l}1.0 \\
1.0\end{array}$ & $\begin{array}{l}\text { SEDIMENT } \\
\text { CONDUCTANCE }\end{array}$ \\
\hline HELP- & & \multicolumn{3}{|c|}{$\begin{array}{l}\text { 1: Straight line } \\
\text { 2: Stepped line } \\
\text { 3: Cubic spline }\end{array}$} & \multicolumn{3}{|c|}{$\begin{array}{l}\text { 4: Scatter diagram } \\
\text { 5: Solid bars } \\
\text { 6: Histogran }\end{array}$} \\
\hline
\end{tabular}

\footnotetext{
INSTRUCT- Enter data in highlighted field(s).

Use carriage return or arrow keys to enter data and move between fields. Use "Accept" command to go to next screen when done entering data.

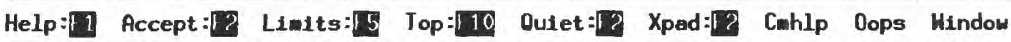

\section{Basic Plot Parameters}

From the "Modify (M)" screen, select the "Basic (MB)" screen, which lists the following options for modifying plot specifications.

$\begin{array}{ll}\text { Axes } & \text { - X-and Y-axis type and limits } \\ \text { Titles } & \text { - above plot } \\ \text { Footnotes } & \text { - below plot } \\ \text { Labels } & \text { - of miscellaneous text } \\ \text { Page } & \text { - layout options } \\ \text { Censored } & \text { - data options } \\ \text { Legend } & \text { - options } \\ \text { Make } & \text { - the plot } \\ \text { Return } & \text { - to previous screen. }\end{array}$

Type the first letter of any option or use the arrow and Enter keys to select and open the screen for the option required. 
From the "Basic (MB)" screen, type "A" (or "a") or use the arrow and Enter keys to bring up the "Axes (MBA)" screen. The "Axes" option provides for specifying axis type, upper and lower limits of the scale, tick-mark intervals, and axis labels. The following options are presented:

$\mathrm{X} \quad$ - axis specifications

Global - Y-axis specifications

Individual - $\mathrm{Y}$-axis specifications.
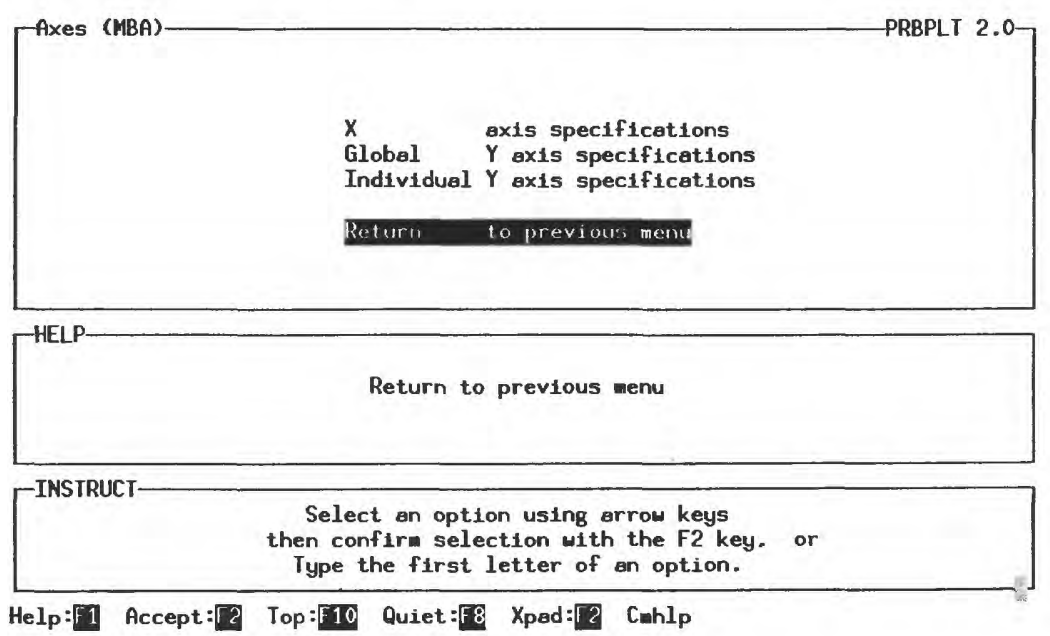

\section{$\underline{\text { X-Axis Specifications }}$}

From the "Axes (MBA)" screen, select the "X-axis (MBAX)" screen. This screen specifies a label to be placed beneath the $\mathrm{X}$-axis. An option also is provided for placing $\mathrm{X}$-axis grid lines on the plot. Use the arrow keys to select the X-axis label option. Enter a label of up to 72 characters. Use a caret $(\wedge)$ in the text to split the label into two lines.

To place an X-axis grid on the plot, select the "Plot X Grid" option and use the space bar to toggle the selection "On" as indicated by the appearance of an "X" in the field to the left of the option name. Use Accept (F2) to return control to the "Axes (MBA)" screen.

\section{Global Y-Axis Specifications}

The "Global" option provides for a single Y-axis to represent all of the variables (curves) to be plotted. From the "Axes (MBA)" screen, select the "Global Y (MBAG)" screen.

\section{Scale Limits}

"Auto" scaling is the default; whereby the system computes and displays the upper and lower scale boundaries based on the range of the data. By using the "Manual" scaling option, the user can specify alternate upper and lower boundaries. Note: The initial scale limits listed for the "Manual" option do not correspond to the extremes of the data in the input data file. To set scale limits in the manual mode, select the "Lower limit" and "Upper limit" fields under "Manual" and enter appropriate values. In subsequent uses of the "Y-Axis (MBAG)" screen in the same session of "prbplt," scale limit values listed in the "Manual" option fields will be the most recent limit values used by the procedure regardless of whether the "Auto" or "Manual" option was used in the previous execution of "prbplt." 


\section{Axis Type}

The Y-axis scale can be either "linear" or "logarithmic" with the default being "linear." Use the arrow keys to select this option and the space bar to toggle the required option "On" as indicated by an "X" in the field to the left of the selected option.

\section{Tick Marks}

A default tick-mark interval is determined based on the range of the data; it can be user specified when the "Manual" option is selected.

\section{Y-Axis Label}

Use the arrow keys to select the "Y-Axis label" option. Enter a label of up to 60 characters in this field.

\section{Plot Grid}

If Y-axis grid lines are desired, then use the arrow keys to select the "Plot Grid" option and then toggle this selection "On" by using the space bar. An "X" in the field to the left of the option name will indicate activation of this option. Use Accept (F2) to return control to the "Basic (MB)" screen.

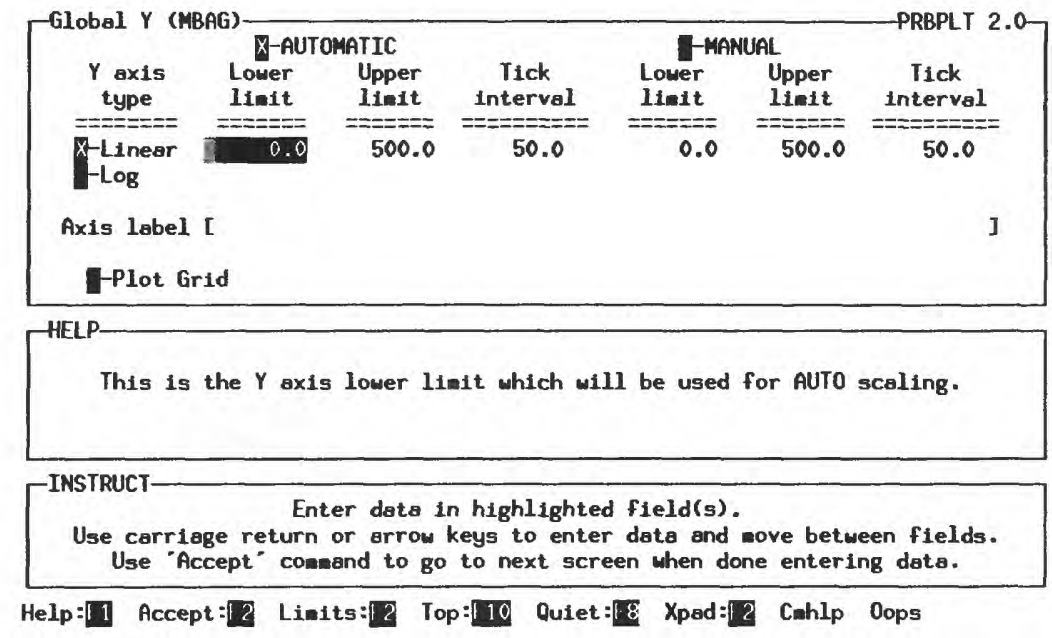

\section{Individual Y-Axis Specifications}

This option is selected to have a Y-axis defined for each of the probability curves to be plotted. Each Y-axis can be specified in the same manner as a "Global Y-axis," with the added option to place an axis on either the left or right side of the plot frame.

From the "Axes (MBA)" screen, select the "Individual Y1 (MBAI)" screen, which prompts for the sequence number of the variable for which a Y-axis is to be specified. Upon entering a number and using the Enter key, the "Individual Y2 (MBAI)" screen is brought up, which is similar to and populated in the same manner as the "Global Y (MBAG)" screen. To place the axis on the right side of the plot frame, select and toggle "On" the "Place on right side" option. An "X" will appear in the field to the left of the option name.

Repeat this process for each variable. Modifications to any Y-axis scale specifications can be made any number of times and in any order by specifying the variable number in the "Individual Y1 (MBAI)" screen. 


\section{Censored Data Options}

By default the "prbplt" program excludes censored data from the analysis. To include censored data (remark codes are disregarded), use the arrow or Tab keys to select (highlight) the "Include censored values" option in the "Censored (MBC)" screen. Use Accept (F2) to accept the selection and return control to the "Basic (MB)" screen.

\section{Legend Options}

To place a legend on the plot describing the line type and symbol used for each curve, use the arrow keys to select the option desired. Toggle the option "On" with the space bar as indicated by the appearance of an "X" in the field to the left of the option name. The legend text label is defined in either the "Curve (MC)" screen or the "Labels (MPTAL)" screen when the partitioned data option is selected in the "Name $(\mathrm{N})$ " screen.
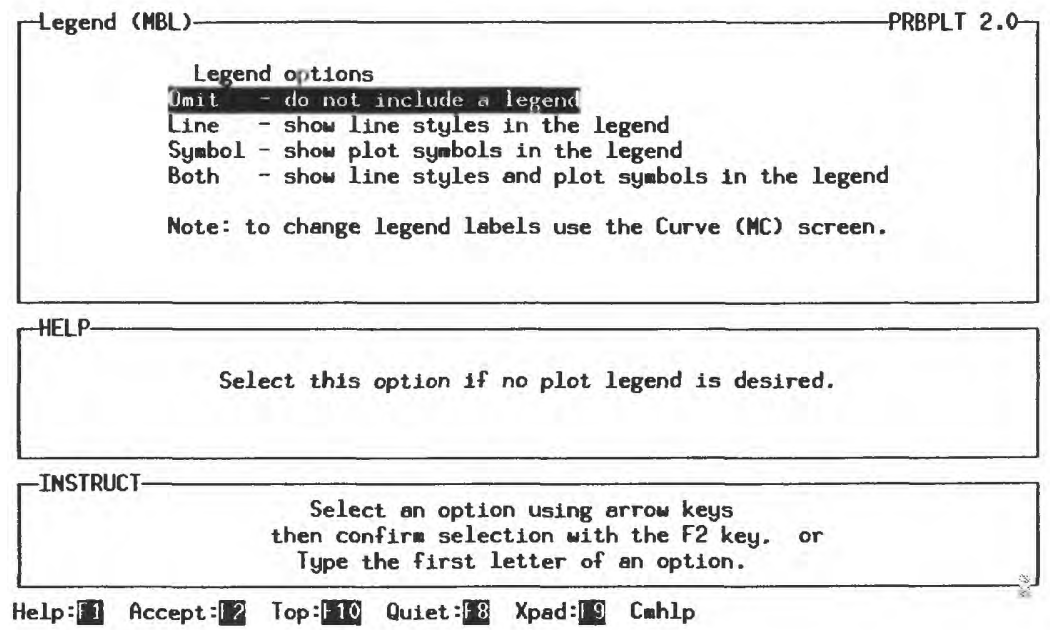

\section{Page Layout Options}

From the "Basic (MB)" screen, select the "Page (MBP)" screen, which provides the following functions:

Page Height

Page Width

Font Style

Plot Page Border

Frame Axis Border

Tick Marks Inside

Horizontal Y-Axis Numbers

Four-Sided Axis Options.

Most options in this list are discussed in the section "Page Layout Options" under "Steps to Using QWGRAF Programs." 

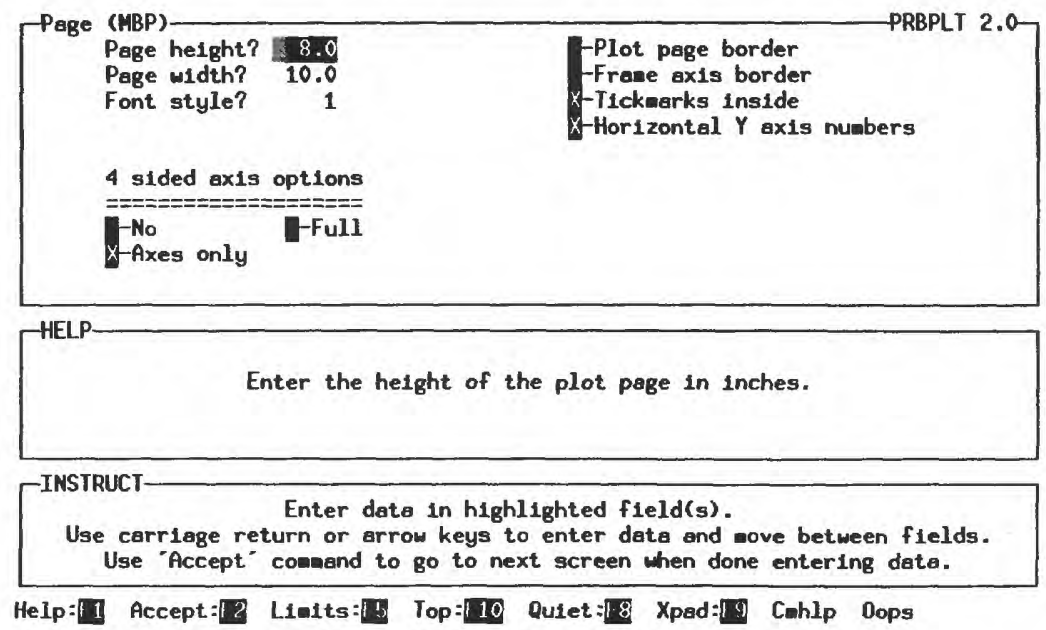

\section{Four-Sided Axis Options}

The four-sided axis option duplicates the X-and Y-axes on the top and right side, respectively, of the plot frame. This option is only applicable when the "Global Y-axis" option is used. The options are as follow:

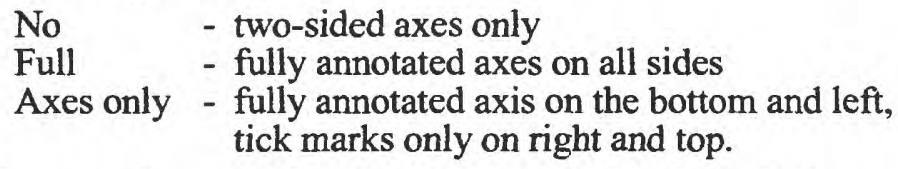

Use the arrow keys to select the option desired and toggle the option "On" with the space bar as indicated by the appearance of an " $\mathrm{X}$ " in the field to the left of the option name.

\section{Make the Plot}

When all arguments for the probability plot have been specified and the appropriate device selected, return control to the "Opening Screen," select the "Make the plot" option, and use the Enter key. If a screen output was selected, then a graphics window opens and the probability plot is presented. If a file output was selected, then a message screen indicates that the output from the procedure has been saved.

\section{Sample Plots}

The following are examples of the types of plots that can be created with the QWGRAF "prbplt" program. These examples are intended to illustrate various options available in the program and do not necessarily conform to publication standards of the Geological Survey. 


\section{QWGRAF}

\section{Example Plots}

PRBPLT

Program: PRBPLT

Plot Specifications: Two Variables; Weibull Plotting Position Model; Percentile Low-High X-Axis Scale Type; Nonexceedance Ranking; Normal Distribution; Automatic Axis Scaling; Individual Y-Axes; Y-Axes Labels; Default X-Axis Label; Explanation Block; Single Title

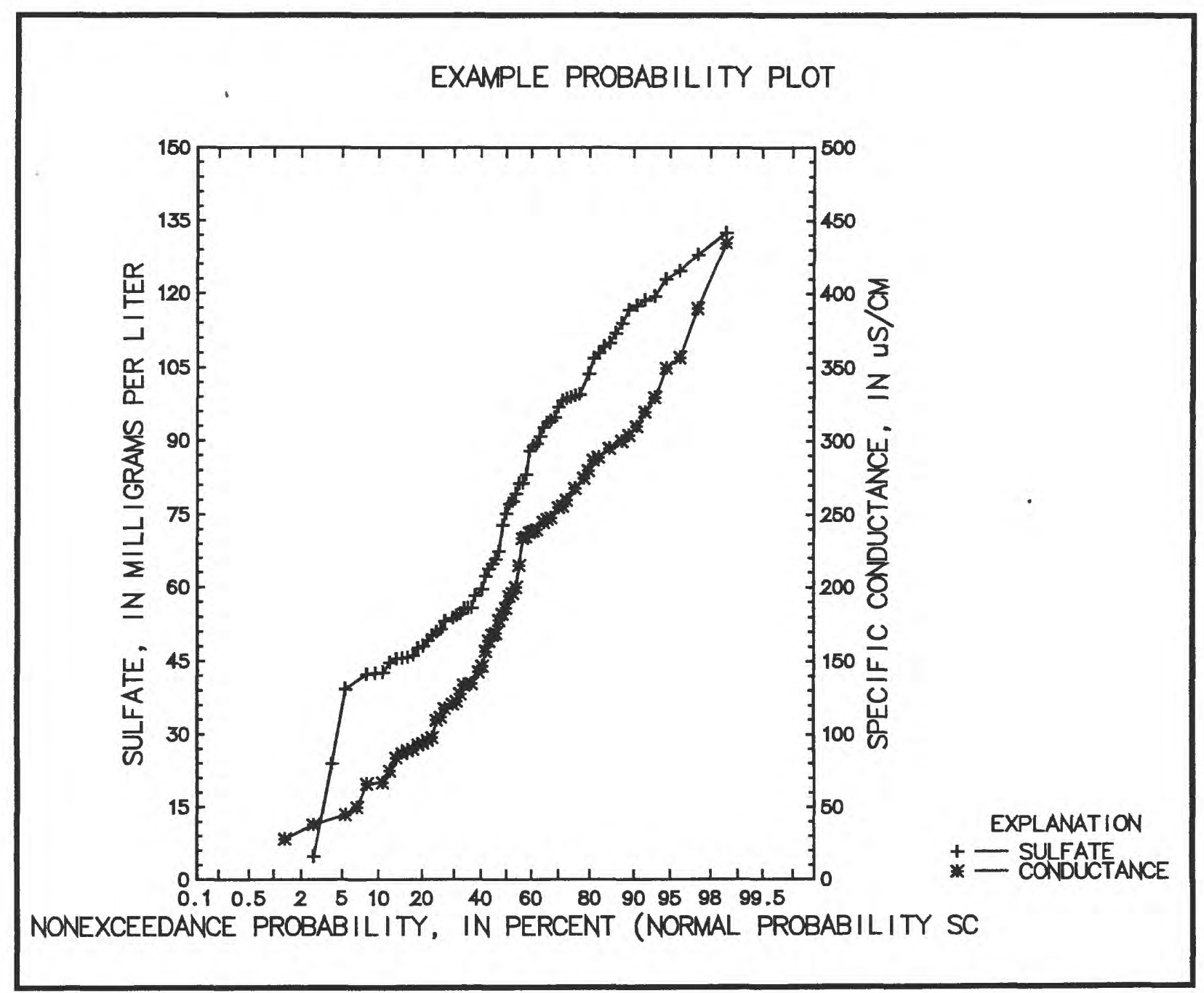




\section{Program: PRBPLT}

Plot Specifications: Two Variables; Weibull Plotting Position Model; Quantile Scale Type; Nonexceedance Ranking; Gumbel Distribution; Automatic Axis Scaling; Individual Y-Axes; Y-Axes Labels; Default $\mathrm{X}$-Axis Label; Explanation Block; Single Title

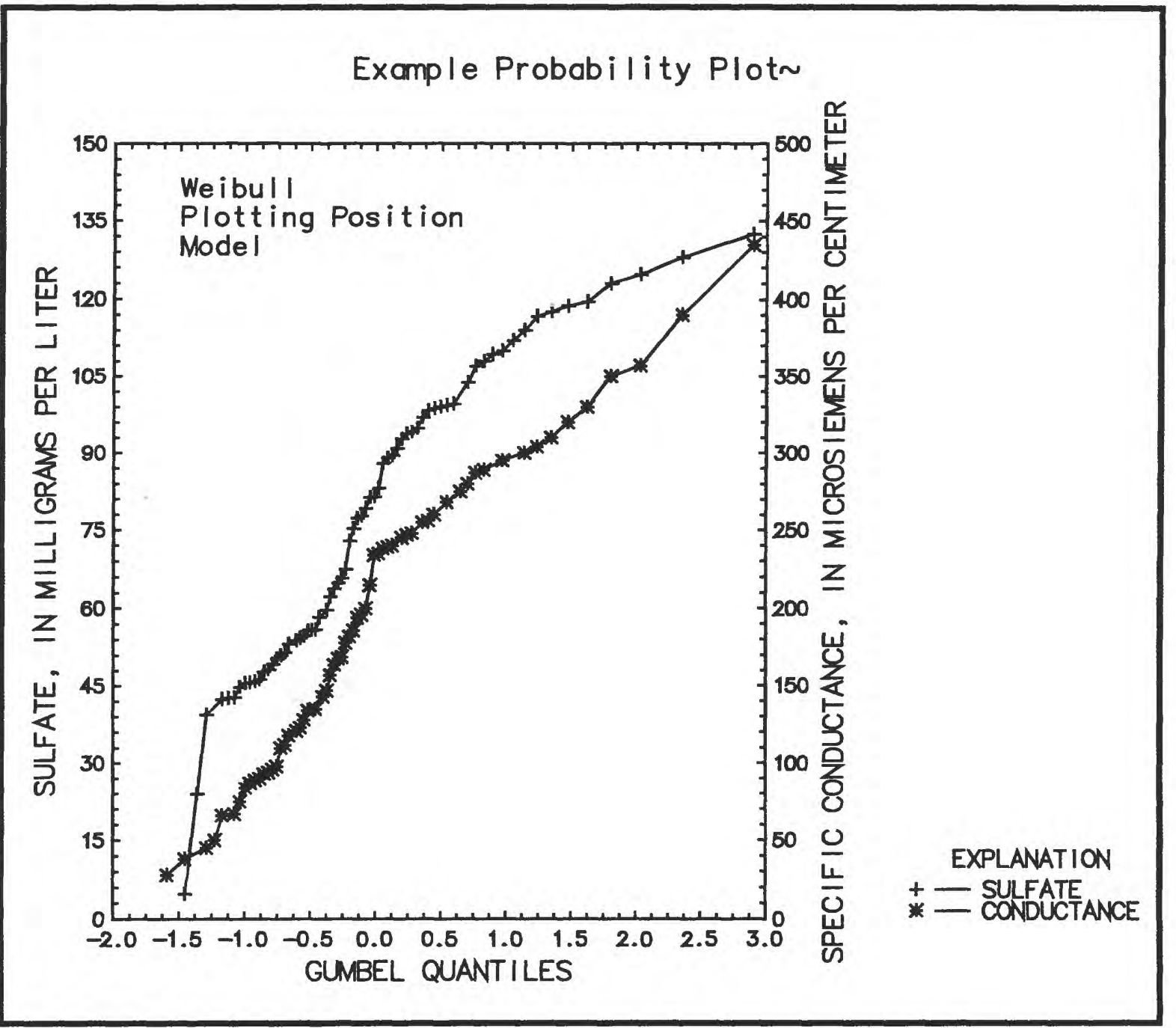


Program: PRBPLT

Plot Specifications: Single Variable Partitioned by State; Weibull Plotting Position Model; Percentile LowHigh Scale Type; Nonexceedance Ranking; Normal Distribution; Explanation Block; Global Y-Axis Scale; Manual Scale Limits; Y-Axis Grid Lines; Y-Axis Label; Default X-Axis Scale Label; Single Title

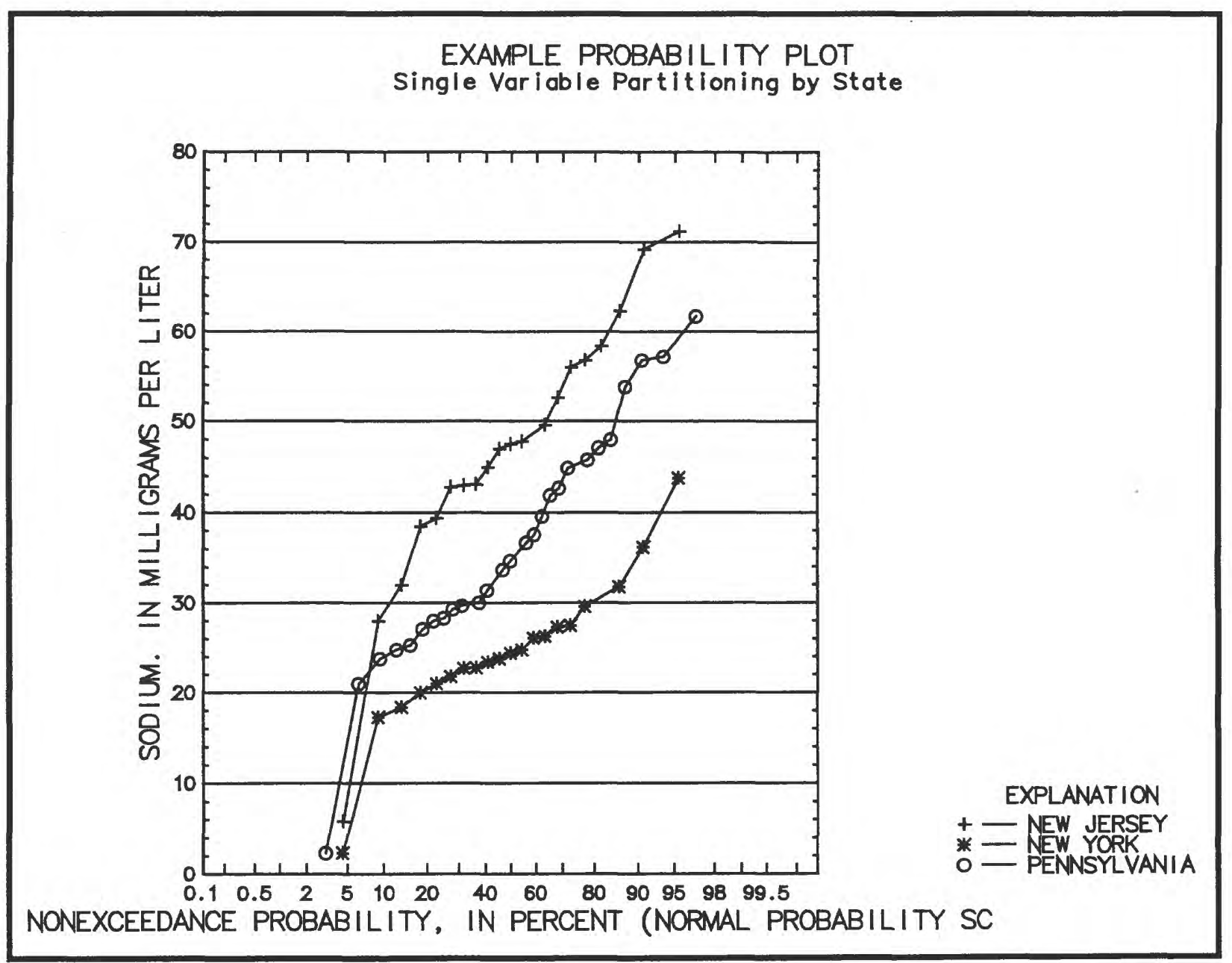




\section{Program: PRBPLT}

Plot Specifications: Weibull Plotting Position Model; Recurrence Interval X Scale Type; Nonexceedance Ranking; Automatic Y-Axis Scaling; Y-Axis Grid Lines; Y-Axis Label; Default X-Axis Label; Single Title

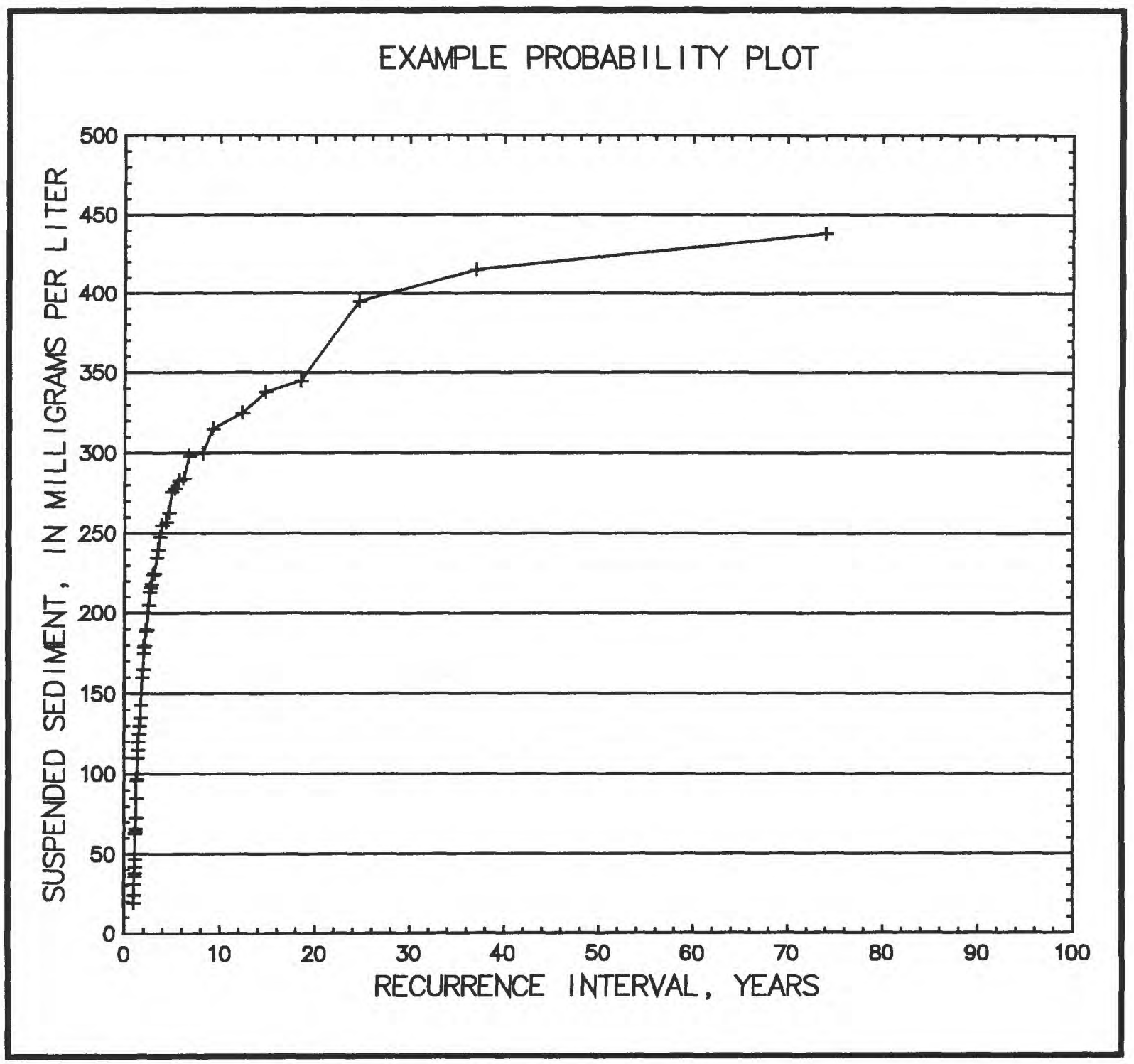




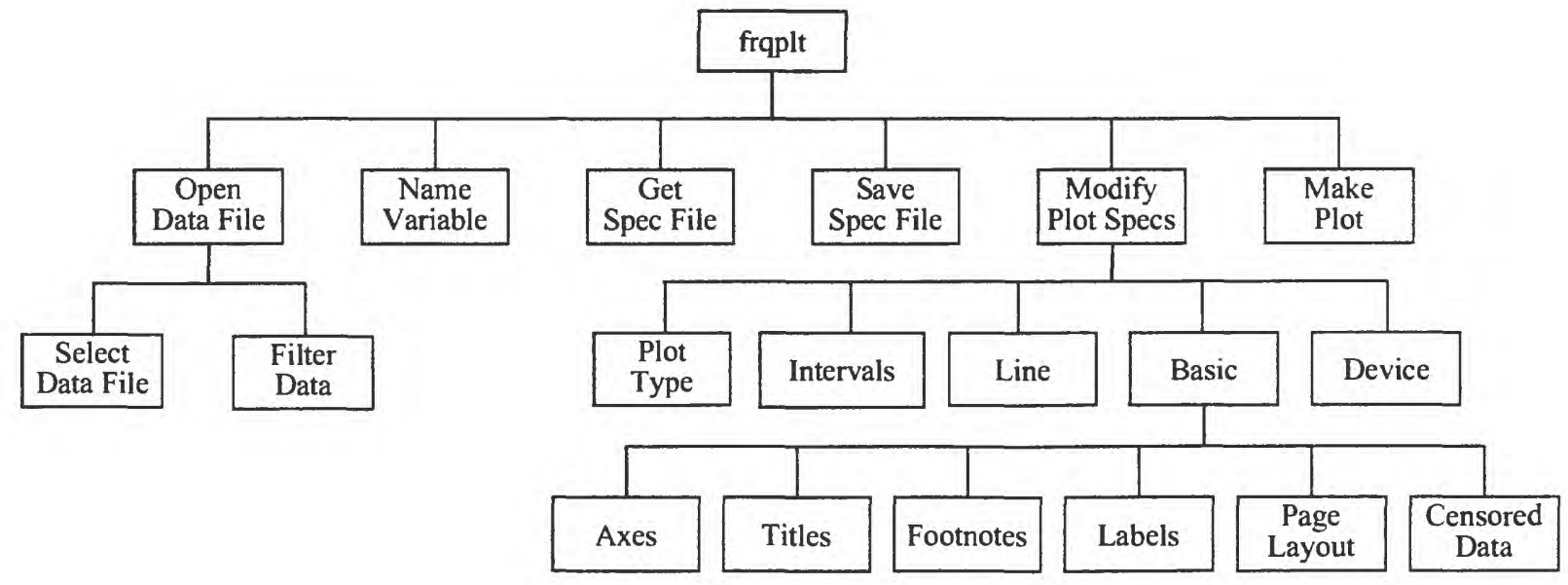

FREQUENCY DISTRIBUTION PLOTS (frqplt)

The "frqplt" program plots frequency distribution functions on the Y-axis versus the data on the X-axis. The frequency distributions available include: FREQUENCY, RELATIVE FREQUENCY, CUMULATIVE FREQUENCY, CUMULATIVE RELATIVE

FREQUENCY, and the EMPIRICAL DISTRIBUTION FUNCTION (EDF). Data are partitioned into one of three interval class types - user-defined intervals, equal intervals, or quantile intervals. The plot can consist of straight lines that connect the user-defined plotting position location of each class, a histogram of each class, or both. The plotting position can be the Lower Bound of the Class, the Midpoint of the Class, or the Upper Bound of the Class. Remarked (censored) data values reported as a limit of quantitation or detection limit can be included or excluded from the plot data set. The Y-axis can be scaled by the program or by user-defined $\mathrm{min} / \mathrm{max}$ limits. Tick-mark intervals can be user defined. Grid lines can be plotted based on the tick-mark interval. There can be up to three title lines and two footnote lines each definable as to size, font, and centering. Up to five user-defined labels (up to 40 characters on multiple lines) can be positioned on the plot. Any number of plot specification files can be saved and used to facilitate plot specification in other "frqplt" sessions.

\section{Background}

Several methods are available for presenting the frequency distribution patterns of the observations in a data set. These methods consist of two elements. The first element partitions the data into interval classes or groups. For user-defined intervals, an appropriate number of intervals is selected and then each interval is manually assigned upper and lower boundary values. For equal intervals the number of classes is selected and the width of each class is determined by dividing the difference between the minimum and maximum values of the data (or by assigning the upper and lower boundaries) by the number of intervals; that is, each interval has the same data width. Quantile intervals have the same number of observations in each interval. The second element involves the computation of a distribution statistic by one of five models for each interval. The data is plotted on the X-axis, and the statistic is plotted on the Y-axis. Rectangular histograms that encompass each interval are plotted and lines can be drawn to connect the lower bound, midpoint, or upper bound points of each histogram. The five models include: (1) FREQUENCY, the number of sample observations within each class; (2) RELATIVE FREQUENCY, the number of sample observations within each class (the frequency) divided by the total number of observations in all of the classes (the sample); 
(3) CUMULATIVE FREQUENCY, the total number of observations in all classes less than or equal to either the lower or upper bound of a given class; (4) CUMULATIVE RELATIVE FREQUENCY, the cumulative frequency divided by the total number of observations in all of the classes (the sample); and (5) EMPIRICAL DISTRIBUTION FUNCTION, a stairstep plot of the cumulative relative frequency of each observation on the $\mathrm{Y}$-axis versus the data value on the $\mathrm{X}$-axis; that is, each observation becomes a class.

\section{Frequency Plot Options}

The options that pertain to the frequency functions supported by the "frqplt" program are found in the following screens:

Function

Frequency Type

Plotting Position Model

Data Intervals

Frequency Distribution Line

Axes

\section{Screen Sequence}

Opening Screen, Modify (M), Plot (MP)

Opening Screen, Modify (M), Plot (MP)

Opening Screen, Modify (M), Intervals (MI)

Opening Screen, Modify (M), Line (ML)

Opening Screen, Modify (M), Basic (MB),

Axes (MBA).

\section{Starting the "frqplt" Program}

The "frqplt" program is invoked by typing "frqplt" on the command line. The "Opening Screen" displays the options that serve as gateways to the screens used to set or modify the frequency plot configuration options.

\section{Load a Specifications File}

To use specifications from a previous "frqplt" session, load a "Specifications" file. From the "Opening Screen," select the "Get (G)" screen. A list of specifications files (extension ".frq") and a field for specifying the file name are presented. Use the arrow keys to select the required file and use either Accept (F2) or the Enter key to accept the selection. The "latest.frq" file contains the program specifications that were used during the most recent execution of the "frqplt" program, whether in the current or previous session.

The "Specifications" file includes the name of the input data file active at the time the specifications file was created. If a new input data file is required, then select the "Open (O)" screen from the "Opening Screen" and select a new input data file.

The "Make the Plot" option can be selected immediately or any of the newly loaded specifications can be changed. Any altered specifications set can be saved to a new file or an old file can be overwritten.

\section{Open Input Data File}

From the "Opening Screen," select the "Open (O)" screen. From the "Open (O)" screen, select the "Select (OS)" screen. Type in a file name or use the arrow, Page Down, and Page Up keys to select a data file. Use either Accept (F2) or the Enter key to accept the file selection and return control to the "Open $(\mathrm{O})$ " screen.

\section{Filter Data}

To restrict the data to be applied to the frequency diagram, use the "Filter" option from the "Open (O)" screen to display the "Filter (OF)" screen, which is used to specify the criteria for selecting the rows to be retained for the plot. 
From the "Opening Screen," select the "Name (N)" screen. Use the arrow and Enter keys to select one variable for the frequency plot. Use Accept (F2) to accept the selection and return control to the "Opening Screen."

\section{Modify Plot Specifications}

After the data file has been opened and the variable of interest named, select the "Modify (M)" screen by typing "M" (or " $m$ ") to bring up the screen directly or use the Tab and arrow keys to select the "Modify compute/plot specifications" option followed by using Accept (F2) or the Enter key. The "Modify (M)" screen lists the following options:

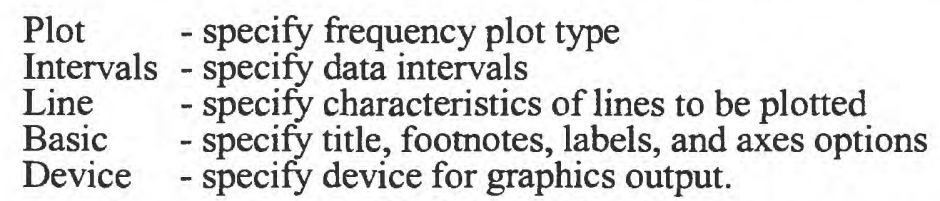

Type the first letter (not case sensitive) of the option to view the next screen or use the arrow keys to select the option and use either Accept (F2) or the Enter key to accept the selection.

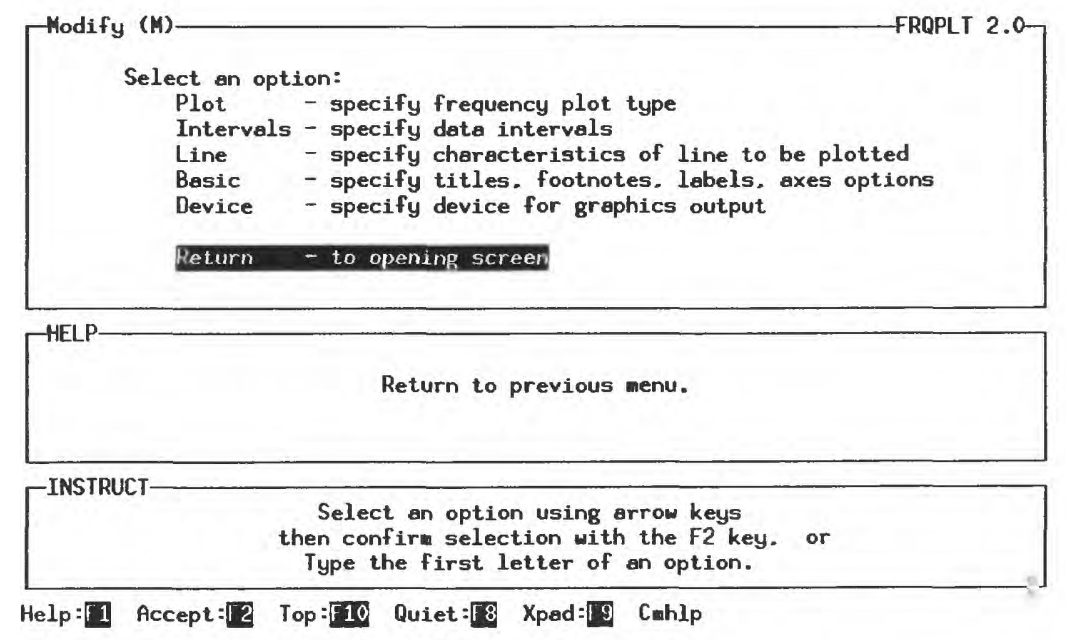

\section{Plot Types}

From the "Modify (M)" screen, select and bring up the "Plot (MP)" screen, which lists the frequency plot types and the plotting position models supported by the program. The frequency plot types supported by the "frqplt" program are as follow:

\section{Frequency \\ Relative frequency \\ Cumulative frequency \\ Cumulative relative frequency \\ Empirical distribution function.}

Use the arrow keys to select the frequency type and toggle the selection "On" by using the space bar key. An "X" will appear to the left of the option description. 


\section{Plotting Position Models}

The plotting position model specifies where the line makes contact within the data interval. The plotting position models supported are as follow:

Lower bound

Midpoint

Upper bound.

Use the arrow keys to select the plotting position model, and use the space bar to toggle this selection "On" as indicated by the appearance of an "X" in the field to the left of the option description. Use Accept (F2) to accept these selections and return control to the "Modify (M)" screen.

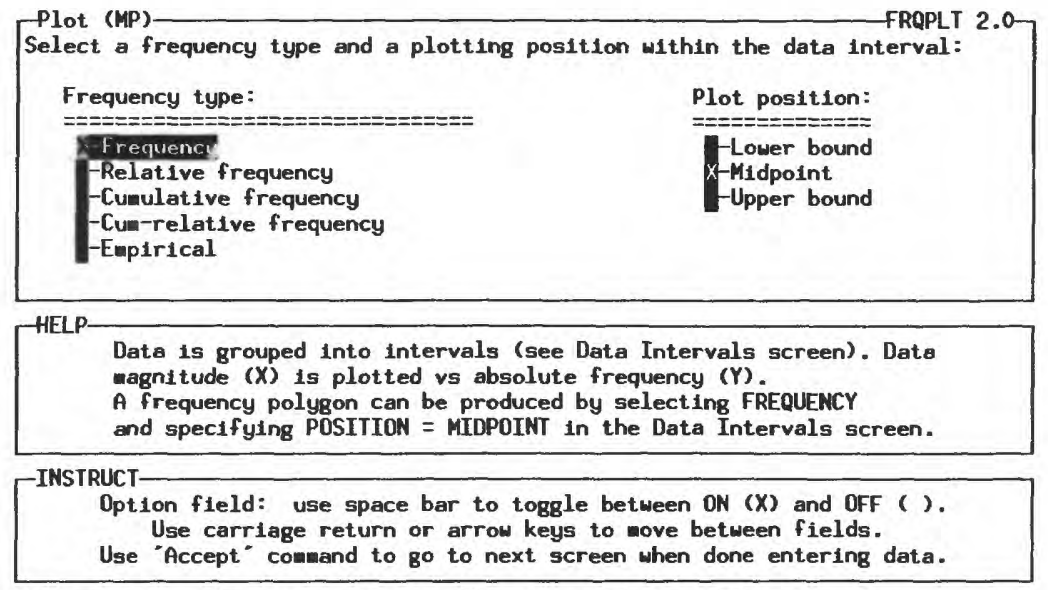

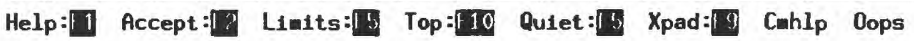

\section{Data Intervals}

The data are ranked and grouped into intervals. Each interval is represented on the frequency plot as a rectangle; the upper and lower bounds of the interval are the right and left edges of the rectangle, respectively. The "Intervals (MI)" screen is used to specify the method to be used to create and populate the intervals.

From the "Modify (M)" screen, select the "Intervals (MI)" screen, which provides three options for computing the data intervals:

Equal - intervals (Equal data ranges in all intervals)

Quantile - intervals (Equal number of observations each interval)

User - intervals (User-defined interval ranges).

Type the first letter (not case sensitive) of the option to view the next screen or use the arrow keys to select the option and then use the Enter key. 


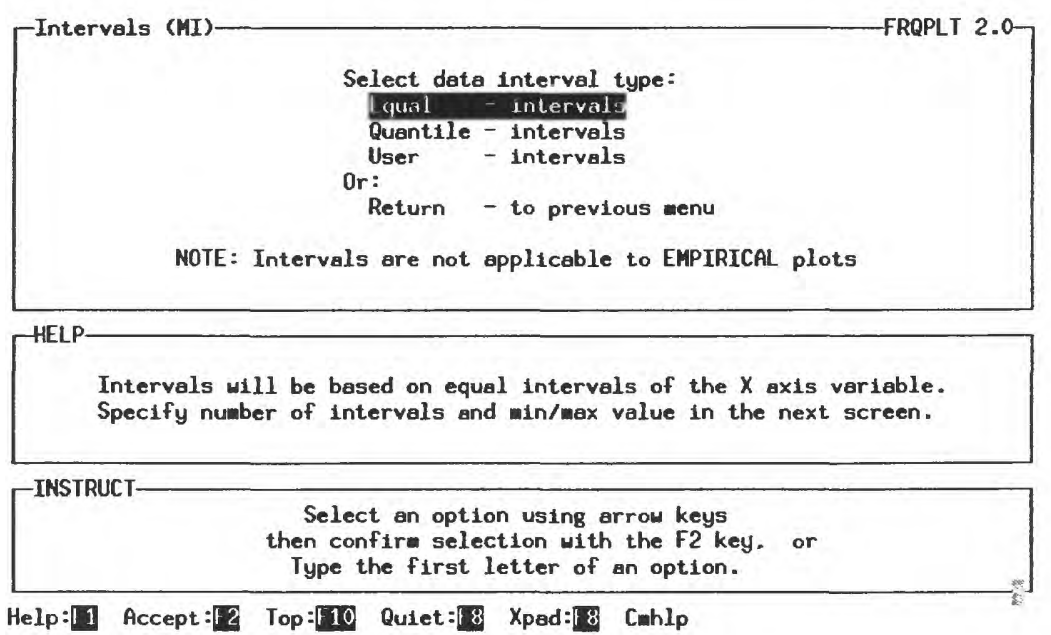

\section{Equal Intervals}

Selection of the "Equal intervals" option brings up the "Equal Intervals (MIE)" screen, which provides for selecting the number of intervals to be used and the range of the data values to be divided equally into those intervals. The program determines and displays the actual maximum and minimum data values for the variable. These values are used by the program to select an upper and lower boundary for the X-axis, when the "Automatic" option is selected. If the "Manual" option is selected, then only those values that fall inclusively within the range specified by the user will be used to create the equal range values for the number of intervals specified. Note that the initial "Manual" minimum and maximum values are system defaults and have no relation to the actual range of data in the input data file. Therefore, the values must be changed to user specifications if the "Manual" option is selected. Also note, in subsequent uses of the "Equal Intervals" screen in the current session of "frqplt," the minimum and maximum values displayed in the "Manual" area will be the values used in the last execution of the program, regardless of whether the "Automatic" or the "Manual" option was selected.

Use the arrow keys to select the "Number of Intervals" option and enter the number of intervals to be used. Use the arrow keys to select either the "Automatic" or "Manual" option. Use the space bar to toggle the selection "On," as indicated by an "X" in the field to the left of the option. For the "Manual" option, use the arrow keys to select the "Minimum" or "Maximum" option. Enter the appropriate values. If this concludes the "Interval" selection process, then use the Enter key or Accept (F2) to accept the selections and return control to the "Modify (M)" screen. 


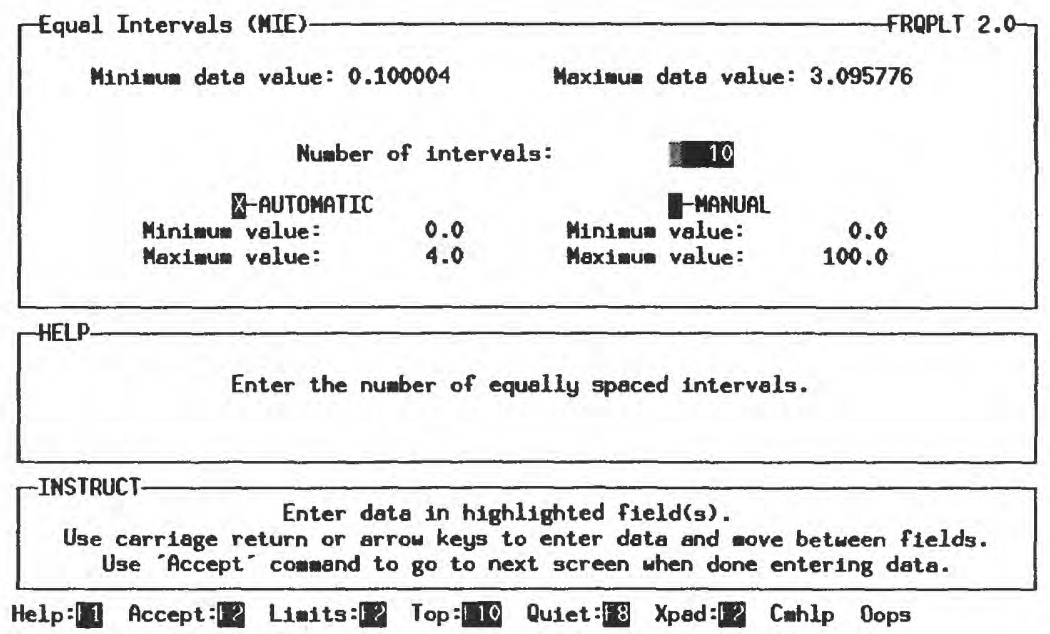

\section{Quantile Intervals}

Selection of the "Quantiles" option brings up the "Quantile Intervals (MIQ)" screen. The only input requirement for this screen is the number of intervals to be used. Based on the number of intervals specified, the program attempts to set interval limits such that each interval contains approximately the same number of data values. It is usually not possible to achieve the same number of values in all intervals. When this is not the case, data values are assigned to intervals in a manner that minimizes the Root Mean Square (RMS) error between the quantized interval cumulative distribution and the ideal cumulative distribution. Consider a case with 23 unique data values grouped into 5 intervals. The ideal population of each interval is $4.6(23 / 5)$, which is physically not possible. The ideal cumulative distribution for each interval is $4.6,9.2,13.8,18.4$, and 23 . To minimize the RMS error, the population of the first interval is defined as:

$$
\mathrm{QP}[1]=\operatorname{INT}(\mathrm{IC}[1]+0.5) \text {, }
$$

where $\mathrm{QP}[1]$ is the quantized population of the interval 1,

IC[1] is the ideal cumulative distribution of interval 1 , and

INT is the integer truncation function.

The remaining populations $(2-\mathrm{N})$ are defined as:

$$
\mathrm{QP}[\mathrm{n}]=\mathrm{INT}(\mathrm{IC}[\mathrm{n}]+0.5)-\mathrm{QP}[\mathrm{n}-1] \text {. }
$$

The quantized interval populations for this example are: 5, 4, 5, 4, and 5. Enter the number of intervals required and use Accept (F2) to accept this value and return control to the "Intervals (MI)" screen. If this concludes the "Interval" selection process, then use the Enter key or Accept (F2) to accept the selections and return control to the "Modify (M)" screen.

\section{User-Defined Intervals}

Selection of the "User Intervals" option brings up the "User Intervals (MIU)" screen. This screen is used to specify specific interval ranges for the frequency plot. Use the arrow keys to select and define the minimum and maximum values for as many intervals as required. These intervals need not be contiguous or in any particular order.

When finished, use Accept (F2) to accept these specifications and return control to the "Intervals (MI)" screen. If this concludes the "Interval" selection process, then use the Enter key or Accept (F2) to accept the selections and return control to the "Modify (M)" screen. 

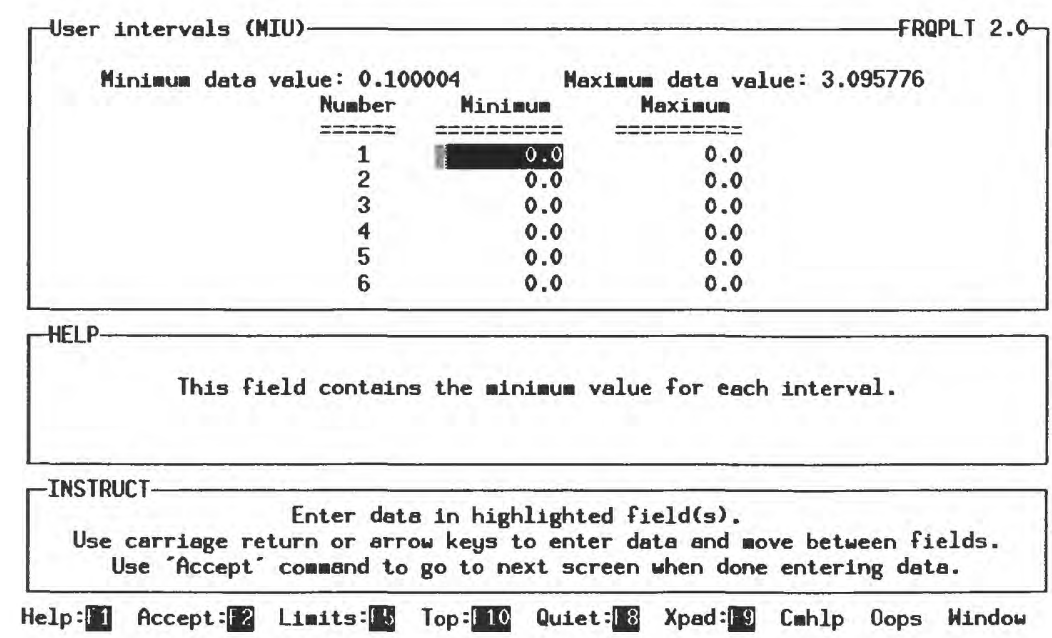

\section{Line Characteristics}

The frequency plot consists of a frequency distribution line, a histogram of the intervals, or both. The "Line" option in the "Modify (M)" screen provides for specifying the pattern, color, and thickness of the lines that form these figures.

From the "Modify (M)" screen, select the "Line (ML)" screen. Use the arrow keys to select an option and enter the appropriate value. Use Help (F1) to bring up the "Help" panel for each option. A list of allowable values (codes or numbers) is presented. Use one of these values to populate the option field. Enter a value of " 0 " (zero) in the "Pattern" field to omit the line from the plot.
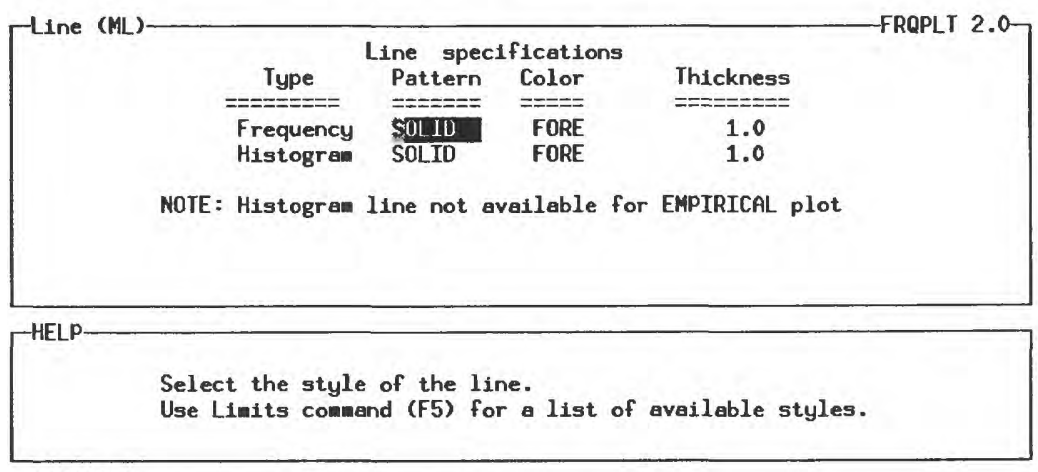

\footnotetext{
TNSTRUCT- Enter data in highlighted field(s).

Use carriage return or arrow keys to enter data and move between fields. Use "Accept" cowand to go to next screen when done entering data.
}

Help:I1 Accept:F2 Limits: Top : 10 Quiet: 8 Xped:I9 Cahlp Dops

\section{Basic Plot Parameters}

From the "Modify (M)" screen, select the "Basic (MB)" screen, which lists the following options for modifying plot specifications:

$\begin{array}{ll}\text { Axes } & -\mathrm{X} \text {-and Y-axis type and limits } \\ \text { Titles } & - \text { above plot }\end{array}$ 


$\begin{array}{ll}\text { Footnotes } & \text { - below plot } \\ \text { Labels } & \text { - of miscellaneous text } \\ \text { Page } & \text { - layout options } \\ \text { Censored } & \text { - data options } \\ \text { Make } & \text { - the plot } \\ \text { Return } & \text { - to previous screen. }\end{array}$

Type the first letter (not case sensitive) of any option or use the arrow and Enter keys to select and open the screen for the option required.
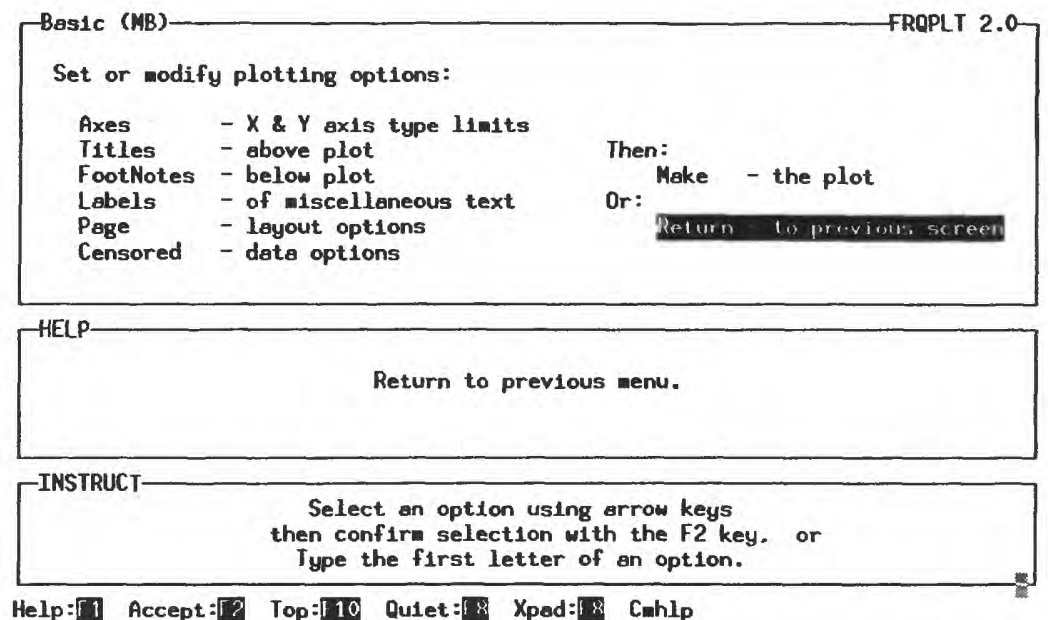

\section{Axes}

From the "Basic (MB)" screen, type "A" (or "a") or use the arrow and Enter keys to bring up the "Axes (MBA)" screen. The "Axes" option provides for specifying the axis type, the upper and lower limits of the scale, the tick-mark interval, and the axis labels. The following options are presented:

$\mathrm{X}$-axis specifications

Y-axis specifications.

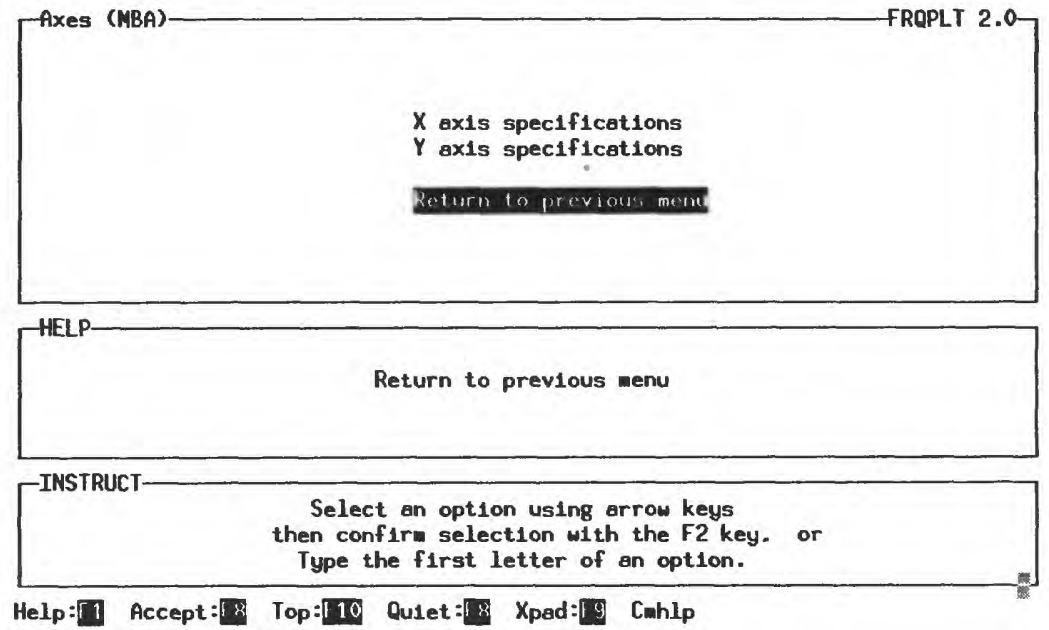




\section{$\underline{X-A x i s ~ S p e c i f i c a t i o n s ~}$}

From the "Axes (MBA)" screen, select the "X-axis (MBAX)" screen.

\section{Axis Limits}

Note: Optional X-axis limits are only applicable for the "Empirical" plot type option (see "Plot (MP)" screen).

The limits of the X-axis scale can be determined by the program or set by the user. Use the space bar to toggle the selection "On" as indicated by the appearance of an " $\mathrm{X}$ " in the field to the left of the option name. Use the arrow keys to select the "Lower limit," "Upper limit," and "Tick interval" options and enter appropriate values. The "Tick interval" should be an equal increment of the $\mathrm{X}$-axis range.

\section{Axis Label}

To place a label beneath the $\mathrm{X}$-axis, use the arrow keys to select the $\mathrm{X}$-axis label option. Enter a label of up to 60 characters.

\section{Grid Lines}

To place X-axis grid lines on the plot, select the "Plot Grid" option and use the space bar to toggle the selection "On" as indicated by the appearance of an "X" in the field to the left of the option name. Specify a color for the grid lines with the "Color" option. Use Help (F1) to bring up the "Help" panel, which lists the available colors and corresponding codes. Specify a line pattern for the grid lines with the "Line Pattern" option. Use Help (F1) to bring up the "Help" panel, which lists the available line patterns and corresponding codes. When finished, use Accept (F2) to return control to the "Axes (MBA)" screen.

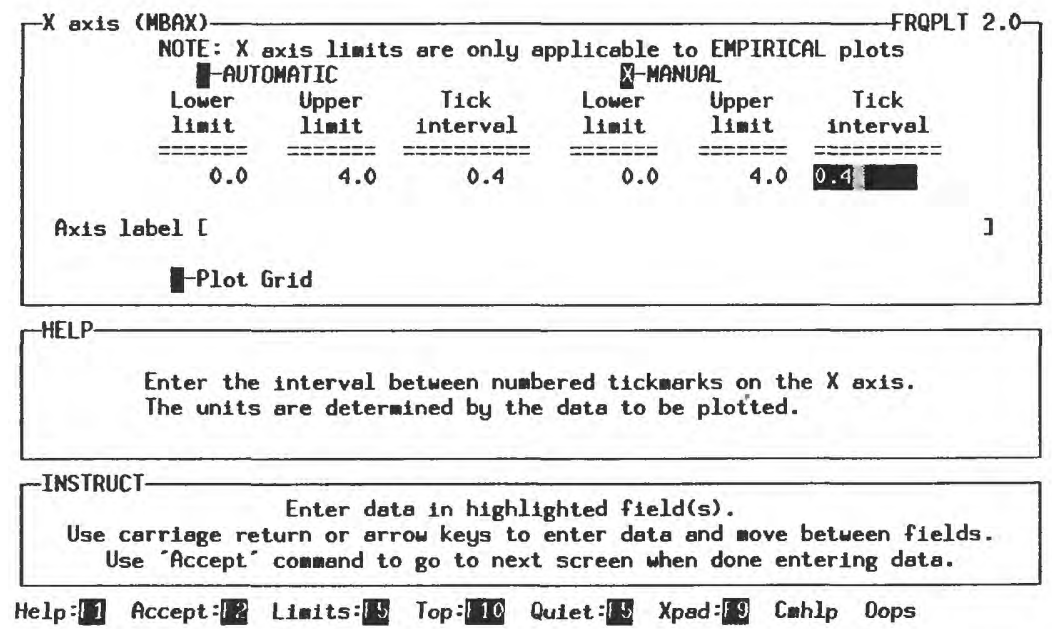

\section{Y-Axis Specifications}

From the "Axes (MBA)" screen, select the "Y-axis (MBAY)" screen. The limits of the $\mathrm{Y}$-axis scale can be determined by the program or set by the user. To set the axis limits manually, use the Enter or arrow keys to select the "Manual" option. Toggle this selection "On" by using the space bar as indicated by the appearance of an " $\mathrm{X}$ " in the field to the left of the option name. Use the arrow keys to select the "Lower limit," "Upper limit," and "Tick interval" options and enter appropriate values. Note, the "Tick interval" should be an equal increment of the $\mathrm{X}$-axis range. 
Axis Label

The "frqplt" program provides default labels for the Y-axis depending on the plot type selected (see "Plot (MP)" screen). These labels are as follow:

Plot Type
Frequency
Relative Frequency
Cumulative Frequency
Cumulative Relative Frequency
Empirical

To place an alternate label to the left of the $\mathrm{Y}$-axis, use the arrow keys to select the Y-axis label option. Enter a label of up to 60 characters.

\section{Grid Lines}

To place Y-axis grid lines on the plot, select the "Plot Grid" option and use the space bar to toggle the selection "On" as indicated by the appearance of an "X" in the field to the left of the option name. Options for "Line Color" and "Line Pattern" also appear when the grid line option is toggled "On." Specify a color for the grid lines with the "Color" option. Use Help (F1) to bring up the "Help" panel, which lists the available colors and corresponding codes. Specify a line pattern for the grid lines with the "Line Pattern" option. Use Help (F1) to bring up the "Help" panel, which lists the available line patterns and corresponding codes. When finished, use Accept (F2) to return control to the "Axes (MBA)" screen.

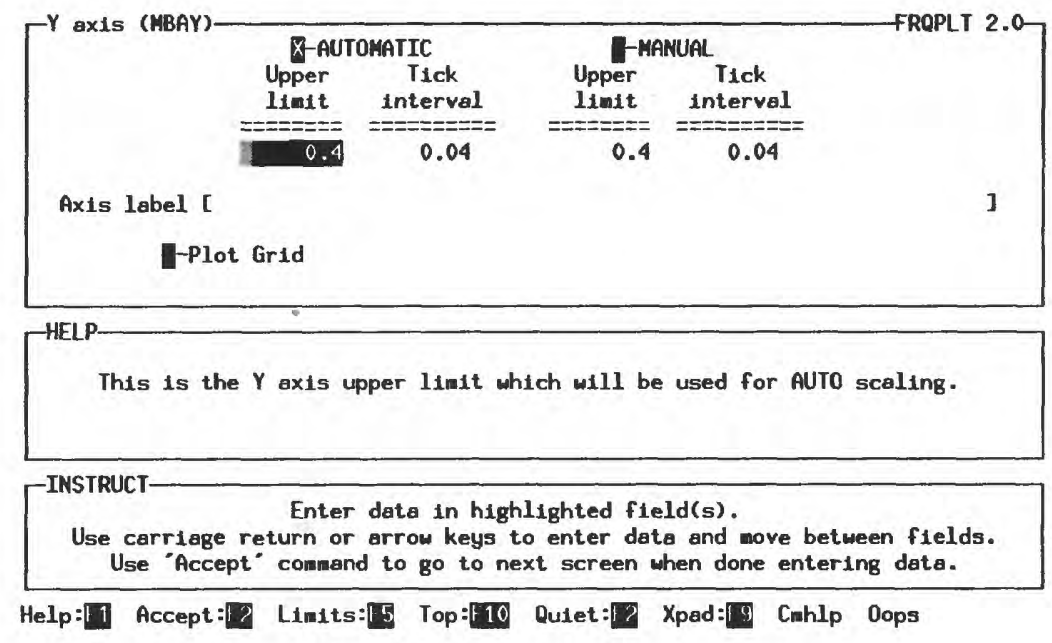

\section{Censored Data Options}

By default the "frqplt" program excludes censored data from the analysis. To include censored data (remark codes are disregarded), use the arrow or Tab keys to select (highlight) the "Include censored values" option in the "Censored (MBC)" screen. Use Accept (F2) to return control to the "Basic (MB)" screen. 


\section{Page Layout Options}

From the "Basic (MB)" screen, select the "Page (MBP)" screen, which provides the following functions:
Page Height
Page Width
Font Style
Plot Page Border
Frame Axis Border
Tick Marks Inside
Horizontal Y-Axis Numbers
Four-Sided Axis Options.

Most options in this list are discussed in the section "Page Layout Options" under "Steps to Using QWGRAF Programs."
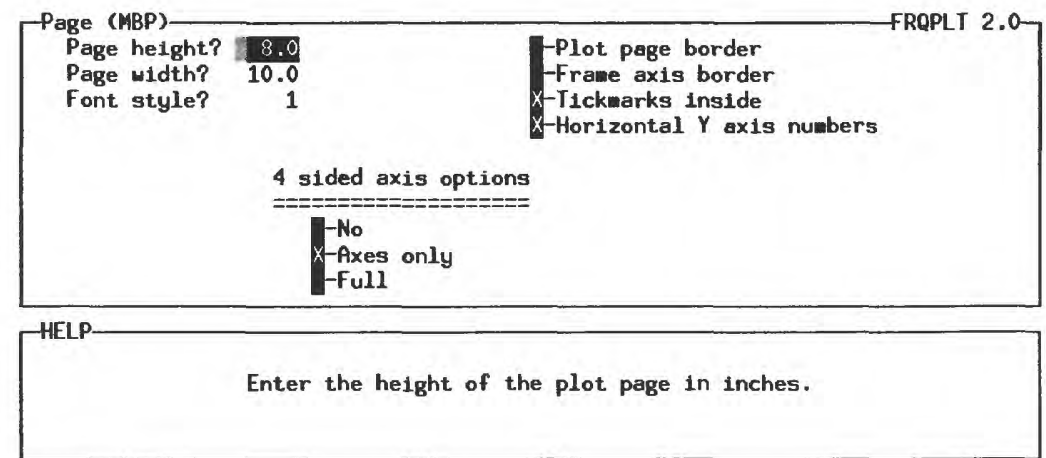

INSTRUCT- Enter data in highlighted field(s).
Use carriage return or arrow keys to enter data and wove between fields.
Use "Accept" comand to go to next screen when done entering data.

\section{Four-Sided Axis Options}

The four-sided axis option provides for duplicating the X-and Y-axes on the top and right side, respectively, of the plot frame. The options available are as follow:

$$
\begin{array}{ll}
\text { No } & \text { - two-sided axes only } \\
\text { Full } & \text { - fully annotated axes on all sides } \\
\text { Axes only } & \text { - fully annotated axis on the bottom and left, } \\
& \text { tick marks only on right and top. }
\end{array}
$$

Use the arrow keys to select the option desired, and toggle the option "On" with the space bar as indicated by the appearance of an " $\mathrm{X}$ " in the field to the left of the option name.

\section{Make the Plot}

When all arguments for the frequency plot have been specified and the appropriate device selected, return control to the "Opening Screen," select the "Make the plot" option, and use the Enter key. If a screen output was selected, then a graphics window opens and the frequency plot is presented. If a file output was selected, then a message screen indicates that the output from the procedure has been saved.

\section{Sample Plots}

The following are examples of the types of plots that can be created with the QWGRAF "frqplt" program. These examples are intended to illustrate various options available in the program and do not necessarily conform to publication standards of the Geological Survey. 
Program: FRQPLT

Plot Specifications: Frequency Option; Frequency Line and Histogram; System Computed Equal Intervals and Number of Intervals; Automatic X-Axis Scaling; X-Axis label; Default Y-Axis Label; Single Title

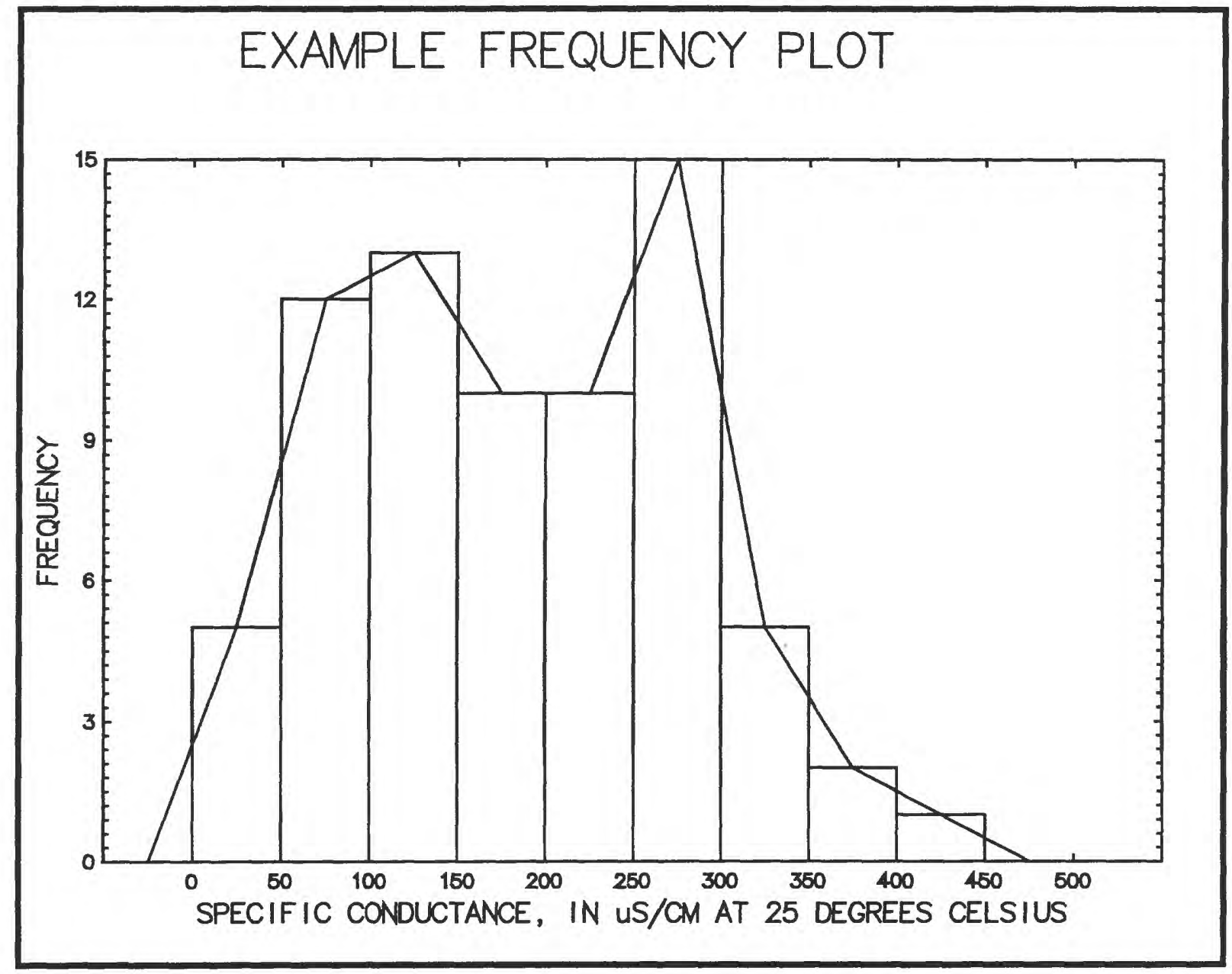


Program: FRQPLT

Plot Specifications: Cumulative Relative Frequency Option; Midpoint Plot Position; System Computed Equal Intervals; User Specified Number of Intervals; Histogram Only; Automatic X-Axis Scaling; X-Axis Label; No Grid Lines; Default Y-Axis Label; Single Title

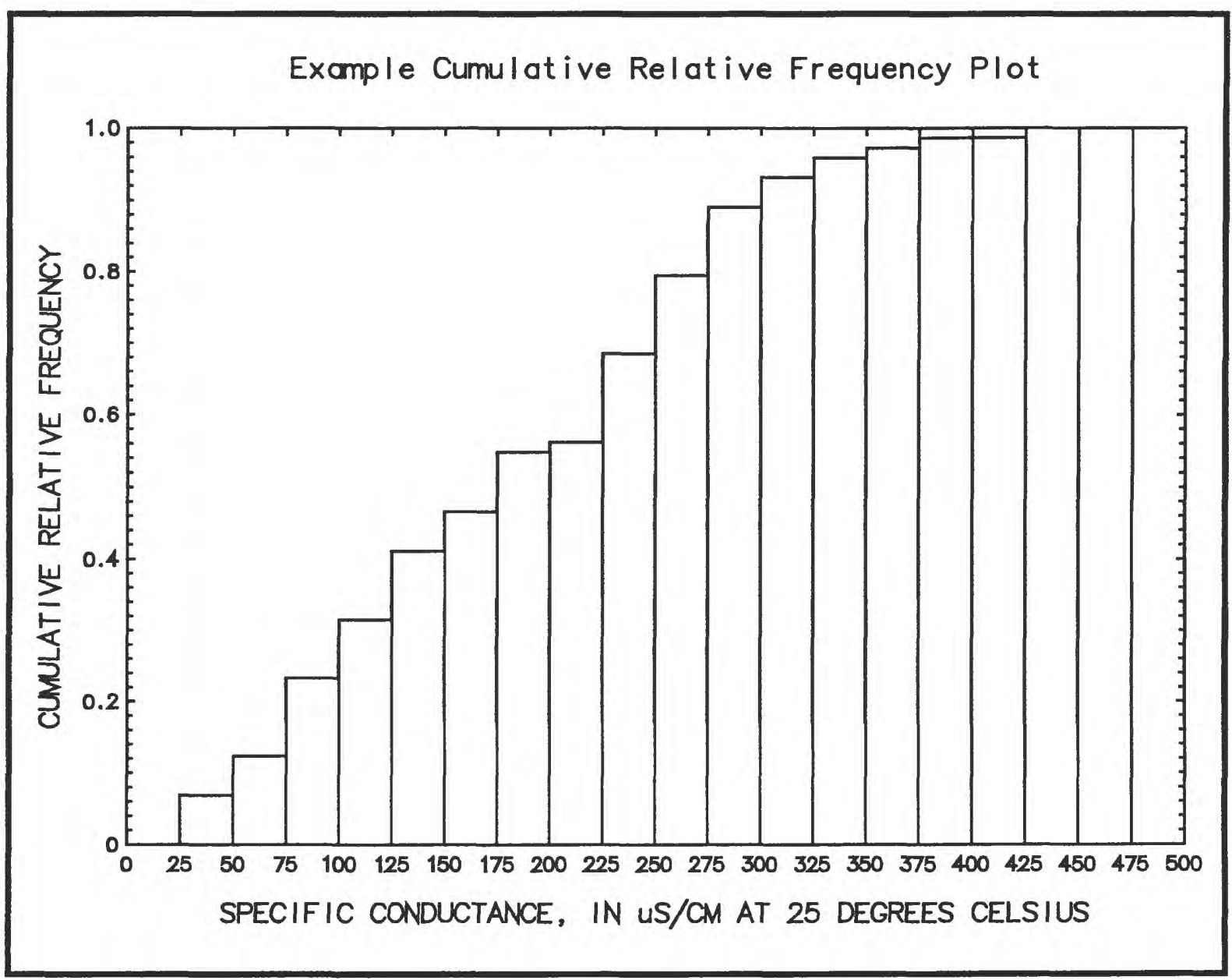


Program: FRQPLT

Plot Specifications: Empirical Distribution Function Option; Automatic X-Axis Scaling; No X-Axis Label; Default Y-Axis Label; Single Title; Single Footnote

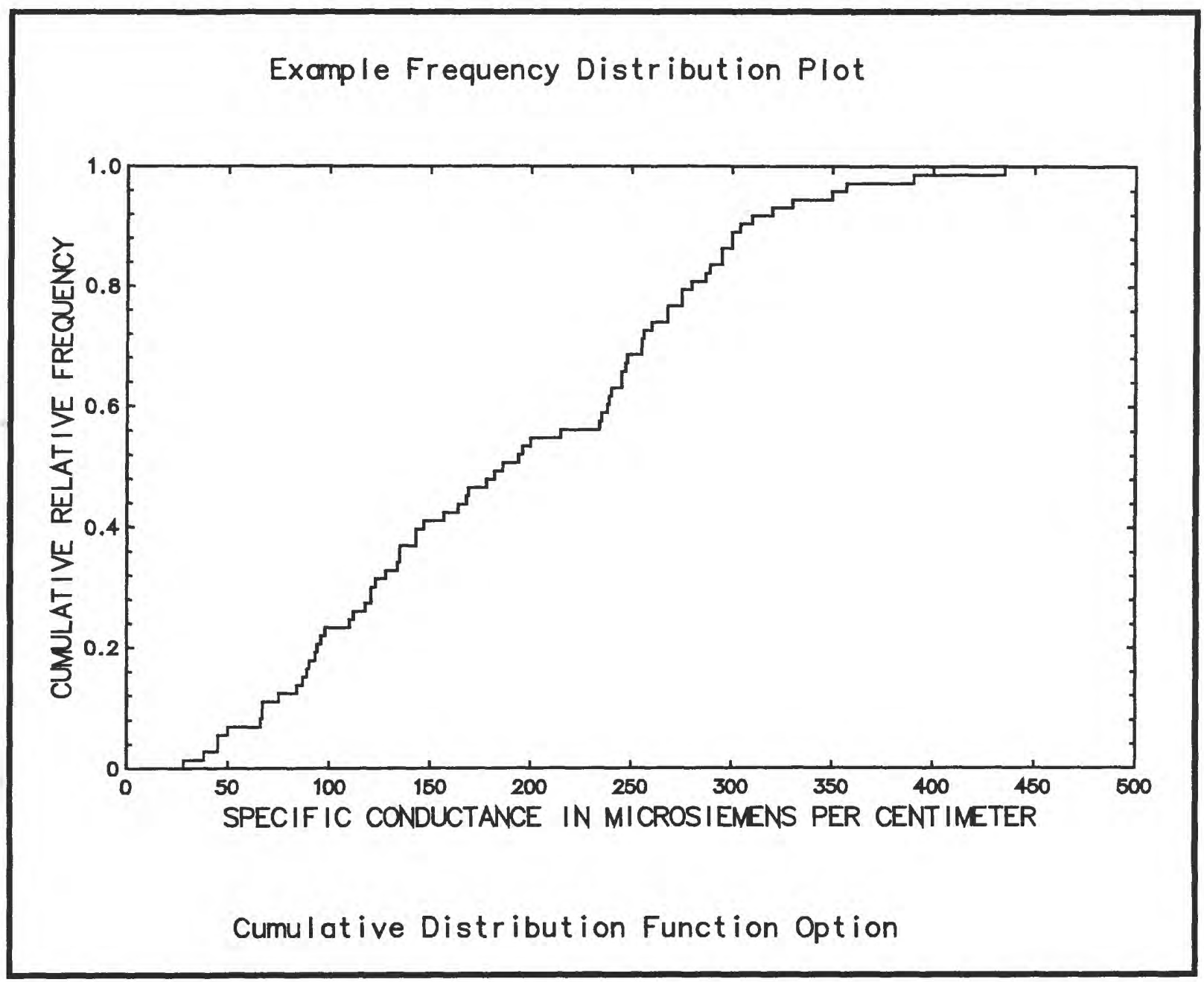




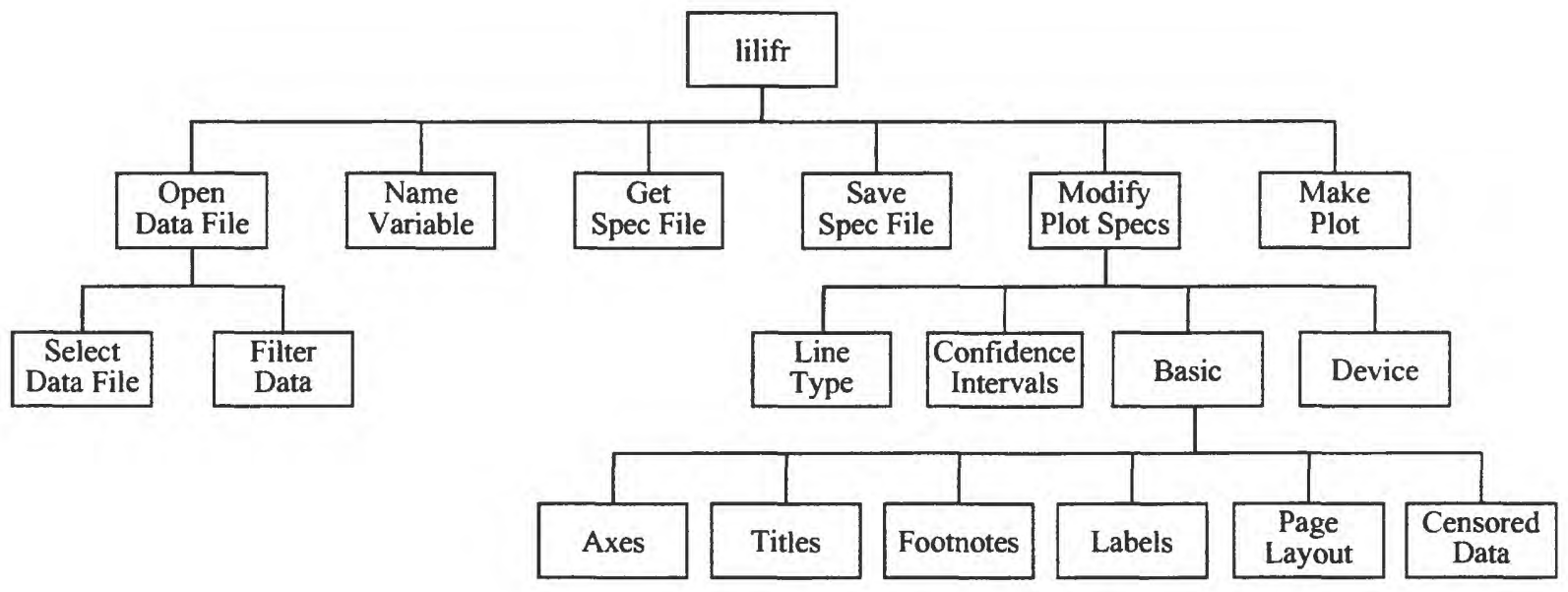

\section{LILLIEFORS TEST FOR NORMALITY (lilifr)}

The "lilifr" program provides a graphical means of determining if a sample is normally distributed. The plot consists of the cumulative relative frequency on the Y-axis and a normalized standard sample values (deviates) on the X-axis. The Empirical Distribution Function (EDF) line for the data and an EDF reference line are user defined relative to line type, color, and thickness. Up to five confidence level lines can be drawn ( 80 percent, 85 percent, 90 percent, 95 percent, or 99 percent) with options for line type, color, line thickness, and labels. Remarked (censored) data values reported as a limit of quantitation or detection limit can be included or excluded from the plot data set. Tick-mark intervals and grid lines are user-defined options. Axes can be drawn on two or four sides with optional annotation. There can be up to three title lines and two footnotes, each definable as to size, font, and centering. Up to five user-defined labels (up to 40 characters on multiple lines) can be positioned on the plot. Any number of plot specification files can be saved and used to facilitate plot specification in other "lilifr" sessions.

\section{Background}

The Lilliefors Test for Normality compares the standard normal distribution function with the sample EDF. The Lilliefors plot is created by plotting the cumulative relative frequency on the Y-axis and the normalized standard sample values as deviates on the $\mathrm{X}$-axis. Computation of the standardized sample values (normalization) is accomplished by subtracting the sample mean from each value and dividing the result by the sample standard deviation, that is:

$$
\mathrm{Z}=(\mathrm{X}-\mathrm{Mean}) / \mathrm{Std} \text { Dev. }
$$

The dashed curve in the middle of the Lilliefors diagram represents a standard normal distribution. This curve is bounded by dotted curves that represent Lilliefors bounds at user-selected confidence levels relative to the sample size of the data set. If the sample EDF (solid line) strays outside the confidence level bounds, then the sample population should be considered not normal. The Lilliefors bounds for this program were taken from tables created from Monte Carlo Methods whereby sets of randomly generated normal deviates were examined for various sample sizes. 


\section{Lilliefors Options}

The options that pertain to the Lilliefors functions supported by the "lilifr" program are found in the following screens:

Function

EDF \& Reference Lines

Confidence Level Bounds

Axes

\section{$\underline{\text { Screen Sequence }}$}

Opening Screen, Modify (M), Line (ML)

Opening Screen, Modify (M), Confidence (MC)

Opening Screen, Modify (M), Basic (MB),

Axes (MBA).

\section{Starting the "lilifr" Program}

The "lilifr" program is invoked by typing "lilifr" on the command line. The "Opening Screen" displays the options that serve as gateways to the screens used to set or modify the Lilliefors plot configuration options.

\section{Load a Specifications File}

To use specifications from a previous "lilifr" session, load a "Specifications" file. From the "Opening Screen," select the "Get (G)" screen. A list of specifications files (extension ".lfr") and a field for specifying the file name are presented. Use the arrow keys to select the required file and use either Accept (F2) or the Enter key to accept the selection. The "latest.lfr" file contains the program specifications that were used during the most recent execution of the "lilifr" program, whether in the current or previous session.

The "Specifications" file includes the name of the input data file active at the time the specifications file was created. If a new input data file is required, then select the "Open (O)" screen from the "Opening Screen" and select a new input data file.

The "Make the Plot" option can be selected immediately or any of the newly loaded specifications can be changed. Any altered specifications set can be saved to a new file or an old file can be overwritten.

\section{Open Input Data File}

From the "Opening Screen," select the "Open (O)" screen. From the "Open (O)" screen, select the "Select (OS)" screen. Type in a file name or select the input data file desired. Use the arrow, Page Down, and Page Up keys to select a data file. Use either Accept (F2) or the Enter key to accept the file selection and return control to the "Open (O)" screen.

\section{Filter Data}

To restrict the data to be used for the Lilliefors diagram, the "Filter" option from the "Open (O)" screen can be used to display the "Filter (OF)" screen used to specify the criteria for selecting those rows that will be retained for use with the program.

\section{Name Variable}

From the "Opening Screen," select the "Name (N)" screen. Use the arrow and Enter keys to select one variable for the Lilliefors plot. Place a "1" in the "Select" field to the left of the variable name. Use Accept (F2) to accept the selection and return control to the "Opening Screen." 


\section{Modify Plot Specifications}

After the data file has been opened and the variable of interest named, select the "Modify (M)" screen by typing "M" (or " $m$ ") to bring up the screen directly; or use the Tab and arrow keys to select the "Modify compute/plot specifications" option followed by using Accept (F2) or the Enter key. The "Modify (M)" screen lists the following options:

Line - specify line type for EDF

Confidence - specify confidence interval options

Basic - specify title, footnotes, labels, and axes options

Device - specify device for graphics output.

Type the first letter (not case sensitive) of the option to view the next screen or use the arrow keys to select the option and use either Accept (F2) or the Enter key to accept the selection.
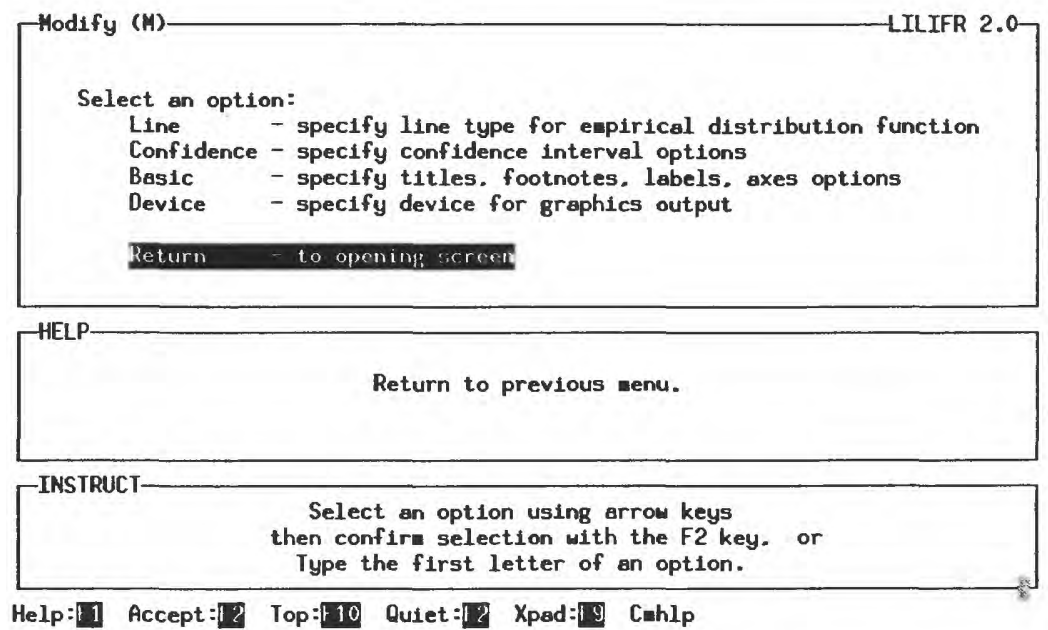

\section{Line Type}

The "Line" option in the "Modify (M)" screen provides for specifying the pattern, color, and thickness of the sample EDF line for the Lilliefors plot. From the "Modify (M)" screen, select the "Line (ML)" screen. Use the arrow keys to select an option and enter the appropriate value. Use Help (F1) to bring up the "Help" panel for each option. A list of allowable values (codes or numbers) is presented. Use these values to populate the option field. 

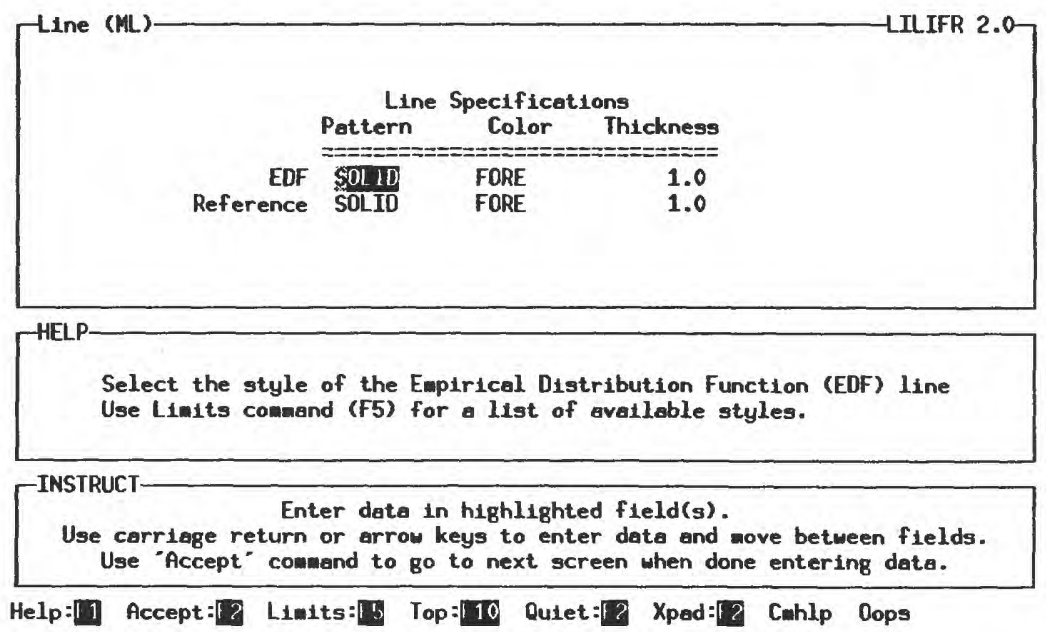

\section{Confidence Interval}

The Lilliefors plot provides for bracketing the sample EDF line with confidence interval lines. Up to five confidence level lines ( 80 percent, 85 percent, 90 percent, 95 percent, or 99 percent) can be plotted. The line type, color, and thickness of these lines can be specified. In addition, a label that indicates the confidence level can be specified.

From the "Modify (M)" screen, select the "Confidence (MC)" screen. Use the arrow keys to select as many confidence level lines as desired and enter the appropriate value as percent in the field.

For each confidence level selected, use the arrow and Enter keys to select the "Line pattern," "Line color," "Line thickness," and "Label" options. Use Help (F1) to bring up the "Help" panel for each option. A list of allowable values (codes or numbers) is presented. Use these values to populate the fields.
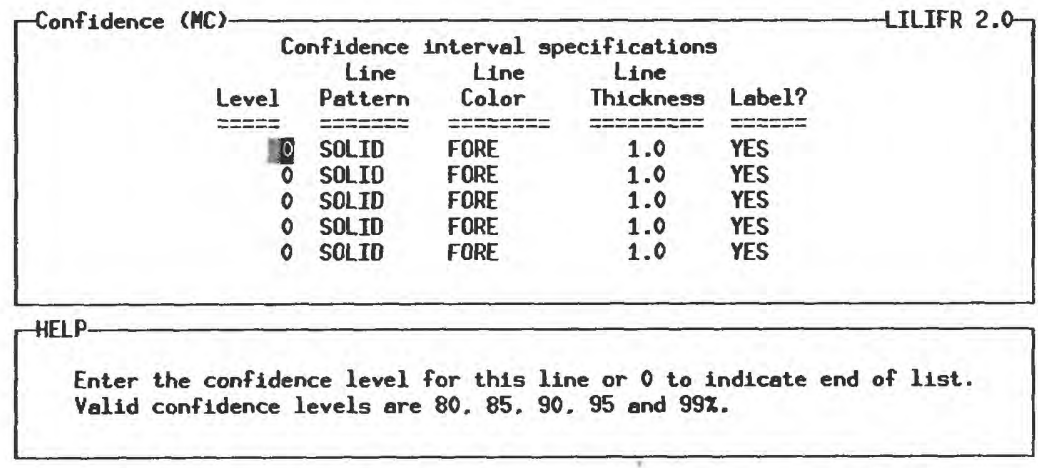

\footnotetext{
TINSTRUCT- Enter data in highlighted field(s).

Use carriage return or arrow keys to enter data and move between fields. Use "Accept" command to go to next screen when done entering data.

Help: 1 Accept: 2 Limits: Top: 10 Quiet: 2 Xpad:- Cahlp Dops Hindow
} 


\section{Basic Plot Parameters}

From the "Modify (M)" screen, select the "Basic (MB)" screen, which lists the following options for modifying plot specifications:

$\begin{array}{ll}\text { Axes } & \text { - X-and Y-axis type and limits } \\ \text { Titles } & \text { - above plot } \\ \text { Footnotes } & \text { - below plot } \\ \text { Labels } & \text { - of miscellaneous text } \\ \text { Page } & \text { - layout options } \\ \text { Censored } & \text { - data options } \\ \text { Make } & \text { - the plot } \\ \text { Return } & \text { - to previous screen. }\end{array}$

Type the first letter of any option or use the arrow and Enter keys to select and open the screen for the option required.

\section{Axes}

The "Axes" option provides options for axis labels, tick-mark intervals, and grid lines for both the X-axis and the Y-axis. From the "Basic (MB)" screen, type "A" (or "a") or use the arrow and Enter keys to bring up the "Axes (MBA)" screen. The following options are presented:

$\mathrm{X}$-axis specifications

Y-axis specifications

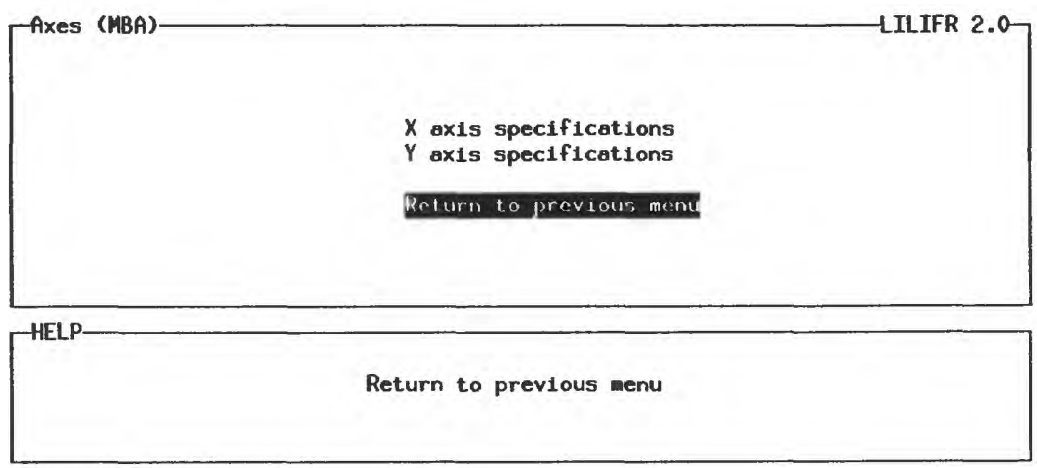

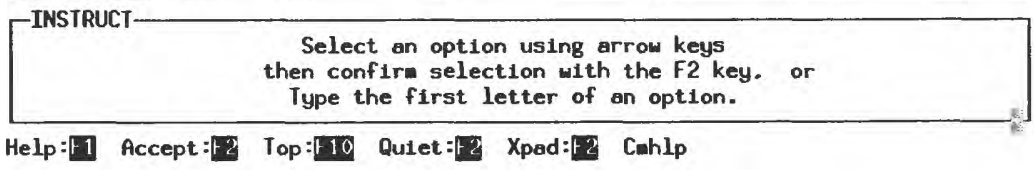

\section{$\underline{X-A x i s ~ S p e c i f i c a t i o n s ~}$}

From the "Axes (MBA)" screen, select the "X-axis (MBAX)" screen. This screen provides options for axis labels (top and bottom), tick-mark intervals, and grid lines for the $\mathrm{X}$-axis.

\section{Labels}

The "lilifr" program provides several default labels for the X-axis depending on whether any confidence interval lines are drawn. If a single confidence interval line is specified (for example, 95 percent), then the top label would default to the following:

\section{5\% LILLIEFORS BOUNDS FOR NORMAL SAMPLES.}


This label would be drawn regardless of the four-sided axis specification (see "Page (MBP)" screen). If there is more than one confidence interval specified, then the top label would default to the following:

\section{LILLIEFORS BOUNDS FOR NORMAL SAMPLES.}

(This label also would be drawn regardless of the four-sided axis option.)

If the user supplies text for the top label, then it is drawn regardless of the four-sided axis option.

If there are no confidence intervals specified, then the following top X-axis label (same as the bottom) is drawn if the four-sided axis option is selected:

\section{STANDARDIZED SAMPLE VALUE.}

To place an alternate label in either position, use the arrow keys to select the $\mathrm{X}$-axis label option desired and enter a label of up to 60 characters.

To delete the label in the top X-axis position, type "BLANK" or "blank" in the "Bottom $\mathrm{X}$-axis label" field.

\section{Tick-Mark Intervals}

Use the arrow keys to select the "Tick-mark interval" option. Enter a tick-mark interval value. The $\mathrm{X}$-axis scale represents standard deviates with a typical range of -4 to +4 .

\section{Grid Lines}

To place X-axis grid lines on the plot, select the "Plot Grid" option and use the space bar to toggle the selection "On" as indicated by the appearance of an " $\mathrm{X}$ " in the field to the left of the option name. Use Accept (F2) to return control to the "Axes (MBA)" screen.
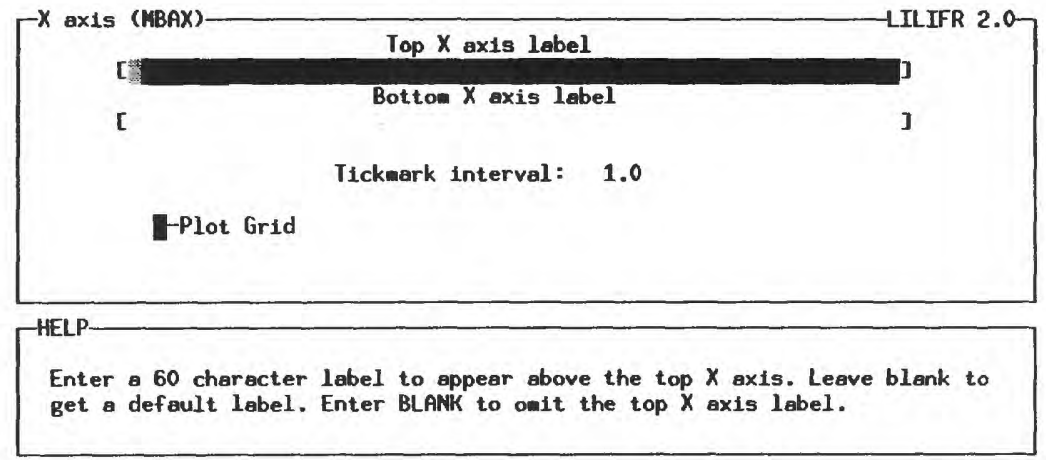

\footnotetext{
\begin{tabular}{|l} 
Enter data in highlighted field(s). \\
Use carriage return or arrow keys to enter data and wove between fields. \\
Use "Accept" command to go to next screen when done entering data.
\end{tabular}
}

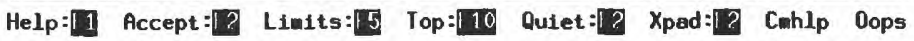

\section{Y-Axis Specifications}

From the "Axes (MBA)" screen, select the "Y-Axis (MBAY)" screen. This screen provides options for an axis label, tick-mark interval, and grid lines for the Y-axis.

\section{Label}

In the absence of a user-supplied Y-axis label, the following default label is plotted on the Y-axis: 


\section{CUMULATIVE RELATIVE FREQUENCY.}

To choose an alternate Y-axis label, use the arrow keys to select the Y-axis label option and enter a label of up to 60 characters. The $Y$-axis label is duplicated on the scale if either the "Axes only" or the "Full" axis option is selected (see "Page (MBP)" screen).

\section{Tick-Mark Interval}

Use the arrow keys to select the "Tick-mark interval" option. Enter a tick-mark interval value. The Y-axis scale represents the cumulative probability with a range of 0 to 1.0.

\section{Grid Lines}

To place Y-axis grid lines on the plot, select the "Plot Grid" option and use the space bar to toggle the selection "On" as indicated by the appearance of an "X" in the field to the left of the option name. Use Accept (F2) to accept the selections to return control to the "Axes (MBA)" screen.

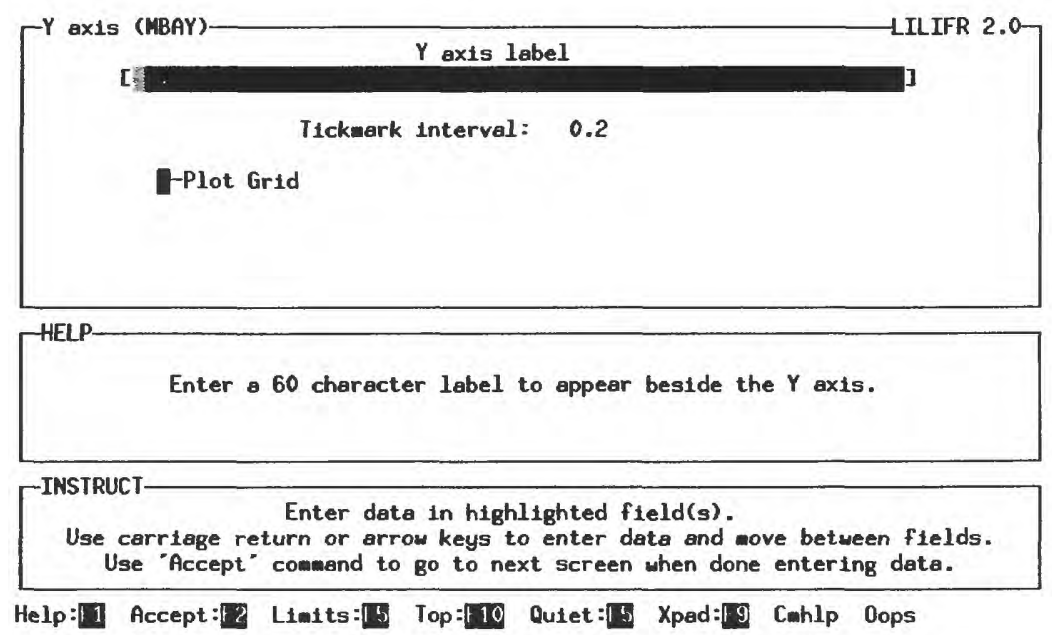

\section{Censored Data Options}

By default the "lilifr" program excludes censored data from the analysis. To include censored data in the plot (remark codes disregarded), use the arrow or Tab keys to select the "Include censored values" option in the "Censored (MBX)" screen. Use Accept (F2) to accept this selection and return control to the "Basic (MB)" screen.

\section{Page Layout Options}

From the "Basic (MB)" screen, select the "Page (MBP)" screen. The "Page (MBP)" screen provides the following functions:

Page Height

Page Width

Font Style

Plot Page Border

Frame Axis Border

Tick Marks Inside

Horizontal Y-Axis Numbers

Four-Sided Axis Options. 
Most options in this list are discussed in the section "Page Layout Options" under "Steps to Using QWGRAF Programs."

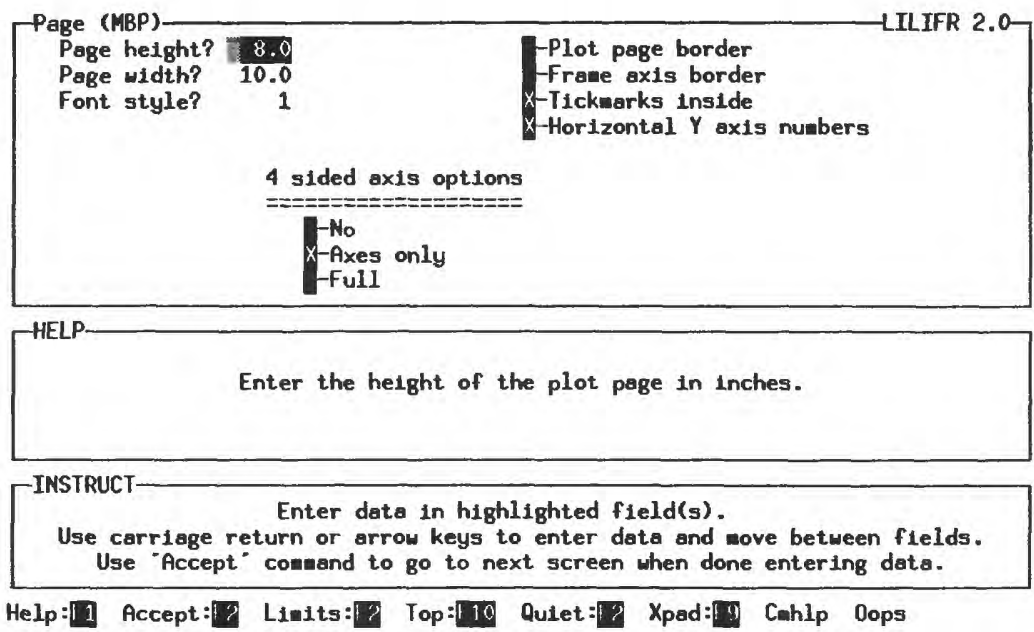

\section{Four-Sided Axis Options}

The four-sided axis option provides for duplicating the $\mathrm{X}$ - and $\mathrm{Y}$-axes on the top and right side, respectively, of the plot frame. The options are as follow:

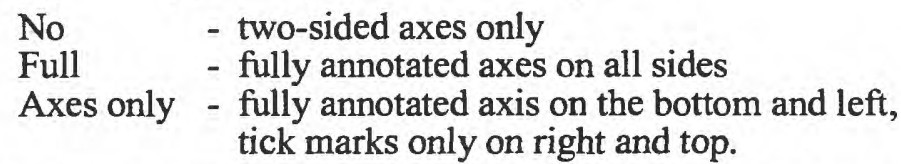

Use the arrow keys to select the option desired, and use the space bar to toggle the option "On" as indicated by the appearance of an " $\mathrm{X}$ " in the field to the left of the option name.

\section{Make the Plot}

When all arguments for the Lilliefors plot have been specified and the appropriate device selected, return control to the "Opening Screen," select the "Make the plot" option, and use the Enter key. If a screen output was selected, then a graphics window opens and the Lilliefors plot is presented. If a file output was selected, then a message screen indicates that the output from the procedure has been saved.

\section{Sample Plots}

The following are examples of the types of plots that can be created with the QWGRAF "lilifr" program. These examples are intended to illustrate various options available in the program and do not necessarily conform to publication standards of the Geological Survey. 
Program: LILIFR

Plot Specifications: Single Title

$$
\text { Example Lilliefors Plot }
$$

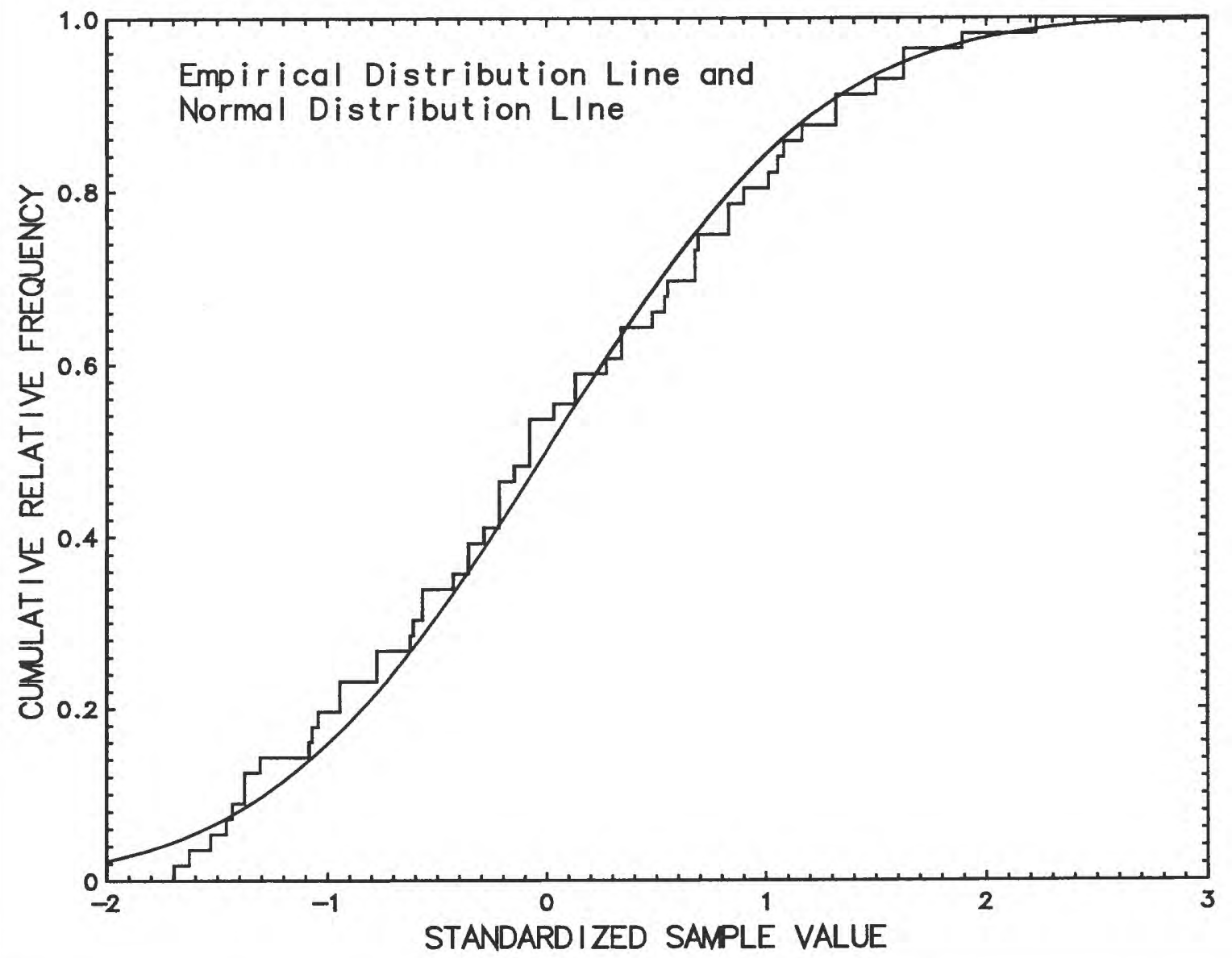


Program: LILIFR

Plot Specifications: 95-percent Confidence Level Line; Confidence Level Label; Top X-Axis Scaling; Top $\mathrm{X}$-Axis Label; Single User-Defined Label; Single Title

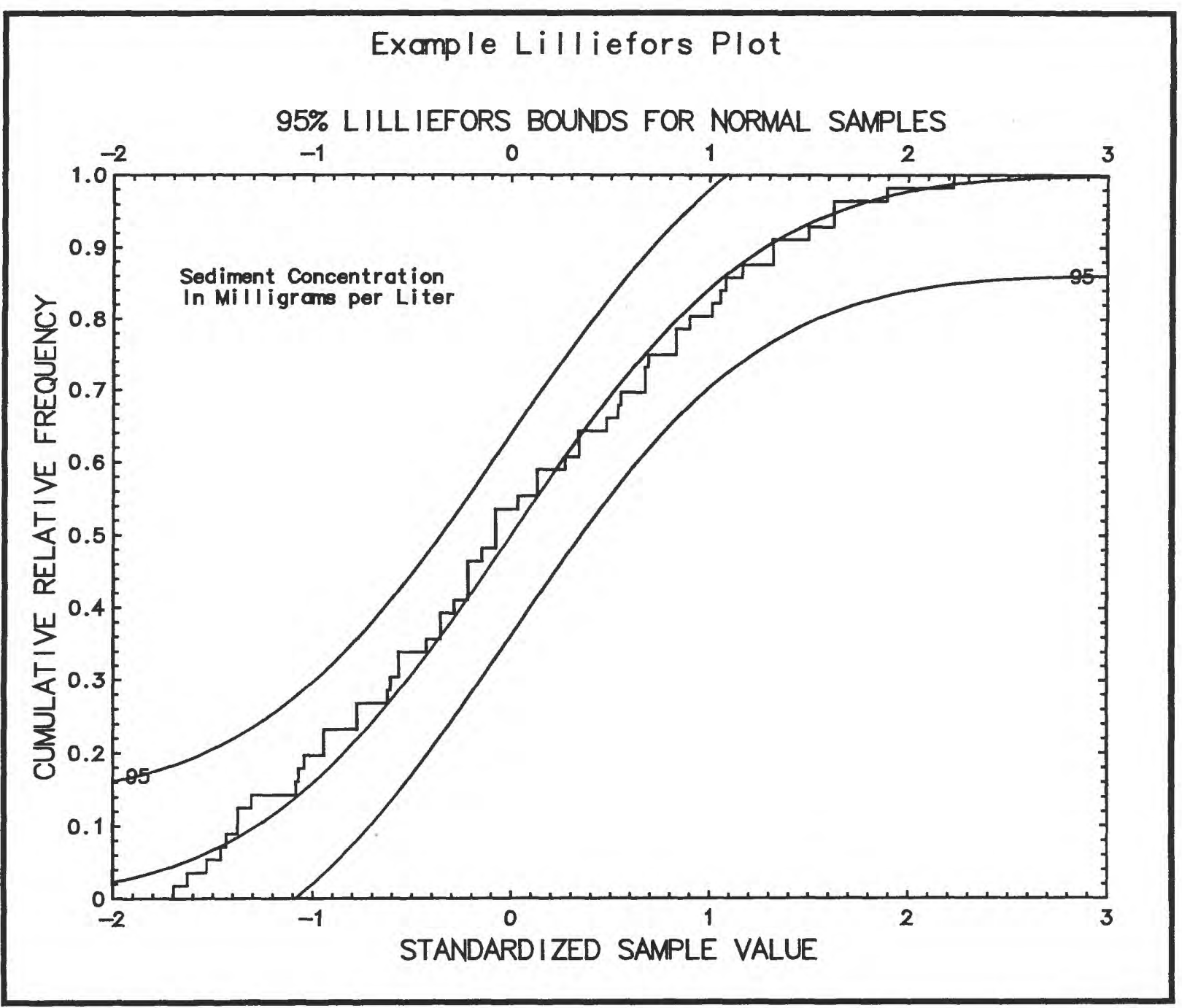




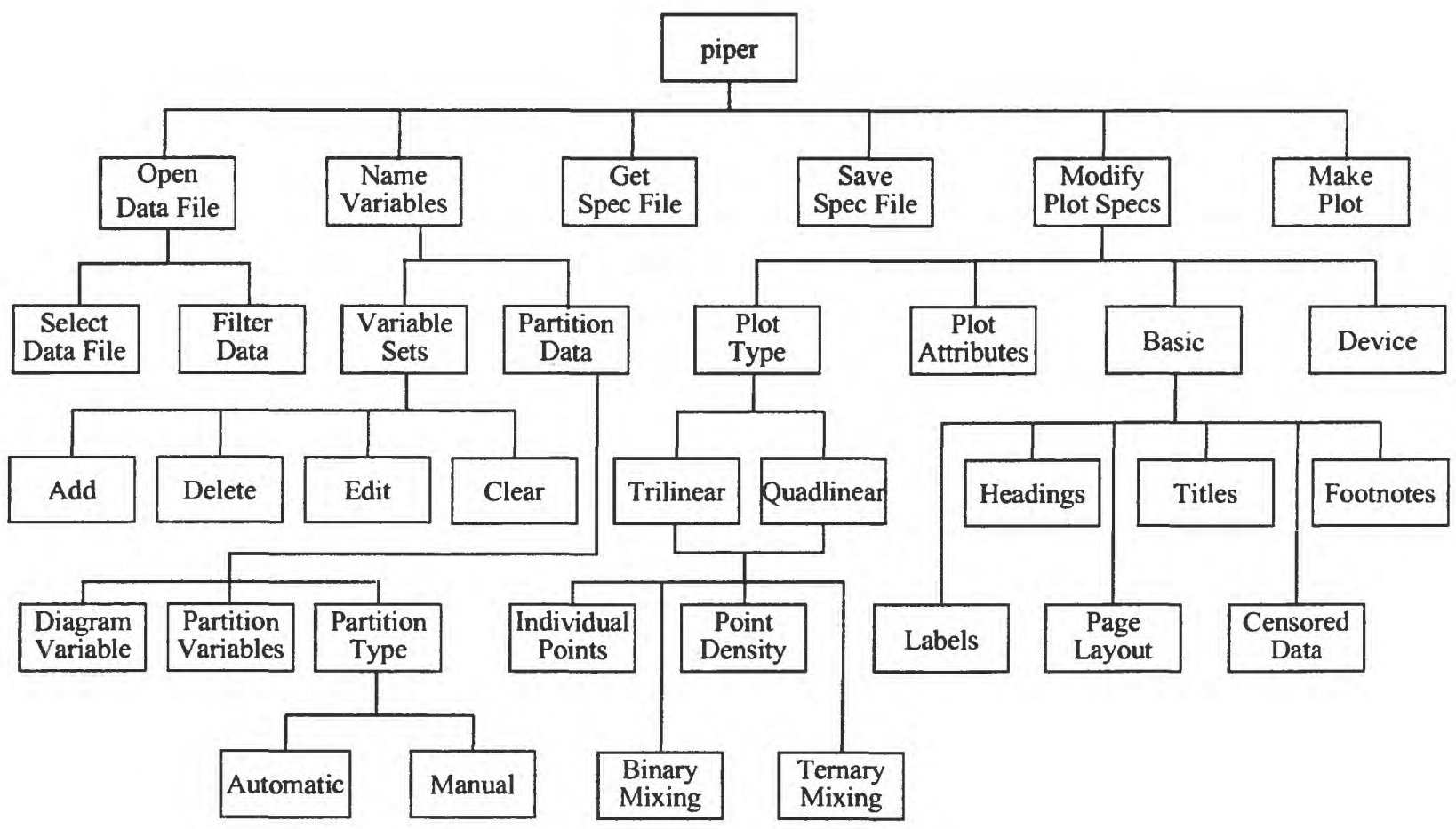

PIPER DIAGRAMS (piper)

A Piper diagram is a hydrochemical facies diagram used to characterize a water body or to compare the chemical character of several water bodies. The "piper" program provides for both Trilinear and Quadlinear diagrams. In addition to the standard display of individual data points, options are provided for displaying reference locations in the form of binary or ternary mixing diagrams. The data also can be displayed as shaded density diagrams where the shading of each minor triangle within the main triangle represents the percentile ranking of the sum of points within the minor triangle relative to the sums of points in the other minor triangles. Each node of the diagram is user defined and can represent a single chemical constituent or a composite of two or more constituents. Data within a diagram can be differentiated on the basis of up to three grouping variables that use combinations of symbols, symbol size, and color. The program provides for up to 90 diagrams on multiple pages. Each page can have up to three lines of diagrams and three diagrams per line. With multiple diagrams there is the option to utilize a different set of variables for each diagram (individual variables can be repeated between sets) or to use one set of variables that has been partitioned into groups of values on the basis of composite values of up to four partition variables. Each group is then represented by a Piper diagram on the plot page. Remarked (censored) data values reported as a limit of quantitation or detection limit can be included or excluded from the plot data set. Titles for each diagram and labels for each node of each diagram are user defined. There can be up to three title lines and two footnote lines each definable as to size, font, and centering. Gridline options include none, 10 percent, and 20 percent. Up to five user-defined labels (up to 40 characters on multiple lines) can be positioned on the plot. Sets of plot specifications can be saved to files and used to facilitate plot configuration in other "piper" sessions. 
Hydrochemical facies are distinct zones that have cation and anion concentrations describable within defined composition categories. Trilinear and Quadlinear Piper diagrams are a graphical means of presenting these categories. A Trilinear Piper diagram is a triangular plot of three variables (single or composite) where each side of the equilateral triangle is the axis for one of the variables. Each axis is labeled from 0 to 100 percent. Because concentrations, expressed as mass per unit volume, between chemical constituents are not additive, the variables are expressed in the normal form; for example, as milliequivalents, which are additive. All three variables, expressed in the normal form, are assumed to sum to 100 percent for each observation. Thus, there are only two degrees of freedom, and each observation can be mapped into a single position within the triangle. The plotted point for each observation is the intersection of three lines, each drawn from its proportionate percentile point on its variable axis, parallel to the axis opposite the 100 percent (or apex) point for that variable. The length of the line drawn from its location in the triangle perpendicular to the axis opposite its apex point is also a measure of that variable's proportionate percentile. The relative positioning of the points within the triangle defines the water types or shows similarities between different water types. A Quadlinear Piper diagram is formed by projecting the points from the two side-by-side triangles into a central diamond, whose upper sides combined with the outer sides of the two triangles form an equilateral triangle. Six variables are required for a quadlinear plot.

\section{Piper Diagram Options}

The "piper" program supports the following piper related functions:

$\begin{array}{ll}\text { Function } & \text { Screen Sequence } \\ \text { Trilinear/Quadlinear } & \text { Opening Screen, Modify (M), Plot (MP) } \\ \text { Data Display Type } & \text { Opening Screen, Modify (M), Plot (MP) } \\ \text { Point Attributes } & \text { Opening Screen, Modify (M), Point Attributes (MA) } \\ \text { Point Density Attributes } & \begin{array}{l}\text { Opening Screen, Modify (M), } \\ \text { Density Attributes (MA) }\end{array} \\ \text { Mixing Diagram Attributes } & \text { Opening Screen, Modify (M), } \\ \text { Mixing Diagram Attributes (MA) } & \text { Opening Screen, Name (N), Partition (NP) } \\ \text { Diagram Partitioning } & \begin{array}{l}\text { Opening Screen, Modify (M), * Attributes (MA) } \\ \text { Variable Partitioning }\end{array} \\ \begin{array}{l}\text { (*= Point or Mixing Diagram) } \\ \text { Legends (Explanations) }\end{array} & \begin{array}{l}\text { Opening Screen, Modify (M), Basic (MB), } \\ \text { Legend (MBL). }\end{array}\end{array}$

\section{Input Data Requirements}

The "piper" program assumes that the data to be displayed in the Piper diagrams are in the equivalent form. See the discussion of "Data Management" in the "General Features of QWGRAF programs" section for suggestions on how to convert concentration data to equivalents.

\section{Starting the "piper" Program}

The "piper" program is invoked by typing "piper" on the command line. The "Opening Screen" displays the options that serve as gateways to the screens used to set or modify the plot configuration options. 


\section{Load a Specifications File}

To use specifications from a previous "piper" session, load a "Specifications" file. From the "Opening Screen," select the "Get (G)" screen. A list of specifications files (extension ".ppr") and a field for specifying the file name are presented. Use the arrow keys to select the required file and use either Accept (F2) or the Enter key to accept the selection. The "latest.ppr" file includes the program specifications that were used during the most recent execution of the "piper" program, whether in the current or previous session.

The "Specifications" file includes the name of the input data file active at the time the specifications file was created. If a new input data file is required, then select the "Open (O)" screen from the "Opening Screen" and select a new input data file.

The "Make the Plot" option can be selected immediately or any of the newly loaded specifications can be changed. The revised set of programs specifications can be saved to a new file or an old file can be overwritten.

\section{Open Input Data File}

From the "Opening Screen," select the "Open (O)" screen. From the "Open (O)" screen, select the "Select (OS)" screen. Type in a file name or select the input data file desired. Use the arrow, Page Down, and Page Up keys to select a data file. Use either Accept (F2) or the Enter key to accept the file selection and return control to the "Open $(\mathrm{O})$ " screen.

\section{Filter Data}

To restrict the data to be applied to the Piper diagram, use the "Filter" option in the "Open $(\mathrm{O})$ " screen to specify the criteria for selecting those rows to be retained for use with the program.

\section{Name Variables}

From the "Opening Screen," select the "Name (N)" screen. The "Name (N)" screen provides the following options:

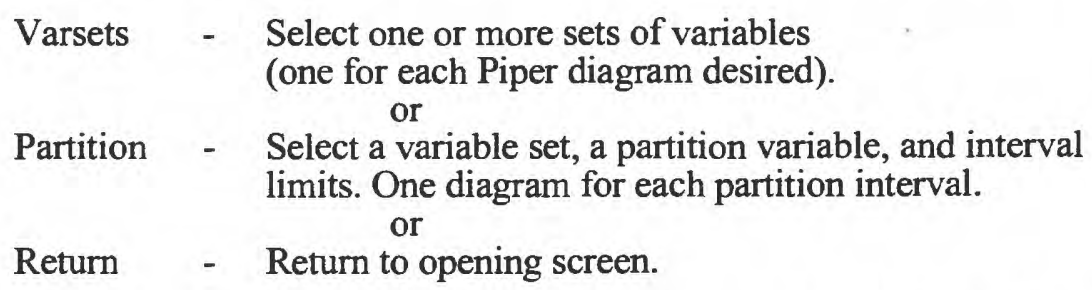

A single Trilinear Piper diagram requires three variables, while a single Quadlinear Piper diagram requires six variables (three for cations and three for anions). A single set of three or six variables results in a single diagram on the plot page (Varsets option). The Varsets option also can be used to define multiple sets of variables (individual variables can be repeated between sets) for the purpose of placing multiple Piper diagrams on a page. The more common approach to multiple diagrams is to take a single set of variables and partition the values into groups on the basis of composite values of up to four partitioning variables (Partition option). Each group is the basis of a Piper diagram. For example, produce a Piper diagram for each State. The "Varsets" and "Partition" functions are described in more detail under "Name (Select) Variables" in the "Shared Features" section. 


\section{Modify Plot Specifications}

After the data file has been opened and the variables of interest named, select the "Modify (M)" screen by typing " $\mathrm{M}$ " (or " $\mathrm{m}$ ") or use the Tab and arrow keys to select the "Modify compute/plot specifications" option followed by pressing Accept (F2) or the Enter key. The "Modify (M)" screen presents the following options:

Plot - specify plot type

Attributes - for data display, symbol styles, shade patterns, etc.

Basic - specify titles, footnotes, labels, and axes options

Device - specify device for graphics output

Return - to opening screen.
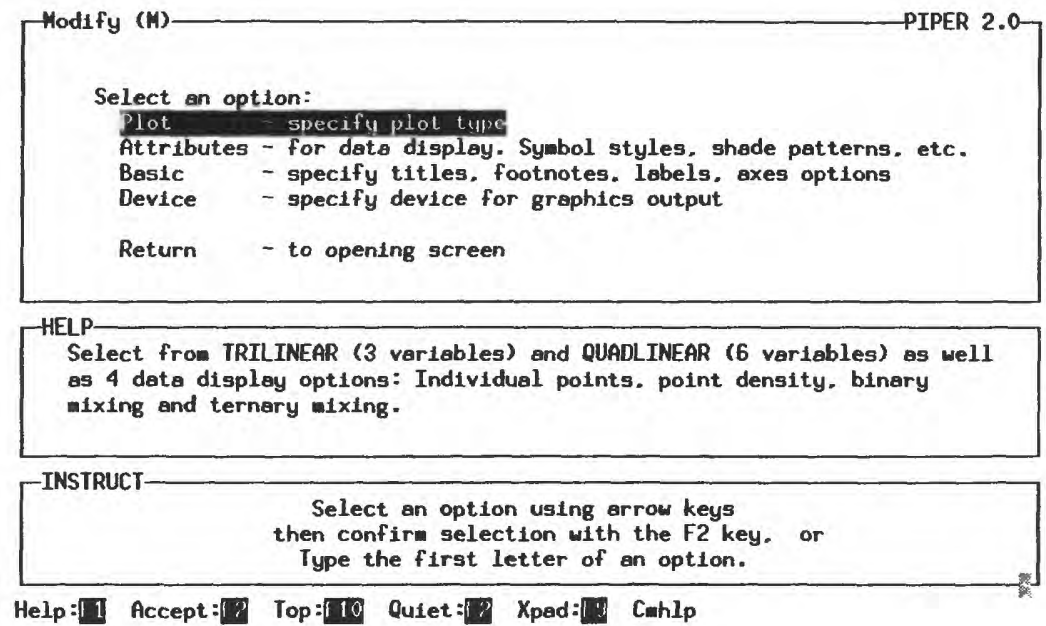

Plot

From the "Modify (M)" screen, select the "Plot (MP)" screen, which presents options for selecting the type of plot and the method of displaying the data in the plot.

\section{Plot Type}

Two plot types are available:

Trilinear

Quadlinear (Default).

Use the arrow keys to select the option desired. Use Help (F1) to obtain a brief description of each diagram type. Use the space bar to toggle "On" the option desired as indicated by a "X" in the field to the left of the option name.

\section{Data Display Method}

Four methods of displaying the data in a Piper diagram are available:

Individual points plotted

Point density shaded

(Default)

Binary mixing diagram

Ternary mixing diagram.

Use the arrow keys to select the option desired. Use Help (F1) to obtain a brief description of each display method. Use the space bar to toggle "On" the option desired as indicated 
by a "X" in the field to the left of the option name. Use Accept (F2) to accept the selection and return control to the "Modify (M)" screen.

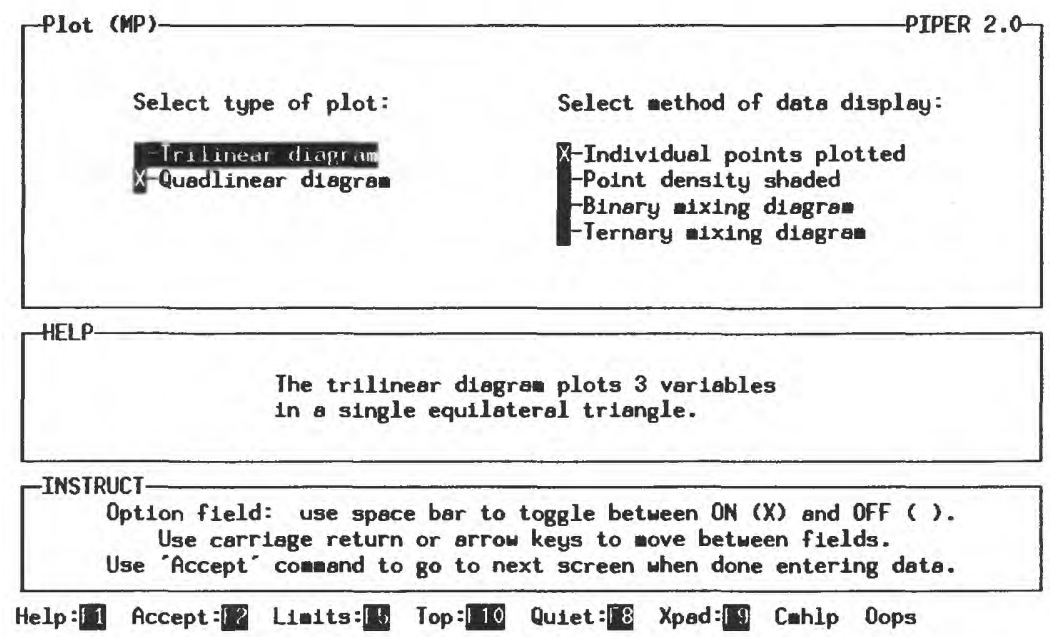

\section{Plot Attributes}

Each of the "Data Display Methods" listed in the "Plot (MP)" screen has its own set of screens relative to the "Attributes" option in the "Modify (M)" screen. Once a "Data Display Method" is selected, selection of the "Attributes" option activates the sequence of screens appropriate for that method.

\section{Individual Points Plotted}

"Individual Points Plotted" is the default method of presenting Piper diagrams; individual data points are represented by a symbol that can be user defined as to type, size, and color. Data within a diagram can be represented by a single set of symbol attributes, or the data can be grouped and each group will then have its own set of symbol attributes. Data can be grouped on the basis of unique composite values of up to three grouping variables. Each grouping variable can control up to three symbol attributes but only if a given symbol attribute is not controlled by one of the other grouping variables. If more than one diagram is to be plotted, then all diagrams can share the same symbol attributes or each diagram can be specified individually.

If the data within a single diagram is to be presented as a single group, that is, all data points will have the same symbol attributes, then the user can return control to the "Opening Screen" and select the "Make" option to generate the plot by using system-default symbol attributes. Otherwise, the user must proceed through the options presented on the "Point Attributes (MA)" screen. From the "Modify (M)" screen, select the "Attributes" option to view the "Point Attributes (MA)" screen.

Note: The partitioning of the data to create multiple diagrams by using either the "Varsets" or "Partition" option in the "Name $(\mathrm{N})$ " screen and the grouping of data within the Piper diagram by using the "Group variable" option in the "Point Attributes (MA)" screen are two independent processes.

\section{Point Attributes}

The "Point Attributes (MA)" screen lists the following options for grouping the data within a Piper diagram and assigning symbol attributes to each group: 
Group - specify variable(s) for grouping data within diagrams

List - specify single or multiple group lists

Specify - symbol type, size, and color.

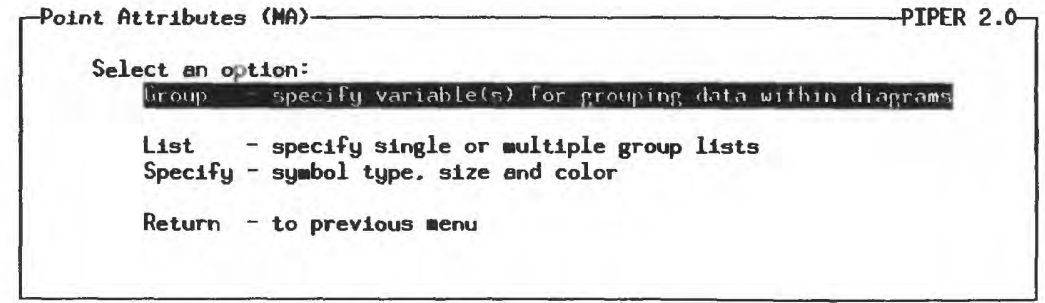

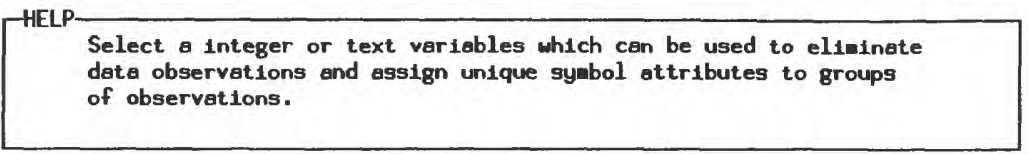

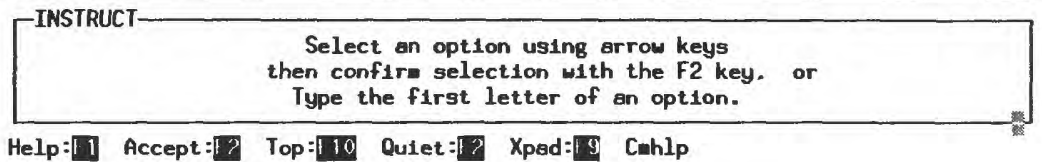

\section{Group Variable}

Use the arrow keys to select the "Group" option and use Accept (F2) or the Enter key to view the "Group (MAG)" screen. Use the Tab, Enter, and arrow keys to select up to three variables to be used as the basis for grouping the data within the diagram. For each grouping variable, enter a number to indicate the combination of symbol attributes (type, size, or color), which will distinguish the groups. Select a combination of symbol attributes for each variable selected such that the sum of the numbers entered is seven or less and that a given symbol attribute is assigned no more than once. The following list and examples demonstrate how the combinations are used:

$$
\begin{array}{llll}
0: \text { deselect } & \text { 1: type } & 2: \text { color } & \text { 3: type+color } \\
\text { 4: size } & \text { 5: type+size } & 6: \text { color+size } & 7: \text { type+color+size. }
\end{array}
$$

If a single grouping variable is selected and assigned a value of seven, then data points associated with each unique value of the grouping variable will be distinguished by a unique combination of symbol type, color, and size. If a single grouping variable is selected and assigned a value of five, then data points for a unique value of the grouping variable will be distinguished by a unique combination of symbol type and size, but all symbols will have the same color.

If two grouping variables are selected and the first variable is assigned a value of one and the second variable assigned a value of two, then data points that represent a unique value of the first variable will be distinguished by symbol type. Data points for each unique value of the second grouping variable will have the same color assigned to all symbols. In this case, all symbols will have the same user-defined size. For two grouping variables, the following combinations are allowed:

$1 \& 2$ or $2 \& 1$ (Size is constant)

$1 \& 4$ or $4 \& 1$ (Color is constant)

$1 \& 6$ or $6 \& 1$

$2 \& 4$ or $4 \& 2$

$2 \& 5$ or $5 \& 2$

$3 \& 4$ or $4 \& 3$. 
If three grouping variables are selected, then one variable should be assigned the value "1" (type), a second variable should be assigned the value "2" (color), and the third variable should be assigned the value "4" (size).

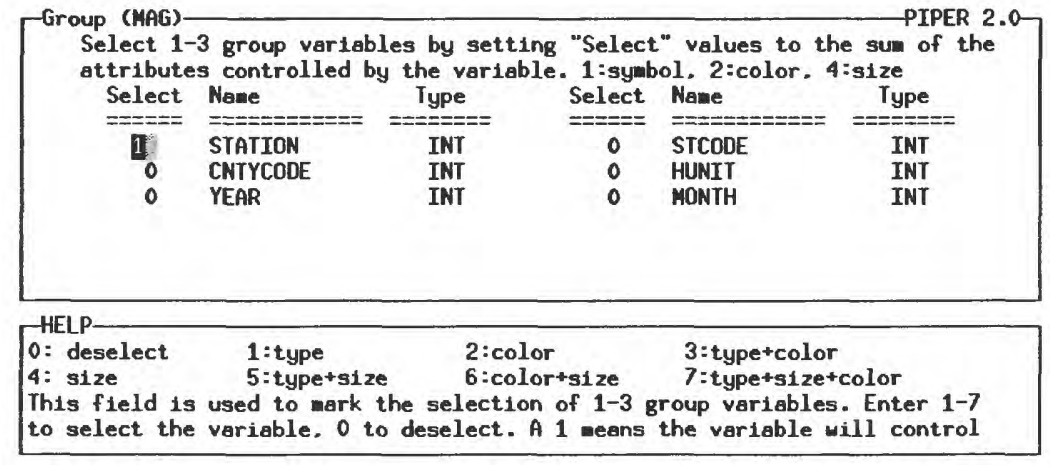

\footnotetext{
TINSTRUCT- Enter data in highlighted field(s).

Use carriage return or arrow keys to enter data and nove between fields. Use "Accept" command to go to next screen when done entering data.

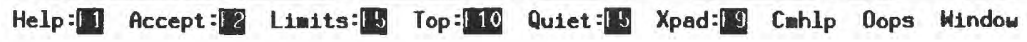

\section{List Single or Multiple Group List}

If either multiple "Varsets" or the "Partition" options are selected in the "Name (N)" screen, then multiple diagrams will be produced. The "piper" program provides for assigning a single list of symbol attribute specifications to all diagrams or each diagram can be specified separately. From the "Point Attributes (MA)" screen, select the "List (MAL)" screen. Use the arrow keys to select either "single" or "multiple." Use Accept (F2) to return control to the "Point Attributes (MA)" screen.
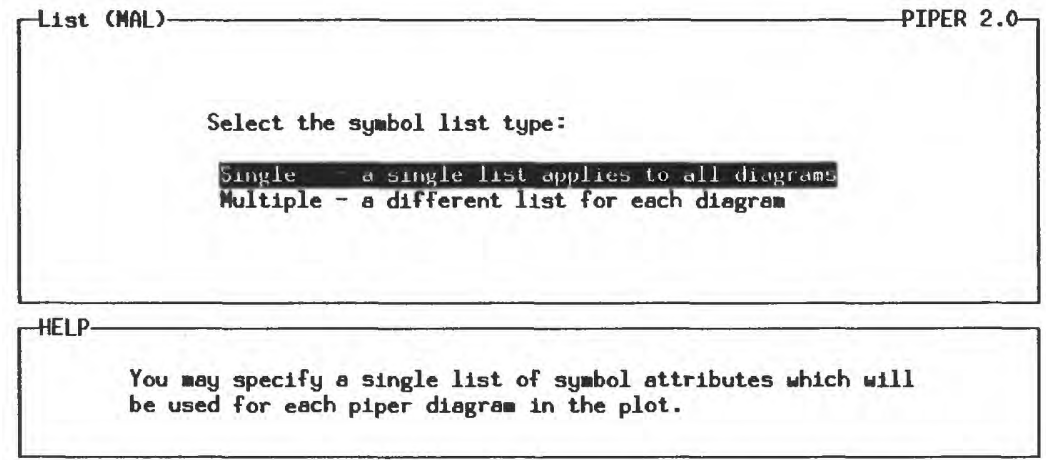

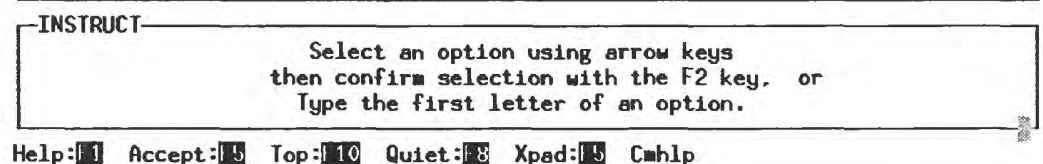




\section{Specify Symbol Type, Size, and Color}

If "single" was selected in the "List (MAL)" screen, then selection of the "Specify symbol type, size, and color" option in the "Point Attributes (MA)" screen will present a single set of "Specify symbol (MAS)" screens for assigning symbol attribute values uniformly to all diagrams. If "Multiple" was selected, then a symbol attribute screen set will be presented for each diagram to be plotted. The number of "Specify symbol (MAS)" screens to be presented within a screen set will depend on the number of grouping variables selected and the combination of symbol attributes assigned to each variable. If there are symbol attributes that remain after the grouping variables have been assigned their attributes and attribute values, then a final "Specify symbol (MAS)" screen is used to assign values to the remaining attributes. These values will be applied to all points in the diagram.

Use the Tab and arrow keys to select a symbol attribute and enter a value in the field. When the values of the "Group Variable" are entered, the system does not search the data file and list all unique values of the variable. The user is responsible for providing the list of values. Any number of values in any order can be entered. The value "REST" can be entered at the end of the "Group Variable" value list to indicate that all data points not represented by the previously entered group variable values will be represented by the symbol attribute value assigned to the "REST" value.

For each symbol attribute, use Help (F1) to bring up the "HELP" panel that lists the allowable values for that attribute. When the symbol type attribute is presented, the user has the option of entering a label (up to 24 characters), which is used in the explanation box on the plot; for example, if the value of the variable STCODE was 34, then "New Jersey" could be entered in the label field.

If the "HELP" panel has more than four lines of information available for a given symbol attribute, then press Help (F1) again to activate the Page Up and Page Down functions (the "Instruct" panel will reflect the change to the page scrolling option). The "Data" panel becomes inactive for data entry. Scroll through the "HELP" panel until the attribute value that is needed is found. Use (F3) to reactivate the Data panel and enter the symbol attribute value in the appropriate field. Use Accept (F2) to go to the next "Specify symbol (MAS)" screen or return control to the "Point Attributes (MA)" screen. Use "Return" to return control to the "Modify (M)" screen.
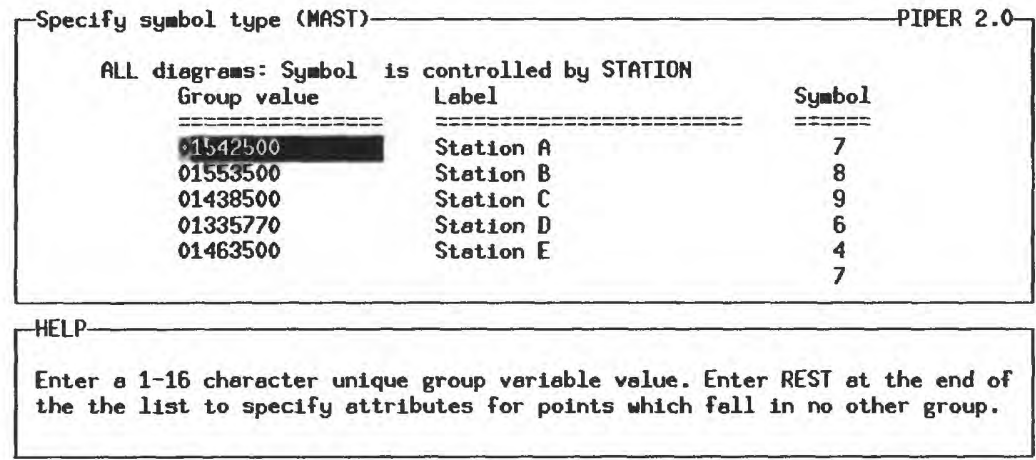

\footnotetext{
INSTRUCT-_ Enter data in highlighted field(s).

Use carriage return or arrow keys to enter data and nove between fields. Use "Accept" comand to go to next screen when done entering data.

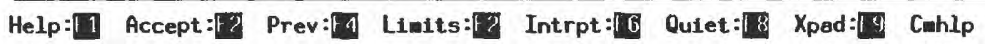



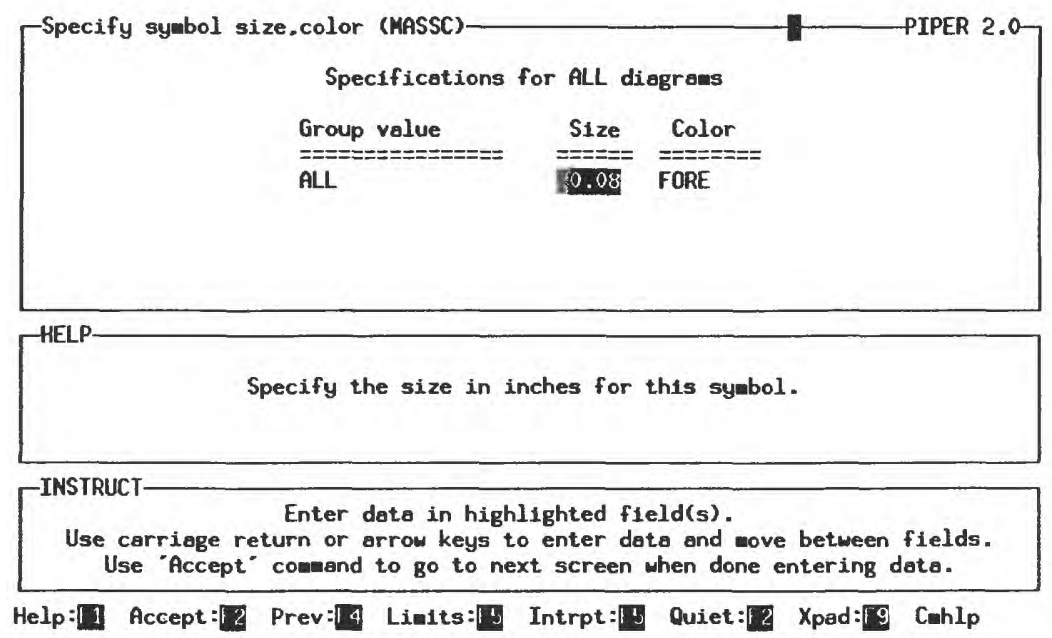

\section{Point Density Shaded Diagrams}

Point Density Shaded Piper diagrams use grid lines (independent of the normal grid line designation specified in the "Page (MBP)" screen) to form a user-defined number of triangular subdivisions within the main triangle, which are shaded according to the ranking of the number of data points in the triangle relative to the other triangles. The number of data points in each small triangle is computed and this number is ranked with the numbers from the other triangles. The ranked data are distributed into a user-defined number of percentile intervals (up to 10). Each percentile interval can be specified as to color and shade pattern. From the "Plot (MP)" screen, select the "Point density shaded" option. Use Accept (F2) to return control to the "Modify (M)" screen.

\section{Density Attributes}

From the "Modify (M)" screen, select the "Density Attributes (MA)" screen, which lists options for selecting the number of axis divisions to use and for specifying the shading intervals and attributes.

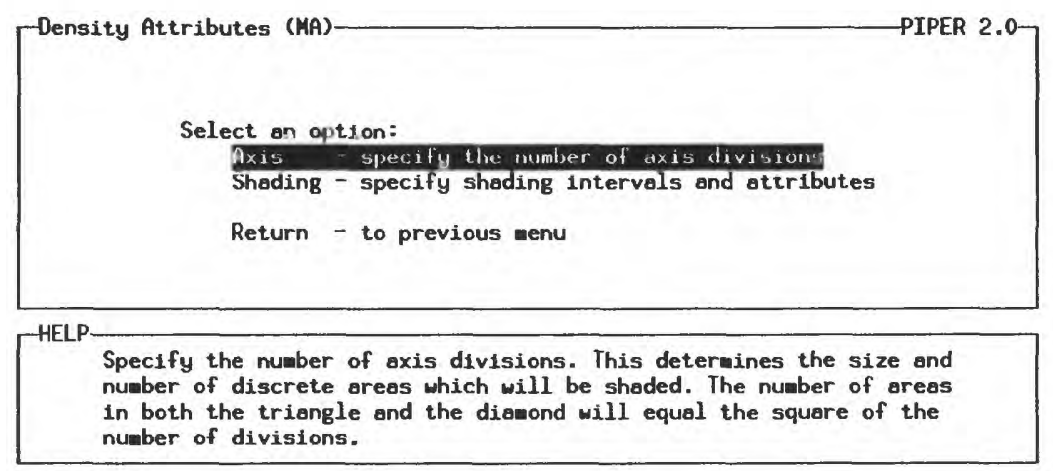

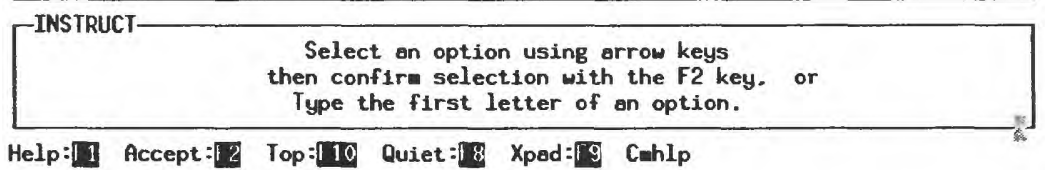




\section{Axis Divisions}

From the "Density Attributes (MA)" screen, select the "Axis (MAA)" screen. The number of axis divisions (default 5) is used to determine the size and number of discrete areas that will be shaded. For a Quadlinear diagram, the number of areas in both the triangles and the diamond will equal the square of the number of divisions specified (default 25). Use Accept (F2) to return control to the "Density Attributes (MA)" screen.

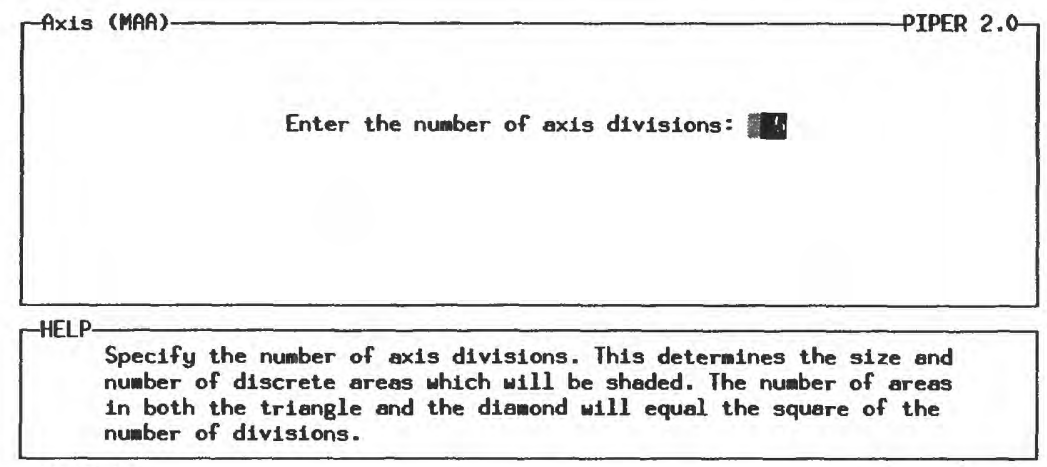

\footnotetext{
[INSTRUCT- Enter deta in highlighted field(s).

Use carriage return or arrow keys to enter data and move between fields. Use "Accept" comand to go to next screen when done entering date.

Help: 1 Accept $:$ Linits $=12$ Top: 10 Quiet $=8$ Xpad: 9 Cahlp Oops
}

\section{Shading Intervals and Attributes}

From the "Density Attributes (MA)" screen, select the "Shading (MAS)" screen, which provides for selecting and characterizing the percentile intervals to be represented on the plot. The default number of intervals is six and each interval is assigned a default maximum interval value, color, and shading pattern. Use the Tab and arrow keys to select the items to be changed. A maximum of ten intervals can be specified. Use Accept (F2) to return control to the "Density Attributes (MA)" screen. Use "Return" to return control to the "Modify (M)" screen.

$\begin{array}{|ccll|}\text { Shading (MAS) } & \begin{array}{c}\text { Maximum } \\ \text { Percent1le }\end{array} & \begin{array}{l}\text { Shading } \\ \text { Color }\end{array} & \begin{array}{c}\text { Shading } \\ \text { Pattern }\end{array} \\ ===-== & =-==== & ==-== \\ 25.0 & \text { RED } & 1 \\ 50.0 & \text { GREEN } & 1 \\ 75.0 & \text { BLUE } & 1 \\ 90.0 & \text { CYAN } & 1 \\ 95.0 & \text { MAGENTA } & 1 \\ 100 . & \text { VELLOH } & 1 \\ 0.0 & \text { BACK } & 1 \\ \end{array}$

HELP-
Specify the maximum xile value for this area. The winiwum $x_{1}$ le will be just
above the next lowest meximum or 0 . Areas which foll within this intervals
min/max percentile ranking for point density will be shaded using its
specified color and pattern. You must provide an interval with max $=100$.

\footnotetext{
INSTRUCT- Enter data in highlighted field(s).
Use cerriage return or arrow keys to enter date and wove between fields.
Use 'Accept' command to go to next screen when done entering date.
} 


\section{Binary and Ternary Mixing Diagrams}

Mixing diagrams are regular Piper diagrams on which selected reference points ( 2 for binary and 3 for ternary) are connected by a line. Data points that fall on the line that connects the binary reference points or within the triangle formed by the ternary reference points are considered to be mixtures of the two or three sources represented by the reference points (see Briel (1993) for additional discussion of mixing diagrams).

\section{Mixing Diagram Attributes}

From the "Modify (M)" screen, select the "Attributes" option to view the "Mixing Diagram Attributes (MA)" screen. The following options are listed:

Partition - data into reference and test sets

Group - specify variable(s) for grouping test data within diagrams

List - specify single or multiple group lists

Specify - symbol type, size, and color

Return - to previous menu.

With the exception of the "Partition data into reference and test sets" option, this screen is processed exactly the same as the "Point Attribute (MA)" screen used for the "Individual points plotted" option selected in the "Plot (MP)" screen; and the "Group," "List," and "Specify" option discussions will not be repeated here. Selection of the "Partition" variable in the subsequent "Partition (MAP)" discussion is a process independent from the selection of the "Grouping" variable in the "Group (MAG)" screen, even though the variable selected in each case could be the same.

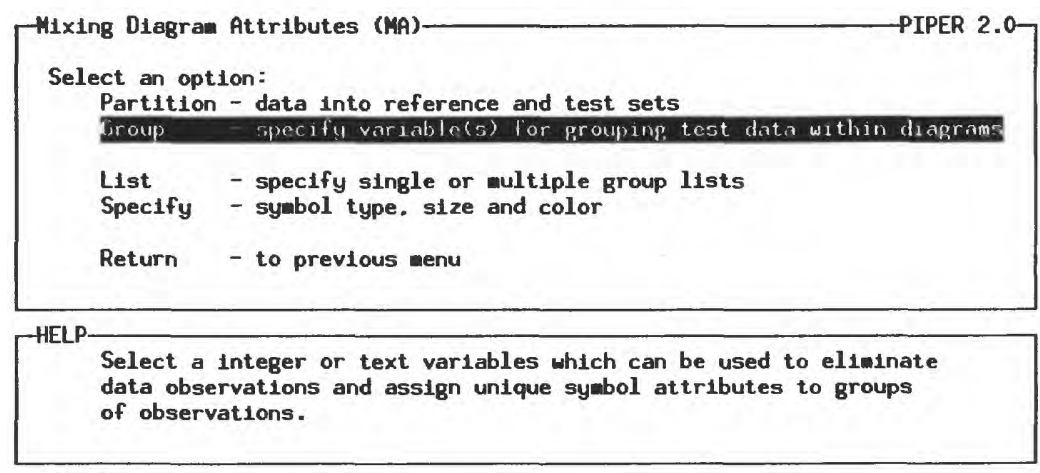

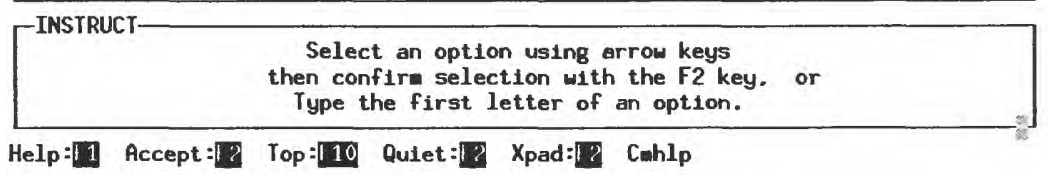


Partition Data into Reference and Test Sets

From the "Mixing Diagram Attributes (MA)" screen, select the "Partition (MAP)" screen. The following options are listed:

Variable - select partition variable

Intervals - specify partition variable values for each group

Attributes - specify reference symbol and line attributes

Return - to previous menu.

Use the arrow keys to select the option required and use Accept (F2). When finished, use Return to return control to the "Mixing Diagram Attributes (MA)" screen.
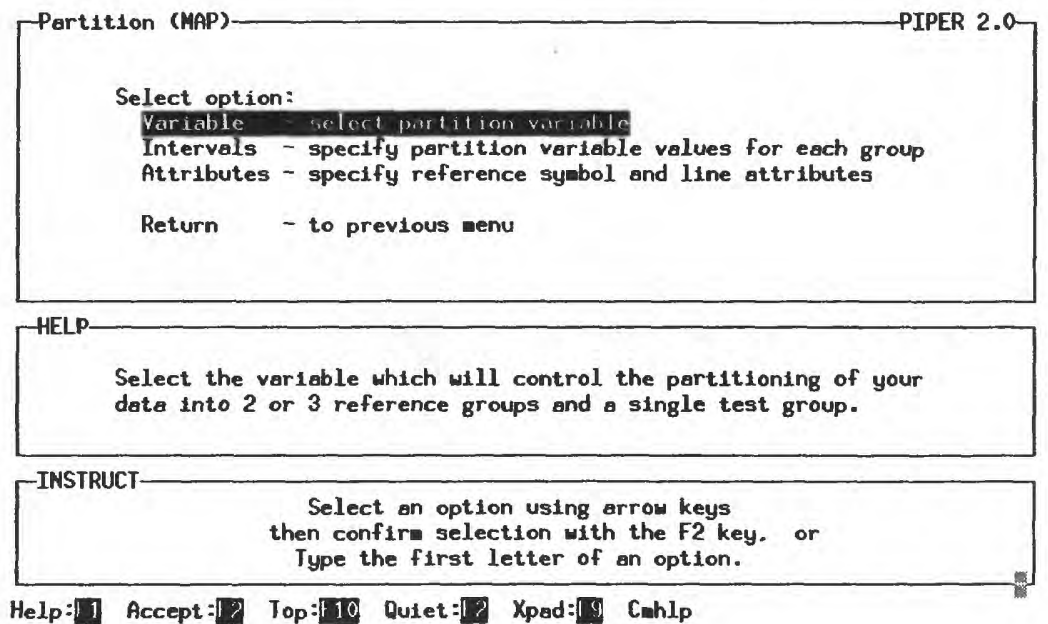

\section{Select Partition Variable}

From the "Partition (MAP)" screen, select the "Variable (MAPV)" screen. Select, by placing a " 1 " in the "Select" column, a single variable whose values will be used to partition data points as reference points or test data points. Use Accept (F2) to accept the selection and return control to the "Partition (MAP)" screen.

\section{Specify Partition Variable Values}

From the "Partition (MAP)" screen, select the "Intervals (MAPI)" screen. Enter a minimum and a maximum value of the partition variable to indicate data points to serve as the first reference point. Do the same for the second and, if necessary, a third reference point (ternary diagram). Similarly, define a range of values to encompass the test data points. It is not necessary to use the entire data set. Additional prefiltering of the data can be done from the "Filter" option in the "Open $(\mathrm{O})$ " screen or by using the filtering option in the "datmgr" program. Use Accept (F2) to accept the selections and return control to the "Partition (MAP)" screen. 

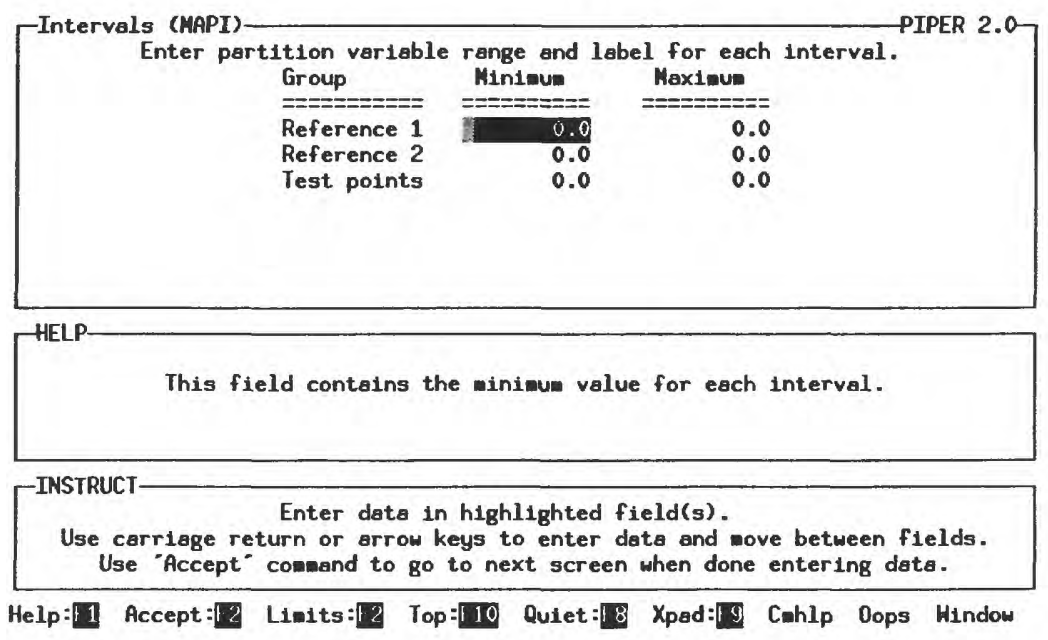

\section{Specify Reference Symbol and Line Attributes}

From the "Partition (MAP)" screen, select the "Attributes (MAPA)" screen to specify the attributes of the reference points symbol and the line(s) that connect them on the plot. Use Help (F1) to list allowable values for these attributes. Make any required changes to the default values listed, including the text to be used in the legend.

If the "HELP" panel has more than four lines of information available for a given symbol attribute, then press Help (F1) again to activate the Page Up and Page Down functions (the "Instruct" panel will reflect the change to the page scrolling option). The Data panel becomes inactive for data entry. Scroll through the "HELP" panel until the attribute value that is needed is found. Press F3 to reactivate the Data panel and enter the symbol attribute value in the appropriate field. Use Accept (F2) to accept the selections and return control to the "Mixing Diagram Attributes (MA)" screen.

Process the "Group," "List," and "Specify" options that follow the instructions given in the section "Point Attributes" for the "Individual points diagrams" data display method. Use Return to return control to the "Modify (M)" screen.

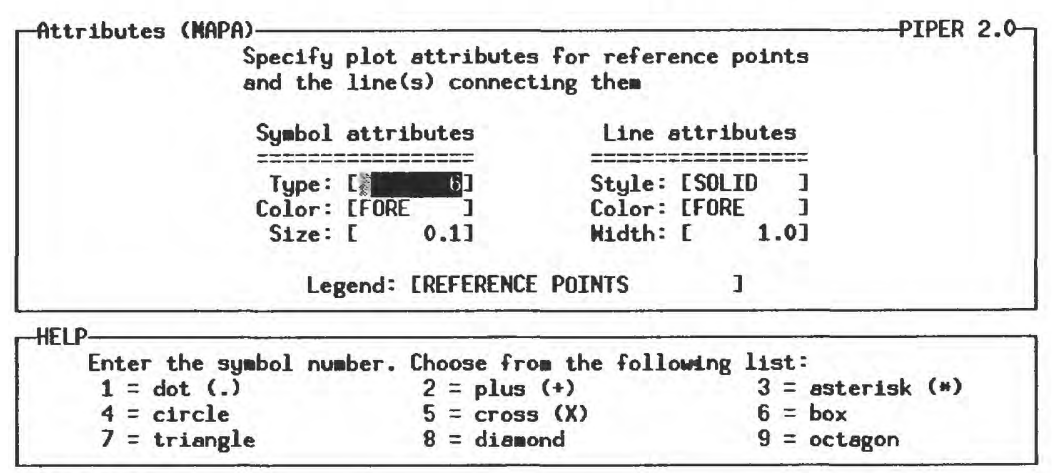

\footnotetext{
-INSTRUCT- Enter data in highlighted field(s).
Use carriage return or arrow keys to enter data and wove between fields.
Use -Accept comeand to go to next screen when done entering data.
} 


\section{Basic Plot Parameters}

From the "Modify (M)" screen, select the "Basic (MB)" screen, which lists the following options:

$\begin{array}{ll}\text { Legend } & \text { - options } \\ \text { Headings } & \text { - for each diagram } \\ \text { Titles } & \text { - above plot } \\ \text { Footnotes } & \text { - below plot } \\ \text { Labels } & \text { - of miscellaneous text } \\ \text { Page } & \text { - layout options } \\ \text { Censored } & \text { - data options } \\ \text { Make } & \text { - the plot } \\ \text { Return } & \text { - to previous screen. }\end{array}$

Type the first letter of any option or use the arrow and Enter keys to select the screen for the option required.
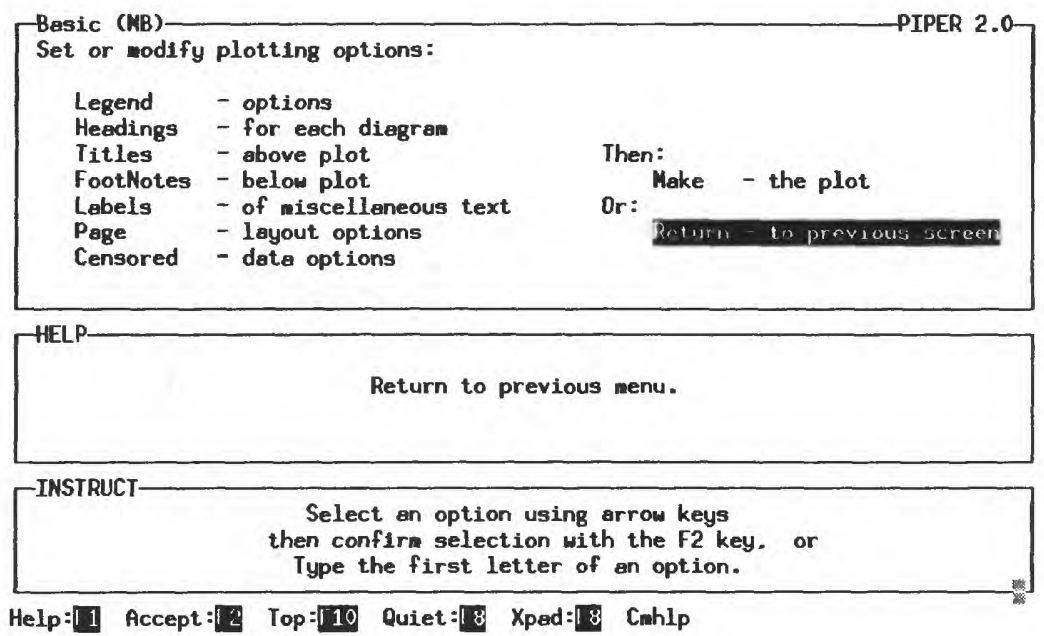

\section{Legends}

Legends can be added either to point symbol or point density piper diagrams. In addition, a statistical block can be added that displays a median value for up to three variables from the data set (not necessarily the cations and/or anions that are displayed in the diagram), the ionic balance, and the period of record. The period of record can be computed from the data set (be sure to have a date variable in the YYMMDD format in the data set) or an arbitrary begin date and end date can be entered if the data set does not have a date variable.

From the "Basic (MB)" screen, select the "Legend (MBL)" screen, which lists the following three options:
Point - symbol legend options
Density - shading legend options
Statistical - explanation block options. 


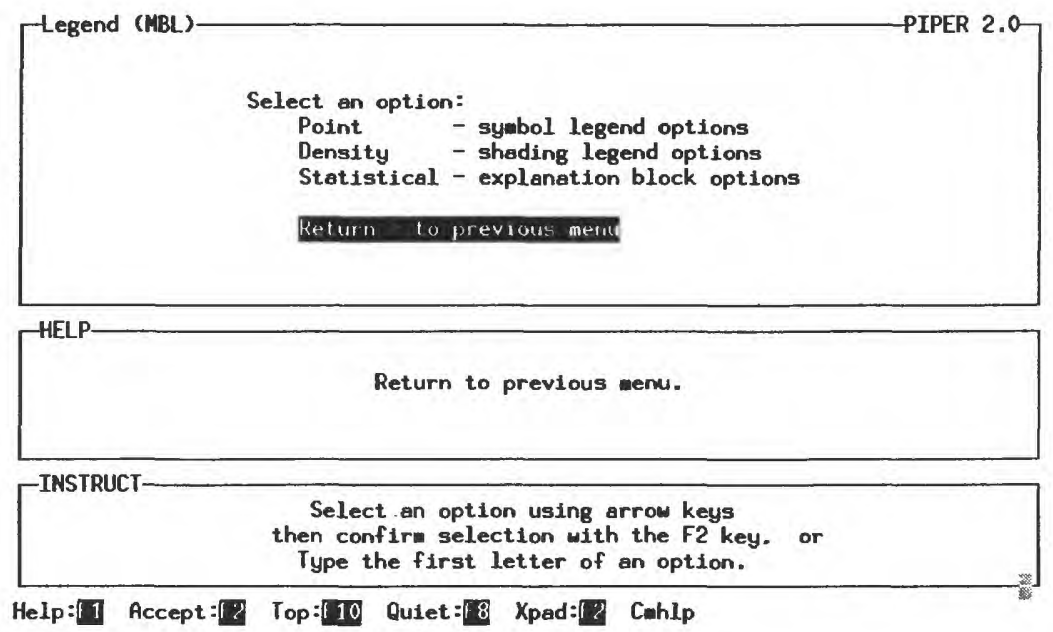

\section{Point Symbol Legend}

From the "Legend (MBL)" screen, use Accept (F2) to select the "Point (MBLP)" screen. The "Include legend" option is, by default, selected and toggled "On" as indicated by an "X" in the field to the left of the option name. Use the space bar to toggle this option "Off," as indicated by a "blank" in the field to the left of the option name.

Use the arrow keys to select the appropriate location (upper left, upper right, split) for the station symbol legend. Use the space bar to place an " $\mathrm{X}$ " in the field to the left of the option name. When the "split" option is selected, the station symbol legend will be evenly split between the upper left and upper right quadrants of the diagram.

Use Accept (F2) to return control to the "Legend (MBL)" screen.

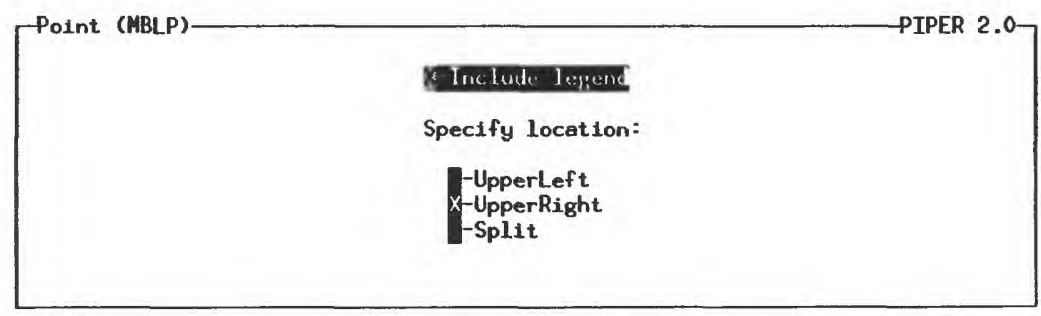

\footnotetext{
Check this box to include a station symbol legend on the diagraw.

Clear the box to omit the legend.

TNSTRUCT-

Dption field: use space bar to toggle between ON $(X)$ and DFF $($ ).

Use carriage return or arrow keys to wove between fields.

Use "Accept" command to go to next screen when done entering data.
}

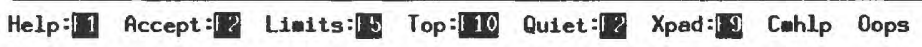

\section{Density Shading Legend}

From the "Legend (MBL)" screen, select the "Density (MBLD)" screen. The "Include legend" option is, by default, selected and toggled "On" as indicated by an "X" in the field to the left of the option name. If a legend for the density diagram is not desired, then use 
the space bar to toggle this option "Off" as indicated by a "blank" in the field to the left of the option name.

\section{Annotation}

Use the arrow keys to select the "annotation" option for the density legend. The "Percentile" option annotates the diagram with the percentile ranking intervals selected in the "Shading (MAS) screen (see "Attributes" option in "Modify (M) " screen). Selection of the "Count \& percentile" option adds the range of sample counts to be found in a given percentile interval. Each shaded polygon of a given color will have a sample count within the range computed for its assigned percentile interval. Use the space bar to toggle "On" the appropriate option as indicated by an " $\mathrm{X}$ " in the field to the left of the option name.

\section{Location}

Use the arrow keys to select the appropriate location (upper left or upper right) for the density legend. Use the space bar to toggle this option "On" as indicated by an "X" in the field to the left of the option name.

Use Accept (F2) to return control to the "Legend (MBL)" screen.
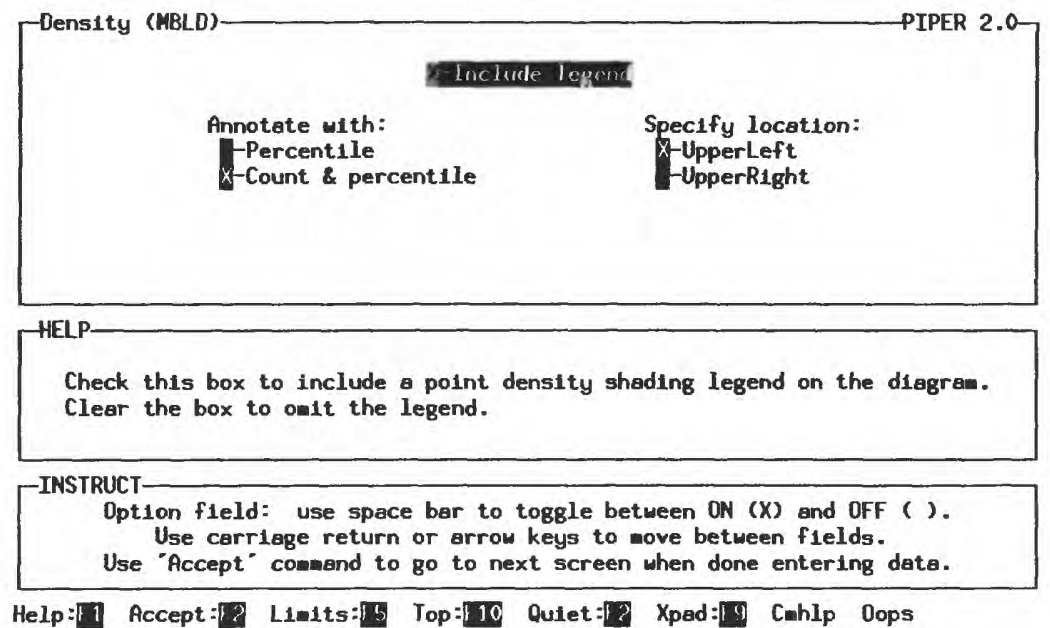

\section{Statistical Explanation Block}

From the "Legend (MBL)" screen, select the "Statistical (MBLS)" screen. By default, the "Include legend" option is selected and toggled "Off" as indicated by a blank in the field to the left of the option name. If a statistical block, including computed median values, ionic balance, and the period of record, is desired, then use the space bar to toggle this option "On" as indicated by a " $\mathrm{X}$ " in the field to the left of the option name.

\section{Statistical Block Location}

Use the arrow keys to select the appropriate location (upper left or upper right) for the location of the Statistical Block legend. Use the space bar to toggle this option "On" as indicated by an "X" in the field to the left of the option name.

\section{User-Specified Period of Record}

With this option the user enters a begin date and end date (YYMMDD format) that is placed on the plot independent of the presence, if any, of a date variable in the data set. Use the Tab and arrow keys to select the "User specified" Period of Record option and then use the space bar to toggle the option "On" as indicated by an " $\mathrm{X}$ " in the field to the left of the option name. 


\section{Compute Period of Record from Data}

With this option the system computes a begin date and end date from the date variable in the input data file. The date variable must be an integer in the YYMMDD format. The date variable is specified in the "Statistics legend labels (MBLS)" screen (see below). Use the Tab and arrow keys to select the "Compute from data" option from the "Period of Record" list. Use the space bar to toggle the option "On" as indicated by an "X" in the field to the left of the option name.

\section{Select Statistical Legend Variables}

If either the "User specified" or the "Compute from data" option is selected for the "Statistical block" option, pressing Accept (F2) brings up the "Statistical legend variables (MBLSV)" screen, which is used to select up to three variables whose mean values will be computed and displayed in the legend. In the case of the "Compute from data" option, this screen also is used to specify the date variable.

Select up to three variables for the mean value computation by entering a " 1,2 , or 3 " in the "Select" field to the left of the variable name of each variable desired. In the case of the "Compute from data Period of Record" option, select the date variable by entering a "4" in the "Select" field.

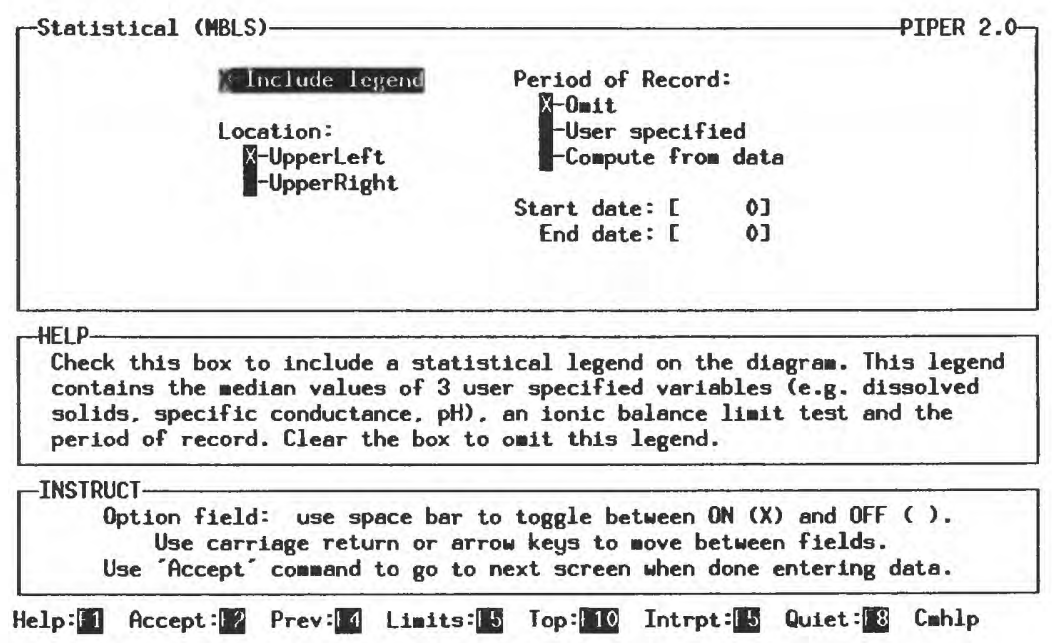

\section{Variable Labels}

After the variables are selected in the "Statistical legend variables (MBLSV)" screen, use Accept (F2) to bring up the "Statistics legend labels (MBLSL)" screen. This screen is used to specify alternate descriptions of the variable names that might be specified as part of the variable description supplied with the data set. Use the Tab and arrow keys to select the "Description" field and enter up to 24 characters of descriptive information about the variable. Next select the "Unit" field and enter up to eight characters to describe the unit of measurement for the variable. The displayed format would look like the following:

Median Dissolved Solids: $223.0 \mathrm{mg} / \mathrm{L}$.

Note that the term "Median" and the median value (: 223.0$)$ are inserted by the system.

Use Accept (F2) to return control to the "Legend (MBL)" screen.

Use Accept (F2) again or Return to return control to the "Basic (MB)" screen. 
Headings

A heading (up to 40 characters) can be specified for each Piper diagram. From the "Basic (MB)" screen, type "H" (or " $h "$ ) or use the arrow keys to select the "Headings (MBH)" screen. For each diagram to be plotted, enter a heading. When finished, use Accept (F2) to return control to the "Basic (MB)" screen. If the diagrams are based on the partitioning of a single set of variables (see "partition" option in the "Name $(N)$ " screen), then the diagram headings also can be specified in the "Headings (MPTAH)" screen accessed from the "Type" option in the "Partition (NP)" screen. Diagram headings that are specified when the partitioning option is invoked will revert to default values (the value of the partitioning variable) whenever any option that affects the partitioning of the data is changed. Use the "Heading (MBH)" screen to reset the desired headings when this occurs.

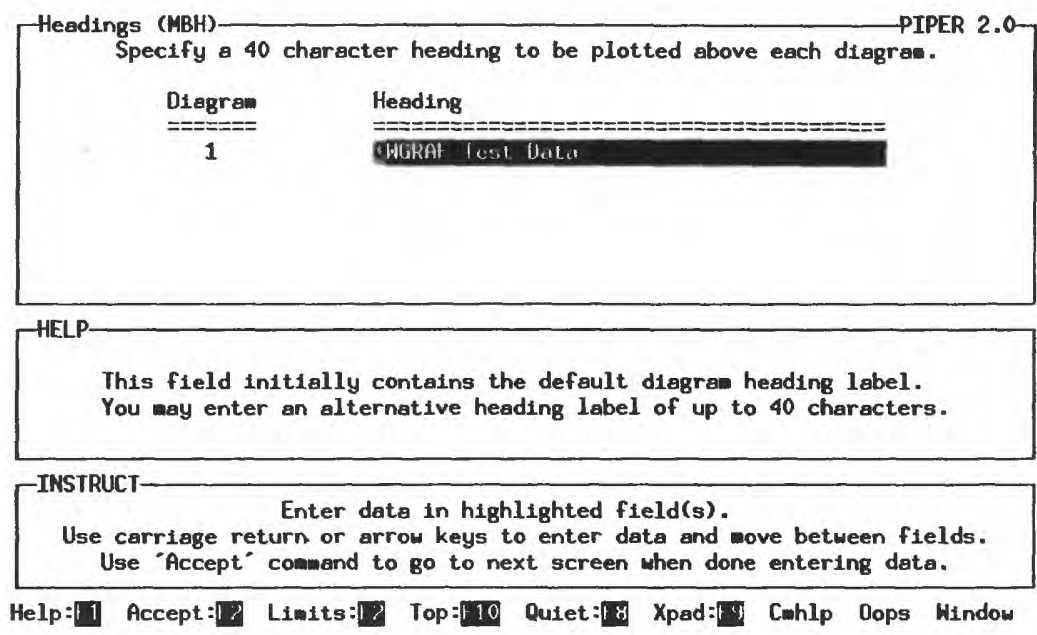

\section{Page Layout Options}

From the "Basic (MB)" screen, select the "Page (MBP)" screen. The "Page (MBP)" screen provides the following functions:

Page

Height

Width

Axis Label

Height

Offset

Diagram Title

Height

Offset

Diagrams in Row 1

Diagrams in Row 2

Diagrams in Row 3

Font Style

Grid Level

Plot Page Border. 
Most options in this list are discussed in the section "Page Layout Options" under "Steps to Using QWGRAF Programs."

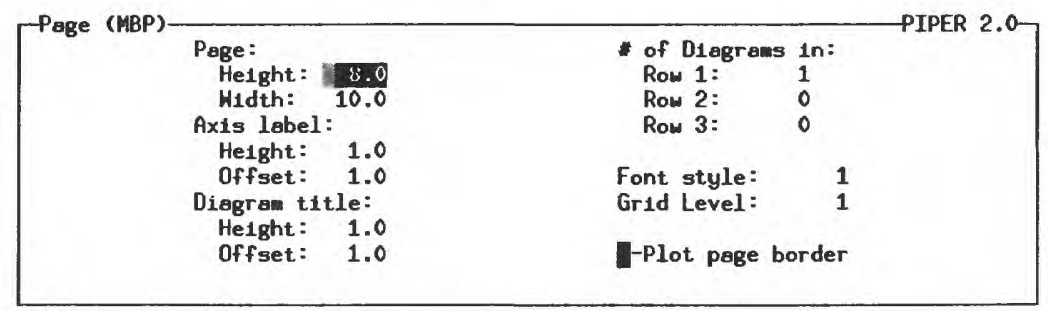

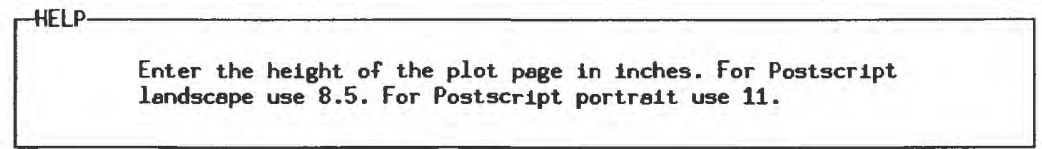

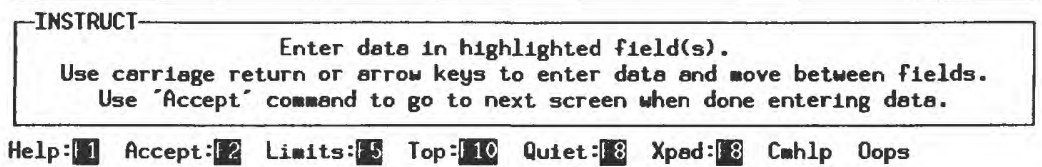

\section{Axis Label}

Height

The labels associated with the variables selected for presentation in the Piper diagram (as assigned in the "Add (NIA)" screen for varsets or the "Diagram (NPD)" screen for partitioned data) are assigned a default size by the system. This size can be changed, but only on a relative basis. Use the Tab or arrow keys to select the "Axis Label Height" option. Enter an alternative factor to replace the default value of "1." A value of 2 would double the height of the label.

\section{Offset}

The space that separates the axis label from the diagram axis is preset by the system. This distance can be changed, but only on a relative basis. Use the Tab or arrow keys to select the "Axis Label Offset" option. Enter an alternative factor to replace the default value of "1." A value of 2 would double the offset distance.

\section{Diagram Title}

\section{Height}

The size of the diagram title specified in the "Heading (MBH)" screen is predefined by the system. This size can be changed, but only on a relative basis. Use the Tab or arrow keys to select the "Diagram Title Height" option. Enter an alternative factor to replace the default value of "1." A value of 2 would double the height of the label.

\section{Offset}

The space that separates the diagram title from the top of the diagram is preset by the system. This distance can be changed but only on a relative basis. Use the Tab or arrow keys to select the "Diagram Title Offset" option. Enter an alternative factor to replace the default value of "1." A value of 2 would double the offset distance.

\section{Number of Diagrams}

The "piper" program provides for up to 90 diagrams on multiple pages. Each page may contain up to nine diagrams distributed in three rows of three diagrams each. The number of pages required will depend on the total number of diagrams and the number of 
diagrams the user wants on each page. Thus, 48 diagrams could be distributed on 48 pages of 1 diagram each, 16 pages of 3 diagrams on each page, 8 pages of 6 diagrams on each page, and so forth.

Use the Enter and arrow keys to select the rows on which diagrams will be placed. Enter the number of diagrams to be placed on each row. If zero is selected for all three rows, then the system will select a default organization.

\section{Grid Level}

Use the Enter and arrow keys to select the "Grid level" option. If the "HELP" panel is not already activated, then use Help (F1) to select the "HELP" panel, which presents the following options that are available for drawing grid lines on the Piper diagram:

0 : No grid line, and tick marks and numbers at 20 -percent intervals.

1: Grid lines, and tick marks and numbers at 20-percent intervals.

2: Grid lines, and tick marks and numbers at 10-percent intervals.

3: Tick marks and numbers at 20-percent intervals, and water-type grid.

Enter the appropriate code number in the "Grid level" field.

\section{Censored Data Options}

From the "Basic (MB)" screen, select the "Censored (MBC)" screen to view the options for censored data. By default the "piper" program excludes censored data from the analysis. To include censored data, use the arrow or Tab keys to select (highlight) the "Include censored values" option. Use Accept (F2) to return control to the "Basic (MB)" screen.

\section{Make the Plot}

When all arguments for the Piper diagram have been specified and the appropriate device selected, return control to the "Opening Screen" and select the "Make the plot" option. Use the Enter key. If a screen output was selected, then a graphics window opens and the Piper diagram is presented. If a file output was selected, then a message screen indicates that the output from the procedure has been saved.

\section{piper Tutorial}

An interactive tutorial is available to guide the user through the basic options of the "piper" program. The user will be guided through the steps required to create several quadlinear Piper diagrams on a page. The data will be partitioned to create a diagram for each State. The diagrams will be annotated with a symbol legend and a summary statistics legend for alkalinity and specific conductance. The tutorial is activated by first typing "Shift @" in the "Opening Screen" of a new "piper" session. When the dialog box comes up, enter the file name found in the tutorial.doc file distributed with the QWGRAF programs. This file is located in the doc subdirectory, which provides specific details on using this and other QWGRAF tutorials.

The tutorial prompts the user for specific keystrokes. The program will only respond when the correct keystroke sequence is entered; that is, incorrect information cannot be entered. Text for labels, titles, and so forth can be entered in either upper or lower case. The system converts everything to upper case.

\section{Sample Plots}

The following are examples of the types of plots that can be created with the QWGRAF "piper" program. These examples are intended to illustrate various options available in the program and do not necessarily conform to publication standards of the Geological Survey. 


\begin{tabular}{|lll|}
\hline QWGRAF & Example Plots & PIPER \\
\hline
\end{tabular}

Program: PIPER

Plot Specifications: Quadlinear Piper Diagram; Data Partitioning Within Diagram; User-Defined Symbols and Sizes; Explanation Block; 20-percent Grid Lines; Two Titles

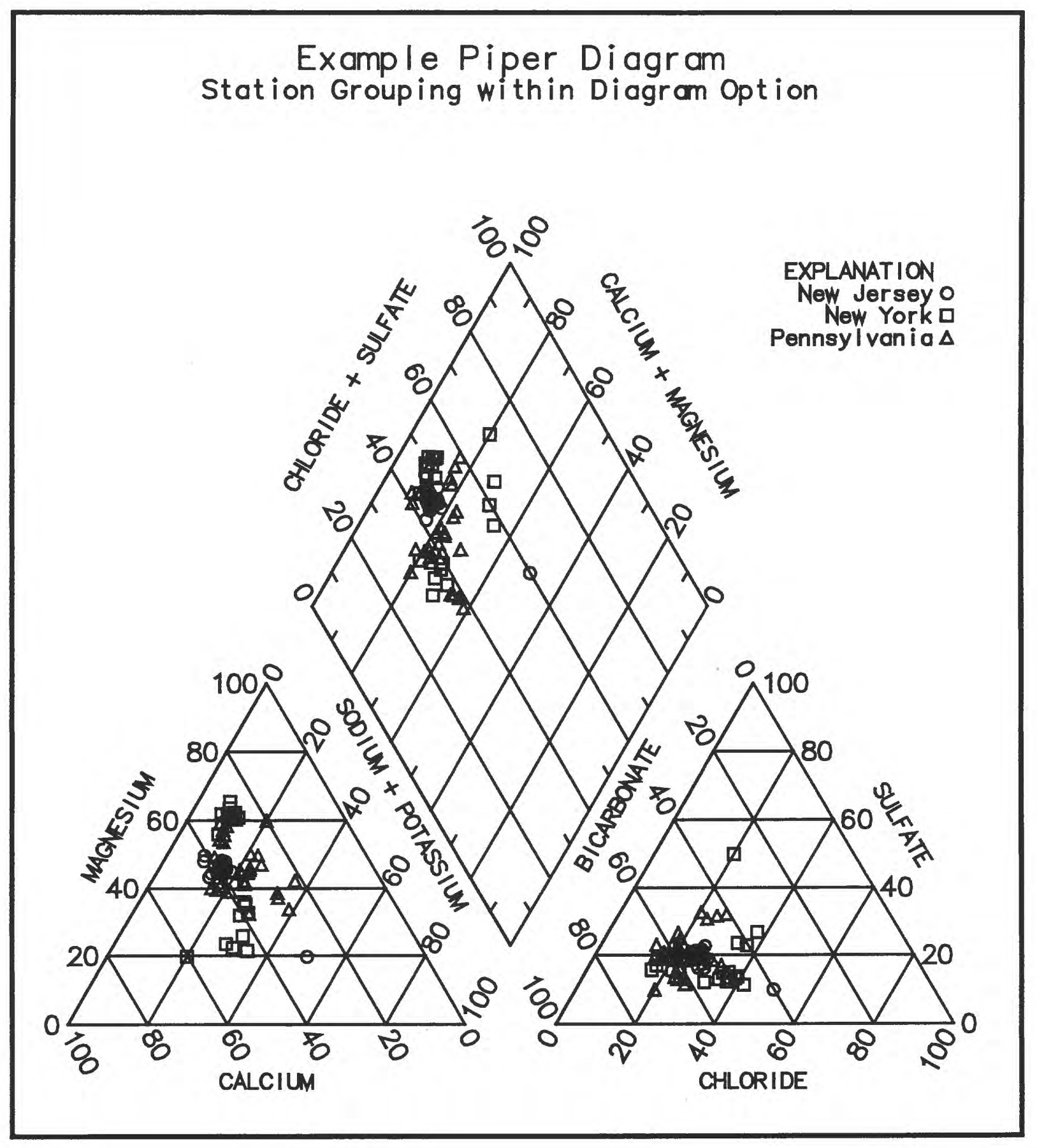

Example Plots 109 


\section{QWGRAF}

\section{Example Plots}

\section{PIPER}

\section{Program: PIPER}

Plot Specifications:Quadlinear Piper Diagram; Multiple Diagrams; Data Partitioning Within Diagram; Explanation Blocks; User-Defined Diagram Labels; Three Titles: 20-percent Grid Lines; User-Specified Symbols and Sizes

\section{Example Piper Diagram} Multiple Diagrans (Partitioning by State)

Partitioning within State by Year
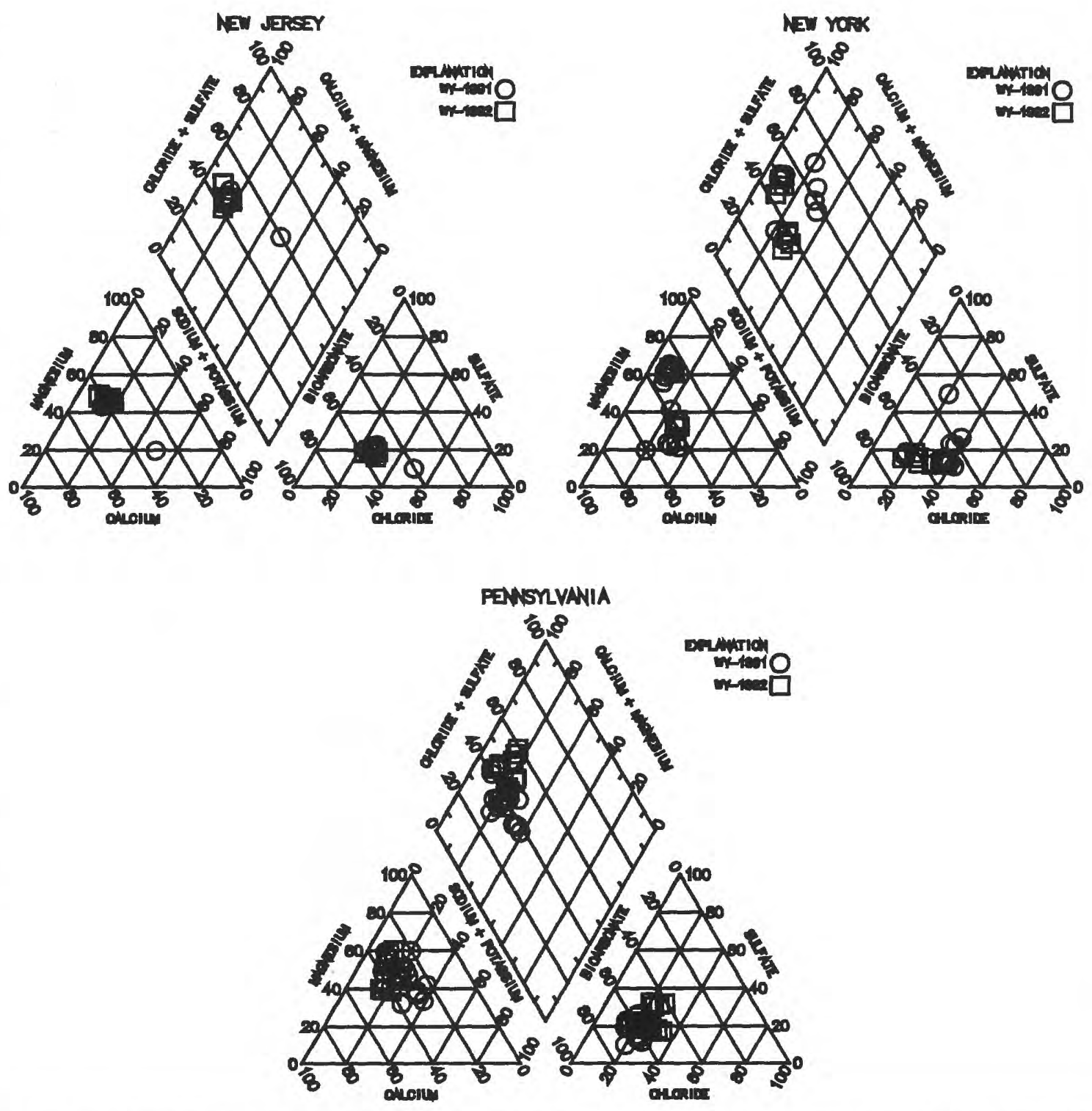
Program: PIPER

Plot Specifications: Quadlinear Piper Diagram; Density Option; User-Specified Percentile Ranges and Shading; Explanation Blocks; 20-percent Grid Lines; Three Titles

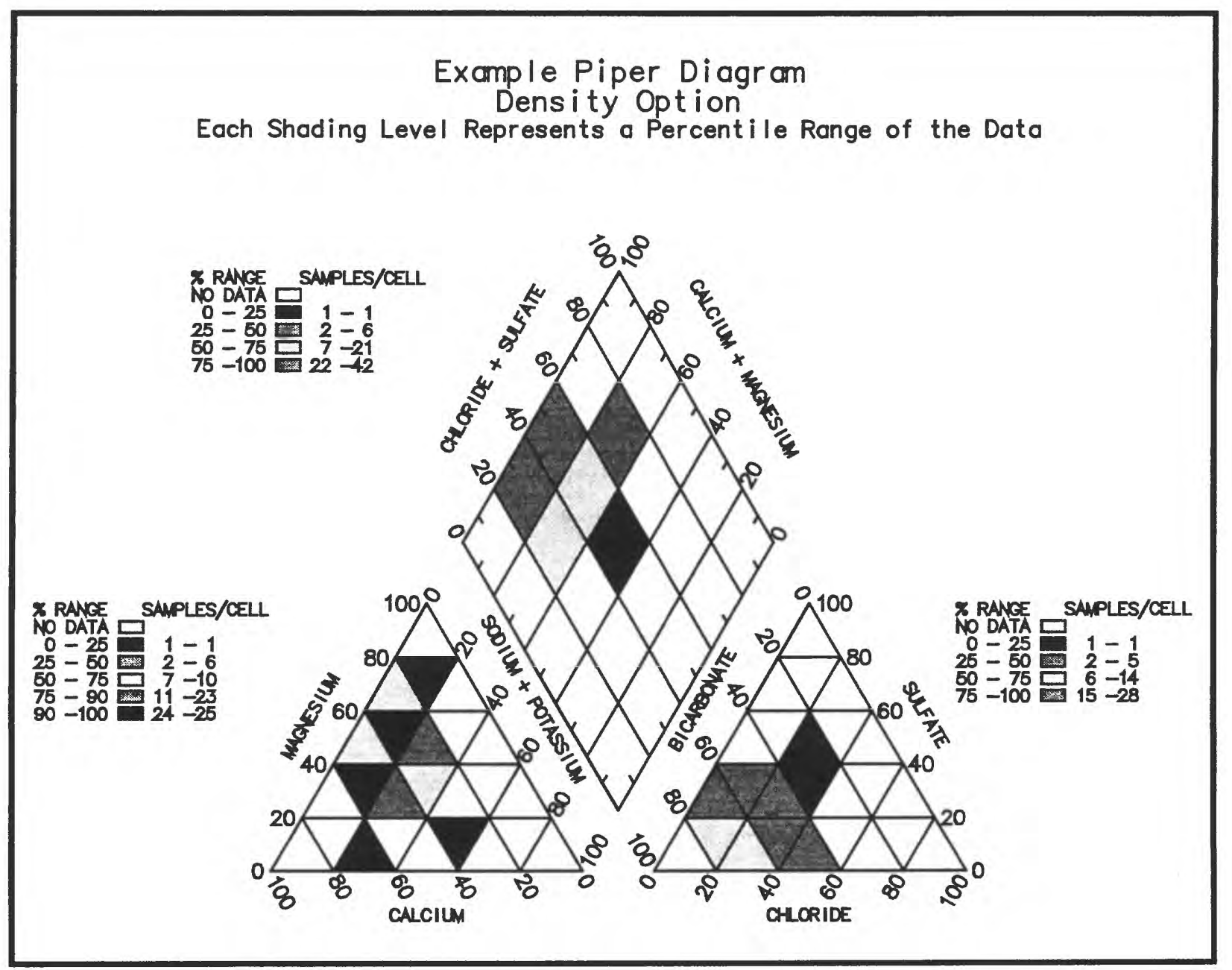


Program: PIPER

Plot Specifications: Quadlinear PIper Diagram; Data Partitioning Within Diagram by Partitioning Variable; User-Specified Symbols and Sizes; Explanation Block; Statistical Legend; 20-percent Grid Lines; One Title

Example Piper Diagram

Optional Statistical Legend

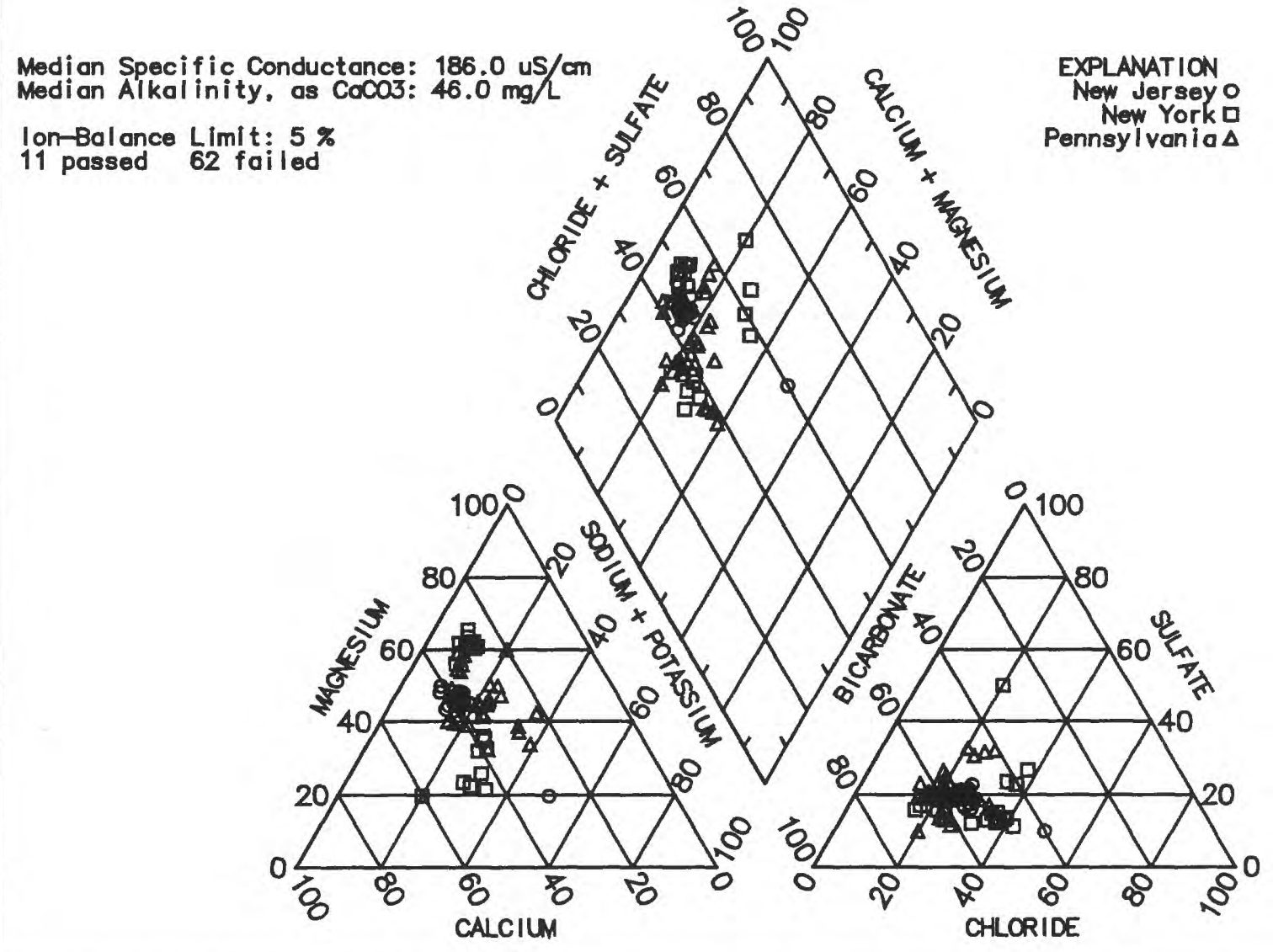


Program: PIPER

Plot Specifications: Quadlinear Piper Diagram; Binary Mixing Option; Data Partitioning Within Diagram by Partitioning Variable (State FIPS Code); User-Specified Symbols and Sizes; Explanation Block; 20-percent Grid Lines; Two Titles

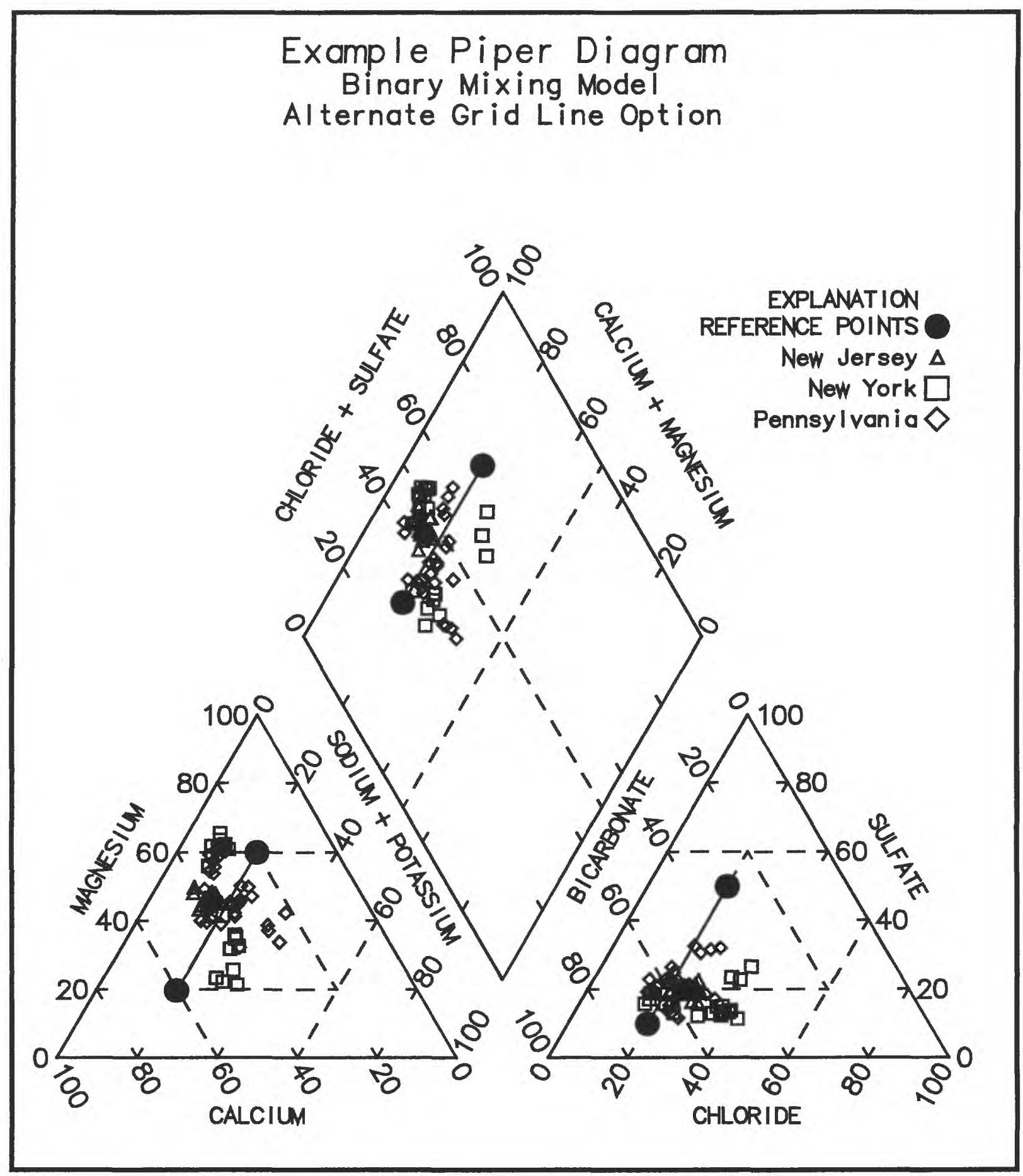




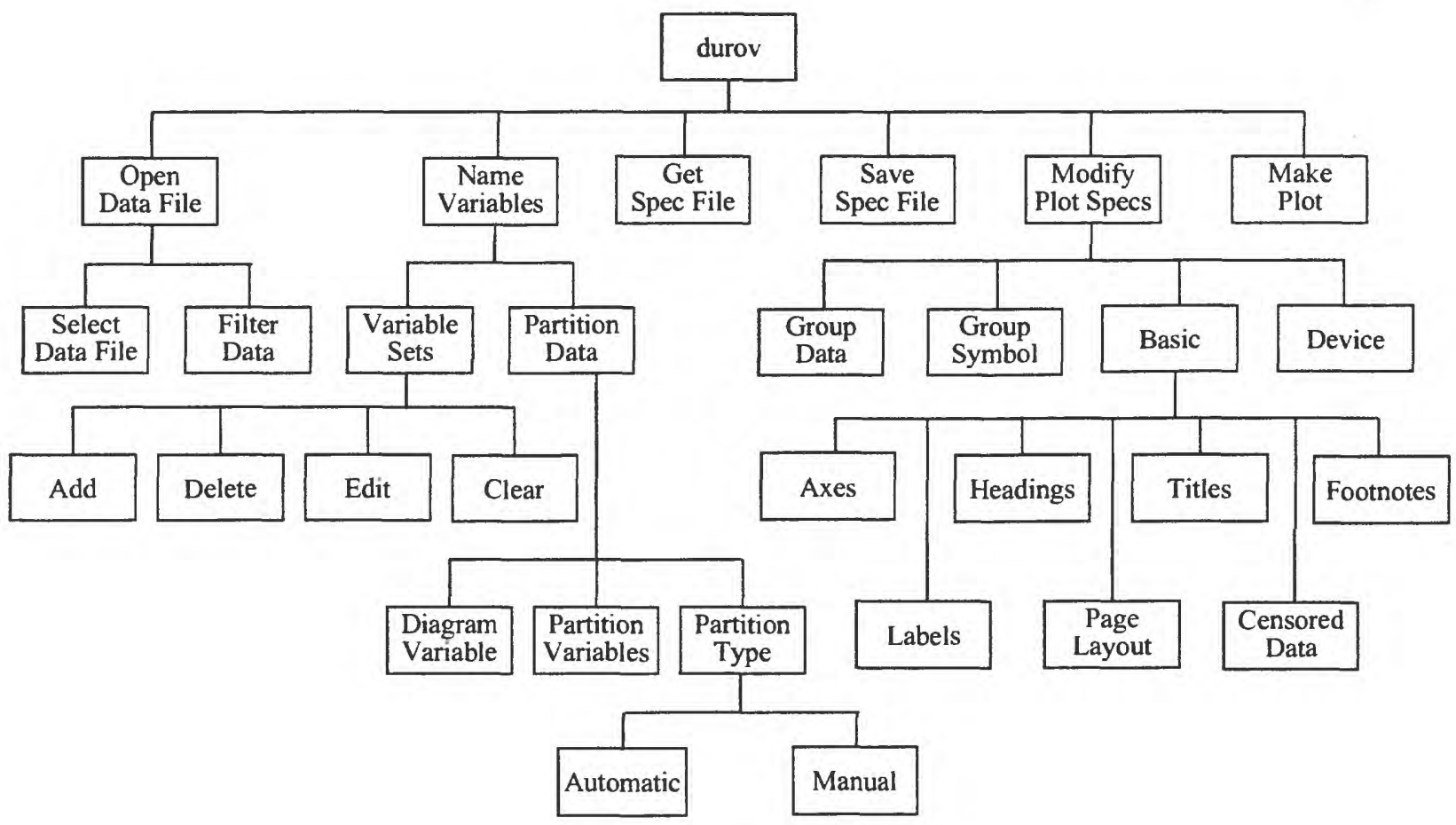

\section{DUROV DIAGRAMS (durov)}

The "durov" program produces Durov diagrams, which are hydrochemical facies diagrams similar to a Quadlinear Piper diagram, except that the intersection of points projected from the two trilinear diagrams are projected to form one of the axes on each of two adjacent $x-y$ plots. The other axis on each of the $x-y$ plots represents another variable from the input data file. Each node of the two trilinear diagrams is user defined and can represent a single chemical constituent or a composite of two or more constituents. Data within a diagram can be differentiated on the basis of a single grouping variable, whose unique values are represented by symbols of user-defined type, size, and color. The program provides for multiple diagrams on a page (up to three lines with up to three diagrams per line). With multiple diagrams there is the option of utilizing a different set of variables for each diagram (individual variables can be repeated between sets) or using one set of variables that has been partitioned into groups of values on the basis of the values of up to four partition variables. Each group is represented by a Durov diagram on the plot page. Remarked (censored) data values reported as a limit of quantitation or detection limit can be included or excluded from the plot data set. Titles for each diagram and labels for each node of each diagram are user defined. There can be up to three title lines and two footnote lines each definable as to size, font, and centering. Grid options include none, 10 percent, and 20 percent. Up to five user-defined labels (up to 40 characters on multiple lines) can be positioned on the plot. Sets of plot specifications can be saved to files and used to facilitate plot configuration in other "durov" sessions.

\section{Background}

Hydrochemical facies are distinct zones that have cation and anion concentrations describable within defined composition categories. In a Durov plot, two trilinear diagrams are arranged so that one side of one triangle is at a 90 -degree angle to one side of the other triangle. The data points from the two triangles are projected until they 
intersect in a central square. These intersection points are then projected as one of the two axes on each of two $\mathrm{x}-\mathrm{y}$ coordinate plots located at the side and bottom of the central square. The other axes on the $x-y$ graphs are represented by other variables from the input data file, such as total dissolved solids, specific conductance, $\mathrm{pH}$, or hardness.

\section{Durov Options}

The "durov" program supports the following Durov functions:

Function

Group Data

Symbol Attributes

Rectangle Axes

\section{Screen Sequence}

Opening Screen, Modify (M), Group (MG)

Opening Screen, Modify (M), Symbol (MS)

Opening Screen, Modify (M), Basic (MB), Axes (MBA).

\section{Input Data Requirements}

The "durov" program assumes that the data to be displayed in the Durov trilinear diagrams are in the equivalent form. See the discussion of "Data Management" in the "General Features of QWGRAF programs" section for suggestions on how to convert concentration data to equivalents. The data to be displayed in the side and bottom $x-y$ coordinate rectangles are normally in standard concentration units.

\section{Starting the "durov" Program}

The "durov" program is invoked by typing "durov" on the command line. The "Opening Screen" displays the options that serve as gateways to the screens used to set or modify the plot configuration options.

\section{Load a Specifications File}

To use specifications from a previous "durov" session, load a "Specifications" file. From the "Opening Screen," select the "Get (G)" screen. A list of specifications files (extension ".drv") and a field for specifying the file name are presented. Use the arrow keys to select the required file and use either Accept (F2) or the Enter key to accept the selection. The "latest.drv" file includes the program specifications that were used during the most recent execution of the "durov" program, whether in the current or previous session.

The "Specifications" file includes the name of the input data file active at the time the specifications file was created. If a new input data file is required, then select the "Open (O)" screen from the "Opening Screen" and select a new input data file.

The "Make the Plot" option can be selected immediately or any of the newly loaded specifications can be changed. Any altered specifications set can be saved in a new file or an old file can be overwritten.

\section{Open Input Data File}

From the "Opening Screen," select the "Open (O)" screen. From the "Open (O)" screen, select the "Select (OS)" screen. Type in a file or select the input data file desired. Use the arrow, Page Down, and Page Up keys to select a data file. Use either Accept (F2) or the Enter key to accept the file selection and return control to the "Open (O)" screen.

\section{Filter Data}

To restrict the data to be applied to the Durov diagram, use the "Filter" option in the "Open (O)" screen to specify the criteria for selecting those rows to be retained for use with the program. This process does not alter the original data file but results in a temporary data file for use with the program. A permanent copy of the filtered data file can be obtained by using the "Filter" option in the "datmgr" program and saving the output in an RDB file. 
From the "Opening Screen," select the "Name (N)" screen. The "Name (N)" screen provides the following options:

Varsets - Select one or more sets of variables (one for each Durov diagram desired).

or

Partition - Select a variable set, a partition variable, and interval limits. One diagram for each partition interval.

or

Return - Return to opening screen.

A single Durov diagram requires eight variables - three variables for the cation list, three variables for the anion list, and two additional variables for the side and bottom rectangles. Normally, a single set of variables is defined that results in a single diagram on the plot page (Varsets option). However, the Varsets option also can be used to define multiple sets of variables for the purpose of placing multiple Durov diagrams on a page. Individual variables can be repeated between sets. The more common approach to multiple diagrams is to take a single set of variables and partition the values into groups on the basis of composite values of up to four partitioning variables (Partition option). Each group is the basis of a Durov diagram; for example, a Durov diagram can be produced for each State. The "Varsets" and "Partition" functions are described in more detail under "Name (Select) Variables" in the section "Shared Features" at the beginning of the report.

\section{Modify Plot Specifications}

After the data file has been opened and the variables of interest named, select the "Modify (M)" screen by typing "M" (or " $m$ ") or use the Tab and arrow keys to select the "Modify compute/plot specifications" option followed by using Accept (F2) or the Enter key. The "Modify (M)" screen presents the following options:

Group - specify variable for grouping data within diagram

Symbol - specify symbol for each group within diagram

Basic - specify titles, footnotes, labels, and axes options

Device - specify device for graphics output

Return - to opening screen.

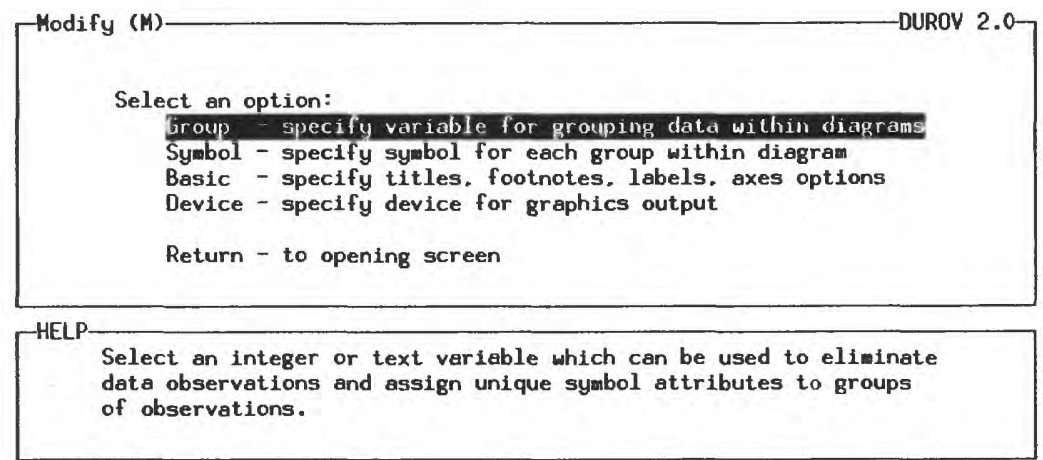

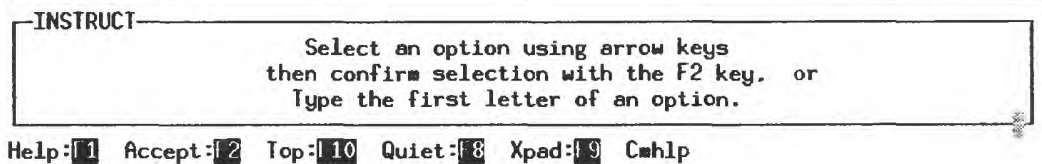




\section{Grouping Variable}

The unique values of the "Grouping Variable" are used to provide partitioning of the data points within the Durov triangles. This would commonly be used to distinguish samples or stations. Each unique value of the "Grouping Variable" is represented by a set of symbol attributes (see "Symbol" option from the "Modify (M)" screen).

From the "Modify (M)" screen, select the "Group (MG)" screen. Use the arrow and Enter keys to select the variable to be used to partition the data. When finished, use Accept (F2) to accept the selection and return control to the "Modify (M)" screen.

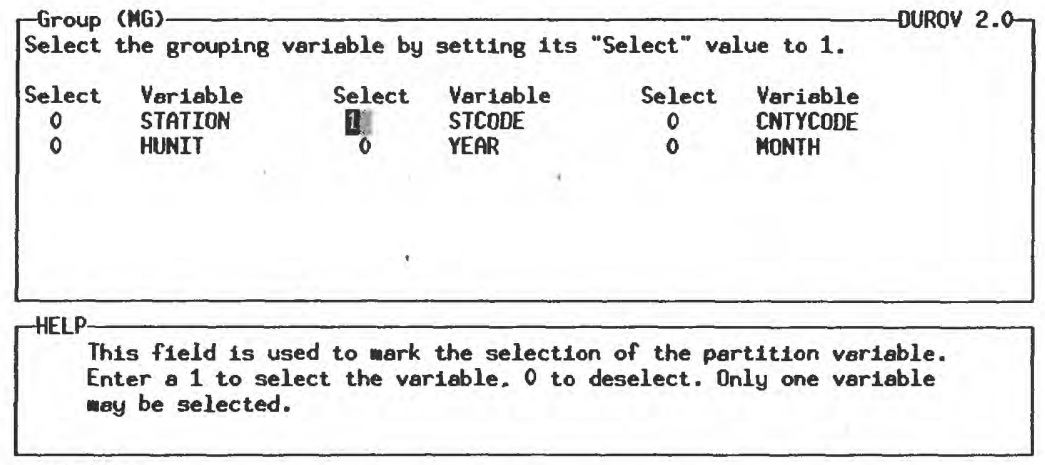

\footnotetext{
INSTRUCT- Enter data in highlighted field(s).
Use carriage return or arrow keys to enter data and wove between fields.
Use "Accept cownand to go to next screen when done entering data.
}

\section{Group Symbol}

The "Symbol" option from the "Modify (M)" screen is used to select the specific values of the grouping variable to be represented in the diagrams. Each value is assigned a symbol type, size, and color. Any combination of type, size, and color can be assigned as long as the combination is unique for each unique value of the filter variable.

From the "Modify (M)" screen, select the "Symbol2 (MS)" screen. The default under "Station number" is "All," which provides for all points in the diagram to be represented by the same symbol with default symbol type, size, and color. An optional label (up to 24 characters) can be specified, as can an alternative symbol type, size, and color.

To assign symbol characteristics to each unique value of the "Station number," use the arrow and Enter keys to select the appropriate items and enter the information required. Once an item is selected, use Help (F1) and Limits (F5) to display the list of allowable values or codes for that item. To page through the list of allowable values, use the Help (F1) again; this allows you to use the Page Down and Page Up keys to scroll through the list. When finished, use (F3) to reactivate the "Data" panel. As many "Station number" values as needed can be specified; the values need not be inclusive, contiguous, or in any particular order. When finished, use Accept (F2) to accept the selections and return control to the "Modify (M)" screen. 


\begin{tabular}{|c|c|c|c|c|}
\hline \multicolumn{5}{|c|}{-Symbol2 (MS) } \\
\hline $\begin{array}{l}\text { Station number } \\
36 \\
42\end{array}$ & $\begin{array}{l}\text { Label } \\
\text { New Jersey } \\
\text { New York } \\
\text { Pennsylvania }\end{array}$ & $\begin{array}{c}\text { Symbol } \\
22 \\
23 \\
24 \\
7 \\
7 \\
7 \\
7\end{array}$ & $\begin{array}{r}\text { Size } \\
.10 \\
.10 \\
0.08 \\
0.08 \\
0.08 \\
0.08 \\
0.08\end{array}$ & $\begin{array}{l}\text { Color } \\
\text { FORE } \\
\text { FORE } \\
\text { FORE } \\
\text { FORE } \\
\text { FORE } \\
\text { FORE } \\
\text { FORE }\end{array}$ \\
\hline
\end{tabular}

Enter a 1-16 character unique station identifier. Enter ALL to specify the symbol type. size and color for all stations. ALL may be used only for the 1st station in the list.

Enter data in highlighted fleld(s).
Use carriage return or errow keys to enter data and wove between fields.
Use 'Accept" command to go to next screen when done entering data.

\section{Basic Plot Parameters}

From the "Modify (M)" screen, select the "Basic (MB)" screen, which lists the following options:

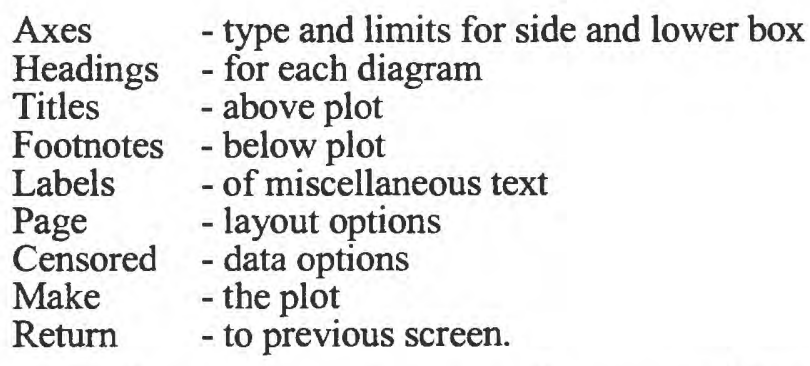

Type the first letter of any option or use the arrow and Enter keys to select the screen for the option required.

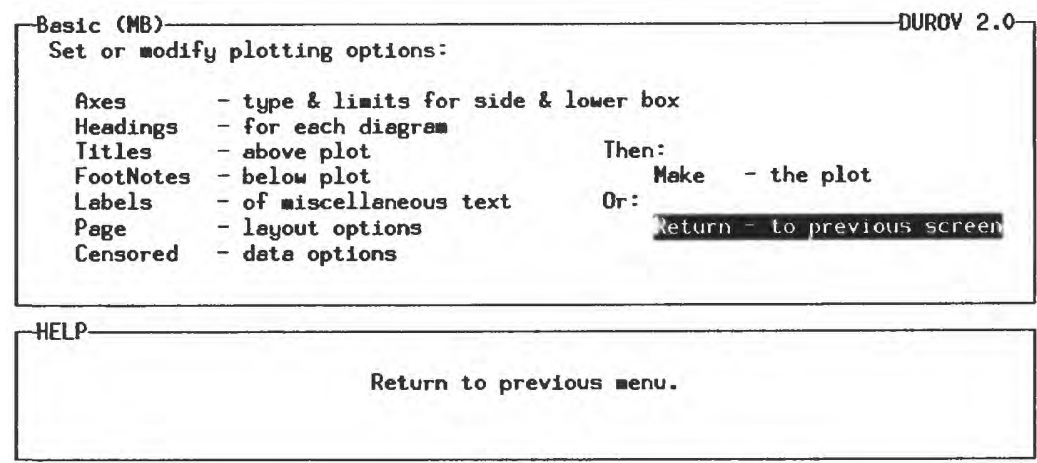

\footnotetext{
INSTRUCT- $\begin{gathered}\text { Select an option using arrow keys } \\ \text { then confirm selection with the F2 key. or } \\ \text { Type the first letter of an option. }\end{gathered}$
Help:11 Accept: Th Top:F10 Quiet:F8 Xpad: 19 Cwhlp
} 


\section{Axes for Side and Lower X-Y Plots}

The Durov plot involves two $\mathrm{x}-\mathrm{y}$ coordinate rectangles onto which the cation/anion pairs from the two trilinear diagrams are projected. The other axis of each rectangle, which can be linear or logarithmic, represents another variable from the data file.

From the "Basic (MB)" screen, select the "Axes (MBA)" screen. Select the "Scaling Method" option to view the "Scaling (MBAS)" screen. Use the arrow keys to select either the "Automatic" or "Manual" option.

If "Automatic" was selected, then use of Accept (F2) or the Enter key brings up the "Automatic (MBASA)" screen. Enter either "Linear" (default) or "Log" for each box selected. Use Accept (F2) to return control to the "Basic (MB)" screen.

If "Manual" was selected, then use of Accept (F2) or the Enter key returns control to the "Axis (MBA)" screen. In turn, select both the "Side Box Limits" and the "Lower Box. Limits" options to bring up a two screen sequence for specifying the axis type, the axis scale lower limit, the axis scale upper limit, and tick-mark interval. The first screen to appear is "Limits1," which queries for the diagram number. This is only applicable when multiple diagrams have been specified by either defining multiple varsets or when the data are being partitioned. The second screen to appear is the "Limits (MBASL)" screen where the actual axis limits are specified.

\section{Axis Type}

Use the arrow keys to select the axis type desired (linear or logarithmic) and then use the space bar to toggle this selection "On" as indicated by the appearance of an "X" in the field to the left of the option name.

\section{Lower Limit}

Use the Enter or arrow keys to select the "Lower Limit" option. Enter a value for the lower limit.

\section{Upper Limit}

Use the Enter or arrow keys to select the "Upper Limit" option. Enter a value for the upper limit.

\section{Tick-Mark Interval}

Use the Enter or arrow keys to select the "Tick-Mark Interval" option. Enter a value for the tick-mark interval.

Use Accept (F2) to return control to the "Axis (MBA)" screen. When finished with the manual axis definition process, use Accept (F2) or the "Return" option to return control to the "Basic (MB)" screen.
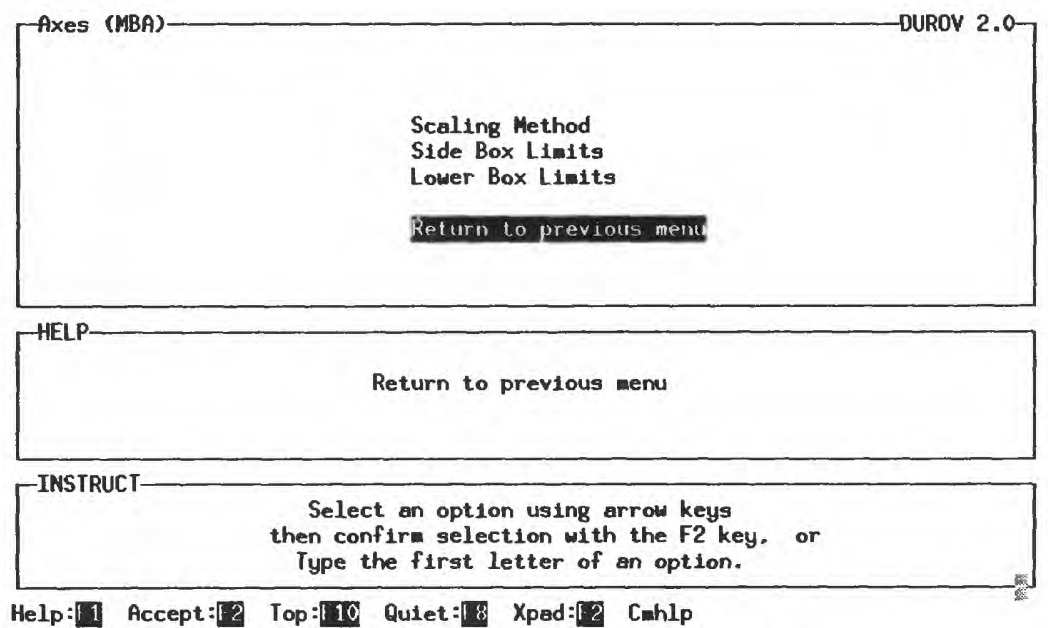

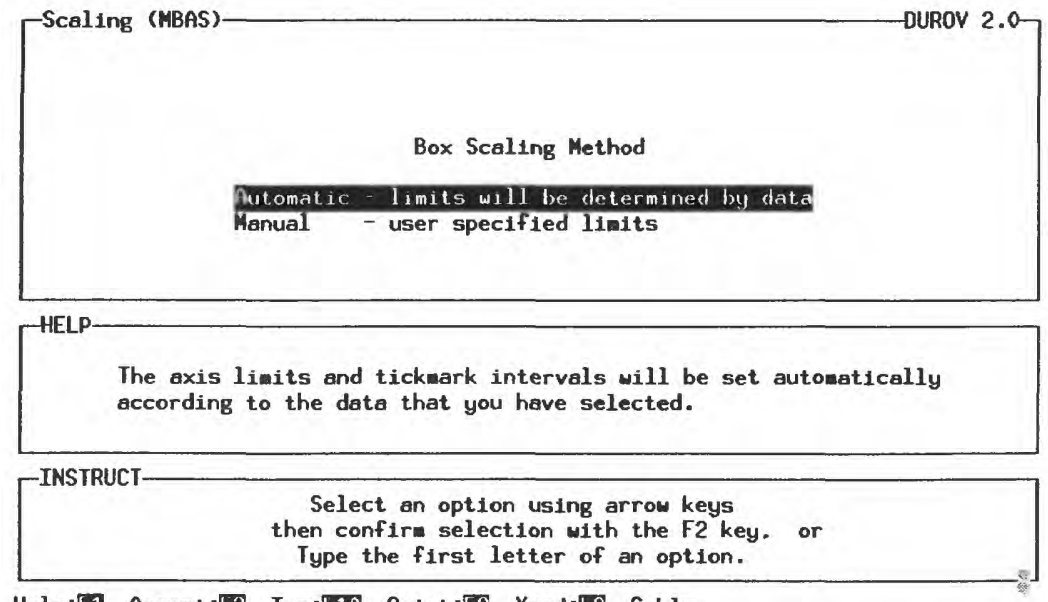

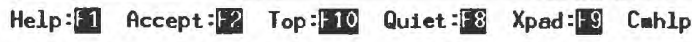

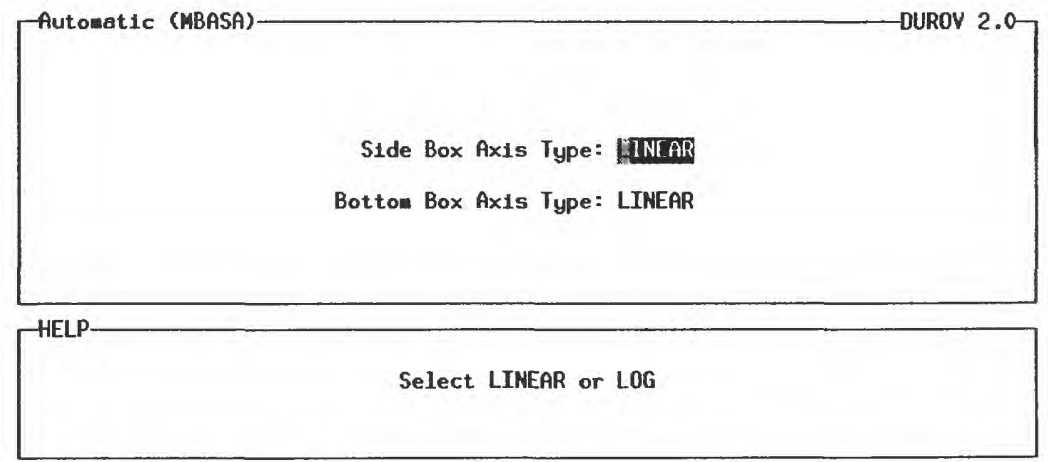

INSTRUCT- Enter data in highlighted field(s).
Use carriage return or arrow keys to enter data and wove between fields.
Use "Accept comand to go to next screen when done entering data.
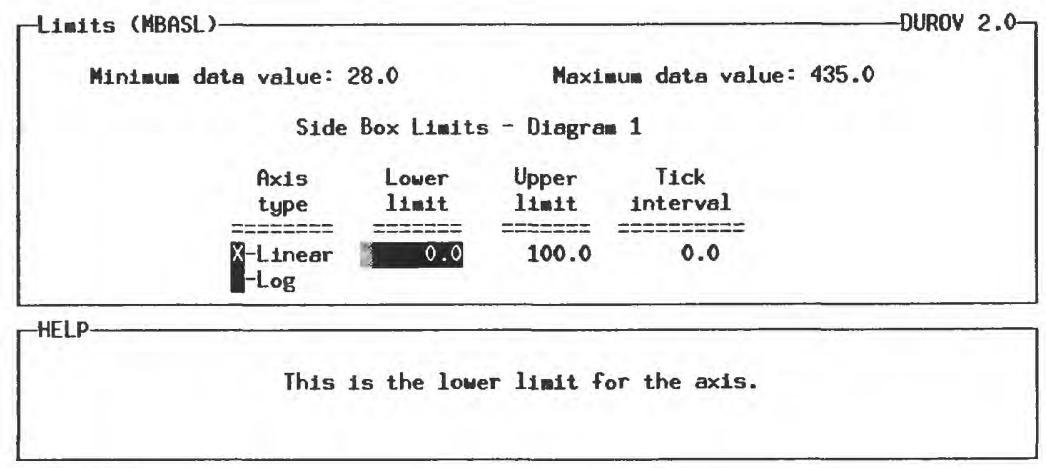

\footnotetext{
TINSTRUCT- Enter data in highlighted field(s).

Use carrlage return or arrow keys to enter data and move between fields. Use "Accept" comand to go to next screen when done entering data.

Help:F1 Accept:F2 Limits:F2 Top:F10 Quiet:F2 Xpad:F9 Cmhlp Dops Dnpg
} 


\section{Headings}

A heading (up to 40 characters) can be specified for each Durov diagram. From the "Basic $(\mathrm{MB})$ " screen, type " $\mathrm{H}$ " (or " $\mathrm{h}$ ") or use the arrow keys to select the "Headings (MBH)" screen. For each diagram to be plotted, enter a heading. When finished, use Accept (F2) to return control to the "Basic (MB)" screen. If the diagrams are based on the partitioning of a single set of variables (see "partition" option in the "Name (N)" screen), then the diagram headings also can be specified in the "Headings (MPTAH)" screen accessed from the "Type" option in the "Partition (NP)" screen. Diagram headings specified when the partitioning option is invoked will revert to default values (the value of the partitioning variable) whenever any option that affects the partitioning of the data is changed. Use the "Heading (MBH)" screen to reset the desired headings when this occurs.

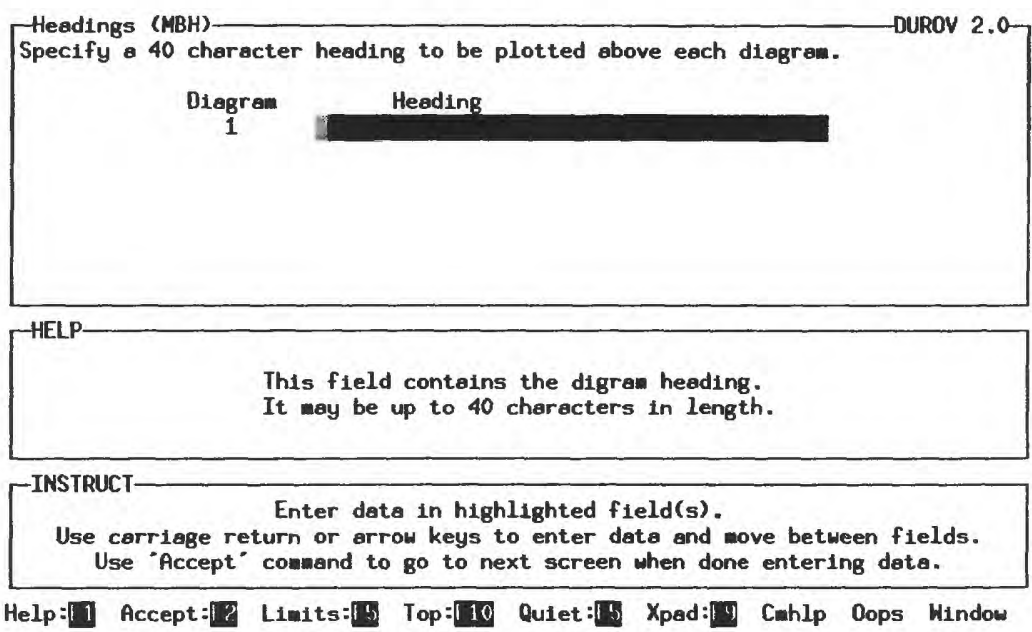

\section{Page Layout Options}

From the "Basic (MB)" screen, select the "Page (MBP)" screen. The "Page (MBP)" screen provides the following functions:

Page Height

Page Width

Font Style

Grid Level

Plot Page Border

Diagrams in Row 1

Diagrams in Row 2

Diagrams in Row 3

Most options in this list are discussed in the section "Page Layout Options" under "Steps to Using QWGRAF Programs."

\section{Grid Level}

Use the Enter and arrow keys to select the "Grid level" option. If the "Help" panel is not already activated, then use Help (F1) to bring up the "Help" panel, which presents the options available for drawing grid lines on the Durov diagram. These options are as follow:

0 : No grid line and tick marks and number at 20-percent intervals.

1: Grid lines, tick marks and numbers at 20 -percent intervals.

2: Grid lines and tick marks and numbers at 10-percent intervals.

Enter the appropriate code number in the "Grid level" field. 

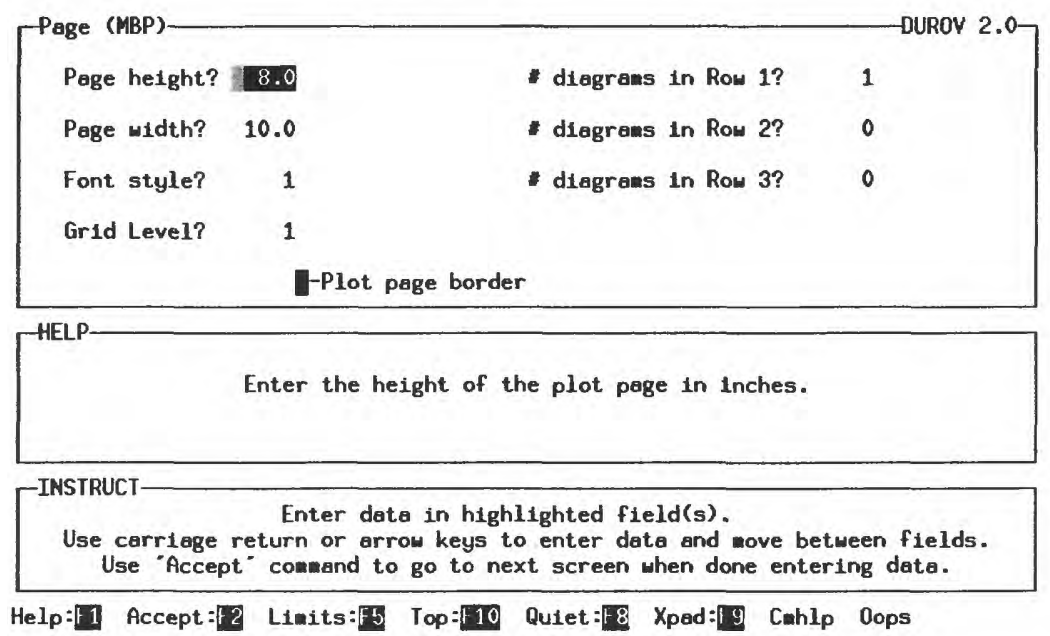

\section{Diagrams in Row 1}

Use the Enter and arrow keys to select the "Diagrams in row 1" option. Enter the number of diagrams to be placed on the first row of the plot page (up to three). Enter zero if you want a default.

\section{Diagrams in Row 2}

Use the Enter and arrow keys to select the "Diagrams in row 2" option. Enter the number of diagrams to be placed on the second row of the plot page (up to three). Enter zero if you want a default.

\section{Diagrams in Row 3}

Use the Enter and arrow keys to select the "Diagrams in row 3" option. Enter the number of diagrams to be placed on the third row of the plot page (up to three). Enter zero if you want a default.

\section{Censored Data Options}

From the "Basic (MB)" screen, select the "Censored (MBC)" screen to view the options for censored data. By default the "durov" program excludes censored data from the analysis. To include censored data, use the arrow or Tab keys to select (highlight) the "Include censored values" option. Use Accept (F2) to return control to the "Basic (MB)" screen.

\section{Make the Plot}

When all arguments for the Durov diagram have been specified and the appropriate device selected, return control to the "Opening Screen," select the "Make the plot" option, and use the Enter key. If a screen output was selected, then a graphics window opens and the Durov diagram is presented. If a file output was selected, then a message screen indicates that the output from the procedure has been saved.

\section{Sample Plots}

The following are examples of Durov plots that can be produced with the QWGRAF "durov" program. These examples are intended to illustrate various options available in the program and do not necessarily conform to publication standards of the Geological Survey. 
Program: DUROV

Plot Specifications: Durov Diagram; No Data Partitioning; Automatic Scaling of Side Boxes

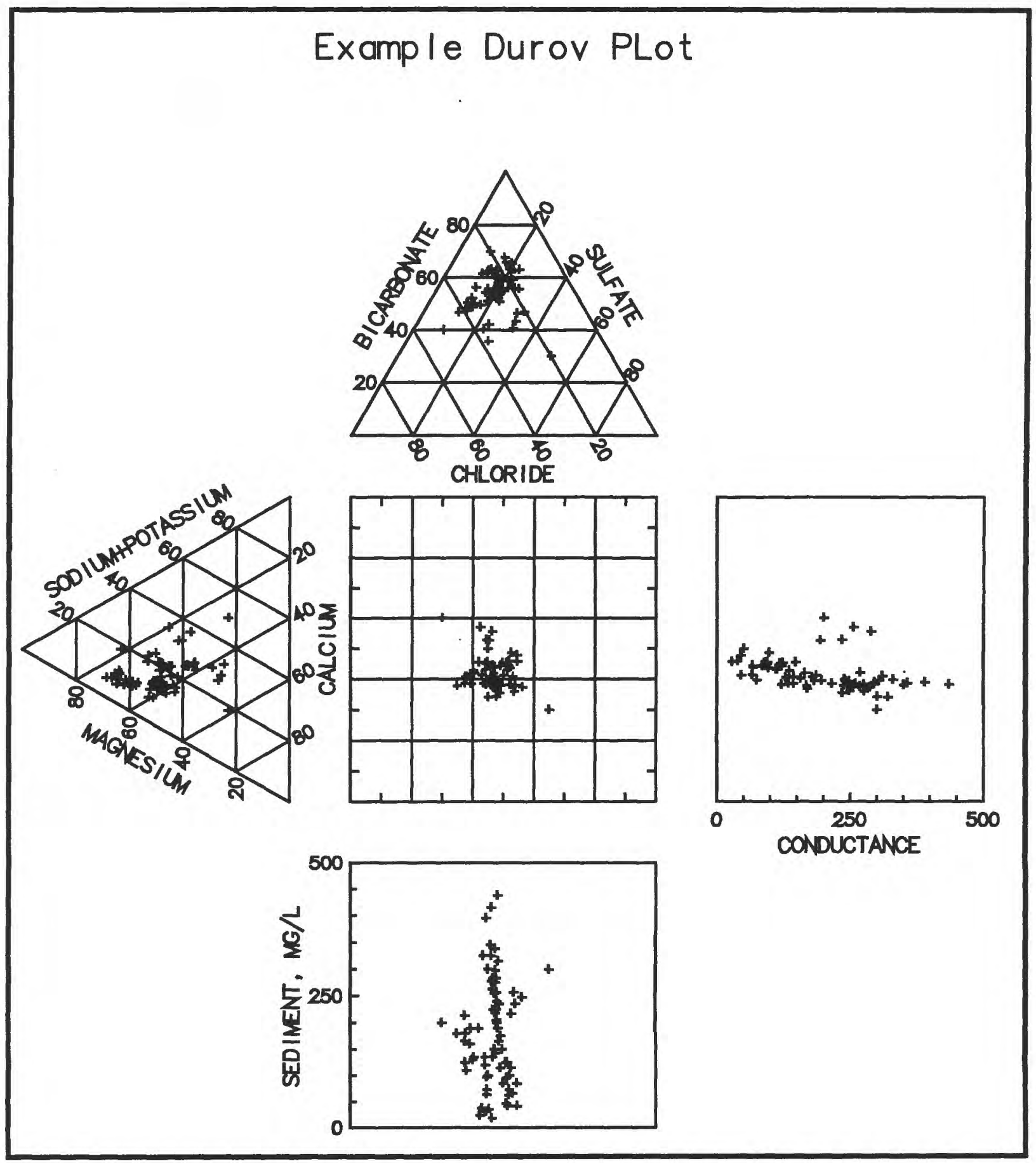




\section{QWGRAF}

Example Plots

DUROV

Program: Durov

Plot Specifications: Durov Plot with Side and Bottom Boxes; Data Partitioning by Station (One Diagram Per Station); Automatic Scaling of Side Boxes; Diagram Labels; 2 Titles

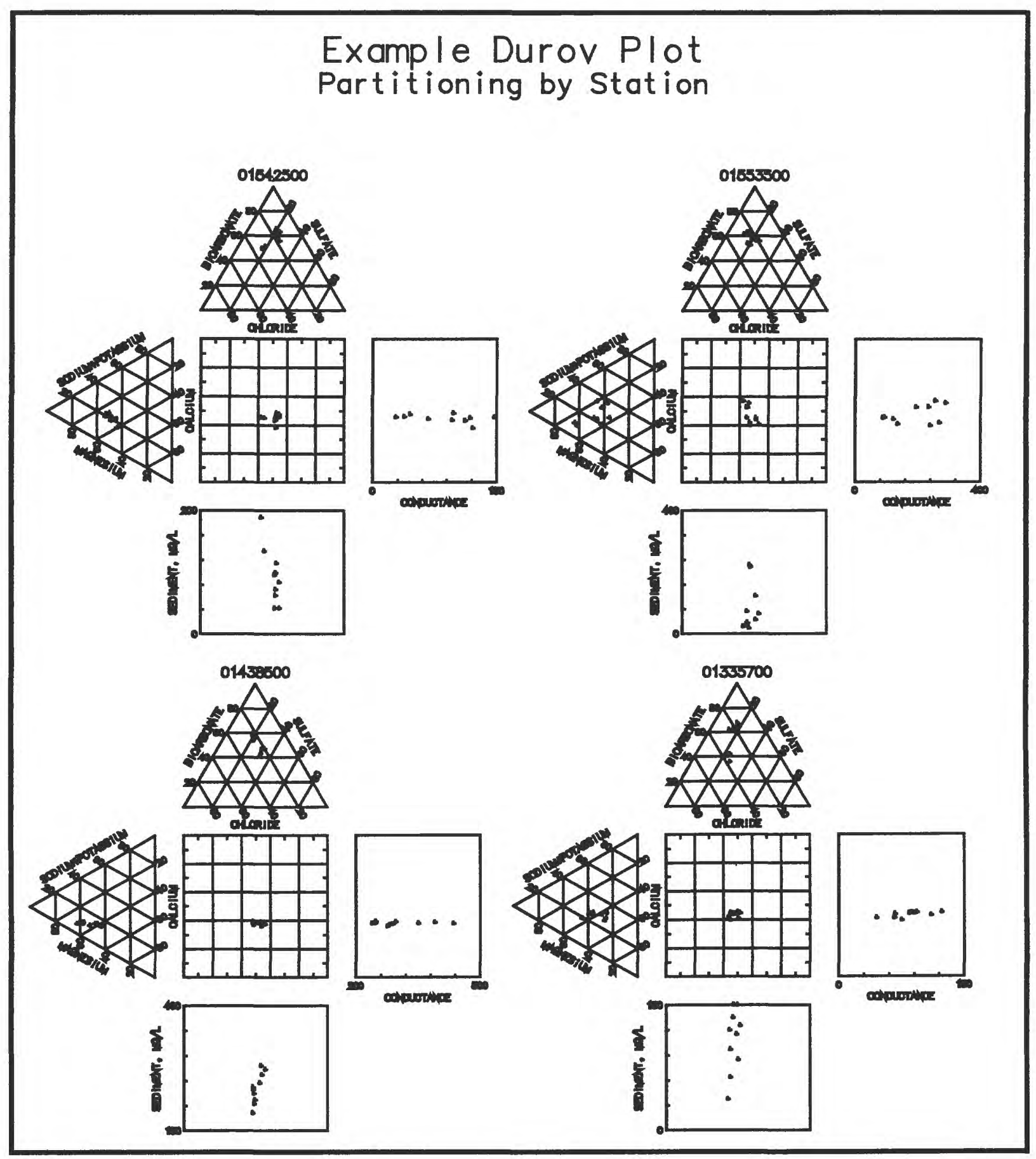




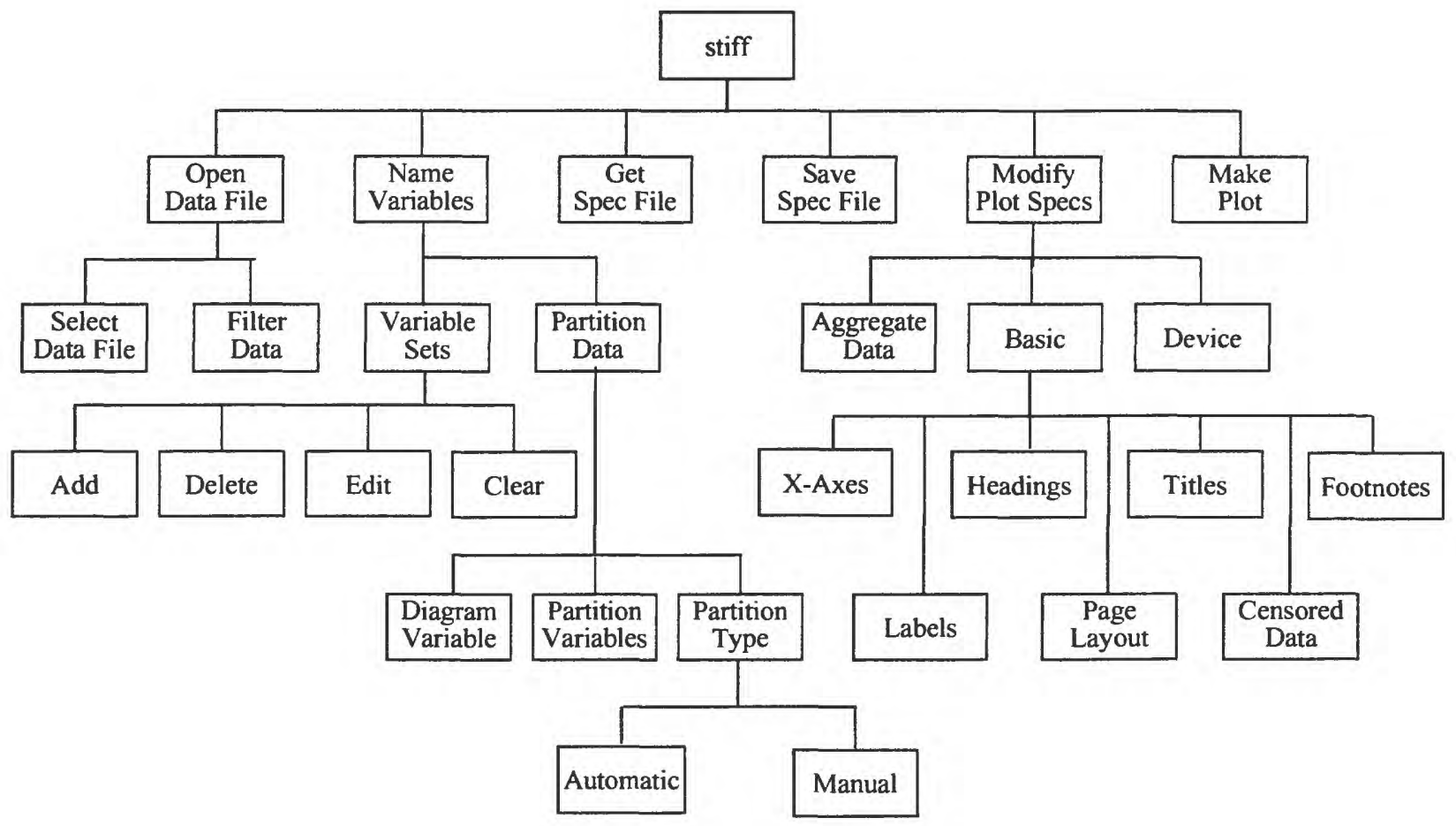

The "stiff" program produces Stiff diagrams, which are a graphical means of comparing the concentrations of anion/cation pairs. Each diagram can display up to five cation/anion pairs. Each node of the diagram is user defined and can represent a single chemical constituent or a composite of two or more constituents. The program provides for plotting up to 15 diagrams on a page - three lines of up to five diagrams per line. With multiple diagrams there is the option of utilizing a different set of variables for each diagram (individual variables can be repeated between sets) or of using one set of variables that has been partitioned into groups of values on the basis of composite values of up to four partition variables. Each group is then represented by a Stiff diagram on the plot page.

Remarked (censored) data values reported as a limit of quantitation or detection limit can be included or excluded from the plot data set. Titles for each diagram and labels for each node of each diagram are user defined. There can be up to three title lines and two footnote lines each definable as to size, font, and centering. Up to five user-defined labels (up to 40 characters on multiple lines) can be positioned on the plot. Any number of plot specification files can be saved and used to facilitate plot specification in other "stiff" sessions.

\section{Background}

A Stiff diagram is a graphical means of comparing the concentrations of major cation/ anion pairs, expressed in milliequivalents. The cation/anion pairs are plotted on horizontal axes (one cation/anion pair per axis) that extend on each side of a vertical axis that represent zero concentration. The points (or nodes) are connected to form an irregular polygon whose shape is a representation of the chemical content of the water body. 


\section{Stiff Diagram Options}

The "stiff" program supports the following Stiff diagram related functions:

Function

Data Aggregation Method Diagram Headings

$\mathrm{X}$-Axis
Screen Sequence

Opening Screen, Modify (M), Aggregate (MA)

Opening Screen, Modify (M), Basic (MB), Headings (MBH)

Opening Screen, Modify (M), Basic (MB), $\mathrm{X}$-Axis (MBX).

\section{Input Data Requirements}

The "stiff" program assumes the data to be displayed in the Stiff diagrams are in the equivalent form. See the discussion of "Data Management" in the "General Features" section for suggestions on how to convert concentration data to equivalents.

\section{Starting the "stiff" Program}

The "stiff" program is invoked by typing "stiff" on the command line. The "Opening Screen" displays the options that serve as gateways to the screens used to set or modify the plot configuration options.

\section{Load a Specifications File}

To use specifications from a previous "stiff" session, load a "Specifications" file (see discussion of "Specifications Files" in the section "Steps to Using QWGRAF Programs"). From the "Opening Screen," select the "Get (G)" screen. A list of specifications files (extension ".stf") and a field for specifying the file name are presented. Use the arrow keys to select the required file and use either Accept (F2) or the Enter key to accept the selection. The "latest.stf" file includes the program specifications used during the most recent execution of the "stiff" program, whether in the current or previous session. There may or may not be other user-defined specifications files available.

The "Specifications" file includes the name of the input data file that was active at the time the specifications file was created. If a new input data file is required, then select the "Open (O)" screen from the "Opening Screen" and select a new input data file.

Program specifications, whether entered via the key board or retrieved from a "Specifications" file, can be changed during the current session. The user may choose to overwrite an existing "Specifications" file or create a new one (see "Save Specifications" option).

\section{Open Input Data File}

From the "Opening Screen," select the "Open (O)" screen. From the "Open (O)" screen, select the "Select (OS)" screen. Type in a file name or select the input data file desired. Use the arrow, Page Down, and Page Up keys to select a data file. Use either Accept (F2) or the Enter key to accept the file selection and return control to the "Open (O)" screen.

\section{Filter Data}

To restrict the data to be used for the Stiff diagram, the "Filter" option from the "Open

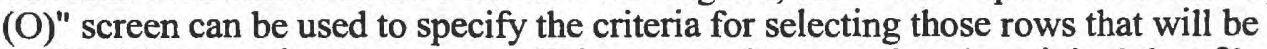
retained for use with the program. This process does not alter the original data file but results in a temporary data file for use with the program. A permanent copy of the filtered 
data file can be obtained by using the "Filter" option in the "datmgr" program and then saving the output in an RDB file.

\section{Name Variables}

From the "Opening Screen," select the "Name (N)" screen, which presents the following options:

Varsets - Select one or more sets of variables

(one for each Stiff diagram desired).

or

Partition - Select a variable set, a partition variable, and interval limits. One diagram for each partition interval.

or

Return - Return to opening screen.

A single Stiff diagram requires at least two cation/anion pairs and can have a maximum of five pairs. A single set of cation/anion pairs produces a single diagram on the plot page (Varsets option). The Varsets option also can be used to define multiple sets of variables (individual variables can be repeated between sets); one Stiff diagram for each set. An alternative approach to multiple Stiff diagrams is to take a single set of cation/anion pairs and partition the values into groups on the basis of composite values of up to four partitioning variables (Partition option). Each group is the basis of a Stiff diagram. For example, produce Stiff diagrams by state and year. The "Varsets" and "Partition" functions are described in more detail under "Name (Select) Variables" in the "Shared Features" section.

\section{Modify Plot Specifications}

After the data file has been opened and the variables of interest named, select the "Modify (M)" screen by typing "M" (or " $m$ ") or use the Tab and arrow keys to select the "Modify compute/plot specifications" option and then use Accept (F2) or the Enter key. The "Modify (M)" screen lists the following options:
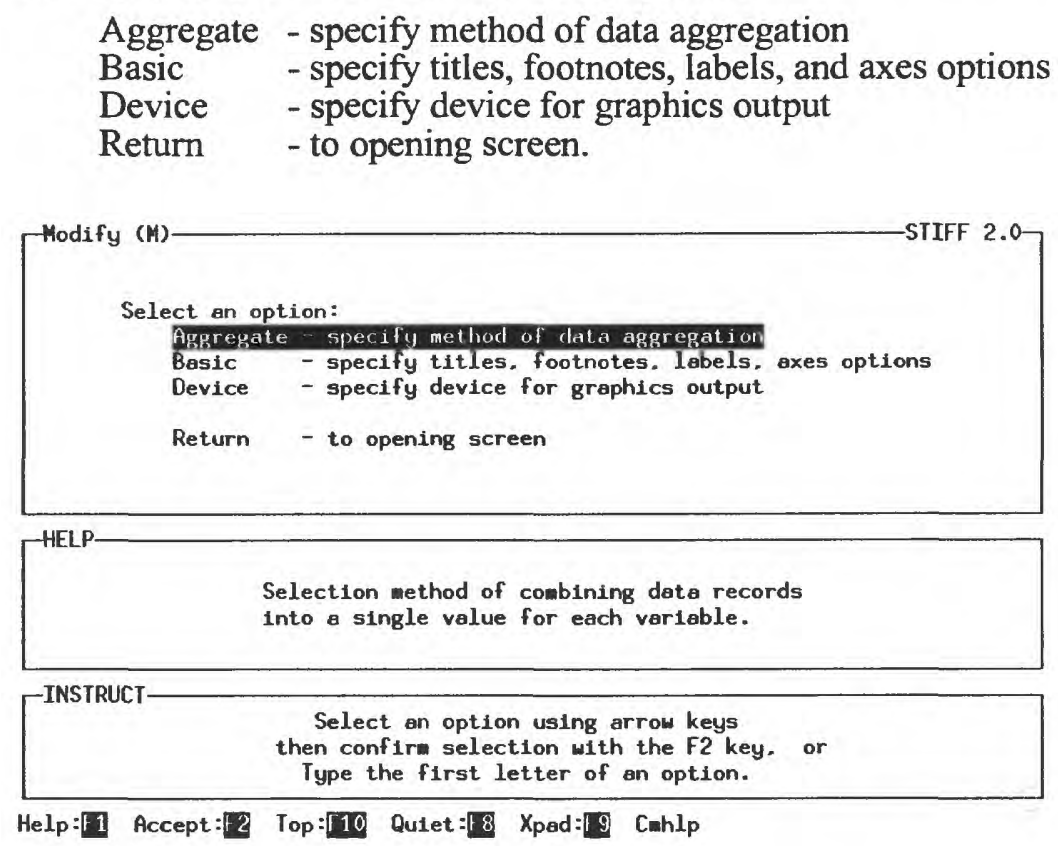


\section{Aggregate Data}

The value used at a node of a Stiff diagram represents a composite value of all the observations in the data file for the constituent (or composite constituent) represented by that node. The "stiff" program provides several methods for aggregating the data to a composite value. These methods are as follow:

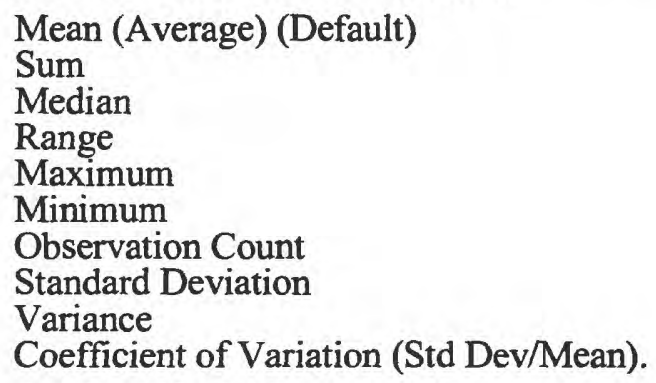

From the "Modify (M)" screen, select the "Aggregation (MA)" screen. Use the arrow keys to select the aggregation method desired and then use the space bar to toggle the selection "On" as indicated by the presence of an "X" in the field to the left of the option description. Use Accept (F2) to accept the selection return control to the "Modify (M)" screen.

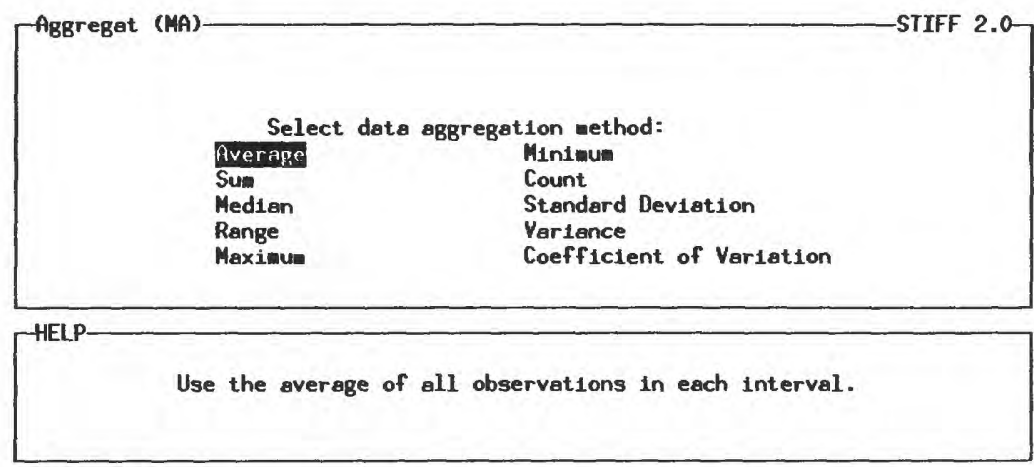

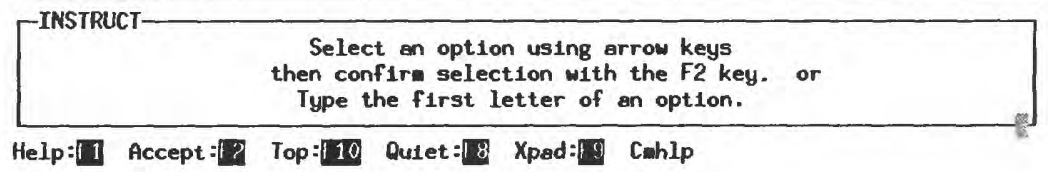

\section{Basic Plot Parameters}

From the "Modify (M)" screen, select the "Basic (MB)" screen, which lists the following options:

$$
\begin{array}{ll}
\text { X-axis } & \text { - limits and label } \\
\text { Headings } & \text { - for each diagram } \\
\text { Titles } & \text { - above plot } \\
\text { Footnotes } & \text { - below plot } \\
\text { Labels } & \text { - of miscellaneous text } \\
\text { Page } & \text { - layout options } \\
\text { Censored } & \text { - data options } \\
\text { Make } & \text { - the plot } \\
\text { Return } & \text { - to previous screen. }
\end{array}
$$

Type the first letter of any option or use the arrow and Enter keys to select the screen for the option required. 

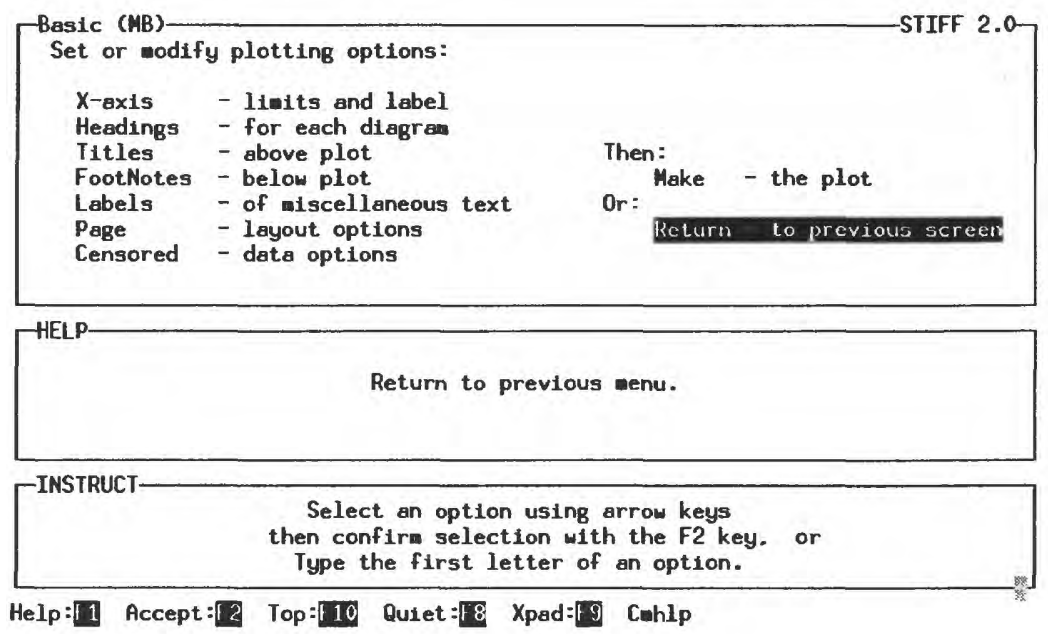

\section{$\underline{\mathrm{X} \text {-Axis }}$}

Each Stiff diagram has an axis at the bottom the diagram, effectively a two-sided axis, the left side of the diagram (normally for, but not necessarily restricted to, anions) and the right side of the diagram (normally for, but not necessarily restricted to, cations). The scale numbering extends from " 0 " at the midpoint of the scale to appropriate limiting values at each end of the scale. The scaling for this axis can be done by the system or its limits can be specified by the user. An axis label also can be specified.

From the "Basic (MB)" screen, select the "X-axis (MBX)" screen. To specify the scaling method, select the "Scaling (MBXS)" screen. Select the scaling option desired and use the space bar to toggle the selection "On" (an "X" appears to the left of the selected option). To specify a label for the axis, use the arrow keys to select the "Axis label" option and enter a label (up to 20 characters). Use Accept (F2) to accept the selections and return control to the "X-axis (MBX)" screen. If "Manual" scaling was selected in the "Scaling (MBXS)" screen, then select the "Axis (MBXA)" screen to set the axis limits for each diagram. Use the "Return" option to return control to the "Basic (MB)" screen.

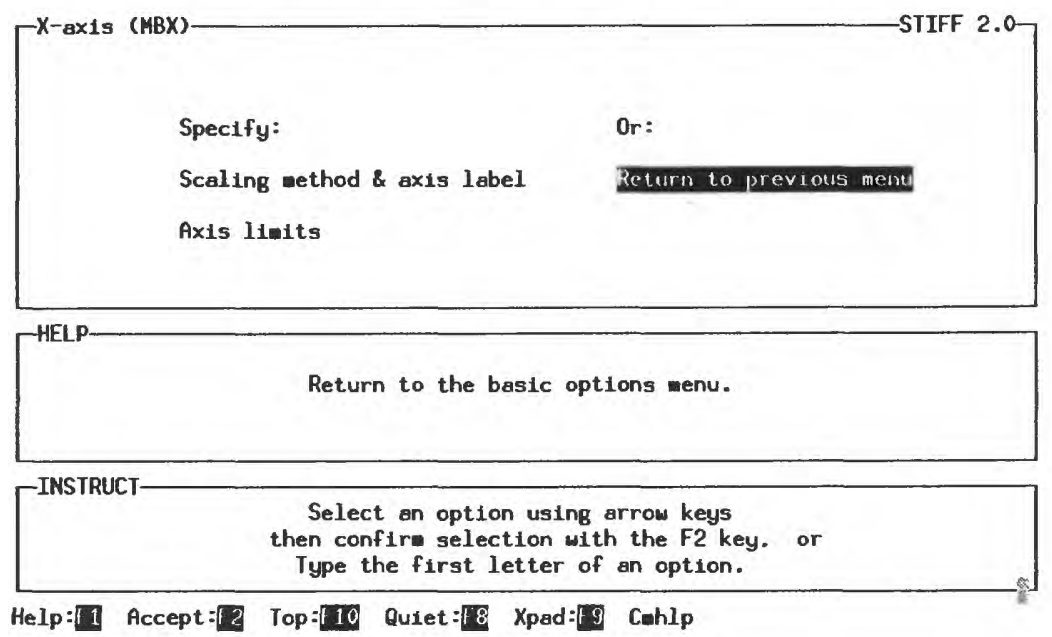



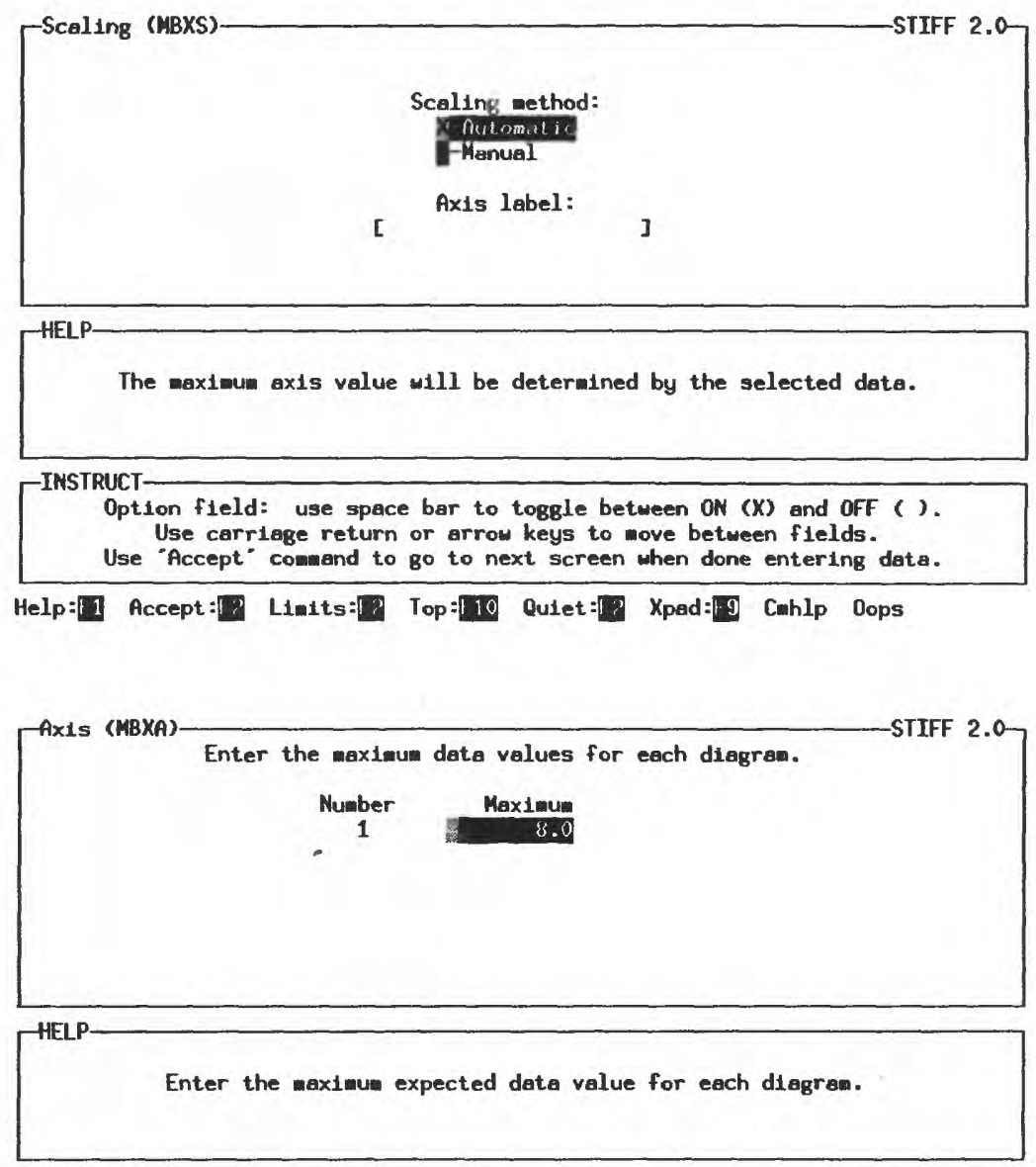

\footnotetext{
TINSTRUCT- Enter data in highlighted field(s).

Use carrlage return or arrow keys to enter data and wove between fields. Use "Accept" command to go to next screen when done entering data.

Help: 1 Accept: Limits: Top : 10 Quiet:F8 Xpad:0 Cahlp Dops Window
}

\section{Headings}

A heading can be specified for each Stiff diagram. From the "Basic (MB)" screen, type "H" (or "h") or use the arrow keys to select the "Headings (MBH)" screen. For each diagram to be plotted, enter a heading (up to 40 characters). When finished, use Accept (F2) to return control to the "Basic (MB)" screen. 


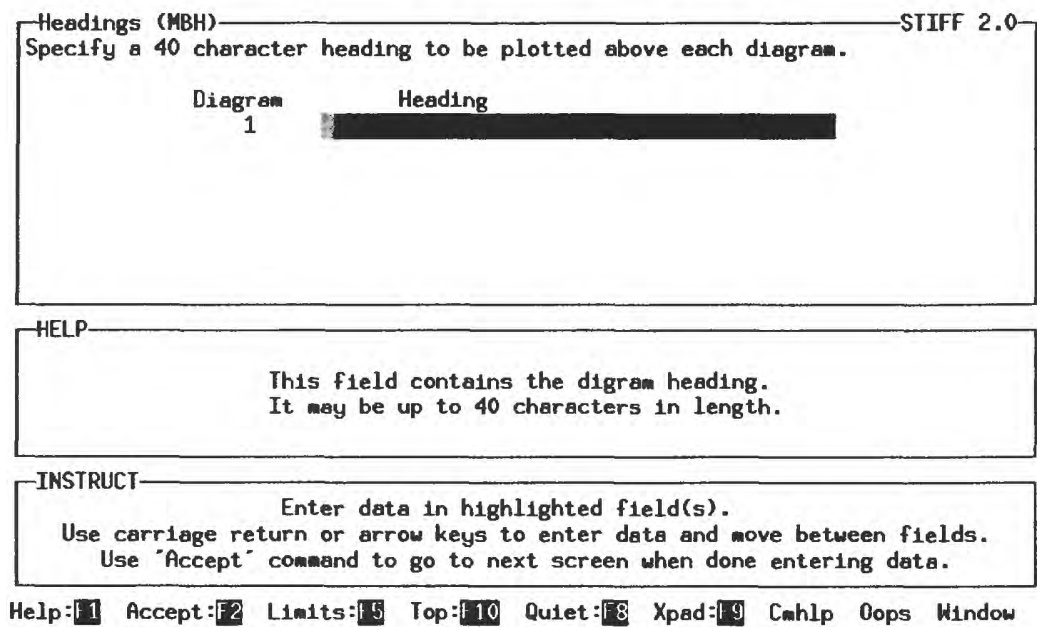

\section{Page Layout Options}

From the "Basic (MB)" screen, select the "Page (MBP)" screen. The "Page (MBP)" screen provides the following functions:

\section{Page Height \\ Page Width \\ Font Style \\ Plot Page Border \\ Diagrams in Row 1 \\ Diagrams in Row 2 \\ Diagrams in Row 3.}

Most options in this list are discussed in the section "Page Layout Options" under "Steps to Using QWGRAF Programs."

\begin{tabular}{|lrlll} 
Page (MBP)- & & & \\
Page height? & 8.0 & \#IIfF 2.0 & \\
Page width? & 10.0 & diagrams in Row 1? & 1 \\
Font style? & 1 & diagrams in Row 2? & 0 \\
-Plot page border & Syagrams in Rou 3? & 0 \\
\end{tabular}

\footnotetext{
Enter the height of the plot page in inches.
INSTRUCT- Enter data in highlighted field(s).
Use carriage return or arrow keys to enter date and move between fields. Use "Accept" command to go to next screen when done entering data.

Help: 1 Accept:F2 Limits: Top : 10 Quiet: 2 Xpad: T Cahlp Oops
} 


\section{Diagrams in Row 1}

Use the Enter and arrow keys to select the "Diagrams in row 1" option. Enter the number of diagrams to be placed on the first row of the plot page (up to five). Enter a value of zero $(0)$ for a default organization.

\section{Diagrams in Row 2}

Use the Enter and arrow keys to select the "Diagrams in row 2" option. Enter the number of diagrams to be placed on the second row of the plot page (up to five). Enter a value of zero $(0)$ for a default organization.

\section{Diagrams in Row 3}

Use the Enter and arrow keys to select the "Diagrams in row 3" option. Enter the number of diagrams to be placed on the third row of the plot page (up to five). Enter a value of zero $(0)$ for a default organization.

\section{Plot Page Border}

To draw a page border around the plot, use the Enter and arrow keys to select this option and then use the space bar to toggle the option "On" as indicated by the appearance of an "X" in the option field.

\section{Censored Data Options}

From the "Basic (MB)" screen, select the "Censored (MBC)" screen to view the options for censored data. By default the "stiff" program excludes censored data from the analysis. To include censored data, use the arrow or Tab keys to select (highlight) the "Include censored values" option. Use Accept (F2) to return control to the "Basic (MB)" screen.

\section{Make the Plot}

When all arguments for the Stiff diagram have been specified and the appropriate device selected, return control to the "Opening Screen," select the "Make the plot" option, and use the Enter key. If a screen output was selected, then a graphics window opens and the Stiff diagram is presented. If a file output was selected, then a message screen indicates that the output from the procedure has been saved.

\section{Sample Plots}

The following are examples of the types of plots that can be created with the QWGRAF "stiff" program. These examples are intended to illustrate various options available in the program and do not necessarily conform to publication standards of the Geological Survey. 


\section{QWGRAF}

Example Plots

STIFF

Program: STIFF

Plot Specifications: Single Variable Set (No Data Partitioning); Data Aggregation by Mean; Single Title; User-Defined Node Labels; Automatic X-Axis Scaling; X-Axis Label

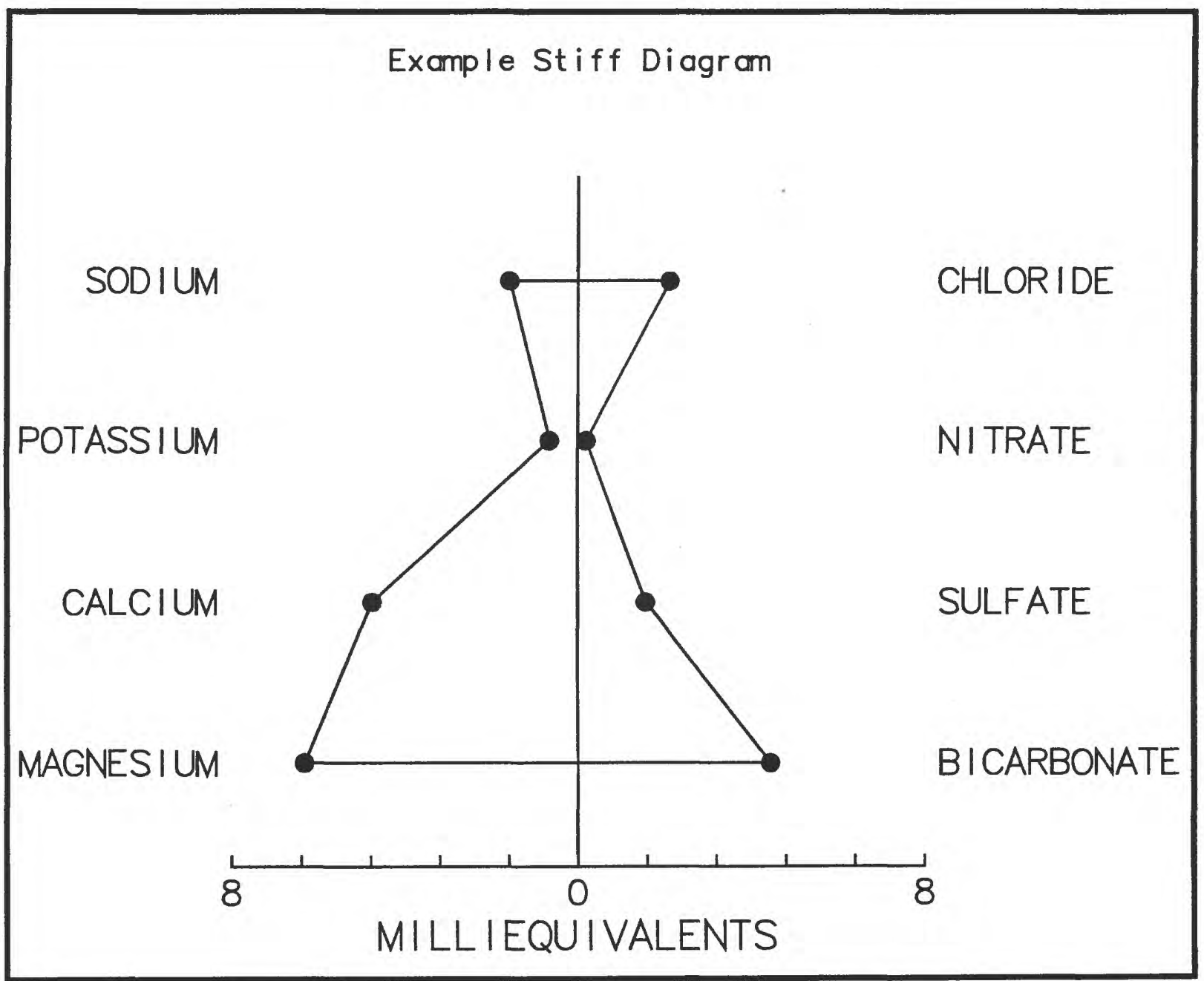




\section{QWGRAF}

\section{Example Plots}

STIFF

Program: STIFF

Plot Specifications: Data Partitioning by State; Data Aggregation by Mean; Two Titles; User-Defined Diagram Headings; Automatic X-Axis Scaling; X-Axis Label

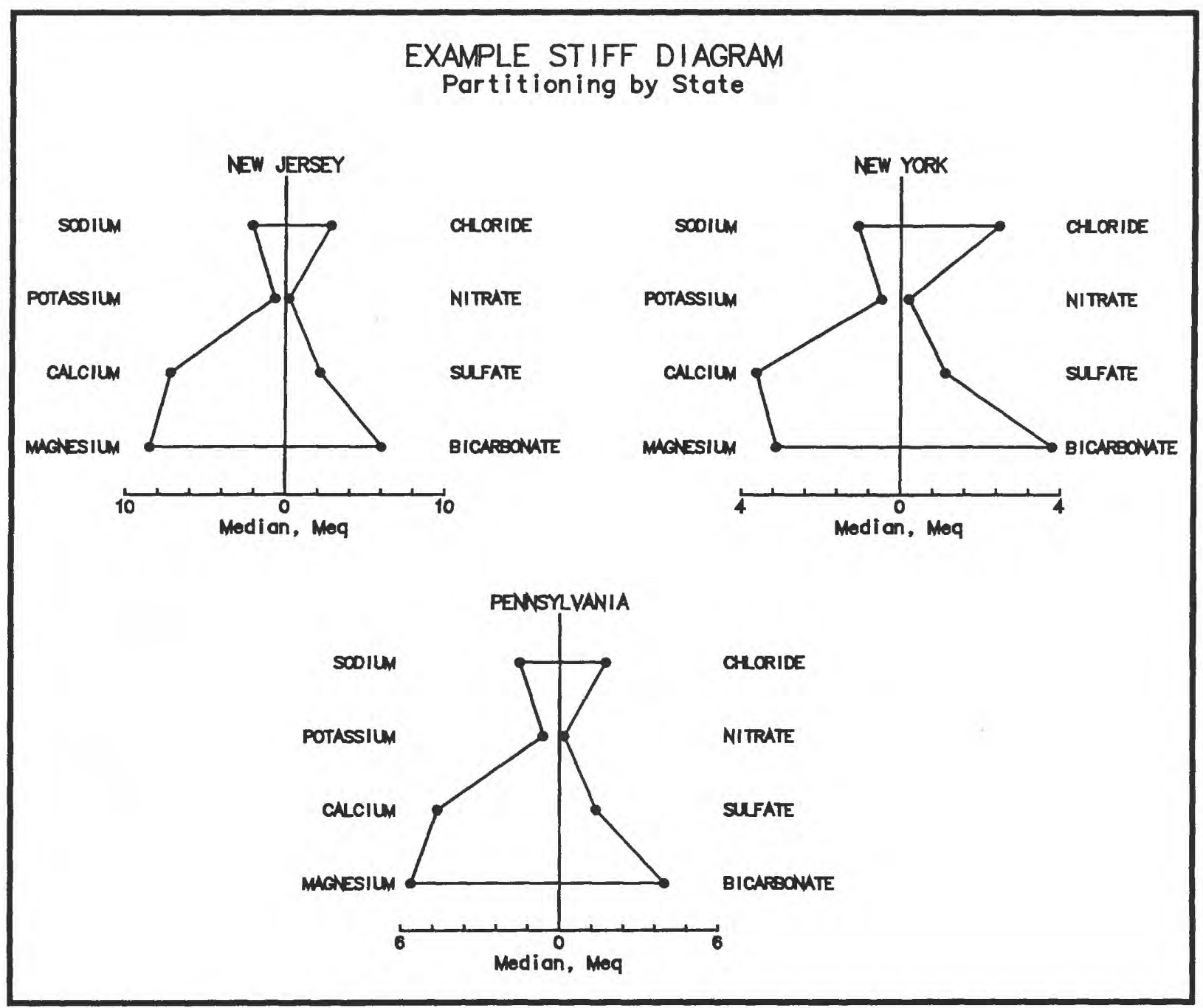




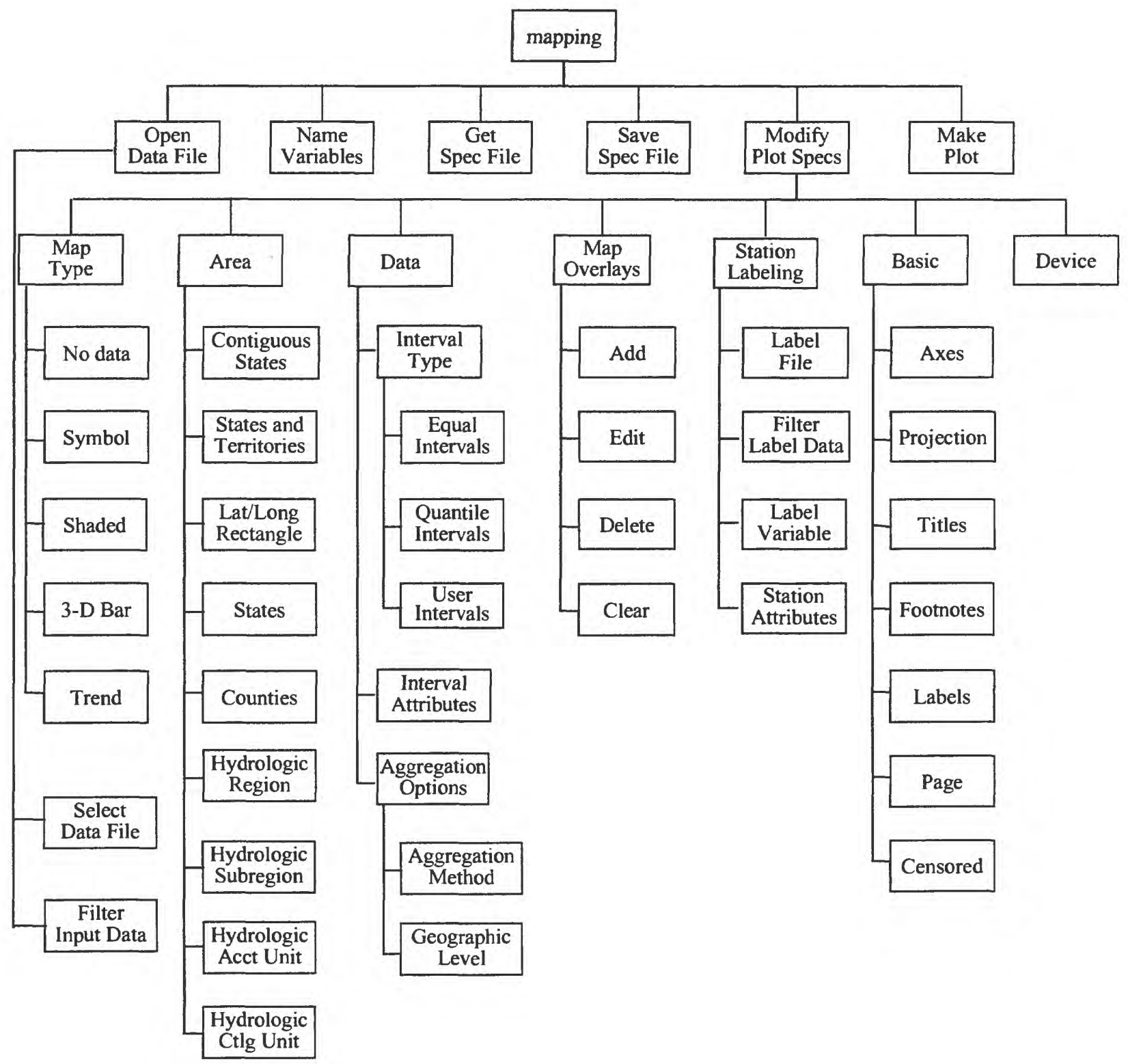

\section{MAPPING (mapping)}

The "mapping" program produces boundary outline maps of the United States and selected territories, States, counties, hydrologic regions, subregions, accounting units, and cataloging units. It also produces user-defined rectangles based on user-supplied latitude/longitude coordinates. Several common map projections are available. The primary boundary can be supplemented with multiple overlays, which include the EPA River Reach file. The "mapping" program has a facility for cross-referencing State and county names with their respective FIPS codes, as well as hydrologic unit codes and names. Each overlay can be defined in terms of line type, thickness, and color. Overlays can be blanked 
or not blanked at the primary boundary. Data can be quantitatively represented on the maps as symbols or bar charts at point locations or by bar charts or shading patterns within areas. The program provides for labeling site locations that utilize values of a variable from either the input data file or a separate variable label file. Label attributes (size, color, font, and angle) can be declared globally or obtained as site-specific values from either the input data file or the variable label file. Remarked (censored) data values reported as a limit of quantitation or detection limit can be included or excluded from the plot data set. Up to five user-defined labels (up to 40 characters on multiple lines) can be positioned anywhere on the plot. There can be up to three title lines and two footnotes, each definable as to size, font, and centering. Any number of plot specification files can be saved and used to facilitate plot specification in other "mapping" sessions.

\section{Mapping Options}

The "mapping" program provides options for the following mapping related functions:

Function

Map Type

Primary Boundary

Overlay Boundaries

Data Display

Station Labeling

Axes/Grids

Map Projection

\section{Screen Sequence}

Opening Screen, Modify (M), Map (MM)

Opening Screen, Modify (M), Area (MA)

Opening Screen, Modify (M), Overlay (MO)

Opening Screen, Modify (M), Data (MD)

Opening Screen, Modify (M), Station (MS)

Opening Screen, Modify (M), Basic (MB),

Axes (MBA)

Opening Screen, Modify (M), Basic (MB), Projection (MBP).

\section{Starting the "mapping" Program}

The "mapping" program is invoked by typing "mapping" on the command line. The "Opening Screen" displays the options that serve as gateways to the screens used to set or modify the plot configuration options.

\section{Input Data Requirements}

The "mapping" program requires an input data file if data are to be mapped or if station locations are to be plotted. An additional file can be accessed to provide labeling information (label variable, font, color, size, and presentation angle) for site locations when such information is not available in the data file. A single variable is specified for point symbol and trend maps and up to four variables are selected for "Area Bar" and "Point Bar" maps. Quantitative mapping of data to point locations or plotting station locations requires the data set to have variables for decimal latitude (DECLAT) and decimal longitude (DECLON). Variables for latitude and longitude in degrees, minutes, and seconds can be converted to DECLAT and DECLON by using the "Create" function in the "datmgr" program. If the input data file is from the NWIS QWDATA system in the FLATOUT format and is accompanied by the "PARNAMES" format definition file, then the LATLG variable is automatically converted to the DECLAT and DECLON variables when read by a QWGRAF program (remember to append the QWFLATOUT files with the ".qwd" and ".qwv" file-name extensions, respectively). The input data file also should contain variables for State code (STCODE), county code (CNTYCODE), and hydrologic unit code (HUNIT) if individual or groups of States, counties, or hydrologic units are to be used as either primary boundaries or overlays. These variables also are used as the basis for aggregating data to these levels for use in "Area Shade" maps. They also can be 
used as partitioning variables for computing data intervals. NOTE: These variable names must be in upper case.

\section{Load a Specifications File}

To use specifications from a previous "mapping" session, load a "Specifications" file. From the "Opening Screen," select the "Get (G)" screen. A list of specifications files (extension ".mpp") and a field for specifying the file name are presented. Use the arrow keys to select the required file and use either Accept (F2) or the Enter key to accept the selection. The "latest.mpp" file contains the program specifications that were used during the most recent execution of the "mapping" program, whether in the current or previous session. There may or may not be other user-defined specifications files available.

The "Specifications" file includes the name of the input data file (if one was used) active at the time the file was created. If a new input data file is required, then select the "Open (O)" screen from the "Opening Screen" and select a new input data file.

The "Make the Plot" option can be selected immediately or any of the newly loaded specifications can be changed. Any altered specifications set can be saved in a new file or an old file can be overwritten.

\section{Open Input Data File}

The default map type upon opening the "mapping" program is a "No Data" map. This map type is used for displaying polygon maps of the States, counties, hydrologic units, and user-defined polygons with various overlays. With this option, no input data file is required. For other map types ("Point Symbol," "Area Shade," "Three-Dimensional (3-D) Bar", and "Trend"), a data file that contains the data to be mapped must be specified. An additional file may be opened from the "Open (MSO)" screen for use with the "Station labeling" option (see "Station" option in "Modify (M)" screen). This option allows a station location to be labeled by using the value of a "label variable" and the selection of four other variables to define the font, color, size, and angle of the label. This option can be used with a "No Data" map, a "Point Symbol" map, or a "Trend" map. For example, in a "Point Symbol" map, the interval data range might be indicated by the size and color of the symbol while the exact value within the interval could be represented in the label.

From the "Opening Screen," select the "Open (O)" screen. From the "Open (O)" screen, select the "Select (OS)" screen. Type in a file name or select the input data file desired. Use the arrow, Page Down, and Page Up keys to select a data file. Use either Accept (F2) or the Enter key to accept the file selection and return control to the "Open $(\mathrm{O})$ " screen.

\section{Filter Data}

To restrict the data to be used for the map, the "Filter" option from the "Open (O)" screen (filter a data file) or the "Open (MSO)" screen (filter a label file) can specify the criteria for selecting those rows that will be retained for use with the program.

\section{Name Variables}

From the "Opening Screen," select the "Name (N)" screen. If the map type is "No Data," which is the default when the mapping program is started, then a message is presented indicating that the "No Data" map has been selected. If any other type of map is to be plotted, then return control to the "Opening Screen" and select the "Modify (M)" screen. From the "Modify (M)" screen, select the "Map (MM)" screen to select the appropriate map type. A map type other than "No Data" must be selected before a variable to map is selected. 
If the map to be plotted is a "Point Symbol" map, "Area Shade" map, or "Trend" map, then a single variable from the input data file must be specified. For a "3-D Bar" map, up to four variables may be selected; each variable is represented as one element in a stacked three-dimensional bar. The bars can be plotted at either specific latitude/longitude locations ("Point Bar") or at the centroids of the selected polygons ("Area Bar").

Use the Enter and arrow keys to select the variables to be mapped. A variable can be deselected by entering a "0" (zero) in the "Select" field. When finished, use the Accept (F2) key to accept the selection(s) and return control to the "Opening Screen."

\section{Modify Plot Specifications}

After the data file has been opened and the variables of interest named, select the "Modify (M)" screen by typing "M" (or "m") or use the arrow keys to select the "Modify compute/ plot specifications" option followed by using the Enter key. The "Modify (M)" screen lists the following options:

$\begin{array}{ll}\text { Map } & \text { - select the type of map } \\ \text { Area } & \text { - select the area to be displayed } \\ \text { Data } & \text { - select data display options } \\ \text { Overlay } & \text { - select polygon or river overlays } \\ \text { Station } & \text { - select station labeling attributes } \\ \text { Basic } & \text { - specify title, footnotes, labels, and axes options } \\ \text { Device } & \text { - specify device for graphics output. }\end{array}$

Type the first letter(s) (not case sensitive) of the selected option or use the arrow keys to select the option and use either Accept (F2) or the Enter key to accept the selection.

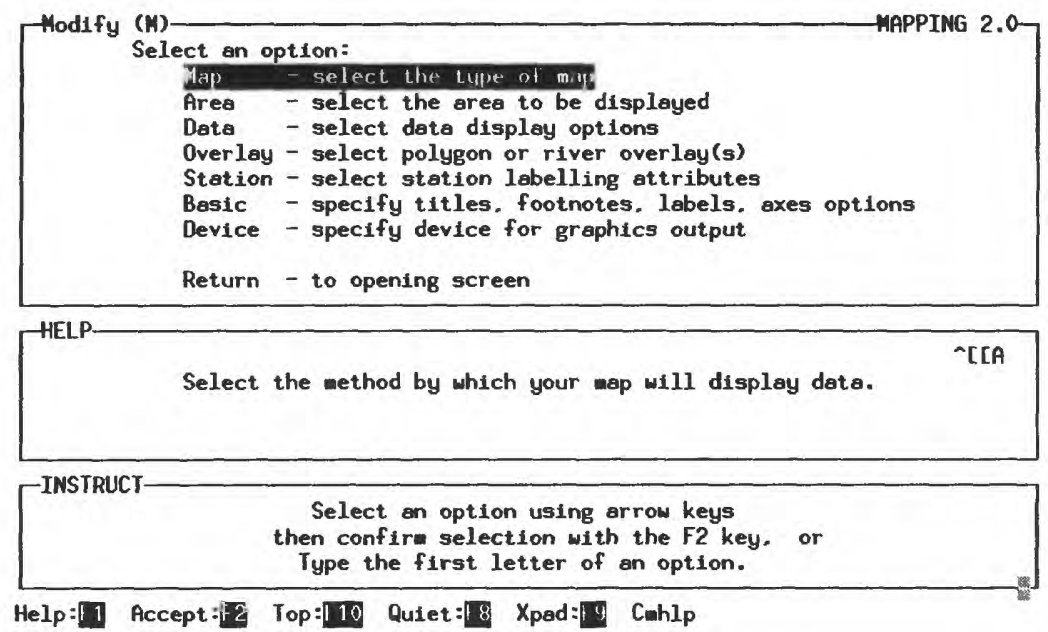

\section{Map Type}

The "mapping" program supports the following map types:

No Data

Point Symbol

Area Shade

3-D Bar (point or area)

Trend (point). 
From the "Map (MM)" screen, use the arrow keys to select the map type desired and use the space bar to toggle this selection "On" as indicated by the appearance of an " $\mathrm{X}$ " in the field to the left of the option name.

When finished, use Accept (F2) to accept the selection and return control to the "Modify (M)" screen.
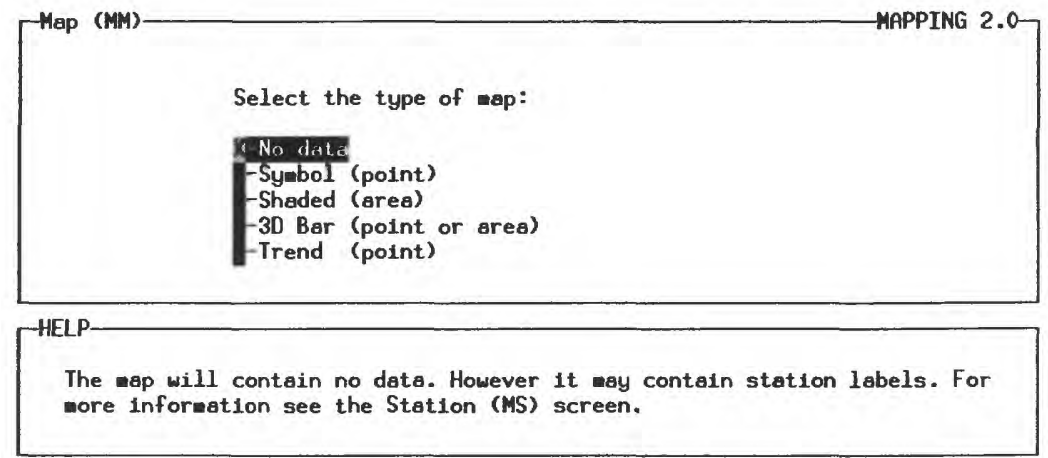

INSTRUCT-
Option field: use space bar to toggle between ON $(X)$ and OFF ().
Use carriage return or arrow keys to wove between fields.
Use "Accept" comand to go to next screen when done entering data.

\section{No Data Maps}

"No Data" maps are plots of primary polygons (USA, States, counties, hydrologic units, or user-defined rectangles) with up to eight optional overlays (see "Overlay" function).

\section{Point Symbol Maps}

"Point Symbol" maps present an aggregated (composite) value of a single variable at specific latitude/longitude locations. The input data file must have the variables DECLAT (decimal latitude) and DECLON (decimal longitude). The data are represented on the map in the form of symbols; the type, size, and color of the symbol represent the range that contains the value of a summary statistic computed for that site (see "Select data display" option for a discussion of the methods available for computing the aggregated values, the number of intervals, and the range of the aggregated values within each interval).

\section{Area Shade Maps}

"Area Shade" maps present color-shaded polygons; each color represents a range of values for an aggregated variable composited for locations within the polygon. For example, the color red might indicate that the mean concentration of nitrate values in a county falls within the interval 5 to $9.9 \mathrm{mg} / \mathrm{L}$, and the color blue would represent the interval 10.0 to $14.9 \mathrm{mg} / \mathrm{L}$. For shading purposes the geographic level to which the data are aggregated and presented may be independent of any primary boundary or overlays. Thus, the color shadings may form their own outlines.

\section{Three-Dimensional Bar Maps}

"Three-Dimensional (3-D) Bar" maps present stacked bars that represent up to four variables either at point locations or at the centroids of polygons. The height and color of each bar in the stack represents the range that contains the value of the computed statistic for a given site or polygon. The map can be adjusted relative to elevation and azimuth angle. 
The height of the bars can be scaled by the program or the user can define the maximum data value to be used for each variable included in the stack.

\section{Trend Maps}

"Trend" maps are similar to "Point Symbol" maps except that the data are presented as either a triangle or an arrow whose size and direction (up or down) are used to indicate the trends magnitude and direction (positive or negative), respectively. The variable used for this map type should contain appropriate trend data. The mapping program cannot compute trend statistics.

\section{Primary Map Display Areas}

The following geographic boundaries may be selected as the primary boundary for a map:

Contiguous 48 States

Contiguous 48 States plus Alaska, Hawaii, and Puerto Rico

Latitude/Longitude Rectangle

List of States

List of Counties

Hydrologic Unit Regions

Hydrologic Unit Subregions

Hydrologic Unit Accounting Units

Hydrologic Unit Cataloging Units.

From the "Modify (M)" screen, select the "Area (MA)" screen. Type the first letter(s) of the option or use the arrow keys to select the option and use the Enter key.

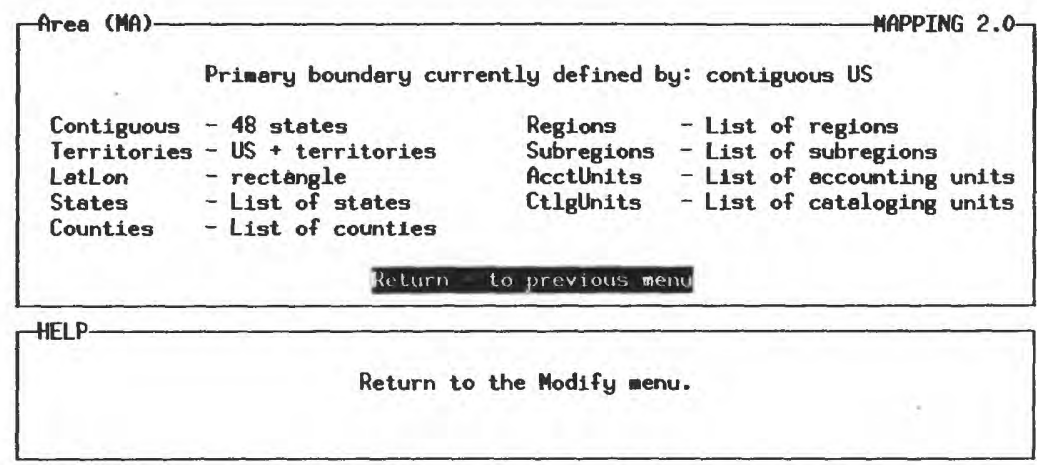

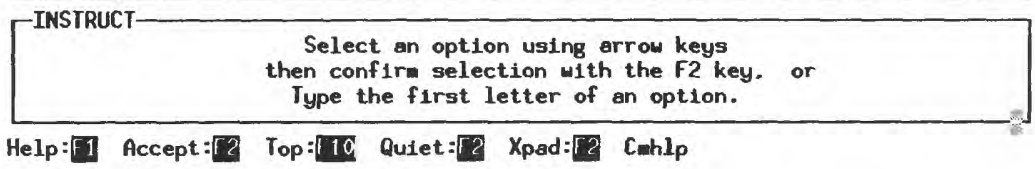

\section{Contiguous 48 States or Contiguous 48 States Plus Alaska. Hawaii, and Puerto Rico}

Selection of the "Contiguous 48 States" or the "Contiguous 48 States plus Alaska, Hawaii, and Puerto Rico" option produces an outline of the 48 States or of the 48 States plus Alaska, Hawaii, and Puerto Rico (in the lower left corner of the map), respectively. Type the first letter(s) of the "Contiguous" (or the "US + Territories") option to select and accept that selection and return control to the "Area (MA)" screen (or the "Territories (MAT)" screen if the "Territories" option was selected). Additionally, the arrow keys can be used to select the option. Then use the Enter key to accept the selection. If the "Contiguous" option is selected, then the system returns control to the "Area (MA)" screen. If the "Territories" option is selected, then the system brings up the "Territories (MAT)" screen. 
The "Territories (MAT)" screen is used to select the territories of Guam, Saipan, and Moen. Use Accept (F2) to return control to the "Area (MA)" screen. Use Accept (F2) again to return control to the "Modify (M)" screen.

\section{Latitude/Longitude Réctangle}

Selection of the "Latitude/Longitude Rectangle" option brings up the "LatLon (MAL)" screen, which is used to set coordinates for a user-defined rectangle.

Use the arrow keys and the Enter key to select each of the four latitude/longitude coordinate fields and enter an appropriate latitude or longitude in decimal degrees. When finished, use Accept (F2) to return control to the "Area (MA)" screen.

\section{Lists of States, Counties, and Hydrologic Units}

Selection of any of the following six options brings up an appropriately named screen from which the specific list of polygon codes can be selected: States (MAS), Counties (MAC), Regions (MAR), SubRegions (MAS), AcctUnits (MAA), CtlgUnits (MAC).

Use the arrow keys to select up to 50 fields ( 24 for hydrologic regions) and enter the appropriate (FIPS) codes for the States, counties, or hydrologic units desired.

\section{Xref - Cross-reference names of political and hydrologic units with FIPS codes}

If the FIPS codes are not known, then use Intrpt (F6) to activate the "Xref Help" screen, which provides a cross reference of the names of political or hydrologic units and their respective FIPS codes. This screen also may be used for determining which political units intersect a particular hydrologic unit or which hydrologic units intersect a particular political unit.

Select the "Lookup Name" option to view the "Find Name/Code" screen. Toggle "On" the polygon type and then use the arrow keys to select the "Enter name or code" field. Enter either a name (not case sensitive) or a code for the polygon desired. The system returns the name and code of the polygon. Repeat as required.

Select the "List Political" option to view the "Xref Polit" screen, which provides for finding political units that intersect with a particular hydrologic unit. Use the arrow keys to select the hydrologic unit type of interest. Use the space bar to toggle "On" the selection. Use the arrow keys to move the cursor to the "Enter hydrologic unit code" field and enter the two-, four-, six-, or eight-digit hydrologic unit code for region, subregion, accounting unit, and cataloging unit, respectively. Next, move the cursor to the appropriate "select political unit type" field and use the space bar to toggle "On" the selection. When finished, use Accept (F2) to bring up the "Xref List" screen that lists the political units that intersect the selected hydrologic unit. Use Accept (F2) to return to the "Xref Help" screen. When finished, use Return to return control to the respective FIPS code screen (State (MAS), Regions (MAR), and so forth), and enter the required FIPS codes.

Select the "List Hydrologic" option to view the "Xref Hydro" screen, which provides for finding hydrologic units that intersect a particular political unit. Use the arrow keys to select the political unit type of interest. Use the space bar to toggle "On" the selection. Use the arrow keys to move the cursor to the "Enter political unit code" field and enter the two-digit (State) or five-digit (State/county) political unit code. Next, move the cursor to the appropriate "select hydrologic unit type" field and use the space bar to toggle "On" the selection. When finished, use Accept (F2) to bring up the "Xref List" screen that lists the hydrologic units that intersect the selected political unit. Use Accept (F2) to return to the "Xref Help" screen.When finished entering the FIPS codes, use Accept (F2) to return control to the "Area (MA)" screen. Use either Accept (F2) or the Enter key to return control to the "Modify (M)" screen. 


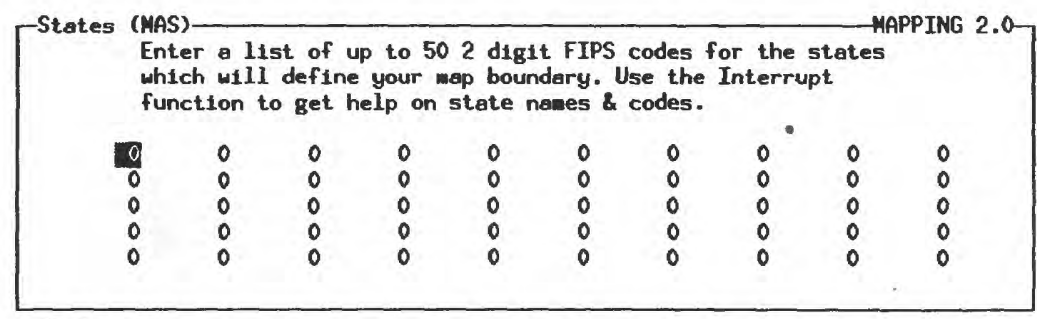
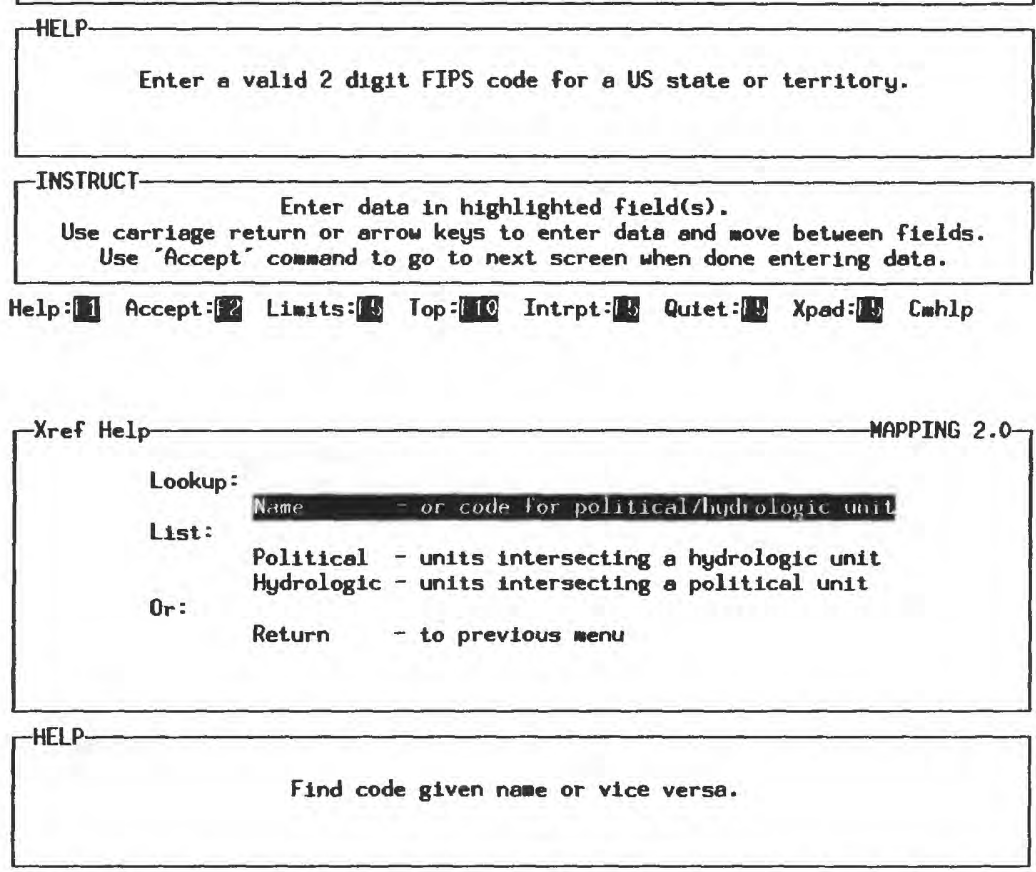

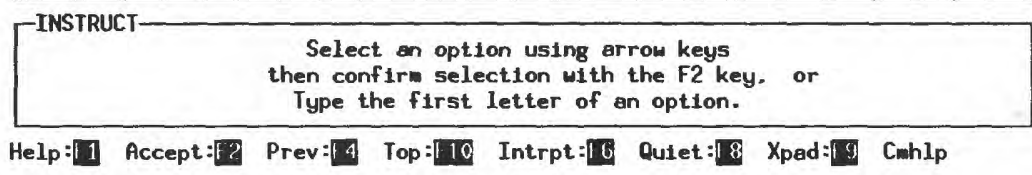

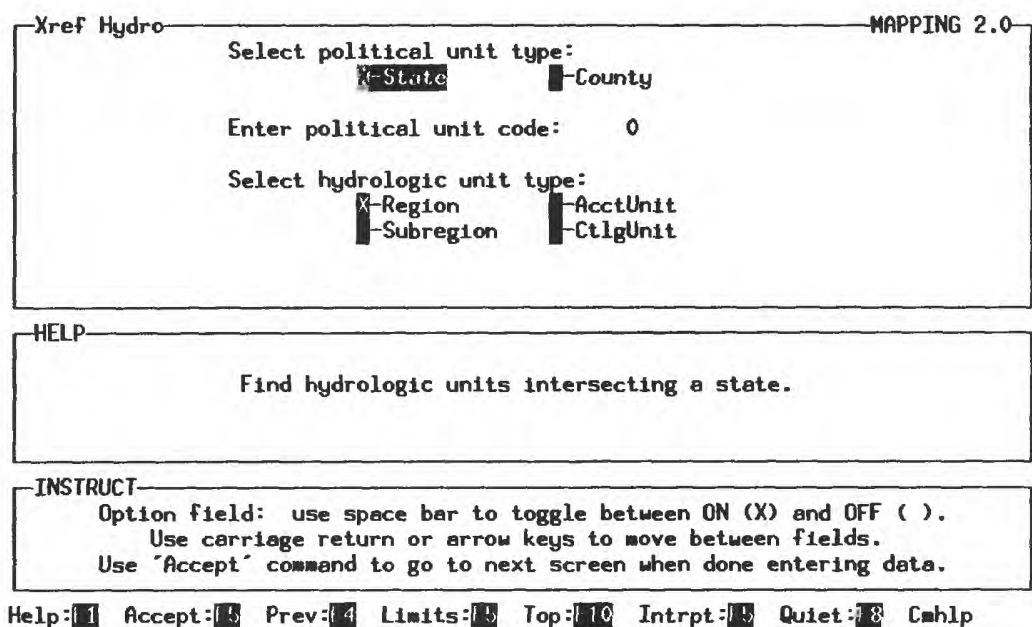




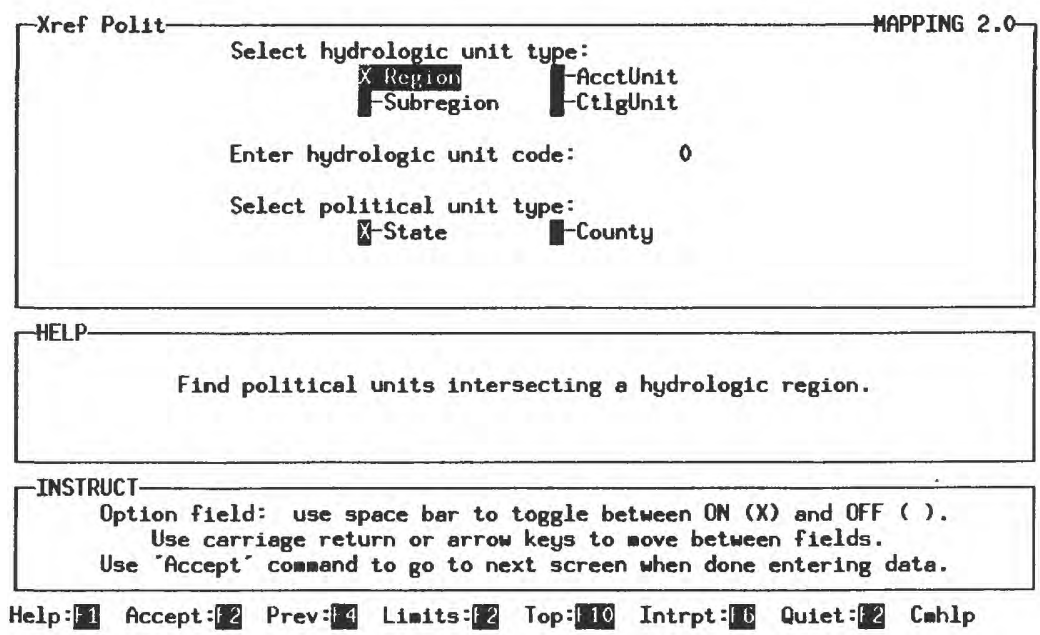

\section{Data Display}

When data are to be mapped, whether to a point location or to a particular polygon level for area shading, the values of a single plot variable are first aggregated to one of nine summary statistics, such as mean. For "Point Symbol" and "Area Shade" maps, the range of the computed statistic is divided into intervals. Each point location is represented by a symbol whose type, color, and size represent a particular data interval. In "Area Shade" maps the data are not only aggregated to a summary statistic but also to a particular geographical level, such as State, county, or one of the hydrologic unit levels. The geographical level of the data aggregation is independent of the primary or overlay polygons, thus the shading pattern can define its own geographical overlay. An optional legend can be drawn for relating the data intervals and the symbols or polygon shades.

For "3-D Bar" maps (point or area), the data for up to four variables are also aggregated, but the computed statistics are not intervalized. After the data aggregation method and geographical aggregation level ("3-D area" map only) are defined, another screen is brought up in which the color and the scaling method for the bar heights are determined. The elevation and azimuth angles for the " $3-\mathrm{D}$ " maps can also be modified. An optional legend can be drawn for relating the data variables and the associated bar colors.

From the "Modify (M)" screen, select the "Data" option. For "Point Symbol" or "Area Shade" maps, a "Data (MD)" screen will appear that displays the following options:

Interval Types

Interval Attributes

Aggregation Options.

For "3-D Point Bar" and "3-D Area Bar" maps, the data interval options are not applicable; thus, the "Data (MD)" screen is skipped and the "Aggregation (MDA)" screen, which is normally brought up by selecting the "Aggregation options" option in the "Data (MD)" screen, is brought up immediately and displays the following options:

Data aggregation method

Aggregation level (Area maps only).

Following the "Aggregation (MDA)" screen for "3-D Point Bar" and "3-D Area Bar" maps, a different "Data (MD)" screen appears in which the specifications for bar scaling and three-dimensional view angles are set. 

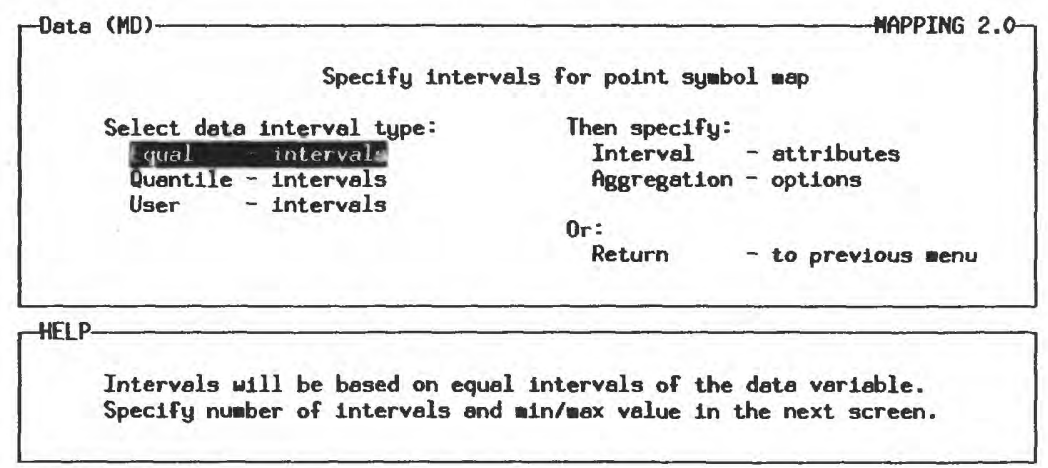

INSTRUCT- $\begin{gathered}\text { Select an option using arrow keys } \\ \text { then confirm selection with the F2 key. or } \\ \text { Type the first letter of an option. }\end{gathered}$
Help:11 Accept: 2 Top: 10 Quiet: 8 Xped: 8 Cahlp

\section{Interval Types}

The following interval types are supported:

Equal - intervals (equal data ranges in all intervals)

Quantile - intervals (equal number of observations each interval)

User - intervals (user-defined interval ranges).

\section{Equal Intervals}

The "Equal Intervals" option computes a summary statistic of the plot variable for each site or polygon. The computed statistic values are then sorted and divided into a userspecified number of equal-sized intervals. The data range used for computing the intervals can be determined by the program or the user can specify the minimum and maximum values from the extremes of the computed statistic.

From the "Data (MD)" screen, select the "Equal (MDE)" screen. The minimum and maximum values of the computed statistic of the plot variable are presented on the first line. The minimum and maximum values used to establish the interval limits are determined from those values by the program and are displayed under the "Automatic" option. Default values presented under the "Manual" option have no relation to the actual data values. These numbers should be changed if the "Manual" scaling option is selected. Note that for subsequent uses of the "Equal (MDE)" screen during the current session of the "mapping" program, both the "Automatic" and "Manual" options will be populated with the minimum and maximum values used in the most recent execution of the program, regardless of whether "Automatic" or "Manual" scaling was chosen.

Use the arrow keys to select the "Number of intervals" option and enter the number of intervals desired. If the minimum and maximum values of the computed statistic are to be manually specified, then use the arrow keys to select the "Manual" option and use the space bar to toggle "On" this selection as indicated by the appearance of an "X" in the field to the left of the option name. Use the arrow keys to select the "Minimum value" and "Maximum value" options and enter the values. These values may represent any range within the actual range of values.

When finished, use Accept (F2) to return control to the "Data (MD)" screen. 

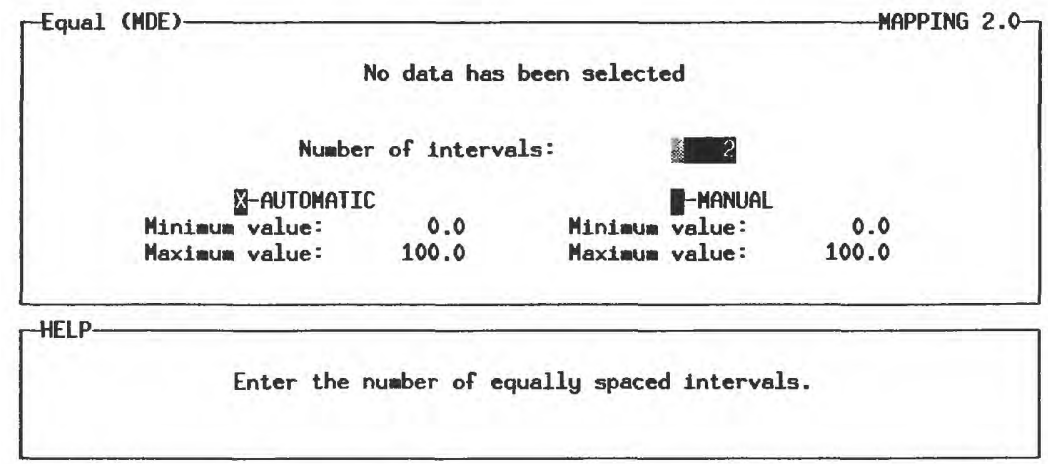

-INSTRUCT- Enter data in highlighted field(s).
Use carriage return or arrow keys to enter data and wove between fields.
Use 'Accept comand to go to next screen when done entering data.

\section{Quantile Intervals}

Selection of the "Quantiles" option displays the "Quantile (MDQ)" screen. The only input requirement for this screen is the number of intervals to be used. Based on the number of intervals specified, the program sets interval limits such that each interval contains approximately the same number of data values. It is usually not possible to achieve the same number of values in all intervals. Data values are assigned to intervals in a manner that will minimize the Root Mean Square (RMS) error between the quantized interval cumulative distribution and the ideal cumulative distribution. Consider a case with 23 unique data values grouped into five intervals. The ideal population of each interval would be $4.6(23 / 5)$, which is not physically possible. The ideal cumulative distribution for each interval is $4.6,9.2,13.8,18.4$, and 23. To minimize the RMS error, the population of the first interval is defined as:

$$
\mathrm{QP}[1]=\mathrm{INT}(\mathrm{IC}[1]+0.5) \text {, }
$$

where $\mathrm{QP}[1]$ is the quantized population of interval 1,

IC[1] is the ideal cumulative distribution of interval 1 , and

INT is the integer truncation function.

The remaining populations $(2-\mathrm{N})$ are defined as:

$$
\mathrm{QP}[\mathrm{n}]=\mathrm{INT}(\mathrm{IC}[\mathrm{n}]+0.5)-\mathrm{QP}[\mathrm{n}-1] \text {. }
$$

Thus, for the above example, the quantized interval populations are $5,4,5,4$, and 5 .

Enter the number of intervals required and use Accept (F2) to accept this value and return control to the "Data (MD)" screen. 

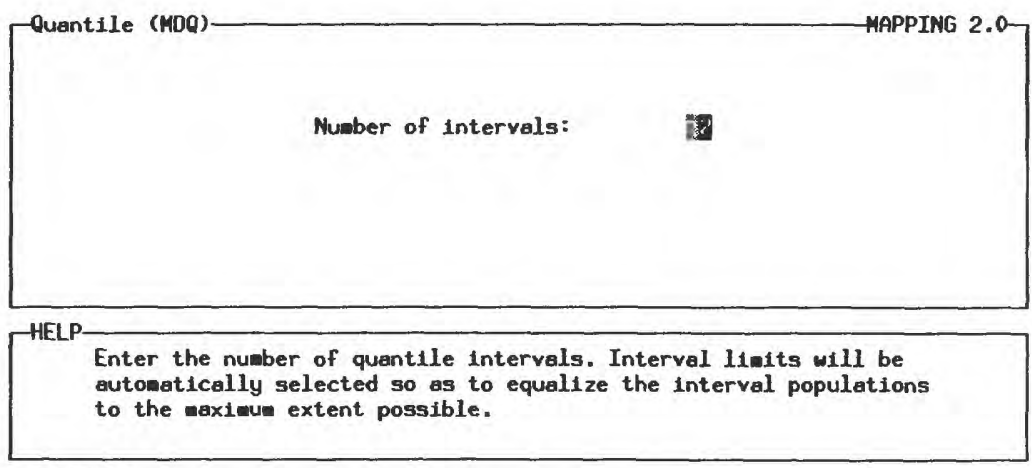

INSTRUCT- Enter data in highlighted fleld(s).
Use carriage return or arrow keys to enter data and nove between fields.
Use Accept comend to go to next screen when done entering data.

\section{User-Defined Intervals}

The "User-Defined Intervals" option permits the user to define the number of intervals to be used and the boundaries of each interval. The intervals need not be contiguous and the interval ranges need not be uniform.

From the "Data (MD)" screen, select the "User (MDU)" screen. The extremes of the computed statistic are listed in the first line. Use the arrow keys to select the "Minimum" and "Maximum" fields and enter the appropriate values. When finished, use Accept (F2) to return control to the "Data (MD)" screen.
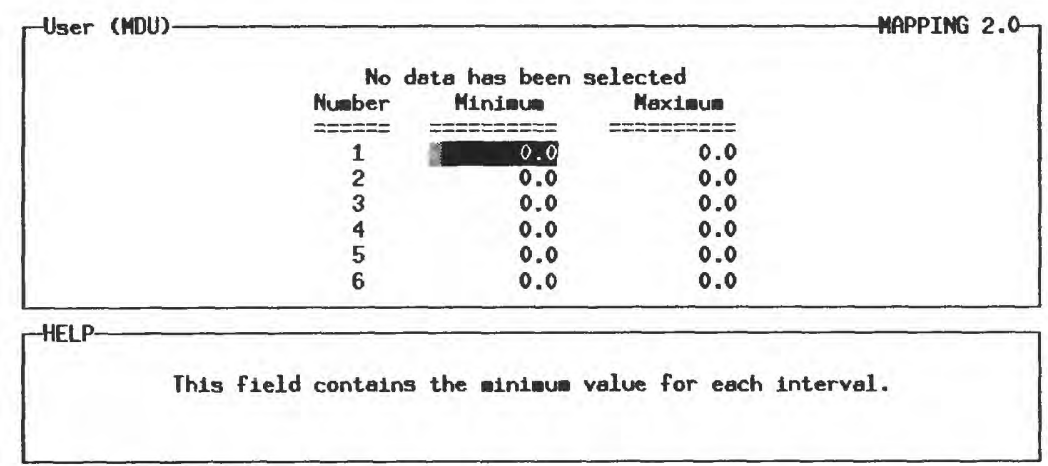

\footnotetext{
INSTRUCT- Enter data in highlighted fleld(s).
Use carriage return or arrow keys to enter data and wove between fields.
Use 'Accept comand to go to next screen when done entering data.
} 


\section{Interval Attributes}

The "Interval Attributes" option specifies the attributes of the symbols to be used to represent the data intervals on the map. The following symbol attributes are specified:

$$
\begin{aligned}
& \text { Type } \\
& \text { Color } \\
& \text { Size. }
\end{aligned}
$$

From the "Data (MD)" screen, select the "Interval (MDI)" screen. For the number of intervals specified, use the arrow and Enter keys to select the field and enter the appropriate symbol type, color code, and size in decimal inches.

Use Help (F1) to view the "Help" panel. Once the "Help" panel is activated, a new Help panel screen appears as each field is selected, listing the appropriate codes for that field.

When the "Help" panel requires more than one page to present additional lines of allowable values, the "Data" panel is deactivated temporarily, which allows the use of the Page Down and Page Up keys for paging through the additional lines. Use (F3) to reactivate the "Data" panel. To reactivate the Page Down and Page Up functions in the "Help" panel, use Help (F1). When finished, use Accept (F2) to return control to the "Data (MD)" screen.

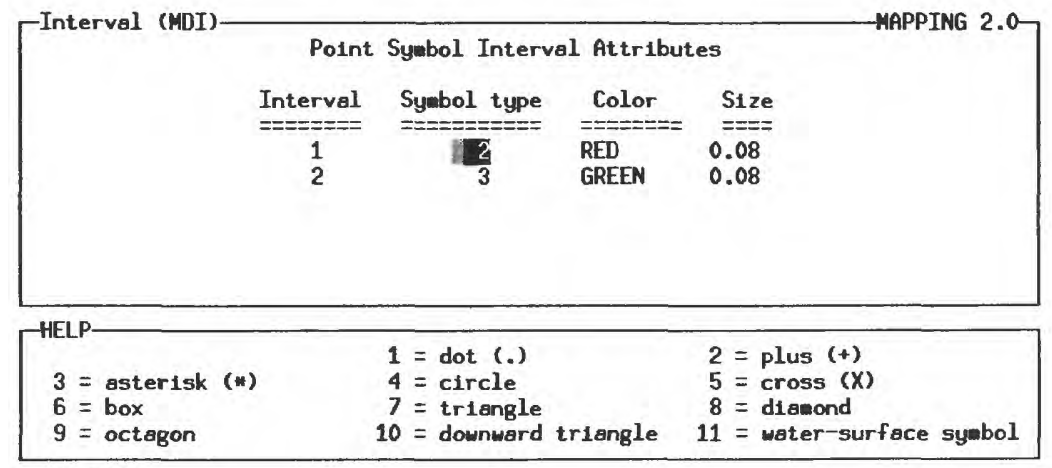

\footnotetext{
INSTRUCT- Enter data in highlighted field(s).

Use carriage return or arrow keys to enter data and move between fields. Use "Accept' comand to go to next screen when done entering data.

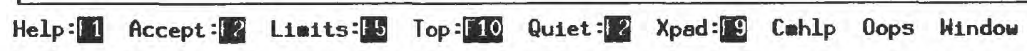

\section{Aggregation Options}

The "Aggregation" option provides the following functions:

Data Aggregation Method

Geographic Level of Aggregation

Note: The "Geographic Level of Aggregation" option is applicable only to "Area Shade" and "Area 3-D Bar" maps.

\section{Data Aggregation Method}

"Data Aggregation" specifies the method used to reduce the number of plot variable values associated with a given site (or area) to a single summary statistic value. The value of this statistic is presented at a point location on the map as a symbol whose type, color, and size reflect the magnitude of the value, or as a shaded polygon, whose color indicates the magnitude. The summary statistics available are as follow: 
Mean (average)
Median
Sum
Minimum
Maximum
Range
Standard Deviation
Variance
Coefficient of Variance (Std Dev * 100/Mean).

Select the "Aggregation (MDA)" screen from either the "Data (MD)" screen ("Point Symbol" or "Area Shade" maps) or by way of the "Data" option from the "Modify (M)" screen ("Point Bar" and "Area Bar" maps). Use the arrow keys to select the "Data Aggregation" method and use the space bar to toggle "On" this selection as indicated by the appearance of an " $\mathrm{X}$ " in the field to the left of the option name.

\section{Geographic Aggregation Level}

If the map type specified in the "Map (MM)" screen is either an "Area Shade" or "3-D Area Bar" map, use the arrow keys to select the geographic level to which the data aggregation will be computed. Use the space bar to toggle "On" the selection. The optional geographic levels are as follow:

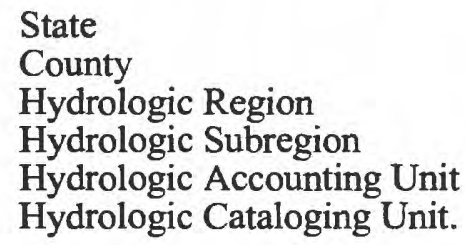

The aggregation level specified here does not have to coincide with either the primary boundary (see "Area (MA)" screen) or any of the overlay boundaries specified in the "Overlay (MO)" screen. In such cases the color shading acts as its own border delineation. Use Accept (F2) to return control to the "Modify (M)" screen ("Point Symbol" and "Area Bar" maps) or proceed to the "Data (MD)" screen for bar scaling and view angle specification ("3-D Point Bar" and "3-D Area Bar" maps).
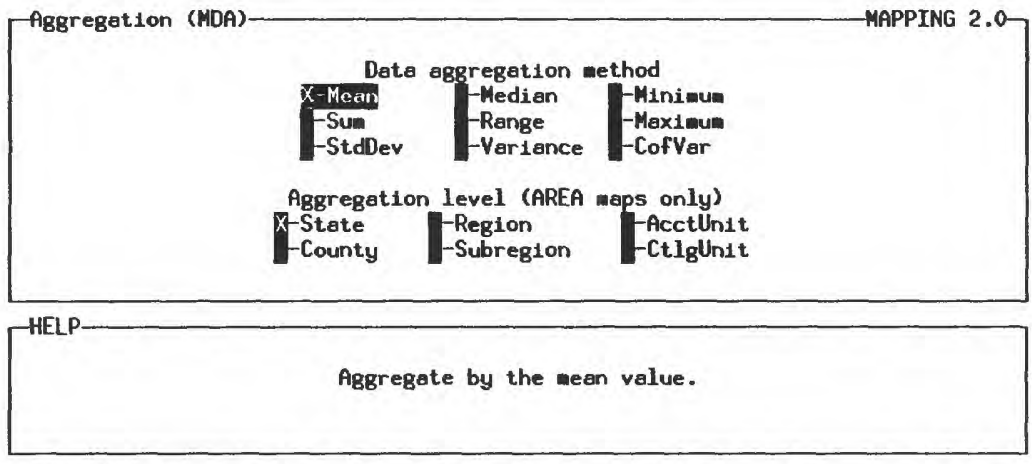

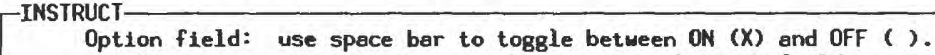

Use carriage return or arrow keys to wove between fields.

Use "Accept" command to go to next screen when done entering data.
}

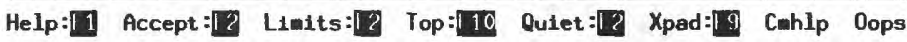




\section{Three-Dimensional Bar Scaling and View Angles}

\section{Bar Scaling}

The "Data (MD)" screen for "3-D Point Bar" and "3-D Area Bar" maps is displayed after using Accept (F2) to accept the "Aggregation (MDA)" screen. Use the Tab and arrow keys to select either "Automatic" or "Manual" scaling for the bars. In manual scaling, by setting the scaling maximum greater than the actual maximum used in automatic scaling, the net effect is to reduce the relative height of the bar on the map. By setting a smaller scaling maximum, a higher bar is produced. Each of the up to four variables is scaled separately.

\section{Elevation Angle and Azimuth Angle}

The default values for elevation angle ( 30 degrees) and azimuth angle (45 degrees) should be suitable for most applications but can be changed to suit user needs. The elevation angle must be set between 5 degrees and 85 degrees. The azimuth angle can have a minimum value of 0 degrees and a maximum value of 90 degrees. Use Help (F1) and Limits (F5) to review view angle specifications.

Use Accept (F2) to return control to the "Modify (M)" screen.

\section{Polygon Overlays}

The primary geographic polygon (see "Area (MA)" screen) can have up to eight overlays specified. An overlay, which can be the primary polygon itself, can be used to specify an alternate line color, pattern, or line thickness. Each overlay has options for the following:

Overlay type

Line specifications

Polygon blanking

Polygon labeling

Selective polygons within a given geographic level.
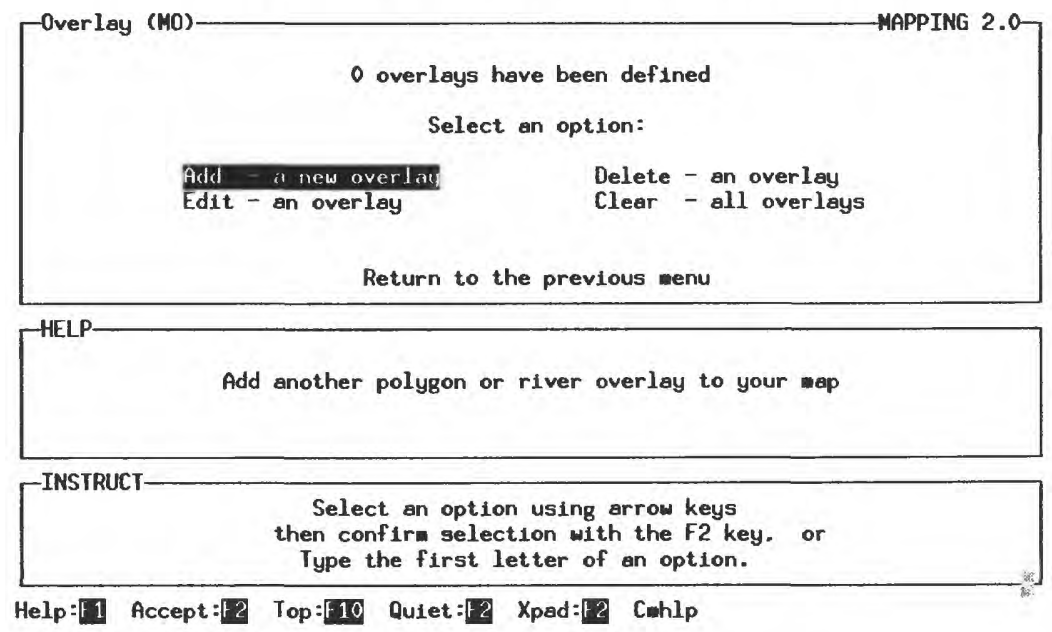

From the "Modify (M)" screen, select the "Overlay (MO)" screen. Use this screen for each overlay desired. This screen provides four options for creating and maintaining geographic overlays. These options are as follow:

Add - a new overlay
Edit - an overlay
Delete - an overlay
Clear - all overlays.

The "Add" discussion applies to either adding or editing an overlay. 


\section{Add an Overlay}

From the "Overlay (MO)" screen, type "A" (or "a") or use the arrow keys to select the option and then use the Enter key.

The following overlay types are supported:

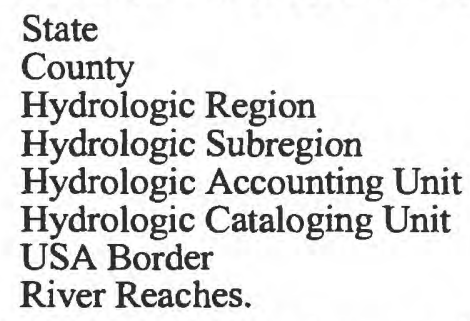

Use the arrow and Enter keys to select the overlay and use the space bar to toggle "On" the selection, as indicated by the appearance of an " $\mathrm{X}$ " in the field to the left of the option name.

If the "River Reaches" overlay is selected, then the screen (in the lower half of the data panel) presents options for specifying the river reach level and the minimum length of reach to be included in the overlay.

\section{Line Specifications}

Line specifications include color, pattern, and relative thickness. The value entered for the "Thickness" option is not an absolute thickness, but rather a multiplication factor of a default thickness.

Use the arrow keys to select a line specification and enter an appropriate code. Use Help (F1) to bring up the "Help" panel. As each line specification field is highlighted, the "Help" panel changes to list the codes and code descriptions available for that option.

\section{Polygon Blanking}

The following blanking options are supported:

POLYGON - Overlay arcs will be clipped at the map boundary polygon

RCTNGL - Overlay arcs will be clipped at the bounding rectangle.

NONE - Each overlay polygon that intersects the map boundary will be plotted in its entirety.

Use the arrow keys to select the "Blanking" option. Use the "Help" panel and type in a blanking option.

\section{Polygon Labeling}

The following polygon labeling options are supported:

NAME - Polygon name will be plotted at the centroid.

CODE - Polygon code will be plotted at the centroid.

NONE - No polygon labels will be plotted (default).

Use the arrow keys to select the "Labels" option. Use the "Help" panel and type in a polygon labeling option.

\section{Selective Polygons Within a Given Geographic Level}

A list of individual polygons within a geographic level overlay, such as counties within States, can be specified. Use the arrow keys to select the "ITEMIZE" option. Enter "YES" in the field and use the Enter key to view the "Itemize" screen. Enter a list of individual FIPS codes. 
If the FIPS codes are not known, then use Intrpt (F6) to activate the "Xref Help" screen, which provides a means of cross referencing the political or hydrologic unit names with their respective FIPS codes. See the previous discussion of the "Xref" function under the "Area" function accessed through the "Modify (M)" screen.

\section{River Reach Specifications}

If the "River Reach" option is selected as the overlay type, then two new options appear in the lower right corner of the "Add (MOA)" or "Edit (MOE)" screen. The "Max level" option specifies the level of the stream relative to the ocean. For example, the Mississippi River is level one (it flows into the ocean), the Missouri River is level two (it flows into the Mississippi), and the Platte River is level three (it flows into the Missouri).

The "Min length" option specifies the minimum length of the streams that will be included in the overlay. This option can be used to eliminate short tributaries. Use the arrow keys to select the option desired and enter a value. When finished, use Accept (F2) to return control to the "Overlay (MO)" screen.

\section{Edit an Overlay}

From the "Overlay (MO)" screen, type "E" (or "e") or use the arrow keys to select the option and use the Enter key. If more than one overlay has been created, then the user is prompted for the number of the overlay to be edited.

\section{Delete an Overlay}

From the "Overlay (MO)" screen, type "D" (or "d") or use the arrow keys to select the option and use the Enter key. The user is prompted for the number of the overlay to be deleted. After the number has been entered, use either Accept (F2) or the Enter key to return control to the "Overlay (MO)" screen.

\section{Clear All Overlays}

From the "Overlay (MO)" screen, type "C" (or "c") and use the Enter key. All existing overlays are cleared and control is returned to the "Overlay (MO)" screen. When finished with the "Overlay (MO)" screen, use either Accept (F2) or the "Return to previous screen" option to return control to the "Modify (M)" screen.

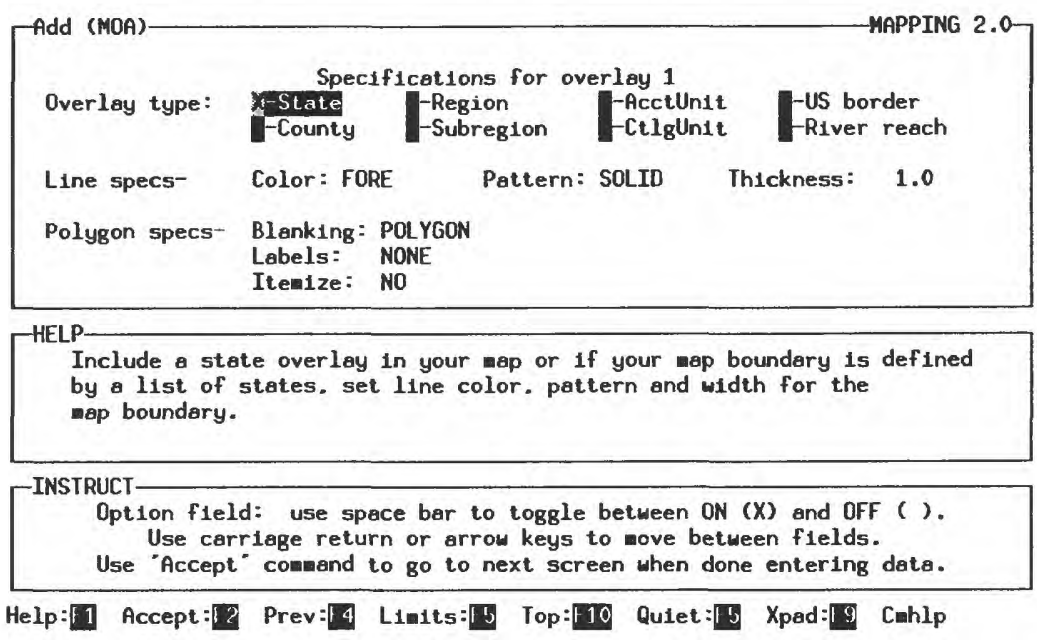




\section{Station Labeling}

The "Station Labeling" option provides for defining labels to be plotted at site locations on the map. Labels can be used with any of the map types, including "No Data" maps. The text plotted in the labels is based on values of either a text or numerical variable. The label characteristics (color, size, font, and presentation angle) can be defined globally (all labels have the same characteristics) or each label can be defined individually based on the values of other label characteristic variables.

If station label variables are used, they can be either in the input data file used for "Point Symbol," "3-D Bar," or "Trend" maps, or in a separate file designated as the label file. If the input data file contains the label information variables and the data variable to be mapped, then the label text variable and the data variable can be the same or different variables. With or without station labels, site locations can be indicated on a "No data" map by specifying a symbol type, color, and size. The decimal latitude and decimal longitude variables, "DECLAT" and "DECLON," must be in the label file, if used, or the data file. From the "Modify (M)" screen, select the "Station (MS)" screen, which lists the following options:

Open - station label file

Filter - with Boolean "where" clause

Name - label variables

Specify - station attributes.

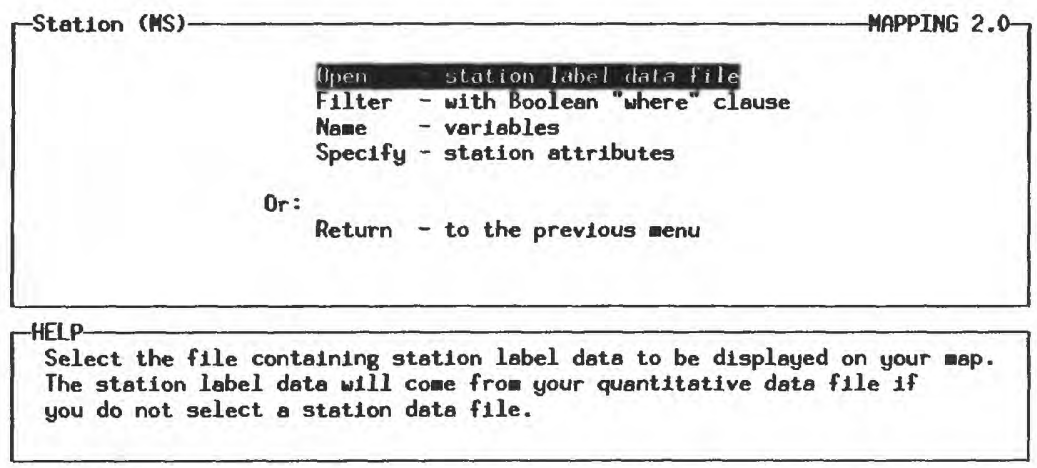

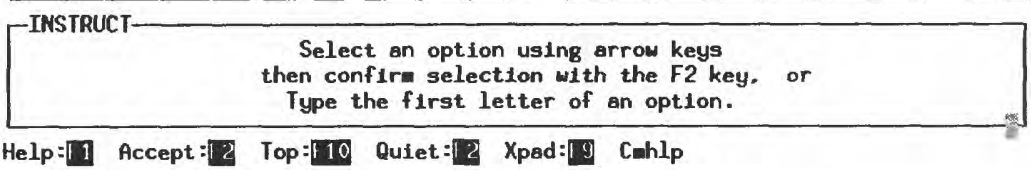

\section{Open Station Label File}

If a separate label file is required, then select the "Open (MSO)" screen. Use the arrow keys to select the file and use Accept (F2) or the Enter key to accept the selection and return control to the "Station (MS)" screen.

\section{Filter Data in Label Variable File}

As in a regular input data file, the data in a separate label file can be filtered by using a Boolean logic clause to select which rows are retained for use in the current application. From the "Station (MS)" screen, select the "Filter (MSF)" screen. This is the screen used for filtering an input data file. Use the arrow keys to select the "Exclude" or the "Include" function and toggle this selection "On" by using the space bar. An " $\mathrm{X}$ " will appear in the field to the left of the selection. Use the arrow keys to select the "Criteria" field and enter a Boolean clause to specify which rows to retain or exclude. Begin each variable name with a "\$" sign. Use Intrpt (F6) to view a list of the variables in the label variable file. When finished with the variable list screen, use Accept (F2) to return control to the "Filter (MSF)" screen to complete the Boolean clause. 
Name Label Variables

To name the variable whose values will show up in the station labels, select the "Name (MSN)" screen from the "Station (MS)" screen. Use the arrow keys to select the label variable and enter a " 1 " in the "Select" field to the left of the variable name. If values for label attributes are to be obtained from the label file (or input data file), then enter a "2" in the select field for the font variable, a " 3 " for the color variable, a "4" for the size variable, and a " 5 " for the angle variable. Global values to these attributes are assigned in the "Label Stations" option in the "Specify (MSS)" screen described subsequently.

\section{Specify Station Attributes}

From the "Station (MS)" screen, select the "Specify (MSS)" screen. This screen initially lists two main options:

Plot station locations

Label stations.

Use the space bar to toggle "On" either or both of these options as indicated by the appearance of an "X" in the field to the left of the option name. After an option is turned "On," additional options appear below the option name to specify the symbol that indicates the site locations, and the label and label attributes, respectively.

\section{Plot Station Locations}

Use the arrow keys to select the options desired and enter an appropriate code. Use Help (F1) to bring up the "Help" panel, which lists the descriptions and code values for the selected attribute.

\section{Label Stations}

If the attributes for the station labels are being obtained from either the input data file or a separate label file, then use the arrow keys to select the "from variable" fields that come from that file (right side of page). Toggle each appropriate selection "On" by using the space bar. An "X" will appear in the field to the left of the attribute name.

If the label attributes are to be assigned globally, that is, the same attribute values will be used for all site locations, then use the arrow keys to select the attribute fields on the left side of the page and enter an appropriate value. Use Help (F1) to bring up the "Help" panel, which lists the descriptions and code values for the selected attribute.

When finished with the "Specify (MSS)" screen, use Accept (F2) to return control to the "Station (MS)" screen. Then use "R" (or "r") or use the arrow keys to select the "Return to previous menu" option and use the Enter key.
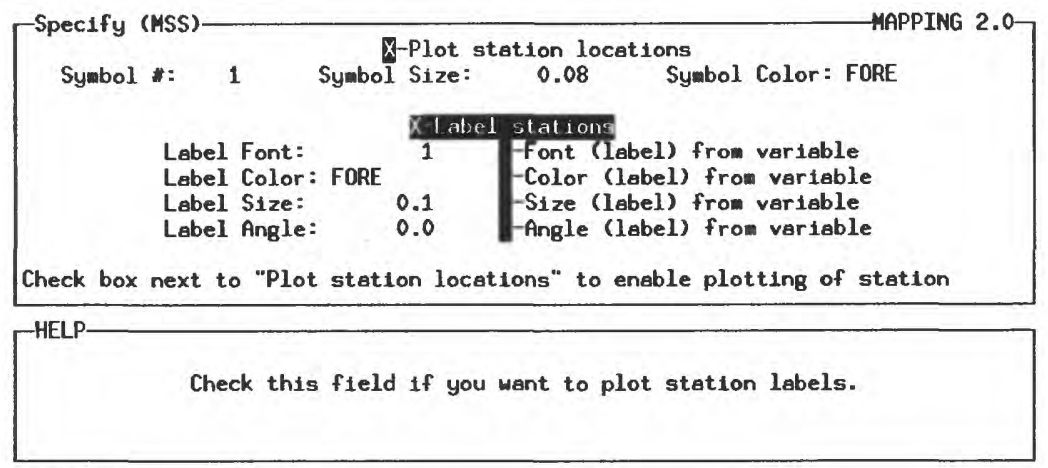

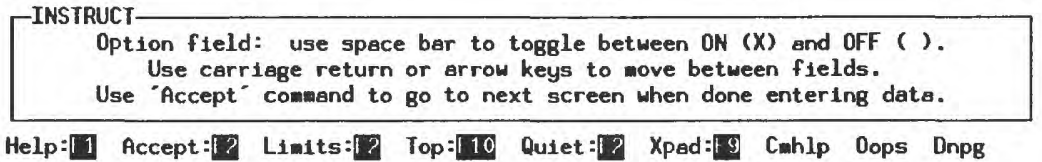




\section{Basic Plot Parameters}

From the "Modify (M)" screen, select the "Basic (MB)" screen, which lists the following options for modifying plot specifications:

$\begin{array}{ll}\text { Axes } & \text { - and grid lines } \\ \text { Project } & \text { - resolution and scaling } \\ \text { Titles } & \text { - above plot } \\ \text { Footnotes } & \text { - below plot } \\ \text { Labels } & \text { - of miscellaneous text } \\ \text { Page } & \text { - layout options } \\ \text { Censored } & \text { - data options } \\ \text { Make } & \text { - the plot } \\ \text { Return } & \text { - to previous screen. }\end{array}$

Type the first letter of any option or use the arrow and Enter keys to select and open the screen for the option required.
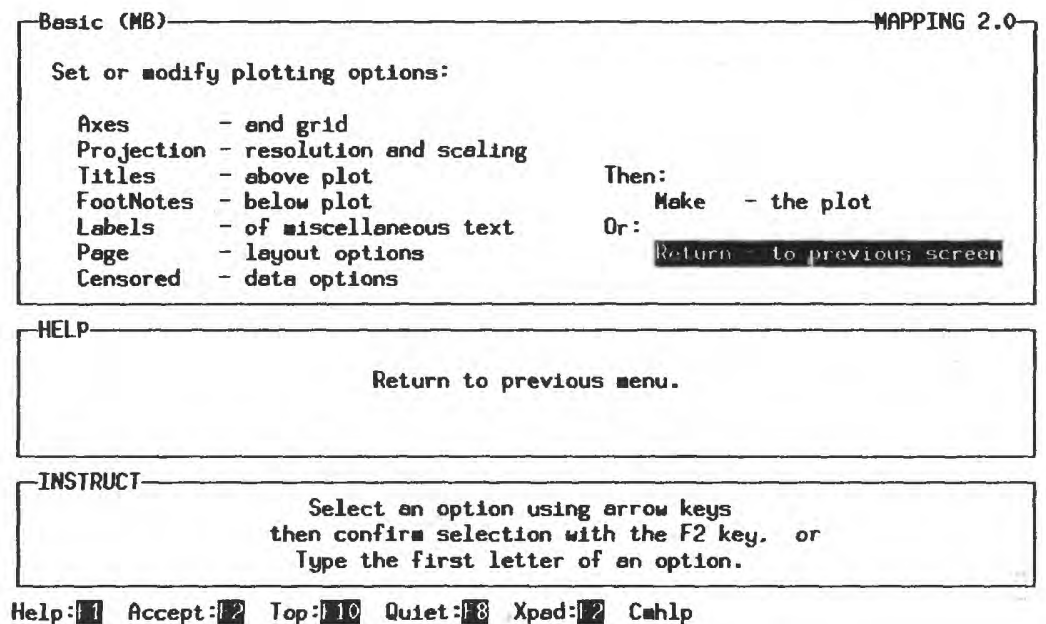

\section{Map Axes and Grid}

A map can be annotated with latitude/longitude axes and grid lines. From the "Basic $(\mathrm{MB})$ " screen, select the "Axes (MBA)" screen. Use the arrow keys to first select the "Plot axes" option and then use the space bar to toggle "On" this option as indicated by the appearance of an " $\mathrm{X}$ " in the field to the left of the option name. When this option is toggled "On," additional options appear on the right side of the screen for specifying the intervals in degrees between the latitude and longitude tick marks.

To specify grid lines at selected latitude and (or) longitude intervals, use the arrow keys to select the "Draw grid" option and then use the space bar to toggle "On" this option. When this option is toggled "On," additional options appear on the right side of the screen for specifying the intervals in degrees between the latitude and longitude tick marks where grid lines will be drawn. When finished, use Accept (F2) to return control to the "Basic (MB)" screen. 


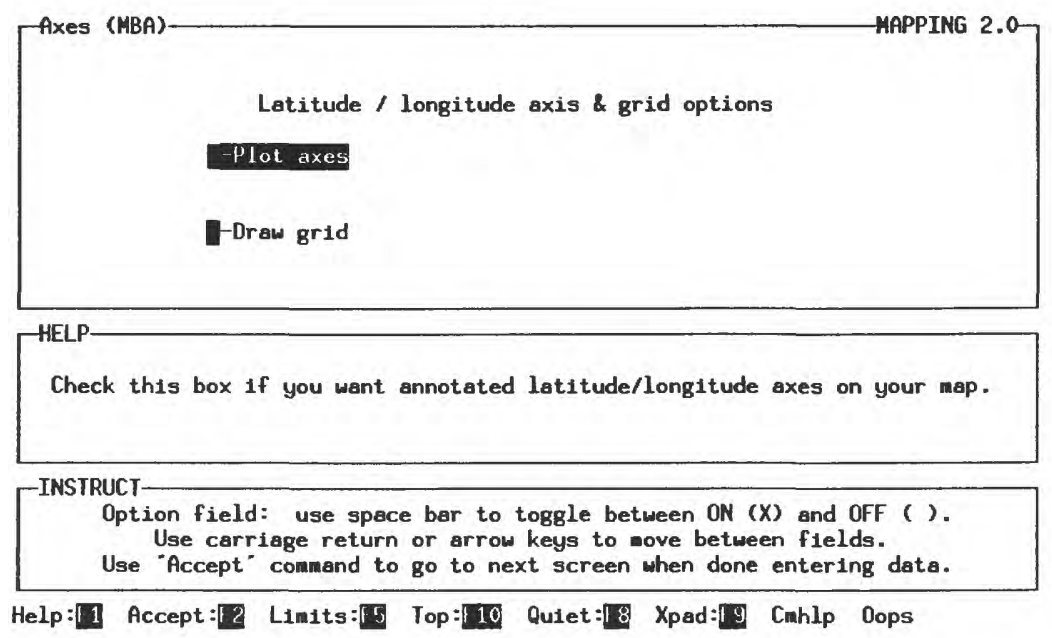

\section{Map Projection}

It normally should not be necessary to modify the system defaults for the map projection. The Albers Equal Area projection with system-determined values for the Central Meridian and Standard Parallels are the default specifications. For those with a need for (and knowledge about) other map projections, the following options are provided:

$\begin{array}{llcc}\text { Map projection } & \begin{array}{l}\text { Central } \\ \text { Meridian }\end{array} & \begin{array}{c}\text { Standard } \\ \text { Parallels }\end{array} & \begin{array}{c}\text { True Scale } \\ \text { Latitude }\end{array} \\ \text { Geodetic (Orthogonal Lat/Long) } & \mathrm{n} / \mathrm{a} & \mathrm{n} / \mathrm{a} & \mathrm{n} / \mathrm{a} \\ \text { Albers Equal Area (default) } & \mathrm{X} & \mathrm{X} & \mathrm{n} / \mathrm{a} \\ \text { Lambert Conformal Conic } & \mathrm{X} & \mathrm{X} & \mathrm{n} / \mathrm{a} \\ \text { Mercator } & \mathrm{X} & \mathrm{n} / \mathrm{a} & \mathrm{X}\end{array}$

From the "Basic (MB)" screen, select the "Projection (MBP)" screen. Use the arrow keys to select the projection and use the space bar to toggle "On" this selection. If applicable, use the arrow keys to select the meridian and latitude options and enter the appropriate values.

\section{Map Scale Units}

The default for map scale units is to fill the available area. If specific scale units are needed, then the following options are available:

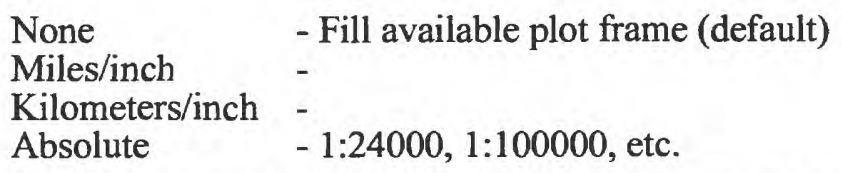

From the "Projection (MBP)" screen, use the arrow keys to select the scale units option and use the space bar to toggle "On" this selection as indicated by the appearance of an "X" in the field to the left of the option name. Then, if applicable, use the arrow keys to select the "Map scale" option and enter a value.

\section{Resolution}

The default resolution for a map is 100 digitized points per inch (dpi). Higher dpi numbers give more detail, but decrease the plot speed. 
From the "Projection (MBP)" screen, use the arrow keys to select the "Resolution" option and enter an alternate value. When finished with the "Projection (MBP)" screen, use Accept (F2) to return control to the "Basic (MB)" screen.
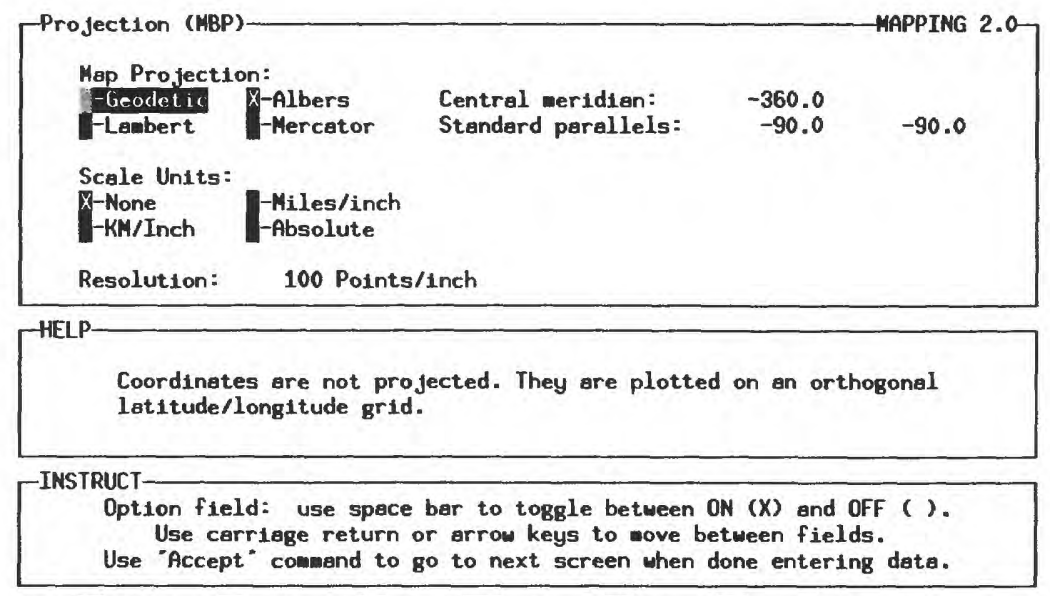

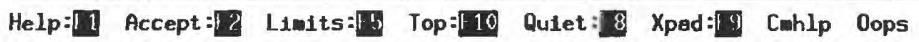

\section{Page Layout Options}

From the "Basic (MB)" screen, select the "Page (MBP)" screen. The "Page (MBP)" screen provides the following functions:
Page Height
Page Width
Font Style
Plot Page Border
Legend Options.

Most options in this list are discussed in the section "Page Layout Options" under "Steps to Using QWGRAF Programs."
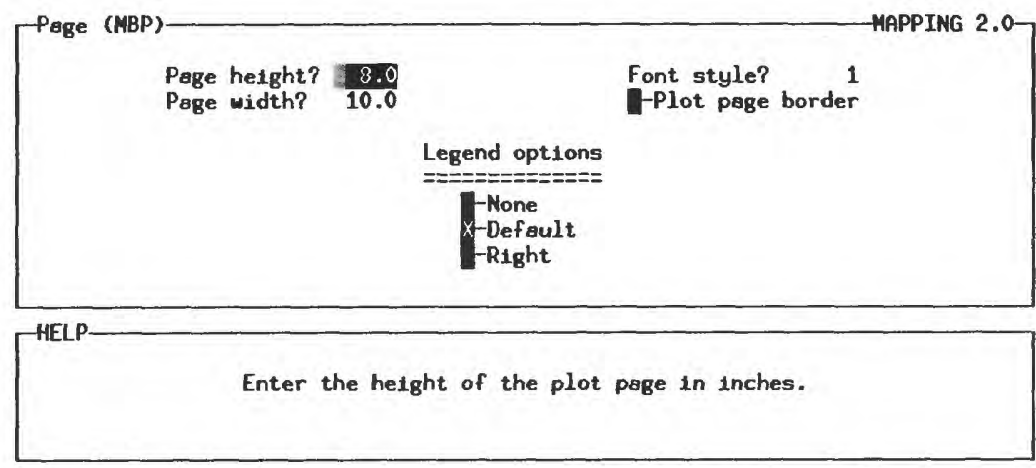

\footnotetext{
INSTRUCT- Enter data in highlighted field(s).

Use carriage return or arrow keys to enter data and wove between fields. Use "Accept" command to go to next screen when done entering date.

Help: I1 Accept: L Limits:
} 


\section{Legend Options}

This option is used to plot a legend that describes the data intervals as represented by the type, size, and color of plotted symbols. Use the arrow keys to select the option desired and toggle the option "On" with the space bar as indicated by the appearance of an "X" in the field to the left of the option name.

\section{Censored Data Options}

From the "Basic (MB)" screen, select the "Censored (MBC)" screen to view the options for censored data. By default the "mapping" program excludes censored data from the analysis. To include censored data, use the arrow or Tab keys to select (highlight) the "Include censored values" option. Use Accept (F2) to return control to the "Basic (MB)" screen.

\section{Make the Plot}

When all arguments for the map have been specified and the appropriate device selected, return control to the "Opening Screen," select the "Make the plot" option, and use the Enter key. If a screen output was selected, then a graphics window opens and the map is presented. If a file output was selected, then a message screen indicates that the output from the procedure has been saved.

\section{mapping Tutorial}

An interactive tutorial is available to guide the user through both basic and selected advanced options of the "mapping" program. The tutorial will use a script file from the "datmgr" program (see tutorial for "datmgr" program), which processes a raw data file through a series of modifications to make it suitable for use by the "mapping" program to create a map of mean $\mathrm{pH}$ values at National Atmospheric Deposition Program sites throughout the United States, Hawaii, Alaska, and Puerto Rico. The station labeling function of the "mapping" program is highlighted. The tutorial is activated by first typing "Shift @" in the "Opening Screen" of a new "mapping" session. When the dialog box comes up, enter the file name found in the tutorial.doc file distributed with the QWGRAF programs. This file is located in the doc subdirectory, which provides specific details on using this and other QWGRAF tutorials.

The tutorial prompts the user for specific keystrokes. The program will only respond when the correct keystroke sequence is entered; that is, incorrect information cannot be entered. Text for labels, titles, and so forth, can be entered in either upper or lower case. The system converts everything to upper case.

\section{Sample Plots}

The following are examples of the types of plots that can be created with the QWGRAF "mapping" program. These examples are intended to illustrate various options available in the program and do not necessarily conform to publication standards of the Geological Survey. 
Program: MAPPING

Plot Specifications: No Data Map; Contiguous 48 States; State Overlay; 2 Titles

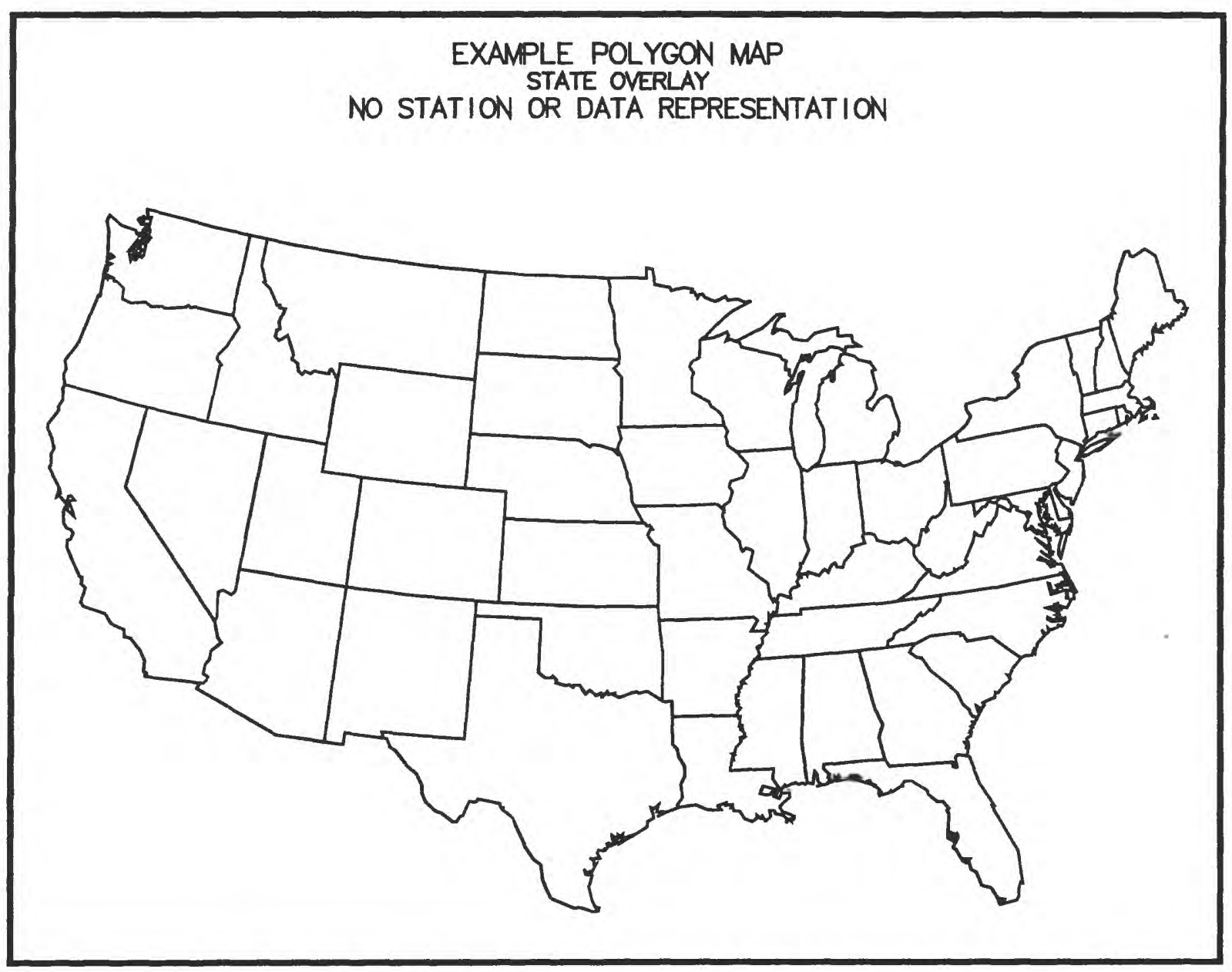


Program: MAPPING

Plot Specifications: No Data Map; USA + Territories; State Overlay; Station Location; Station Labeling With Value of Variable; 2 Titles

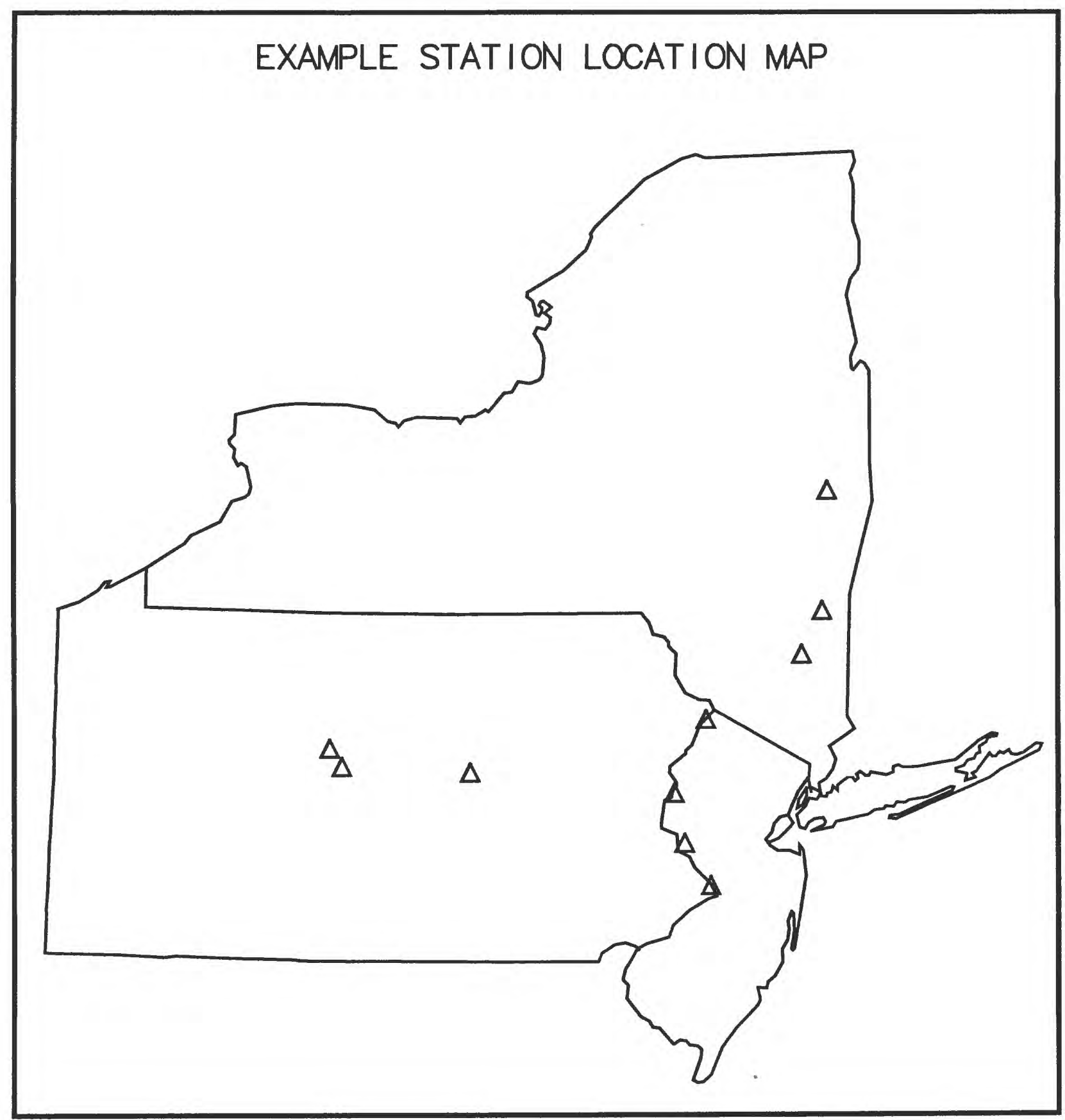


Program: MAPPING

Plot Specifications: 3-D Point Symbol Map; 2 Variables; 3 Titles; List of States, Hydrologic Accounting Unit Overlay; Explanation Block

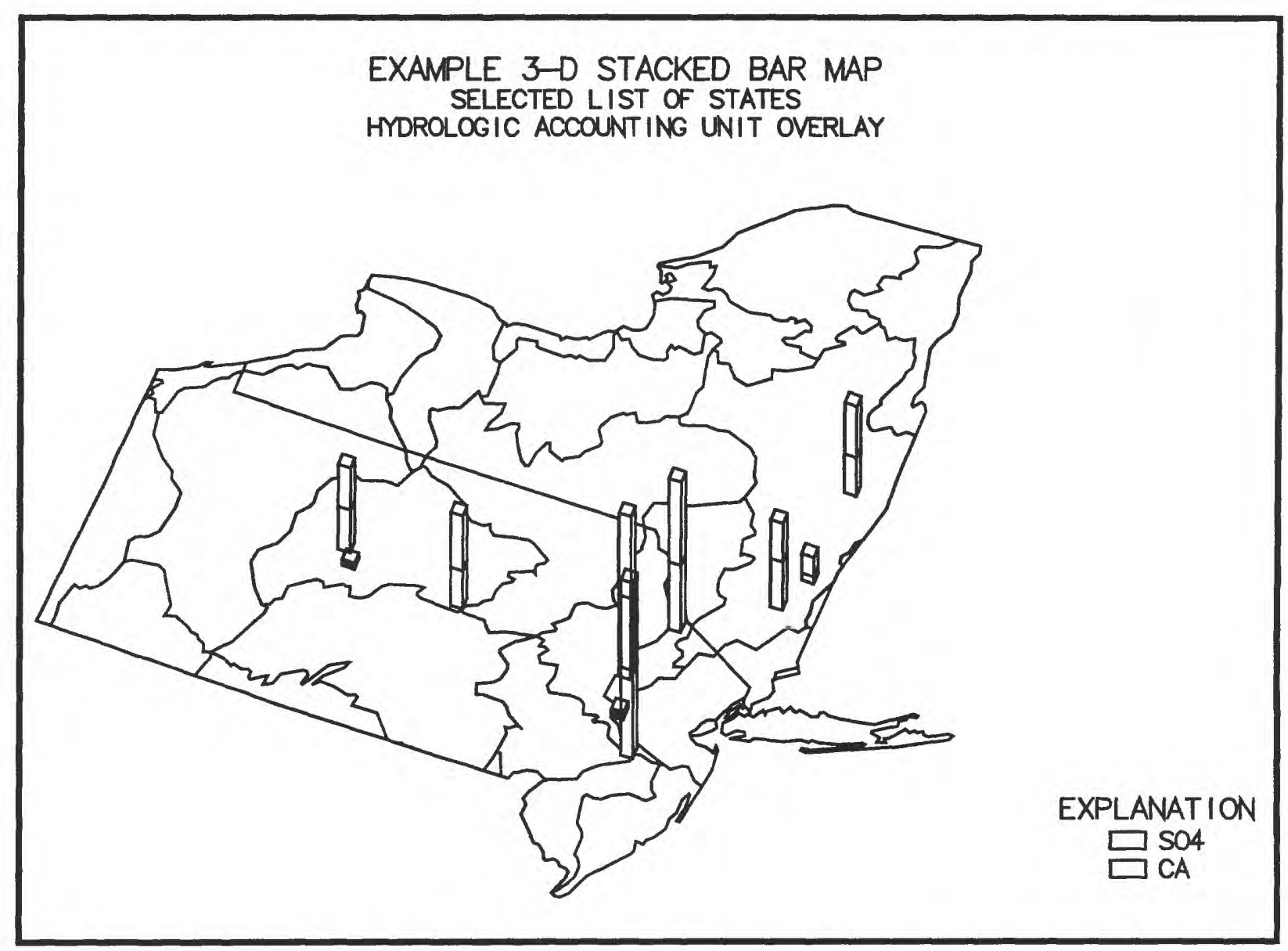


Program: MAPPING

Plot Specifications: Point Symbol Map; Equal Data Intervals; List of States; County Overlay; 2 Titles; 1 Footnote; Explanation Block

\section{EXAMPLE POINT SYMBOL MAP \\ Select List of States \\ County Over lay}

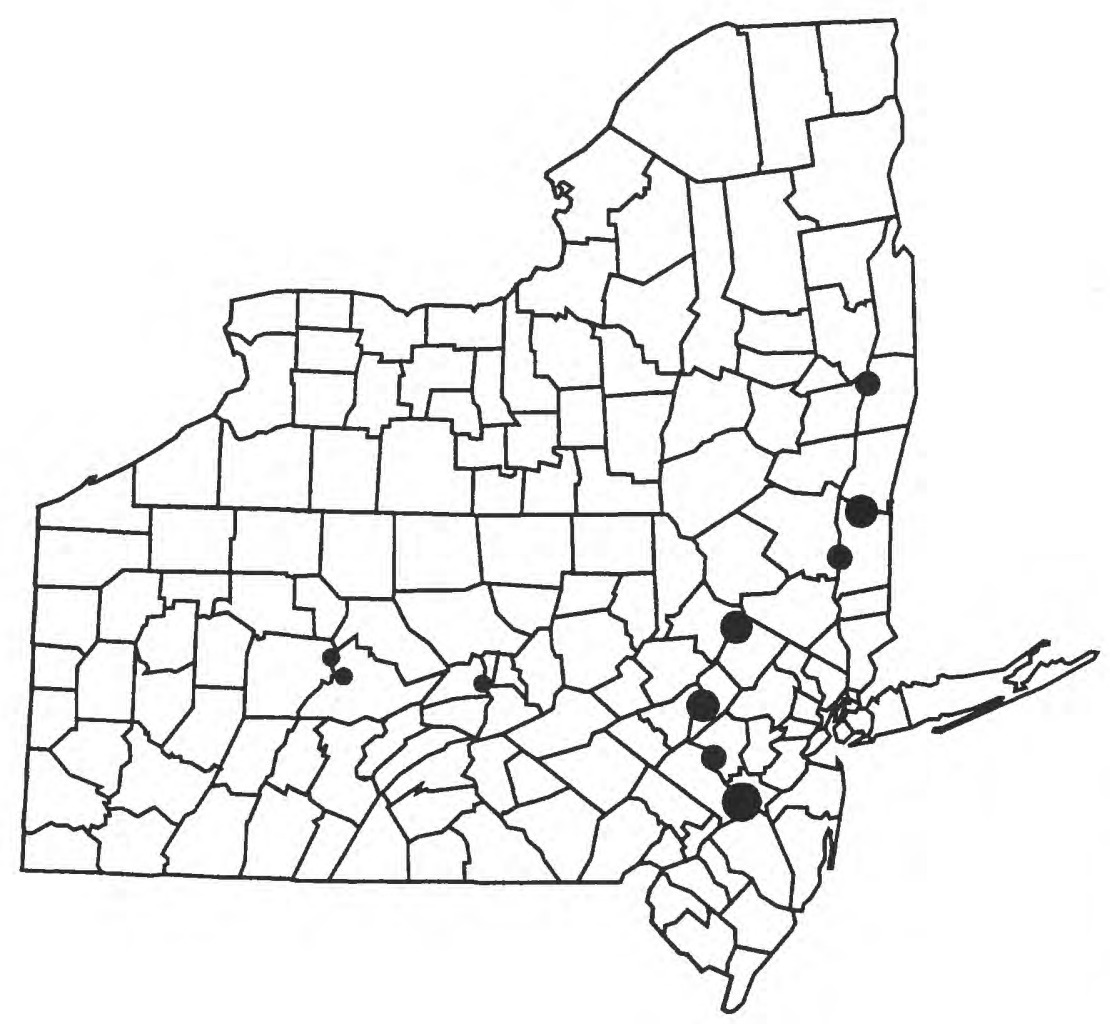

EXPLANATION

- $0-100$

$100-200$

$200-300$

$300-400$

Figure 1.-Sediment concentration, $\mathrm{mg} / \mathrm{L}$ 


\section{Program: MAPPING}

Plot Specifications: Area Shade Map; List of States; County Overlay; Data Aggregation by County Mean; 1 Title (Centered); 1 Footnote (Left Justified); Explanation Block

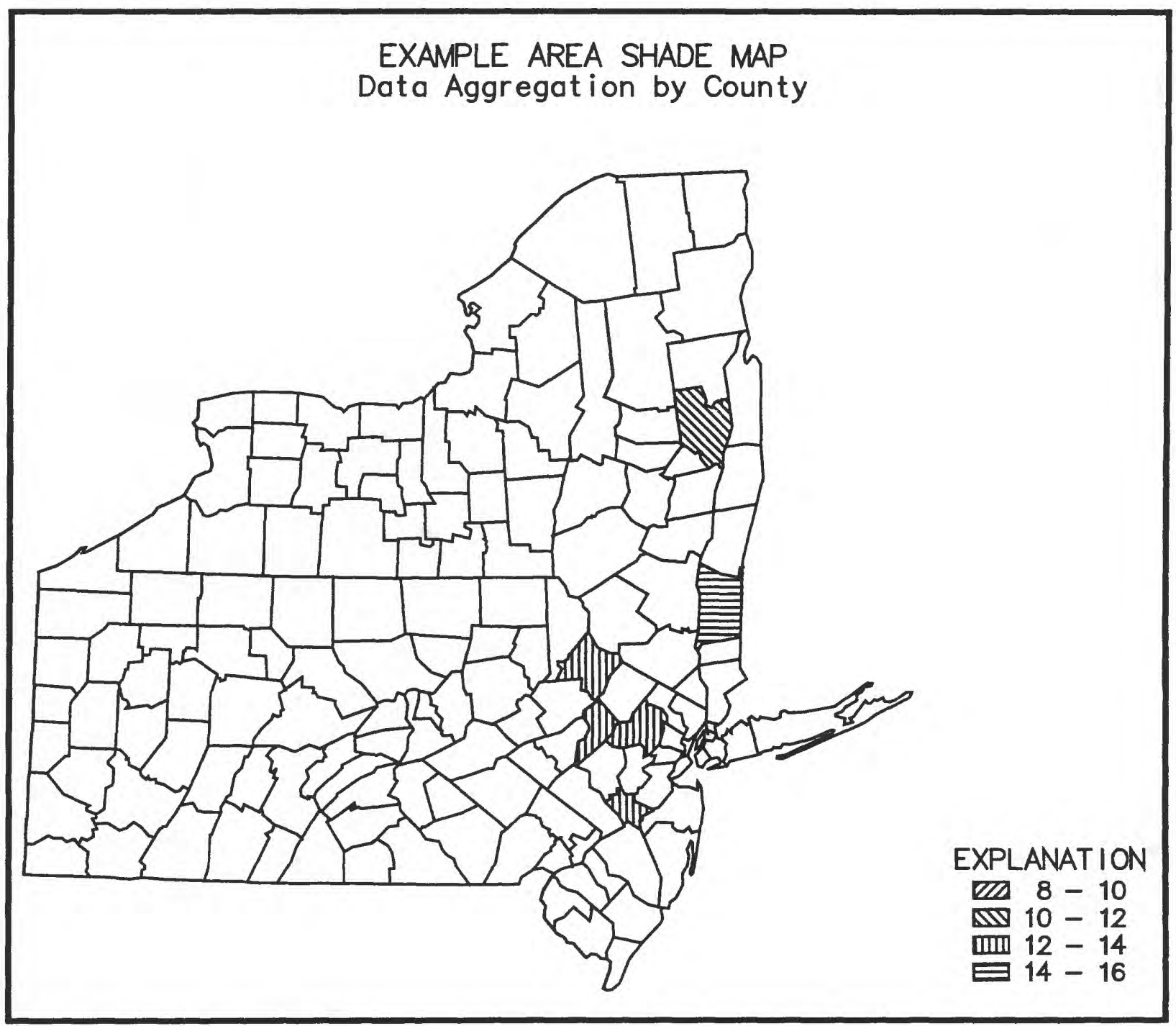


Program: MAPPING

Plot Specifications: No Data Map; Script File Used as Input File for Station Location and Station Labeling Information; USA + Territories Primary Boundary; State Overlay; Two Titles

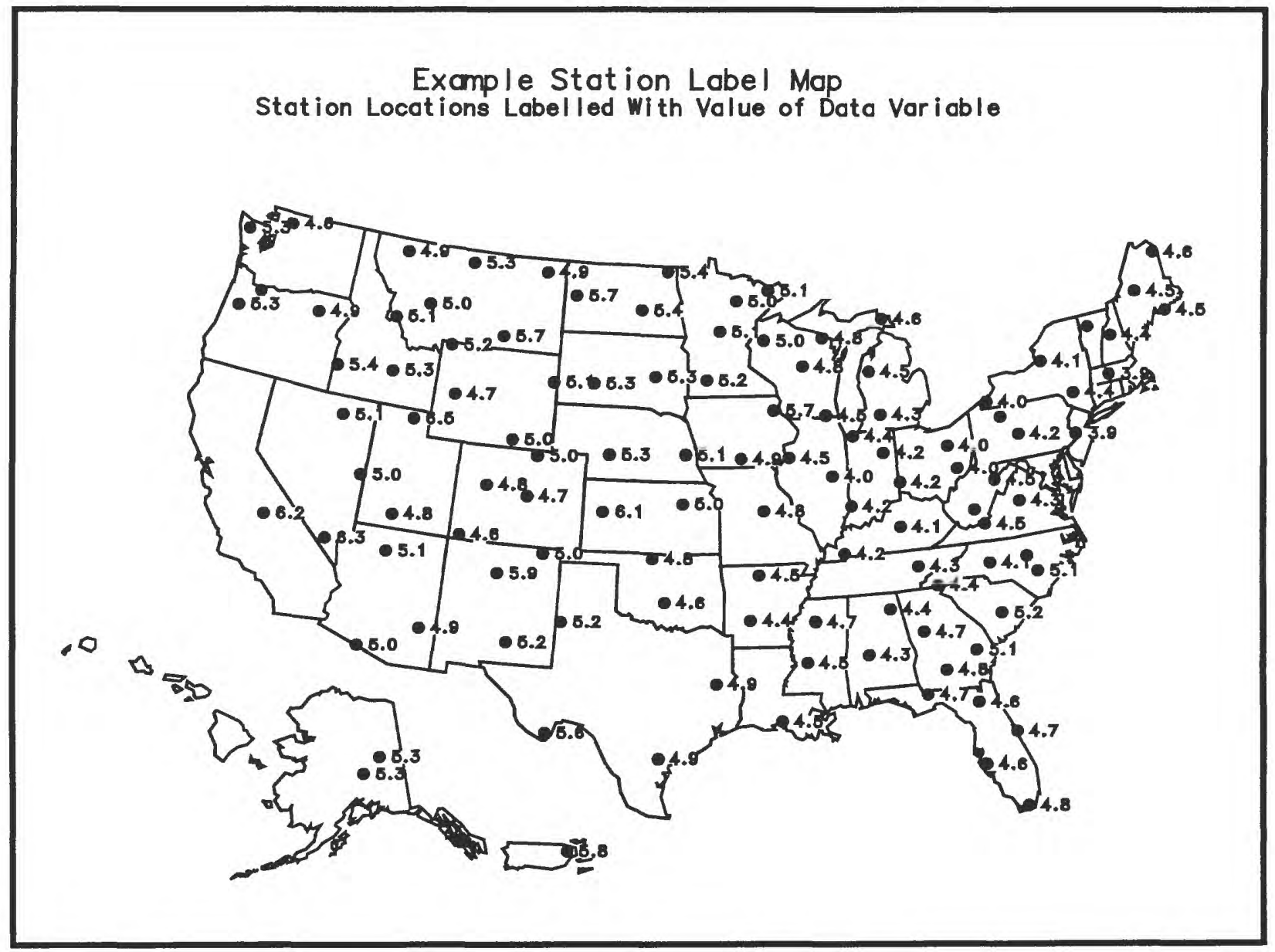




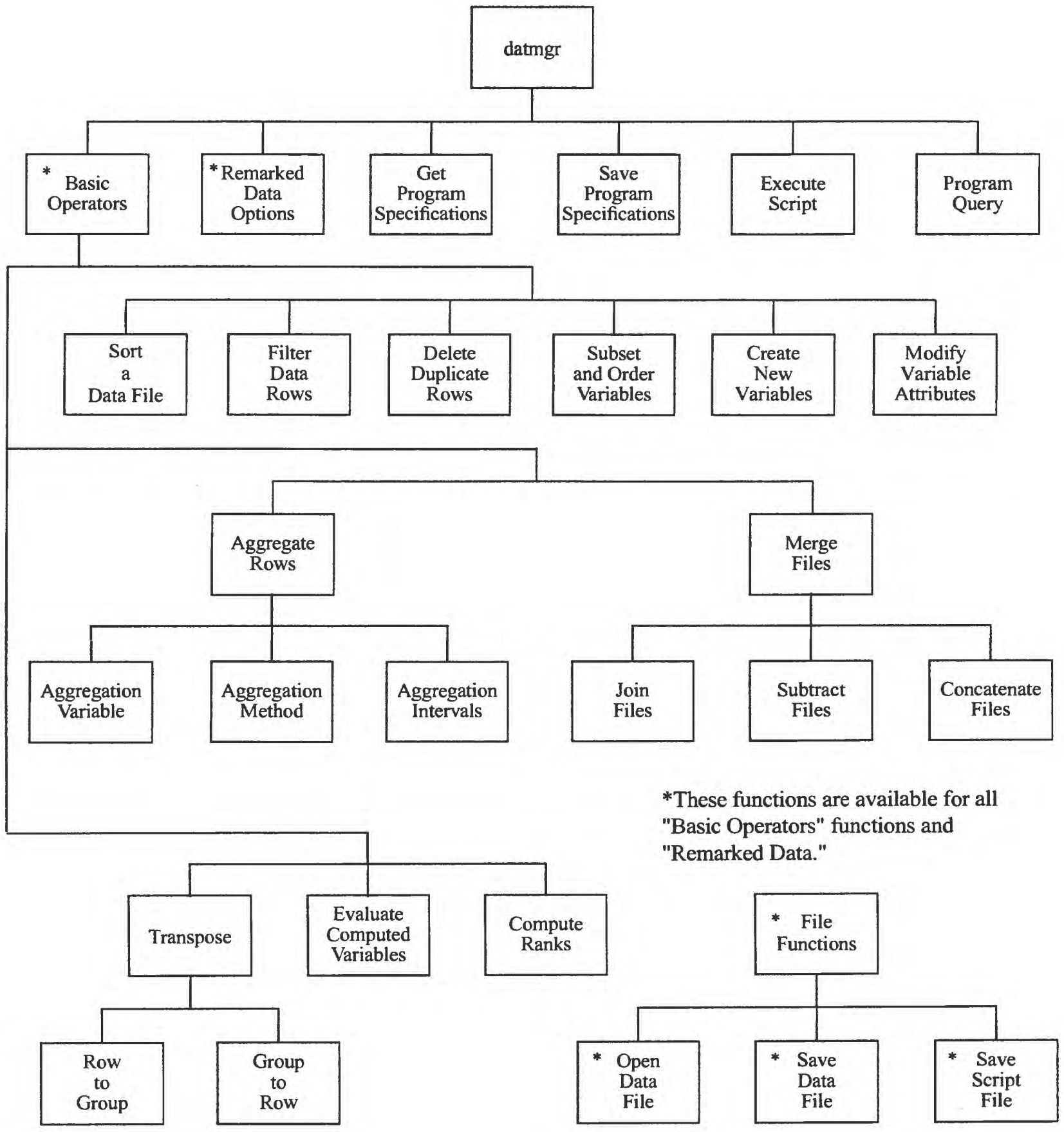




\section{DATA MANAGER (datmgr)}

Data Manager (datmgr) is an interactive program providing a toolkit for modifying data files used with QWGRAF programs or other programs that use RDB files (see "The RDB File" discussion in Appendix C). The "datmgr" program is actually an interface to the RDB system, which consists of ASCII files in the RDB file format and a library of data manipulation operators. Scripting languages, such as Perl and Python, can be used with the RDB system. The interface, which has the same look and feel as the other QWGRAF programs, minimizes the need to learn and use the complex syntax of a scripting language to access the files. Output from the "datmgr" program is either or both an RDB data file or a script file; both of which can be used as input files for QWGRAF programs.

\section{datmgr Tutorial}

Four interactive tutorials are available to guide the user through many of the options available in the "datmgr" program. A tutorial is activated by first typing "Shift @" in the "Opening Screen" of a new "datmgr" session. When the dialog box comes up, enter the file name found in the tutorial.doc file distributed with the QWGRAF programs. This file is located in the doc subdirectory, which provides specific details on using this and other QWGRAF tutorials.

The first tutorial is based on an actual case study where a raw data file containing "junk" text in the data fields is processed through several steps to produce a script file to be used as input to the QWGRAF mapping program (see description of tutorial for the mapping program). The steps involve sorting, filtering out inappropriate observations, creating some new variables, aggregating the data to an "average" value, joining with an auxiliary site location file, and outputting the result as a script file, which is used as the input file to the mapping program.

The second tutorial converts a QWFLATOUT file (".qwd" file extension) and its associated format definition file (".qwv" extension) to RDB format.

The third tutorial converts an ASCII file (".dta" file extension) and its associated format definition file (".dtv" extension) to RDB format. New variables defined in the format definition file, but not actually in the data file, are computed and added to the RDB file.

The fourth tutorial also converts the same ASCI file above to RDB, however, this time the new variables defined in the format definition file are not computed and added as columns in the RDB file. Instead, the algorithms themselves are transferred to the header definition line in the RDB file. A QWGRAF program then computes these variables as needed in the procedure.

The tutorials prompt the user for specific keystrokes. The programs will only respond when the correct keystroke sequence is entered; that is, incorrect information cannot be entered. Text for labels, titles, and so forth, can be entered in either upper or lower case. The system converts everything to upper case.

\section{The "Opening Screen"}

The Data Manager program is invoked by entering "datmgr" on the command line. The first screen to appear is the "Opening Screen," which lists the following options:

$\begin{array}{ll}\text { Get } & \text { - specifications from file } \\ \text { Save } & \text { - current specifications } \\ \text { Basic } & \text { - operators } \\ \text { Remarked } & \text { - data options } \\ \text { Execute } & \text { - a script } \\ \text { Query } & \text { - for general information } \\ \text { Return } & \text { - to operating system. }\end{array}$


The "Basic - operators" option is the main gateway to the eleven primary operators supported by the "datmgr" program. The other options apply to all of the primary operators in a global sense, especially the "Remarked - data options" option, which should be used before any of the primary operators to retain or delete remark codes.
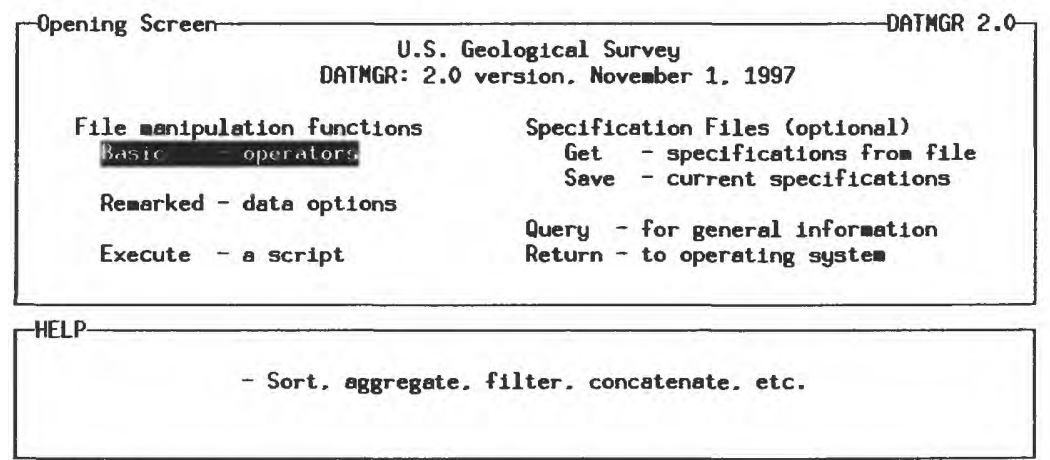

\begin{tabular}{|c|} 
INSTRUCT- \\
Select an option using arrow keys \\
then confirm selection with the F2 key. or \\
Type the first letter of an option.
\end{tabular}

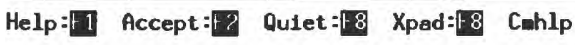

\section{Basic Operators}

The "datmgr" program provides eleven operators for modifying data files. These operators utilize RDB operators and functions, and thus, are available in command line mode (see Appendix $\mathrm{F}$ for a complete description of the functions and their usage syntax).

Within a given "datmgr" session, each operator can be invoked in any sequence as many times as required. Output from each operator is either an RDB file or a Script file, or both. Output from one operator usually is used as input to the next operator. Script from the current operator is appended to script from the previous operator, which was appended to script from its preceding operator in repeating series. Thus, care should be taken to sequence the operators logically when creating a complex script.

From the "Opening Screen," select the "Basic (B)" screen. The following operators are listed:

$\begin{array}{ll}\text { Sort } & \text { - a file } \\ \text { Aggregate } & \text { - a file } \\ \text { Row } & \text { - filtering } \\ \text { Variable } & \text { - subset/reorder } \\ \text { Create } & \text { - new variables } \\ \text { Evaluate } & \text { - computed variables } \\ \text { Modify } & \text { - variable attributes } \\ \text { Transpose } & \text { - row to group / group to row } \\ \text { Merge } & \text { - files (join, subtract, or concatenate) } \\ \text { Delete } & \text { - duplicate rows } \\ \text { Compute } & \text { - ranks (percentiles) } \\ \text { Return } & \text { - to opening screen. }\end{array}$

From the "Basic (B)" screen, enter the first letter(s) (not case sensitive) of the operator or use the arrow keys to select the operator and then use Enter.

When finished, type "R" (or " $r$ ") to return control to the "Opening Screen" or use the arrow keys to select the "Return to opening screen" option and use Enter. 

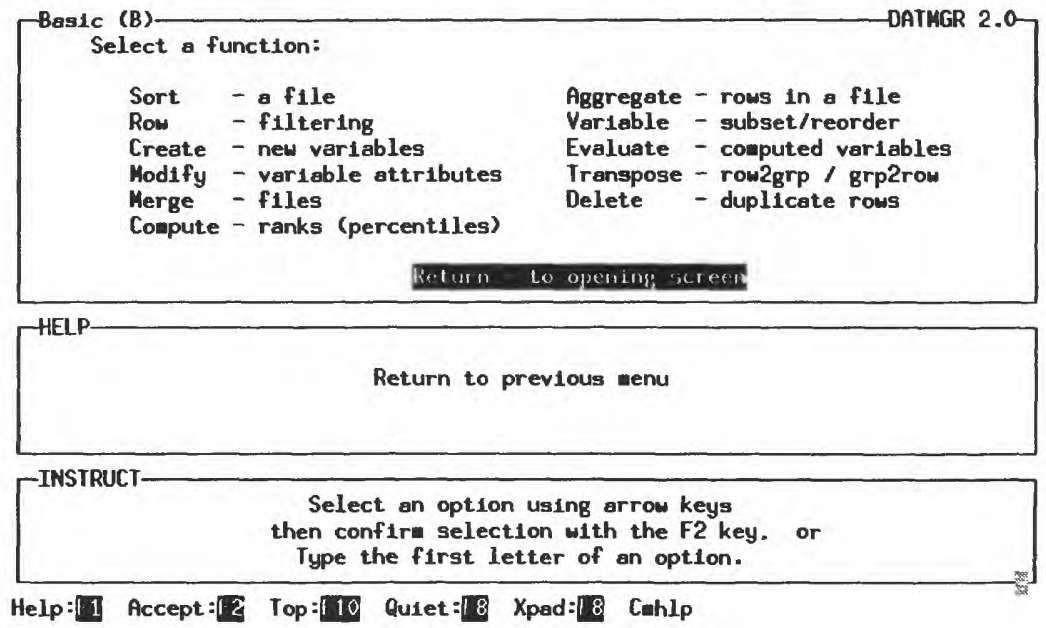

\section{Get Specifications}

To use specifications from a previous "datmgr" session, load a "Specifications" file (see discussion of "Specifications Files" in the section "Steps to Using QWGRAF Programs"). From the "Opening Screen," select the "Get (G)" screen. A list of specifications files (extension ".dmg") and a field for specifying a file name are presented. Use the arrow keys to select the required file and use either Accept (F2) or the Enter key to accept the selection. The "latest.dmg" file includes the specifications used for the most recent execution of the "datmgr" program, whether in the current or previous session.

The "Specifications" file includes the name of the input data file used when the file was created. If a new input data file is required, select the new file using the "Open input data file" option from the "Opening Screen."

\section{Save Specifications}

Program specifications, whether manually entered or retrieved from a "Specifications" file, can be changed as many times as required during the current session. Specifications can be saved for reuse by either overwriting an old "Specifications" file or creating a new one. From the "Opening Screen," select the "Save (S)" screen. Enter a file name or pathname and file name. Use either Accept (F2) or the Enter key to accept the file name. The system prompts the user for permission to overwrite an existing file.

\section{Input Files}

Each of the primary operators available in the "datmgr" program requires that an input file be specified. The input file can be a data file in one of the formats supported by QWGRAF, including RDB, or a script file discussed subsequently. For the first operator invoked during a given "datmgr" session, the field used to specify the input file name in the respective "File (B*F)" screen will be blank and a file name must be entered and selected. For each subsequent operator invoked in the current session of "datmgr," the output file from the last operator becomes the default input file for the new operator. The default file name can be changed. NOTE: If the user stays within a given operator after executing an operator and creating an output file of the results, the input file name for the next execution of the operator is the original input file name, not the output file name just created. The user must return to the "File $\left(\mathrm{B}^{*} \mathrm{~F}\right)$ " screen to specify and select the input file required. 


\section{Output Options}

The "datmgr" program provides an output option for each operator. For example, if "remarked (censored)" data are removed from the input file using the "Remarked Data Options" operator; then selected columns are removed from the file using the "Subset and order variable" operator followed by aggregating the data by State code using the "Aggregate" operator; it will be necessary to specify an output option for each of these operators. The output can be either an RDB data file or a script file (see discussion subsequently). Note that the actual file manipulation process is initiated when the name of the output file is accepted. To abort the execution of the current operator, blank out the output file name with a single space followed by using Accept (F2) or Enter, or respond with "no" to the prompt asking permission to overwrite a file.

\section{RDB Script Files}

An alternative to specifying an RDB data file as output is to create a script file. A script file is an RDB file (.rqs extension) but contains no data lines. Scripts of the RDB operators and arguments are stored in sequence in the RDB comment lines at the beginning of the file. The script is created in a continuous background process, whether or not an actual script file is created. The script is appended as each operator is invoked. If the input file used for a "datmgr" operator also is a script file, it is appended with the script generated in the current operator. Thus, if the output file is also to be a script file and the name specified is the same as the input script file, then the new file contains the original script plus the appended script from the current operator.

\section{Execute a Script File}

Once a "Script" file has been created, "datmgr" provides an option for executing the script in order to review the results before the script is used as an input file for another "datmgr" operator or QWGRAF program. The "Execute - a script" option in the "Opening Screen" creates an RDB file containing the final form of the data as processed by the script. Any new variables created as a result of the scripting process, but whose values are not actually required by the scripting process are not evaluated. The algorithm(s) used to create these variables, along with the variable characteristics (name, data type, precision), however, are stored in the RDB file header lines as described in the discussion of the RDB system and its WRD extensions in Appendix C. Computed variables are only evaluated when specifically required by a QWGRAF program.

From the "Opening Screen," select the "Execute - a script" option to bring up the "Execute (E)" screen. Select the "Input - script file" option to bring up the "Input (EI)" screen, which lists the script files (".rqs" extension) in the current directory. Type in or select the script file to be executed. When the file selected is accepted (use Accept (F(2) or Return), control is returned to the "Execute (E)" screen. Execute the script by selecting and accepting the "Execute - a script" option. The system will display the "Execute (EE)" screen in which you will enter a name for the RDB file that will contain the results of the script process. The system automatically adds the ".rdb" extension. 

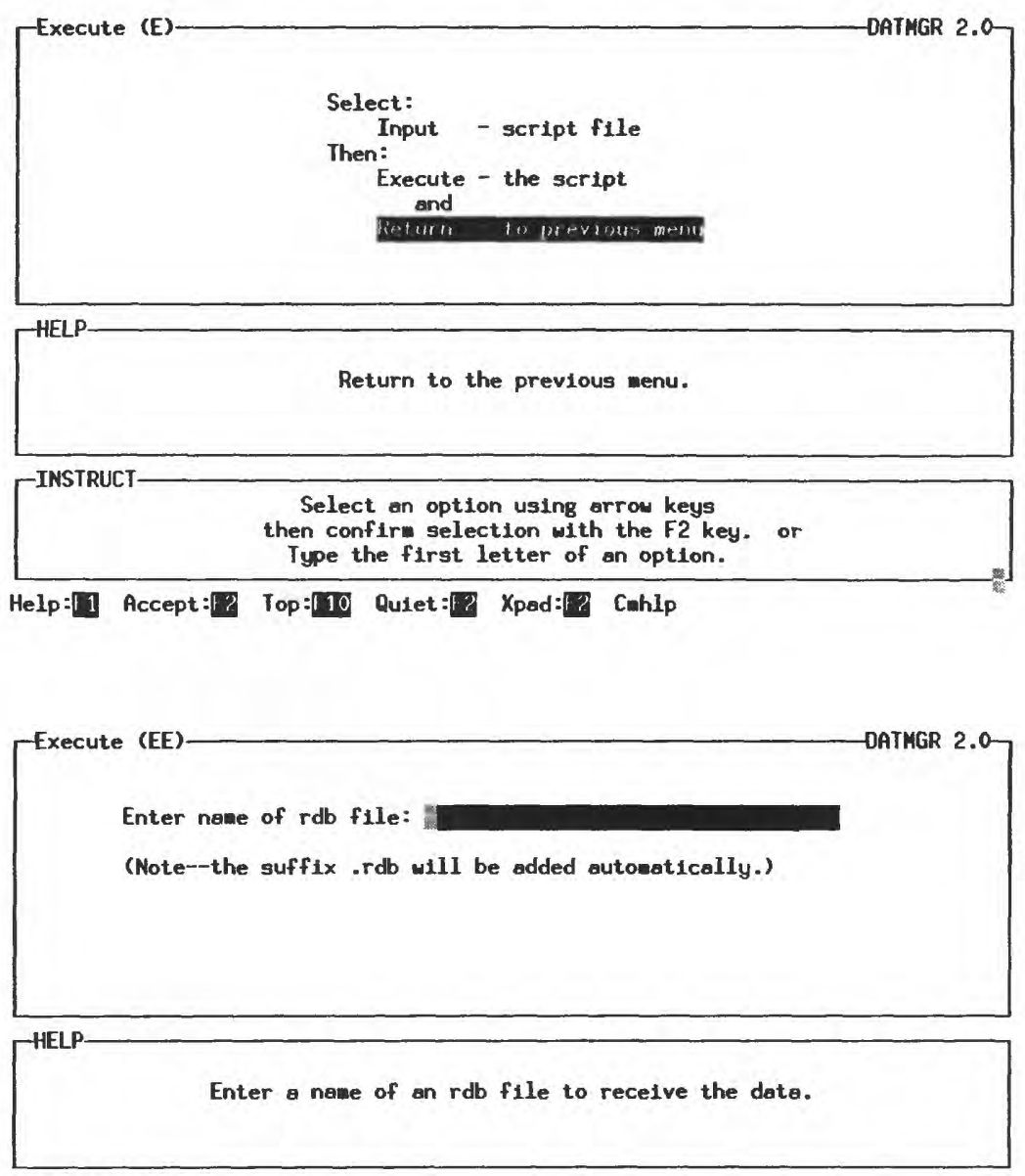

\footnotetext{
INSTRUCT- Enter data in highlighted field(s).

Use carriage return or arrow keys to enter data and cove between fields. Use "Accept" comand to go to next screen when done entering data.

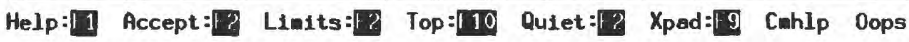

\section{Executing the Script from Command Line}

A script can be executed from the command line as in the following examples:
rdbqry <file_name.rqs
Output to screen
rdbqry <file_name.rqs | ptbl
(computed variables not evaluated)
Pipe to "ptbl" for output to screen
(computed variables not evaluated)
rdbqry <file_name.rqs $\mid$ compcol $-\mathrm{v} \mid \mathrm{ptbl}$
Pipe to "compcol" to evaluate
computed variables; pipe to "ptbl".

\section{Script Files as Input Files to QWGRAF Programs}

A script file can be used as input to any QWGRAF program. When the program is executed, the script is executed first reconstructing the data manipulation operators used to produce the required data file. 


\section{Remarked (Censored) Data Options}

The "Opening Screen" provides an option for remarked (censored) data. This option is different than similar options available in the other QWGRAF programs because it provides for modifying or removing the remark codes assigned to the censored values as opposed to just including or excluding the data from an analysis. From the "Opening Screen," select the "Remarked (R)" screen.

\section{Select Input Data File for Censored Data}

From the "Remarked (R)" screen, select the "File (RF)" screen. The file selected here could be a script file to which additional script will be added. If a data file or script file was created by a previous "datmgr" operator, its file name will appear in the "Enter file name" field; otherwise this field will be blank. Select or type in a new file name and use either Accept (F2) or the Enter key to accept the file name and return control to the "Remarked (R)" screen.

\section{Remarked Data Options}

The following options are provided for handling censored data values, including values computed from censored values:

Keep remark codes - Remark codes will be retained with the detection limit (reporting) values.

Delete remark codes - The remark codes will be removed from the data. If this option is selected, one of the following options must be specified.

a. Retain value - The detection (reporting) limit values will be retained and considered real values.

b. Set as missing - The value is removed from the file and the field is set as "missing."

Set ingest default - Use the above specification as the remark handling option for the filters converting other data formats to RDB.

From the "Remarked (R)" screen, select the "Remark (RR)" screen from which the remarked data options are specified. Note that when the "Delete remark codes" option is selected and toggled "On" with the space bar, the screen changes to bring up two additional options. When finished, use Accept (F2) to return control to the "Remarked (R)" screen. Use Accept (F2) again to return control to the "Opening Screen."

The option selected can be applied to just the current session of "datmgr" or it can be set as the default remark handling option when other data formats are converted to RDB. To set this option, use the space bar to Toggle "On" the "Set ingest default" option. This option will remain in effect until changed in another "datmgr" session. 

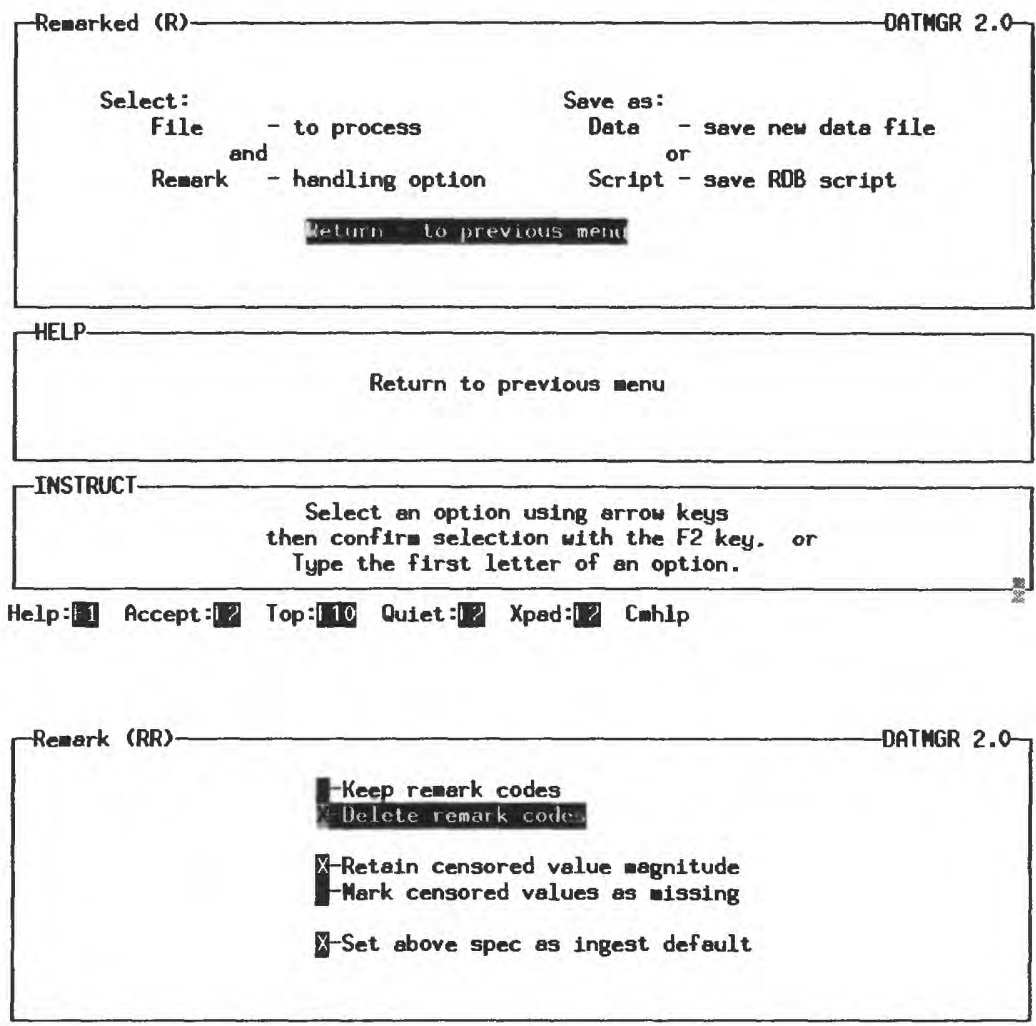

\footnotetext{
Eliminate remark codes frow file. If you select this box you nust also specify the disposition of censored values in the file.

TINSTRUCT

Option field: use space bar to toggle between $O N(X)$ and OFF ( ). Use carriage return or arrow keys to wove between fields.

Use "Accept" comend to go to next screen when done entering data.

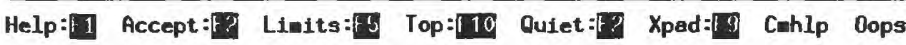

\section{Save an RDB Data File from "Remarked Data" Option}

To create an output data file from the "Remarked Data options" process, select the "Data (RD)" screen from the "Remarked (R)" screen. Enter a file name in the field. Use either Accept (F2) or the Enter key to accept the selection. The system appends the ".rdb" extension to the file name. The system prompts the user for permission to overwrite an existing file. Pathnames may be used to place the resultant file in a selected directory.

\section{Save an RDB Script File from "Remarked Data" Option}

To save the script from the "Remarked data options" operator, save it to a new script file or append it onto an existing script file. From the "Remarked (R)" screen, select the "Script (RS)" screen. If the input file to this operator was an RDB script file, its file name will appear in the entry box. Use Accept (F2) or the Enter key to accept this selection. The new script file will contain the old script with the new script appended. If a new script file is required, enter a new file name and use Accept (F2) or the Enter key. The system appends the ".rqs" extension to the file name. The system prompts the user for permission to overwrite an existing file. Pathnames may be used to place the resultant file in a selected directory. 


\section{Sort a Data File}

The "Sort" operator provides for sorting a data file by one or more variables. Each sort field can be designated as either "ascending" or "descending." From the "Basic (B)" screen, select the "Sort (BS)" screen.

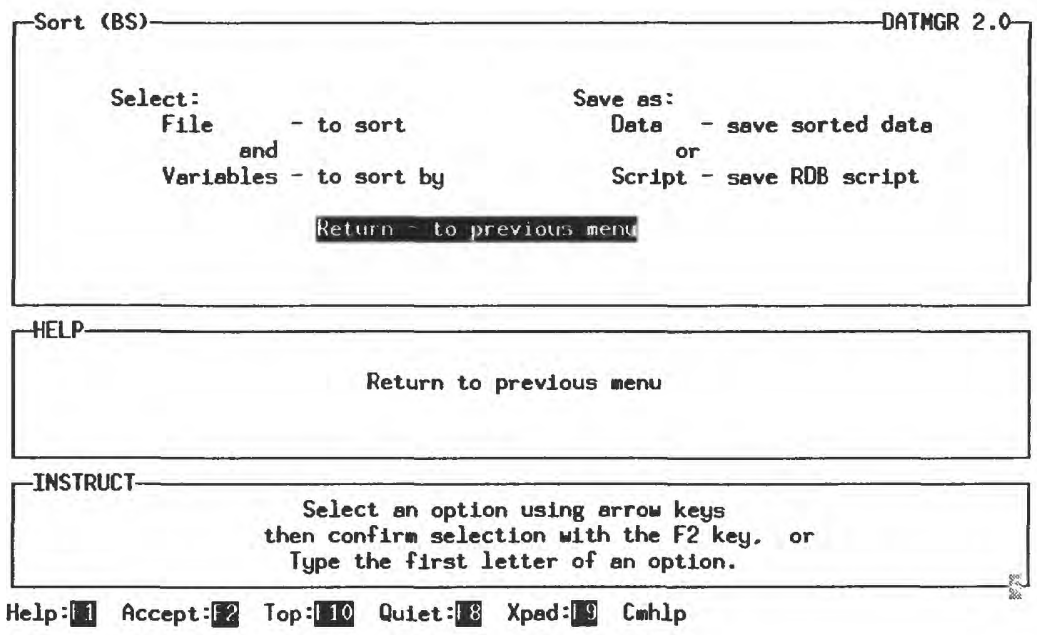

\section{Select a File to be Sorted}

From the "Sort (BS)" screen, select the "File (BSF)" screen for selecting the data file to be sorted. This file could be a script file to which additional script for the sort operator will be added. If a data file or script file was created by a previous "datmgr" operator, its file name will appear in the "Enter file name" field; otherwise this field will be blank. Select or type in a new file name and use either Accept (F2) or the Enter key to accept the file name and return control to the "Sort (BS)" screen.

\section{Select Sort Variables}

From the "Sort (BS)" screen, select the "Variables (BSV)" screen for naming the sort variables. The sort variables are marked for selection in the order of nesting with the highest level (or primary) sort variable being given a value of "1" in the "Select" column. For an ascending sort, the sort order value is preceded with a " + " sign (the plus sign is optional), while a descending sort order value is preceded with a "-" (minus) sign. Use Enter to highlight the next variable.

\section{Save an RDB Data File}

To create an output data file from the "Sort a data file "operator, select the "Data (BSD)" screen from the "Sort (BS)" screen. Enter a file name in the field and use either Accept (F2) or the Enter key to accept the selection. The system appends the ".rdb" extension to the file name. The system prompts the user for permission to overwrite an existing file. Pathnames may be used to place the resultant file in a selected directory.

\section{Save an RDB Script File}

To save the script from the "Sort" operator, save it to a new script file or append it onto an existing script file. From the "Sort (BS)" screen, select the "Script (BSS)" screen. If the input file to this operator was an RDB script file, its file name will appear in the entry box. Use Accept (F2) or the Enter key to accept this selection. The new script file will contain the old script as well with the new script appended. If a new script file is required, 
enter a new file name and use Accept (F2) or the Enter key. The system appends the ".rqs" extension to the file name. The system prompts the user for permission to overwrite an existing file. Pathnames may be used to place the resultant file in a selected directory. Select the "Return to previous menu" option to return control to the "Basic (B)" screen.

\section{Aggregate a Data File}

Aggregation is the process whereby rows of a data set are grouped by the values of a partitioning (grouping) variable and then condensed to a single row where the value of each observation in a column is a computed statistic of the condensed rows, such as mean, median, or observation count. The determination of the number of groups to be created and the value ranges of the partitioning variable that these groups represent can be accomplished in the following ways:

1. The number of groups can be set equal to the number of unique values of the partitioning variable.

2. The range of values of the partitioning variable can be divided into a userdefined number of groups, each group of which has an equal range of values of the partitioning variable. The minimum and maximum values of the partitioning variable can be determined by the system or the user can select the minimum and maximum values.

3. Specific interval ranges are defined, where the ranges between intervals (groups) need not be the same and can even overlap. The number of intervals is equal to the number of individual ranges defined.

From the "Basic (B)" screen, select the "Aggregate (BA)" screen, which lists the following functions:

$\begin{array}{ll}\text { File } & \text { - to aggregate } \\ \text { Variable } & \text { - to control aggregation } \\ \text { Method } & \text { - for combining records } \\ \text { Intervals } & \text { - of aggregation } \\ \text { Censored } & \text { - data option } \\ \text { Save } & \text { - data file } \\ \text { Save } & \text { - script file. }\end{array}$
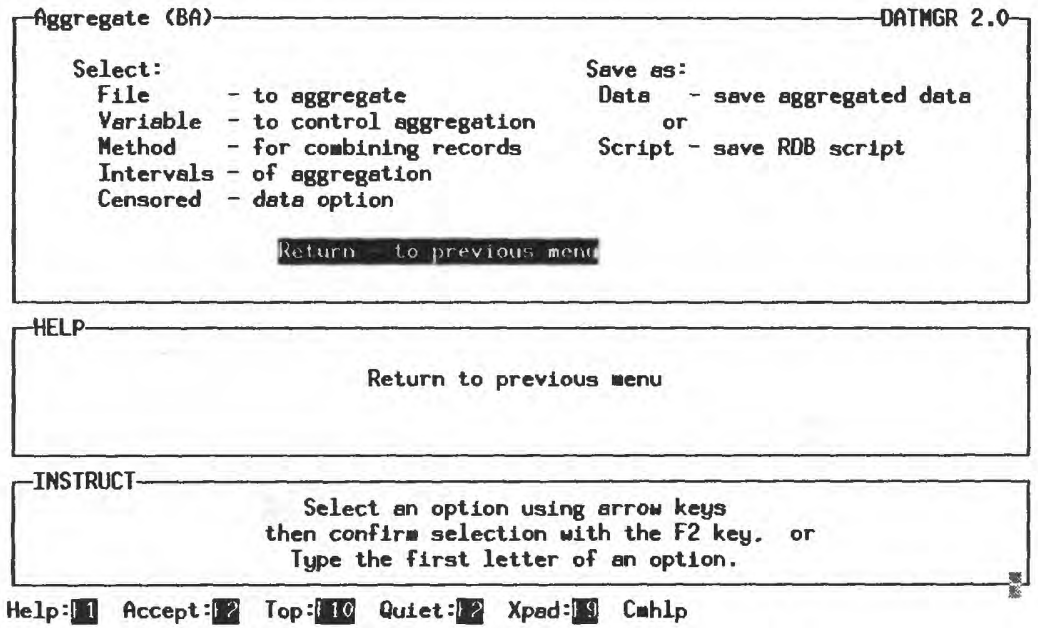


\section{Select Input File to Aggregate}

From the "Aggregate (BA)" screen, select the "File (BAF)" screen from which the input data file is selected. This file could be a script file to which additional script for the aggregate operator will be added. If a data file or script file was created by a previous "datmgr" operator, its file name will appear in the "Enter file name" field; otherwise this field will be blank. Select or type in a new file name and use either Accept (F2) or the Enter key to accept the file name and return control to the "Aggregate (BA)" screen.

\section{Select Variables to Control Aggregation}

From the "Aggregate (BA)" screen, select the "Variable (BAV)" screen from which the partitioning or grouping variables are selected. Use the arrow keys and the Enter key to select up to four variables. For more than one partition variable, order the variables from the highest to the lowest level of nesting by entering $1,2,3$, or 4 in the "select" column field to the left of the appropriate variable names. A variable can be deselected by entering a " 0 " (zero). If more than one aggregation variable is selected, the only "Interval type" option available is "Unique." Use Accept (F2) to return control to the "Aggregate (BA)" screen.

Caution: The aggregation process can have unexpected results when using "real" variables as aggregation variables. The system only uses a certain number of digits in a real number and any number with a larger number of digits is truncated to the number of digits used by the system. Thus, using large real variables as aggregation variables can result in aggregation groups containing numbers not appropriate for that group. This situation may occur when an integer number, such as a date or station ID, is declared a real variable. Such numbers with different digits in the last few positions can be considered "equal" by the system, resulting in their being assigned to the wrong group, such as when the group is based on "unique" values of the aggregation variable. To avoid this situation, use double precision real or integer variables.

\section{Method of Combining Records}

From the "Aggregate (BA)" screen, select the "Method (BAM)" screen to select whether a single aggregation method is to be applied globally to all variables or whether each variable is to be assigned its own method. A global method should be selected first followed by assigning alternate methods to selected variables, if required.

\section{Single (Global) Aggregation Method}

Select the "Global (BAMG)" screen, which lists the following methods of aggregation:

Average (Mean) (AVG)
Sum (SUM)
Median (MED)
Range (RNG)
Maximum (MAX)

Minimum (MIN) Observation Count (CNT)

Standard Deviation (STD)

Variance (VAR)

Coefficient of Variation (COV).

Use the arrow keys to select the method desired and use Accept (F2) to accept the selection and return control to the "Method (BAM)" screen. Select "Return to previous menu" option to return control to the "Aggregate (BA)" screen. 

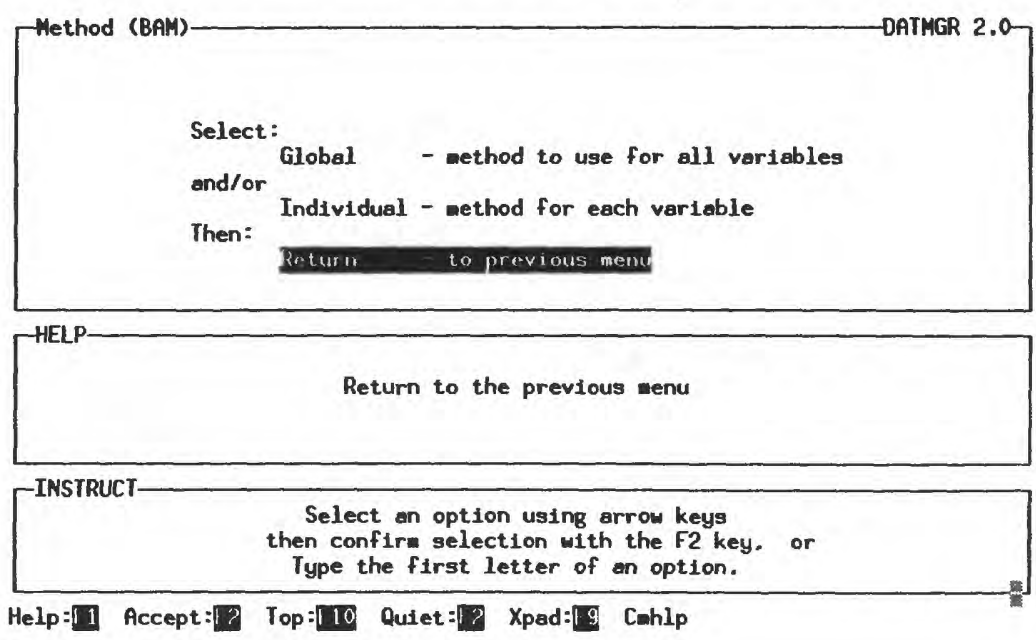

Help: 11 Accept: I? Top: 10 Quiet: 1 Xpad: 9 Cahlp

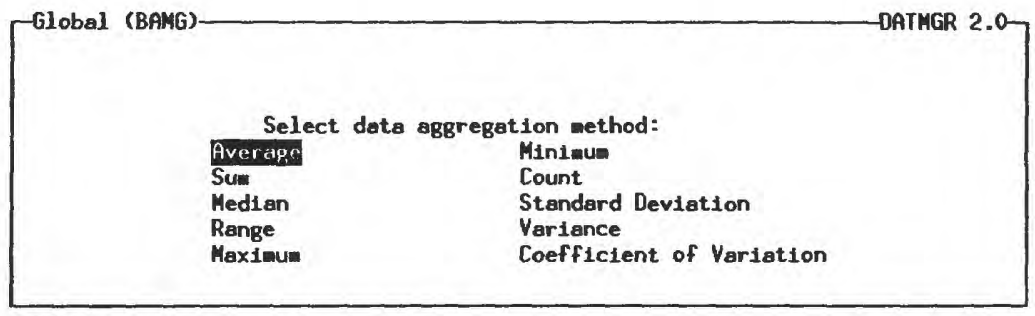

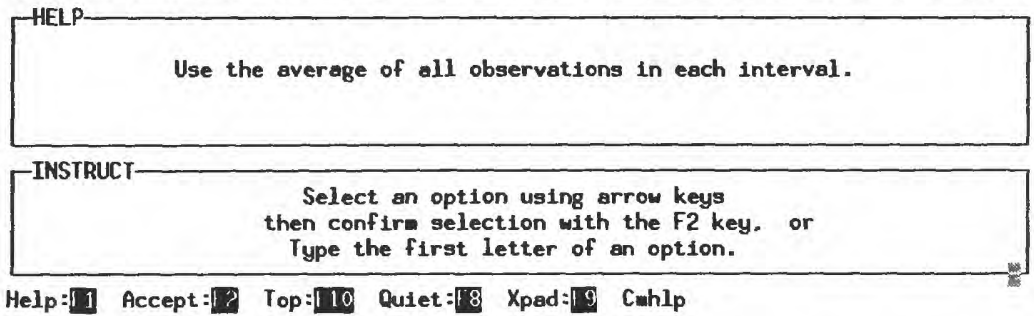




\section{Individual Variable Aggregation Method}

Select the "Individual (BAMI)" screen. For each variable to be aggregated by a method other than the default method indicated in the "default" column, type in the selected three-character code. Use Help (F1) to view the "Help" panel with a list of the three-character codes. Use Accept (F2) to return control to the "Method (BAM)" screen. Select "Return to previous menu" option to return control to the "Aggregate (BA)" screen.

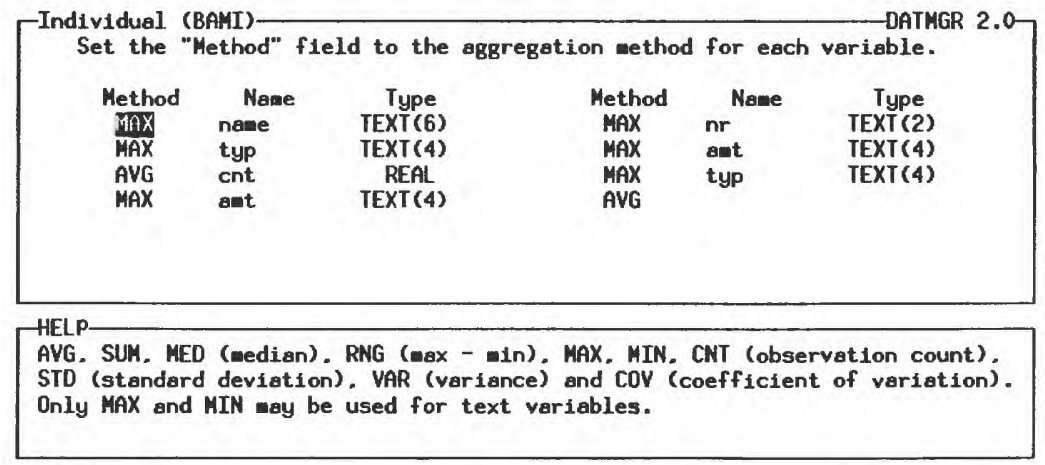

\footnotetext{
INSTRUCT- Enter data in highlighted field(s).

Use carriage return or arrow keys to enter data and wove between fields. Use "Accept" comeand to go to next screen when done entering data.

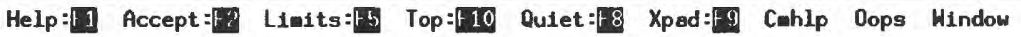

\section{Establish Interval Boundaries for the Aggregation}

From the "Aggregate (BA)" screen, select the "Intervals (BAI)" screen to select the method to determine the number of intervals to be computed and the range of values in each interval. The "Interval" methods are as follow:

$$
\begin{array}{ll}
\text { Unique } & \text { - values of aggregation variables } \\
\text { Equal } & \text { - size intervals } \\
\text { User } & \text { - defined intervals. }
\end{array}
$$
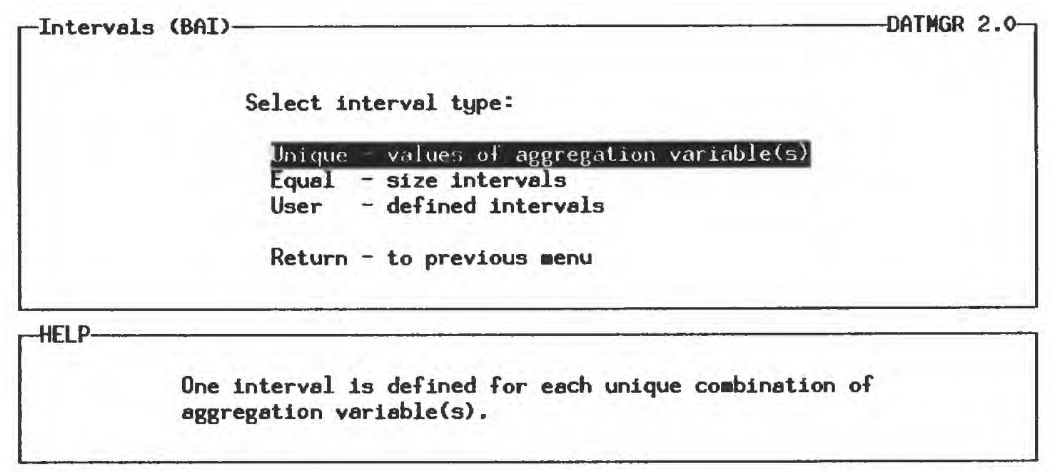

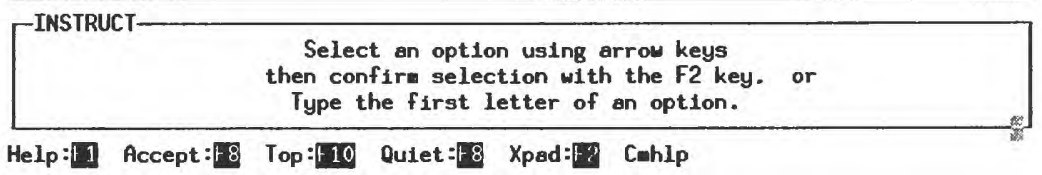




\section{Unique Values of Aggregation Variables}

From the "Intervals (BAI)" screen, select the "Unique" option if an interval is to be created for each unique value of the combination of aggregation variables. Note that "Unique" is the only interval option allowed when more than one variable is used to define the aggregation grouping.

In most cases, the unique values of the partitioning variables adequately define the number of intervals. However, it also is possible to further partition the unique values of any or all of the partition variables. For example, in the case of the Hydrologic Unit Code, there are four levels imbedded in the eight-digit integer number. If unique values of the entire number are used with the "Unique" option, the intervals would be based on the Cataloging Unit (eight digits). By using a special extension of the "Unique" option (select the "Unique (BAIU)" screen), it is possible to have the interval based on Region (the first two digits of the code), Subregion (the first four digits in the code), or Accounting Unit (the first six digits in the code). In the "Unique (BAIU)" screen, an integer divisor can be specified to truncate the eight-digit number to form the higher level partitioning factor; for example, use 1000000 for Region; 10000 for Subregion; and 100 for Accounting Unit. A different divisor can be specified for each partition variable. Use Accept (F2) to return control to the "Intervals (BAI)" screen.
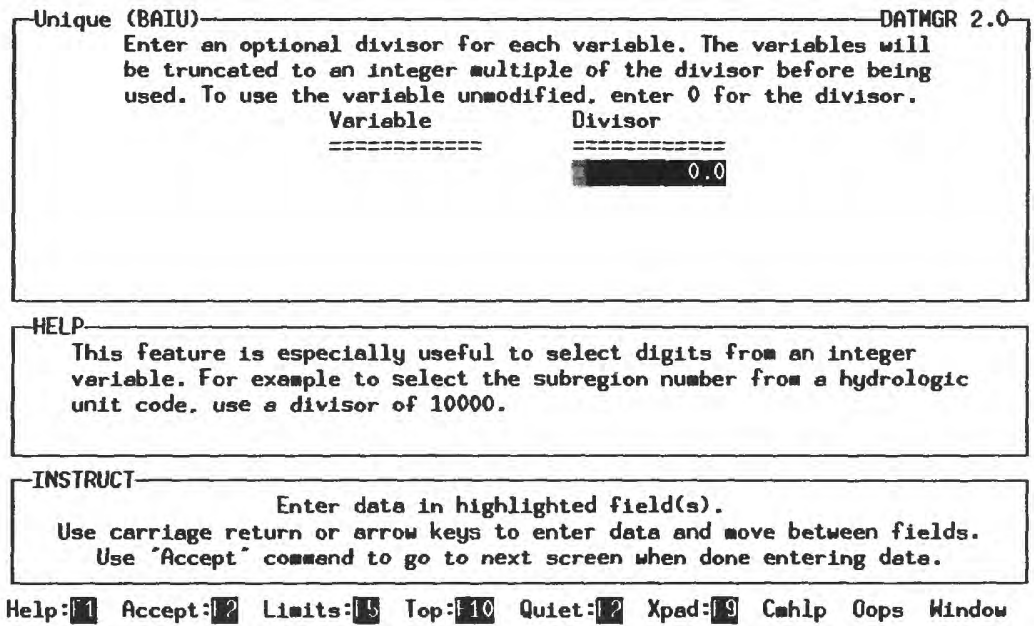

\section{Equal Intervals}

From the "Intervals (BAI)" screen, select the "Equal (BAIE)" screen. Use the arrow keys to highlight the "Number of Intervals" option. Change the default value as required. If the "Automatic" option is selected, the system will compute the range of the partitioning variable and divide this range into the number of intervals specified. Each interval will have an approximately equal range of values of the partitioning variable.

If the "Manual" option is selected, the user specifies the minimum and maximum values (range) of the partitioning variable. This range is then divided by the specified number of intervals to determine the equal magnitude range of each interval. Use Accept (F2) to return control to the "Intervals (BAI)" screen. 

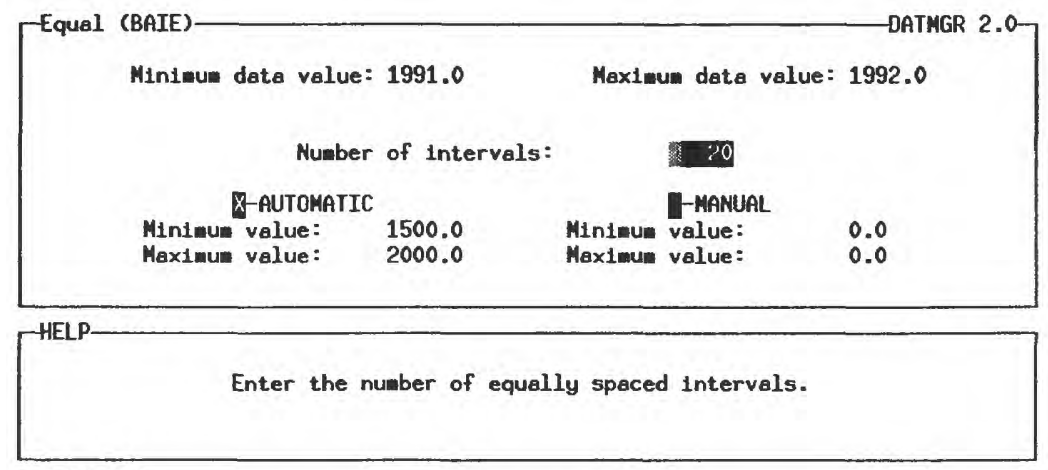

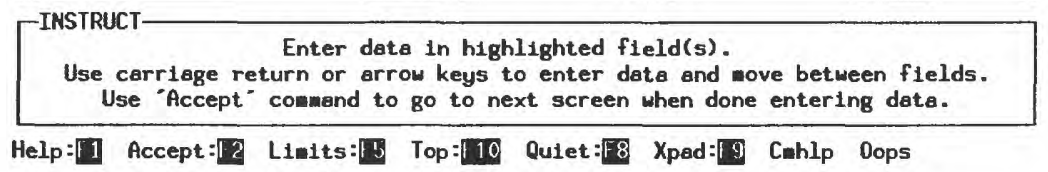

\section{User-Defined Intervals}

From the "Intervals (BAI)" screen, select the "User (BAIU)" screen. Use the Enter and arrow keys to enter minimum and maximum values for as many intervals as required. The intervals need not be contiguous. Use Accept (F2) to return control to the "Intervals (BAI)" screen. Select the "Return to previous menu" option to return control to the "Aggregate (BA)" screen.

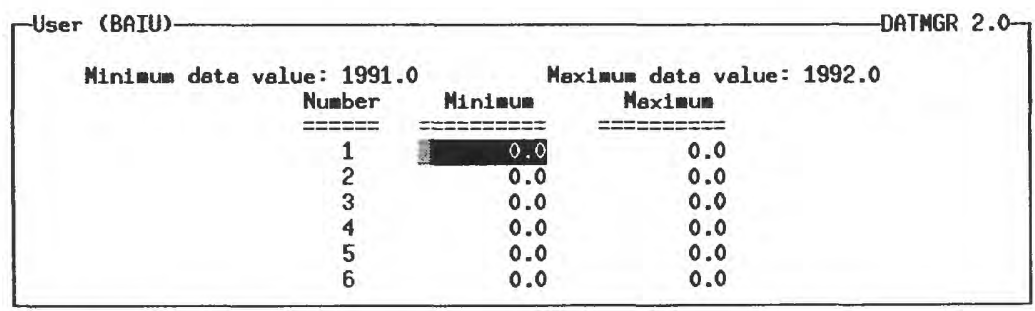

\footnotetext{
This field contains the iniwu value for each interval.
This
INSTRUCT- Enter data in highlighted field(s).
Use carriage return or arrow keys to enter data and wove between fields. Use "Accept" consand to go to next screen when done entering data.

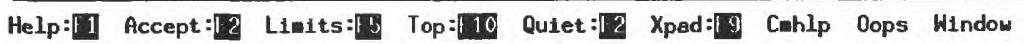

\section{Censored Data Option}

By default, the aggregate operator excludes censored data from the analysis. To include censored data in the aggregation, from the "Aggregate (BA)" screen, select the "Censored (BAC)" screen and then use the arrow or Tab keys to select (highlight) the "Include censored data" option. Use Accept (F2) to accept the selection and return control to the "Aggregate (BA)" screen. 
Save an RDB Data File

To create an output data file from the "Aggregate" operator, select the "Data (BAD)" screen from the "Aggregate (BA)" screen. Enter a file name in the field. Use either Accept (F2) or the Enter key to accept the selection. The system appends the ".rdb" extension to the file name. The system prompts the user for permission to overwrite an existing file. Pathnames may be used to place the resultant file in a selected directory.

\section{Save an RDB Script File}

To save the script from the "Aggregate" operator, save it to a new script file or append it onto an existing script file. From the "Aggregate (BA)" screen, select the "Script (BAS)" screen. If the input file to this operator was an RDB script file, its file name will appear in the entry box. Use Accept (F2) or the Enter key to accept this selection. The new file will contain the old script with the new script appended. If a new file is required, enter a new file name and use Accept (F2) or the Enter key. The system appends the ".rqs" extension to the file name. The system prompts the user for permission to overwrite an existing file. Pathnames may be used to place the resultant file in a selected directory. Select the "Return to previous menu" option to return control to the "Basic (B)" screen.

\section{Filter Data Rows}

The "Row" operator provides a filtering process for selecting rows in a data file that meet certain criteria. The criteria are specified in the form of Boolean expressions entered in the "Criteria (BRC)" screen. A comprehensive suite of Boolean Logic operators and connectors is available for constructing the filtering criteria. The criteria statement can be as simple or complex as required with appropriate use of connectors and parenthetical groups. Provision is made to either include or exclude rows on the basis of the criteria statement. From the "Basic (B)" screen, select the "Row (BR)" screen.

\section{Select Input Data File}

From the "Row (BR)" screen, select the "File (BRF)" screen. This file could be a script file to which additional script for the filter operator will be added. If a data file or script file was created by a previous "datmgr" operator, its file name will appear in the "Enter file name" field; otherwise this field will be blank. Select or type in a new file name and use either Accept (F2) or the Enter key to accept the file name and return control to the "Row (BR)" screen.

\section{Filter Criteria Syntax}

From the "Row (BR)" screen, select the "Criteria (BRC)" screen. Although at this time the syntax used for the "datmgr" filter criteria operator is based on the Perl scripting language, usually only the basic arithmetic operators and connectors are needed to construct the required search criteria. For a detailed description of Perl programming, see the textbook Programming Perl (Wall and Schwartz, 1991). The filter criteria syntax entered in the "Criteria (BRC)" screen is passed to an RDB operator, "perltbl" written in Perl. This operator is described in the RDB Operators section of Appendix C; Appendix $\mathrm{C}$ also provides details on the available operators and the usage syntax.

To view a list of variable names from the current input data file (or script) that might be used in constructing the filter criteria, use Intrpt (F6). Use Accept (F2) to return control to the "Criteria (BRC)" screen.

To view a list of the functions, operators, and connectors that are available for constructing the filter criteria, use Help (F1) if the "Help" panel is not already activated. Use the down-page key, Dnpg (;d), and the up-page key, Uppg (;u), to scroll through the list. When finished, use (F3) to reactivate the data panel. Note: depending on when the "Help" panel is activated, use Help (F1) again to activate the Dnpg and Uppg functions, the status of which is indicated in the "Instruct" panel at the bottom of the screen. 
The name of each variable used in the criteria expression must be prefaced with a "\$" sign. Some functions are prefaced with an " $\&$ " while others are not. The " $\&$ " prefaced functions are nonstandard Perl functions created especially for the "perltbl" operator.

\section{RDB Functions}

The following is a list of the commonly used RDB functions:

Function Description

\begin{tabular}{|c|c|}
\hline$+-* 1$ & Addition, subtraction, multiplication, and division \\
\hline$\$ a \% \$ b$ & Modulo division (remainder of variable $\$ a$ divided by variable $\$$ b) \\
\hline I \& 1 & Bitwise "or," "and," and exclusive "or" \\
\hline$\gg<<$ & Bitwise shift right, and bitwise shift left \\
\hline ** & Exponentiation \\
\hline$\$ a . \$ b$ & String concatenation (Append string variable $\$ b$ to variable $\$ a$ ) \\
\hline$\$ a \times \$ b$ & Value of variable \$a strung together b times \\
\hline$++\$ a$ & Increment variable $\$ a$ by 1 \\
\hline$-\$ \mathrm{a}$ & Decrement variable $\$ a$ by 1 \\
\hline$\| \& \&$ & Logical "or," "and" \\
\hline ! \$a & Logical "not" (True if variable \$a is not true) \\
\hline$=1=$ & Numeric equality and numeric inequality \\
\hline eq ne & String equality and string inequality \\
\hline$<$ & Numeric less than and numeric greater than \\
\hline lt $\mathrm{gt}$ & String less than and string greater than \\
\hline$<=>=$ & Numeric less than or equal to and numeric greater than or equal to \\
\hline le ge & ring less than or equal to and string greater than or equal to. \\
\hline
\end{tabular}

\section{RDB Numeric Functions}

The following is a list of the commonly used numeric functions available:

Numeric

Function

$\sin (\mathrm{X})$

$\tan (\mathrm{X})$

$\& \operatorname{acos}(\mathrm{X})$

$\operatorname{atan} 2(\mathrm{Y}, \mathrm{X})$

$\exp (X)$

$8 \log 10(\mathrm{X})$

$\& \max (X \ldots)$

\&floor(X)

$\operatorname{rand}(\mathrm{X})$

\section{Description}

sine of $\mathrm{X}$

tangent of $\mathrm{X}$

inverse cosine of $X$

inverse tangent of $\mathrm{Y} / \mathrm{X}$

e to the power of $X$

base $10 \log$ of $X$

maximum value

nearest integer $<=X$

random number $(0-X)$
Numeric

Function Description

$\cos (\mathrm{X}) \quad$ cosine of $\mathrm{X}$

$\& \operatorname{asin}(\mathrm{X})$

$\& \operatorname{atan}(X)$

$\operatorname{sqrt}(X)$

$\log (\mathrm{X})$

$\& \min (X \ldots)$

$\& \operatorname{nint}(X)$

$\operatorname{int}(\mathrm{X})$ inverse sine of $X$

inverse tangent of $X$ square root of $\mathrm{X}$ natural (base e) $\log$ of $X$

minimum value nearest integer to $X$ integer part of $X$

\section{RDB String Functions}

The following is a list of the commonly used string functions available:

String Function

len(string)

substr(string, length)

index(string, substring)

\section{Description}

Get length of a string value.

Get a substring. If length is omitted, then get to end of string.

Get position of substring in string. 


\section{Date/Time Functions}

The date function provides considerable flexibility for formatting a variable containing a date or a date and time. The variable should be a text variable where the date is listed first followed by time (optional). Any of the following forms would be recognizable by the date/time functions listed subsequently:

Oct 12,1994

15 November 86

$01 / 01 / 94$

941217

$06 / 17 / 8516: 45$

Date/Time Function

\&canonical_date(\$date)

\&year(\$date)

\&month(\$date)

$\&$ day(\$date)

\&hour(\$date)

\&minute(\$date)

\&water_year(\$date)

\&dow(\$date)

\&date_parts(\$date)

\&days(\$date)

\&date_time (\$days)
(October 12, 1994)

(November 15, 1986)

(January 1, 1994)

(December 17, 1994)

(4:15 pm on June 17,1985$)$.

\section{Description}

Convert a date to canonical form

(yyyy/mm/dd hh:mm:ss).

Extract the year from the date.

Extract the month from the date.

Extract the day from the date.

Extract the hour from the date.

Extract the minute from the date.

Extract the water_year from the date.

Get the day of the week (1-7) from the date.

Get array of date components from date and place in listed variables $(\$ y, \$ m, \$ d, \$ h, \$ m n, \$ s)$.

Get the number of days between 1901/01/01 and \$date.

Get the date that is \$days later than 1901/01/01.

\section{Data Validation Functions}

Function

\&isNumber(expr)

\&is Date(expr)

\section{Description}

Tests whether expression is a number.

Tests whether expression is a date.

Syntax Example - An example of the filter criteria syntax as entered in the "Criteria (BRC)" screen follows:

\section{Filter Type: Retain}

Criteria: \&isNumber(\$RAINFALL) \& \&

\&isNumber(\$PH) \&\& \$RAINFALL $>=0.01 \& \&$

$\$ P H>=0.01$

Explanation: Retain a row in the file if the variable RAINFALL is a number greater than or equal to $0.01 \mathrm{AND}$ if the variable $\mathrm{PH}$ is a number greater than or equal to 0.01 ; otherwise, remove the row from the output file.

Use Accept (F2) to return control to the "Row (BR)" screen. 


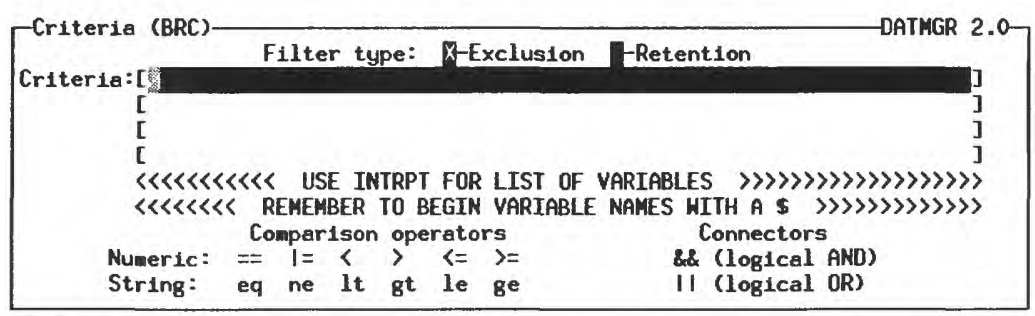

HELP- Enter a line of filter criteria. Remember to begin each variable name with \$. You may use parentheses to force evaluation order. Operators:

II \&\& Logical OR. AND

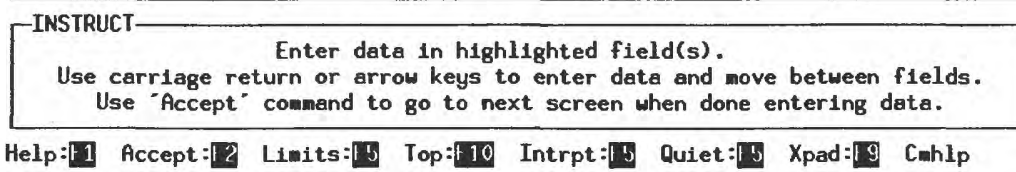

\section{Save an RDB Data File}

To create an output data file from the "Row filtering" operator, select the "Data (BRD)" screen from the "Row (BR)" screen. Enter a file name in the field. Use either Accept (F2) or the Enter key to accept the selection. The system appends the ".rdb" extension to the file name. The system prompts the user for permission to overwrite an existing file. Pathnames may be used to place the resultant file in a selected directory.

\section{Save an RDB Script File}

To save the script from the "Row filtering" operator, save it to a new script file or append it onto an existing script file. From the "Row (BR)" screen, select the "Script (BRS)" screen. If the input file to this operator was an RDB script file, its file name will appear in the entry box. Use Accept (F2) or the Enter key to accept this selection. The new file will contain the old script with the new script appended. If a new script file is required, enter a new file name and use Accept (F2) or the Enter key. The system appends the ".rqs" extension to the file name. The system prompts the user for permission to overwrite an existing file. Pathnames may be used to place the resultant file in a selected directory. Select the "Return to previous menu" option to return control to the "Basic (B)" screen. 


\section{Variable Subset/Reorder}

The "Variable Subset/Reorder" operator provides a process to select certain variables (columns) from the input data file, reorder them, and place them in a designated output data file or provides for their selection in a script.
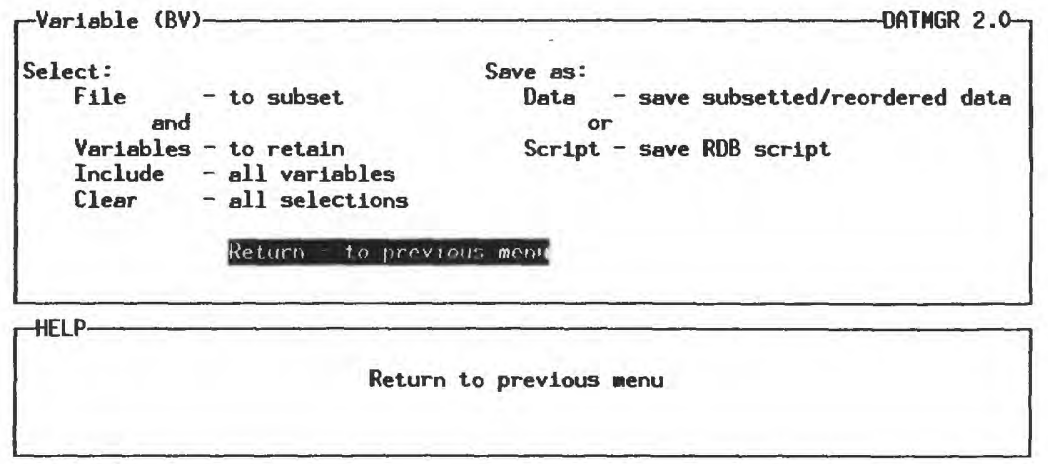

INSTRUCT- $\begin{gathered}\text { Select an option using arrow keys } \\ \text { then confirm selection with the F2 key, or } \\ \text { Type the f1rst letter of an option. }\end{gathered}$
Help: 1 Accept: Top: 110 Quiet: 8 Xpad:19 Cohlp

\section{Select Input Data File}

From the "Basic (B)" screen, select the "Variable (BV)" screen. From the "Variable (BV)" screen, select the "File (BVF)" screen. This file could be a script file to which additional script for the subset/reorder operator will be added. If a data file or script file was created by a previous "datmgr" operator, its file name will appear in the "Enter file name" field; otherwise this field will be blank. Select or type in a new file name and use either Accept (F2) or the Enter key to accept the file name and return control to the "Variable (BV)" screen.

\section{Select Variables to Retain}

From the "Variable (BV)" screen, select the "Variables (BCV)" screen. From the list of variables, use the arrow keys to highlight the variables to be retained in the output data file. Order the variables by populating the "Select" field with the appropriate numbers $(1-N)$. Deselect any unneeded variables by entering a " 0 " (zero) in the "Select" field. If the "Include all variables" option is used initially to include all variables, deselecting variables not to be retained may be easier than selecting individual variables.

To start over, use the "Clear all variables" option to deselect all variables.

\section{Save an RDB Data File}

To create an output data file from the "Variable subset/order" operator, select the "Data (BVD)" screen from the "Variable (BV)" screen. Enter a file name in the field. Use either Accept (F2) or the Enter key to accept the selection. The system appends the ".rdb" extension to the file name. The system prompts the user for permission to overwrite an existing file. Pathnames may be used to place the resultant file in a selected directory.

\section{Save an RDB Script File}

To save the script from the "Variable subset/reorder" operator, save it to a new script file or append it onto an existing script file. From the "Variable (BV)" screen, select the "Script (BVS)" screen. If the input file to this operator was an RDB script file, its file 
name will appear in the entry box. Use Accept (F2) or the Enter key to accept this selection. The new script file will contain the old script with the new script appended. If a new script file is required, enter a new file name and use Accept (F2) or the Enter key. The system appends the ".rqs" extension to the file name. The system prompts the user for permission to overwrite an existing file. Pathnames may be used to place the resultant file in a selected directory. Select the "Return to previous menu" option to return control to the "Basic (B)" screen.

\section{Create New Variables}

The "Create" operator provides for the creation of new variables (columns) in the output data file. A full range of mathematical functions and comparison operators are available to construct the required computational algorithms. Any number of new variables can be added to a file. In a given "datmgr" session, newly created variables can have the algorithms edited or deleted. Variables created in other "datmgr" sessions are modified using the "Modify" operator in the "Basic (B)" screen.

Output options include the following:

1. Define, compute, and store the values for the new variables in the output data file.

2. Define the variable and algorithm, but do not compute the values. The resulting data file will have the variable definitions and algorithms stored in the "Column definition" lines of the RDB file, but there will be no data columns for the new variables. If this file type is used as an input file for another QWGRAF program, the program makes the necessary computations if a computed variable is needed for the program.

3. Create a script file containing the algorithm. See discussion of script files in the section "Output an RDB Script File."
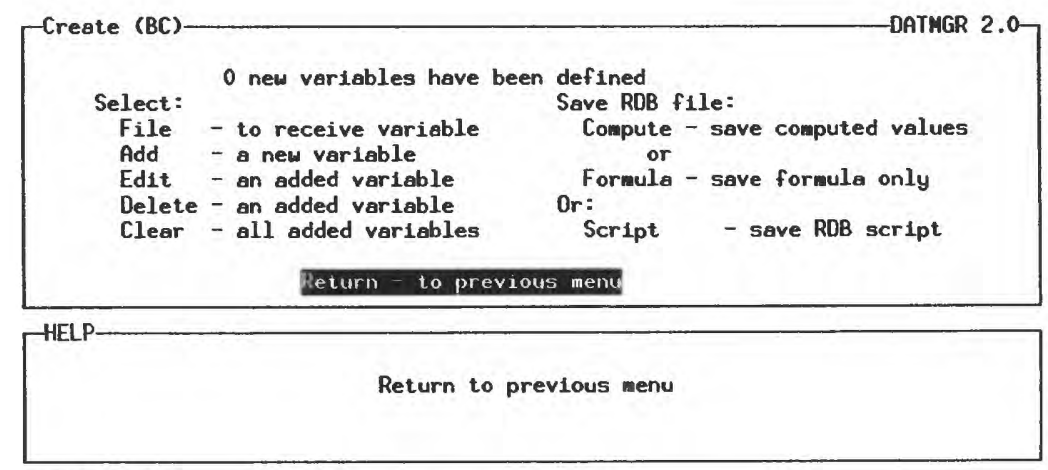

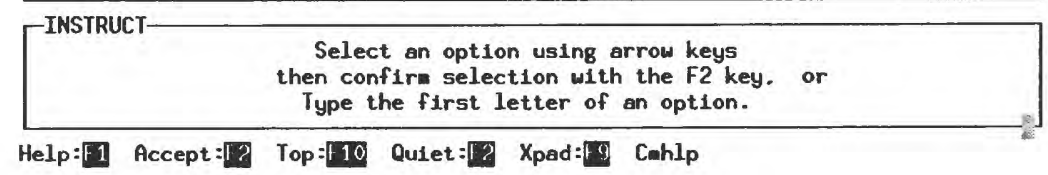

\section{Select a File to Receive New Variable}

From the "Basic (B)" screen, select the "Create (BC)" screen. From the "Create (BC)" screen, select the "File (CF)" screen. This file could be a script file to which additional script for the "Create" operator will be added. If a data file or script file was created by a previous "datmgr" operator, its file name will appear in the "Enter file name" field; otherwise this field will be blank. Select or type in a new file name and use either Accept 
(F2) or the Enter key to accept the file name and return control to the "Create (BC)" screen.

\section{Add a New Variable}

From the "Create (BC)" screen, select the "Edit (BCE)" screen in which the characteristics and algorithm of the new variable will be defined.

\section{Variable Name}

Use the arrow keys to position the cursor in the "Name" field and enter the name for the new variable (12 characters or less). This name should not conflict with the name of an existing variable in the file.

\section{Variable Type}

Use the arrow keys to position the cursor in the desired "variable type" field and use the space bar to place an "X" in the field. Use the left arrow and Enter keys to reposition the cursor if the variable type is to be changed.

\section{Variable Description}

Use the arrow keys to move the cursor to the "Description" field and enter a description of the new variable (optional).

\section{Variable Code}

Use the arrow keys to move the cursor to the "Code" field, which will contain the algorithm definition code. As an aide to constructing the algorithm, use Intrp (F6) to view a list of variable names from the current input data file (or script) that might be in the algorithm. After viewing the variable names, use Accept (F2) to return control to the "Edit (BCE)" screen.

Enter the name of the new variable preceded by a "\$" and followed by an " = " sign. Enter the algorithm to compute the new variable utilizing Perl syntax. To view a list of operators and functions available for creating algorithms, see the previous discussion in the "Row Filtration" section or use Help (F1) to activate the "Help" panel. Use Dnpg (;d) and Uppg (;u) to scroll through the list. When finished, use (F3) to return control to the data panel. See also the section "ASCII Flat Files to RDB" in Appendix C and the discussion of the RDB operator "perltbl" in Appendix E. The following syntax rules apply to the creation of algorithms:

1. Precede each variable name used in the algorithm with a "\$" sign.

2. Multiple lines are permitted, but each line must end with a semicolon (;).

Note: The use of semicolons as line terminators in algorithm construction (see also datmgr "Modify" operator) is in conflict with use of the semicolon as the escape character for QWGRAF screen functions (see discussion of the "User Interface" in the "How QWGRAF Works" section). To reconcile this conflict, override the function key escape character by placing either of the following lines in your TERM.DAT file in the current directory.
TRMDLM 35
(\#) becomes the escape character
TRMDLM 33
(!) becomes the escape character

It is a good idea to remove the above line from the TERM.DAT file when no longer needed.

3. It is not necessary to enclose either individual variables or the entire computational statement in parentheses, but parentheses can be used to define selected preference. 
4. for loops - The following is an example of the syntax used for a "for loop":

for $(\$ i=1 ; \$ i<10 ; \$ i++) \quad\{\$ x=\operatorname{sqrt}(y * 115.6) ; \$ y=\log 10 ; \$ s=\$ x+\$ y\}$;

Explanation: for \$i equal 1 to 9, with increments of 1 , compute values of $x, y$, and $s$; that is, while $i$ is less than 10 , increment by $1 . . .$.

Note: The $\$ i++$ syntax is shorthand for $\$ i=\$ i+1$

$\$ i+=2$ would be shorthand for $\$ i=\$ i+2$

5. if/then/else clauses - The following is an example of an if/then/else clause:

if(x gt 100.0) $\{y=100.0+x ; j=j+1\}$ else if ( $x$ gt 500); $\{y=200+x$;

$\mathrm{j}=\mathrm{j}+2\}$ else $\{\mathrm{y}=\mathrm{x} ; \mathrm{j}=1\}$;

Some examples of algorithm syntax are as follow:

$\$$ na $(\mathrm{meq})=\$$ na $* 0.04348$

$\$ D E C L A T=\$ l a t d e g+\$ l a t m i n / 60 .+\$ l a t s e c / 3600$.

Explanation: Decimal latitude computed from 3 variables

\$DECLAT $=\operatorname{int}(\$ l a t d m s / 10000)+\operatorname{int}((\$ l a t d m s \% 10000) / 100) / 60 .+$; (\$latdms\%100)/3600.

Explanation: Decimal latitude from a single six-digit integer number using "Modulo" (\%) and integer (int) functions to extract and format components into decimal form; for example, 483202 to 48.53389. Note use of semicolon to break up line.

Use Accept (F2) to return control to the "Create (BC)" screen. If one or more of the source variables contains at least one remarked value, use of Accept (F2) brings up the "Remark (BCER)" screen to specify a censored data option for the variable just created.

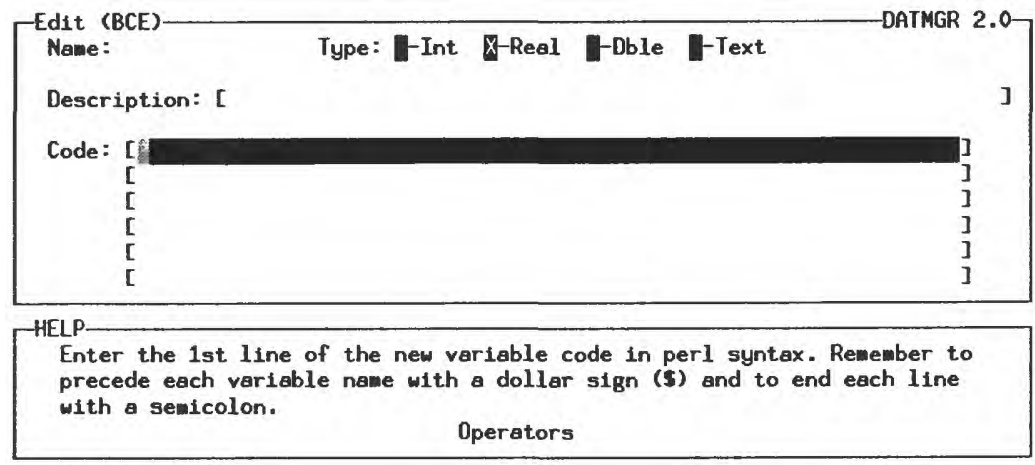

\footnotetext{
TINSTRUCT- Enter data in highlighted field(s).

Use carriage return or arrow keys to enter data and move between fields. Use "Accept" command to go to next screen when done entering data.

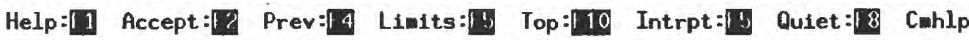




\section{Censored Data Options}

By default, an algorithm used to compute a new variable does not consider remark codes that may be associated with any of the source variables used in the computation. Thus, computed values are based on the values of the source variables and are not remarked. This is true whether the new variable is created on ingest by the ".dta" and ".ftr" data conversion filters (see discussion of the ".dtv" and ".ftv" format definition files in Appendix E) or by the "Create" operator in the "datmgr" program. If remarked data are detected in any of the variables used in the algorithm, the "Remark (BCER)" screen is presented with the following options for treating the computed values:

Omit - Do not include remark codes for this variable (Default)

Less than - Use < as a remark code

User defined - Use the remark code specified below

Remark code: [Z].

Use arrow keys to select an option. A user-defined remark code can be any single printable character ( $\mathrm{Z}$ is default). Remarked values can be included or excluded from any subsequent "datmgr" operator or QWGRAF program using the "Censored data" option. Use Accept (F2) to return control to the "Create (BC)" screen.

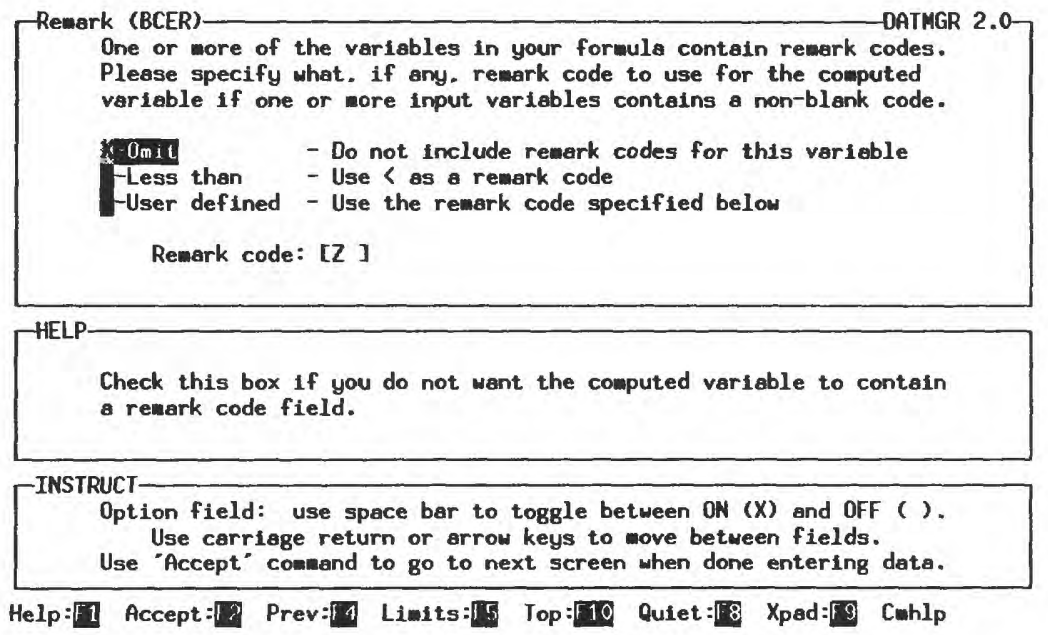

\section{Edit a New Variable}

Variables created in the current session of "datmgr" can be edited. Note: do not use the "Modify" operator to edit variables created in the current "datmgr" session; use the "Modify" operator for variables created in a previous session. From the "Create (BC)" screen, select the "Edit" option to bring up the "Edit (BCE)" screen (it is the same screen used in the "Add" option). Change the variable characteristics as desired. When modifying any of the code lines, place the cursor at the beginning of the line and type the new line in its entirety. The cursor cannot edit individual characters or words. Use Accept (F2) to accept the changes and bring up the "Remark (BCER)" screen. Use the arrow keys to make changes to the censored data options. Use Accept (F2) to return control to the "Create (BC)" screen. 


\section{Delete a New Variable}

To delete a variable created in the current "datmgr" session, select the "Delete (BCD)" screen. The system prompts the user for the number of the variable created in the current session and then to verify that the variable is to be deleted. Enter "0" (zero) to return control to the "Create (BC)" screen without deleting a variable.

\section{Clear All New Variables}

To remove all variables created in this session, select the "Clear (BCC)" screen. The system prompts the user to verify that all created variables are to be deleted. If no new variables have been created, then the system returns control to the "Create (BC)" screen with the "Add a new variable" option highlighted.

\section{Save an RDB Data File}

If a new data file is required after new variables are created, two options are available.

\section{Save Computed Values}

The output RDB file from the "Create" operator can be populated with the original data values and computed values for any variables defined by algorithms. These algorithms could have been defined in the current session of "datmgr" or previously defined in the algorithm extension (<perl> keyword) of the format definition line of the input RDB file (see also the discussion in Appendix D on algorithm definition in format definition files for the ".dta" and ".ftr" file types).

\section{Save Formula}

In the interest of saving disk space, the output file need not be populated with the computed variables but rather with algorithms defined to compute the values for these variables stored in the column definition line of the output RDB file. If the computed variables are required in a subsequent QWGRAF program, the values are computed.

From the "Create (BC)" screen, select either the "Compute (BCC)" or "Formula (BCF)" screen. The system prompts for the name of the output RDB file to be saved. The system appends the ".rdb" extension to the file name. The system will query for permission to overwrite an existing file. Pathnames may be used to place the resultant file in a selected directory.

\section{Save an RDB Script File}

To save the script from the "Create new variables" operator, save it to a new script file or append it onto an existing script file. From the "Create (BC)" screen, select the "Script (BCS)" screen. If the input file to this operator was an RDB script file, its file name will appear in the entry box. Use Accept (F2) or the Enter key to accept this selection. The new file will contain the old script with the new script appended. If a new script file is required, enter a new file name and use either Accept (F2) or the Enter key. The system appends the ".rqs" extension to the file name. The system will query for permission to overwrite an existing file. Pathnames may be used to place the resultant file in a selected directory. Select the "Return to previous menu" option to return control to the "Basic (B)" screen. 


\section{Evaluate Computed Variables}

The "Evaluate" operator provides an opportunity to convert a script or RDB file containing algorithms for creating variables to a data file with columns populated with the computed values.

\section{Select Input Data File to be Evaluated}

From the "Basic (B)" screen, select the "Evaluate (BE)" screen. From the "Evaluate (BE)" screen, select the "Input (BEI)" screen. If a script file was created by a previous "datmgr" operator, its file name will appear in the "Enter file name" field; otherwise this field will be blank. Use the arrow and Enter keys to select an input file name or type in a new file name. Use either Accept (F2) or the Enter key to accept the file name and return control to the "Evaluate (BE)" screen.

\section{Evaluate the Input Data File}

From the "Evaluate (BE)" screen, select the "Evaluate (BEE)" screen. Enter a file name in the field and use either Accept (F2) or the Enter key to accept the selection. The system appends the ".rdb" extension to the file name. The system prompts the user for permission to overwrite an existing file. Pathnames may be used to place the resultant file in a selected directory.

\section{Modify Variable Attributes}

The "Modify" operator provides for changing the name, data type, description, or algorithm of a variable created in a previous session of "datmgr." Use the "Edit" function in the "Edit (BCE) screen" from the "Create - new variables" operator to edit variables created in the current session of "datmgr."
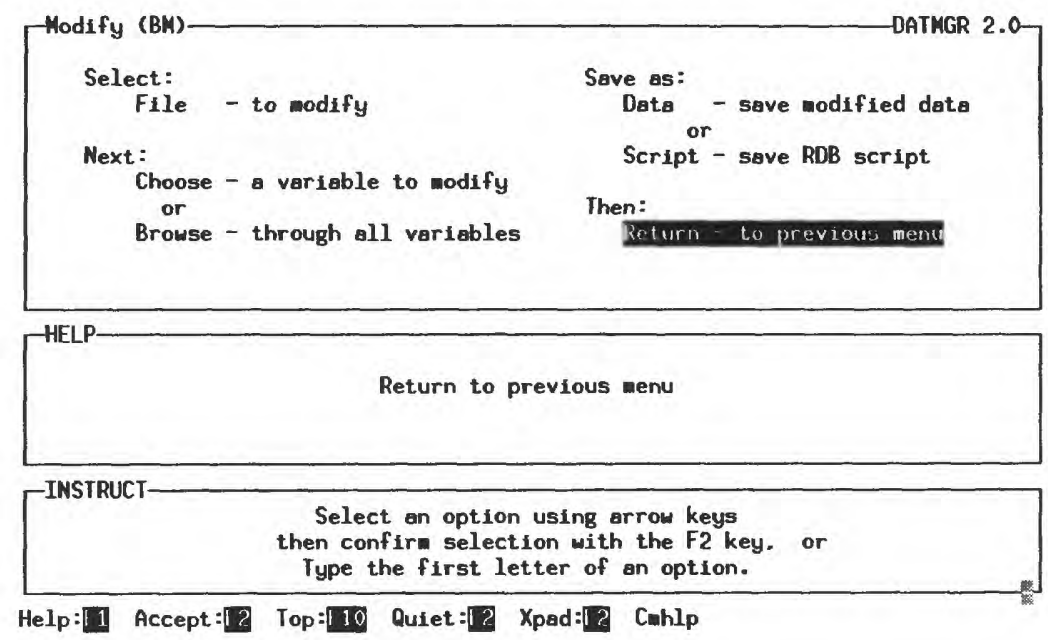

\section{Select Input Data File}

From the "Basic (B)" screen, select the "Modify (BM)" screen. From the "Modify (BM)" screen, select the "File (BMF)" screen. The file to be selected could be a script file to which additional script for the modify operator will be added. If a data file or script file was created by a previous "datmgr" operator, its file name will appear in the "Enter file name" field; otherwise this field will be blank. Select or type in a new file name and use either Accept (F2) or the Enter key to accept the file name and return control to the "Modify (BM)" screen. 


\section{Choose a Variable to Modify}

From the "Modify (BM)" screen, select the "Choose a variable to modify" option to view the "Variable (BMV)" screen. Select one variable to modify by entering a "1" in the "Select" field. Use Accept (F2) to select the variable and bring up the "Edit (BMVE)" screen (same screen used for the "Create" operator). Use the Enter and arrow keys to highlight the fields to be modified. Enter any new text or field values. Use the space bar to toggle "On" or "Off" the variable type option required. When changing the algorithm code, the entire field must be reentered. The arrow keys cannot be used to selectively position the cursor to enter or replace characters.

Note: Changing the variable name does not create a new variable, it just changes the name of the existing variable. Use Accept (F2) to accept the changes.

Note: Use of multiple lines in the definition of an algorithm requires the use of a semicolon to terminate each line, which is in conflict with use of the semicolon as the function key escape character. Change the escape character by placing a line in the TERM.DAT file in the current directory (see discussion in "Create Variables" section).

\section{Modify Censored Data Option}

If the modified variable contains remarked values, use the Accept (F2) key in the "Edit (BME)" screen to bring up the "Remark (BMER)" screen. Use the arrow keys to select a censored data option and toggle "On" that option using the space bar. Use Accept (F2) to return control to the "Modify (BM)" screen. Use the arrow keys to select the "Return to previous menu" option and use Return to return control to the "Basic (B)" screen.

\section{Browse Existing Variables}

If several variables are to be changed, it may be more efficient to use the "Browse" operator to move from one variable to another. Select the "Modify (BMV)" screen. The first variable is presented and appropriate changes can be made to the variable attributes. Use Accept (F2) to view the next variable. Use Prev (F4) to move backward in the list of variables. Use Intrpt (F6) to exit the "Browse" operator.

\section{Save the Modified RDB Data File}

To create an output data file from the "Modify variable attributes" operator, select the "Data (BMD)" screen from the "Modify (BM)" screen. Enter a file name in the field. Use either Accept (F2) or the Enter key to accept the selection. The system appends the ".rdb" extension to the file name. The system prompts the user for permission to overwrite an existing file. Pathnames may be used to place the resultant file in a selected directory.

\section{Save an RDB Script File}

To save the script from the "Modify variable attributes" operator, save it to a new script file or append it onto an existing script file. From the "Modify (BM)" screen, select the "Script (BMS)" screen. If the input file to this operator was an RDB script file, its file name will appear in the entry box. Use Accept (F2) or the Enter key to accept this selection. The new file will contain the old script with the new script appended. If a new script file is required, enter a new file name and use Accept (F2) or the Enter key. The system appends the ".rqs" extension to the file name. The system prompts the user for permission to overwrite an existing file. Pathnames may be used to place the resultant file in a selected directory. Select the "Return to previous menu" option to return control to the "Basic (B)" screen. 


\section{Transpose}

The "Transpose" operator provides for splitting a row of data into multiple rows, "row2grp," or merging several rows into a single row, "grp2row."

In "row2grp" (Row to Group), selected variables (columns) are split into groups, each containing an equal number of variables. Each "split" group may be defined as a specific set of variable names or the groups may be defined on the basis of pattern searches. Caution should be used when using multiple search patterns (one for each group) to ensure that the pattern criteria will result in the same number of variables being assigned to all groups. The variables assigned to each group need not be contiguous in the input file. The output file will have a column created for each "split" group plus a column for each of the other variables as selected by the user. The latter are referred to as "collected" variables.

The number of rows in the output data file will be equal to the number of variables specified for the "split" groups multiplied by the number of rows in the input data file. If the "split" groups contain three variables, then three rows will be created in the output file for each row in the input file. Each row in the output data file will contain one value from each "split" group plus the value of each "collected" variable from that same row.

Collected values, thus, get repeated in each of the rows originating from a given row in the input file.

For example, if the input data file has two "split" groups of three variables each, plus two additional "collected" variables, there will be four columns in the output file, one column for each "split" group and one column for each of the "collected" variables. If the input file consists of four rows, there will be 12 rows in the output file.

In "grp2row" (Group to Row) rows from the input file are grouped, each group forming the basis of a single row in the output file. The number of rows and columns produced in the output file depends on the method used to collect the rows. The number of columns created is also based on which variables are declared "collected" and which are declared "carried." Variables not collected are considered carried. Normally, carried variables are assumed to have the same value in all rows of the input data file that get grouped into a given row of the output file. If this is not the case, the output from the group2row operator will result in lost data for these variables. Such variables should either be declared "collected" or removed from the input data file (the "Variable" operator in "datmgr" can be used to remove selected variables from a file). One column is created in the output file for each carried variable.

In the first approach to collecting rows, the row collection groups are based on a repeating sequence of a fixed number of rows in the input file as specified by the user. One row will be created in the output file for each group of collected rows. The number of columns created will be equal to the number of collected variables multiplied by the number of rows in a row collection group. A column also will be created for each variable not listed in the "collect" list, that is, each carried variable. The names of the columns based on the collected variables are created by concatenating a sequential row group number to the collected variable names, for example, flow_1, flow_2, year_1, year_2.

An alternative approach to collecting rows is to base the groups on the unique values of an indexing or label variable and a set of grouping (by) variables upon which the file is sorted. The user can specify a subset of the index variable values. The index variable may be in the current input file or an alternative file. A column is created for each user-designated collected variable multiplied by the number of unique index variable values. The column names from this process are created by appending the value of the index variable to the name of the collected variable; for example, flow_013981, flow_013982, sediment_013981, sediment_013982. Additional columns are created for each carried variable, each grouping (by) variable, and the index variable. The number of rows created 
in this approach is based on the number of unique composite values of the sort variable(s) and the number of repeated index values that equate to a given sort value.

For further discussion and some examples of the "Group to Row" and "Row to Group" operators, see the discussion on the operators "row2grp" and "grp2row" in Appendix C. From the "Basic (B)" screen, select the "Transpose (BT)" screen, which lists the two row/ column transposition options available:

\section{Row to Group \\ Group to Row.}

From the "Transpose (BT)" screen, select either the "Row (BTR)" screen or the "Group (BTG)" screen.

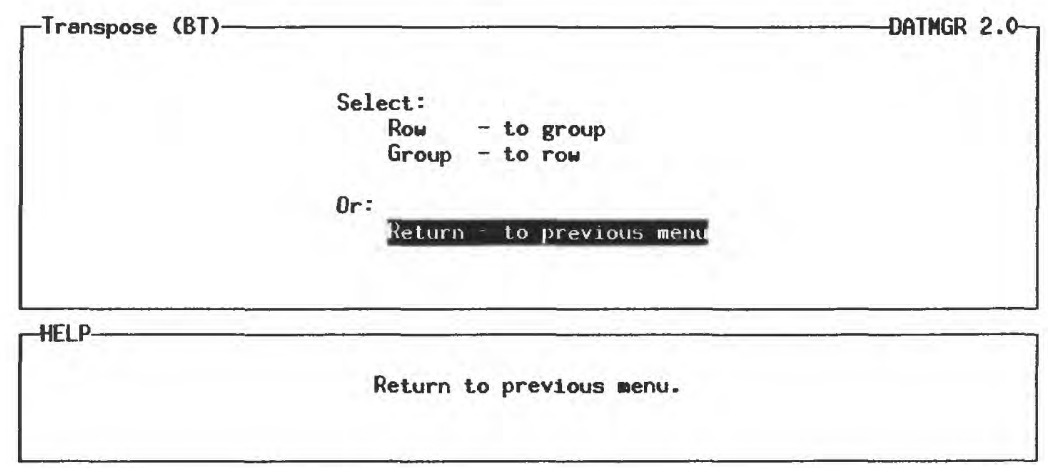

$\begin{aligned} & \text { INSTRUCT- } \\ & \text { then confirm selection with the F2 key. or } \\ & \text { Type the first letter of an option. }\end{aligned}$
Help: 1 Accept: 2 Top: 10 Quiet: 8 Xpad: 2 Cahlp 


\section{Row to Group}

The "Row (BTR)" screen lists the following options available for the "Row to Group" operator.

$\begin{array}{ll}\text { File } & \text { - to process } \\ \text { Variable } & \text { - splits } \\ \text { Pattern } & \text { - splits } \\ \text { Index } & \text { - variable (optional) } \\ \text { Save Data } & \text { - save data in RDB file } \\ \text { Save Script } & \text { - save RDB script. }\end{array}$

The main option to be specified in the "Row to Group" operator is the method used to create the split groups, which ultimately determines the number of columns in the output file, as well as the number of rows. The "Variable splits" option sets the user-determined number of groups and the list of variables to be included in each group. The "Pattern splits" option creates groups based on fitting one or more text patterns to all available variable names. In either case, all groups must have the same number of variables.
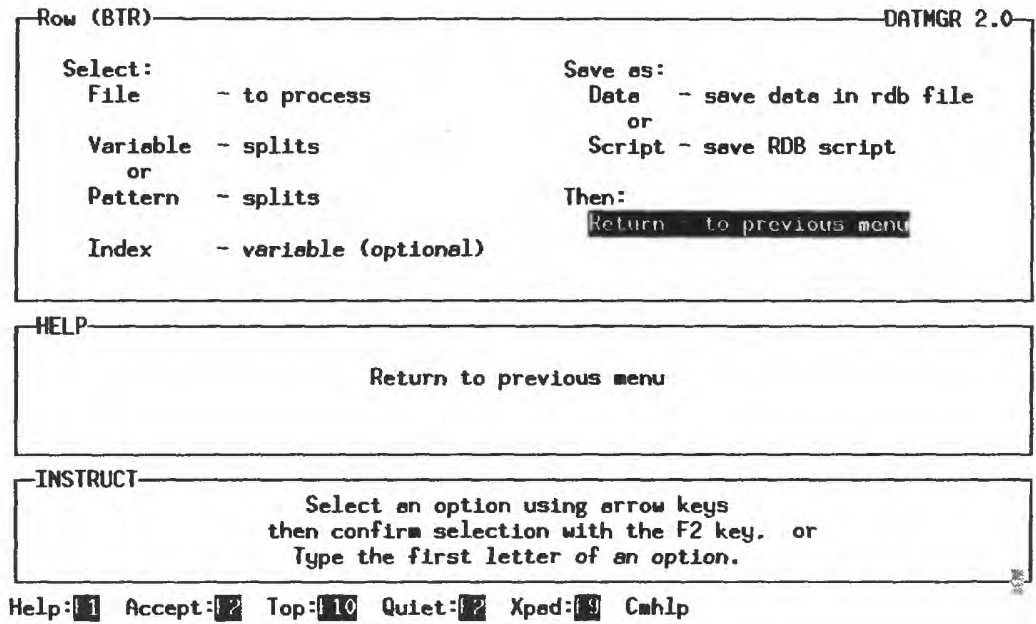

\section{Select Input File}

From the "Row (BTR)" screen, select the "File (BTRF)" screen. This file could be a script file to which additional script for the transpose operator will be added. If a data file or script file was created by a previous "datmgr" operator, its file name will appear in the "Enter file name" field; otherwise this field will be blank. Select or type in a new file name and use either Accept (F2) or the Enter key to accept the file name and return control to the "Row (BTR)" screen.

\section{Variable Splits}

From the "Row (BTR)" screen, select the "Variable (BTRV)" screen, which provides the following options for creating and managing the groups of variable names:

$$
\begin{array}{ll}
\text { add } & \text { - a new group of variables } \\
\text { edit } & \text { - a group of variables } \\
\text { delete } & \text { - a variable group } \\
\text { clear } & \text { - all variable groups. }
\end{array}
$$



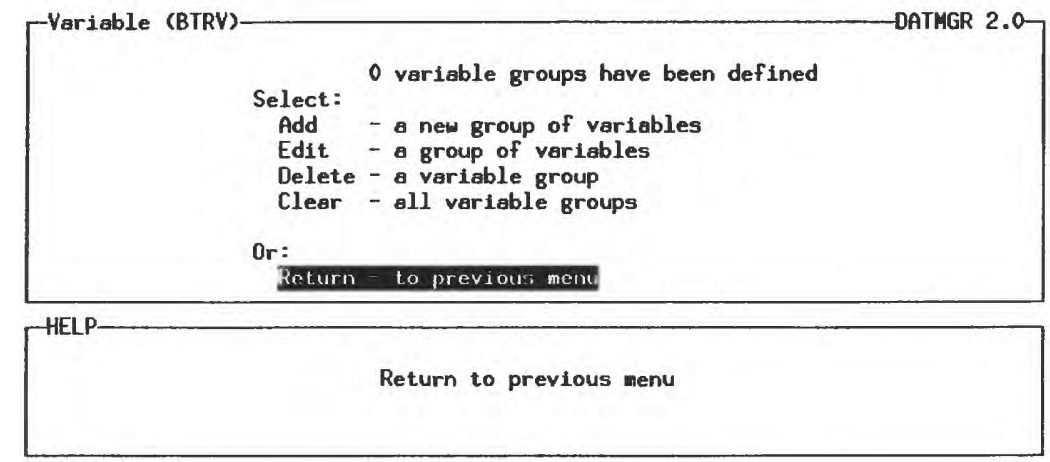

IINSTRUCT-
$\begin{gathered}\text { Select an option using arrow keys } \\ \text { then confirm selection with the F2 key. or } \\ \text { Type the first letter of an option. }\end{gathered}$
Help:1 Accept: 2 Top: 10 Quiet: 12 Xpad: 9 Calp

\section{Add a New Group of Variables}

Select the "Add" option as many times as necessary to create the number of groups required. The "Add" option uses the "Edit (BTRVE)" screen to list the variables in the data file. Designate up to eight variables for the group by entering a number (1 to 8$)$ in the appropriate "Select" fields. The variables may be selected in any order. A variable can be deselected by entering a "0" (zero).

\section{Edit or Delete Groups}

Each variable group created is given a sequential number used by the "Edit" and "Delete" options to select the group to be edited or deleted, respectively. The "Edit" and "Delete" options use the "Which number" screen to specify the group number. If the "Edit" option is selected, the "Edit (BTRVE)" screen is used to modify the group by selecting or deselecting (enter " 0 " (zero) in the "Select" field) the appropriate variables. If the "Delete" option is selected, the group specified is deleted and control is returned to the "Row (BTR)" screen.

\section{Clear All Variable Groups}

From the "Variable (BTRV)" screen, select the "Clear all variable groups" option. All groups will be deleted and the "Variable (BTRV)" screen is refreshed, permitting the user to add other groups. Select the "Return" option to return control to the "Row (BTR)" screen. 


\section{Pattern Splits}

From the "Row (BTR)" screen, select the "Pattern (BTRP)" screen. Enter a pattern definition for each specified group. Remember that the "Pattern Split" option requires split groups to have the same number of variables. To use the "Pattern" option, define text patterns in "regular expressions," which are the expressions entered in the "Pattern" field in the "Pattern" screen. Many "regular expressions" have been defined, with variations among UNIX and other operating systems. The RDB operator used to implement the QWGRAF "row2grp" operator accepts most of these expressions. The following meta characters can be used to form matching expressions:

\section{Meta Character \\ Description}

(period)

[] (Brackets)

$\wedge$ (Caret)

\$ (Dollar)

- (Hyphen)

Examples:

"a.c"

"a[bc]d"

"ab[^cd]e"

[0-9]

"^Start of line"

"End of line\$"

[a-z]

[A-Za-z]

[^A-Za-Z0-9]
Matches any character except newline.

Defines a set of characters, any one of which will satisfy the match requirement.

When the caret is used as the first meta character in an expression, the expression matches only at the beginning of the line. If the caret is used as the first character of the set, it excludes the set from matching.

When the dollar sign is used as the last meta character, the expression matches only at the end of the line.

If the hyphen is used between two characters in a set, a range of ASCII characters is specified.

$$
\begin{aligned}
& \text { matches "abc" "a2c" or "a c" } \\
& \text { matches "abd" "acd" but not "axd" } \\
& \text { could match "abae" or "ab4e" } \\
& \text { matches any single digit } \\
& \text { matches expression only when it begins a line } \\
& \text { matches expression only when it ends a line } \\
& \text { matches any lower case alphabetic character } \\
& \text { matches any alphabetic character } \\
& \text { matches any non-alphanumeric character }
\end{aligned}
$$

For a more detailed discussion of regular expressions, see the textbook Programming Perl (Wall and Schwartz, 1991, p. 24-29).

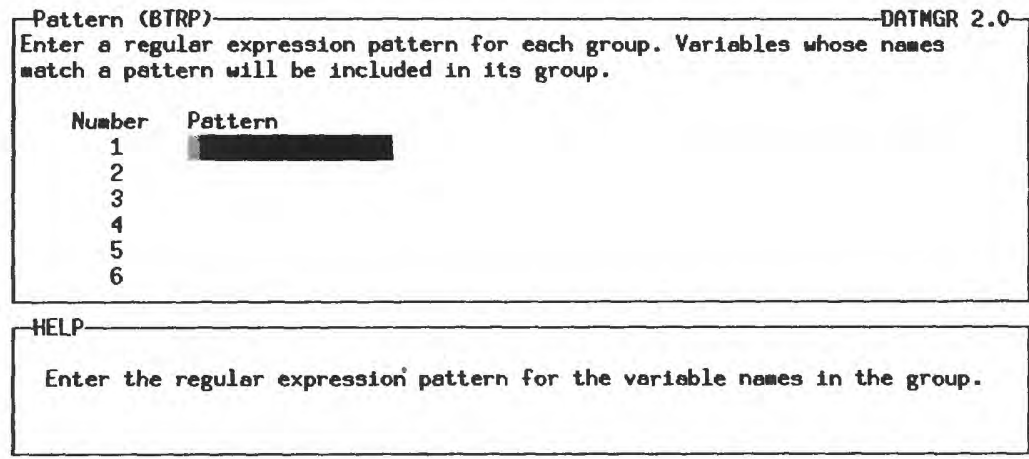

\footnotetext{
TINSTRUCT- Enter data in highlighted field(s).

Use carriage return or arrow keys to enter data and wove between fields. Use "Accept" command to go to next screen when done entering data.

Help: 11 Accept: 12 Limits: Top $=10$ Quiet: 8 Xpad: 9 Cahlp Oops Hindow
} 


\section{Index Variable}

The "Index variable" feature is optional for "row2grp" and is used to create an extra column in the output file to contain a system-generated integer index number. This index relates the rows in the output file to their respective source rows and columns in the input file. Each sequential group of values in the "index column" represents, in sequential order, each group of variables in a given row in the input data file as defined by the "Split" groups. In addition, rows in the output data file with the same index value come from the same column in the input data file. The "index" values can be used with the values of any of the "collected" variables, that is, those variables not included in any of the "splits" groups, to identify the specific row in the input data file from which a row in the output file originated.

From the "Row (BTR)" screen, select the "Index (BTRI)" screen. Enter a name (12 characters or less) in the field. This variable name must not conflict with any other variable in the input file. Use Accept (F2) or the Enter key to return control to the "Row (BTR)" screen.
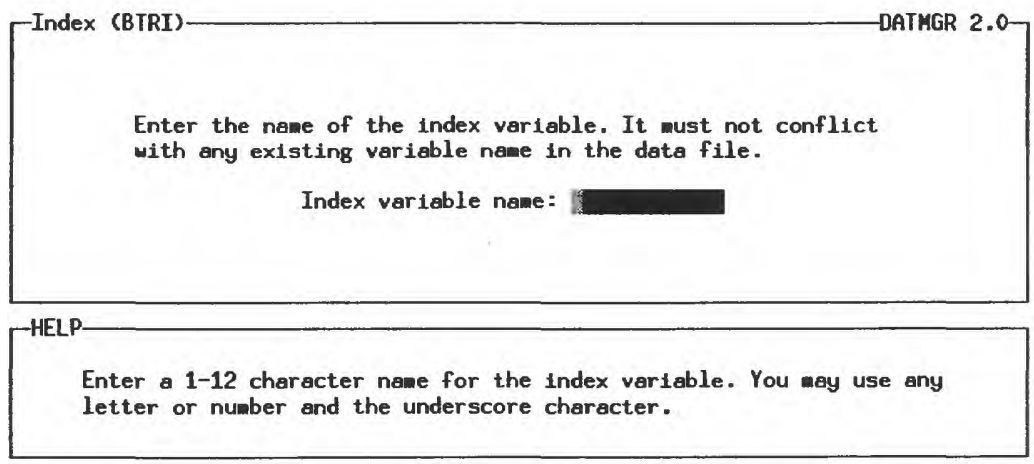

\footnotetext{
INSTRUCT- Enter data in highlighted field(s).
Use carrlage return or arrow keys to enter data and wove between fields.
Use "Accept" comand to go to next screen when done entering data.
} 


\section{Group to Row}

From the "Transpose (BT)" screen, select the "Group (BTG)" screen, which presents the following options:

1. Select the file to process.

2. Specify the method by which the rows will be collected into groups:

a. consecutive row groups

b. value of index variable.

3. Specify the variables to be collected.

4. Specify the "Grouping" (or "By") variables and the sorting order.
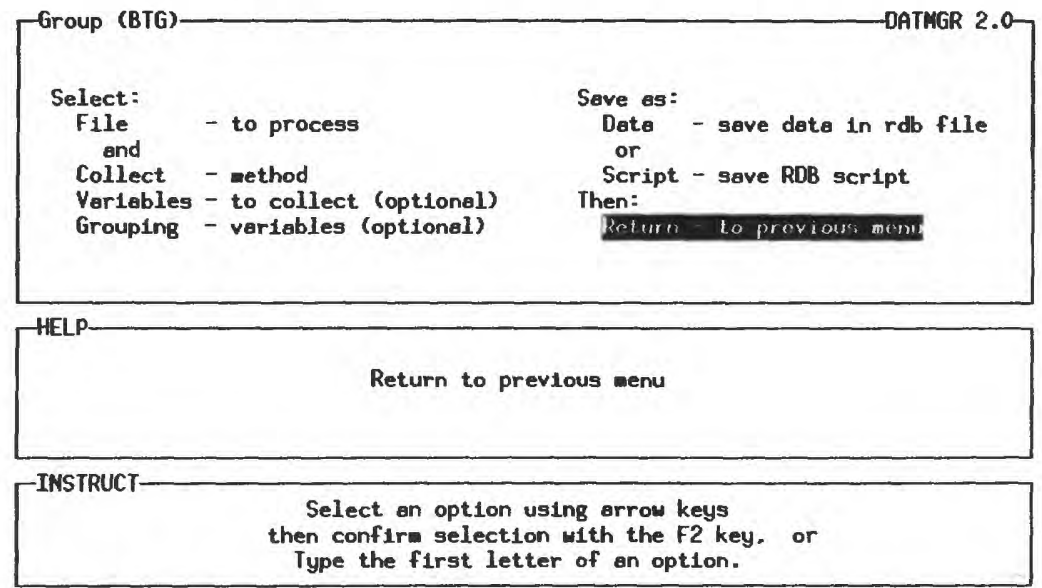

Help: 1 Accept: [1] Top: 110 Quiet: 18 Xpod: 18 Cuhlp

\section{Select Input File}

From the "Group (BTG)" screen, select the "File to process" option and use Enter to view the "File (BTGF)" screen. This file could be a script file to which additional script for the "transpose" operator will be added. If a data file or script file was created by a previous "datmgr" operator, its file name will appear in the "Enter file name" field; otherwise this field will be blank. Select or type in a new file name and use either Accept (F2) or the Enter key to accept the file name and return control to the "Group (BTG)" screen. 


\section{Row Collection Methods}

Two methods are available for collecting rows - Consecutive Rows or Indexing Label Variable. From the "Group (BTG)" screen, select the "Collect method" option and use Enter. From the "Collection (BTGC)" screen, select either the "Consecutive (BTGCC)" screen (see discussion in "Consecutive Rows Method" section) or the "Label (BTGCL)" screen (see discussion in "Label Variable Method" section).
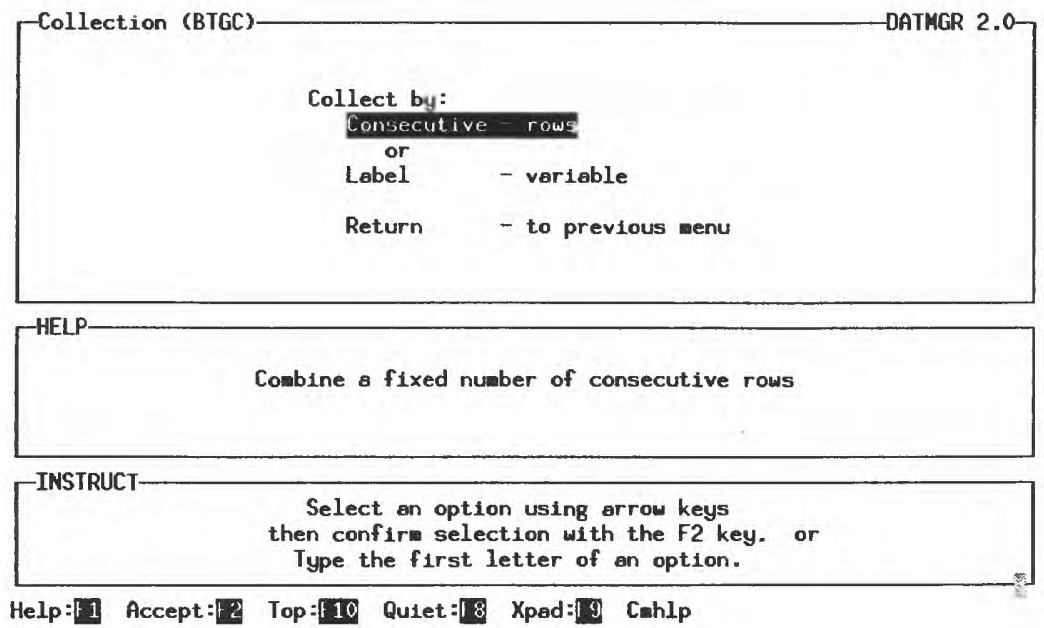

\section{Consecutive Rows Method}

Row collection groups will be formed by sequentially collecting a fixed number of rows from the input file into groups. From the "Consecutive (BTGCC)" screen, enter the number of consecutive rows to be placed in each group and use Enter. To specify selected variables to be "collected" (the default is all variables), select the "Variables (BTGV)" screen (see "Variables to be Collected" section).

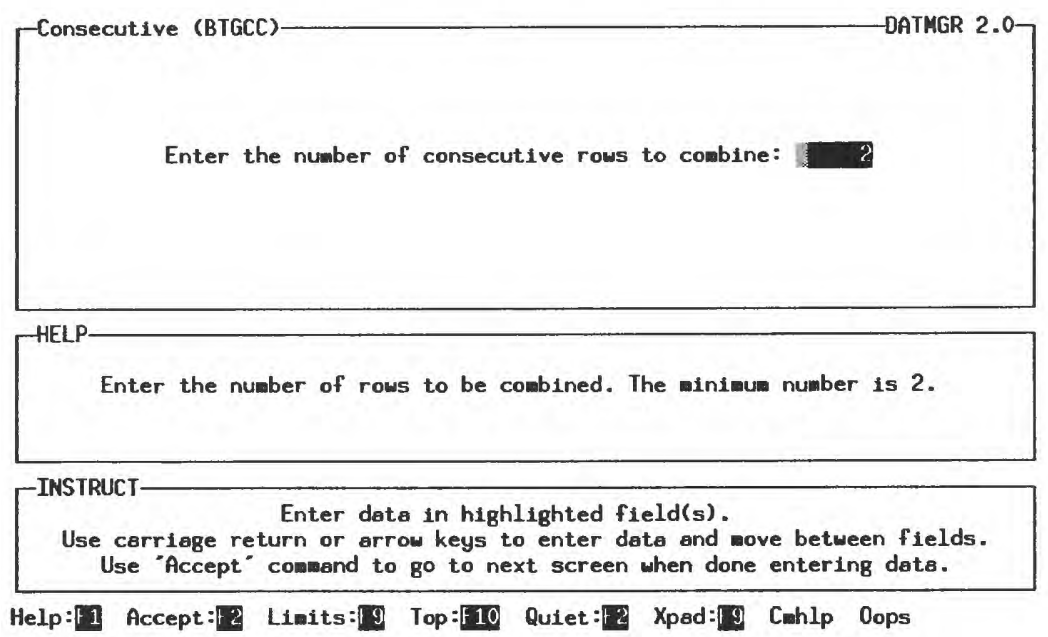


Label (Index) Variable Method

The unique values of a specified "Index" or label variable and a set of grouping (by) variables will be used as the basis for the number of rows and columns to be created. The "Index" variable can come from the input data file or an alternate file. From the "Group (BTG)" screen, select the "Row collection (BTGR)" screen followed by the "Label (BTGRL)" screen. The variable to be used as the index label is chosen from this screen followed by indicating the source file that includes this variable.

\section{Choose a Label Variable}

From the "Label (BTGRL)" screen, first select the "Label - choose a label variable" option and use Enter. The "Label (BTGRLL)" screen appears. Using the Enter and arrow keys, select one variable to be used as the "Label variable" by entering a "1" (one) in the appropriate "Select" field. Use Accept (F2) to accept the variable and return control to the "Label (BTGRL)" screen.

\section{Specify Index Values}

If the index variable is in the input data file, use the "Values" option to specify the list of unique values to be used. If no values are specified in the "Values" option, then all unique values will be used.

From the "Label (BTGRL)" screen, select the "Values" option and use Enter to view the "Values (BTGRLV)" screen. Use the arrow keys to position the cursor at the appropriate "Index value" fields, and enter values for the label variable that will be concatenated to the "Collected" variable names to form new column names in the output file. When finished, use Accept (F2) to accept these values and return control to the "Label (BTGRL)" screen.

\section{Read index Values from Alternate File}

If the index values are to be read from an alternate file, from the "Label (BTGRL)" screen, select the "File" option and use Enter to view the "File (BTGRLF)" screen. Use the arrow keys to select the name of the file containing the index values. The variable in the file containing the index values must have the same name as indicated in the "Label" option described previously.

\section{Variables To Be Collected}

From the "Group (BTG)" screen, select the "Variables (BTGV)" screen. Select the specific subset of variables to be collected by setting the "Select" field in the order the variables are to be collected and arranged in the output file. Use the Enter and arrow keys to move between variables. When finished, use Accept (F2) to return control to the "Group (BTG)" screen.

\section{Select Grouping Variables}

The "Grouping variables" option in the "Group (BTG)" screen defines the number of rows in the output file as the number of combined unique values of the "Grouping" ("By") variables (up to four, in sorting order). The program sorts the input file on these grouping variables. The grouping variables and the sorting order should be selected to prevent unexpected results in the output file. From the "Group (BTG)" screen, select the "Grouping (BTGG)" screen. Use the Enter key and arrow keys to highlight the "Select" fields in sequential sort order. Set the primary sort field to " 1 " followed by sequential numbers (up to 4) for the other variables in nested order. When finished, use Accept (F2) to return control to the "Group (BTG)" screen. 


\section{Save an RDB Data File}

To create an output RDB data file from the "Transpose" operator, select the respective "Data (BTRD)" or "Data (BTGD)" screens from either the "Row (BTR)" screen or the "Group (BTG)" screen. Enter a file name in the field. Use either Accept (F2) or the Enter key to accept the selection. The system appends the ".rdb" extension to the file name. The system prompts the user for permission to overwrite an existing file. Pathnames may be used to place the resultant file in a selected directory.

\section{Save an RDB Script File}

To save the script from the "Transpose" operator, save it to a new script file or append it onto an existing script file. From the "Row (BTR)" screen or the "Group (BTG)" screen, select the respective "Script (BTRS)" or "Script (BTGS)" screen and use Enter to view the "Script" screen. If the input file to this operator was an RDB script file, its file name will appear in the entry box. Use Accept (F2) or the Enter key to accept this selection.

The new file will contain the old script with the new script appended. If a new script file is required, enter a new file name and use Accept (F2) or the Enter key. The system appends the ".rqs" extension to the file name. The system prompts the user for permission to overwrite an existing file. Pathnames may be used to place the resultant file in a selected directory. Select the "Return to previous menu" option to return control to the "Transpose (BT)" screen and again to return control to the "Basic (B)" screen.

\section{Merge Data Files}

The "Merge" operator provides for creating an output file of merged records from two files. The available "Merge" operators are as follow:

Relational Join

Relational Subtract

Concatenation.

From the "Basic (B)" screen, select the "Merge (BM)" screen.
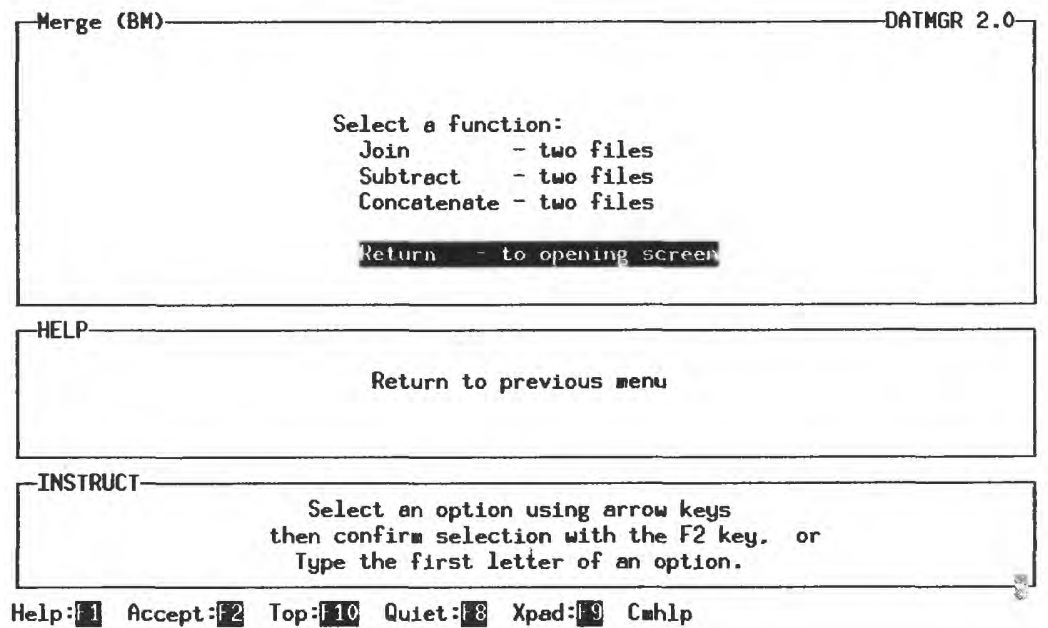

\section{Relational Joins}

From the "Merge (BM)" screen, select the "Join (BMJ)" screen. The "Join" operator provides three options for the matching criteria by which records from one file are merged (appended) horizontally to records of a second file. The output file may contain more records than either of the input files and some records may have several "missing" 
values if a record in one file could not be matched with a record in the second file. The matching criteria may involve up to eight variable pairs from the two data files.
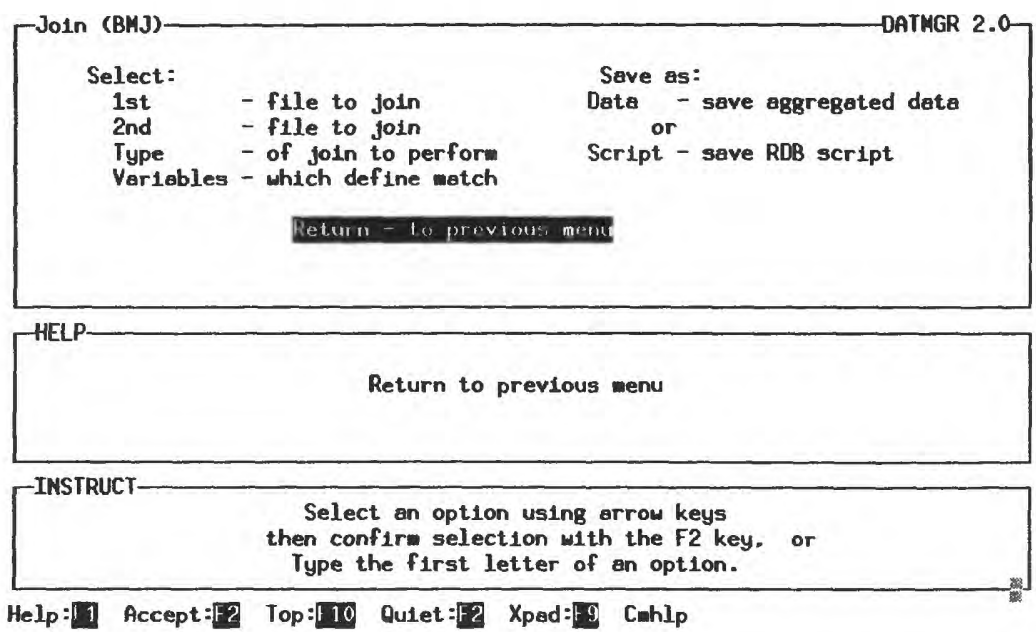

\section{Select Input Data Files to be Joined}

From the "Join (BMJ)" screen, select the "1st (BMJ1)" screen to select the first of the two files to be joined. This file could be a script file to which additional script for the join operator will be added. If a data file or script file was created by a previous "datmgr" operator, its file name will appear in the "Enter file name" field; otherwise this field will be blank. Select or type in a new file name and use either Accept (F2) or the Enter key to accept the file name and return control to the "Join (BMJ)" screen.

A similar process is used for the "Second file to join" operator. From the "Join (BMJ)" screen, select the "2nd (BMJ2)" screen to select the second of the two files used in the join operator.

\section{Select Join Type}

Based on the type of join specified, records from the two input files that meet the matching criteria are combined in the output file. From the "Join (BMJ)" screen, select the "Type (BMJT)" screen. Four types of "Join" are supported:

1. Natural - The output file from the two joined files will contain one record for each pair of matching records in the input files. Records from either input file may contribute to multiple matches in the other file.

2. One to One - The output file will contain one record for each pair of matching records in the input files. Each input record, however, may contribute to only one match.

3. Master/detail - Every record from file 1 is included in the output file. If there is no match in file 2 for a record, variables from the second file are set to missing in the output record.

4. Cartesian - Every record from each file is included in the joined file. If there is no match, variables are set to missing in the output file.

Use the arrow keys to select the option desired and use Enter. 

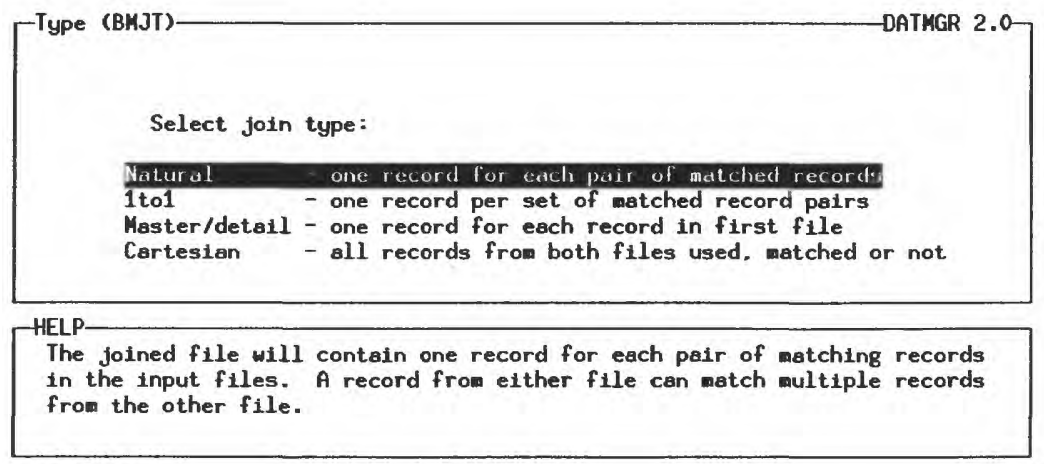

INSTRUCT-
$\begin{gathered}\text { Select an option using arrow keys } \\ \text { then confirm selection with the F2 key. or } \\ \text { Type the first letter of an option. }\end{gathered}$
Help: 1 Accept: 2 Top: 10 Quiet: 8 Xpad: 8 Cahlp

\section{Select Variables to Control Join}

Selected pairs of variables (if values match in both input files) control which records are combined and added to the output file. From the "Join (BMJ)" screen, select the "Variables (BMJV)" screen. Variables from both files are presented in side-by-side columns. Using the Enter and arrow keys to highlight the "Select" column, select up to eight variables from the first file to be matched, one on one, with an equal number of variables in the second file by assigning the same number to the paired "Select" fields in each column. The variable names do not have to match.

\section{Relational Subtract}

From the "Merge (BM)" screen, select the "Subtract (BMS)" screen. The "Subtract" operator writes records from the first input file to the output file when there is no match in the second input file.

From the "Merge (BM)" screen, select the "Subtract (BMS)" screen.
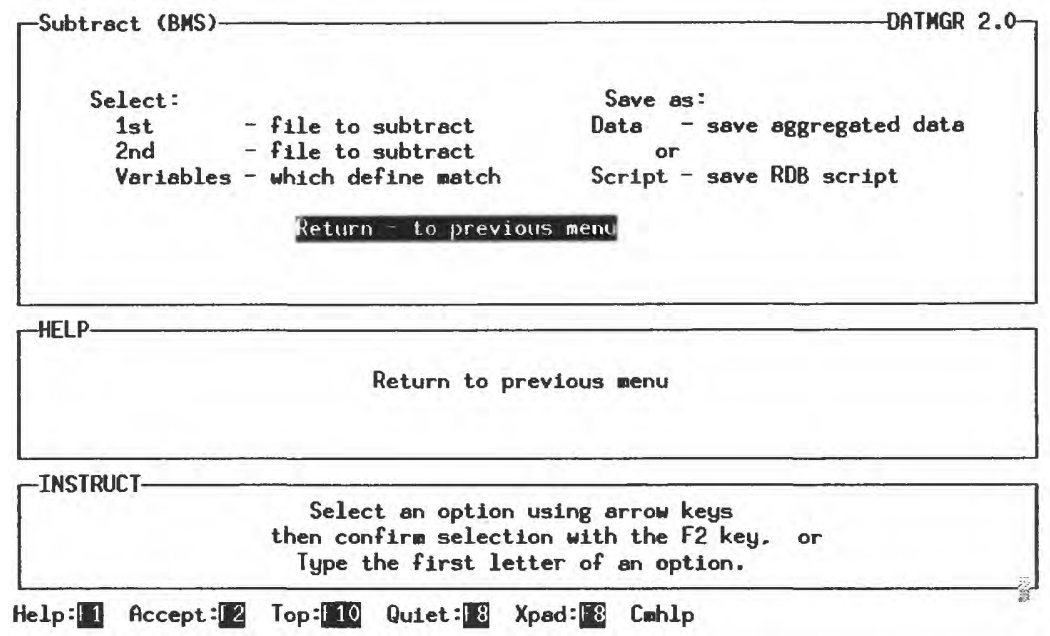


\section{Select Input Data Files to Subtract}

From the "Subtract (BMS)" screen, highlight the "First file to subtract" option and use Enter to view the "1st (BMS1)" screen. This file could be a script file to which additional script for the subtract operator will be added. If a data file or script file was created by a previous "datmgr" operator, its file name will appear in the "Enter file name" field; otherwise this field will be blank. Select or type in a new file name and use either Accept (F2) or the Enter key to accept the file name and return control to the "Subtract (BMS)" screen.

A similar process is used for selecting the second file for the "Subtract" operator. From the "Subtract (BMS)" screen, select the "2nd (BMS2)" screen to select the second of the files to be used in the "Subtract" operator.

\section{Select Variables to Control Subtract}

Selected pairs of variables from the two input files are used to control which records from the first input file are written to the output file when there is no match. From the "Subtract (BMS)" screen, select the "Varset (BMSV)" screen. Variables from both files are presented in side-by-side columns. Using the Enter and arrow keys to highlight the "Select" column, select up to eight variables from the first file to be matched, one on one, with an equal number of variables in the second file by assigning the same number to the paired "Select" fields in each column. The file names do not have to match.

\section{Concatenation}

From the "Merge (BM)" screen, select the "Concatenate (BMC)" screen. The "Concatenate" operator adds the observations of selected variables from one file (file 2) to the same variables in another file (file 1). Options include restricting the output file to those variables that are present in both files or including nonmatching variables in the output file with "missing" values assigned to records from the file not having equivalent variables in the other file.

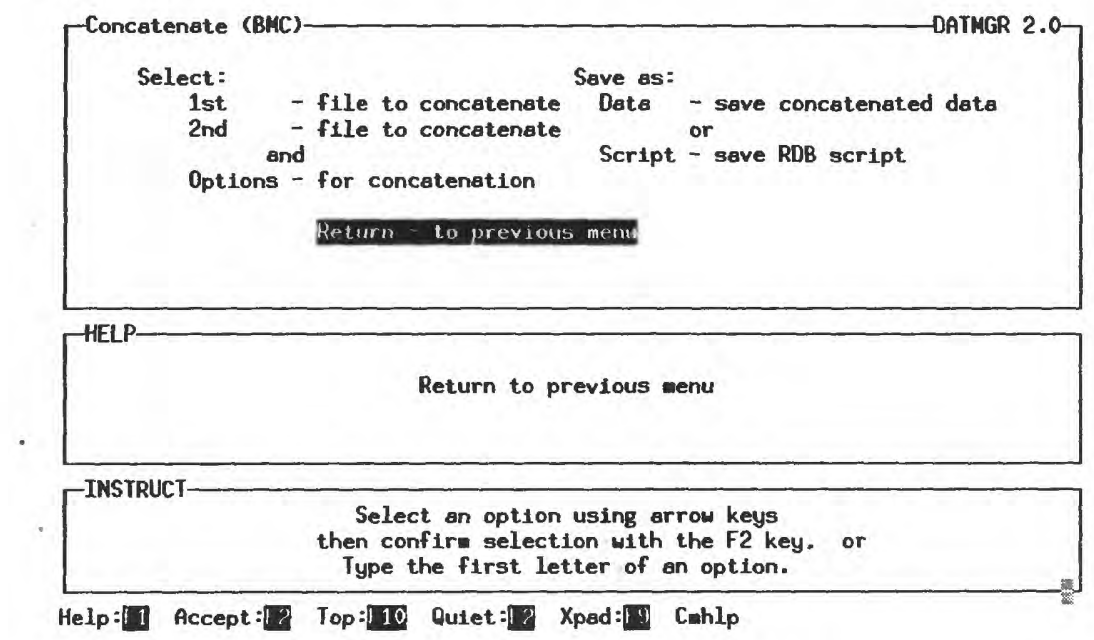

\section{Select Input Data Files to be Concatenated}

From the "Concatenate (BMC)" screen, select the "1st (BMC1)" screen to select the file to which the second file will be concatenated. This file could be a script file to which additional script for the "concatenate" operator will be added. If a data file or script file was created by a previous "datmgr" operator, its file name will appear in the "Enter file name" field; otherwise this field will be blank. Select or type in a new file name and use either Accept (F2) or the Enter key to accept the file name and return control to the "Concatenate (BMC)" screen. 
A similar process is used for the "Second file to join" operator. From the "Concatenate (BMC)" screen, select the "2nd (BMC2)" screen to select the file that will be concatenated to the first file.

\section{Options for Concatenation}

Two options are available for selecting variables to be included in the output data file from the concatenation process:

All variables from both files

Only variables common to both files.

From the "Concatenate (BMC)" screen, select the "Options (BMCO)" screen. First, enter the name of a variable to be added to the output file that will contain the name (pathname) of the file from which the record (row) originates. Next, select the option desired and use the space bar to toggle the selection "On" as indicated by the appearance of an "X" in the field to the left of the option name.
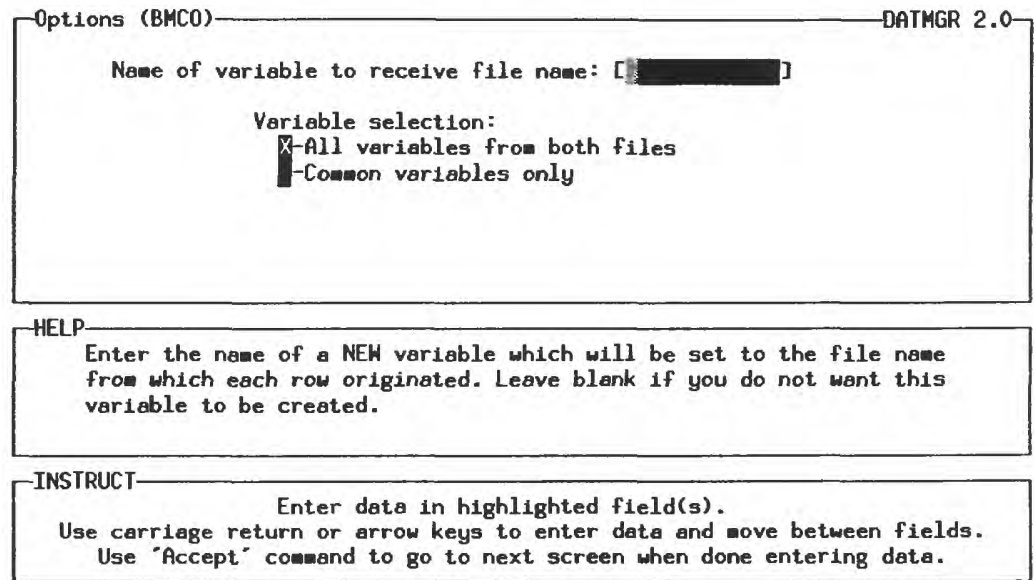

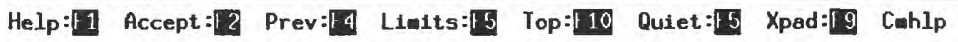

\section{Save an RDB Data File}

To create an RDB file from the "Merge" process, select the "Data (BM*D)" screen from the appropriate "Join (BMJ)," "Subtract (BMS)," or "Concatenate (BMC)" screen, where "**" would be "J," "S," or "C," respectively. Enter a file name in the field. Use either Accept (F2) or the Enter key to accept the selection. The system appends the ".rdb" extension to the file name. The system prompts the user for permission to overwrite an existing file. Pathnames may be used to place the resultant file in a selected directory.

\section{Save an RDB Script File}

To save the script from the "Merge" operator, save it to a new script file or append it onto an existing script file. From the "Join (BMJ)," "Subtract (BMS)," or "Concatenate (BMC)" screen, select the appropriate "Script $\left(\mathrm{BM}^{*} \mathrm{~S}\right)$ " screen, where "*" would be "J," "S," or "C," respectively. If the input file to this operator was an RDB script file, its file name will appear in the entry box. Use Accept (F2) or the Enter key to accept this selection. The new file will contain the old script with the new script appended. If a new script file is required, enter a new file name and use Accept (F2) or the Enter key. The system appends the ".rqs" extension to the file name. The system prompts the user for permission to overwrite an existing file. Pathnames may be used to place the resultant file in a selected directory. Select the "Return to previous menu" option twice to return control to the "Basic (B)" screen. 


\section{Remove Replicate Rows}

The "Merge" operator, and especially the concatenation option, can result in replicate rows in the output data file. The "Delete duplicate rows" operator removes replicate rows. The replication criteria may involve one, more than one, or all of the variables in the input data file.

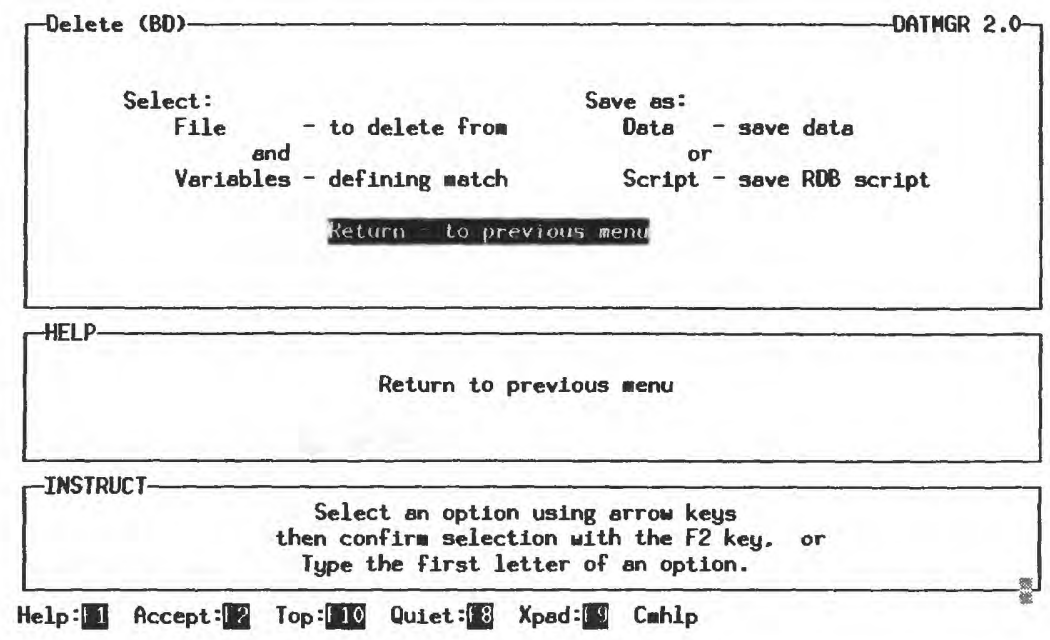

\section{Select an Input Data File}

From the "Basic (B)" screen, select the "Delete (BD)" screen. From the "Delete (BD)" screen, select the "File (BDF)" screen. If a data file or script file was created by a previous "datmgr" operator, its file name will appear in the "Enter file name" field; otherwise this field will be blank. The file indicated could be a script file to which additional script for the "delete duplicate rows" operator will be added. Use either Accept (F2) or the Enter key to accept this selection or type in a new file name and use either Accept (F2) or the Enter key to accept the new file name.

\section{Variables Defining Match}

Any (or all) of the variables in the input data file can be used with the matching criteria for removing replicate rows. The variables can be selected in any order. Only the selected variables will be used to determine equivalent values between records. From the "Delete (BD)" screen, select the "Variable (BDV)" screen. Use the arrow and Enter keys to select the variables. Enter a positive number in the "Select" field or deselect a field by entering a " 0 " (zero). When finished, use Accept (F2) to accept the selections and return control to the "Delete (BD)" screen.

\section{Save an RDB Data File}

To create an RDB file from the "Delete duplicate rows" operator, select the "Data (BDD)" screen from the "Delete (BD)" screen. Enter a file name in the field. Use either Accept (F2) or the Enter key to accept the selection. The system appends the ".rdb" extension to the file name. The system prompts the user for permission to overwrite an existing file. Pathnames may be used to place the resultant file in a selected directory.

\section{Save an RDB Script File}

To save the script from the "Delete duplicate rows" operator, save it to a new script file or append it onto an existing script file. From the "Delete (BD)" screen, select the "Script (BDS)" screen. If the input file to this operator was an RDB script file, its file name will 
appear in the entry box. Use Accept (F2) or the Enter key to accept this selection. The new file will contain the old script with the new script appended. If a new script file is required, enter a new file name and use Accept (F2) or the Enter key. The system appends the ".rqs" extension to the file name. The system prompts the user for permission to overwrite an existing file. Pathnames may be used to place the resultant file in a selected directory. Select the "Return to previous menu" option to return control to the "Basic (B)" screen.

\section{Compute Ranks (Percentiles)}

The "Compute ranks" operator converts a column of observed values to a list of values representing the following percentiles:

\section{$0.10 .51225102025 \quad 5075809095989999.599 .9$}

The output data file will contain 17 rows of data, one for each percentile. For small data sets, the data value for a particular percentile may be set to "missing" because the percentile ranks at the lower and upper percentiles are undefined. An optional variable can be named to hold the percentiles (as percentages) in the output data file. The following options are provided for treating censored data:

Use detection (reporting) limit as a real data value

Treat censored values as missing values

Predict ranks below detection limit (Helsel MLE Technique).

\section{Percentile Algorithm}

The algorithm used to calculate the percentile values is defined as follow:

Let:

$\mathrm{X}(\mathrm{n}) \quad=$ sorted (ranked) data values

$\mathrm{n} \quad=$ total frequency (number of observations)

$\mathrm{j}=$ fraction of data less than or equal to the stated percentile

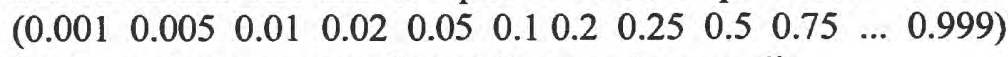

$\mathrm{P}_{\mathrm{j}} \quad=$ the data value corresponding to the stated percentile.

Then:

$$
\begin{array}{ll}
A & =j *(n+1) \\
B & =\operatorname{int}(A) \quad \text { integer value of "A") } \\
C & =A-B \\
P_{j} & =X(B)+C *(X(B+1)-X(B)) .
\end{array}
$$

Note: For small data sets, values for $\mathrm{P}_{\mathrm{j}}$ may be undefined at the lower and upper percentile values. Thus, values for $P_{j}$ are only calculated when:

$$
\begin{aligned}
& \mathrm{B}>=1 \\
& \mathrm{~B}<=\mathrm{n}-1
\end{aligned} \text { and }
$$

Undefined values for $\mathrm{P}_{\mathrm{j}}$ are set as "missing" in the output data file. 

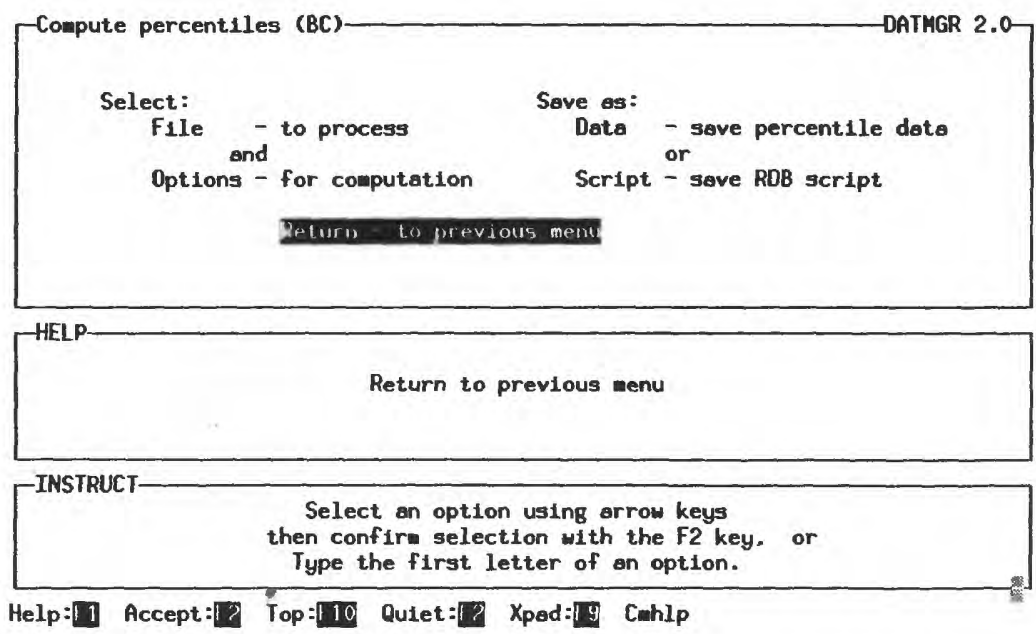

\section{Select an Input Data File}

From the "Basic (B)" screen, select the "Compute ranks (BC)" screen. From the "Compute ranks (BC)" screen, select the "File (BCF)" screen. If a data file or script file was created by a previous "datmgr" operator, its file name will appear in the "Select a file for input" field; otherwise this field will be blank. The file indicated could be a script file to which additional script for the compute operator will be added. Use either Accept (F2) or the Enter key to accept this selection or type in a new file name and use either Accept (F2) or the Enter key to accept the new file name.

\section{Options for Computing Percentiles}

From the "Compute ranks (BC)" screen, select the "Options (BCO)" screen.

\section{Variable to Receive Percentile Ranking}

A new variable can be added to the output data file to contain the percentile values as percentages. To select such a variable, type the variable name (12 characters or less) in the "Name variable" field.

\section{Censored Data Handling Method}

The following options are available for treating censored data in the computation of percentiles.

Use censored value as data value

Treat censored value as a missing value

Predict value below detection limit.

The first option disregards the remark code and treats the value in the field as a real value. The third option uses the same parameter estimation routine (Helsel MLE) as the "boxplt" program to estimate the percentile value below the detection limit. Use the arrow keys to select an option and toggle this selection "On" using the space bar. When finished, use Accept (F2) to accept these selections and return control to the "Compute ranks (BC)" screen. 

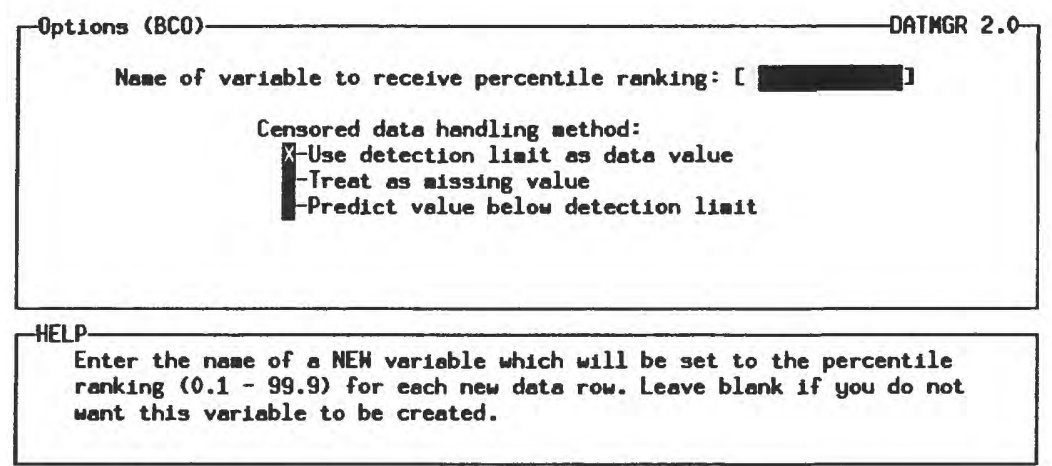

INSTRUCT- Enter data in highlighted field(s).
Use carriage return or arrow keys to enter data and wove batween fields.
Use 'Accept comand to go to next screen when done entering data.

\section{Save an RDB Data File}

To create an RDB file from the "Compute ranks" operator, select the "Data (BCD)" screen from the "Compute ranks (BC)" screen. Enter a file name in the field. Use either Accept (F2) or the Enter key to accept the selection. The system appends the ".rdb" extension to the file name. The system prompts the user for permission to overwrite an existing file.

Pathnames may be used to place the resultant file in a selected directory.

\section{Save an RDB Script File}

To save the script from the "Compute ranks" operator, save it to a new script file or append it onto an existing script file. From the "Compute ranks (BC)" screen, select the "Script (BCS)" screen. If the input file to this operator was an RDB script file, its file name will appear in the entry box. Use Accept (F2) or the Enter key to accept this selection. The new file will contain the old script with the new script appended. If a new script file is required, enter a new file name and use Accept (F2) or the Enter key. The system appends the ".rqs" extension to the file name. The system prompts the user for permission to overwrite an existing file. Pathnames may be used to place the resultant file in a selected directory. Select the "Return to previous menu" option to return control to the "Basic (B)" screen. 
Briel, L.I., 1993, Documentation of multiple-technique computer program for plotting major-ion composition of natural waters: U.S. Geological Survey Open-File Report 93-74, 88 p.

Chilingar, G.V., 1956, Durov's classification of natural waters and chemical composition of atmospheric precipitation in USSR-A Review: American Geophysical Union Transactions, v. 37, no. 2, p. 193-196.

Helsel, D.R., 1990, Less than obvious, statistical treatment of data below the detection limit: Environmental Science \& Technology, v. 24, no. 12.

Knobel, L.L., and Leahy, P.P., 1981, DUROV, a FORTRAN program for plotting chemical parameters on a hydrochemical facies diagram: U.S. Geological Survey Water Resources Division Bulletin, OctoberDecember, p. 34-41.

Maddy, D.V., Lopp, L.E., Jackson, D.L., Coupe, R.H., and Schertz, T.L., 1989, National Water Information System User's Manual, v. 2, chap. 2, Water Quality System: U.S. Geological Survey Open-File Report 89-617, 212 p.

Manis, R., Schaffer, E., and Jorgensen, R., 1988, UNIX relational database management: Englewood Cliffs, N.J., Prentice Hall.

Piper, A.M., 1944, A graphic procedure in the geochemical interpretation of water analyses: American Geophysical Union Transactions, v. 25, p. 914-923.

1953 [Revised], A graphic procedure in the geochemical interpretation of water analyses: U.S. Geological Survey Ground Water Notes, Geochemistry, no. 12, 14 p.

Stiff, H.A., Jr., 1951, The interpretation of chemical water analysis by means of patterns: Journal of Petroleum Technology, v. 3, no. 10, p. 15-17.

Wall, Larry, and Schwartz, Randall, 1991, Programming Perl: Sebastopol, Calif., O'Reilly \& Associates, $465 \mathrm{p}$.

Zaporozec, A., 1972, Graphical interpretation of water-quality data: Ground Water, v. 10, no.2, p. 32-43. 
APPENDIXES

REFERENCES 213

- PAGr 215 follows. 
Three files are associated with the interaction between the user and the program. The optional TERM.DAT file is used to change some aspects of how the program operates; the PROC.LOG file, for example, PIPER.LOG, records keystrokes entered during a program session; and error and warning messages are written to the ERROR.FIL file if problems arise during a program session. The ERROR.FIL file also can contain messages that document the processing sequence of the program.

TERM.DAT The TERM.DAT file includes the user's preferences used to override the default values of parameters that define the configuration of the computer system. The program looks for a TERM.DAT file in the current directory. The parameters include terminal type, the program response to the Enter key, and graphics options. Table A.1 describes the TERM.DAT parameters that show the default values as set for DOS-based computers, for DG/UX-based (Data General/UNIX) computers, and for other UNIX-based computers. A parameter is overridden by specifying its keyword and the new value in the TERM.DAT file. One record is used to define each parameter-with the keyword beginning in column 1 and the value beginning in column 8 .

Many of the TERM.DAT parameters control graphical output, such as the specification of a graphics device, text font, symbol size, and background color. The values assigned to the graphics parameters depend on the software library used to implement the Graphical Kernel System (GKS). For information on overriding the graphics parameters on DOS-based computers, see the README file distributed with the program. For other computer platforms, see the README documentation that accompanies the program and the GKS library documentation for your system.

Table A.1. TERM.DAT parameters

\begin{tabular}{|c|c|c|c|c|c|}
\hline \multirow[b]{2}{*}{$\begin{array}{l}\text { Parameter } \\
\text { keyword }\end{array}$} & \multicolumn{3}{|c|}{ Default values } & \multirow[b]{2}{*}{ Valid values } & \multirow[b]{2}{*}{ Description } \\
\hline & DOS & DG/UX & $\begin{array}{l}\text { Other } \\
\text { UNIX }\end{array}$ & & \\
\hline TRMTYP & $\mathrm{PC}$ & VT100 & VT100 & $\begin{array}{l}\text { PC } \\
\text { VT100 } \\
\text { OTHER }\end{array}$ & Terminal type. \\
\hline MENCRA & NEXT & NEXT & NEXT & $\begin{array}{l}\text { NONE } \\
\text { DOWN } \\
\text { NEXT }\end{array}$ & Program response to Enter key. \\
\hline USRLEV & 0 & 0 & 0 & 0 to 2 & $\begin{array}{l}\text { User experience level: } 0=\text { lots, } 1=\text { some, } \\
2=\text { none. }\end{array}$ \\
\hline GRAPHS & YES & YES & YES & $\begin{array}{l}\text { NO } \\
\text { YES }\end{array}$ & $\begin{array}{l}\text { Is a software library for graphics } \\
\text { generation available? }\end{array}$ \\
\hline GKSDIS & 1 & 1100 & 4 & $0-9999^{a}$ & $\begin{array}{l}\text { GKS code number for workstation type for } \\
\text { display terminal. }\end{array}$ \\
\hline GKSPRT & 2 & 102 & 4 & $0-9999^{a}$ & $\begin{array}{l}\text { GKS code number for workstation type for } \\
\text { printer device. }\end{array}$ \\
\hline GKSPLT & 3 & 7475 & 4 & $0-9999^{\mathrm{a}}$ & $\begin{array}{l}\text { GKS code number for workstation type for } \\
\text { pen plotter. }\end{array}$ \\
\hline GKSMET & 4 & 24 & 4 & $0-9999^{a}$ & GKS code number for metafile. \\
\hline GKPREC & CHAR & STROKE & CHAR & $\begin{array}{l}\text { STRING } \\
\text { CHAR } \\
\text { STROKE }\end{array}$ & Text precision. \\
\hline GKSCFT & 1 & 1 & 1 & -9999 to $9999^{a}$ & Text font for screen. \\
\hline GKPRFT & 1 & -2808 & 1 & -9999 to $9999^{a}$ & Text font for printer. \\
\hline GKPLFT & 1 & 1 & 1 & -9999 to $9999^{a}$ & Text font for plotter. \\
\hline BCOLOR & BLACK & BLACK & BLACK & $\begin{array}{l}\text { WHITE } \\
\text { BLACK } \\
\text { OTHER }\end{array}$ & $\begin{array}{l}\text { Background color of graphics screen } \\
\text { display. }\end{array}$ \\
\hline BGRED & 0 & 0 & 0 & 0 to 100 & $\begin{array}{l}\text { Percent red for background of graphics } \\
\text { screen display if BCOLOR=OTHER. }\end{array}$ \\
\hline
\end{tabular}


Table A.1. TERM.DAT parameters-Continued

\begin{tabular}{|c|c|c|c|c|c|}
\hline \multirow[b]{2}{*}{$\begin{array}{l}\text { Parameter } \\
\text { keyword }\end{array}$} & \multicolumn{3}{|c|}{ Default values } & \multirow[b]{2}{*}{ Valid values } & \multirow[b]{2}{*}{ Description } \\
\hline & DOS & DGUX & $\begin{array}{l}\text { Other } \\
\text { UNIX }\end{array}$ & & \\
\hline BGREEN & 0 & 0 & 0 & 0 to 100 & $\begin{array}{l}\text { Percent green for background of graphics } \\
\text { screen display if BCOLOR=OTHER. }\end{array}$ \\
\hline BGBLUE & 0 & 0 & 0 & 0 to 100 & $\begin{array}{l}\text { Percent blue for background of graphics } \\
\text { screen display if BCOLOR=OTHER. }\end{array}$ \\
\hline SYMSIZ & 100 & 100 & 100 & 1 to 10000 & Symbol size ratio in hundredths. \\
\hline TXTEXF & 133 & 100 & 100 & 0 to 200 & Text expansion factor in hundredths. \\
\hline TXTCHS & 0 & 0 & 0 & 0 to 200 & Text character spacing in hundredths. \\
\hline
\end{tabular}

a. Refer to GKS documentation and README file supplied with the program to determine valid values for these parameters.

The value of the MENCRA parameter and the data panel type control how the program responds when the Enter key is pressed. Table A.2 describes the program response for the various combinations of MENCRA values and data panel type. The default value for the MENCRA parameter is "NEXT."

Table A.2. MENCRA values and corresponding program response

\begin{tabular}{|c|c|c|}
\hline MENCRA & Data panel contents & Program response when Enter is pressed \\
\hline \multirow[t]{4}{*}{ NEXT } & menu & $\begin{array}{l}\text { Same as if Accept (F2) were executed: the highlighted menu } \\
\text { option is selected and the program advances to the next screen. }\end{array}$ \\
\hline & form & $\begin{array}{l}\text { For all but the last field on the form, causes the cursor to advance } \\
\text { to the next field. For the last field on the form, the response is the } \\
\text { same as for Accept (F2)-the program advances to the next } \\
\text { screen. (Note: usually the rightmost, lowest field is the "last" field } \\
\text { in the form. Occasionally, however, it is not!) }\end{array}$ \\
\hline & table & $\begin{array}{l}\text { For each row, the cursor advances across the fields in the row. For } \\
\text { all but the last row, the cursor advances from the last field in the } \\
\text { row to the first field of the next row. For the last row, the cursor } \\
\text { advances to the beginning of the last row. (Use Accept (F2) to } \\
\text { advance to the next page of the table.) }\end{array}$ \\
\hline & text & $\begin{array}{l}\text { Same as if Accept }(\mathrm{F} 2) \text { were executed-the program advances to } \\
\text { the next screen. }\end{array}$ \\
\hline \multirow[t]{4}{*}{ NONE } & menu & No effect-it is ignored. \\
\hline & form & $\begin{array}{l}\text { Cursor is advanced to the next field. When the cursor is in the last } \\
\text { field, the cursor cycles back to the first field. }\end{array}$ \\
\hline & table & $\begin{array}{l}\text { For each row, the cursor advances across the fields in the row. For } \\
\text { all but the last row, the cursor advances from the last field in the } \\
\text { row to the first field of the next row. For the last row, the cursor } \\
\text { advances to the beginning of the last row. (Use Accept (F2) to } \\
\text { advance to the next page of the table.) }\end{array}$ \\
\hline & text & No effect-it is ignored. \\
\hline \multirow[t]{4}{*}{ DOWN } & menu & $\begin{array}{l}\text { Same as if the down arrow were pressed: the highlight bar moves } \\
\text { to the next menu option. When the highlight bar is on the last } \\
\text { menu option, it returns to the first menu option. }\end{array}$ \\
\hline & form & $\begin{array}{l}\text { Cursor is advanced to the next field. When the cursor is in the last } \\
\text { field, the cursor cycles back to the first field. }\end{array}$ \\
\hline & table & $\begin{array}{l}\text { For each row, the cursor advances across the fields in the row. For } \\
\text { all but the last row, the cursor advances from the last field in the } \\
\text { row to the first field of the next row. For the last row, the cursor } \\
\text { advances to the beginning of the last row. (Use Accept (F2) to } \\
\text { advance to the next page of the table.) }\end{array}$ \\
\hline & text & No effect-it is ignored. \\
\hline
\end{tabular}


Figure A.1 shows an example TERM.DAT file that overrides the default BLACK background color of the graphics display. The parameter BCOLOR is set to OTHER and the parameters BGRED and BGBLUE are set to 40 and 60 , respectively. The resulting graphics screen display background will be a shade of purple. In addition to the parameters described in table A.1, there are five parameters that can be used to set the foreground and background colors of parts of the program screen on DOS-based computers. These parameters are described in table A.3.

Figure A.1. Example TERM.DAT file.

BCOLOR OTHER

BGRED 40

BGREEN 0

BGBLUE 60

Table A.3. TERM.DAT parameters for color display (DOS-based computers)

\begin{tabular}{cccl}
\hline $\begin{array}{c}\text { Parameter } \\
\text { keyword }\end{array}$ & $\begin{array}{c}\text { Default } \\
\text { value }\end{array}$ & $\begin{array}{c}\text { Allowable } \\
\text { values }^{\mathrm{a}}\end{array}$ & \multicolumn{1}{c}{ Description } \\
\hline CLRFRS & 15 & 0 to 15 & Standard foreground color. \\
CLRBKS & 1 & 0 to 15 & Standard background color. \\
CLRFRE & 7 & 0 to 15 & Foreground color for error messages in instruction panel. \\
CLRBKE & 4 & 0 to 15 & Background color for error messages in instruction panel. \\
CLRFRD & 14 & 0 to 15 & Color of first letter of commands in command mode. \\
\hline
\end{tabular}

a. 0-black, 1-blue, 2-green, 3-cyan, 4-red, 5-magenta, 6-brown, 7-light gray, 8-dark gray, 9-bright blue, 10-bright green, 11-bright cyan, 12-bright red, 13-bright magenta, 14-yellow, or 15-white

PROC.LOG A file named PROC.LOG, for example, PIPER.LOG, is created each time the program is run. This file contains the sequence of keystrokes entered during a program session. It may be used as an input to the program in a later session. To save the sequence of keystrokes, you must rename the file because the program will overwrite the PROC.LOG file in the next session of the program. Keystrokes of nonprinting keys, such as the backspace and function keys, are represented in the file by special codes. Table A.4 lists each code and its definition. Menu options are chosen in one of two ways-either press the first letter(s) of a menu option, or position the cursor with the arrow keys and then Accept (F2). The use of the first method will make the log file easier to interpret and modify. The use of the letter " $x$ " for making option selections also will help. Table A.5 contains an annotated example log file for a program session that opens a WDM file and gets a summary of the contents of the file.

Table A.4. Codes used for nonprinting characters in a log file

\begin{tabular}{ccc|ccc}
\hline Code & $\begin{array}{c}\text { User's } \\
\text { keystroke }\end{array}$ & $\begin{array}{c}\text { Associated } \\
\text { command }\end{array}$ & Code & $\begin{array}{c}\text { User's } \\
\text { keystrokes }\end{array}$ & $\begin{array}{c}\text { Associated } \\
\text { command }\end{array}$ \\
\hline$\# 227$ & $;$ or Esc & & $\# 401$ & F1 & Help \\
$\# 208$ & Backspace & $\# 402$ & F2 & Accept \\
$\# 213$ & Return or Enter & $\# 403$ & F3 & \\
$\# 301$ & $\uparrow$ & $\# 404$ & F4 & Prev \\
$\# 302$ & $\downarrow$ & $\# 405$ & F5 & Limits \\
$\# 303$ & $\rightarrow$ & $\# 406$ & F6 & Intrpt \\
$\# 304$ & $\leftarrow$ & $\# 407$ & F7 & Status \\
$\# 307$ & Page Up & $\# 408$ & F8 & Quiet \\
$\# 308$ & Page Down & $\# 409$ & F9 & Xpad \\
& & $\# 410$ & F10 & Top \\
\hline
\end{tabular}

a. The Escape key is only functional on DOS-based computers. 
Table A.5. Description of example log file

\begin{tabular}{|c|c|c|}
\hline Line & Contents & Explanation \\
\hline 1 & fotest.wdm & $\begin{array}{l}\text { The File option was selected from the Opening screen menu. The Qpen option } \\
\text { was selected from the File (F) screen menu. The file name test.wdm was entered } \\
\text { in the Open (FO) screen. }\end{array}$ \\
\hline 2 & \#402 & $\begin{array}{l}\text { Accept, function key F2, was executed, which causes the test.wdm file to be } \\
\text { opened. }\end{array}$ \\
\hline 3 & s & The Summarize option was selected from the File (F) screen menu. \\
\hline 4 & \#402 & $\begin{array}{l}\text { After the summary of the contents of the test.wdm file had been viewed, } \\
\text { Accept, function key } \underline{F 2} \text {, was executed. }\end{array}$ \\
\hline 5 & $\pi$ & $\begin{array}{l}\text { The Return option was selected from the File (F) screen menu. The Return } \\
\text { option was selected from the Opening screen menu. }\end{array}$ \\
\hline
\end{tabular}

Log files can be used to repeat a task, or they can be modified to perform a similar task. A log file may contain all of the keystrokes required for a complete program session, or the keystrokes for part of a program session. For example, all of the keystrokes needed to plot the results of a model run can be saved and then reused for the next model run. A subset of a session might consist of the keystrokes required to select a particular group of data sets; this log file would be used whenever that data were needed.

A log file may be entered at any point in the program by first typing "@"; then type the name of the log file in the small panel that appears. The program will run using the input from the file just as if it were being typed in. The program and the log file must be synchronized; if the keystrokes in the log file get out of sync with the program, then the program may produce unpredictable results. A common cause of synchronization problems involves output files. If an output file, which did not exist when the log file was generated, exists when the log file is read by the program, then the program may ask if it is OK to overwrite the existing file. Because the keystrokes required to answer this question are not in the log file, the program will probably do something unexpected. 


\section{APPENDIX B. Glossary of Terms}

Aggregation. The process of condensing multiple rows of a data file into a single row with the resultant value of each column representing a computed statistic of the condensed row values; for example, mean, median, or number of observations.

Attribute. An object used to characterize and identify an element in a data base.

Block. A string of data values in a time-series data set with a header value that indicates the date of the first value, number of values in the string, and quality code of the string.

Buffer. An array of data-set numbers.

Censored data. A data value remarked with a code that indicates a qualification of the value; for example, a "less than" $(<)$ remark code would indicate that the actual value of the stored constituent is less than the value reported.

Command file. A text file of a sequence of responses to screens.

Concatenation. A vertical relation join in which a column of one file is matched and appended to a column in a second file. In QWGRAF the matching of columns is by the column name assigned in the column name line of the RDB file.

Data compression. When a sequence of time-series values are the same, the value and the number of those values are stored instead of repeated storage of the same value.

Data-set number. A number from 1 to 32,000 assigned to a data set on a WDM file.

Direct-access file. A file in which records can be randomly accessed, written or read.

Flat file. A sequential text file of rows and columns of data.

GKS (Graphical Kernal System). An ANSI and FIPS graphics standard implemented by many software vendors with a set of Fortran callable subroutines.

Log file. A file created by the program to store the sequences of responses to menus.

Menu. Text written to the terminal for requesting input.

Message file. A read only, direct-access file that stores the questions, help information, and valid responses for menus, as well as the attribute names, definitions, and characteristics.

Quality code. A number from 0 to 30 tagged to time-series data and used by some data process options to describe the quality of the data, with 0 being best and 30 being worst.

Partition variable. A variable in the data set whose unique values are used as the basis for grouping the rows in the data set. The grouped rows may or may not be further aggregated.

Relational join. The horizontal merging of records from two or more files that are written to an output file if there is a value match in each of one or more designated sets of common variables (not necessarily the same name) in the source files.

Relational subtract. Records from the first of two files are written to an output file when there is no value match between any of the designated pairs of common variables between the two files.

RDB file. A data formatting system based on TAB delimited ASCII files that contain comment and column definition information at the top of the file. The RDB system comes with a robust toolkit of mathematical and operator functions for modifying and managing the data. RDB functions are invoked through scripting languages such as Perl and Python.

RDB operator. Programs that perform unique functions on the data in an RDB file. RDB operators are grouped into data movers, report generators, and utilities.

Screen. The 24-row (line) by 80-column (character) image on the display monitor or in an $\mathrm{X}$ window on the display monitor.

Script file. A special RDB file that contains no data rows but a script of Perl commands imbedded in the $\mathrm{RDB}$ file comment lines. This file is read by the RDB operator "rdbqry," which then executes the script to perform a sequential list of operations on the RDB data file(s) specified within the script. Script files can be used as input files to any QWGRAF procedure. 
Specifications file. A file that contains the complete list of specifications, which includes the source data file name, that are passed as arguments to a QWGRAF program. There are three types of Specification files-latest, default, and user defined. Specification files, of which there can be any number, can be called into a program at any time to replace the current specifications.

TERM.DAT file. User's configuration file used to modify the computer system options for the program.

Time series. Values of a measured or calculated variable over time at regular or irregular intervals.

WDM (Watershed Data Management). A binary direct-access file and associated software for storage, retrieval, and management of hydrologic and related data.

Window. A subset of a screen bounded by a drawn rectangle. 
QWGRAF programs operate internally on data files formatted in RDB. RDB is a publicdomain relational data-base management system that is based on user-editable ASCII (flat) files and a library of tools for managing and modifying the data. RDB is highly portable and will run on UNIX, MS-DOS, or Macintosh computers. Scripting languages such as Perl and Python can be used in command line mode to access and modify RDB files.

The RDB system was chosen as the underlying data-formatting system for QWGRAF programs because of the potential use and transferability of files between QWGRAF programs and other UNIX and DOS based systems. RDB data-management functions also are used in selected options in QWGRAF programs.

The RDB system is generally accessible in command line mode through the use of relatively complex syntax that requires a significant learning curve not suitable for all levels of potential users of the QWGRAF system. Thus, QWGRAF includes an interactive program "datmgr" that provides a wide range of data manipulation capability, which with very minor exceptions, does not require the user to have any knowledge or experience with RDB syntax or any of the available scripting languages used with RDB. Functions available in "datmgr" include sorting, aggregation of data by a row grouping variable and a summarization statistic (mean, median, or sum), definition of algorithms for creating new variables, relational algebra joins with other data files, concatenation of files, Boolean logic selection of specific rows, column subsetting, and row-to-column and column-to-row transformations.

The RDB system views the form of the data file as that of a relation or table, with rows and columns of information. There is no effective limit on the number of columns (variables) or the number of rows in a data set. A unique aspect of an RDB file is the use of specialized lines of self-documentation inserted at the top of the file. This documentation, which also is editable, can serve informational needs as well as functional needs (see subsequent section on "RDB FILE EXTENSIONS").

\section{RDB Data File Structure}

An RDB file is an ASCII file; therefore, it can be manipulated by regular UNIX utilities and edited with standard text editors. An RDB data file consists of comment lines, header lines, and data lines.

\section{Comment Lines}

Comment lines are indicated by a pound (\#) sign in the first column, a space in the second column, followed by user supplied text. Any number of comment lines may be used. Comment lines also provide a functional role to store scripts generated by the "datmgr" program (see subsequent discussion on "RDB Scripts").

\section{Header Lines}

RDB files require two header lines. The fields in the first header line contain the variable names to be assigned to the data columns in the file. The second header line contains the characteristics to be assigned to the variables, such as field length, data type and variable description. Special RDB file extensions have been implemented for use by QWGRAF programs to provide additional variable definition capabilities (see subsequent discussion on "RDB Structure Extensions."

\section{Column (Variable) Names}

Variable names used in QWGRAF programs are limited to 12 characters. RDB file variable names are separated by a TAB. Alphabetic, numeric, and the underscore ("-") characters are the best choices for use in variable names. Variable names should include at 
least one alphabetic character and they are case sensitive; that is, "COUNT" is different from "Count."

Although other nonalphanumeric characters, such as, the percent sign (\%), colon (:), the "@" sign, equals (=), comma (,), dot (.), and dash (-), are generally acceptable for use in RDB names, problems can arise when using these characters in names to be used by some of the RDB and Perl operators utilized by the "datmgr" program. The pound (\#) sign is acceptable if it is not the first character in a variable name; otherwise, the line would be considered a comment line.

The TAB character is never to be used in column names, nor should internal spaces or UNIX I/O redirection characters $(<,>$, or I ) be used.

\section{Data Definition Lines}

The data definition line contains a repeating sequence of fields, one group of fields for each variable. The first field in each group defines the default RDB specifications for column width, data type, and alignment justification. The column width must be specified; data type and alignment are optional. The data definitions in this field take the form of adjacent characters in a single word (see subsequent discussion on "Example Data Type Definitions"). The remaining fields in a group contain additional definitions of the variable provided by RDB file extensions. Each variable definition group must be separated by a TAB.

\section{Field Width}

The width of each field is specified by a numeric count and can have a maximum value of "12."

\section{Data Types}

Data types supported in standard RDB files include string $(\mathrm{S})$, numeric $(\mathrm{N})$, or month (M). Default is type "string." In RDB files used by QWGRAF, the column extension facility (see subsequent discussion of "RDB Structure Extensions") provides for more detailed data-type definitions including integer, real, double, or text (with defined field length).

\section{Printout Justlfication}

Printout justification is specified by " $<$ " for left justification and " $>$ " for right justification. If not specified, the string and month types will be left justified and numerics will be right justified. Printout justification is not utilized in QWGRAF programs.

\section{Example Data Type Definitions}

The following are examples of data definitions as they would appear in a standard RDB file.

$8 \mathrm{n}<\quad-$ The variable is an 8-digit numeric (integer or real) left justified.

$8 \mathrm{n} \quad-$ The variable is an 8-digit numeric (integer or real) right justified.

$15 \mathrm{~S}>\quad-15$-character string right justified (default).

\section{Data Lines}

RDB file data lines consist of rows and columns of observations (values). There can be one or more columns (variables) in a row, and there can be any number of rows in an RDB file. QWGRAF programs, however, are generally limited to 10,000 rows. Data columns are separated by a TAB. Therefore, data values must not contain TAB characters. All rows in the data lines of an RDB file are terminated with RETURN (NEWLINE) characters. The missing values are indicated by consecutive TAB markers. 


\section{RDB Structure Extensions}

RDB files can be implemented with extensions to provide additional column definition capability. Applications, such as QWGRAF, must be designed to utilize these extensions. Each column can have its own unique set of extensions; thus, the additional data definition field(s) provided in an RDB file extension follow the applicable default column definition word field on the second header line.

The additional functionality provided by these extensions does not alter or compromise the functionality of the standard RDB column definition fields if the file is used in nonQWGRAF applications. Any QWGRAF data definition text that follows the default RDB data definition word field would simply be interpreted as miscellaneous text.

\section{Keywords}

Each optional data definition field in an RDB file extension is identified by a keyword in angle brackets (<keyword $>$ ) at the beginning of the field. The keywords are recognized by the QWGRAF programs, which then interpret the information that follows the keyword to assign the intended characterizations to the data in that column. The following extensions have been developed specifically for the QWGRAF programs.

\section{Data Types <dtype}

Standard RDB supports only three types of variables-numeric $(\mathrm{N})$, string $(\mathrm{S})$, and month (M). Through the use of the "<dtype>" extension, QWGRAF supports the following additional data types:

$$
\begin{array}{lll}
\text { <dtype> } & \text { int } & \text { (integer) } \\
\text { <dtype> } & \text { real } & \text { (floating point, single precision) } \\
\text { <dtype> } & \text { double } & \text { (floating point, double precision) } \\
\text { <dtype > } & \text { text X } & \text { (text, where "X" is field length). }
\end{array}
$$

\section{Algorithms <perl>}

The <perl $>$ extension permits the storage of algorithms for defining and computing new variables to be added to the data file. While this can be done manually with a text editor, the QWGRAF "datmgr" program also provides for defining the algorithms for new variables. The "datmgr" program presents a screen to the user in which the column characteristics (name, type, and length), as well as the algorithm, are specified.

In either case, the column definition and the algorithm for the new variable are stored in the RDB file, but the actual data values are not stored. The values will be computed when needed by one of the QWGRAF programs. See Appendix D on "Conversion of Other Data to RDB" and the sections in Appendix F on the "compcol" and "perltbl " operators for more information and details on the Perl syntax used to formulate the algorithms. An example of an algorithm as stored in an RDB file follows:

$$
<\text { perl }>\text { \$calcium_meq }=\$ \text { calcium } * 0.0449 \text {. }
$$

\section{Variable Descriptions <descr}

The $<$ descr $>$ extension provides for user-supplied text to further characterize the variable; for example,

$$
<\text { descr }>\text { Sodium, mg/L. }
$$

\section{Example RDB Tables}

Tables C. 1 and C. 2 show examples of RDB files as viewed with an editor. The TAB character is represented by " $<\mathrm{T}>$ " and the RETURN character is represented by " $<\mathrm{R}>$." It is important to note that only actual data are stored in the data fields, with no leading or trailing blanks. Null values would be indicated by consecutive TAB characters. 
Table C.1 is the simplest form of the RDB file and contains no comment lines (or imbedded scripts) or column extensions. Note column names, column definitions, and data values are separated with TAB's $(<\mathrm{T}\rangle)$.

Table C.1. A simple RDB table

NAME $<$ T $>$ COUNT $<$ T $>$ TYP $<$ T $>$ AMT $<$ T $>$ OTHER $<$ T $>$ RIGHT $<R>$

$6<\mathrm{T}>5 \mathrm{~N}<\mathrm{T}>3<\mathrm{T}>5 \mathrm{~N}<\mathrm{T}>8<\mathrm{T}>8<\mathrm{R}>$

Bush $<\mathrm{T}>44<\mathrm{T}>\mathrm{A}<\mathrm{T}>133<\mathrm{T}>$ Another $<\mathrm{T}>\mathrm{This}<\mathrm{R}>$

Hansen $<\mathrm{T}>44<\mathrm{T}>\mathrm{A}<\mathrm{T}>23<\mathrm{T}>$ One $<\mathrm{T}>\mathrm{I} \mathrm{s}<\mathrm{R}>$

Jones $<\mathrm{T}>77<\mathrm{T}>\mathrm{X}<\mathrm{T}>77<\mathrm{T}>$ Here $<\mathrm{T}>\mathrm{O}$ N $<\mathrm{R}>$

Perry $<\mathrm{T}>77<\mathrm{T}>\mathrm{B}<\mathrm{T}>244<\mathrm{T}>$ And $<\mathrm{T}>\mathrm{The}<\mathrm{R}>$

Hart $<\mathrm{T}>77<\mathrm{T}>\mathrm{D}<\mathrm{T}>1111<\mathrm{T}>$ So $<\mathrm{T}>$ Right $<\mathrm{R}>$

Holmes $<\mathrm{T}>65<\mathrm{T}>\mathrm{D}<\mathrm{T}>1111<\mathrm{T}>\mathrm{O} \mathrm{N}<\mathrm{T}>\mathrm{E}$ dge $<\mathrm{R}>$

Table C. 2 is an RDB file that contains a single comment line, a column name line, a column definition line that contains extensions for data type and algorithm definition, and 15 data lines. Names in the variable name row and column definitions, which include the algorithm definitions, are separated by TAB's $(<T\rangle)$. Note that three algorithms are defined, but the data columns for these variables are empty. When required by another QWGRAF program, the variables will be computed. The slashes at the end of the column name and definition lines indicate that in the actual ASCII file information on the line following the slash would continue on the same line.

Table C.2. RDB table with column definition extensions

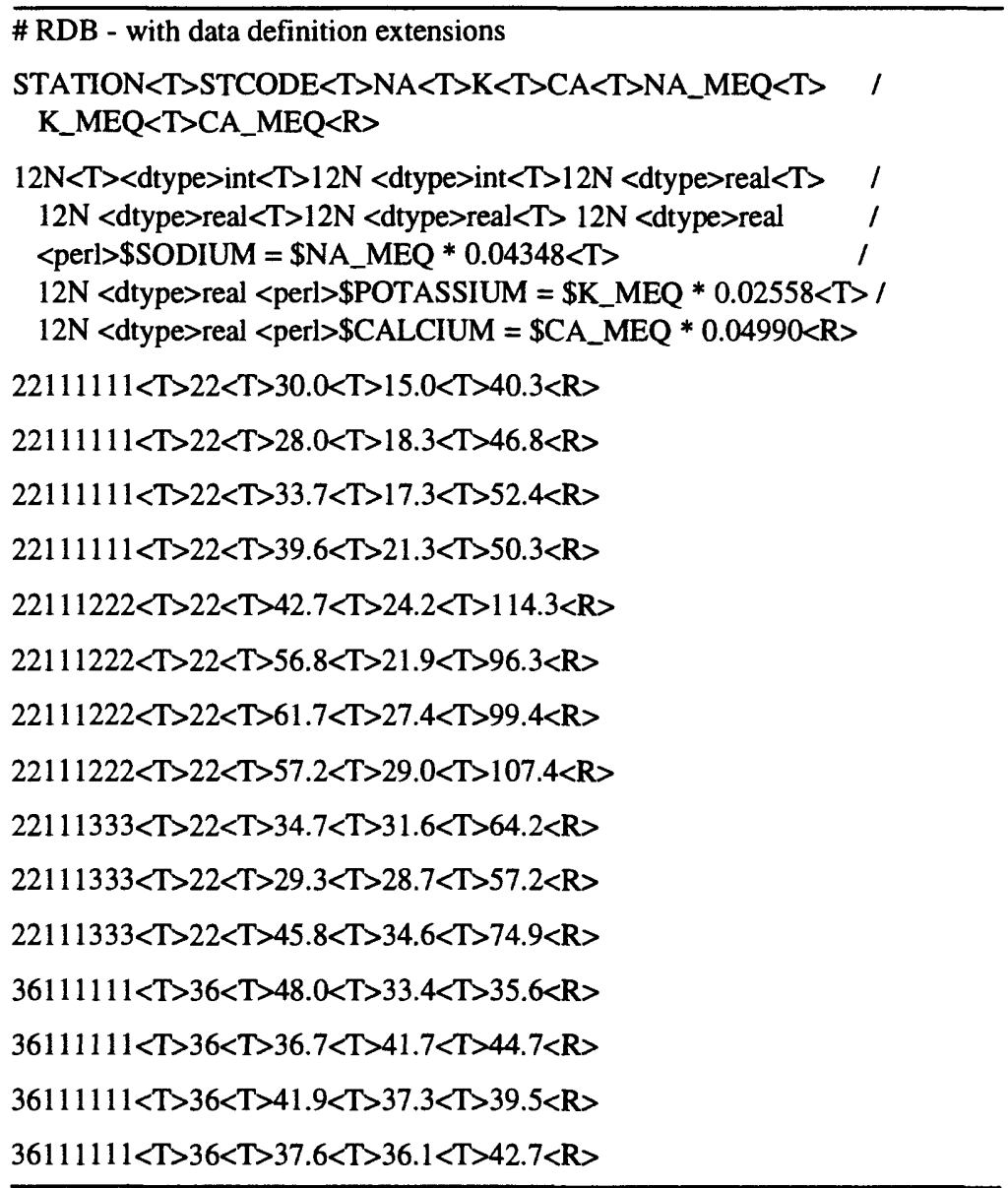




\section{RDB Scripts}

As the user develops a data-management scenario in the "datmgr" program, the program captures the various RDB commands used and creates an executable script. This script contains the information a QWGRAF program needs to read the input data file, convert the data to the RDB format, and make the required modifications to the data. The resultant file that contains the script (described subsequently) can then be used as an input file to any QWGRAF program.

The "datmgr" program creates a script each time the program is used. Thus, the user does not need to know or use any RDB command line syntax or any of the scripting languages that operate on RDB files. Even though the script is being generated continuously, the user decides whether to save the script for future use. When saved, the script is stored in the comment lines of a special RDB file identified by the ".rqs" file-name extension. Script lines in this file are distinguished from the other comment lines (comment lines begin with the \# sign) by the use of a beginning ("\# begin script") and an ending ("\# end script") keyword line.

A script file does not contain data lines, only the instructions to generate the data required by a QWGRAF program. Thus, it can be used as the input file to any QWGRAF program. The "datmgr" program provides an option to execute a script. The output from this process is an RDB data file that contains the data in its final form.

A more detailed discussion of the script function is given in the description of the "rdbqry" operator in the "OPERATORS" section in Appendix F.

Table C. 3 is an example of an RDB script file that contains a Perl script in the comment lines. Note the absence of data lines. All data values whether populated in the original source file or to be computed by an algorithm in some step of the script process will be produced from this script for use by the QWGRAF program. Note that the slash at the end of some lines indicates that the following line is a continuation of the preceding line in the actual ASCII file.

Table C.3. RDB file used to store a script generated by QWGRAF "datmgr" program

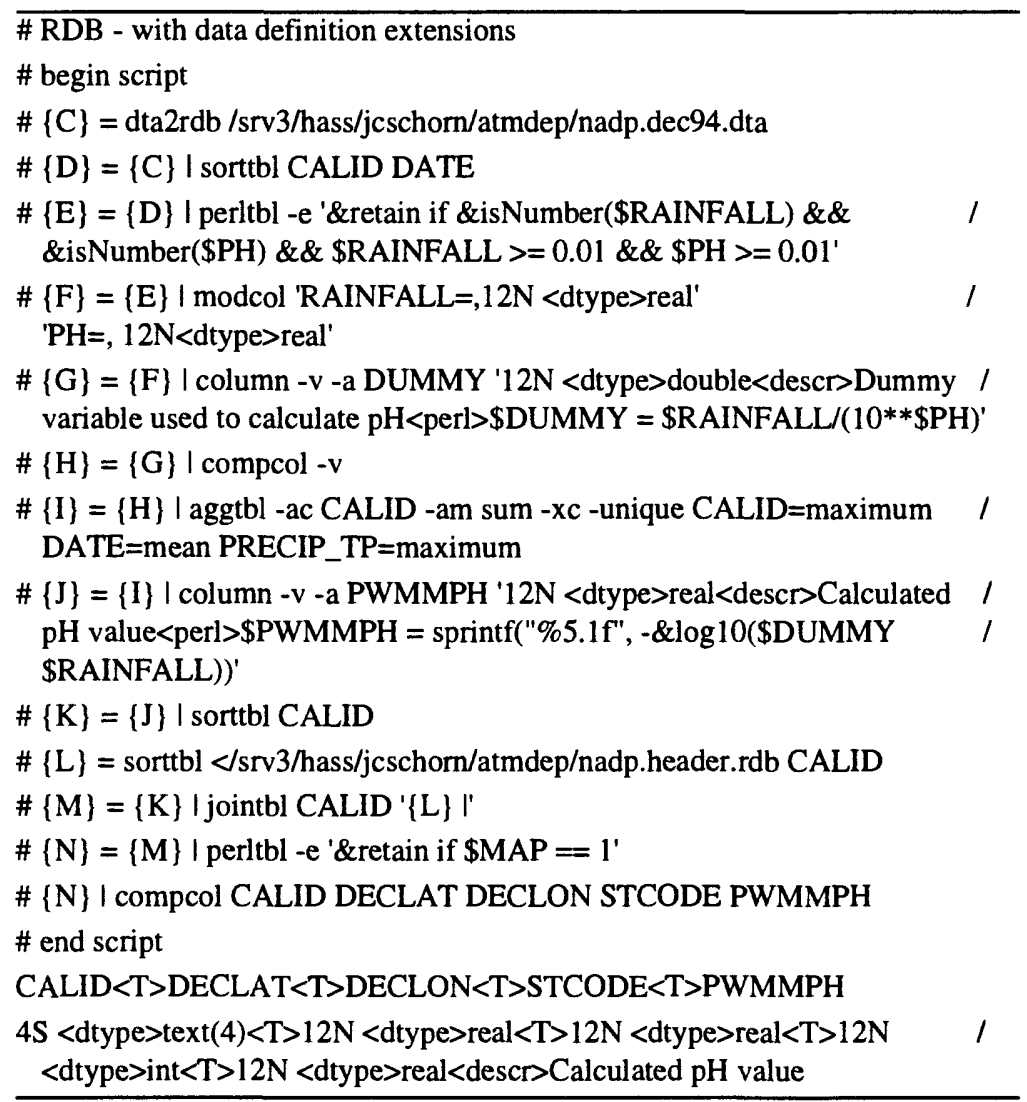


All programs in QWGRAF operate internally on RDB files; thus, it is often necessary to convert other data formats to the RDB format. Several options are available for converting other formats to RDB. Users familiar with RDB syntax and scripting languages, such as Perl or Python, can develop their own conversion routines. Selected RDB operators have been created to convert various file formats to RDB. Macros can be created to convert files used with statistical programs to RDB. A procedure is described to convert spreadsheet files to RDB. Finally, special filter programs, implemented as RDB operators, have been developed that permit QWGRAF programs to detect selected data formats and convert them to RDB format. The discussions in Appendix D focus on the descriptions of these other file formats and how the data and inherent functional utility in these files can be translated to RDB files to meet the data-management needs of the user. With very few exceptions, it should not be necessary for the typical QWGRAF user to use or even be aware of RDB, its operators, or complex command line syntax in order to prepare data for use with the QWGRAF programs. The QWGRAF interface provides most of the data-management tools required. For documentation purposes and for those so inclined, Appendix E provides detailed discussions on the command line syntax and use of the macros and RDB operators used by QWGRAF to create and modify RDB files.

\section{RDB Tables from Third Party Systems}

Some third party packages use data files that are not readable by regular text editors; thus, to create a filter to convert these formats to RDB is usually impractical. The alternative is to develop internal programs (macros) to output a file in RDB format. Conversion macros for the three packages listed below have been created and are described in more detail in Appendix E.

Package $\quad \underline{\text { RDB Macro }}$

Statit tblwrite

SAS sasrdb.sas

Tactician An RDB macro for Tactician is not available; but the procedure described in Appendix E will produce an RDB file from Tactician, as well as several other commercial spreadsheet programs.

\section{RDB Table Conversion Operators}

Several RDB operators have been created for converting common formats to RDB. These operators were created for the RDB user and are not a formal part of the QWGRAF system. However, because the files created by these operators are RDB, they can be used in QWGRAF applications. These operators are described briefly below and in the section on "Standard RDB Operators for Data Format Conversions" in Appendix E.

Operator name: 2 tabs

Description:

Converts an ASCII file with columns separated by delimiters other than TABS to an RDB tab-delimited file. The output file must be edited to add header lines that describe the column names (line 1) and the column type, width, and justification (line 2).

Operator name: f2tbl

Description:

Reads data by using a Fortran-style format string.

Operator name: tsql

Description:

Converts an Ingres table to an RDB file. 


\section{QWGRAF Data Format Filters}

QWGRAF programs detect and convert other selected data formats to RDB format. These conversions are accomplished by special filter programs that operate transparent to the QWGRAF user. These programs are implemented as RDB operators (see table D.1 below), and can be used outside of QWGRAF in command line mode to convert data files (see Section III in Appendix E).

Each data file to be used by a QWGRAF program is identified as to its format by a special three-character extension to the file name. If a requested data file has no identifiable extension, then it is assumed to be an RDB file. If the file-name extension matches an available filter, then no additional action is required by the user as each QWGRAF program recognizes the format type and invokes the appropriate filter to make the conversion.

The file format conversion process results in the creation of a temporary RDB file used by the QWGRAF program. If a permanent copy of the RDB file is required, then it can be created either by using the filter operator in command line mode or by invoking one of the functions in the QWGRAF "datmgr" program and outputting the result to a new RDB file. An RDB file created in "datmgr" will be identified by the ".rdb" added to the root file name. Table D.1 lists the format filter programs currently available for QWGRAF. Also listed for reference purposes is the name of the RDB operator created to implement the filter. Each filter is discussed in detail subsequently.

\section{Format Definition Files}

Some data formats can use an accompanying file to provide information to characterize the variables in the data file. The Format Definition File provides for assigning names to data columns, specifying data types, adding additional descriptive information, and depending on the format, defining additional functionality, such as algorithms to create new variables. These files are associated with the data files by having the same root name as the data file and a unique three-character file-name extension (see table D.1).

Table D.1. Input file formats recognized by QWGRAF programs

\begin{tabular}{|c|c|c|c|}
\hline $\begin{array}{l}\text { File-name } \\
\text { extension }\end{array}$ & $\begin{array}{l}\text { Format } \\
\text { definition } \\
\text { extension }\end{array}$ & $\begin{array}{l}\text { RDB } \\
\text { operator }\end{array}$ & Description \\
\hline.$r d b$ & none & none & $\begin{array}{l}\text { Default format used by QWGRAF programs. RDB files are } \\
\text { ASCII data arranged in rows and columns delimited by } \\
\text { TAB's. Special rows at top of file contain comments, } \\
\text { variable names, and column definitions. }\end{array}$ \\
\hline dta & dtv & $\mathrm{dta} 2 \mathrm{rdb}$ & $\begin{array}{l}\text { Blank or text delimited files of rows and columns of real, } \\
\text { integer or text data. Use of format definition file is optional. }\end{array}$ \\
\hline.$q w d$ &.$q w v$ & qwd2rdb & $\begin{array}{l}\text { ASCII files from NWIS-I QWDATA FLATOUT program. } \\
\text { Requires format definition file created by QWGRAF } \\
\text { program, but extension name changed to .qwv. }\end{array}$ \\
\hline str & none & str2rdb & $\begin{array}{l}\text { NWIS-I } 1 \text { and * cards from USGS WATSTORE and NWIS- } \\
\text { I systems. Does not use format definition file. }\end{array}$ \\
\hline ftr & .ftv & $\mathrm{ftr} 2 \mathrm{rdb}$ & $\begin{array}{l}\text { ASCII file output from Fortran program. Requires a format } \\
\text { definition file similar to .dtv file, but first line must contain } \\
\text { the Fortran format statement. }\end{array}$ \\
\hline .pst & .psv & pst2rdb & $\begin{array}{l}\text { PSTAT format output from USGS NWIS-I QWDATA } \\
\text { system. Requires format definition file created by } \\
\text { QWDATA program, but extension name changed to .psv. }\end{array}$ \\
\hline.$p c t$ & none & none & $\begin{array}{l}\text { ASCII file for the "boxplt" program that contains percentile } \\
\text { values along with axis label and other information in a non- } \\
\text { columnar format. }\end{array}$ \\
\hline
\end{tabular}




\section{ASCII Flat File to RDB}

QWGRAF provides a filter (dta2rdb operator) to convert ASCII flat files that are organized as simple rows and columns of numeric data values to RDB. An ASCII data file can exist alone or be accompanied by a format definition file (see discussion below), which provides additional capability to define the characteristics of the variables (columns) in the resultant RDB file.

\section{File-Name Extensions}

The conversion of this type of file to the RDB format will occur when a QWGRAF program detects a file name that has been appended with the ".dta" extension; for example, my_file.dta. If a format definition file is used with the ".dta" file, then it must have the extension ".dtv" added to the root file name; for example, my_file.dtv.

\section{Missing Values}

Missing values in a ".dta" file can be designated by any of the following indicators:

$$
\text { " * " " " " - " " -- " " -- " " -0- " " -999999 " (leave quotes off). }
$$

In the newly created RDB file, a missing value is indicated by a null field; that is, two consecutive TAB markers.

\section{Censored Data}

Data values in a file may be accompanied by a single character remark code. For example, values that represent an analytical detection limit (or reporting level) are preceded by the "less than" (<) sign; for example, $<1$. Other remark codes are permitted. If the data file is not accompanied by a format definition file (see discussion below), then the file is assumed to have censored data and the resulting RDB file will include indicators for the censored data.

If censored data indicators are to be included in the $\mathrm{RDB}$ file, then an additional variable (one-character text variable) is created in the RDB file for each real variable with at least one value remarked. Each new variable will have the same root name as its corresponding data variable but will have the name appended with the "_R" extension. For example, for a variable in a ".dta" file not accompanied by a format definition file, the corresponding remark variable name in the RDB file would have the form VAR00X_R; while a remark variable for a variable defined in a format definition file would have $\mathbf{a}$ form such as CALCIUM_R.

\section{Format Definition File}

An ASCII flat file can stand alone or be accompanied by a format definition file, which provides options for alternative column delimiters, assigning alternative column names and data types, defining algorithms for additional variables, handling of remarked (censored) data, and miscellaneous descriptive text.

\section{Variable Names}

In the absence of a format definition file, variables in the data file are assumed to be numeric (real). Upon conversion to RDB, the columns are given names in sequential order from left to right of the form:

$$
\text { VAR001 VAR002 VAR003 .....VARNNN. }
$$

If a format definition file is present, then the first field is used to define alternative names for the data columns in the RDB file. Variable names are limited to 12 characters and are subject to the same guidelines discussed under the heading "Column Names" in the section on "The RDB File."

\section{Keywords}

The remaining functionality to be imparted to the RDB file via the format definition file involves the use of keywords to identify the beginning of a functional definition in the file. The currently available keywords include: $\langle$ dtype $\rangle,\langle$ delimiter $\rangle,\langle$ descr $\rangle,\langle$ rmk $\rangle$, $<$ perl $>$. Note the use of the " $<>$ " characters to enclose the keyword. Keywords and their 
associated functional descriptions can be strung together on a single line (separated by at least one space - not a tab), or each can be placed on its own line for improved readability. The keyword (with $<>$ characters) must be the first item on any additional lines that follow the line with the variable name definition; otherwise, it will be assumed that the line represents a new variable definition.

\section{Delimiter (<delimiter>)}

In the absence of a format definition file or if the <delimiter $>$ keyword line (first line in the format definition file) is not present, then the data columns in the data file are assumed to be separated by one or more blanks. The columns do not need to be neatly arranged.

If the data file is accompanied by a format definition file, then provision is made to define any of three data column delimiters - one or more blanks, a TAB, or a single alphanumeric character. To define the delimiter, put the keyword <delimiter $>$ in the first line of the format definition file followed by one or more spaces and one of the following - space, tab, or $\mathrm{X}$, where $\mathrm{X}$ is any single printable upper- or lower-case alphanumeric character.

$$
\text { <delimiter> space <delimiter> tab <delimiter }>x \text {. }
$$

\section{Data Types (<dtype>)}

Valid data types are int, real, double, and text( $x)$, where $x$ is length of the text field. Data fields for text variables with embedded blanks must be enclosed in double quotes.

$$
<\text { dtype }>\text { int }<\text { dtype }>\text { real }<\text { dtype }>\text { double }<\text { dtype }>\text { text(35). }
$$

\section{Miscellaneous Text <descr $>$}

The <descr $>$ keyword provides for adding miscellaneous text to a variable description line. This text can be used to provide a detailed description of the variable. This text does not need to be enclosed in quotes.

\section{Computed Variables (<perl>)}

The format definition file also provides for the definition of variables to be added to the output RDB file. The form of the ASCII data file itself has no provision for indicating variables to be computed; however, the format definition file can contain the information required to define and compute the new variables. This information is transferred to the column definition lines of the output RDB file, which in turn is interpreted by the QWGRAF program for actual computation of the data values when required. The computed variables will be assigned a missing value code (null field) if any source value in the data file used in the algorithm is missing. A missing value also is assigned to a computed variable if a source value is remarked and remarked data are indicated by the program to be excluded from the current application.

The algorithm syntax involves RDB functions by using the Perl scripting language (see discussion of this syntax in the section "Row Selection" of the "datmgr" program documentation and the discussion of the "perltbl" operator in Appendix F). All variable names must begin with the "\$" sign. Table D.3 shows an example of this syntax.

\section{Remarked (Censored) Data (<rmk >)}

If a format definition file is present and the $<\mathrm{rmk}>$ keyword is not present for a given variable, then the program assumes the data for that variable are NOT censored, regardless of the existence of remark codes in the data column. The presence of the $<\mathrm{rmk}>$ keyword, which does not have any arguments, indicates that remark variables are to be included in the RDB file. The <rmk> keyword is ignored for TEXT and computed variables. Computed variables can be associated with an appropriate remark code that use the "Modify" function in the "datmgr" program. 


\section{Example Data File}

Table D.2 is an example of a basic ASCII data file that contains space-delimited data. This table could be read and used without a format definition file.

Table D.2. ASCII space delimited data file

\begin{tabular}{rrrrr}
\hline 22111111 & 22 & 30.0 & 15.0 & 40.3 \\
22111111 & 22 & 28.0 & 18.3 & 46.8 \\
22111111 & 22 & 33.7 & 17.3 & 52.4 \\
22111111 & 22 & 39.6 & 21.3 & 50.3 \\
22111222 & 22 & 42.7 & 24.2 & 114.3 \\
22111222 & 22 & 56.8 & 21.9 & 96.3 \\
22111222 & 22 & 61.7 & 27.4 & 99.4 \\
22111222 & 22 & 57.2 & 29.0 & 107.4 \\
22111333 & 22 & 34.7 & 31.6 & 64.2 \\
22111333 & 22 & 29.3 & 28.7 & 57.2 \\
22111333 & 22 & 45.8 & 34.6 & 74.9 \\
36111111 & 36 & 48.0 & 33.4 & 35.6 \\
36111111 & 36 & 36.7 & 41.7 & 44.7 \\
36111111 & 36 & 41.9 & 37.3 & 39.5 \\
36111111 & 36 & 37.6 & 36.1 & 42.7 \\
\hline
\end{tabular}

\section{Example Format Definition Files}

Table D.3 is an example of a format definition file that could be used with table D.2.

Table D.3. Format definition file for use with data file in table D.2

\begin{tabular}{|c|c|c|c|}
\hline <delimiter & SPACE & & \\
\hline STATION & $<$ dtype $>$ & INT & \\
\hline STCODE & $<$ dtype $>$ & INT & \\
\hline NA & $<$ dtype $>$ & REAL & $<$ descr $>$ SODIUM IN MG/L \\
\hline $\mathbf{K}$ & $<$ dtype $>$ & REAL & $<$ descr $>$ SODIUM IN MG/L \\
\hline CA & $<$ dtype $>$ & REAL & $<$ descr $>$ SODIUM IN MG/L \\
\hline NA_MEQ & $<$ dtype $>$ & REAL & $<$ perl $>\$ N A * 0.04348$ \\
\hline \multicolumn{4}{|c|}{$<$ descr $>$ Sodium in milliequivalents } \\
\hline K_MEQ & $<$ dtype $>$ & REAL & $<$ perl $>\$ K * 0.02558$ \\
\hline \multicolumn{4}{|c|}{$<$ descr $>$ Potassium in milliequivalents } \\
\hline CA_MEQ & $<$ dtype $>$ & REAL & $<$ perl $>\$ C A * 0.04990$ \\
\hline
\end{tabular}

\section{NWIS-I QWDATA QWFLATOUT File to RDB}

Data files created by the QWFLATOUT option in the USGS NWIS-I QWDATA program can be converted to RDB format (qwd2rdb operator). The file created by the

QWFLATOUT option also has an associated format definition file with the same root file name as the data file, but with the extension "PARNAMES."

The QWFLATOUT format is discussed in detail in Maddy and others (1989, p. 4-6 to 4-11).

\section{File-Name Extensions}

For use with QWGRAF, the QWFLATOUT file name must have the ".qwd" extension added and the format definition file must have the "PARNAMES" extension changed to ".qwv"; for example, my_file.qwd and myfile.qwv.

\section{Format Definition File}

The QWFLATOUT format definition file identifies variables in the QWFLATOUT data file in two ways. The USGS NWIS_IQWDATA data base system identifies data elements by the five-digit parameter codes developed by the U.S. Environmental Protection 
Agency (EPA) for use in the EPA STORET system. These parameter codes are used as variable names in the ".PARNAMES" format definition file created by the QWDATA program. Upon conversion to the RDB format, the parameter code is prefixed with a " $\mathrm{P}$ " to form the RDB variable name; for example, P00430. As in the case of the ASCII data file, a variable with one or more remarked values results in the creation of a remark variable in the RDB file of the form P00430_R.

Some QWDATA data elements require the creation of a special class of parameter codes not supported in the EPA parameter code system. These data elements, some of which are numeric and others which are alphanumeric, are assigned five-character names, such as STATE and HUNIT.

\section{Parameter Code Conversions}

Some of the alphanumeric parameter codes from the NWIS-I QWDATA system are converted to alternate names and (or) data formats in the RDB file. For example, the 15-digit LATLG variable contains latitude and longitude in degrees/minutes/seconds. This variable is converted to two variables, DECLAT and DECLON, which are the latitude and longitude decimal equivalents of degrees/minutes/seconds. The decimal form is required in the QWGRAF "mapping" program. Thus, when a QWDATA format definition file is encountered, NWIS-I variables are converted as shown in table D.4.

Table D.4. Conversion criteria for QWDATA alpha parameter codes

\begin{tabular}{lllll}
\hline QWDATA & RDB & Data & \multicolumn{2}{c}{ Format change } \\
name & name & $\begin{array}{l}\text { Dape } \\
\text { typem) }\end{array}$ & (to) \\
\hline STAID & STATION & text(15) & & \\
SNAME & STA_NAME & text(50) & & \\
STATE & STATE_CODE & int & & \\
CNTYC & CNTYCODE & int & SSCCC & CCC \\
DATES & BEGIN_DATE & int & YYYYMMDD & YYMMDD \\
TIMES & BEGIN_TIME & int & HHMM & HHMMSSS \\
EDATE & END_DATE & int & YYYYMMDD & YYMMDD \\
ETIME & END_TIME & int & HHMM & HHMMSSS \\
LATLG & DECLAT & real & DDMMSS & DDD.DDDD \\
& DECLON & real & DDDMMSS & DDD.DDDD \\
AGNCY & AGENCY & int & & \\
ASRCE & ANAL_SOURCE & int & & \\
ASTAT & ANAL_STATUS & int & & \\
CTBDA & C_DRAINAGE & real & & \\
DISTR & DIST_CODE & int & & \\
GUNIT & GEOL_UNIT & int & & \\
HSTAT & HYD_COND & int & & \\
HUNIT & HYD_UNIT & int & & \\
LOCAL & LOC_WELL_NO & text(26) & & \\
MEDIM & SAMP_MEDIUM & int & & \\
SAMPL & RECORD_NO & int & & \\
SITEC & SITE_TYPE & int & & \\
STYPE & SAMP_TYPE & int & & \\
\hline
\end{tabular}

\section{Miscellaneous Text}

The format definition file from the QWDATA program includes variable descriptions, which are implemented as miscellaneous text identified by the $<$ descr $>$ keyword in the RDB file. 


\section{Data File Format}

The QWDATA data file consists of a series of 10 character fields, one for each variable retrieved from the QWDATA data base. The output file contains rounded numbers in the format shown in table D.5.

Table D.5. Output format from QWDATA FLATOUT program for data in a QWDATA data file identified with EPA parameter codes

\begin{tabular}{llll}
\hline Remark & $(1)$ & - col. & 1 \\
Value & $(1)$ & - cols. & $2-10$ \\
Remark & $(2)$ & - col. & 11 \\
Value & $(2)$ & - cols. & $12-20$ \\
$\cdot$ & & & \\
$\cdot$ & & & \\
$\cdot$ & & & \\
Remark & $(\mathrm{N})$ & & \\
Value & $(\mathrm{N})$ & & \\
\hline
\end{tabular}

Some alpha parameter codes are exceptions to the above format (mainly text variables that require more than 10 spaces) and therefore use multiple blocks of 10 spaces to hold fields longer than 10 spaces. Table D.6 shows the format for alpha parameter code text variables. Note that the first column in the first block of a multi-block set is always blank.

Table D.6. Output format from QWDATA FLATOUT program for alpha parameter code text variables

\begin{tabular}{ll}
\hline LATLG & Uses last 19 columns of two 10-space blocks; positions 3-9 contain the latitude and \\
positions $12-19$ contain the longitude. \\
LOCAL & Uses last 19 columns of two 10-space blocks. \\
SNAME & Uses last 49 columns of five 10-space blocks. \\
\hline
\end{tabular}

\section{Missing Values}

The missing value indicator for QWDATA (-999999) is recognized and is converted to a null field in the RDB file.

\section{Remarked (Censored) Data}

If remark codes are present in the data file, then an additional variable (one-character text variable) is created in the RDB file for each real variable with at least one value remarked. Each new variable will have the same root name as its corresponding data variable, but it will have the variable name appended with the "_R" extension; for example, P10010_R.

\section{NWIS-I QWCARDS 1 and * CARDS to RDB}

The NWIS-I QWCARDS program is used to transfer water-quality data from the National Water-Quality Laboratory to the NWIS-I QWDATA system. The data are transferred in sets of card images. A set of card types $(1, *, 5,7, \mathrm{X}, \mathrm{M}$, and \#) contain the information to describe each analysis record. Only the 1 and * cards are interpreted by the filter (str2rdb operator) that converts this format to the RDB format.

The QWCARDS 1 and *-card format is discussed in detail in the USGS publication entitled "NWIS National Water Information System, User's Manual, Volume 2, Chapter 2. Water-Quality System, p. 4-6 to 4-11."

\section{File-Name Extension}

A data file that contains 1 and * cards must have the ".str" extension added to the file name. There is no format definition file used with the QWCARDS 1 and *-card format. 


\section{Data File (Card Type 1)}

The Card Type 1 contains QWDATA header information in a fixed field format. Table D.7 shows how the fields are interpreted for conversion to RDB. Fields not indicated are ignored.

Table D.7. Conversion criteria for 1 and * cards Card Type 1 fields

\begin{tabular}{lll}
\hline Column(s) & OWDATA & QWGRAF \\
\hline $2-16$ & STAID & STATION \\
17 & MEDIM & SAMP_MEDIUM \\
$18-23$ & DATES & BEGIN_DATE \\
$24-27$ & TIMES & BEGIN_TIME \\
$28-31$ & EDATE & END_DATE (Note: month and day) \\
$32-35$ & ETIME & END_TIME \\
$36-43$ & GUNIT & GEOL_UNIT \\
44 & ASTAT & ANAL_STATUS \\
45 & ASRCE & ANAL_SOURCE \\
46 & HSTAT & HYD_COND \\
47 & STYPE & SAMP_TYPE \\
48 & & HYD_EVENT (No alpha code defined) \\
$64-68$ & AGNCY & AGENCY \\
\hline
\end{tabular}

\section{Data File (Card-Type *)}

Card-Type * contains the laboratory determinations for each of the parameter codes and any associated qualifiers (remarks, quality assurance codes, laboratory method codes, and precision codes). The filter captures only the remark code and laboratory method code, which are the first and third elements in the qualifier list that follow the value. Table D.8 shows a typical " 1 and *" card format record.

Table D.8. Example of a single analysis in QWCARDS 1 and *-card format

\begin{tabular}{l}
\hline 01064140 98810180930 H9999 19 USGS \\
*P00010=11.700(:I: :3),P00020=14.000(:I: :2),P00025=760.000(:I: :3), \\
*P00027=1028.00(:I: :4),P00028=80020.0(:H: :5),P00060=415.00(:I: :3), \\
*P00076=3.200( :H:A:2),P00095=122.000(:I: :3),P00300=10.5000(:I: :3), \\
*P00400=7.100(:I: :2),P00403=7.6000(:H:A:3),P00410=26.000(:I: :2), \\
*P00452=0.00(:I: :2),P00453=29.000(:I: :2),P00608=.060(:H:B:3), \\
*P00610=.040( :H:B:3),P00613=.010(1:H:B:3),P00625=.600(:H:A:2), \\
*P00631=.100(:H:B:3),P00665=.040(:H:B:3),P00666=.040(:H:B:3), \\
*P00671=.020( :H:B:3),P00915=6.600(:H:D:2),P00925=1.100(:H:C:2), \\
*P00930=16.000(:H:C:2),P00935=1.300(:H:B:2),P00940=14.00(:H:E:2), \\
*P00945=13.000(:H:D:2),P00950=.100(1:H:B:2),P00955=3.100(:H:D:2), \\
*P01000=10.000(<:H:A:2),P01030=2.000(<:H:A:2),P01049=25.00(::A)
\end{tabular}

\section{Remarked (Censored) Data}

If remark codes are present in the " 1 and *" card data file, then an additional variable (one-character text variable) is created in the RDB file for each real variable with at least one censored data value. Each new variable will have the same root name as its corresponding data variable, but the variable name will be appended with the "_R" extension; for example, P10010_R.

\section{Fortran to RDB}

ASCII files created by output format statements in Fortran programs are convertible to RDB (ftr 2 rdb operator). The filter requires both a data file and a format definition file.

\section{File-Name Extensions}

The data file output from a Fortran program must have the ".ftr" file-name extension. The format definition file must have the same root name and the ".ftv" file-name extension. 


\section{Missing Values}

Missing values in a ".ftr" file can be designated by any of the following indicators: " *" " " " " " " " " " "-. " " - " " " " 999999 " (leave quotes off).

In the newly created RDB file, a missing value is indicated by a null field; that is, two consecutive TAB markers.

\section{Format Definition File}

In general, the format of the ".ftv" file is similar to that of the ".dtv" file except that the $<$ delimiter $>$ keyword is not supported. Another difference is that the first line in the format definition file contains the Fortran style format statement used by the filter to define the field location, field size, and data type of each variable. This line may be the only line in the file or it can be followed by ".dtv" type format definition lines. If there are no format definition lines, then the columns are named VAR001, VAR002 ...

VARNNN with their data types discerned from the Fortran format string.

\section{Format String}

The format string may contain any number of parenthetically nested groups each with an associated multiplier. The following fields are supported:

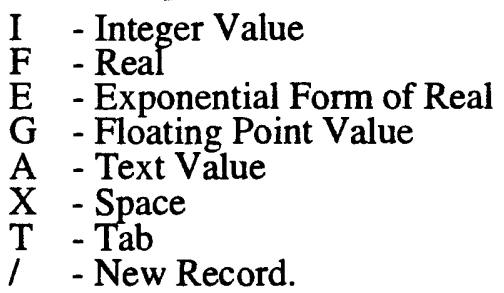

An example ".ftv" file for a six column data file would be as follow:

2(2I3,F6.2)

VAR1 <dtype >int

VAR2 <dtype $>$ int

VAR3 < dtype >real

VAR4 <dtype>int

VAR $5<$ dtype $>$ int

VAR6 < dtype>real.

The "/" field may be used to skip input records or to cause the logical input data record to span multiple physical input records. For example, if the format statement in the previous ".ftv" file was changed to the following:

$2(2 \mathrm{I} 3, \mathrm{~F} 6.2) /$,

then every other record starting with record 2 would be skipped. Similarly, a format statement of the following type:

2I3,F6.2/2I3,F6.2,

would cause two input records to be joined horizontally into a single output record.

\section{Remarked (Censored) Data}

For censored data, the Fortran format statement must include an additional alpha field for each variable that could be censored, for example,

I2,A1,F6.1

STATE $<$ dtype $>$ int

SODIUM $<$ dtype $>$ real $<$ rmk $>$.

The I2 field is used to parse STATE, the A1 field is assumed to contain the remark code for SODIUM, and the F6.1 field is used to parse SODIUM. Note that even though the format specified for the remark code must precede the data format specifier, the position of the remark code within the actual data record may be controlled by the use of the TAB field. In the previous example, if the data record contained a two-digit integer followed by a six-digit real with a trailing remark code, then the following format string could be used:

I2,T9,A1,T3,F6.1.

The position of the "T9, A1" format relative to the "T3, F6.1" format in the string tells the program to get the remark code from the field that follows the data value. 


\section{Data File Format}

The data file produced as output from a Fortran program is a fixed-format, space- delimited ASCII file. It is important to retain the column alignment defined by the Fortran format statement in the format definition file.

\section{PSTAT (from QWDATA) to RDB}

The PSTAT file output from the QWDATA system and the accompanying format definition file are convertible to RDB (pst 2 rdb operator). The remark code summary statistics file is not utilized in the conversion.

\section{File-Name Extensions}

The data file from the QWDATA PSTAT option does not have a specified file-name extension. For use with the PSTAT to RDB filter the file name must have the ".pst" extension added to the root file name. The format definition file is initially created with the extension, CMND, which must be changed to ".psv" for use with the filter. The resulting file names would be of the form: my_file.pst and my_file.psv.

\section{Format Definition File}

The first line of the format definition file is ignored by the filter. The five fields indicated on the second line represent the five alpha parameter codes-STAID, DATES, TIMES, EDATE, and ETIME - from the NWIS-I QWDATA system. Upon conversion, these variables, which represent the five fields on the header line of each analysis in the PSTAT data file, are assigned the following variable names in the RDB file:

$$
\text { STATION, BEGIN_DATE, BEGIN_TIME, END_DATE, and END_TIME }
$$

The remaining lines of the format definition file consist of 80-character records that contain five-digit EPA parameter codes (prefixed with a "P"), each separated by a space (not a TAB). As with the ".qwd" filter, the parameter codes prefixed with a "P" constitute the variable names assigned to the data columns in the RDB file; for example, P00010.

\section{Data File Format}

The PSTAT data file consists of multiple analyses, each consisting of a header record and one or more data records. The header record consists of five fields for the variablesSTATION, BEGIN_DATE, BEGIN_TIME, END_DATE, and END_TIME-as discussed above, with respective field lengths of $16, \overline{8}, 4,8,4$ and space in between. Each data record contains a maximum of eight fixed-length data value fields. The field length is nine spaces and each field is preceded by a blank space. Table D.9 is an example of a PSTAT format definition file and table D.10 is an example of a PSTAT data file.

\section{Missing Values}

Missing values in a PSTAT data file are indicated by a single dash (-).

Table D.9. Example of a PSTAT format definition file

BUILD file_name.pstP, FILE file_name.pst

VARS STATION.NUMBER:C DATE:C TIME:C END.DATE:C END.TIME:C P00010 P00025 P00065 P00076 P00080 P00095 P00300 P00301 P00310

Table D.10. Example of a PSTAT data file

\begin{tabular}{|c|c|c|c|c|c|c|c|}
\hline 01408500 & 199 & 0315 & & - & & & \\
\hline $\begin{array}{l}6.200 \\
1.500\end{array}$ & 752.00 & 6.320 & - & - & 72.00 & 10.400 & 85.069 \\
\hline 01408500 & 199 & 0623 & & - & & & \\
\hline $\begin{array}{l}20.00 \\
<1.0\end{array}$ & 758.00 & 3.350 & - & - & 81.00 & 7.500 & 82.964 \\
\hline
\end{tabular}




\section{Remarked (Censored) Data}

Remark codes in the PSTAT data file are placed to the left side of the value, not at the beginning of the field (see last value in table D.10). If remark codes are present in the data file, then an additional variable (one-character text variable) is created in the RDB file for each real variable with at least one value remarked. Each remark variable will have the same root name as its corresponding data variable but will have the name appended with the "_R" extension; for example, P00010_R.

\section{PSTAT Filter Extension}

The PSTAT output from the NWIS-I QWDATA system supports only five alpha parameter codes-STAID, DATES, TIMES, EDATE, and ETIME. The QWGRAF PSTAT filter, however, supports all the alpha parameter codes defined by the QWDATA system except ADDPC and CALCV. Thus, the user has the option of editing a PSTAT file to include data identified with other alpha codes. The variable names used in the output RDB file use the same alpha code names used in the ".qwd" filter and are converted to the appropriate format used in QWGRAF programs; for example, LATLG to DECLAT and DECLON. Any values for text variables must be enclosed in quotes, whether the text string has imbedded spaces or not.

\section{Percentile Data for "boxplt" Program to RDB}

The QWGRAF "boxplt" program reads an additional specially formatted file that contains percentile values instead of observed values. This file contains text strings for use as plot titles and axis labels, an indicator for the number of plots to be generated, and a detection limit value to be applied to all variables.

\section{File-Name Extensions}

An input data file with the percentile format must have the extension ".pct" appended to the end of the file name; for example, myfile.pct.

\section{Format Definition File}

The ".pct" file format does not require a format definition file.

\section{File Format}

The format for an input data file that contains data as percentiles is as follow:

Line 1:

Plot title, 1-72 characters beginning with first non-blank.

Line 2:

$\mathrm{X}$-axis title, 1-72 characters beginning with first non-blank.

Line 3:

Y-axis title, 1-72 characters beginning with first non-blank.

Line 4:

Number of Plots (N), integer from 1 to 25.

Line 5:

Detection (reporting) limit, a real number.

In the current version of boxplot this value applies to all variables.

Lines 6 - N+5: One line for each variable. Line order represents plot order. Columns 1-12 contain the label for variable to be diagrammed.

The remaining fields are for the statistics and are free format except the numbers must be separated by at least one blank.

The first field after the label contains the number of observations for this variable from the original data file. This number is also used as a label.

The next five fields contain the values that correspond to the $\mathrm{P} 10, \mathrm{P} 25, \mathrm{P} 50, \mathrm{P} 75$, and $\mathrm{P} 90$ percentiles. The P10 and P90 fields are optional and can be replaced with a single dash (-).

The final field, also optional, is used for the arithmetic mean of the observations. It is not necessary to use a single dash if the mean is missing. 


\section{APPENDIX E. Data Format Conversion Macros and RDB Operators}

\section{RDB Files from Third Party Packages}

RDB from Description: There is no macro or RDB operator involved here, just a process to create Spreadsheets the RDB file definition within a spreadsheet file.

Example: Using the spreadsheet cells, do the following:

1. Put 0 or more rows at the top as comments starting with a pound (\#) sign.

2. Add a row of RDB column names.

3. Add a row of RDB column definitions.

4. Add 0 or more rows of data. (Do not worry about delimiters here, just use Tactician cells. RDB Tab delimiters will be specified in the "Save As" function.)

5. Pull down the "File" menu and select "Save As ...."

6. Change "Save File of Type" to "Delimited File."

7. Click on "OK."

8. Edit the pathname to save the file, if necessary.

9. Make sure delimiter is "Tab" and click on "OK."

RDB from Description: The Statit macro, tblwrite, takes the resident file in Statit and outputs it to Statit an RDB file.

Syntax: tblwrite output.rdb variable list

\section{Standard RDB Operators for Data Format Conversions}

The following RDB operators are not utilized by the QWGRAF programs, but can be used in command line mode to create RDB files from the source file types indicated.

2TABS Description: Converts an ASCII file with columns separated by delimiters other than TABS to an RDB tab-delimited file. The output file must be edited to add the RDB header lines describing the column names (line1) and the column type, width, and justification (line 2).

Syntax: 2tabs <input_file [options] >output_file.rdb

Options:

-s Data columns separated by single space.

-ss Data columns separated by two or more spaces.

$-d$ " $\quad$ Data columns separated by delimiter text identified within the double quotes. 
F2TBL Description: Reads data using a FORTRAN-style format string. The format string is specified using the -format option. Column names may also be specified using the -names option.

Syntax: f2tbl [options] <input_file >output_file.rdb

Example:

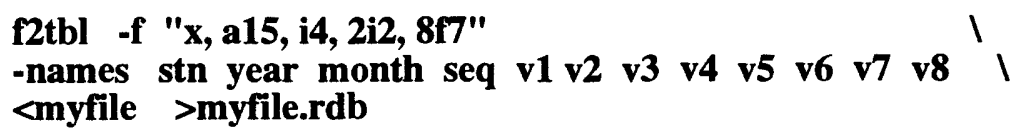

Options (abbreviations are in parentheses):

$\begin{array}{ll}\text {-(f)ormat "string" } & \text { FORTRAN format string } \\ & \text { Field types - n, N, f, F, e, E, g, G, i, I } \\ & \text { Skip option - x, x2, 3x6, x8, 4x8 } \\ & \text { Tab option - t, t4, T4, T15, 3T15 } \\ & \text { Skip line option - I } \\ & \text { Definitions and control options can be grouped } \\ & \text { using parentheses and the groups can be repeated } \\ \text { by preceding them with a number. } & \\ \text {-names list } & \text { List of column names. } \\ \text {-pattern "reg exp" } & \text { When reading files with f2tbl, lines can be filtered } \\ & \text { using a regular expression specified with the } \\ & \text {-pattern option. The expression between the } \\ \text { delimiters (" ") can be simple text strings or more } \\ \text { complex patterns can be specified using the full } \\ \text { range of meta characters with regular expressions. } \\ \text { Specify a pattern used to recognize missing values. } \\ \text { If the missing option is used, then any data values, } \\ \text { regardless of type, that match the pattern are } \\ \text { converted to missing values; that is, null values } \\ \text { (two consecutive TAB markers). }\end{array}$

If the pattern begins and ends with a "/," then the text between the slashes matches values containing that substring. Without the slashes the pattern string must match the entire value being compared.

TSQL Description: Converts an Ingres table to an RDB file.

Syntax: tsql databasename -rdb -table tablename >outputfile.rdb

\section{RDB Operators for Data Format Conversions}

With the exception of the "rdb2dta" operator, the following data format conversion filters-implemented as RDB operators-are utilized by the QWGRAF programs to convert other data formats to the RDB format used internally by all QWGRAF programs. The filters are invoked automatically when a QWGRAF program recognizes a threecharacter extension at the end of the file name. The descriptions below are intended to supplement the functional information given in the main text of this report and to describe the syntax used in command line mode of RDB and the various scripting languages. See the section entitled "Conversion of Other Data to RDB" for detailed discussion of the various data and format definition files recognized by QWGRAF. 
RDB2DTA Description: Although most data conversion operations conducted by users of the QWGRAF programs involve getting data converted to RDB, there may be an occasional need to convert an RDB file to ASCII form. The "rdb2dta" filter is not formally available within QWGRAF programs (including "datmgr") at the present time; however, it is available in command line mode as an RDB operator.

Syntax: rdb2dta <infile outfile -dlm [options] -fw [X]

Arguments:

input file

output file

-dlm [options]

$-f w[X]$
The source file, which is read from STDIN, must be preceded by the "<" sign.

The output file is written to STDOUT and does

NOT require the file name to be preceded by the " $>$ " sign. A ".dtv" (format definition file) with the same root name will also be created.

The following options are available for the "-dlm" delimiter option:

$$
\begin{aligned}
& \text {-dlm space } \\
& \text {-dlm tab }
\end{aligned}
$$$$
\text { -dlm x ("x" may be any ASCII character) }
$$

"-fw" is the field width of numeric variables. The default value for " $\mathrm{X}$ " is 12 .

\title{
Example: rdb2dta <myfile.rdb myfile.dta -dlm* -fw 16
}

DTA2RDB Description: The ".dta" format applies to files that are essentially rows and columns of data as ASCII characters. These files, which can be read with any text editor, contain no header records or other self-defining information. All fields are considered to be numeric (floating point) and are separated by at least one space. In the absence of a format definition file, all variables in the resultant RDB file are assigned generic names such as VAR01, VAR02 ... VARnn. For each column containing at least one remarked (censored) data value, an additional variable will be created in the RDB file by appending "_ $R$ " to the variable name; for example, VAR01_R. Missing values in the ".dta" file may be indicated by any of the following:

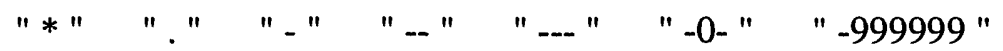

If the file is accompanied by a format definition file (same file name but appended with the extension ".dtv"), then several options are available to the user to impart special characteristics to each variable. The rows in the format definition file represent in sequential order each variable in the data file. The columns in the format definition file consist of the name of the variable in the first field (12 characters or less) followed by zero or more keywords and keyword arguments. Keywords must be enclosed in " $<>$ " characters. The following keywords are available.

Keywords:

\author{
<delimiter> - Unlike the other keywords, the <delimiter $>$ keyword \\ applies to the file as a whole and therefore is placed on the \\ first line of the format definition file. The <delimiter $>$ \\ keyword provides for specifying the delimiter between \\ the columns in the input data file. The following \\ delimiters are supported. \\ $<$ delimiter $>$ space \\ $<$ delimiter $>$ tab \\ $<$ delimiter $>$ any single character \\ Note: "any single character" means any single printable \\ character, such as "*", "I", "\&", ".", "_", "I", etc.
}


<dtype> - This keyword defines the data type to be assigned to the variable. The following data types are supported:

$$
\begin{aligned}
& <\text { dtype }>\text { int } \\
& <\text { dtype }>\text { real } \\
& <\text { dtype }>\text { double } \\
& <\text { dtype }>\text { text( } x)
\end{aligned}
$$

Note: Text variables with embedded blanks must be enclosed in double quotes. The " $x$ " in the "text" data type indicates the maximum width of the text variable.

$<$ rmk $>\quad$ - If a data file is accompanied by a format definition file but the $<\mathrm{rmk}>$ keyword is NOT present for a given variable, then the system assumes that the corresponding column in the data file does not have any remarked data even if the data are actually remarked. The presence of this keyword tells the system that if the corresponding column in the data file has at least one remarked value, then a remark variable is to be created in the RDB file for this variable. The form of the remark variable name consists of the data variable name appended with "_R"; for example, calcium_R. The $<$ rmk $>$ keyword has no arguments.

$<$ rmk $>$

<descr $>\quad-$ The $<$ descr $>$ keyword provides for adding miscellaneous text as a descriptor of the variable. This text does not need to be enclosed in quotes and has no functionality within the QWGRAF programs.

\section{<descr> Calcium, water, filtered, $\mathrm{mg} / \mathrm{l}$}

$<$ perl $>\quad-$ The $<$ perl $>$ keyword provides for the definition of new variables to be added to the RDB file. A new variable is created using the algorithm which follows the <perl> keyword. The algorithm can use the values from any of the other variables in the input data file. In the algorithm, all variable names must begin with the "\$" sign. The algorithm syntax mimics the Perl scripting language, which in turn utilizes RDB functions. For a discussion of this syntax please refer to the discussion of the "perltbl" operator later in this appendix. See also the textbook Programming Perl (Wall and Schwartz, 1991).

$$
\begin{aligned}
& <\text { perl> \$ca_meq }=\text { \$CALCIUM } * 0.04990 \\
& <\text { perl> \$DISCH_LOG }=\text { \&log10 }(\$ D I S C H)
\end{aligned}
$$

Note: In the above example the "log 10 " function is preceded by the " $\&$ " sign, which means it is not a standard RDB function and was created for use in the local implementation of RDB.

The command line syntax does not require including the format definition file name on the command line as the operator looks for the same root file name with the ".dtv" extension. The ".rdb" extension for the output RDB file is also optional in command line mode.

Syntax: dta2rdb input_file >output_file

Example: dta2rdb myfile.dta >myfile.rdb 
QWD2RDB Description: The NWIS-I QWDATA system provides an option for producing an ASCII output file for use in applications not integrated with the QWDATA system. The output file is accompanied by a format definition file (".PARNAMES" file-name extension must be changed to ".qwv") similar in function to the ".dtv" format definition file used with a ".dta" file. The "qwd2rdb" filter reads these files and converts the format to the RDB format used by QWGRAF programs. In command line mode the use of the ".rdb" extension for the output RDB file name is optional.

Syntax: qwd2rdb input file >output file [rmk_switch]

Arguments:

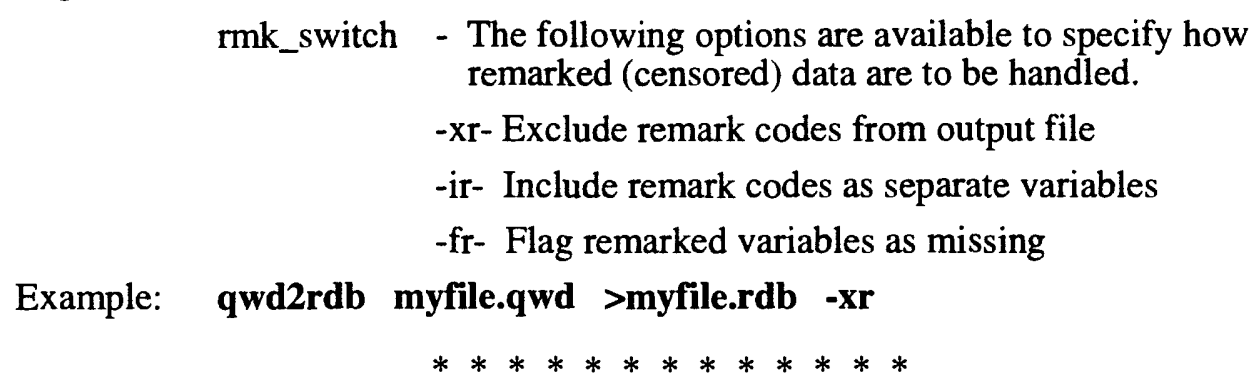

STR2RDB Description: The "str2rdb" operator converts files with the 1 and *-card format used in the NWIS-I system to RDB. Only the Type 1 and Type * cards are supported. This format does not provide for the use of an accompanying format definition file. The file-name extension used with the 1 and *-card files is ".str." In command line mode the ".rdb" extension for the output RDB file is optional.

Syntax: str2rdb input_file >output_file

Example: str2rdb myfile.str >myfile.rdb

PST2RDB Description: The "pst2rdb" operator converts files created in the PSTAT format by the PSTAT option in the NWIS-I QWDATA system to RDB. The output from this option does include a format definition file. The data file must have the ".pst" extension added to the root file name and the format definition file name must have the ".CMND" extension replaced with the ".psv" extension. The command line syntax does not require including the format definition file name on the command line as the operator looks for the same root file name with the ".psv" extension. The ".rdb" extension for the output RDB file is also optional in command line mode.

Syntax: pst2rdb input_file >output_file

Example: pst2rdb myfile.pst >myfile.rdb

FTN2RDB Description: The "ftn2rdb" operator converts files created by Fortran programs using Fortran formatting lines to RDB. The output file from this process is an ASCII file similar to a ".dta" file with spaces as the delimiter. Thus, this file type can be associated with a format definition file which is exactly the same as the ".dtv" format definition file except for the inclusion of the Fortran formatting statement on the first line of the file. The filter then sets data types based on this format string. The "delimiter" function is not supported. If the formatting line is the only line in the file, then the variables are assigned generic names of the form: VAR01 VAR02 VAR03 ... VARnn. Otherwise, the format definition file has the same potential functionality as the ".dtv" format definition file; that is, variable names, data types, and keywords.

The command line syntax does not require including the format definition file name on the command line as the operator looks for the same root file name with the ".ftv" extension. The ".rdb" extension for the output RDB file is also optional in command line mode.

$\begin{array}{llll}\text { Syntax: } & \text { ftn2rdb input_file >output_file } \\ \text { Example: } & \text { ftn2rdb } & \text { myfile.ftn } & >\text { myfile.rdb }\end{array}$


The RDB operators described below are used by the QWGRAF programs to perform the indicated modifications of the data in the input data file. The purpose of the descriptions given here is to supplement the functional information given in the main text of this report and to describe the syntax to use these operators in regular command line of RDB and the various scripting languages. The QWGRAF interface incorporates the indicated options available with each operator.

SORTTBL Description: The "sorttbl" operator sorts a data file by one or more columns. Each column can be sorted in ascending or descending order using the "-r" option

Syntax: sorttbl [options] [-r] column [[-r] column] $\ldots<$ input file

Options (abbreviations are in parentheses):

-c $\begin{aligned} & \text { Check that the RDB file is sorted on the selected columns. Give } \\ & \text { no output unless the file is out of sort. } \\ & \text {-(h)elp } \\ & \text {-r }\end{aligned} \begin{aligned} & \text { Print this "help" information. } \\ & \text { Descending order. Applies to the column following only. } \\ & \text {-u }\end{aligned} \begin{aligned} & \text { Retain only those rows that have a unique set of values for the } \\ & \text { indicated key columns; that is, remove replicate rows. }\end{aligned}$
Example: sorttbl COUNT -r TYP < sample

Explanation: Sort the file "sample" first by column "COUNT" in ascending order and then by column "TYP" in descending order.

\section{COLUMN}

Description: The "column" operator selects (subsets) columns (variables) by ordered name and outputs an RDB file with these columns. This can be used effectively to select, order, add, delete, or duplicate columns.

Syntax: column [options] list

Options (abbreviations are in parentheses):

$\begin{array}{ll}\text {-(e)dit } & \text { Edit option used by "etbl" operator (not a QWGRAF function). } \\ \text {-(h)elp } & \text { Print this "help" information. } \\ \text {-v } & \text { Inverse option. Selects all columns except those named in "list." }\end{array}$ The "list" argument is normally a list of column names.

If "list" contains a name in a triplicate of the form "-c NAME NEWNAME," then the column name specified in the input RDB file as NAME will be changed to "NEWNAME" in the output RDB file.

Note: The above name change function is handled in the "Modify" function in "datmgr." If "list" contains a name in a triplicate of the form "-a NAME DEFN," then a new (null) column is added to the output RDB file at that point in the output name list. It will be assigned the name "NAME" and the definition will be indicated in the "DEFN" field; for example, $12 \mathrm{~N}$.

Note: The above name change function is handled in the "Create" function in "datmgr."

\section{Example: column STATID DATE STCODE -c HCO3 BICARBONATE SODIUM}


COMPCOL Description: The "compcol" operator selects columns by name and order and outputs an RDB file with just these columns. This operator can be used to select, order, add, delete, or duplicate columns.

The operator "compcol" is essentially the same as the RDB "column" operator except that null fields for newly defined columns can be populated with computed data values. With the "column" operator, new variables can be defined, but the data fields are "null" fields requiring them to be populated externally with an editor. The "datmgr" program in

QWGRAF provides for the creation of an RDB file similar to the type created with the "column" operator, except that the algorithm to compute the actual data values is stored in the optional documentation area of the second header line in the RDB data file, an RDB extension created for QWGRAF. The operator "compcol" evaluates all computed variables and outputs a file containing the computed values. The output file does not contain the algorithm definition as there is no point in keeping it once the values are computed. The user has the option to overwrite the original file or create a new file.

Below is an example of an RDB file configured by "datmgr" for input to "compcol." The columns "Na" and " $\mathrm{K}$ " are populated, but the column "Na_K" is only defined; that is, there are no data for this column in the data rows. Note, in the output file the data field for "NA_K" is populated and the algorithm definition is no longer stored.

\section{Syntax: compcol [options] list <input_file >output_file}

Options (abbreviations are in parentheses):

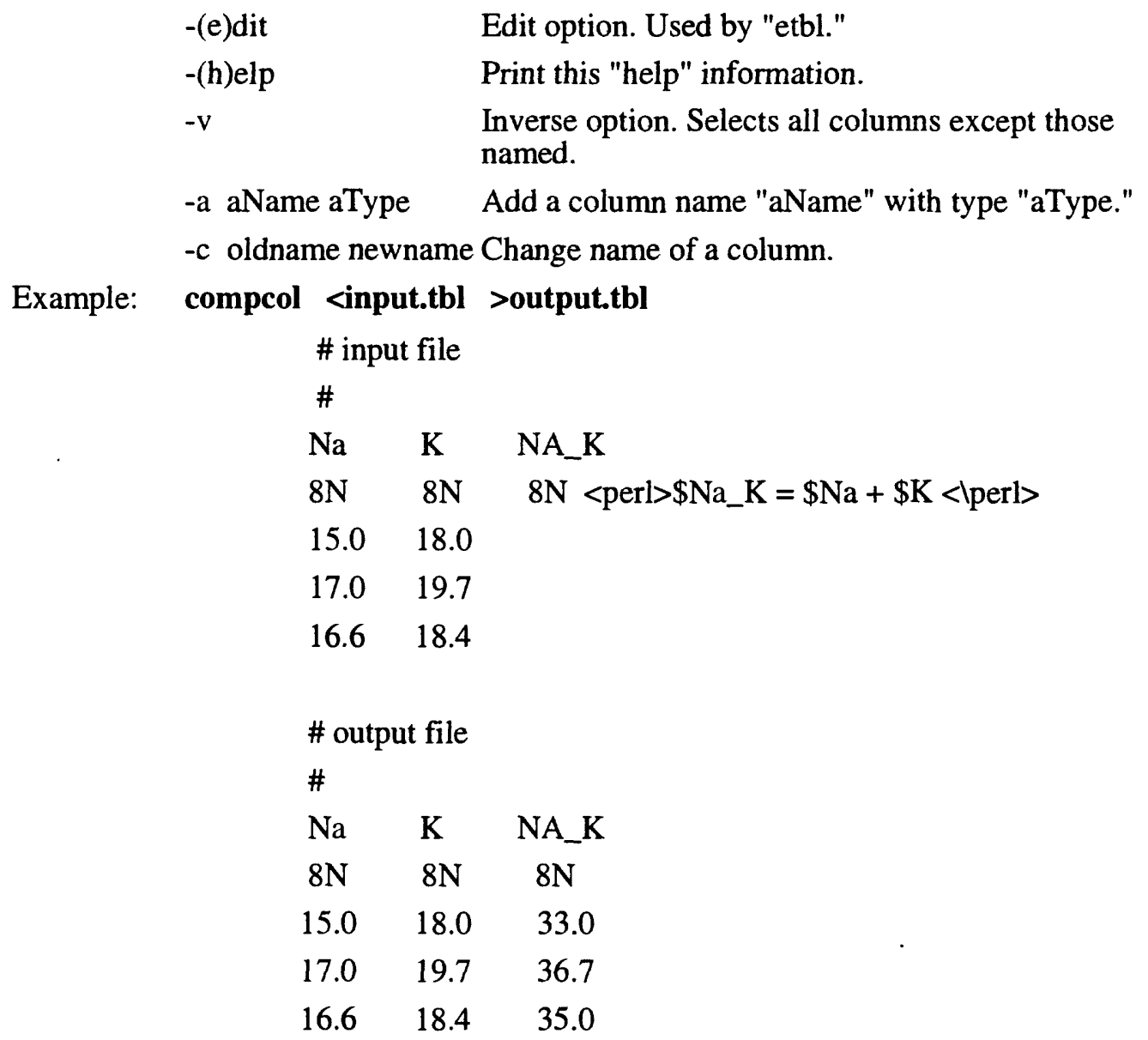

Explanation: The original file had two variables- $-\mathrm{Na"}$ and "K"-plus a definition for a third variable-_Na_K." The output file will have three variables-"Na", "K", and "NA_K." The algorithm to 
compute the new variable is included (via editor or by the QWGRAF datmgr program) to the right of the column definition for the "Na_K" variable. Note the syntax relative to the $<$ perl $>$ and $<$ perl> delimiters. Multiple statements can be included between these delimiters, including IF/THEN/ELSE clauses. RDB variable names must have the prefix "\$."

The value "list" in the syntax is normally a list of column names. If "list" contains a triplicate of the form "-c NAME NEW," then column name "NAME" will be changed to "NEW." If "list" contains a triplicate of the form "-a NAME DEFN," then a new null (empty) column is added to the data set, with name "NAME" and column definition "DEFN." An algorithm (preceded by a space) can follow the "DEFN" in which case the actual data values will be computed for the output file.

\section{Example: compcol NAME COUNT < sample >new_sample}

Explanation: Select from the data set "sample" all observations in columns named "NAME" and "COUNT." Output result to "new_sample."

\section{Example: compcol -a LENGTH 10 < sample >new_sample}

Explanation: Add a new column named "LENGTH" with a size of 10 to the data set called "sample." If an algorithm is specified for computing this variable, then the computations are done prior to outputting to the new file "new_sample."

MODCOL Description: The "modcol" operator changes the attributes of a column in an RDB file. This operator is used for the "Modify" function in the QWGRAF "datmgr" program. The column attributes that can be changed with "modcol" include the variable name, the standard RDB attributes for data type and field length, for example $8 \mathrm{~N}$, and the keyword attributes implemented as RDB file extensions in the QWGRAF system, that is <dtype >, $<$ descr $>$, and $<$ perl $>$.

Syntax: modcol <input_file >output_file oldname=[newname] [, newattri] ...

Example: modcol <in.rdb >out.rdb V1=DATE V2=TIME, '8N < dtype>real' V3=, '8N <dtype $>$ int'

Explanation: Changes the name of column V1 to DATE, the name of column V2 to TIME and its type to real. The name of column V3 remains unchanged, but its type is changed to integer. Note the use of single quotes in the attribute descriptions. Quotes are required to enclose the inclusive attribute descriptions for a given column because each attribute description (meta data) is followed and preceded by one or more blanks. 
Description: The "jointbl" operator provides for relational joins-Natural, One to One, Master/Detail, or Cartesian - of two or more RDB data files based on specified columns.

Natural - The output file from the two joined input files will contain one record for each pair of matching records in the input files. Records from either input file may contribute to multiple matches in the other file.

One to One - The output file will contain one record for each pair of matching records in the input files. Each input record, however, may contribute to only one match.

Master/Detail - Every record from file 1 is included in the output file. If there is no corresponding match in file 2 for a given record, then the variables from the second file in the output record are set to missing.

Cartesian - Every record from each file is included in the output file. If there is no corresponding match in the other file, then the variables from the other file are set to missing.

The -fill option can be used to request that rows without matching cases be included, but does not request cartesian product.

File joins can be based on different column names in the two RDB files by using the [=col.name] option.

The order of columns in the output RDB file will be (1) the join columns from the RDB file_1, (2) the other columns from RDB file_1, then (3) the other columns from RDB file_2.

Syntax: jointbl [options] <file_1 col.name[=col.name_2] ... file_2

Options (abbreviations are in parentheses):

-c Do a cartesian cross-product join.

-(h)elp Print this "help" information.

-md Do a "Master/Detail" join rather than a natural join.

-1 to1 Do a one-to-one join. If duplicate key values are found in the two files, then rows are matched in order of occurrence.

-fill 1 Fill in null values for variables from file 2 if no match for row in file 1.

-fill 2 Fill in null values for variables from file 1 if no match for row in file 2 (implied by -md or -c).

-fill Fill in with null values for unmatched rows.

Examples:

RDB file named sample:

$\begin{array}{llll}\text { name } & \text { nr } & \text { typ } & \text { amt } . \\ 6 & 2 & 4 & 4 \\ \text { Bush } & 1 & \text { A } & 133 \\ \text { Bush } & 2 & \text { A } & 134 \\ \text { Hansen } & 3 & \text { A } & 143 \\ \text { Hobbs } & 4 & \text { B } & 144 \\ \text { Hobbs } & 5 & \text { B } & 144 \\ \text { Jones } & 6 & \text { C } & 155 \\ \text { Perry } & 7 & \text { D } & 244 \\ \text { Perry } & 8 & \text { D } & 311\end{array}$


RDB file named samplej2:

$\begin{array}{llll}\text { name } & \text { cnt } & \text { typ } & \text { amt } \\ 6 & 5 \mathrm{~N} & 4 & 5 \mathrm{~N} \\ \text { Hobbs } & 41 & \text { A } & 141 \\ \text { Hobbs } & 42 & \text { BB } & 142 \\ \text { Hobbs } & 51 & \text { BB } & 144 \\ \text { Hobbs } & 43 & \text { CC } & 143\end{array}$

The command to do a natural join of samplej and samplej2 on column name is as follow:

\section{jointbl <samplej name samplej2}

and the result is shown in table F.1.

Table F.1. Natural join of rdb files samplej and samplej2

\begin{tabular}{lllllll}
\hline name & nr & typ & amt & cnt & typ & amt \\
6 & 2 & 4 & 4 & $5 \mathrm{~N}$ & 4 & $5 \mathrm{~N}$ \\
Hobbs & 4 & $\mathrm{~B}$ & 144 & 41 & $\mathrm{~A}$ & 141 \\
Hobbs & 4 & $\mathrm{~B}$ & 144 & 42 & $\mathrm{BB}$ & 142 \\
Hobbs & 4 & $\mathrm{~B}$ & 144 & 51 & $\mathrm{BB}$ & 144 \\
Hobbs & 4 & $\mathrm{~B}$ & 144 & 43 & $\mathrm{CC}$ & 143 \\
Hobbs & 5 & $\mathrm{~B}$ & 144 & 41 & $\mathrm{~A}$ & 141 \\
Hobbs & 5 & $\mathrm{~B}$ & 144 & 42 & $\mathrm{BB}$ & 142 \\
Hobbs & 5 & $\mathrm{~B}$ & 144 & 51 & $\mathrm{BB}$ & 144 \\
Hobbs & 5 & $\mathrm{~B}$ & 144 & 43 & $\mathrm{CC}$ & 143 \\
\hline
\end{tabular}

The command to do a "master-detail" join of the same two rdb files on column name is as follow:

$$
\text { jointbl -md < samplej name samplej2 }
$$

and the result is shown in table F.2.

Table F.2. Master-detail join of rdb files samplej and samplej2

\begin{tabular}{lllllll}
\hline name & nr & typ & amt & cnt & typ & amt \\
6 & 2 & 4 & 4 & $5 \mathrm{~N}$ & 4 & $5 \mathrm{~N}$ \\
Bush & 1 & $\mathrm{~A}$ & 133 & & & \\
Bush & 2 & $\mathrm{~A}$ & 134 & & & \\
Hansen & 3 & $\mathrm{~A}$ & 143 & & & \\
Hobbs & 4 & $\mathrm{~B}$ & 144 & 41 & $\mathrm{~A}$ & 141 \\
Hobbs & 4 & $\mathrm{~B}$ & 144 & 42 & $\mathrm{BB}$ & 142 \\
Hobbs & 4 & $\mathrm{~B}$ & 144 & 51 & $\mathrm{BB}$ & 144 \\
Hobbs & 4 & $\mathrm{~B}$ & 144 & 43 & $\mathrm{CC}$ & 143 \\
Hobbs & 5 & $\mathrm{~B}$ & 144 & 41 & $\mathrm{~A}$ & 141 \\
Hobbs & 5 & $\mathrm{~B}$ & 144 & 42 & $\mathrm{BB}$ & 142 \\
Hobbs & 5 & $\mathrm{~B}$ & 144 & 51 & $\mathrm{BB}$ & 144 \\
Hobbs & 5 & $\mathrm{~B}$ & 144 & 43 & $\mathrm{CC}$ & 143 \\
Jones & 6 & $\mathrm{C}$ & 155 & & & \\
Perry & 7 & $\mathrm{D}$ & 244 & & & \\
Perry & 8 & $\mathrm{D}$ & 311 & & & \\
\hline
\end{tabular}


Using the original two files, the command to do a one-to-one join is as follow:

$$
\text { jointbl -1to1 <samplej name samplej2 }
$$

and the results are shown in table F.3.

Table F.3. One-to-one join of rdb files samplej and samplej2

\begin{tabular}{lllllll}
\hline name & nr & typ & amt & cnt & typ & amt \\
6 & 2 & 4 & 4 & $5 \mathrm{~N}$ & 4 & $5 \mathrm{~N}$ \\
Hobbs & 4 & $\mathrm{~B}$ & 144 & 41 & $\mathrm{~A}$ & 141 \\
Hobbs & 5 & $\mathrm{~B}$ & 144 & 42 & $\mathrm{BB}$ & 142 \\
\hline
\end{tabular}

The command to do a one-to-one join with fill is as follow:

$$
\text { jointbl -1to1 -fill <samplej name samplej2 }
$$

and the result is shown in table F.4.

Table F.4. One-to-one join of rdb files samplej and samplej2 with fill

\begin{tabular}{lllllll}
\hline name & $\mathrm{nr}$ & typ & amt & cnt & typ & amt \\
6 & 2 & 4 & 4 & $5 \mathrm{~N}$ & 4 & $5 \mathrm{~N}$ \\
Bush & 1 & $\mathrm{~A}$ & 133 & & & \\
Bush & 2 & $\mathrm{~A}$ & 134 & & & \\
Hansen & 3 & $\mathrm{~A}$ & 143 & & & \\
Hobbs & 4 & $\mathrm{~B}$ & 144 & 41 & $\mathrm{~A}$ & 141 \\
Hobbs & 5 & $\mathrm{~B}$ & 144 & 42 & $\mathrm{BB}$ & 142 \\
Hobbs & & & & 51 & $\mathrm{BB}$ & 144 \\
Hobbs & & & & 43 & $\mathrm{CC}$ & 143 \\
Jones & 6 & $\mathrm{C}$ & 155 & & & \\
Perry & 7 & $\mathrm{D}$ & 244 & & & \\
Perry & 8 & $\mathrm{D}$ & 311 & & & \\
\hline \multicolumn{7}{l}{} \\
& & $* * * * * * * * * * * * *$ &
\end{tabular}

SUBTBL Description: The "subtbl" operator writes to an output file only those rows from the first of two designated input files based on a "no match" between the (Key) column(s) specified. The designated key columns between the two input files need not have the same name.

Note: The two RDB files must be sorted on the key columns in order for a subtract operation to function correctly.

Syntax: $\quad$ subtbl $<$ rdbfile_1 column[=column_2] ... rdbfile_2

Options (abbreviations are in parentheses):

-(h)elp Print this "help" information.

-m Use all matching column names. Both files must be sorted on the matching names in the order that they appear in the "rdbfile_1"

Example: subtbl <file_a cost $=$ price file_b file_c

Explanation: Exclude all rows from file_a in the output file, "file_c," where the value of "cost" in "file_a" is equal to the value of "price" in "file_b"; that is, only those rows from "file_a" that do not have a match are written to "file_c." 
CATTBL Description: The "cattbl" operator concatenates two or more files vertically, copying all column names or just those columns common to all files to the output file (standard output). In command line mode, the syntax permits any number of files to be concatenated in a single step, while the "datmgr" program permits only two files to be concatenated at a time. Thus, the use of the "-pattern" option to select all files with common text in file names is not permitted in "datmgr."

Syntax: cattbl [options] file1 file2 ...

Options (abbreviations are in parentheses):

\begin{tabular}{|c|c|}
\hline$-1($ able $) l$ & $\begin{array}{l}\text { Create an additional variable in the output } \\
\text { file, named "name," that contains the name of } \\
\text { the file from which that particular row comes } \\
\text { from. }\end{array}$ \\
\hline -p(attern) reg expression & Use files that match the regular expression. \\
\hline$-m($ etal $)$ & $\begin{array}{l}\text { Only use the column definition information } \\
\text { from the first occurrence of a column name } \\
\text { (in the first file read) in constructing the } \\
\text { column characteristics of a column appearing } \\
\text { in the output file. This option is turned on in } \\
\text { "datmgr" and cannot be turned off. If this } \\
\text { option is not specified in command line } \\
\text { mode, then all column information for all } \\
\text { occurrences of a given column name are } \\
\text { combined together. }\end{array}$ \\
\hline$-u($ nion $)$ & $\begin{array}{l}\text { Copy all column names which appear in all } \\
\text { input files. If a column name in one file is not } \\
\text { matched with a column name in another file, } \\
\text { then the rows in the file not having the } \\
\text { column name are populated as "missing". }\end{array}$ \\
\hline -inter(sect) & $\begin{array}{l}\text { Copy only the column name which appears } \\
\text { in all input files. }\end{array}$ \\
\hline
\end{tabular}

Example: cattbl -1 source $-\mathbf{i}$ apples oranges

PERLTBL Description: Similar to the "compute" operator, except that "perltbl" uses the Perl scripting language, while "compute" uses the Python language. The "perltbl" operator provides for data transformations and data selection in an RDB file using C- or Fortran-like syntax. The operator "perltbl" includes the ability to add and rename columns as in the column command, and the ability to select rows, as in the row command.

"pertbl" statements consist of variable names, functions, and operators.

Each statement is applied to each row of data in the input data.

Variable names in "perltbl" statements begin with "\$." Using this prefix allows variables to be distinguished from the many predefined Perl functions and keywords.

Perl operator tests :

\begin{tabular}{llll} 
Numeric Test & String Test & & Description \\
\hline$==$ & eq & & Equal to \\
$!=$ & ne & Not equal to \\
$>$ & gt & Greater than \\
$>=$ & ge & Greater than or equal to \\
$<$ & it & Less than \\
$<=$ & le & Less than or equal to \\
$<=>$ & $\mathrm{cmp}$ & Not equal to, with signed return
\end{tabular}


Perl logical operators :

\begin{tabular}{|c|c|c|}
\hline Operator & Function & Description \\
\hline$\$ a \& \& \$ b$ & And & True if $\$ a$ is true and $\$ b$ is true \\
\hline$\$ a \| \$ b$ & Or & $\$ a$ if $\$ a$ is true, otherwise $\$ b$ \\
\hline ! \$a & Not & True if $\$ a$ is not true \\
\hline
\end{tabular}

Perl arithmetic operators :

\begin{tabular}{|c|c|}
\hline Operator & Function Description \\
\hline$\$ a+\$ b$ & Add $\$ a$ to $\$ b$ \\
\hline$\$ a-\$ b$ & Subtract $\$ a$ and $\$ b$ \\
\hline$\$ a * \$ b$ & Multiply $\$ a$ and $\$ b$ \\
\hline$\$ a * * \$ b$ & Raise $\$ a$ to the power of $\$ b$ \\
\hline $10 * * \$ b$ & Raise 10 to the power of $\$ b$ \\
\hline$\$ a / \$ b$ & Divide $\$ a$ by $\$ b$ \\
\hline$\$ a \% \$ b$ & Remainder of $\$ \mathrm{a} / \$ \mathrm{~b}$ (Modulus) \\
\hline$\$ a=\$ b$ & Assign value of $\$ b$ to $\$ a$ \\
\hline$\$ a+=\$ b$ & Increment $\$ \mathrm{a}$ by $\$ \mathrm{~b}$ \\
\hline$\$ a-=\$ b$ & Decrement $\$ a$ by $\$ b$ \\
\hline$++\$ a(\$ a++)$ & Increment $\$ a$ by 1 before reference (after reference) \\
\hline$--\$ a \quad(\$ a--)$ & Decrement $\$ a$ by 1 before reference (after reference) \\
\hline$\$ a=\$ b$ & Append string $\$ b$ to $\$ a($ see $(\$ a . \$ b)$ below \\
\hline$\$ a . \$ b$ & Values of $\$ a$ and $\$ b$ as one long string \\
\hline$\$ a \times \$ b$ & Value of $\$ a$ strung together $\$ b$ times \\
\hline
\end{tabular}

Functions in perltbl: There are two kinds of functions used in "perltbl": Perl functions and "perltbl" functions. Perl functions have ordinary names without a prefix, such as "log" and "exp"; "perltbl" functions have a " $\&$ " prefix, which is used by Perl to distinguish non-built-in functions, such as $\& \log 10$.

\section{Numeric functions :}

$\begin{array}{ll}\text { Function } & \text { Description } \\ \cos (\$ a), \sin (\$ a) \ldots & \text { Trigonometric functions } \\ \log (\$ \mathrm{x}) & \text { Log base } \mathrm{e} \\ \& \log 10(\$ \mathrm{x}) & \text { Log base 10 } \\ \exp (\$ \mathrm{x}) & \text { Raise "e" to the power of \$x } \\ \operatorname{int}(\$ \mathrm{x}) & \text { Get integer part of \$x } \\ \operatorname{rand}(\$ \mathrm{v}) & \text { Return a random number between 0 and \$v }\end{array}$

String functions: In the functions below "string" can represent a real character string, a variable containing a string, or a computed character string value. String literals can be enclosed in single or double quotes. 
Function

len(string)

substr(string, start, length)

index(string, substring)

\section{Description}

Get the length of a value

Get a substring; if length is omitted, then get to end of string

Get position of substring in string

Date_Time Functions: The Perltbl date function provides considerable flexibility for formatting the \$date variable, which can include both the date and time. This is basically a text variable, where the date is listed first followed by time (if present). Some common examples of date formats supported in the \$date variable would include the following:

Oct 12, 1994

15 November 86

01/01/94

941217

$06 / 17 / 8516: 45$

Function

\&canonical_date(\$date)

\&year(\$date)

$\&$ month $(\$$ date $)$

$\&$ day $(\$ d a t e)$

\&hour(\$date)

\&minute(\$date)

\&water_year(\$date)

\&dow

\&date_parts(\$date)

\&days(\$date)

\&date_time(\$days)
(Jan. 1, 1994)

(Dec. 17, 1994)

(4:15 pm on June 17,1985$)$

Description

Convert a date to canonical form (yyyy/mm/dd hh:mm:ss)

Extract the year from the date

Extract the month from the date

Extract the day from the date

Extract the hour from the date

Extract the minute from the date

Extract the water year from the date

Get the day of the week (1-7) from date

Get array of date components from date $(\$ \mathrm{y}, \$ \mathrm{~m}, \$ \mathrm{~d}, \$ \mathrm{~h}, \$ \mathrm{mn}, \mathbf{s})=$ \&date_parts(\$date)

Get the number of days between 1901/01/01 and \$date

Get the date that is \$days later than 1901/01/01

\section{Data Validation Functions:}

Function

\&isNumber(expr)

\&isDate(expr)
Description

Tests whether expression is a number

Tests whether expression is a date 
Miscellaneous Functions:

Function
\&delete
\&retain
for loops

sprintf("\%m.nx," exp)

\section{Description}

Delete rows based on condition statements

Retain only those rows that meet conditions

"DO" loops for iterations over integer

indexes, e.g.

$$
\text { (for } \$ I=1 ; \$ i=10 ; \$ I=\$ 1+1)
$$

or for iterations over elements of an array, e.g.

(for\$v (@array)

Used to specify the output field format for variables, where $m \& n$ are optional sizes of the output field format whose interpretation depends on the type of field specified by $x$.

$\begin{array}{ll}\text { x Value } & \text { Description } \\ \text { s } & \text { String } \\ \text { c } & \text { Character } \\ \text { d } & \text { Decimal number } \\ \text { ld } & \text { Long decimal number } \\ \text { u } & \text { Unsigned decimal number } \\ \text { lu } & \text { Long unsigned decimal number } \\ \text { x } & \text { Hexadecimal number } \\ \text { lx } & \text { Long hexadecimal number } \\ \text { o } & \text { Octal number } \\ \text { lo } & \text { Long octal number } \\ \text { e } & \text { Exponential format floating-point } \\ & \text { number } \\ \text { f } & \text { Fixed point format floating-point } \\ & \text { number } \\ \text { g } & \text { compact format floating-point } \\ & \text { number }\end{array}$

Syntax: perltbl -e [options 'statement1; statement2; ...; statement'

Options (abbreviations are in parentheses):

-(h)elp

-(b)egin 'code'

-(l)ast 'code'

-(e)xecute 'code'

-begin_file name

-last_file name

-(a)dd variable_name

-(c)hange variable name new_name
Print this 'help' information.

Perl code to execute at start of file.

Perl code to execute at end of file.

Perl code to execute for each row.

Perl variables are used for each column.

- File of Perl code to execute at start of file. May be abbreviated to -bf.

File of Perl code to execute at end of file. May be abbreviated to -lf.

Add a variable to dataset, as in column.

Change a variable name 
-(d)ebug

-(w)arn

-(n)oprint
Print out generated code for row, but do not execute.

Print diagnostic warnings on standard error.

Do not write an output data file. This is similar to the Perl -n option.

Examples:

Code: $\quad$ perltbl -e ' $\$ f 1=\& \log 10(\$ f 1) ; \$ f 2=\& \log 10(\$ f 2)$ '

Explanation: Convert $\mathrm{f} 1$ and $\mathrm{f} 2$ to logs base 10 .

Code: $\quad$ perltbl $-\mathrm{e}$ ' $\$$ total $=\$$ f $1+\$ 2$ '

Explanation: Set 'total' to sum of $\mathrm{f} 1$ and $\mathrm{f} 2$.

Code: $\quad$ perltbl -e '\$s $+=\$$ flow; $\$$ flow $=\#$ '

Explanation: Replace 'flow' with a running sum of flows.

Code: $\quad$ perltbl -e '\$flow $=\$$ flow +100 if \&isNumber( $\$$ flow)'

Explanation: Add 100 to all non-missing flow values.

Code: $\quad$ perltbl -e '\&delete if ! \&isNumber(\$flow)'

Explanation: Delete rows with missing data for 'flow'.

Note: ! is 'Not' operator, that is, if ! \&isNumber means 'if not true'.

Code: $\quad \$ p H=$ sprintf $(" \% 5.2 f ", \$ p H)$

Explanation: Overwrite the existing value of $\$ \mathrm{PH}$ but restrict the length of the field to 5 characters rounded to 2 decimal places.

Code: $\quad \$ \mathrm{Ca}=(\$ \mathrm{Ca}<0) ? 0.0: \$ \mathrm{Ca}$

Explanation: A conditional assignment. Set all negative values of $\$ \mathrm{Ca}$ to 0.0 .

ROW2 GROUP
Description: The Row2Group function splits a row of data into several rows. One or more groups of columns (variables), called "split groups", are defined in the input file. All groups have the same number of variables. This number determines the number of rows to be created in the output file for each row in the input file. For example, consider the example below where the input file contains three rows with two split groups, each containing three variables. There is also one other variable not assigned to any split group. The output file will have nine rows (there are three rows in the input file and the size of the split groups is three). There will be three columns in the output file, one for each split group and one for the other column, which is "carried" over. The data values from a given line and split group in the input file are now in the same column. The values of the variable not assigned to a split group are repeated in each line of the output file corresponding to the originating line in the input file.

The "-split" option is used to specify a list of variables in a split group. The variables in a split group need not be contiguous in the input file. By using the "-pattern" option, an expression (usually in quotes) can be passed as an argument for finding those variables whose names contain or match the expression. Caution should be used with the "pattern" option because split groups must have the same number of variables.

The "index" option is used to request the creation of a column in the output data file containing an integer index whereby each row generated in the output data file from a given row in the input data file has a unique index number and all rows in the output data file with the same index number represent the same column grouping established by the "split" groups. 
Syntax: row2group <input_file >output_file [options]

Options:

-(i)ndex name

-(h)elp

-(s)plit list

-(p)attern varname
Name of index variable (to be created).

Print this "help" information.

Specify list of variables to split.

Specify a list of variables using a variable name "varname."

Example: For the following file called sample.rdb:

$\begin{array}{lllllll}\text { date } & \text { var01p } & \text { var02q } & \text { var03p } & \text { var04q } & \text { var05p } & \text { var06q } \\ 2 \mathrm{n} & 9 \mathrm{n} & 9 \mathrm{n} & 9 \mathrm{n} & 9 \mathrm{n} & 9 \mathrm{n} & 9 \mathrm{n} \\ 1 & 0.8 & 14 & 0.3 & 1.019 & 0.5 & 0.487 \\ 2 & 0.3 & 25 & 0.2 & 1.469 & 0.1 & 1.844 \\ 3 & 0 & 18 & 0 & 0.988 & 0 & 0.376\end{array}$

the command:

row2group <sample.rdb -split var01p var02q var03p I -split var04q var05p var06q

produces:

$\begin{array}{lll}\text { date } & \text { var01p } & \text { var04q } \\ 2 \mathrm{n} & 9 \mathrm{n} & 9 \mathrm{n} \\ 1 & 0.8 & 1.019 \\ 1 & 14 & 0.5 \\ 1 & 0.3 & 0.487 \\ 2 & 0.3 & 1.469 \\ 2 & 25 & 0.1 \\ 2 & 0.2 & 1.844 \\ 3 & 0 & 0.988 \\ 3 & 18 & 0 \\ 3 & 0 & 0.376\end{array}$

Explanation: Two "split" groups are defined in the command line resulting in the creation of two columns in the output file. An additional column is produced for the variable not in a "split" group (date). The size of the split group is three; thus, three rows are created in the output file for each row in the input file. The names of the columns representing the "split" groups are based on the column name of the first variable in each split group; that is, var01p and var04q.

Note the output data set is written to the screen because there is no redirection (">") command to a file.

Example: The following command:

row2group <sample.rdb -index col -pattern 'p\$' -p 'q\$' । $>$ new.rdb 
produces the following data file, new.rdb:

$\begin{array}{llll}\text { date } & \text { col } & \text { var01p } & \text { var04q } \\ 2 \mathrm{n} & 6 \mathrm{n} & 9 \mathrm{n} & 9 \mathrm{n} \\ 1 & 0 & 0.8 & 14 \\ 1 & 1 & 0.3 & 1.019 \\ 1 & 2 & 0.5 & 0.487 \\ 2 & 0 & 0.3 & 25 \\ 2 & 1 & 0.2 & 1.469 \\ 2 & 2 & 0.1 & 1.844 \\ 3 & 0 & 0.0 & 18 \\ 3 & 1 & 0.0 & 0.988 \\ 3 & 2 & 0.0 & 0.376\end{array}$

Explanation: The output is directed to a file, new.rdb, containing four columns, one for each group defined by the "pattern" option, i.e., variable names ending in " $p$ " and " $q$ ", respectively, one for the "carried" variable (date), and one for the "index" column (col). The index column serves as a cross index of rows in the output file to rows in the input file. All values in each sequential group of rows in the output file come from the same row in the input file. All values in each column of the output file having the same index column value come from the same column in the input file.

\section{GROUP2 ROW}

Description: The "group2row" operator combines data from several rows into a single row, which has more columns than the input file. An output row consists of a column for each "carried" variable, a column for each grouping ("by") variable, plus a column for each "collected" variable multiplied by a factor whose value depends on which method of row selection is used.

"Carried" variables are specified in the "-carry" option. They are assumed to have the same value for each composite value of the "collected" variables. If the "carried" values are not the same, then data are lost in the output file. There will be one column created in the output file for each carried variable.

Grouping variables are specified in the "-by" option. The input file should be presorted in grouping variable order. There will be one column created in the output file for each grouping variable.

Certain of the columns (variables) in the input file are designated "collected." Each collected variable will form the basis of several columns in the output file, the number of which will depend on which method of row selection is used.

There are two approaches to collecting rows from the input file. First, the rows are divided into groups each containing the same number of rows. Use the "-rows" option to specify the number of rows in a group. The number of rows in the output file is equal to the number of groups collected. The number of columns created in the output file will be equal to the size of a row group multiplied by the number of variables collected (-collect option).

The second approach to collecting rows is to use the unique values of an index or label variable. A column is created in the output file for each collected variable multiplied by the number of unique values of the index or label variable (-labels option). Index values can also be read from an alternate file using the "if file (col)" option. If the "if" option is used, then the index variable can be specified using either the "-labels" option or the "if" option. Specific values of the index variable can be specified using the "-index list" option. 
The number of rows created in the output file is based on the number of unique composite values of the grouping (by) variable(s) and the number of index values that equate to a given grouping value. There may be multiple occurrences of a given "index" value for a given grouping value.

The output column names for those columns based on collected variables will be a concatenation of the index value to the end of the collected variable name.

\section{Syntax group2row [options] list}

Options (abbreviations are in parentheses):

-(r)ows number

-(1)abels lname

-(b)y list

-(i)ndex list

-(in)put file

-sorted sfile

-if file [col]

-(h)elp

-(ca)rry calist

$-(\mathrm{co})$ llect list
Specify number of rows to collect from input file, which in turn will determine the number of columns to be created in the output file for each collected variable.

Specify a variable whose unique values will be used to create a column for each collected variable. These values will also be concatenated to a collected variable name to form the new column name. These values must be capable of forming valid $R D B$ names.

Specify grouping variables. Data will be collected by groups defined by consecutive rows with identical values of the grouping (by) variables. Each unique value of the "by" variable results in a row in the output file.

Specify select values of the "labels" variable to collect. Each listed unique value of the "index" variable is used to create a new column in the output file for each collected variable from the input file.

Read input from the specified input file, rather than from standard input. If the "-labels" options is used, then column indexes will be derived by finding all unique values in column "Iname" of the input file.

Same as -input option, but data are already sorted, so suppress automatic sort. May be abbreviated -si.

Read index values from file.

Read values from "col" if specified, otherwise read from column specified with the "labels" option.

Print this "help" information.

Carry variables are listed in "calist." Carry variables are assumed to have the same values for each of the collected variables, and thus are not duplicated on output. Use of the -carry option could result in loss of data on output.

Specify list of variables to collect. Each "collect" variable will form the basis of a column for each unique value of the specified label variable. The names of the new columns will be a concatenation of the "collect" column name and the value of the "label" variable. If more than one "collect" column name is listed, then the number of new columns 
created will be the product of the number of unique values in the "label" column and the number of "collect" variables listed. If this option is not used, then all columns not mentioned in the -by or -carry options will be collected.

Example: For the following input data set, sample.rdb:

$\begin{array}{lll}\text { station } & \text { year } & \text { flow } \\ 8 \mathrm{~s} & 4 \mathrm{n} & 10 \mathrm{n} \\ 013985 & 1980 & 100 \\ 013985 & 1981 & 120 \\ 013985 & 1982 & 125 \\ 013985 & 1983 & 200 \\ 013985 & 1984 & 50 \\ 013985 & 1985 & 500 \\ 013981 & 1980 & 10 \\ 013981 & 1981 & 20\end{array}$

the command:

$$
\text { group2row <sample.rdb -r } 2 \text {-co year flow }
$$

produces:

$\begin{array}{lllll}\text { station } & \text { year_1 } & \text { year_2 } & \text { flow_1 } & \text { flow_2 } \\ 8 \mathrm{~s} & 4 \mathrm{n} & 4 \mathrm{n} & 10 \mathrm{n} & 10 \mathrm{n} \\ 013985 & 1980 & 1981 & 100 & 120 \\ 013985 & 1982 & 1983 & 125 & 200 \\ 013985 & 1984 & 1985 & 50 & 500 \\ 013981 & 1980 & 1981 & 10 & 20\end{array}$

Explanation: The RDB command used the -r option telling the system to use two row groups from the input file. The "collected" columns were "year" and "flow"; thus, each of these columns now forms the basis for two columns in the output file. Note the concatenation of the "_1" and "_2" to the column names "year" and "flow."

Example: Using the same input file, the following RDB command:

$$
\begin{aligned}
& \text { Sorttbl <sample.rdb year | group2row -l station -b year । } \\
& \text {-i } 013985013981 \text {-co flow }
\end{aligned}
$$

produces:

$\begin{array}{lll}\text { year } & \text { flow_013985 } & \text { flow_013981 } \\ 4 \mathrm{n} & 10 \mathrm{n} & 10 \mathrm{n} \\ 1980 & 100 & 10 \\ 1981 & 120 & 20 \\ 1982 & 125 & \\ 1983 & 200 & \\ 1984 & 50 & \\ 1985 & 500 & \end{array}$


Explanation: The input file is first sorted by year and the results piped to the "group2row" operator. The -by option specifies that a row in the output file be created for each unique value of "year" in the input file. Three columns were created in the output file, one for the "by" variable ("year"), and one each for each unique value of the label 'station' (-1 option). These new columns are based on the "collected" column "flow" from the input file. Thus, the column names are concatenations of "flow" and the unique values in the "station" column. Note, that missing values are placed in the fields where no "flow" value exists for a given station.

AGGTBL Description: The "aggtbl" operator generates an aggregated version of an RDB file. Aggregation of a data file involves either of two approaches; (1) all records that have a unique set of values within one to four grouping variables (columns) will have a statistic computed for each group of records that share the same value of the composite grouping variable; or (2) a statistic is computed for each group of records that fall into each of the defined ranges of the designated interval variable. The aggregated data file output will contain fewer rows than the input data file.

Aggregated values are computed by one of the aggregation methods-mean, median, sum, minimum, maximum, observation range (maximum minus minimum), observation count, standard deviation, variance, or coefficient of variation.

For example, starting with a data set containing several years of water-quality sample data, create another data set with the mean values of major constituents by year within each county. Or, using the intervals function (-user or -equal) compute the maximum value for each constituent in the year intervals 1970-74, 1975-79, 1980-84, and 1985-89.

\section{Syntax: aggtbl <input_file [options] [Intervals] [Methods] >output_file}

Options (abbreviations are in parentheses):

$$
\begin{array}{ll}
\text { (h)elp } & \text { Print this "help" information } \\
\text {-ac COL1[/DIV1] [..., } & \text { Specifies the name of up to four variables, whose } \\
\text { COL4[/DIV4]] } & \begin{array}{l}
\text { unique composite values control the aggregation } \\
\text { and a divisor for each variable for extracting a } \\
\text { higher level of aggregation. }
\end{array}
\end{array}
$$

Note: Only one variable may be selected when either the -equal or -user option is selected.

If the -unique option is selected, then an optional argument may be specified for each variable (DIV1...DIV4). The primary purpose of this argument is to attain a higher level of aggregation by reducing the value of the variable by powers of ten; that is, truncate the variable from the right side. For example, to aggregate on the basis of the Accounting Unit (first six digits of the Hydrologic Unit Code), use a divisor of 100 to truncate the last two digits. To aggregate on Hydrologic Region (first two digits of the Hydrologic Unit Code), use a divisor of 1000000 .

Example syntax: -ac HUNIT/100 
-am METHOD

-unique

-equal

-auto

-user

-ni NUM

$-\mathrm{xc}$

Interval Options:

-intv MN MX
Specifies the default aggregation method.

METHOD may be one of the following: mean, median, sum, minimum, maximum, range, count, stdev (standard deviation), variance, cv (coefficient of variation). Different methods may be specified for individual variables. Minimum and maximum are the only valid aggregation methods for text variables.

Generate a single aggregated record for each unique set of values of the specified aggregation variables. This is the only option available for multiple aggregation variables or for aggregation on a text variable. Not to be used when either -equal or -user are specified.

Selects equal width aggregation intervals. Requires the -ni option and either one interval specifier (-intv) or the -auto option.

Note: Not applicable when aggregation involves more than one variable, that is, when -unique is specified.

Used in conjunction with -equal to specify computation of interval limits based on range of the aggregation variable.

Selects user specified aggregation intervals. Requires the -ni option and one interval specifier (-intv) for each interval.

Note: Not applicable when aggregation involves more than one variable, that is, when -unique is specified.

Specifies the number of aggregation intervals. Used in conjunction with the -equal and -user options.

Exclude censored data from aggregation computations. The default is to include censored data.

Specify the lower and upper bounds of an aggregation interval. Used in conjunction with the -user option. The number of -intv arguments required is specified by the -ni option.

Methods:

NAME $=$ METHOD

Specify aggregation method on a per variable basis. Use this option for those variables for which a different method is desired than is indicated as the default method for all other variables, that is, as specified in the -am option. NAME is an RDB variable name, Method is the aggregation method (see -am option).

\section{Example: aggtbl < project -ac STATE, COUNTY, YEAR -unique -am mean -xc > project.agg}

Explanation: Group the records in the file "project" by STATE, COUNTY, and YEAR. Compute the mean of the data values within each group within each column. Exclude all censored data. Output results to a new file "project.agg." 
PCTLRDB Description: The "pctlrdb" operator generates a percentile version of an RDB file. The output file will contain only the numeric variables from the input file. The output file should contain exactly 17 rows corresponding to the following percentile values.

$$
\begin{array}{lllllllllllllllll}
0.1 & 0.5 & 1 & 2 & 5 & 10 & 20 & 25 & 50 & 75 & 80 & 90 & 05 & 98 & 99 & 99.5 & 99.9
\end{array}
$$

Syntax: pctlrdb <input_file >output_file

Options (abbreviations are in parentheses):

$$
\begin{array}{ll}
\text {-(h)elp } & \begin{array}{l}
\text { Print help information on this operator } \\
\text {-rc NAME }
\end{array} \\
& \begin{array}{l}
\text { Specifies a column name (up to } 12 \text { characters) to be added } \\
\text { to the output file to contain the percentile ranking for each } \\
\text { row. }
\end{array} \\
\text {-xc } & \begin{array}{l}
\text { Exclude remarked (censored) data from the percentile } \\
\text { computations. }
\end{array} \\
\text {-ic } & \begin{array}{l}
\text { Include remarked (censored) data in the percentile } \\
\text { computations. }
\end{array} \\
\text {-pc } & \begin{array}{l}
\text { Include remarked (censored) data in the percentile } \\
\text { computations, but use Helsel's (1990) statistical technique } \\
\text { to predict what the censored value will be. This technique } \\
\text { utilizes regression and a combination of robust probability } \\
\text { and distributional MLE procedures for computing the mean, } \\
\text { standard deviation, median, and interquartile range (IQR). }
\end{array}
\end{array}
$$

Example: pctlrdb my_input_file.rdb -rc Percentile >my_output_file.rdb

RMKFILT Description: The "rmkfilt" operator is used as a specialized routine within the QWGRAF programs as part of the remark code function. If the user decides to exclude remark codes for consideration in the analysis, then a decision is made to either remove all rows in the file that contain at least one remarked value or replace the value in each remarked field with a "missing value" indicator; that is, a NULL value in an RDB file. In the latter case the number of rows in the data file does not change.

\section{Syntax: rmkfilt <input_file [-m] output_file}

Options (abbreviations are in parentheses):

$-\mathrm{m} \quad$ Set data value to missing (NULL) for all remarked (censored) observations. If this option is omitted from the command line, then any row containing at least one remarked value will be removed from the output file.

$$
\text { -(h)elp Print help information on this operator. }
$$

When the option to exclude remarked data from the file is selected, the columns established in the RDB file to hold the remark codes are removed from the output file. 
Description: The "rdbqry" operator executes an RDB query (script) file created in the QWGRAF "datmgr" program. An RDB query file contains a query script imbedded in the comment area of an RDB file. Although this is by definition and formatting an RDB file, it contains no data rows, just the comment lines containing the script, the column name line, and the column definition line. The query script is distinguished from other comments by the beginning and ending script lines - "\#begin script" and "\#end script." Non-script comment lines may appear before and after the section containing the script lines. The script may consist of any number of token definition lines and must contain exactly one base pipe definition line.

A user-defined token consists of any alphanumeric string within curly brackets. A token definition line consists of a token followed by an equal sign followed by RDB commands, which may include references to other token definition lines. The pipe definition line performs the last operations leading to the creation of the output file; that is, the data file actually used by the program using the script as an input put file. The token definition statements and base pipe definition may appear in any order. Tokens may be referenced before they are defined, but each referenced token must be defined. Circular references will result in an error.

The syntax that follows is for command line use and the "execute an RDB query file" option in the "datmgr" program. Its use in this form would result in the creation of an RDB data file. The use of a query file as an input file to a QWGRAF program, however, does not result in the creation of a permanent RDB data file. A temporary data file that disappears when the program ends is created.

\section{Syntax: rdbqry [options] <rdbquery >output_file.rdb}

Options (abbreviations are in parentheses):

$$
\text { -(h)elp Print this "help" information }
$$

Example: The following is an example of a script that can be imbedded in the comment lines of an RDB file either with a text editor or by using the QWGRAF "datmgr" program.

$$
\begin{aligned}
& \text { \# rdb } \\
& \text { \# } \\
& \text { begin script } \\
& \#\{\mathrm{~A}\}=\text { sorttbl }<\mathrm{t} 1 \text {.rdb name } \\
& \#\{\text { B }\}=\text { sorttbl }<\text { t2.rdb name } \\
& \begin{array}{l}
\#\{C\}=\{B\} \mid \text { perltbl }-e \text { '\&delete if \$name eq Mike' \# filter } \\
\#\{A\} \mid \text { jointbl name ' }\{\mathrm{C}\} \text { l' }
\end{array} \\
& \text { \# end script } \\
& \text { \# } \\
& \text { \# The following two lines are the column names (first line), and data width } \\
& \# \text { and type groups (second line) of the output file from the above script. } \\
& \text { name rank serial } \\
& \begin{array}{lll}
16 & 8 & 12 \mathrm{~N}
\end{array}
\end{aligned}
$$

Explanation: The above script, which would be found in the file identified in the argument < rdbquery (see syntax), sorts two RDB files (t1.rdb and t2.rdb), filters (deletes) from the second file rows where column name equals "Mike," and then joins the two files outputting the result to a new file identified in the argument $>$ output_file.rdb. Note the syntax of the $\{C\}$ token in the pipe definition line. The jointbl operator is expecting a file name here. In this case we are piping the result of the $\{C\}$ token operation as the input file to jointbl. Thus, the entire expression ' $\{C\}$ l' must be enclosed in quotes in order to be passed as a single argument to jointbl.

Following the comment area (no more \# signs) the RDB query file should contain a standard RDB header defining the column names, data widths, and types. While this header is neither required nor used by "rdbqry," it can be used by application programs to determine the contents of the query without incurring the overhead of executing the script. 
QWGRAF programs have the option of creating three output file formats.

\author{
Postscript File \\ HPGL File (can be used with in-line plotters) \\ CGM File
}

Postscript files can be imported into FrameMaker; however, the graphic images cannot be printed from FrameMaker. HPGL and CGM files also can be imported into FrameMaker, however these images can be modified and scaled with the FrameMaker graphic tools and then printed. The procedures for importing HPGL and CGM files into FrameMaker are as follow:

\title{
I. QWGRAF HPGL to FrameMaker
}

\section{A. Create HPGL file}

1. Complete and verify plot specifications.

2. Make the plot on the screen to confirm plot specifications.

3. Select "Device" option in "Modify" screen.

4. Select "Plotter (HPGL)" option. Use Accept (f2) or the Enter key to accept selection.

5. Enter a file name with the ".hpgl" file-name extension.

6. Use Accept (F2) to return to main screen.

7. Make the plot again.

B. Import HPGL file into FrameMaker

1. In the FrameMaker document place the cursor at the desired insertion point.

2. From the "File" menu click on "Import."

3. With the left mouse button down drag the cursor to "File" dialog box and release button.

4. From the import file dialog box single click on the file to be imported (must have ".hpgl" extension).

5. Click on "Copy into document."

6. Click on "Import."

\section{Modify frames and objects}

At this point you may want to use the imported graphic as is or you may want to prepare it for subsequent modification or scaling by using the FrameMaker graphic tools. The preparation procedure involves removing extraneous text frames and rectangles; making modifications to individual objects; grouping the objects; scaling the grouped object; and then positioning the object. The steps to import and modify a HPGL files into FrameMaker are as follow:

1. The imported graphic has two text frames. Remove the outer frame as follow:

2. Click left mouse button on edge of inner text frame to select it.

3. From "Edit" menu click on "Cut" to temporarily remove the inner frame and place it on the clipboard.

4. Select the remaining text frame and delete it with the backspace key (do not cut it).

5. Paste the cut text frame from step 3 above into same location.

6. With the text frame selected, drag the side and top handle bars to expand the sides of the text frame away from any objects within the frame.

7. When sufficient space has been provided, select all of the graphics objects within the frame (use a Marques selection). Then from the Graphics menu click on "Group."

8. With the grouped object selected, use the "Scale" option in the "Tools" dialog box to resize the grouped object within the text frame. From the "Scale" dialog box select a scaling factor (in percent). Click on "Scale" to set the new scaling. Scaling can also be done by dragging the handle bars of the grouped object. 
9. As required, reposition the rescaled grouped object within the text frame by clicking the left mouse button while holding down the "Ctrl" key and then dragging the object to the desired location within the text frame.

10. If required, resize the text frame by first selecting it and then dragging the handle bars to effect the appropriate size. If desired, fill in the text frame border with an appropriate line type, thickness and color by using the "Pen" menu box, color menu box, and "Pts" menu box in the "Tools" dialog box.

11. Color graphics can be printed on a color laser printer. For a gray scale effect, print the document on the regular laser printer.

12. When the grouped graphic object has been "ungrouped"; individual objects can be selected and modified by using the graphics tool box. Depending on the graphics program used to generate the graphic, objects that were expected to be filled may appear to be unfilled. Click on the opaque (black) square in the fill menu box. If the object does not fill or does not fill with the right color, then click on the color box and then click on the appropriate fill color when the box that lists the available colors comes up.

\section{QWGRAF CGM to FrameMaker}

\section{A. Create a CGM File}

1. Complete and verify plot specifications.

2. Make the plot on the screen to confirm plot specifications.

3. Select "Device" option in "Modify" screen.

4. Select "CGM" option. Press "Enter" key to accept selection.

5. Enter a file name into the space indicated and add the ".cgm" file-name extension.

6. Return to main screen and make the plot again.

B. Import CGM file into FrameMaker

1. In your FrameMaker document place the cursor at the desired insertion point.

2. From the "File" menu click on "Import."

3. With the left mouse button down drag cursor to the "File" dialog box and release the button.

4. From the "Import file" dialog box single click the left mouse button on the file to be imported (must have ".cgm" extension).

5. Click on "Copy into document."

6. Click on "Import."

\section{Graphic is missing}

The first thing you will notice with an imported CGM file is that the intended graphic does not appear to have been imported into the document. The graphic is indeed there, but you have to go through some additional steps to bring it forward.

\section{Modify frames and objects}

1. The imported graphic appears to be an empty text frame. Note that the text frame is selected with the handle bars displayed.

2. Just inside the text frame click the left mouse button. The text frame is deselected and another set of handle bars appears, which indicates the presence of an invisible text rectangle (not a text frame) inside of which is also the invisible graphic from QWGRAF.

3. Use the backspace key to delete (do not cut) the text rectangle.

4. Click on the text frame to select it.

5. From the "Edit" menu click on "Select all in frame." The handle bars for all the individual objects of the graphic from QWGRAF now appear, but not the graphic objects themselves.

6. From the "Graphics" menu click on "Tools" or click on the triangle in the upper portion of the right scroll bar to bring up the "Tools" menu.

7. Click on "None" in the "Fill" box. Nothing will happen at this point. 
8. Click on the desired pattern (usually opaque(black)) in the "Pen" box. Nothing happens.

9. Click on the "Color" box and then click on the appropriate color in the "Color" box (usually black). The QWGRAF graphic now appears as several individual objects and they are still selected.

10. If the graphic is satisfactory, then click on the "Group" option in the "Tools" menu.

11. Usually a CGM file is not brought into FrameMaker as cleanly as a HPGL file. It may be necessary to modify and move individual objects. With all of the objects selected, click on an empty area to deselect the objects. Click on individual objects to select them and make the necessary modifications.

12. When finished click on "Select all in frame" from the "Edit" menu and then click on "Group" in the "Graphics Tools" menu.

13. With the grouped object selected, use the "Scale" option in the Graphics Tools dialog box to resize the grouped object within the text frame. From the "Scale" dialog box select a scaling factor (in percent). Click on "Scale" to set the new scaling.

14. As required, reposition the rescaled grouped object within the text frame by clicking the left mouse button while holding down the "Ctrl" key and then dragging the object to the desired location within the text frame.

15. If required, resize the text frame by first selecting it and then dragging the handle bars to effect the appropriate size.

16. Color graphics can be printed on a color laser printer. For a gray scale effect, print the document on the regular laser printer. 


\title{
APPENDIX H. Concentration Conversion Factors
}

\section{Concentration to Normal/Molar Form}

\author{
Grams to Equivalents/Moles
}

Milligrams to Milliequivalents/Millimoles

Micrograms to Microequivalents/Micromoles

\begin{tabular}{|c|c|c|c|c|c|}
\hline Constituent & Normal & Molar & Constituent & Normal & Molar \\
\hline Alkalinity (as $\mathrm{CaCO} 3$ ) & 0.01998 & 0.00999 & Magnesium $\left(\mathrm{Mg}^{+2}\right)$ & 0.08229 & 0.04114 \\
\hline Aluminum $\left(\mathrm{Al}^{+3}\right)$ & 0.11119 & 0.03706 & Manganese $\left(\mathrm{Mn}^{+2}\right)$ & 0.0364 & 0.01821 \\
\hline Ammonium $\left(\mathrm{NH}_{4}^{+1}\right)$ & 0.05544 & 0.05544 & Manganese $\left(\mathrm{Mn}^{+3}\right)$ & 0.05461 & 0.01821 \\
\hline Antimony (Sb) & & 0.00821 & Mercury $\left(\mathrm{Hg}^{+2}\right)$ & 0.00997 & 0.00499 \\
\hline Arsenic $\left(\mathrm{As}^{+5}\right)$ & 0.06674 & 0.01335 & Molybdenum $\left(\mathrm{Mo}^{+6}\right)$ & 0.06254 & 0.01042 \\
\hline Barium $\left(\mathrm{Ba}^{+2}\right)$ & 0.01456 & 0.00728 & $\operatorname{Nickel}\left(\mathrm{Ni}^{+2}\right)$ & 0.03408 & 0.01704 \\
\hline Beryllium $\left(\mathrm{Be}^{+2}\right)$ & 0.22192 & 0.11096 & Nitrate $\left(\mathrm{NO}_{3}^{-1}\right)$ & 0.01613 & 0.01613 \\
\hline Bicarbonate $\left(\mathrm{HCO}_{3}{ }^{-1}\right)$ & 0.01639 & 0.01639 & Nitrite $\left(\mathrm{NO}_{2}^{-1}\right)$ & 0.02174 & 0.02174 \\
\hline Boron $\left(\mathrm{B}^{+3}\right)$ & 0.27750 & 0.0925 & Phosphate $\left(\mathrm{PO}_{4}^{-3}\right)$ & 0.03159 & 0.01053 \\
\hline Bromide $\left(\mathrm{Br}^{-1}\right)$ & 0.01252 & 0.01252 & Phosphate $\left(\mathrm{HPO}_{4}^{-2}\right)$ & 0.02084 & 0.01042 \\
\hline Cadmium $\left(\mathrm{Cd}^{+2}\right)$ & 0.01779 & 0.0089 & Phosphate $\left(\mathrm{H}_{2} \mathrm{PO}_{4}^{-1}\right)$ & 0.01031 & 0.01031 \\
\hline Calcium $\left(\mathrm{Ca}^{+2}\right)$ & 0.0499 & 0.02495 & Phosphorus $\left(\mathrm{P}^{+5}\right)$ & 0.16143 & 0.03229 \\
\hline Carbonate $\left(\mathrm{CO}_{3}^{-2}\right)$ & 0.03333 & 0.01666 & Potassium $\left(\mathrm{K}^{+1}\right)$ & 0.02558 & 0.02558 \\
\hline $\operatorname{Cesium}\left(\mathrm{Cs}^{+1}\right)$ & 0.00752 & 0.00752 & Rubidium $\left(\mathrm{Rb}^{+1}\right)$ & 0.0117 & 0.0117 \\
\hline Chloride $\left(\mathrm{Cl}^{-1}\right)$ & 0.02821 & 0.02821 & Selenium $\left(\mathrm{Se}^{+6}\right)$ & 0.07599 & 0.01267 \\
\hline Chromium $(\mathrm{Cr})$ & 0.1154 & 0.01923 & Silica $\left(\mathrm{SiO}_{2}\right)$ & & 0.01664 \\
\hline Dichromate $\left(\mathrm{Cr}_{2} \mathrm{O}_{7}^{-2}\right)$ & 0.00926 & 0.00463 & Silver $\left(\mathrm{Ag}^{+1}\right)$ & 0.00927 & 0.00927 \\
\hline Cobalt $\left(\mathrm{Co}^{+2}\right)$ & 0.03394 & 0.01697 & Sodium $\left(\mathrm{Na}^{+1}\right)$ & 0.0435 & 0.0435 \\
\hline Copper $\left(\mathrm{Cu}^{+2}\right)$ & 0.03147 & 0.01574 & Strontium $\left(\mathrm{Sr}^{+2}\right)$ & 0.02283 & 0.01141 \\
\hline Fluoride $\left(\mathrm{F}^{-1}\right)$ & 0.05264 & 0.05264 & Sulfate $\left(\mathrm{SO}_{4}^{-2}\right)$ & 0.02082 & 0.01041 \\
\hline Hydrogen $\left(\mathrm{H}^{+1}\right)$ & 0.99212 & 0.99212 & Sulfide $\left(\mathrm{S}^{-2}\right)$ & 0.06238 & 0.03119 \\
\hline Hydroxide $\left(\mathrm{OH}^{-1}\right)$ & 0.0588 & 0.0588 & Thorium $\left(\mathrm{Th}^{+4}\right)$ & 0.01724 & 0.00431 \\
\hline Iodide $\left(\mathrm{I}^{-1}\right)$ & 0.00788 & 0.00788 & Titanium $\left(\mathrm{Ti}^{+3}\right)$ & 0.06267 & 0.02089 \\
\hline Iron $\left(\mathrm{Fe}^{+2}\right)$ & 0.03581 & 0.01791 & Uranium $\left(\mathrm{U}^{+6}\right)$ & 0.02521 & 0.0042 \\
\hline Iron $\left(\mathrm{Fe}^{+3}\right)$ & 0.05372 & 0.01791 & Vanadium (V) & & 0.01963 \\
\hline Lead $\left(\mathrm{Pb}^{+2}\right)$ & 0.00965 & 0.00483 & $\operatorname{Zinc}\left(\mathrm{Zn}^{+2}\right)$ & 0.03059 & 0.0153 \\
\hline Lithium $\left(\mathrm{Li}^{+1}\right)$ & 0.14407 & 0.14407 & & & \\
\hline
\end{tabular}




\section{APPENDIX I. Conversion Factors}

\begin{tabular}{|c|c|c|}
\hline To convert from & To & Multiply by \\
\hline Milligrams per liter & Tons per acre-feet & 0.00136 \\
\hline Milligrams per liter & Tons per day & second-feet $x 0.002697$ \\
\hline Quarts (U.S.) & Liters & 0.9463 \\
\hline${ }^{\mathrm{a}}$ Second-foot days & Acre-feet & 1.983471 \\
\hline Second-foot days & Gallons per day & 646,317 \\
\hline${ }^{\mathrm{b}}$ Second-feet & Gallons per minute & 448.8 \\
\hline Gallons per minute & Liters per second & 0.06309 \\
\hline${ }^{c}$ Acre-feet & Gallons & 325,851 \\
\hline Acre-feet & Cubic feet & 43,560 \\
\hline Acre-feet & Cubic meters & $1,233.5$ \\
\hline Cubic feet & Cubic meters & 0.028317 \\
\hline Cubic feet & Gallons & 7.481 \\
\hline $\mathrm{Ca}^{+2}$ & $\mathrm{CaCO}_{3}$ & 2.4973 \\
\hline $\mathrm{Mg}^{+2}$ & $\mathrm{CaCO}_{3}$ & 4.118 \\
\hline $\mathrm{HCO}_{3}{ }^{-1}$ & $\mathrm{CaCO}_{3}$ & 0.8202 \\
\hline $\mathrm{CO}_{3}^{-2}$ & $\mathrm{CaCO}_{3}$ & 1.6679 \\
\hline${ }^{d} \mathrm{HCO}_{3}{ }^{-1}$ & $\mathrm{CO}_{3}^{-2^{3}}$ & 0.4917 \\
\hline $\mathrm{NO}_{3}{ }^{-1}$ & $\mathrm{~N}$ & 0.2259 \\
\hline $\mathrm{N}$ & $\mathrm{NO}_{3}{ }^{-1}$ & 4.4268 \\
\hline $\mathrm{NO}_{2}^{-1}$ & $\mathrm{~N}$ & 0.3045 \\
\hline $\mathrm{N}$ & $\mathrm{NO}_{2}{ }^{-1}$ & 3.2845 \\
\hline $\mathrm{NH}_{4}{ }^{+1}$ & $\mathrm{~N}$ & 0.7765 \\
\hline $\mathrm{N}^{4}$ & $\mathrm{NH}_{4}^{+1}$ & 1.2878 \\
\hline $\mathrm{PO}_{4}^{-3}$ & $P^{4}$ & 0.3261 \\
\hline $\mathrm{P}^{4}$ & $\mathrm{PO}_{4}^{-3}$ & 3.0662 \\
\hline
\end{tabular}

${ }^{\mathrm{a}} 1 \mathrm{sec}$-foot/day $=1$ cubic foot per second for 24 hours

${ }^{b} 1$ sec-foot $=1$ cubic foot per second

c 1 acre-foot $=$ an area of 1 acre 1 foot deep

d In the reaction $2 \mathrm{HCO}_{3}^{-1}+\mathrm{H}_{2} \mathrm{O}=\mathrm{CO}_{3}^{-2}+\mathrm{H}_{2} \mathrm{O}+\mathrm{CO}_{2}(\mathrm{~g})$ for computing total dissolved solids 